Jens N. Faaborg

Animaux domestiques
dans la littérature
narrative française
au Moyen Âge

Museum Tusculanum Press

Université de Copenhague

2006

[e-Book] 
(C) Museum Tusculanum Press et l'auteur, 2006

Conseiller auprès de l'éditeur: Hans Peter Lund

Mise en pages par Pernille Sys Hansen

Police: Lino Letter

ISBN 8763504642

Publié avec le soutien financier de

The Danish Research Council for the Humanities [Le conseil de recherche des lettres et sciences humaines du Danemark]

Museum Tusculanum Press

Université de Copenhague

Njalsgade 94

DK-230o Copenhague S

www.mtp.dk 


\section{Table de matières}

Introduction 4

1. Les animaux I 13

1.1. Les chevaux - une categorie à part 15

2. Les animaux II 147

2.1. Autres mammifères 147

2.2. Les oiseaux 200

3. La vie des animaux 226

3.1. Appréciation des animaux 226

3.2. Traitement des animaux 240

3.3. Nourriture des animaux 258

3.4. Etables, bergeries etc. $\quad 271$

3.5. « Langues» 274

4. Utilisation des animaux 277

4.1. Utilisation des animaux vivants $\quad 278$

4.2. Utilisation des produits d'animaux vivants $\quad 312$

4.3. Utilisation des produits d'animaux morts $\quad 318$

5. Animaux dans les idées, la langue et l'imagination

5.1. Comparaisons 334

5.2. Métaphores 354

5.3. Proverbes, dictons, locutions $\quad 357$

5.4. Allusions à fables et légendes $\quad 364$

5.5. Jeux d'enfants $\quad 367$

5.6. Euvres d'art $\quad 368$

6. Conclusion $\quad 371$

7. Tables 374

7.1. Table I 374

7.2. Table II 377

7.3. Table III $\quad 386$

7.4. Table Iv 392

8. Index 396

8.1. Chevaux 396

8.2. Autres mammifères 404

8.3 Oiseaux 411

9. Bibliographie $\quad 416$

9.1. Textes étudiés $\quad 416$

9.2. Ouvrages consultés $\quad 428$ 


\section{Introduction}

Si quelques-uns des auteurs de textes narratifs en ancien et moyen français n'hésitent pas à mentionner des représentants de la faune sauvage dont ils savent en général seulement qu'ils sont féroces et hostiles aux hommes, tels que lions et tigres (1), un plus grand nombre d'entre eux s'étendent plutôt sur d'autres animaux sauvages qu'ils connaissent mieux parce qu'il s'agit d'animaux du pays, tels que loups et renards, lièvres et cerfs, ou même vivant près des hommes, comme rats et souris. Ce sont là des animaux avec lesquels les hommes sont obligés de vivre, qu'ils craignent et détestent et dont ils essaient de se débarrasser, ou dont ils se servent pour leur nourriture ou leurs vêtements. Le renard vole les poules du paysan, le loup lui tue un mouton ou un veau ; les souris font des dégâts à la campagne aussi bien qu'en ville où elles mangent le blé et rongent les boiseries. Lièvres et cerfs fournissent de la viande, surtout pour les repas des nobles, les insectes bourdonnent, les petits oiseaux chantent :

(...) cil qui aiment en tel maniere püent estre resemblable a parpillon qui tant se trait envers la clarté de la chandeille que il art. Troie, 198, 23-5.

Et les petis oisiaus savaiges

Fait dous chanter per se boucaiges ; Florimont, 8621-2. Cf. ib., 4231-2.

Ce fu a un matin, que l'aube iert esclarcie, que li oiselez chantent et la rose est florie ; Rou, II, 3183-4. Cf. Narbonnais, 5 .

Les hommes doivent se protéger contre beaucoup de ces animaux, ils les chassent et les tuent et profitent ainsi de leur viande, de leurs peaux, ou bien ils constatent simplement leur existence sans y réfléchir.

Mais ce sont les animaux domestiques qui l'emportent - et de loin - dans nos textes. La distinction entre animaux sauvages et animaux domestiques s'avère d'ailleurs difficile pour certaines catégories.

Les lapins sont régulièrement mentionnés dans les descriptions des repas, mais dans quelques-unes des occurrences du terme, nous hésitons à voir des animaux élevés en garenne, c'està-dire domestiqués : 
Perchevaus molt bien servis fu :

Viandes ont sainnes et netes,

Ploviers et partris et anetes,

Qui ont eü de la rimee,

Et conins norris en ramee

Tenres qui ont cras le regnon, Cont. P., I, 4974-9.

En ce qui concerne les oiseaux, il est quelquefois difficile aussi de vérifier s'il s'agit d'animaux sauvages ou d'animaux domestiqués. C'est le cas des cygnes, des faisans, des perdrix et des paons, dont on utilise la viande et les plumes. Ils apparaissent régulièrement avec des oiseaux sauvages de sorte que l'on serait tenté de les compter parmi le produit de la chasse, p. ex. :

«Aporte li a mengier a planté

Et pain et vin et piment et claré,

Grues et jantes et poons en pevré. » Orange, 172-4.

Tel vie pas apris n'avoie,

Quant je chiéz mon pere mennoie,

Mes viandes chieres et fines,

Chapons en rost, oisons, gelines,

Cynnes, paons, perdris, fesanz,

Herons, butors qui sont plesans,

Et venoisons de maintes guisez

A chiens courans par force prises : Anjou, 1105-12.

Nous pensons néanmoins que les paons se trouvaient dans la basse-cour avec poules et canards. Ce sont des oiseaux qui, originaires de l'Inde, se sont répandus à l'état domestique dans toute l'Europe. En plus, ils apparaissent si souvent aux repas décrits dans nos textes qu'il n'aurait guère été possible d'en trouver assez s'il avait fallu les chasser dans la nature.

Quant aux faisans et aux perdrix, il est presque certain qu'ils sont sauvages : ils sont désignés comme la proie des oiseaux prédateurs ou mentionnés en compagnie d'autres oiseaux sauvages et rien n'indique qu'ils aient été élevés dans des faisaneries ou ailleurs. Nous ne les compterons donc pas parmi les animaux domestiques :

Quant Moranz vit dant Guilliaume venir

Plus le desire que faucons la perdris $\mathrm{Mez}, 1036-7$.

Ploviers et faisans et pastez

Fait li sires sanz plus atendre

Aporter et la nape estendre. Cont. P., I, 6170-2. 
Les cygnes se trouvent mentionnés tantôt avec des animaux sauvages, tantôt avec des animaux domestiques, surtout des paons. Dans le lai Milun, le protagoniste possède un cygne extraordinaire qui semble bien apprivoisé et qu'il appelle « mon » cygne :

Si a maint cygne et maint faisant,

Et foison de pain beluté, Galeran, 6788-9. Cf. Anjou, 1105-12.

Li uns porte char d'ors et li autres lardé

Ou bon cisne rosti ou poon enpevré. Barbastre, 6452-3.

Char de poons et cisnes, et vin viez et claré. Renaut, 10643.

Un cisne aveit k'il mut ama,

Le brief li ad al col lié

E dedenz la plume muscié.

Un suen esquïer apela,

Sun message li encharga.

«Va tost, » fet il, « change tes dras!

$\mathrm{Al}$ chastel m'amie en irras,

Mun cisne porteras od tei ;

Garde quë en prengez cunrei, Lais, Ix, 164-72.

Parfois, ils sont même présentés comme la proie d'oiseaux prédateurs (2), ainsi dans les vers suivants de $\mathrm{Mez}$ et de Roche :

A mon faucon avoie .I. cine pris, $M e z, 4426$.

A ce[l] faucon qu'i[l] porte a abatu .j. cine,

Li faucons s'i encharne, par vertu s'i afiche ;

Li filz au duc descent, qui son oisel delivre ;

Li ber revot monter quant Hardre[z] li escrie :

«Si m'aït Diex, vassal, c'est molt grans lecherie

"Quant ou vivier mon pere venez prenre les cines : Roche,

3741-6. Cf. ib., 3755-7.

Le deuxième extrait montre que ces grands oiseaux vivaient, au moins en partie, dans des viviers privés. Le terme vivier a normalement le même sens que dans la langue moderne (É. Littré, IV, p. 2514: «Pièce d'eau courante ou dormante dans laquelle on nourrit du poisson »), ou il désigne simplement un étang :

es vivers prendre les peissuns

e es forez les veneisuns ; Rou, III, 891-2.

Et si sont les gaaigneries,

Li vivier et les praeries, Cont. P., I, 6203-4. 
Mais il peut également désigner une volière ou un enclos pour les oiseaux :

Par desous la fenestre en.$j$. vivier s'asi[s]t

Et fist tous les oiseax remüer et fremir. Roche, 4205-6.

Si tenoit sur son poing un faucon joli,

Si vit en un vivier .j. malart acroupi : Brun, 2758-9.

Il nous paraît donc permis de conclure que les cygnes (même celui de Milun) vivaient dans la nature aussi bien que dans les étangs et les lacs près des habitations humaines - comme de nos jours - mais rien ne prouve que même dans ces derniers cas ils étaient domestiqués. Ceci fait que nous ne nous croyons pas autorisé à les compter parmi les animaux domestiques.

Dans le domaine des oiseaux prédateurs, le problème se présente un peu différemment. Nos textes présentent un certain nombre d'exemples équivoques où l'on ne peut pas dire avec exactitude s'il s'agit d'animaux sauvages ou d'animaux domestiqués, comme :

Et se ainssi fu ce n'est mie merveille, car tout aussi comme la loe doute l'esprevier tout ainsi douterent cil de hors l'empereour et Daphus qui son compains estoit. Helcanus, 139.

Kanqu'il pooient l'un sor l'autre destendre

Ne s'espargnoient nes k'esprevier caille ; Enfances O., 5367-8.

Mais, dans la plupart des occurrences, il est tout à fait clair qu'il s'agit d'oiseaux apprivoisés qui appartiennent à quelqu'un, que l'on utilise pour la chasse, dont on peut faire cadeau à quelqu'un etc. Dans notre exposé, nous ne tiendrons compte, évidemment, que des exemples présentant ces oiseaux apprivoisés :

Et prist esprevier mué

Que il meïsmes ot mué,

Et maine .II. chienès petiz, $M R$, LVII, 37-9.

Au departir li a doné

.I. esprivier .v. fois müé,

Si li dona .I. blanc levrier,

.I. confanon et .I. destrier. Rigomer, 6543-6.

Le terme oiseau s'emploie naturellement très souvent pour parler des oiseaux sauvages (voir p. 4). Mais assez régulièrement nous le voyons utilisé pour désigner des oiseaux domestiqués, le plus souvent des faucons ou d'autres oiseaux de chasse - parfois 
mentionnés avec des chiens. Les extraits de Bueve montrent clairement son rôle de dénominateur commun : il est question des mêmes oiseaux dans les deux cas :

Et vint ostoirs et vint faucons müés Bueve, I, 4378.

Les chevaus ont maintenant establés

Et les oiseus sor les perces posés ib., 4410-1.

Ou mon oisel sur mon poign pestre : Galeran, 3885 .

Il ont laz et oisiauz et grant mute de chiens ; Roche, 3195. Cf.

ib., 3741-3; 3753-4;3813.

Ensemble aloient bien sovent

A desduit de chiens et d'oisealz. Durmart, 70-1

Telz gens ne sont pas fauconniers,

Ainçois sont bien d'oiseaux murtriers. Deduis, 477-8. Cf. passim.

S'il ert hom qui amast delit,

Ne chiens ne oisiaus ne deduit,

En tote France n'a, je cuit,

Home qui tant en ait apris Escoufle, 6526-30.

Quant vint a l'endemain, chascun s'abilla, parlant et devisant de ce que plus luy estoit, Gerard de chiens et d'oiseaulx, (...)

Cent, 26, 374-6.

Moult ert sires de venoisons ;

$\mathrm{S}^{\prime}$ avoit ses chiens et ses oisiaus ; $M R, \mathrm{xx}, 20-1$.

Asés avrés chiens et oisiaus,

Muls et chevaus riches et biax Partonopeu, 1455-6.

« Les chiens et les oiseus ne peut il oublier : Aiol, 7125.

Gliglois repaisoit un oissiel. Gliglois, 628. Cf. ib., 608-16 ; 639 ; 1109 et passim.

Puis apiela un damoisiel,

Qui sor son puing tint un oisiel ;

Müés estoit de tierche mue. Violette, 160-2.

mais aussi d'autres oiseaux : 
Une crasse oul avoit en mue ;

C'est .I. oisel de prime vere.

Or orrez ja con le provoire

Fu bien servi de sa crasse oie ; MR, cxLIII, 8-11.

En bien .vii. kages ou en .viii.

Pendent li oisel as fenestres. Escoufle, 5520-1.

Le terme beste se trouve assez fréquemment pour désigner les animaux, sauvages aussi bien que domestiques. Parlant des chansons de geste, Friedrich Bangert, Die Tiere im altfranzösischen Epos, p. 5, a observé qu'ordinairement beste ne désigne que les quadrupèdes, observation que nous voyons corroborée par les extraits suivants :

Escrit i sont et bestes et oisel Narbonnais, 3831.

Deus ne fist beste ne oisel

Ne en cest siecle point de bel

Ne en cest mont point de deduit,

Dont vos n'i trovissies estruit. Cristal, 8663-6.

Nous ne tiendrons pas compte, bien sûr, des exemples où il est question indubitablement d'animaux sauvages.

Dans le premier exemple suivant il n'est pas tout de suite clair de quels animaux parle le texte - c'est la suite qui nous révèle qu'il s'agit d'un loup et d'une brebis ; dans le fabliau cL (Le dit du soucretain), l'homme regrette d'avoir perdu entre autres choses ses bestes, peut-être ses vaches :

"Palamidés, fait il, se Dex vous saut, veïstes vous onques en un lieu metre ensamble deus bestes qui naturaulement s'entrehaïssent, ki longement se tenissent em pais ? - »(...) Mout a ore plus grant discorde entre le leu et le berbis k'il n'a entre Palamidés et Tristran, (...) Et si sai je tout chertainnement que je vi ja caoir en une fosse assés parfonde le leu et le berbis, la meïsmes u li leu l'emportoit. Et quant li leus vit et reconnut $\mathrm{k}^{\prime} \mathrm{il}$ estoit caüs en la fosse dont il ne pooit issir et il se vit emprisonné, il devint si mauvais et si couars durement k'il ne fourfist rien a la berbis, la u il estoit en la fosse avoeuc lui seul a seul. Et se la berbis eüst point de hardement en soi, je croi bien tout chertainnement k'il n'i eüst ja desfensse qu'ele ne le peüst ochirre, si avoit li leus du tout perdu le cuer et le hardement. TP, III, 151, 5-8, 19-20 et 22-31.

Ensamble avons eü maint bien

Et mainte joie : or n'avons rien. 
Dras ne chevaus ne nulles bestes,

Fors la meson desor nos testes : $M R, \mathrm{CL}, 81-4$.

C'est d'ailleurs toujours le contexte qui nous donne au moins une idée sur la nature des bestes mentionnées. Dans Lancelot, I, XxI, 5 , nous lisons que le valet a vu des vaches et des brebis, et Aucassin présente les mêmes animaux. L'extrait de Merlin présente un vacher et ses bêtes, sans aucun doute des vaches et des veaux. Dans les deux exemples de TP ainsi que dans le premier exemple de Claris, il s'agit probablement de moutons étant donné la présence du berger, tandis que le second exemple du même texte ainsi que celui d'Eneas nous présentent un cheval. C'est aussi le cas du premier exemple tiré de $M R$, (Du prestre qu'on porte ou De la longue nuit), car le vilain parle de sa jument. Le fabliau cxLV ( $D u$ prestre et $d u$ leu) ne nous renseigne pas sur l'espèce des animaux - nous savons seulement qu'ils appartiennent à un vilain. Dans le fabliau cxi (De la damoisele qui n'ot parler de foutre qui n'eust mal au cuer), les bestes de l'autre vilain sont probablement des bœufs préparés pour le labourage. Le dernier exemple ci-après, tiré du même fabliau, est particulier : le terme beste ne désigne pas un animal mais le sexe masculin (au vers 173 du fabliau, le sexe est appelé polain et, au vers 184, cheval) :

(...) li vaslet fiert des esperons cele part ou il avoit veues les bestes, si trove deus pastors seans sor deus jumentes megres (...) Lancelot, I, XxI, 5 .

(...), si s'endormi dusqu'au demain a haute prime que li pastorel iscirent de la vile et jeterent lor bestes entre le bos et la riviere, (...) Aucassin, xviII, 5-7.

(...), s'il trovoit mé bués ne mes vaces ne mes brebis en ses pres n'en sen forment, (...) $i b .$, xxII, 17-8.

Et a l'issir de laiens encontra li rois un vachier qui amenoit bestes a l'abbeie. Merlin, II, 221.

A celui point li avint qu'il s'acointa de pastours, ki gardoient bestes u bois. TP, I, 168, 13-4.

Dont lou avint k'il trouverent pastours en une mareschiere, ki gardoient bestes, et estoient li pastour dusques a dis, li un viel et li autre jovene. $i b$. . II, 39, 3-6.

Lors a choisi par aventure

Bestes, qui aloient pesant ; 
Cele part torne maintenant,

Le bergier prent a apeler Claris, 9419-22.

Li dis ainsi con par dangier,

Que ja mes ne le serviroie

$\mathrm{Ne}$ avec lui ne demorroie

Ne ne monteroie sor beste. $i b ., 25991-4$.

Ele an ot antrovez les pans,

que li parut li destre flans,

et chevalchot un palefroi

qui soz li moine grant esfroi.

Unques ne fu tant gente beste : Eneas, 4045-9.

Le deüst on por chou tuer

Qu'il estoit montés sor ma bieste ? MR, LxxxIX, 532-3.

Li leu ses bestes estrangloit $i b .$, cxLV, 26 .

Li vilains estoit en la cort,

Ses bestes atire et atorne

Et sa busche au soloil retorne : ib., cxI, 50-2.

Se beste entroit dedanz mon pré

Por boivre en la fontaine clere,

Tantost corneroit li cornerre

Por faire li honte et paor $i b$, ib., 154-7.

Le terme bétail désigne plusieurs espèces d'animaux domestiques :

(...) le roy Cormorand faisoit assembler le bestial du pays de l'autour, c'est assavoir chevaulx et beufz et vaches, moutons et brebis, (...) Ogier, 223.

Vaiches et bestiailles c'on a fait achater. Lanson, 4696 .

Quant aux poissons, nous ne doutons pas que les hommes médiévaux en ont tiré profit. Nos textes montrent qu'ils en ont mangé la viande, mais même tenus dans les viviers, comme c'était certainement souvent le cas (voir p. 6), les poissons ne peuvent guère être considérés comme des animaux domestiqués : ils sont restés sauvages, mais dans un univers dont les hommes ont créé les limites. Ils ne font donc pas objet de nos recherches.

Pas plus que les abeilles, loin d'être domestiqués et pourtant si utiles aux hommes, à qui elles fournissent l'édulcorant principal de l'époque et la cire (3). Nous n'avons trouvé dans nos textes 
que trois mentions de ces insectes, dans Ogier et Jehan de Saintré dans une situation particulière : Gautier les utilise pour punir le templier Godebeuf et pour lui faire avouer son crime, madame du Perche veut punir la « dame » de Jehan de Saintré pour son infidélité :

Or vous diray de la mouche de quoy vous faites la plainte pour lui. Je vous dy qu'elle est doulce et debonnaire devant et fele et crüeuse par derriere, car elle porte le miel en la bouche et en la queue porte le venin, sy que elle point et envenime. Bérinus, 161.

A ces parolles Gaulthier le fit despouiller tout nu et luy oster la chemise et le fist atacher a une coulompne par ses sergens et lui fist oyndre tout son corps de myel, yeulx, nez et bouche. Puis fist lacher deux veisseaulx de mouche a myel qui se getterent sur luy si aprement que par tout son corps n'y avoit lieu ne place qui n'en fut tout avironné (...) Ogier, 225.

(...), et si dy oultre, s'il estoit vray, que telle dame devroit estre despoillee toute nue dez la ceinture en amont et toute reze, puis oindre de miel, puis menee par la ville afin que les mouches li courissent et la picassent, (...) Jehan de S., 305, 32 - 306, 3.

Il est remarquable que Friedrich Bangert, qui énumère tant d'animaux, sauvages aussi bien que domestiqués (et même surnaturels), ne mentionne pas les abeilles. Il a relevé quelques exemples d'autres insectes (op. cit., p. 229) dont il dit qu'ils importunent les hommes et les bêtes.

1 Il y a lieu de remarquer que parfois des animaux surnaturels se mêlent à ces animaux sauvages ; c'est le cas entre autres dans Thèbes, 9955-6 : treuvent serpanz et granz dragons, / treuvent lieparz et granz lyons ; (...). Dans le même roman, au vers 5013, l'auteur présente des zèbres traînant une charrette : Le curre traient quatre azoive, (...).

2 Cf. Friedrich Borchert, Die Jagd in der altfranzösischen Literatur, p. 57.

3 Peter Dinzelbacher, Mittelalter, dans Mensch und Tier in der Geschichte Europas, p. 182 : «Die Imkerei bot nicht nur fast den einzigen Süßstoff, sondern auch ein Konservierungsmittel und das begehrte Wachs ; (...) ». 


\section{Les animaux I}

Quels sont les animaux domestiques que nous trouvons dans les textes étudiés ? Dans sa thèse, L'animal dans la littérature française au XII et au XIII siècles, p. 234, Jean Bichon énumère une série d'animaux domestiques ayant leur place dans différents textes (c'est nous qui soulignons) : « Parfois, souvent même, l'animal figure dans le récit parce qu'il fait partie, sans autre, du cadre dans lequel se meuvent les personnages ; (...) Des bœufs hersent un champ : on s'adresse aux bouviers pour savoir si la troupe est passée par là (Perceval, 84). Le jeune Perceval, qui se rend à Carlion, demande son chemin à un charbonnier menant son âne (Perceval, 836). S'étant levé matin, il assiste à un combat entre un faucon et une bande d'oiseaux sauvages (Perceval, 4172). (...) La nuit tombe, le voyageur cherche un gîte : il s'oriente vers les habitations en écoutant les aboiements des chiens et les chants des coqs (Fresne, 144). Cligès a besoin d'un prétexte pour expliquer qu'il se rende si souvent dans la maison de campagne où Fénice habite secrètement : il y a mis, dit-il, un autour en mue (Cligès, 6236 et sv.). Un cheval est si décharné et si affaibli que les mâtins guettent sa chute pour le dévorer (Perceval, 3708) ».

Pour les classes sociales supérieures, les animaux constituent une partie importante des richesses, comme il appert dans l'extrait suivant :

« Toz dus et contes et molt riches terriers :

« N'i a celui qui n'ait .v. escuiers ;

«En destre font mener les bons destriers,

« Et granz avoirs trouser sor les somiers ;

"Portent faucons, ostoirs et espreviers,

« Chiens et brachez, et viautres et levriers. Aymeri, 2132-7.

Nous constatons que dans la plupart des textes étudiés les chevaux prédominent (1) ; ceci n'est pas du tout étonnant étant donné que la plupart de ces textes racontent la vie des chevaliers, des rois, des nobles, pour qui le cheval est absolument nécessaire : il leur sert de moyen de transport, il est indispensable pour les tournois et la guerre et aussi pour la chasse. Le chevalier est un " guerrier noble qui combat à cheval » (définition proposée par Jacqueline Picoche dans le Dictionnaire étymologique du français, p. 951).

Perrine Manne ouvre son article Images du cheval à la ferme (Senefiance 32, p. 339) en écrivant que « Si le cheval est très pré- 
sent aussi bien dans les textes que dans l'iconographie du Moyen Age, c'est qu'il est d'abord le compagnon du chevalier. »

Selon Jean Flori, Chevaliers et chevalerie au Moyen Age, p. 105, « le chevalier doit évidemment posséder une monture, ou mieux deux, plus tard cinq à sept chevaux de guerre (destriers) et un ou généralement deux écuyers chargés de porter ses armes, s'occuper des chevaux et veiller à fournir au chevalier un cheval de rechange en cas de perte ; à Hastings, le duc Guillaume eut ainsi trois chevaux tués sous lui. » et Michel Pastoureau écrit, page 123 de La Vie quotidienne en France et en Angleterre au temps des Chevaliers de la Table Ronde : « (...) il apparaît bien qu'un chevalier convenablement équipé possède au moins : un cheval de route, sur lequel il voyage, un cheval de somme, qui transporte ses armes et son attirail, et un ou deux chevaux spécialement réservés au combat. Curieusement, les juments semblent avoir été jugées inaptes à ce dernier emploi. »

Selon Fr. Schmidt, Das Reiten und Fahren in der altfranzösischen Literatur, p. 2, les chevaliers et toutes les personnes appartenant à la classe noble se trouvent toujours montés sur des chevaux, tandis que les membres des classes inférieures possèdent rarement une monture et sont, par conséquent, obligés de se déplacer à pied. D'où le dédain de la société chevaleresque pour les piétons.

Le cheval est tellement intégré à la vie des nobles, que l'on ne peut pas s'imaginer l'un sans l'autre, et apprendre à monter et à maîtriser sa monture faisait partie de l'enfance de tous les garçons nobles :

Quant il orent .v. ans, si lez font chevauchier

Et quant il en ont .vi. bien galopent destrier Gui de N., 117-8.

La suprématie des chevaux se voit clairement quand on compare le nombre d'occurrences des différents termes qui les désignent à celui des autres animaux : dans pour ainsi dire tous les cas, leur pourcentage est nettement supérieur. Il est évident que dans les chansons de geste et dans la plupart des romans d'aventure, où il y a de nombreuses scènes de bataille, les chevaux prédominent (normalement 8 à 9 chevaux sur 10 occurrences d'animaux domestiques). Par contre, dans Cent et Deduis, où il n'y a pas une seule scène de bataille, ils ne représentent que respectivement un peu plus de 30\% et env. $7 \%$ des occurrences. Les deux extrêmes sont Ille, Pontieu, Vair P. et Mort Artu, où il n'y a que des chevaux, et Vergi, où se trouve un seul animal, le petit chien de la châtelaine. Ombre ne présente que trois animaux : un cheval, un destrier et une colombe sauvage (cette dernière ne compte évidemment pas pour nous).

Et non seulement les chevaux sont mentionnés plus souvent 
que les autres animaux domestiques, mais ils se présentent sous un grand nombre de dénominations. Il est donc licite de placer les chevaux dans une catégorie à part.

\subsection{Les chevaux - une categorie à part}

Les chevaux sont, avec les chiens, les seuls animaux domestiques dont on distingue les races et dont les textes nous fournissent toute une série de dénominations différentes. A quelques exceptions près, ils sont aussi les seuls animaux que l'on distingue par des noms propres.

Le nombre des dénominations différentes va de un seul cheval (dans Silence et Turpin) à vingt-trois (dans Roussillon), mais il varie beaucoup ; voici des exemples : Barbastre 19, Athis 15, Lion 13. Tristan de N. et Aspremont 12, Renaut 11, Inconnu et Mez 10, Enfances G., Aymeri, Rigomer et Gui de B. 9, Orson 8, Aiol et Mort Aymeri 7, Espees 6, Joufroi, Cleomadés et Meliacin 5, Eracle 4, Meraugis et Orange 3, Diable et Ombre 2.

Dans la majorité des cas, c'est le terme cheval qui prédomine, ainsi dans Rigomer 260 occurrences sur un total de 322 chevaux, Roche 63 sur 87 (13 occurrences de destrier), Diable 33 sur 37, Espees 117 sur 137 (4 exx. de destrier), Cleomadés 227 sur 282 (10 exx. de destrier), Meliacin 166 sur 180 (4 exx. de destrier), Eracle 59 sur 85 (aucun ex. de destrier), Floovant 48 sur 86, Perceval 101 sur 169, Mort Artu 89 sur 107, Athis 229 sur 359, Gormont 16 sur 29.

Dans plusieurs textes pourtant (il s'agit presque uniquement de chansons de geste), c'est le terme destrier qui l'emporte; $\mathrm{p}$. ex. : Ami en présente 25 occurrences contre 10 de cheval, Aspremont 109 contre 107, Barbastre 106 contre 91, Raoul 130 contre 42, Orson 23 contre 21, Otinel 42 contre 20, Enfances G. 58 contre 28, Orange 9 contre 6, Florence 78 contre 39, Charroi 12 contre 3, Couronnement 32 contre 22, Jourdain 39 contre 7, Lycorne 48 contre 23, Narbonnais 93 contre 41, Aymeri 74 contre 18, Mort Aymeri 69 contre 16, Macaire 29 contre 15.

Dans Le Vair Palefroi nous avons relevé 37 occurrences du terme palefroi et seulement 5 de cheval, dans Pontieu 4 occurrences de palefroi et 2 de cheval.

Dans le premier volume de l'édition de Tristan en prose, à quatre exceptions près (palefroi, chien, braque, bête), le terme cheval est le seul à représenter les animaux domestiques. Il s'y présente 131 fois, dont 130 dans des scènes avec des chevaliers ou d'autres nobles, une fois monté par le harpiste qu'Iseut envoie en Bretagne : 
Quant li harperres, ki avoec lui estoit, voit que Kahedins est mors en tel maniere il n'ose avoec lui demourer, ains vient a son ceval et monte, (...) TP, I, 164, 11-3.

Certains termes, dont cheval, destrier, palefroi, roncin, sommier sont les plus fréquemment employés, sont toujours employés comme substantifs. On constate qu'il est possible de placer deux de ces termes ensemble; il nous semble qu'il faut en conclure qu'ils tiennent tous les deux la place du noyau dans un syntagme substantif. Il y a lieu de noter la rime dans ces exemples et aussi le fait que palefroi désigne un cheval qui sert au déplacement des hommes :

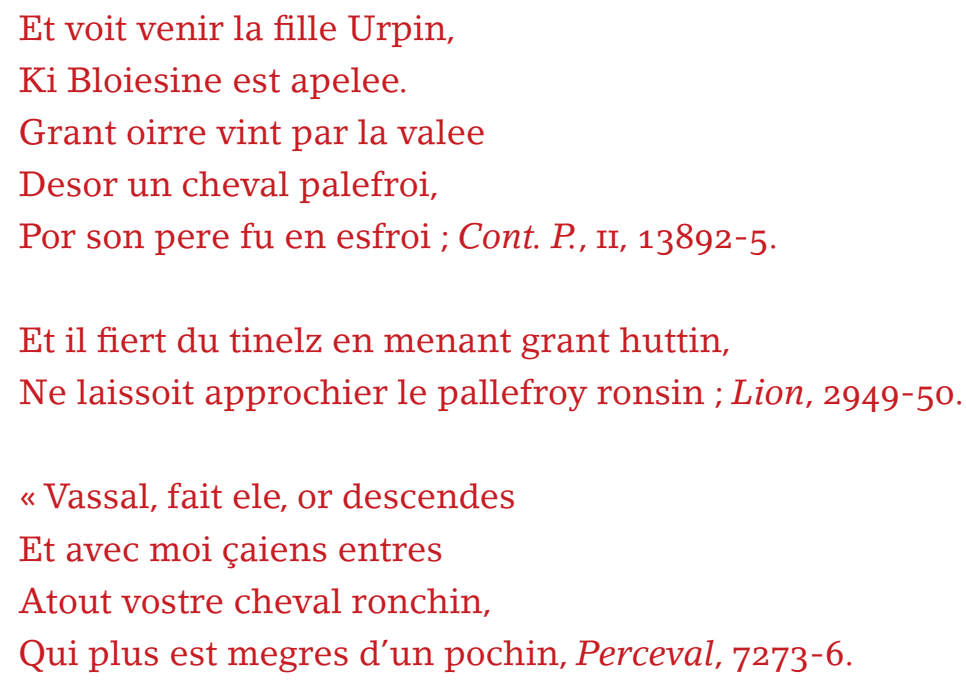

Les autres termes désignant un cheval, dont voici la liste des plus usuels : arabi, aragon, auferrant, bai, bauçant, chaceor, coureor, coursier, ferrant, gascon, liart, morel, ros, sor, vair, vairon, se trouvent employés tantôt seuls, c'est-à-dire comme substantifs, tantôt comme épithètes à l'un des termes du groupe cheval, destrier etc., tantôt mis ensemble dans un syntagme substantif.

Il y a lieu de noter que toutes les dénominations du dernier groupe indiquent la robe, la race/l'origine ou une qualité des chevaux en question (ou les deux ; voir p. ex. p. 30, Joufroi, 402-3, un exemple qui indique et la robe et la race d'un destrier). Certaines d'entre elles peuvent servir comme un nom propre. Dans ces derniers cas, il est parfois difficile de préciser s'il s'agit du nom d'un cheval, généralement indiqué par un majuscule initial, ou simplement d'une épithète, car le nom aussi bien que l'adjectif ou le substantif peuvent être précédés par un article, p. ex. :

Baudous, li filz au duc d'Angay,

Y est sur le courant Liart : Galeran, 5620-1. 


\section{El liart sist d'Esclavonie}

qui fu bons pour chevalerie. Thèbes, 6327-8.

Leis une roche voit tenir lo Bausant. Enfances G., 456.

Et mesire Gavains adrece

Viers lui le cief de l'auferant,

Et il guencist sor le bauçant, Rigomer, 7286-8.

L'ordre des termes en question est souvent facultatif : nous trouvons, en effet, rouz liart en face de liart rouz, sor bauçant en face de bauçant sor, auferrant destrier en face de destrier auferrant, mais avec certains termes toujours le même ordre, p. ex. auferrant corsier :

Aprés brocha chascun son auferrant corsier ; Barbastre, 2272.

Floovans a broicié son auferrant corsier, Floovant, 404.

Nous notons aussi que ces mêmes termes se trouvent employés liés par et :

Icil .vir. roi montent sor les destriers,

Qui trestot furent auferrant et corsier. Mez, 3976-7.

Tout compte fait, il nous semble impossible de décider définitivement lequel des termes d'une telle construction est le noyau, lequel l'épithète. Tout au plus peut-on affirmer que certains termes sont plus souvent épithètes, d'autres plus souvent noyau, à moins qu'on ne veuille conclure, comme pour cheval palefroi et palefroi roncin (voir supra Cont. P., II, 13892-5 et Lion, 2949-50), qu'il s'agit d'un syntagme substantif à deux termes dans la place du noyau?

Les différentes dénominations se remplacent parfois pour désigner un seul et même cheval (voir aussi infra s.v. cheval), comme nous le voyons p. ex. dans Aliscans : v. 6349 auferrant = v. 6351 destrier $=$ v. 6353 cheval $;$ Cristal vv. 4232/4688 destrier $=$ vv. 4227/4699 cheval ; Lion : v. 22599 l'arragon = v. 22601 destrier gascon $=$ v. 22606 destrier arabit et, dans le même texte, v. 3047-8 destrier gascon = v. 3049 l'aragon. Fr. Schmidt, op. cit., p. 10, a remarqué que ce phénomène se trouve avant tout dans les textes tardifs (fin du XIII ${ }^{\mathrm{e}}$ et XIV ${ }^{e}$ siècles); nous avons constaté la même fluctuation dans des textes plus anciens. Que les races des chevaux soient ainsi confondues ne semble pas gêner les auteurs.

Il est évident que bien souvent les termes désignant les chevaux sont accompagnés d'épithètes soulignant la qualité ou la valeur des animaux en question : bon, riche, grand, isnel, de prix etc. 
Des syntagmes prépositionnels comme de Sulie, d'Espaigne etc. marquent l'origine des animaux. Aucune de ces expressions ne se trouve jamais comme noyau dans des syntagmes substantifs.

Il n'est pas sans intérêt de noter qu'assez fréquemment les chevaux sont présents dans les textes, sans être nommés. C'est le cas entre autres avec les verbes monter, descendre, chevaucher, dont nous présentons ici quelques-unes des très nombreuses occurrences. Le verbe déchevaucher est beaucoup plus rare que les autres :

- Sire, font il, or montés donques, Barisel, 137.

«Sire, font il, car descendés, ib., 165.

Li provos les fist tous descendre Gliglois, 1881.

Congié prennent a Charle, chascuns monte an la sele ; Saisnes, 954

Si monterent li chevelier. Florimont, 478. Cf. ib., 1611.

Illuec sont a pié descendu. ib., 1282.

Tant chevacherent per vertu ib., 1265. Cf. ib., 1280.

Atant s'en tornent brochant a esperon. Aliscans, 5835 .

Maintenant ilueques s'em part

Li seneschaux. qui molt est tart

Que il se reçoit desarmez,

Poignant s'en vait tous abrivez,

Jusqu'a la fontaine ne fine Floriant, 351-5.

Et tant vasal vilment descevalcier Aspremont, 9460.

Le même phénomène se retrouve dans Enfances $O$. Le texte décrit la dévastation du pays : celle-ci comporte le manque de nourriture aux animaux, c'est-à-dire avant tout aux chevaux des armées :

Vous savez bien que tout avons gasté

Celui paÿs, et dou lonc et dou lé ;

Ne trouveront, s'il ne l'ont aporté,

Orge n'avainne, ne forrage ne ble,

Ne chose nule dont soient governé. Enfances O., 617-21.

Un petit extrait d'Erec nous donne une idée du nombre exorbitant de chevaux à la cour du roi Arthur - à moins qu'il ne s'agisse 
d'une des fréquentes exagérations des romans. Diomedès, lui, dit simplement qu'il a un nombre suffisant, sans préciser :

Ainçois que noune fust sonnee,

Ot adobé li rois Artus

Quatre cenz chevaliers et plus

Toz filz de contes et de rois.

Chevax dona a chascun trois, Erec, 6652-6.

Mais je ne sui mie a grant soufrete de destriers, quar je en ai assés, vostre merci. Troie, 152, 49-51.

Certains chevaux sont décrits comme des animaux extraordinaires devant lesquels leurs propriétaires ou d'autres personnes sont en admiration. De même que certains textes nous présentent de longues descriptions des adolescents et des adolescentes idéaux, nous y trouvons des présentations d'un cheval idéal, comme les suivantes :

Li destrers est e curanz e aates,

Piez a copiez e les gambes ad plates,

Curte la cuisse e la crupe bien large,

Lungs les costez e l'eschine ad ben halte,

Blanche le cue e la crignete jalne,

Petites les oreilles, la teste tute falve ;

Beste nen est nule ki encontre lui alge. Roland, 1490-6. Cf.

Gaufrey, 944-50.

Ne ses cevaus, çou m'est avis,

Plus blans que une flors de lis

Estoit trestous fors les orelles,

Mais celes sont andeus vermelles.

(...)

Et li cevaus remest plus blans

Que cines en eve noans.

(...)

La veïssiés ces demoiseles

Et les dames et les puceles

Tout entour le ceval venir ;

Volentiers le veulent vëir.

Por çou que il estoit si biaus,

L'aplennient de lor mantiaus.

Les iox, le chief et les narines

Li torcierent de lor ermines,

Le col, le crupe et les costés.

Onqes de ceval qi fu tes, 
Ne prisent dames tel conroi,

Qu'eles font del ceval le roi. Rigomer, 12989-92, 13027-8 et

13053-64.

Mais pour ce qu'il estoit bien eure,

Monta sans plus faire demeure

Sus un cheval apert et aspre ;

Et fu couvers d'un blanc dïaspre

Par dessus l'autre couvreture.

Li chevax de grant estature

Estoit et de grande vistece,

A une teste longe et seche,

A uns iex gros, parfont fendus;

Et fu grans et bien estendus,

Et ot forte eschine a merveilles,

A unes petites oreilles

Et un col gros et espés pis ;

Des mustiaus ne fu pas du pis,

Kar il les ot gros et foitables,

Jambes fermes et bien estables,

Et fu bien par terre enjointiés

N'ert pas toute jour afaitiés

Des piés com je voi mains chevaus,

Kar por courre tertres ne vaus

Tous desferrés au lonc du jour,

Ne fust il ja mis assejour,

Tant ot les ongles durs et fors,

Et tant refu grans ses esfors.

S'ot il uns piés trop bien jointis,

Sains et roons et bien faitis,

Et larges dehors et dedens ;

Et ot grande bouche et bons dens,

Et s'avoit si tres bone alaine

Que pour souffrir travail ne paine

N'ot onques mais nus chevaus tele ;

La crupe avoit et large et bele,

A une rains trop vertüeusez

Que ja ne fussent dolereusez

D'un chevalier armé porter.

Trop se faisoit bel deporter

A regarder si bele taille.

Unes hanchez ravoit, sans faille,

Larges et grosses et faitichez ;

S'ot unes espaules massicez

Dont il ne fu pas pis seans ;

Onques chevaus miex poursivans

$\mathrm{Ne}$ fu ne de taille plus fine. 
Il ot une petite crine

Qui durement li avenoit.

Li chevaus apers se tenoit,

Qui bien sentoit le cuer de lui :

Il n'ot en la place celui

Qui bien ne le loast a certes.

Unes narrines ot ouvertes,

Dont il rouflait si fierement

Qu'il sambloit trestout vraiement

K'il deüst une tour abatre.

La en avoit tex .xximI.

Ki sour lui n'osassent monter

Ne pres d'une lance habiter. Meliacin, 11863-918. Cf. Chevalerie d'O., 11454-65.

Athis en porte li liarz,

Qui n'est fuitis ne trop coarz,

Mes delivres, forz et isneaus.

Piez ot coupez et plaz musteaus,

Cuisse reonde et secorciee

Et la crine corte et deugiee,

Ample le nés et refroncié

Et l'uel espés et röellié,

Le flanc bien gros, la crope lee,

Le col voutiz, teste apilee,

Meigre et treitice et corte oreille ;

Bon est et beaus a grant merveille ;

Plus tost s'en vet, quant il le broche,

Que nus quarreaus, quant il descoche. Athis, 16787-80o.

La reine Camille est montée sur un palefroi hors du commun : non seulement il jette " grant esfroi » autour de lui, mais les différentes parties de son corps présentent toutes les couleurs possibles (cf. Fr. Schmidt, op. cit., pp. 39-40) :

Ele an ot antrovez les pans,

que li parut li destre flans,

et chevalchot un palefroi

qui soz li moine grant esfroi.

Unques ne fu tant gente beste :

come noif ot blanche la teste,

lo top ot noir, et les oroilles

ot anbedos totes vermoilles,

lo col ot bai et fu bien gros,

les crins indes et vers par flos ;

tote ot vaire l'espalle destre

et bien noire fu la senestre ; 
les piez devant ot lovinez

et fu toz bruns par les costez ;

soz le vantre fu leporins

et sor la crope leonins

et fu toz noirs desoz les auves ;

les dos james devant ot falves,

les dos desriers vermalz com sans ;

les quatre piez ot trestoz blans,

noire ot la coe une partie,

l'altre blanche, tote crespie,

lo pié copé, les james plates :

molt fu bien faiz et bien aates.

Li palefroiz fu bien anblanz,

et li froins fu molt avenans ; Eneas, 4045-70.

Dans Gaufrey, l'auteur présente un cheval dont la particularité lui vaut d'être remarqué par les gens :

Et li cheval sous li estoit de tel semblant :

L'un costé avoit taint aussi comme arrement,

Et l'autre resembloit coton, tant estoit blanc ;

Une petite corne avoit $\mathrm{u}$ front devant.

Le cheval Cornuet l'apeloient la gent ; Gaufrey, 4912-6.

A d'autres, les auteurs attestent des traits humains : ils sont courageux, ils sont forts, ils sont intelligents et astucieux, tel le bon destrier de Renaut de Montauban. L'auteur de Renaut souligne à plusieurs reprises que ce cheval est fée (2) ; il dit qu'il comprend les paroles « com se ce fust .I. hon » - et quoiqu'il ne sache pas parler, il est quand même capable de se faire comprendre. Il peut même exprimer ses sentiments : lorsque la famine menace les assiégés et que Renaut se sent obligé de tuer Bayart qu'il aime tant, le cheval se met à genoux devant son maître :

Et puis monte el cheval qui lui fu apresté :

Onques Dex ne fist beste de la soe bonté,

Si out a non Baiart, ice fu verité,

Por corre .xxx. leues ne seroit ahané

Por ce qu'en Normendie fu le cheval faé. Renaut, 884-8.

Li vair destrier d'Espaigne, quant se sent alegié,

Il entra es galoz si a tant esploitié

Que il a .I. grant tertre par devant li poié.

Et quant Bayart le voit, mult ot le cuer irié,

Aprés le vair d'Espaigne es le vos eslaissié,

Et a consieut le vair, n'i a gaire targié,

Par la crigne le prent si l'a avant sachié. 
Tant a Bayart le vair tiré et maistrié

Tote lor ambleüre sunt el champ reperié.

(...)

Li ribaut fu hardiz si est avant passé

Et a sesi Renaut par l'estrieu neelé.

Quant Bayart le senti si a le pié levé,

.I. tel cop li dona le cuer li a crevé.

(...)

Quant Amaugis le voit si descent maintenant,

Le destre pié Bayart delia a itant.

« Bayart, ce dist Renaut, trop alez belement :

"Se vos passent issi, blasme i avrez mult grant!»

Quant Bayart ot Renaut si le vet escoutant,

Ensement l'entendi com mere sen enfant,

Il froncha des narilles, le chief vet escoant ;

Renaut lesche la regne, Bayart s'en vet corant,

Tot le col estendu vet terre porpernant.

(...)

Oez des .ii. chevaux com il ont esploitié !

Quant virent lor seingnor si forment chaploier,

Li uns cort contre l'autre com fussent enragié,

Des denz s'entremordoient et feroient des piez,

Autresi se conbatent comme dui chevalier.

(...)

Quant Bayart se regarde si vit Rollant a terre,

Il a levez les piez bruiant comme tanpeste,

Fiert le cheval Rollant en l'oreille senestre,

Ou il vousist ou non, li fist ronpre la teste.

(...)

De voir le savez bien, franc chevalier baron,

Que Baiart fu faez, li destriers aragons,

Si entent la parole com se ce fust .I. hon.

A Renaut est venuz enz el bruillet roont,

Qui estoit endormiz comme traveilliez hon ;

Baiart ne puet parler, ne dire o ne non,

Renaut hurte del pié, que il ot gros et lonc,

Et fiert si durement en l'escu au lion

De l'un chief jusqu'en l'autre li esmie et deront.

Renaut si s'esveilla et sailli contremont,

$i b ., 4107-15,5050-3,5160-8,7840-4,8721-4$ et 9703-12. Cf. $i b$. ,

7734-9.

«Montaubant vos dourai et les murs qui sont bloi,

« Et Bayart mon cheval que j'ain autant com moi,

(...)

Il a pris .I. coutel, en l'estable en entra,

Quant Bayart l'a veü, de joie sautela, 
Por l'amor son seingnor grant joie demena.

(...)

Puis revint a l'estable a Bayart l'arragon,

Ferir le vout el cuer d'un coutel a bandon,

Quant devant li se mist Bayart a genoillon.

Quant Renaut l'a veü, si dolent ne fut onc,

Il ne le ferist puis por tot l'avoir del mont ; ib., 11028-9,

12111-3 et 12124-8.

Et il y en a d'autres qui sont presque aussi exceptionnels :

Li cevax Hue vit l'Amauri u pré ;

Cele part vint, que n'a soing d'arester.

Et li cevaus Amauri le dervé

A moult grant friente vers le Huon mené,

Mais li Huon n'i a preu aresté.

Des piés devant commença a grater,

De ciaus derriere commence a regreter ;

Un si grant caup a l'Amauri donné,

Emmi le front l'a tant bien asené

Que il li fist andeus les iex voler

Et de la teste le cervele verser.

Et li cevaus ne pot plus endurer,

Ains caï mors a tere emmi le pré. Huon, 1812-24.

Marchegai ot la noisse des quivers Sarrasins,

S'ot les escus as elmes et as lances tentir :

Tel deul fait li chevaus a poi n'esrage vis,

Del destre piet grata et durement heni,

Et demaine tel noise q'Aiols s'en esperi. Aiol, 5063-7. Cf. Che-

valerie d'O., 6237-8; 6250-2.

Quant l'entendi Elies, por poi qu'il n'est dervés :

Il a pris un baston par sa ruiste fierté,

Sore li est corus, qu'il le voloit tuer :

«Lechiere, » dist li dus, « mar l'ossastes penser,

«Que Marchegai fu mors, mes destriers sejornés :

« Jamais autres si boins ne sera recovrés.

« Issiés fors de ma tere, ja plain pié n'en tenrés.

(...)

Se li fist devant lui Marchegai amener.

Li chevals estoit cras, si ot plains les costés,

(...)

Mais d'une cose furent Borgengon engingié,

Que le ceval Aiol ont ariere laissié.

Uns Lonbars le saisi, sel vaut aplanoier :

Li cevaus aperçoit que Aiols n'ert che nient, 
Le Lonbart a tué a anbedeus ses piés.

Vers Lengre[s] s'en retorne devement eslaissiés,

La porte li ovri Asses li Beruiers,

Et Marchegai i entre : ains ne fu tex destriers !

Plus seut tous tans de guere que mavais chevaliers. $i b$., $8265^{-}$

71, 8285-6 et 8539-47.

Quant li chevalz ot Guillame parler,

Tant fort s'esfroie et tant c'est desraé

Siauz ke le tienent ait toz escravanté.

Voilent ou non si l'ont laisiét aler ;

Per mi la presse des Françoiz est torneiz,

Dusc'a Guillame ne se volt arester.

Adonc se sunt ambedui retrové ;

Onkes n'oïstes de dous amins parler,

Ne ne veïstes si grant joe mener,

Comme Guillames ai Bauçant l'abrivé. Enfances G., 1410-9.

Suz ciel n'est rien tant ignel,

Ne leppart ne nis cheveroil

Ne dromedaire ja tant n'alast

Que cist destrers tost nel passast ;

Ja mar crendrez braz de mer

Que ne puissez sur lui passer ;

Se vus de ço ne me creez,

Ben voil que vus l'assaiez;

Mais me custume ad le cheval,

Dunt meint home ad eu mal :

Suz ciel n'est home qui l'aproçast,

Qu'il an eire nel devorast,

Se jo nun, qui l'ai gardee,

Qui sur tote rien l'ai amé. Gui de W., 6211-24.

Ses boens chevals grat(e) e henist,

Yder soufraine son cheval,

Pus le retient en un estal.

Bien set qu'il sent alcone chose ; Yder, 3573-6.

Et Arondiaus le connut au parler,

Henist si fort, la terre fait croler ; Bueve, I, 3430-1.

Le bon chevalier Ogier dispose de deux chevaux intelligents: d'abord Broyfort et plus tard Papillon; celui-ci est fée :

(...) et ainsi qu'il fut sur le chemin ou il devoit passer si advisa son cheval Broyfort qui l'attendoit ne plus ne moins que le serviteur eut attendu son maistre. Dont Ogier se trouva tout 
resioux. Et dist a Broyfort: tu as gagné d'estre bien pensé aujourd'uy car tu m'a esté loyal ; si monta a coup dessus. Ogier, 81.

Et quant Ogier vit son cheval se pensa que ce n'estoit pas Broifort. Mais le cheval lui fist bonne recongnoissance et lui hannist et baye la gueule et frape des piez en terre $i b ., 140$.

Et quant il eut tourné et viré assés, le cheval qui nommé estoit Papillon vint devers lui hennissant s'agenoullant devant lui. Et par plusieurs fois se couchoit devant lui. Et quant par plusieurs fois Ogier entendit le signe qu'il vouloit qu'il montast dessus (...) ib., 269.

Et adonques son cheval Papillon, qui avoit entendement, se leva sur ses piez de derriere et leur courut apres moult isnellement ; (...) ib., 296.

Mais il y a aussi des descriptions de chevaux plus "normaux", p. ex. ceux des chevaliers de Charlemagne, de Tristan et de Sagremor qui ont peur de l'imprévu, le destrier Vairon de Baudouin, pourtant si fort et si courageux, et le cheval de Lancelot qui entre en conflit avec un autre cheval :

De la noyse e des barberes se espounterent nos chivaus e tournerent le dos e s'en fuyerent si ke les chivalers ne les poeyent reteiner. Turpin, 794-6.

Et il crolloit tous, a la verité dire, pour le tres grant randon dont li cevaliers venoit ; et d'autre part li cevaus, qui grans et sejournés estoit et isniaus durement et avoit acoustumé souventes fois a courre desour le pont, vint illuec corrant conme rage. Le ceval mesire Tristran, qui le passage n'avoit pas acoustumé et qui sentoit que li pons crolloit desous lui, ne vient pas par tel acuellement ne par tel desmesurance : la coustume li fait mout mal ; et li cevaus si est duis et li autres non. TP, vII, 13, 8-16.

Li chevaus, qui paissoit encor

Lors qu'il a le cerf perceü,

Si grant paour en a eü,

Ensi comme une mue beste

Qui lieve contremont la teste

Et lance oultre, et s'enfuit au bois. Méliador, 28416-21.

Il choisisrent Vairon, qu'est joste la ramee,

Atachié a un rain par la resne doree.

Lors lor fu bien avis borse eüssent trovee ; 
Plus de .c. en i poignent a une randonee.

Li chevax ot l'effrois comme beste bersee ;

De la paor q'il a, a sa resne tiree

Que ou nou de la branche est rompue et fausee;

Corant s'an va vers Rune, mainte foiz l'a passee. Saisnes,

3974-81.

Lors descent et atache son cheval auques pres de l'autre et se couche de l'autre part de la fonteinne. Et ne demora guieres que li chevaliers s'esveilla por la noise des deus chevax qui s'entrecombatoient ; Mort Artu, 74, 15-9.

Nous venons de voir, pages 21-22, un cheval très bariolé ; la plupart du temps, pourtant, nous nous trouvons en présence d'animaux à robe unie. Assez souvent les auteurs aiment mettre une série assez importante de chevaux de différentes couleurs côte à côte, vraisemblablement pour rendre ainsi leurs descriptions plus intéressantes et plus vives :

Maint bon destrier sort et bausent

Blanc et gris et noirs et ferrant Chauvency, 415-6.

Je ai trois palefroiz mout buens,

Onques meillors n'ot rois ne cuens,

Un sor, un vair et un baucent. Erec, 1383-5.

Tant bon cheval bauçain[t] et sor,

Fauves et noirs et blans et bais, ib., 2152-3. Cf. ib., 2340 ; Vair

P., $167-80$.

chevauls ont gaaigniés blans et baucens et sors ; Rou, II, 3294.

et gaaigner destriers blans et vairs et ferrans, ib., 3360. Cf.

Narbonnais, 3912.

Mes a oes le suen cors demainne

Quatre divers destriers an mainne,

Un sor, un fauve, un blanc, un noir. Cligés, 4241-3.

Vu la grande intimité entre cheval et maître, il n'est certainement pas étonnant que celui-ci aime sa monture, qu'il le soigne ou, plutôt, le fait soigner par les écuyers. Nous traiterons de ce thème au chapitre 3.

Voici maintenant la liste de dénominations que nous avons relevées dans nos textes, y compris les noms propres de chevaux et un nombre d'adjectifs qui caractérisent les chevaux (3). Nous laissons de côté tout ce qui est dit des harnois et nous limitons 
strictement à ce qui a trait aux animaux : origine, couleur, qualités.

\subsubsection{Les termes qui sont toujours substantifs}

a. amoravi.

A.-J. Greimas, Dictionnaire de l'ancien français, p. 29, explique : " $1^{\circ}$ Sarrasin. $-2^{\circ}$ Cheval de bataille. " Concernant l'exemple de Narbonnais, voir notre commentaire à arabi, p. 82, ib., 4171-2 et 7329 .

Monte tost sor Morel, le bon amoravi,

Si pren vairon en destre : ains deus meillours ne vi. Partonopeu-C, 2043-4.

Es vos poingnant le preu conte Aymeri.

Bien fu armé desor l'Amoravy, Narbonnais, 4868-9.

\section{b. augalie.}

De ce terme nous ne pouvons présenter qu'un seul exemple qui montre bien qu'il est question d'un cheval, mais rien d'autre :

Corsolz s'en est tornez fuiant sor l'augalie,

Aïmer et Hernaut l'enchaucent par envie. Barbastre, 6722-3.

A.-J. Greimas, op. cit., p. 22, explique : "Nom donné aux souverains d'Orient » et " Trône de souverain d'Orient ». Faut-il peutêtre y voir une allusion à « calife » et lui donner le sens de « cheval oriental »?

c. cheval.

Le terme cheval sert généralement de dénominateur commun, c'est-à-dire qu'il peut remplacer chacune des autres appellations. Ceci implique que bien souvent un seul et même animal est appelé tantôt palefroi, tantôt cheval, ou tantôt destrier, tantôt cheval, etc. - le choix peut dépendre du rythme ou de la rime.

Nous en voyons un exemple dans Roche, où le jeune Landri est assis, au vers 2475, sur un arragon; sept vers plus loin, on le retrouve sur un destrier qui est probablement le même animal (quoique dans l'entretemps ait eu lieu « .j. abateïs fier ») et qui, au vers 2483 déjà, est appelé simplement cheval.

Voici d'autres exemples du phénomène (voir aussi p. $36 \mathrm{MR}$, xI, 151-2, 178 et 194-5) :

Metés les sieles sor les corans destriers,

Desc'a Bordele vous couvint cevauchier ; 
(...)

- « Baron, dist Karles, or ça venés avant,

Metés les sieles es palefrois amblant,

(...)

Et li mesaige ne se vont atargant,

Desc'a Bordele ne vont resne sacant,

Ens la vile entrent sor les cevax corant,

Puis en monterent sus el palais luisant ; Huon, 292-3, 305-6 et 322-5.

Ensint chevalche Galehout mas et pensis qu'il ne dit mot n'a Lancelot n'a autrui, tant que ses palefrois est tos tressuans. Et lors est entrés en un chemin perrous et li chevals fu durement cargiés del chevalier qui sor lui fu grans et pesans et plains de doleros pensés, et si refu encombrés de la malvese aleure que il aloit : Lancelot, I, II, 5.

chascuns li sali a l'estrier.

Il sali jus du bon destrier,

si l'a livré au marechal

et cil atorne le cheval

de fain, d'avaine et de litiere.

(...)

quant armés fu, lors vint aval.

On li amaine son cheval,

lors monte el bon destrier de pris Cont. P., III, 15623-7 et

15727-9.

De terre ens ou cheval salli

- Onques n'en seut gré as estriers - ;

$\mathrm{Si}$ estoit ce .I. moult biaus destriers

C’on li avoit laiens bailliet. Méliador, 10719-22.

Congié demendent, es chevax sont monté,

(...)

Chascuns chevauche bon mulet sejorné,

Ou palefroi richement enselé ; Aymeri, 1565 et 1576-7.

qant un prestres vint a cheval

(...)

Mout tost se mist vers le ronci ;

(...)

Lors li saut entre les arçons,

et li polains fu esfraez,

(...)

et si me rant mon palefroi Renart, IV, 11722, 11809, 11826-7 et 11889 
Son chevals li ont amené,

Un mult buen vair destrier gascon, Joufroi, 402-3.

la sele met en son cheval,

ses armes prent, monte el destrier, Thèbes, 1238-9.

A tant de sa main destre soigne son vis et croise,

Et broche le destrier qu'est de terre espanoise,

Et li chevax se lance an Rune plaine toise. Saisnes, 1531-3.

Tarquines broche le destrier

Qu'il ot delivre et mout legier,

Point lo cheval et esperone, Athis, 7483-5.

Li cheval estoient d'Espaigne

Molt par ierent bel li destrier ; Claris, 8204-5.

Gliglois apielle un escuier :

«Va, sy me mainne cest destrier

A mon oste, car jou t'en proy,

Et se li di jou li envoi. »

Cil prent le cheval et le maine,

L'oste le done a bone estraine. Gliglois, 2465-70.

(...) : et aussi qu'il prendra et amenera en bataille ung autre coursier, car cestuy n'est pas ung cheval mais est ung dyable. Ogier, 303.

Mais nous trouvons aussi des cas où l'auteur fait la distinction $\mathrm{p}$. ex. entre cheval et destrier et entre cheval et palefroi :

Puis s'adobent ensemble li chevalier hardi

Et montent es chevaus et es destriers de pris. Roche, 2276-7.

Chascuns a point qui cheval, qui destrier, Couronnement, 1504 .

et biax chevax et biax destriers, Thèbes, 2988.

E par ces rens i vunt curant

Meint bon cheval e meint destrer Ipomedon, 3896-7.

herberjeürs e pautoniers,

ki chevaus meinent e destriers. Rou, III, 2993-4.

descenduz est du palefroi,

son cheval quiert, l'en li ameine ; ib., 8480-1. 
Mes n'i merron cheval ne palefroi

Ne blanc hauberc ne heaume d'Aminois Orange, 323-4.

Enselé et appareillié

Li cheval et li palefroi

Sanz estormie et sanz desroi ; Vair P., 918-20.

Chevaus menrons et palefroiz Athis, 9117.

A ces parolles es vos .I. mesagier,

mais il n'avoit sergent ne chevalier,

ne [n'ot] cheval, palefroit ne destrier ;

tos est deschaus, n'ot chauce ne chaucier, Loquifer, 951-4.

Nen oit çival, palafroi ne destrer ; Macaire, 2324. Cf. ib., 2573-

$4 ; 3572$.

Illueques ont assés conquis

Destriers, cevaus et palefrois. Blancandin, 5902-3. Cf. ib., 2075 ; 6033.

Que le terme cheval ait vraiment la fonction de dénominateur commun pour toutes les espèces d'animaux de selle, nous le voyons corroboré dans Roche : lorsque la princesse Salmadrine demande au messager Outré quels chevaux lui et son compagnon prendront pour aller en France, il répond qu'ils ont « deux mulets »; elle lui propose alors «.ij. dromadaires coranz et abrivez » que nous retrouvons plus loin où revient le terme cheval pour les désigner :

A piet sont descendu au mauberin degré ;

La defors si laiss[i]erent les chevaus por garder.

Li dromadaire furent estanc et tressüé

Et li mès sont forment travaillé et pené. Roche, 1624-7.

Les dromadaires leur sont volés par Malingre et Hardré, mais pour l'écuyer qui annonce ce vol à son maître, il s'agit de chevaux :

Pristrent les dromadaires qu'il truevent establez, ib., 1696.

«A la moie foi, sire, mal vos est encontré :

"Perdu sont li cheval que deviez garder

«A ces .ij. pelerins que vos ici veez. » ib., 1706-8.

Dans Loquifer se trouve un passage que l'on peut lire de la même manière ; mais il est possible aussi de l'interpréter différemment : le dromadaire est plus grand et plus rapide que n'importe quel 
cheval, c'est-à-dire que pour voyager vite il faut préférer les dromadaires aux chevaux :

Loquifers ist premerains de sa nef, avoc lui sont .IIII. roi coronés ; son dromadaire ont devent lui moné, selle ot le dos de fin acié tenpré, li estrier sont de fin or noielé, li anés ot .xxir. pouz de les ; il n'est cheval de la soe bonté, il n'ait si grant en la crestienté.

Ans cort autant con .I. falcons müé, Loquifer, 998-1006.

Dans beaucoup de textes, le terme cheval désigne régulièrement le cheval de bataille, c'est-à-dire qu'il remplace destrier :

Il a mandeit az senechal

Que faisce ameneir son cheval

Et ses armes tost aporteir, Florimont, 545-7.

Li rois command[e] a encelleir

Son cheval et fait aporteir

Ses armes illuec en la place, $i b ., 587-9$. Cf. $i b ., 3345 ; 4871$.

« Feitez moi tost armer .c. de vos compengnons

Et vestir les haubers desous les auquetons

Et monter es chevax auferrans et gascons ; Gui de N., 969-71.

Je irai joster, nel lerroie

Por riens. Ça mes armes! » Tantost

L'arment et cil s'en vet de l'ost

Sor .I. cheval plus noir que more. Meraugis, 5400-3.

Un chevalier trop bien monté

Sor un grant cheval abrievé. Durmart, 1815-6.

Adont se sont li Rommain armé et monterent sur les chevaus de garde, et on mena leurs pallefrois en destre. Laurin, 1624-6.

Nous pensons qu'il faut comprendre l'extrait suivant, de Saisnes, ainsi : même le moins riche des guerriers en question a un cheval, ou bien un palefroi, ou bien un destrier. Cf. un exemple semblable avec oisel, p. 304, Saisnes, 1442-5: 
Tuit li pire ont cheval, palefroi ou destrier,

Et totes riches armes qi a roi ont mestier. Saisnes, 2282-3.

Nous ne tenons pas compte des 28 occurrences de chevalet, dans Cleomadés, où le terme désigne le cheval artificiel en ébène du nain Crompart. Le même terme se trouve, à la rime, dans le fabliau xxxvı, parallèlement à cheval, et également dans Cent :

Uns povres Mercier, sanz revel,

I vint à tot son chevallet ;

$\mathrm{N}^{\prime}$ avoit besasse ne valet ;

Petite estoit sa mercerie.

"Que ferai-je, Sainte Marie, »

Dist li Merciers, « de mon cheval ? » $M R$, xxxvI, 18-23.

Si devez savoir que tantost il monta sur son chevalet,

(...) Cent, 5, 58-9. Cf. ib., 84, 21.

d. destrier.

É. Littré (Dictionnaire de la langue française, II, p. 1117) définit le terme ainsi : " Dans le langage de la chevalerie et des guerres du moyen âge, cheval de bataille. » et explique son origine : « (...) de dextra, dextre, main droite, à cause que l'on conduisait ces chevaux avec la main droite avant de les monter pour la bataille. » Cette explication se retrouve, avec une précision supplémentaire, dans le Dictionnaire Général, p. 717 : « (...) de destre, main droite (lat. dextera), le cheval de bataille étant conduit de la main droite par l'écuyer quand le chevalier ne le montait pas (...) Cheval de bataille au moyen âge. » R. Grandsaignes d'Hauterive est encore plus précis quand il écrit dans son Dictionnaire d'ancien français, p. 178 : «Destrier, gros cheval de bataille, tenu de la main droite par l'écuyer, et que le chevalier montait lorsqu'un danger se présentait (...) »- définition qui a été reprise presque mot à mot par A.-J. Greimas, op. cit., p. 187 : « Gros cheval de bataille, mené de la main droite par l'écuyer et que le chevalier ne montait que lorsqu'un danger se présentait. »Voir aussi Fr. Schmidt, op. cit., p. 6.

Plusieurs extraits de textes corroborent parfaitement ces définitions :

Lors encontre Percevaus un vallet qui estoit fors et legiers et cevauchoit un ronchin et menoit un ceval en destre noir conme meure. $T P$, vIII, 44, 1-3.

La lance dist qu'il li aport

Et que son cheval li estraigne,

Celui qu'il maine en destre, et praigne

Son palefroi et si le maint. Perceval, 5668-71. 
Et li rois est montés sor .I. palefroi et fait mener en destre .I. grant cheval (...) Lancelot, III, xxxIx, 1.

Mes sire Gauvains fu armez, et si fist a deus escuiers mener an destre deus destriers. Charrete, 254-6.

.v. escuiers a chascune d'aus mené,

Qui bien estoient garni et conreé ;

En destre moinent maint destrier abrivé, Aymeri, 1580-2. Cf. $i b ., 2134 ; 2707-8$.

Et li sires de laiens li fist amener son destrier en destre (...) Merlin, II, 200.

Lugain, l'écuyer d'Yder, ayant à mener cinq destriers, laisse les quatre aller devant lui et tient le cinquième à droite du cheval sur lequel il est monté :

Des cinc destriers ke Luguain meine

Eirent li quater devant lui,

Areisné furent dui et dui

Le quint en destre par le frein

Meine [de]juste lui Luguain. Yder, 1437-41.

Nous avouons ne pas bien suivre l'explication du terme que donne Peter Dinzelbacher dans son long article sur l'homme et l'animal au Moyen Age. Il prétend, en effet, qu'en ancien français le terme destrier proviendrait du fait que le chevalier était obligé de faire courir son étalon (seul admis comme cheval de bataille (4)) en galop à droite, car c'était là le seul moyen de tirer le plein effet de la lance tenue à droite, sans risque d'être blessé par le choc en retour causé par la collision (5).

Que le destrier soit le cheval de bataille est confirmé par un grand nombre de descriptions de batailles dans nos textes. Voici un petit choix des exemples qui montrent tout à fait clairement que c'est destrier qui désigne le cheval de bataille :

(...), e vist les champs coverts de chevalers, esquiers, serjauntz e vadlets, les uns armés sur destrés, les uns a pié, (...) Lors escrie ces chevalers e les comanda armer e mounter lur destrers,

(...) Fouke, 12, 23-5 et 29-30.

Sire James de Normandye e ces quinze compaignouns chevalers se armerent molt richement e tot de blaunche armure, e 
furent tous noblement mountéz de blancz destrers (...) ib., 70, $23-6$.

chascun fu bien armé et sist sor bon destrier, Rou, II, 3222 .

«Vez le vos la as armes, sor cel ferrant destrier, Parise, 1901.

"Si encore un jor le truis en tornei,

« Ke mun destrer puisse acurser vers lui, Otinel, 72-3.

« Et soient en sa robe .IIII.c. compagnon

"Adobez de lor armes sor les destriers gascon.

(...)

«Que le voise servir a .m. vassaux en selle,

"Armez de bones armes sor destriers de Castelle ? Renaut, 77-8 et $243-4$.

« Barons, alez avant et si les desfiez. »

Et cil li respondirent : « Si com vos commandez. »

Des palefroiz descendent, es destriers sunt montez,

Cele part est venuz chascun d'elx toz armez.

(...)

Des palefroiz descendent, es destriers sunt montez, $i b ., 3225-$

8 et 4520 . Cf. passim.

"S'an nos assaut, trés bien nos desfandon. »

Et cil responent : «A Deu beneiçon! »

Chascuns descent del mulet arragon,

Es destriers montent sanz plus d'arestoison, Aymeri, 2736-9.

Ez vous Charlon poignant par la bataille,

Ne sambloit pas estre rois de frapaille :

Sor un destrier sist plus blanc que toaille,

Fort et seür et de tres fine taille,

Norris avoit esté en Cornouaille, Enfances O., 5401-5.

Or conmença ledit duc Nayme de Baviere de monter sur ung puissant destrier pour aler marcher sur les Sarrazins,

(...) Ogier, 20.

La veïssiez ces chevaliers garnir,

Ces blanz haubers endosser et vestir,

Ces palefrois et ces mulés guerpir,

Et veïssiez es bons destriers saillir. Mez, 8841-4. 
Mais il y a aussi un certain nombre d'exemples où le terme destrier désigne la monture servant à se déplacer, comme dans les fabliaux XI et LXII :

A tant ez-vos en la meson

Son ami qui l'est venuz querre ;

Du palefroi mist piet à terre,

(...)

Si me montez sor vo cheval ;

(...)

A tant ont veu avaler

Le chastelain sor son destrier, $M R, \mathrm{xI}, 151-2,178$ et 194-5.

S'encontrent .I. chapelain

Seur .I. balai palefroi amblant.

(...)

Si est montez sor le destrier,

Mès trop li sont cort li estrier, ib., LXII, 56-7 et 205-6.

Dans Erec, Mort Artu et Floriant, nous voyons le terme employé dans la description de scènes de chasse, dans Floriant même pour désigner la monture d'un écuyer sorti à cheval pour son plaisir :

Sor un destrier estoit montez : Erec, 94.

Si avint que li veneeur le roi chaçoient un grant cerf et l'avoient acueilli en la forest ; il vint a la fonteinne por estanchier sa soif, car assez avoit esté chaciez d'une part et d'autre. Et quant il se $\mathrm{fu}$ feruz en la fonteinne, uns archiers qui estoit montez seur un grant destrier et venoit grant piece devant touz les autres, quant il fu auques pres de li, si trest cele part por lui ferir par mi le piz ; Mort Artu, 64, 27-35.

S'en vait li cers, s'est ens entré.

$\mathrm{Li}$ rois est au palais venus,

S'est de son destrier descendus, Floriant, 8208-10. Cf. ib., 36770.

A tant ez vous .I. escuier

Qui chevauchoit .I. bon destrier,

Le roi salua hautement,

Puis li dist : «Sire, a moi entent.

J'estoie alez esbannoier

Jouste ce bois sor mon destrier

Si encontrai .I. païssant, $i b .$, 3627-33. 
Dans le fabliau de Berengier au lonc cul, c'est d'abord un chevalier, plus tard sa femme, qui monte un destrier ; Eremborc qui désire se battre aux côtés de son mari Renier et de son filleul Jourdain refuse le palefroi, qui est la monture normale des dames - elle préfère un bon destrier :

Puis s'en montois sor .I. destrier;

(...)

El destrier monte, si s'en va,

(...)

Atant sor son cheval remonte

La dame, et en meson s'en va. $M R$, XcIII, 17, 146 et 232-3.

Va s'an Jordains qui gaires ne se targe.

Damme Erenbors la cortoise et la large

Ne voloit mie que sans li en alaissent.

N'en mainne mie palefrois qui soit lasches,

Ainz est montee le bon destrier d'Arrabe,

En son poing tint un roit espié qui taille. Jourdain, 965-70.

Dans Amadas, on place le héros blessé sur un destrier qui marche tout doucement ; Eneas fait transporter le cadavre de Pallas dans une litière portéee par quatre destriers ; le duc Richart, prisonnier, est lié sur un destrier et emmené comme une marchandise :

A tant ont pris le damoisel

A fine force, et a martire

Le font liier et a grant ire ;

Angousseus en sont et dolent

Puis l'ont monté isnelement

Sus un souef amblant destrier,

Derriere lui un cevalier Amadas, 1902-8.

Colchier lo fist sor la litiere, quatre destriers ot a la biere, desor lui mist un covertor Eneas, 6125-7.

As diz ont toz lor armes despoillié ;

Le duc Richart i ont pris et leié.

Tot altresi come cofre en somier

L'en ont mené sor un corant destrier.

De ci a l'ost ne voldrent atargier. Couronnement, 2184-8.

Dans Renaut, dans Roche et dans Inconnu, un messager arrive sur un destrier rapide. Mais il faut noter aussi que, plus loin, le dernier de ces trois textes souligne que des chevaliers qui ne portent 
ni armes ni boucliers sont montés sur des palefrois et non sur des destriers : ceux-ci servent donc indubitablement à la bataille :

«Eslisons .I. mesage, Flamenc ou Berruiier,

«Et si soit bien armez sor son corrant destrier, Renaut, 115-6.

Et uns mesage vint poignant tot abrivez

Sor .I. fauve destrier qui estoit pomelez, ib., 980-1.

A tant es vos .j. mès sor .j. corrant destrier ; Roche, $2431=i b$., 2455.

A tant es vos un mesagier,

Qui vient avant sor son destrier, Inconnu, 71-2.

Quatre jornees, voire plus,

Avoit chevauchié la roïne,

Quant a l'issir d'une gaudine

A trové quatre chevaliers

Sor lor palefrois, sans destriers ;

Escus ne armes ne portoient, $i b .$, 5058-63.

Dans Tristan de N., nous voyons le jeune Doon se plaindre de ne pas avoir de destrier pour participer à une joute - mais pourquoi fait-il la distinction entre un destrier et un arragon, car normalement (comme nous le montrent p. ex. les extraits de Mez et de Barbastre) ce dernier terme lui aussi désigne un cheval de bataille ? Est-ce la rime qui est en jeu?

Si vous donrai .xx. destriers d'Arragon

Et .ii. somiers chargiez de bons mangons. » Mez, 13494-5.

Et Guibert lesse corre, si fiert Anbefelon

Que tot abat ensemble et lui et l'arragon. Barbastre, 5991-2.

Le bastart de Nanteul fut plain de marrison

De ce qu'i n'eust monnoye qui voulsist se poy non,

Në armeüre nulle, ne destrier n'arragon

Pour aller a la jouste conquerre l'arragon. Tristan de N., 5080-3.

Dans Aiol, nous avons trouvé une scène avec des larrons traînés à la queue de destriers, de même que Huon mentionne cette forme de punition; nous notons que, dans les deux cas, notre terme se trouve à la rime : 
Il vinrent as larons, nes vaurent laisier :

Cele part les trainent as keues des destriers ; Aiol, 7006-7.

Je jugeroie, si me puist Dieu aidier,

Se lez voullez vous trestuit ottroier,

Que Hue rait son paiis et son fiez,

Et Gerart soit traÿnnés a destrier, Huon, 9985-8.

e. doine.

Dans son dictionnaire de l'ancien français, A.-J. Greimas ne donne ce terme que comme adjectif signifiant « avare ». Dans le sens de « cheval », il doit être bien rare car nous n'en avons trouvé qu'une seule occurrence, dans Barbastre, où il se trouve parallèlement à bauçant, bai corant, arabi corant et aux noms de cheval Liart, Atengnant, Baligant et Bruiant. Le sens ne fait aucun doute : il s'agit bien d'un cheval de bataille, mais nous ne savons pas du tout si le terme indique des qualités particulières - l'épithète poisant se trouverait avec n'importe quel autre terme de cheval de bataille.

Aymeris s'est armé, si sailli el baucent,

Et Guillaumes d'Orenge desor le bai corant,

Bueves des Conmarchis sor le doine poisant,

Et Sanses sor Liart, et Hue en Atengnant,

Renier de Monhermer sor le vair Baligant,

Navari de Toulouse desor le sor Bruiant,

Et Geufroi l'Engevin sor l'arrabi corant, Barbastre, 172-9.

\section{f. estalon.}

Nous n'avons relevé qu'une occurrence du terme ; il désigne évidemment un cheval de bataille (voir pp. 14 et 34). Quant à l'affirmation de Jean Froissart que les Allemands nourrissent mal leurs chevaux de bataille, nous ne l'avons trouvée confirmée nulle part dans nos textes. Friedrich Bangert n'a pas trouvé d'occurrences du terme dans les chansons de geste. C'est pourquoi il prétend, op. cit., p. 21, qu'il n'y existe pas de terme pour désigner le cheval mâle et que l'on s'est servi du terme destrier par opposition à jument.

Et se li cheval fuissent fade,

Malagrené et mal nouri,

Si com cil estalon pouri

Sur quoi uns alemans tournoie,

Il feussent cheü en la voie, Méliador, 10751-5.

g. gazele.

Un seul exemple, tiré de Barbastre. Kurt Baldinger, Dictionnaire étymologique de l'ancien français, $\mathrm{G}_{3}, 1974$, cite, p. 420, le même 
extrait et demande : « animal exotique ?, ou le cheval comparé à une gazelle?» :

Estes vos un paien pongnant parmi les prez,

Desus une gazele fu s'ensengne levez, Barbastre, 6624-5.

h. genet.

Le terme, rare, désigne un petit cheval espagnol.

Le roy lui envoia un tresbel et tres puissant coursier puillois et deux tresbaus genez de l'Andelosie, (...) Jehan de S., 135, 25-7.

(...), messire Enguerrand lui envoya un tres bel coursier d'Espaigne et un tresbel genet de l'Andelosie, (...) $i b ., 136,9-11$.

\section{i. gramadone.}

G. Perrie Williams, dans une note à la page 211 de son édition du Bel Inconnu, écrit au sujet de ce terme : " nom d'espèce ou nom propre de cheval (?). » Nous n'avons trouvé que l'exemple dont il parle. Faut-il approcher le terme de doine, peut-être précédé de " grand »? Quoi qu'il en soit, il est question d'un cheval de bataille :

Sor gramadone fu armés. Inconnu, 1135.

\section{j. haquenée.}

Un autre terme assez rare :

Puis monterent li chevalier

Qui vont moult bien apparillier,

Sus une hagenée amblant,

Hermondine au tres doulz samblant. Méliador, 1008-11. Cf.

ib., 3198-200.

(...) trois cens escus dont les $C$ seront pour une bonne hacquenee ou pour un bon cheval que premiers donnerez a Madame (...) Jehan de S., 72, 17-9. Cf. ib., 71, 23-4 et passim.

Les deux heraulx estoent tous deux vestus d'ung riche drap d'or, montez sur deux acquenees blanches, (...) Jehan de P., 45, 6-7. Cf. ib., 62, 23-6.

(...) ; dont le Jouvencel ne fist que rire et dist qu'il avoit grant envie de chevaucher hacquenée et que onques convoitise ne fut bonne. Jouvencel, II, 132. 
La bonne religieuse a pou s'elle osa disner a son aise, tant avoit haste de nuncier a madame ces nouvelles; et a l'ayde de sa bonne hacquenée, et du grand desir qu'el a d'estre a l'ostel, s'avança si bien que madame l'abbesse fut trestoute esbahie de si tost la revoir. Cent, 21, 38-43.

k. iocor.

Encore un terme que nous n'avons trouvé que dans un seul texte. Wendelin Foerster, le premier éditeur de Li Chevaliers as deus espees, note à la page 394 de son livre : « 2679 iocor vom Pferde, ebenso 3487, mir unbekannt (...) » Nous n'y voyons pas plus clair que Foerster. L'adjectif «blanc », employé dans les deux occurrences, indique peut-être qu'il s'agit d'un cheval très apprécié :

Commanda a son escuier

Le blanc iocor atorner, Espees, 2678-9.

Puis dist k'il uoist apareillier

Le blanc iocor et l'amaint

En cil iardin. Cil ne se faint

De li faire ses volentes. $i b .$, 3486-9.

1. ive.

Le terme ive (< equa) a été éliminé par son synonyme jument (Jacqueline Picoche, op. cit., p. 272). Il est employé beaucoup moins fréquemment que celui-ci.

Voici les occurrences que nous en avons relevées. Dans les trois premiers exemples, de Thèbes et de Gui de B., sont soulignés le fait de mettre au monde un poulain et les circonstances surnaturelles de l'engendrement. Deux exemples montrent que l'on utilise les ives pour la punition :

Il ot cheval Thideüs,

qui valoit bien cent mars et plus,

ne tel cheval ne nasqui d'ive

pour corre par terre une live! Thèbes, 8565-8.

Un cheval a qui mout vet tost ;

sor mer fu nez, en haute rive,

engendrez de noitun et d'yve.

Noitum marin estoit son pere,

yve sauvage fu sa mere.

Quant vint au terme qu'il dut nestre,

lez unne roche, sor senestre

se torna l'yve a quelque paine ;

de lez la roche en mi l'araine 
sor l'erbe fresche et sor le fain

se delivra de cel poulain. ib., 8968-78. Cf. $i b ., 8995-6$.

Et fu d'une ive fiere et de tygre engendré,

Qui ne menjue [mie] d'avoine ne de blé, Gui de B., 2338-9.

« Il te fera ou col une grant hart noer,

«A la queue d'une yve te fera traïner. $i b .$, 1931-2.

A la coue d'une ieve li tient le chaon,

Par rues le traïne[nt] [et] aval et amont ; Roche, 4024-5.

Ja ne tornerez cele part

Que trop volentiers ne vos sive.

Car fust ore li ronchis ive

Que l'escuier tolu avez! Perceval, 7152-5.

Aymeri, qui présente quatre exemples du terme, l'oppose d'abord à cheval ayant probablement ici le sens d'étalon, mais montre plus tard que l'ive sert régulièrement comme cheval de bataille. Voir à ce propos jument, p. $4^{2}$ :

Tel i ot ive a queue recopée

Ou haut cheval a grant teste levée. Aymeri, 1631-2.

Et Savariz qui se fu trez arriere,

Esperonna sa grant ive corsiere

Et vait ferir Hugon par de derriere ib., 1762-4. Cf. ib., 1777-8 ;

1898-9.

m. jument.

La conversation entre le loup et la jument, dans Renart, nous montre que la jument travaille pour le paysan : elle est habituée au travail pour un maigre salaire et ne se laisse pas tenter par la promesse vague de froment, d'avoine ou d'orge que lui fait le loup - elle préfère continuer à paître " près d'un blé » (en plus, il ne faut pas oublier les mauvaises expériences avec le loup qu'ont les animaux domestiques) :

se volez estre en ma conpaigne,

nos ferïons mout grant gahaigne ;

assez vos donroie a mangier

de quel que avrïez plus chier,

ou bon froment, ou bone avaine,

ou bon orge, a quel que paine ; 
(...)

Car vos porpansez, damoisele,

de ce vilain qui si vos tue

et vos fait traire a la charrue ;

(...)

si serez fors d'autrui dangier :

ne vos estovra charrïer,

ne ça ne la porter nul fais ;

a toz jorz mais vivroiz en pais.

- Sire Isangrin, se je peüsse,

vo conpaignie chier eüsse,

mais je ne puis corre n'aler,

por ce voil ici pasturer :

de mon pié destre par derriere

passai ier en une charriere,

une espine me feri enz. Renart, v, 15009-14, 15018-20, 15027-

37.

D'autres textes nous présentent des situations semblables (voir aussi p. 255, Wistasse, 198-207) :

C'est uns vilains, c'est .I. bouviers ;

Les jumenz seut chacier devant ; $M R$, xxIv, 698-9.

Jadis estoit uns vilains riches,

Qui mout estoit avers et chiches ;

Une charrue adès avoit,

Tos tens par lui la maintenoit

D'une jument et d'un roncin ; $i b .$, LxxIv, 1-5.

Lés un fossé passe, si voit

Une jument paissant au fons ;

Li fossés n'iert lés ne parfons.

U li jumens paist en celée

Illuec gisoit, tieste clinée,

.I. vilains ki entour son brac

De son cavestre ot fait .I. lac,

Por se jument tenir plus choie.

Chius ki portoit le prestre en voie,

Arieste lés le jumentiele. $i b .$, LxxxIx, 474-83.

Atant prent Galahos de luy congié et s'en vait chevauchant dusques au bas vespre tant que il ataint vachiers qui estoient vestu de robes de religion; (...) Et li uns dist que il li mousteroit. Lors met el trot une grant jument sor coi il seoit (...) Lancelot, III, xxx, 16-7. Cf. ib., I, xxI, 5. 
La jument est sans aucun doute une bête de somme. Pourtant nous la voyons aussi utilisée pour le voyage - mais pas par des nobles. Lorsque Guillaume au court nez veut faire croire qu'il est un marchand, il monte sur une jument :

Et chevaucha une jument molt foible ; Charroi, 1042.

Li clerc ont tost l'uis desfermé,

Si ont le sac dedanz gité :

Après ont mis en un prael

La jumant, joste le choisel.

(...)

Le sac lieve sor la jumant

O sa fame qui li aida,

(...)

Qant durent prendre lo fromant,

Ne trovent ne sac ne jumant. $M R$, cxix, $61-4,94^{-5}$ et $105^{-6}$.

Vos dirai d'un provoire .I. conte,

Si con GUERINS le nos raconte,

Qui au marchié voloit aler :

Sa jument a fait ensseler,

Qui granz estoit et bien peüe :

.II. ans l'ot li prestres tenue ;

$\mathrm{N}^{\prime}$ avoit gaires ne soi ne fain.

Assez avoit aveine et fain. $i b .$, xcII, 3-10.

D'un prestre conte qui s'esmut

A un marchié o aler dut.

Sa jumant a fait enseler

(...)

Et li prestres tot à droiture

Sa jumant i a fait ganchir, $i b .$, cxIII, 1-3 et 14-5.

Atant ez voz, si con moi samble,

Un provoire sanz plus de gent,

Qui chevalchoit une jument, ib., cXLVII, 252-4.

Un extrait du fabliau des .IIII. souhais saint Martin nous semble prouver que le terme peut désigner tout simplement un cheval. C'est un vilain qui parle et, comme nous l'avons vu plus haut, son cheval est normalement une jument, par conséquent il lui est naturel d'appeler un cheval jument :

Se deïssiez que fusse un ours,

Ou asnes, ou chievre, ou jument,

Jel seroie tout esraument. $M R$, cxxxIII, 82-4. 
Dans Tristan de $N$. et Laurin, nous avons relevé des exemples qui nous semblent la preuve de la distinction entre " cheval de bataille » et « cheval de travail ». Le terme cheval a ici, à notre avis, le sens d'étalon, préféré pour les tournois et les guerres (cf. pp. 14 et 34) et étant par conséquent le cheval par excellence des chevaliers, tandis que la jument est celui des paysans. Mais Aliscans montre qu'une jument peut très bien servir pour le combat; le texte se sent pourtant obligé de souligner la qualité extraordinaire de l'animal en question, ce qui nous fait penser que la situation est exceptionnelle. Voir aussi brehaigne :

Du cheval dessendi et la belle combra,

Jus du cheval la mist et Tristan s'avalla,

Sans cheval ne jument en place demoura, Tristan de N.,

10421-3.

Adont n'espargna il ne cheval ne jument : (...) Laurin, 9135-6.

Margot venoit mout aïreement,

N'ot pas destrier, ainz chevauche jument ;

Ne la donast por .m. livres d'argent,

Et l'un et l'autre sont noir com arrement.

Plus tost cort l'ive ne vole oisel volant ; Aliscans, 5924-8.

Lorsque les juments étaient devenues trop vieilles pour accomplir les durs travaux, on les abattait et en mangeait la viande - mais sans l'apprécier beaucoup, comme le montrent le fabliau De deux Angloys et de l'anel et un proverbe :

Il sanble char de viel jument.

Se fu asnel que ge voi ci,

Ainz fu anel vostre merci. $M R$, xLVI, 88-90.

Mauveise viande feit vielle jument. Proverbes, 1210.

Une seule occurrence du terme, dans le fabliau cxxinI, nous montre une jument avec un poulain ; Aiol emploie jument comme une injure :

Li blans jumens an capelain

Gist là hors atout .I. polains, $M R$, cxxiII, 433-4.

«Va t'en de chi, Lonbart, li cors Dei mal te fache !

« Tant as mangiet compeus de soris et de rates,

« Et tant de le composte, de presure et de rapes,

«Jument me sambles plain(s) u asne [u porc] u vache. Aiol, 8860-3. 
Il y a certainement lieu de souligner qu'à peu d'exceptions près, les exemples du terme jument que nous venons de présenter sont tirés de textes décrivant la vie du peuple, spécialement des paysans. Ceci met en relief le fait que la jument était, à côté du bœuf, l'animal du travail champêtre et ne servait que rarement à d'autres tâches. Ceci corrobore les affirmations de Fr. Schmidt qui écrit, op. cit., p. 11, que la jument est souvent mentionnée à côté du roncin (voir le fabliau Lxxiv ci-dessus) et qu'elle est méprisée comme celui-ci parce qu'elle est la bête de travail du paysan.

n. palefroi.

É. Littré, op. cit., III, p. 909, nous donne une explication détaillée du terme et de son emploi dans l'ancienne langue : "Cheval de parade. On distinguait anciennement les chevaux en destriers, qui étaient les chevaux de bataille; en palefrois, qui étaient les chevaux de marche ordinaire pour les voyages; et en roussins, qui étaient les chevaux de somme et de travail. (...) Il se disait surtout des chevaux que montaient les dames. " Le Dictionnaire Général, p. 166o, est beaucoup plus court : «Anciennt. Cheval de marche (par opposition à destrier). » A.-J. Greimas et R. Grandsaignes d'Hauterive écrivent tous les deux " Cheval de marche, ou de parade ».

C'est bien avec le sens de " cheval de marche " que nos textes présentent le terme. En voici quelques exemples :

Et enmena avoeuc li Galaad qui a mervelles estoit biaus enfes de son aage ; si le porta uns esquiers devant lui sour un palefroi fort et isnel et bien portant. TP, vI, 24-7.

Et s'il velt a cheval monter,

Si lo fai monter davant toi.

Ne voil qu'ait autre palefroi

Fors che lo tuen, ne autre ator ; Joufroi, 2568-71. Cf. ib., 2859-62.

En son palefroi est montez,

S'en est a les noces alez; ib., 3535-6. Cf. ib., 3608-10.

Jouglés, qui ot la matinee

dormi a Tref come borjois,

vint sor un palefroi norrois

aprés le gentil bacheler. Dole, 2174-7. Cf. ib., 2434-6.

Or est le roy Ganors ens es pors arrivés ;

.I. palefroi li fu maintenant aprestéz ;

Par son estrief à or li est le roy montéz,

Dame Aye seur .I. mul qui bien fu afeutréz. 
Il ont tant chevauchié qu'il viennent as degrés ; Gui de N.,

75-9.

Mais Hector les convoia hors de la salle tant qu'il monterent en lor pallefrein, si s'en vont avau la ville, (...) Troie, 87, 6-8.

Voir aussi : $M R$, xvI, 81-2 ; LXII, 56-7 ; Aiol, 1259-60 ; Barbastre, 5760-1 ; Ipomedon, $361 ; 10407$ et passim ; Fouke, 51, 28-30 ; Claris, 5740-1 ; Lycorne, 2788-9 ; Protheselaus, 2908-9 ; 9427-9 ; Papegau, 7, $24 ; 17,12$ et passim ; Orson, $28 ; 1264 ;$ Rou, I, 262 ; II, 3037-8 ; Narbonnais, 672-4 ; 2372 ; Godin, 11941-2 ; 12506 ; 14430 ; Mort Aymeri, $571 ;$ Laurin, $7335 ; 12445 ; 12615$ et passim.

Il est vrai aussi que les femmes montent sur des palefrois; dans ces cas-là, les chevaux sont souvent connus pour leur marche agréable. En voici quelques-uns des très nombreux exemples tirés de nos textes :

Quant ele fu a tere venue, ele monta sour un parlefroi ki mout estoit biaus et cointes et ricement apareilliés de toutes coses, (...) TP, II, 80, 4-6.

(...), il lour avint adonc k'il encontrerent une damoisele ki venoit toute seule, cevauçant sour un grant palefroi ferrant tout le grant cemin de la forest, si grant oirre com ele pooit traire de l'ambleüre du palefroi. $i b .$, II, 89, 12-5.

Une porpre d'or estelee

Ot vestue, si fu orlee

Tout entor a bendes d'orfroi,

Et sist sor un blanc parlefroi

Qui molt venoit soef amblant. Cont. P., I, 1981-5. Cf. ib., I, 3132-3.

Et la pucele isnelement

Descent et son palefroi laisse, ib., II, 7460-1. Cf. $i b .$, II, 9837-8 ; II, 9843-4 ; II, 13892-5.

Quant il les vit molt en fu liés,

Car entre eles conut s'amie

Qui menoit cele conpaignie

Et sist sor un blanc palefroi ;

Souef anbloit et sans desroi,

El blanc fu de noir pumelés, Inconnu, 3942-7. Cf. ib., 147-8 ; 167-9. 
Li palefrois si se desroie,

Sour qu'ele siet, a desmesure,

Mais si souef va l'ambleüre

Que daintiés est de l'esgarder. Amadas, 4706-9.

(...), es vous venant grant oirre une damoisiele monte[e] seur un blanc petit palefroi, (...) Merlin, II, 110.

Delez le tre ai je fait atachier

Un palefroi ambleour bel et chier,

A frain d'orfroi et a sele a or mier,

Sor quoi ferés la pucele puier,

Se nous povez conquerre au bran d'acier ; Enfances O., 2682-

6. Cf. $i b ., 8146-7$.

Ayglentine la bele ne se vout atargier,

Isnelement monta seur .I. palefroi chier - Gui de N., 984-5.

Cf. $i b ., 1359$.

Et chevauchoit un palefroi

que l'autrier li tramist un roi.

Mout bien emblant et bien delivres,

son pris estoit de cinc cent livres,

et fut touz noirs, fors que les hanches

et les espaules qu'il ot blanches,

et les costez et les oreilles

et les jambes qui sont vermeilles. Thèbes, 4069-76. Cf. ib.,

4097-100.

Dans un nombre considérable des exemples où une dame monte sur un palefroi, l'épithète norrois s'accole à notre terme. Nous supposons qu'il est question de petits chevaux norvégiens (d'autant plus qu'il y a aussi des occurrences avec l'épithète petit). Il y a quelques rares occurrences avec les épithètes gascon et breton, mais les palefrois norrois sont de toute évidence les montures préférées des dames. Nous avons trouvé quelques exemples où ils sont montés par des hommes (voir supra, Dole, 2174-7) :

Mais, ensi qu'il se combatoient, atant es vous vers aus venir une damoisele sour un palefroi noirois (...) TP, VI, 118, 21-2.

Une courtoise damoisele,

Qui molt ert avenanz et bele,

Desus .I. palefroi norrois, Claris, 810-2. Cf. ib., 23718-9.

Gui dist à Ayglentine, le preus et le courtois :

'Damoisele, brochiés le palefroi norrois - Gui de N., 1487-8. 
Rois Loth et rois Baudemagus

Sont vers Blanchandine venus,

Par la regne la vont prenant

Del noirois palefrois amblant. Floriant, 6007-10.

Desor un palefroi norrois Blancandin, 685 .

A tant en vont andui ensamble,

La mule la damoisele amble

Plus tost c'uns palefrois norois. Cont. P., I, 1887-9.

De l'un un palefroi norrois Erec, 2386. Cf. ib., $2615 ; 4126$.

Voir aussi : Cont. P., III, 14843-5 ; MR, III, 186-7 ; cxxv, 134-5 ; TP, I, 178, 23-4 ; I, 180, 56-8; III, 5, 7-9 ; III, 185, 5-6 et passim ; Joufroi, 2139-44 ; Rigomer, 37-40 ; 110-1；1391-7 ; Cleomadés, 5749-54 ; 5760-2 ; Meliacin, 7654-5 ; 13351-2 ; Ille, 1051 ; Berte, 197-9 ; Helcanus, $8 \mathrm{a} ; 130$; 177 ; Athis, 7120-1 ; Lycorne, 3513-4 ; Protheselaus, 6155-6 ; 11451-2 ; Yder, 2629-30 ; 3182-3 ; Lion, 8785-6 ; 32739-40 ; Tristan de N., 4441-2 ; 15299 ; Auberon, 871-4 ; Eracle, 46 ; Couci, 6210-2 ; Violette, 783-4 ; Lancelot, vII, Ia, 16 et passim ; Queste, 12, 22-4 ; 129, 28-9 ; Troie 65, 20-1 ; Deduis, 10435-43 ; Wistasse, 1199200 ; Mort Artu, 95, 13-4 ; 96, 1 ; 130, 54-6 ; 170, 4 ; Atre, 1504-5 ; 2495-6 et passim; Laurin, 5681-3 ; 5817-8 et passim ; Charrete, 202-4 ; 733-4 et passim ; Perceval, 6715-6 ; 7067-9 ; Méliador, 2214 ; 7823 et passim ; Blancandin, 541-2 ; Lais, v, 472-3 ; 548-52 ; Erec, 79-80 ; 1178 ; Papegau, 6, 34-5.

Dans plusieurs textes il y a des exemples où deux palefrois portent une litière. Il peut être question d'y transporter des blessés, des dames etc. :

Quant il ot ensi une piece esté, ne demoura pas granment qu'il vit venir une litiere $u$ gisoit uns cevaliers que doi palefroi portoient. TP, VIII, 15, 1-3.

Et une litiere menoient

Couuerte d'un uermeil samit,

S'erent doi palefroi petit

Atele deuant et deriers. Espees, 1902-5.

Et uns seus cheualiers gisoit

Entre eles en une litiere,

Rice et noble de grant maniere,

Sor .ij. blans petis palefrois

Si dolereus et si destrois $i b ., 9250-4$. 
Blancheflour la roÿne ont en litiere mis

Entre deus palefrois qui furent de grant pris,

Car ne pot chevauchier, tant fu ses cuers maris. Berte, 2367-9.

Et le quens de Namur grant destresse endura,

Dessus une litiere tantost on l'emporta. Tristan de N., 20583-4.

Et quant il s'est grant piece esperiz, si voit venir, en une litiere que portoient dui palefroi, un chevalier malade qui mout se plaignoit angoisseusement. Queste, 58, 15-7.

Et prie de bon cuer a Dieu

Qu'il le respast. A tant l'ont pris

Entre lor braz, el lit l'ont mis

Sor .II. chevaus qui n'erent pas

Bredif. A tant s'en vont le pas Meraugis, 4746-50.

Quant li rois aproche de la forest a mains que l'en ne trairoit d'un arc a .III. fies, si en voit hors issir une litiere sor .II. palefrois qui tost et soef le portent. (...) Et quant ele aproche, si voit dedens .I. chevalier armé de toutes armes ne mais que d'escu et de hiaume dont il n'a point. Li chevaliers fu navrés de .II. tranchans de lanches (...) Lancelot, viI, xxira, 2.

Atant a s'espee chainte, si lache son hiaume isnelement et fait ateler .II. palefrois a une litiere qu'il avoit fait faire tantost, si fait lever le cors son fil, car laissier nel i vaudra pas. ib., vII, xIva, 31 et passim.

Dans Jehan, deux palefrois servent de chevaux de somme; une quarantaine de vers plus loin, ils sont appelés sommiers (cf. pourtant p. 75) :

Car deus palefrois biaus et grans

A fait chargier d'estrelins blans,

Si le fist a Jehan donner, Jehan, 1991-3.

Robins en mena un sommier

Et un autre li messagiers $i b ., 2035-6$.

Un exemple de Rigomer montre que les palefrois n'étaient pas vraiment doués pour les batailles (cf. p. 35, Renaut, 3225-8), et Gauvain s'étonne de trouver une lance et un bouclier (signes de combat) près d'un chêne auprès duquel il voit un petit palefroi : à son avis, palefroi et armes ne vont pas bien ensemble. La même opinion se retrouve dans Eliduc, où les chevaliers désarmés se 
déplacent sur des palefrois. Mais dans Aspremont nous lisons que, faute de mieux, c'est-à-dire de destriers (ici appelés aussi cheval), on peut toujours prendre des palefrois - sans qu'on ait besoin de parler d'humiliation, comme le fait Jean Flori, op. cit., p. 105 : "Combattre avec une mule, et plus encore un palefroi (cheval de parade) ou, pire, un roncin ou un sommier (chevaux de trait ou de somme) constitue pour un chevalier une humiliation que les plus démunis ne peuvent parfois éviter. »

Il seoit sour .I. palefroi

Qui auques fu de grans errois,

Si s'abuisa tant qu'il cäi,

Et li chevaliers autresi Rigomer, 8719-22.

$\mathrm{Au}$ chaisne vit un escu pendre

Et dalez une lance droite.

D'esrer vers le chaisne s'esploite

Tant que dalez le chesne vit

Un palefroi noiret petit,

Si li vint molt a grant merveille,

Que ce n'est pas choses pareille,

Que pas n'avienent, ce li samble,

Armes et palefrois ensamble.

Se li palefrois fust chevax,

Dont quidast il qu'aucuns vassax,

Qui por son los et por son pris

Alast errant par le païs,

Eüst montee cele angarde. Perceval, 6526-39.

Quant il avrunt fet lur eschec,

Si returnerunt par ilec ;

Desarmez sur lur palefrez

S'en revunt [il] soventefez, Lais, xII, 177-80.

Dites lor tot que il vignent o moi :

Qui n'a ceval si monte en palefroi

Ou il acore a pie tot le sabloi. " Aspremont, 4852-4.

El palefrois monta qui n'a destrier : ib., 4958.

Nous pensons que l'extrait suivant, de Lancelot, donne une idée de la situation normale des préparatifs d'un combat: le chevalier Lancelot se dirige vers la cour monté sur un palefroi, un écuyer le suit avec son cheval « en destre », c'est-à-dire avec son destrier :

Et qant il est toz armez fors de son chief et de ses mains, si monte sor un palefroi et s'en vait a la cort grant aleure, (...) ; si 
fu Lyonyaus ses coisins delez lui qui li porte son escu et son hiaume, et uns autres escuiers li porte son glaive et maine son cheval en destre. Lancelot, III, IV, 1.

Par contre, on semble bien avoir pu se servir des palefrois pour la chasse, comme nous le montre l'extrait suivant de Renaut :

Sovent vont en riviere desor lor palefrois,

Portent ostors muez et faucons montenois

Donc prenent ces oisealx a merveillos esplois. Renaut, 2538-

40.

Même pour le voyage, les destriers sont parfois plus rapides que les palefrois. Nous le voyons dans l'extrait suivant d'Amadas, où les compagnons du protagoniste, pour attraper celui-ci, descendent des palefrois et montent sur les destriers :

Et cil les palefrois guerpirent

Et es destriers mult tost salirent. Amadas, 1839-40.

C'est dans ce même texte que se trouve la description du palefroi idéal : il est beau, il est blanc, il est rapide, il est agréable à monter :

Ne demoura c'un seul petit

C'un palefroi revit passer

Qui bien faisoit a regarder,

Car il n'estoit mie tondus,

Ains ert trop cointement crenus.

Grans ert et biaus, ce m'est avis,

N'ot si bel en trente païs ;

De cors, de membres ne de teste,

Ne vit nus hom si gente beste

Ne qui mix doie avoir bon los

De bonté, que mult a le dos

Combre a mesure pour porter

La sele a droit sans remüer

Costes et flans, crupe a raison

Large et lee sans mesprison,

Ample narine, les oels gros ;

Nes ert de gale et de souros,

De blancheur resanbloit ermine ;

En portraiture n'en cortine

N'en fu ainc nus de sa biauté,

Si vous di bien la verité.

Viste ciere ot comme d'orguel,

Col enarcie et large entroel ; 
La rue fait toute fremir

Et les cailliaus le fu salir ;

Tant par va tost a desmesure,

Si bel, si souef, l'ambleüre,

C'autres cevaus pas ne peüst

Si aler se si fait ne fust.

Nus ne le voit ki bien ne die

$C^{\prime}$ ainc tel beste ne fu en vie

Nul jor, a certes ne a gas. ib., 4174-205.

Anjou nous présente le comte de Bourges allant à pied, parce qu'il n'a pas de palefroi ; le texte se moque un peu de lui en décrivant le mauvais état de ses semelles et en les comparant à des palefrois mal ferrés :

Si fu legier a aprester

Pour cheminer et pour errer,

Quer pallefroi n'ot a fferrer

Ne coursier c'on li maine en destre,

Et en tel point souloit il estre ;

Ainz chevauche sus lez semelles,

Qui ne sont ne bonnes ne belles,

Quer les quaillox les ont trestoutes

Et dessireez et derroutes.

Mal est ferréz cilz pallefroiz, Anjou, 5592-601.

La distinction entre chevaux et palefrois se retrouve dans plusieurs textes. Cheval a probablement le sens de destrier :

Ore est Sagrenors en esfroi,

Car n'a cheval ne palefroi, Claris , 12143-4.

Car roys Laris lor fist donner

Palefroiz et chevaux et robes : $i b .29743-4$.

Hennissent cil cheval, cil mul, cil palefroi. Roche, 355.

Qui dontra mais destrier, hiaume, escu ne lance,

Palefroi ne cheval, fenon ne conoissance! Lanson, 3206-7.

Le palefroi a amené,

Si est le pucele montee ;

Et Gavains a sa teste armee,

Si est montés sor son ceval. Atre, 1504-7.

Si met la sanbue et la sele

Sor le palefroi la pucele 
Et puis ensele les chevals

Et si lor lace les poitrals ; Durmart, 2289-92. Cf. ib., 2995-6 ;

6625-30.

Et il fait la damoisele monter le palefroi et il remonte en son cheval et s'em part d'illuec. Lancelot, v, Lxxxvi, 3. Cf. ib., Iv, LXXXIII, 40.

Et chil est deschendus du palefroi ou il seoit, si est montés sor un cheval, $i b$., vII, xva, 25.

Ly chevaliers quant il le voit, Del palefroy est descendus, Encontre Biauté est venus.

Primierement l'a salüee

Et puis entre ses bras combree,

Sour son palefroy le leva,

Son cheval prist et sus monta,

A Biauté revint bonnement,

La resne del palefroy prent. Gliglois, 1234-42.

Durmart distingue nettement entre palefroi et destrier tout en affirmant qu'ils peuvent être de valeur égale :

Ne metent pas en nonchaloir

Le palefroi ne le destrier, Durmart, 3134-5.

Li rois sist sor un palefroi

Qui mout estoit riches et grans

Et beaz et fors et bien anblans ;

Mout valoit bien un bon destrier

Por un grant taz de gent percier. $i b .$, 7208-12.

L'extrait suivant, de L'Atre périlleux, présente la distinction «classique » : la demoiselle est montée sur un palefroi, le chevalier sur un destrier et le valet sur un roncin :

La damoisele fait monter

Que Gavains o lui amena

Sor un bon palefroi qu'ele a.

Gavains monta sor son destrier,

Et le vaslet, qui ert legier,

Et qui moult volentiers le fist,

La damoisele Escanor prist

De devant lui sor le roncin. Atre, 2494-501.

Pontieu distingue les palefrois des roncins et des sommiers : 
Palefrois et roncis et somiers je vous liverrai asés, et autre avoir. - Pontieu, 69-70.

Comme les destriers, les palefrois peuvent servir pour la punition (cf. pp. 38-39). L'exemple de Claris est particulier en ceci qu'il s'agit de la punition d'un mort pour lequel on montre le mépris en attachant sa tête coupée à la queue du palefroi et en la traînant sur le sol :

Mes en la fin torna a faille

Li chevaliers et fu ocis.

Gales li Chauz le chief a pris,

A la pucele en fet present ;

Et ele le prist bonement,

Par les cheveus le tire et sache ;

Et puis molt fierement l'atache

A la coe du palefroi ;

Lors broche et fiert par grant derroi

Le palefroi, sor quoi seoit ;

Le chef en tel point trainoit ; Claris, 23840-50.

Les palefrois de relais sont menés à droite, comme les destriers :

Cele li porte et si li mainne

An destre un palefroi mout buen. Yvain, 2976-7. Cf. ib., 3084-5.

\section{o. poulain.}

Dans toute une série d'exemples, le terme ne désigne pas « le petit d'un animal » (définition donnée par R. Grandsaignes d'Hauterive et d'A.-J. Greimas, op. cit.), mais plutôt un jeune cheval (6). Tel est le cas dans plusieurs fabliaux, dans lesquels on leur met des charges mêmes très lourdes (des cadavres) sur le dos, et aussi dans d'autres textes :

Atant va le poulain saisir ;

Si li a la sele sus mise :

(...)

Et li poulains en travers saut

Une heure bas, et autre haut,

Si que nus ne le puet tenir. $M R$, cxxiII, 442-3 et 453-5.

«Va, si m'ameine mon polain,

Se g'ai le moine dont lier

Ge cuit, g'en ferai chevalier. »

Martinot le polein ameine ;

(...) 
Et li poulein a tant erré

Que il est entrez en la porte. $i b .$, cxxxvi, 756-9 et 774-5.

Eracles coisist un poulain

qui quatre dens encor tenoit

et avoit canqu'il convenoit

a tel ceval con il demande.

(...)

Vint mars vous donrai del poulain ; Eracle, $1352-5$ et 1479.

Mout par est li poulains isniax, Thèbes, 9027.

Si savoit moult bien poindre ou cheval ou polain. Bâtard, 3847 .

Le poulain du fabliau cxxxvi est d'ailleurs appelé palefroi au vers 780 et cheval au vers 804, de même que celui dans Renart, où le terme désigne la monture d'un prêtre, est appelé cheval v. 11722, roncin v. 11809 et palefroi v. 11889 :

Lors li saut entre les arçons,

et li polains fu esfraez, Renart, Iv, 11826-7.

Les exemples suivants nous montrent que le poulain peut être un jeune animal désinvolte qui n'est pas encore habitué à la selle, ni aux éperons, ni au frein :

Il a ceans un poulain dru,

Qui mout destruit avoine et fainc

Ne onques n'ot sele ne frainc,

Et si ne fu poins d'esperons : $M R, \mathrm{CL}, 55^{2-5}$.

Petit poulain fu apellés

Par son non Morel le Desreés.

Unne moie suer le nourri,

Petit poulain et si vus di

Nus hons ne le chevaucera

Fors que vous et si vous fera

De grans honneurs qu'il se definne. Lycorne, 6213-9.

Nous remarquons que, tout comme pour jument, le terme apparaît presque uniquement dans des fabliaux, c'est-à-dire qu'il est question d'un terme ayant droit de cité surtout dans le monde bourgeois et paysan. Même dans Eracle, où poulain apparaît 24 fois, nous nous trouvons dans ce monde roturier, car le jeune Eracle achète le poulain extraordinaire à un paysan. 
p. poutrel.

Ce terme a le même sens que poulain : « jeune cheval ». Dans le second extrait de Roussillon, nous voyons un syntagme substantif à deux noyaux : cheval ferrant et poutrel. Il est à noter que le terme se trouve à l'assonance :

Cuit bien bons cevalers se desensel

U envers u a denz de son podrel Roussillon, 1577-8.

Portet escut e lance nou de Bordel[1],

E chevauche un cheval ferrant poutre[1] ; ib., 5233-4.

Li Sarrazins le referit isnel,

Mort l'abatit tantost de son poutrel. Jourdain, 1667-8.

Et, quant Milles le voit, si broche le poitrel, Florence, 4046. Cf. $i b ., 4050$.

.I. en i ot sor .I. poutriel

Fort et hardi et mout isnel. Rigomer, 13497-8. Cf. ib., 16380-1.

Chascun à son poueir a brochié le poutrel,

As roiaus vont jouster le pendant d'un vauchel - Gui de N., 1428-9.

poutrels orent de Capadoce, qui n'ont mehaig, jale ne boce, d'un merveillos haraz de mer, et molt sont legier a armer ; Eneas, 3935-8.

Quant a la porte fu dessendu du poutrel,

A la porte a feru grant cop d'un grant martel, Brun, 1879-80.

De Lyverie fu amenés poutrel,

Plus a durs ongles que n'est acier ne fer. Bueve, II, 1902-3. Cf. ib., 4949 ; III, $5960 ; 10648$.

Or fu Gautiers à pié enz an prael ;

Voit Amboyn, ne li fu mie bel,

Qui esloingniez li fu sor le potrel, Gaydon, 7963-5. Cf. ib., 9304.

Li aumachours chevauchoit un poutrel qui mout ambloit doucement et isnel. Godin, 8650-1. Cf. ib., 11021 . 
q. roncin.

A.-J. Greimas, op. cit., p. 572, définit ce terme ainsi : « Cheval de somme et de trait, généralement de peu de valeur : (...) », tandis que R. Grandsaignes d'Hauterive, op. cit., p. 520, écrit seulement "Cheval de charge et de trait ». Dans le tome ix de son édition de Lancelot, Alexandre Micha définit, p. 308 : " cheval de somme et de trait, c'est une monture dépréciée ".

Plusieurs exemples tirés de nos textes viennent corroborer ces définitions et l'on ne s'étonne pas de rencontrer les roncins dans un contexte roturier ; comme ces animaux ont peu de valeur, on les apprécie peu et les traite conformément. Nous trouvons d'ailleurs les roncins parallèlement à d'autres bêtes de somme, comme sommiers et mules/mulets.

Si menoit jarbes à loier

D'un roncinet de povre coust,

Qu'il avoit très devant aoust

Moult mal péu, et bien pené,

Et si en avoit amené

Son blé ainz l'aout, por l'orage.

Poi ot avaine, et poi forage,

Et por argent qu'il en vout prendre,

Se penssa qu'il le menra vendre ;

(...)

Li vilains son roncin atorne,

Et frote, et conroie, et estrille ;

En .I. blanc chevestre de tille,

Le maine sanz sele et sanz frain,

Bien sanble roncins mors de fain ;

(...)

Si en trait .I. roncins fors,

Qui n'estoit mie des plus fors

C'onques vi, ne des plus vaillanz,

Ainz estoit maigres et taillanz,

Dos brisié, mauvès por monter ;

Les costes li pot-on conter ;

Hauz ert derrière, et bas devant,

Si aloit d'un pied sousclochant,

Dont il n'estoit preu afaitiez ;

$\mathrm{N}^{\prime}$ estoit reveleus ne haitiez,

N'il n'avoit talent de hennir.

(...)

Mès vez ci roncin bien vendable,

Fols est qui le tient en estable ;

Bons est par tout où l'en l'aderce,

Bons en charrue, bons en erce, 
Et bons ès trais et ès limons, $M R$, xIII, 30-40, 44-8, 83-93 et 121-5. Cf. p. 40, ib., LXXIV, 1-5.

«Vostre chevaus n'est mie des miex corans :

«L'autre jor nen ert mie si rabiant,

«Ains resambloit ronchin a paisant, Aiol, 4228-30.

Tout sont mis a desconfiture

Quant Perchevaus par aventure

Vint par le forest chevalchant

Desor un noir ronchi bauchant,

Maigre, pelu, redois et las ; Cont. P., I, 4321-5.

E cil reporta un espé

Dunt li fers fut mut ruuillé,

E chevauche un megre runcin ; Ipomedon, 7781-3. Cf. ib., 8331-6.

(...), atant es vous venir sour un maigre et las rouchin trotant un vilain qui amenoit un sien fil, jovene enfant de l'aage de quinze ans, sour une povre jument. Merlin, II, 69.

Meis mis peres n'a, bien le sai,

Fors son destrier e un roncié, Yder, 713-4.

La pauvreté du jeune Durmart se trouve soulignée par le choix du terme roncin ; l'épithète " trottier », qui se retrouve dans Orson, indique à notre avis que le cheval est incapable d'aller au galop :

Entre lui et son escuier

N'avoient c'un ronci trotier. Durmart, 555-6.

A un garçon toli un viel roncin trotier,

Si s'an fuit a Biauvaiz tot le plenier santier. Orson, 2162-3.

Dans Perceval, Gregoreas emmène le bon destrier de Gauvain qui devra monter sur le roncin d'un valet; une trentaine de vers plus loin, nous lisons la description de ce roncin qui n'est certainement pas digne du chevalier célèbre. Un troisième extrait nous montre qu'il est complètement inapte comme cheval de bataille - il n'est pas étonnant que Gauvain s'en plaigne. Dans Méliador, le chevalier Gracien devant se battre descend du roncin et monte sur son coursier ; une scène semblable se trouve dans Aiol :

Le Gringalet je en menrai,

Que plus ne m'en puis or vengier ; 
$\mathrm{Au}$ ronchin le t'estuet cangier

Dont l'escuier as abatu, Perceval, 7136-9.

El ronchin ot molt laide beste :

Graisle ot le col, grosse la teste,

Longues oreilles et pendans ;

Et de viellece ot toz les dans,

Que l'une levre de le boche

De plain doit a l'autre n'atoche.

Les oex ot trobles et oscurs,

Les piés crapeus, les costez durs,

Toz depechiés a esperons.

Li ronchis fu maigres et lons,

S'ot maigre crupe et torte esquine. $i b .$, 7161-71.

Et mesire Gavains l'atent,

Si s'afiche si roidement

Sor les estriers que il en ront

Le senestre tot en roont ;

Et il a le destre guerpi,

S'atent le chevalier ensi

$\mathrm{C}^{\prime}$ onques li ronchis ne se muet,

N'esperoner tant ne le puet

Que il le puisse removoir.

«He ! las ! fait il, si mal seoir

Fait en ronchin a chevalier,

Quant il velt d'armes esploitier. » $i b ., 7335-46$.

Li chevaliers si bien s'esploite

Qu'il vient la, ne plus il ne targe,

Le glaive ou poing, ou col la targe,

Bien armés et faiticement,

Pour faire .I. grant commencement.

Sitost qu'il fu mis ou chemin,

Il descendi de son roncin.

Ses escuiers errant li baille

Son coursier ; il monte sans faille. Méliador, 4865-73.

Puis laisent les roncins ens el pré estraier.

Si saillirent es seles des auferans corsiers : Aiol, 4807-8.

La charrette sur laquelle monte Lancelot est tirée par un roncin :

(...) ; si vint cele part grant aleure, si ataint cele karete a quelque paine et voit sor les limons .I. nain cort et gros et reskignié qui cache a une corgie .I. viel ronchin qui estoit dedens les limons. Lancelot, III, XxxvI, 24. 
On trouve néanmoins un certain nombre de cas présentant une autre idée des roncins et qui montrent que ceux-ci peuvent même parfois servir de monture aux chevaliers (parfois à défaut de destrier) et aux dames; moins souvent nous les voyons employés pour la chasse. Nous constatons que ces roncins, qu'ils soient montés par des chevaliers ou par leurs valets, sont souvent dits grands et forts et rapides - le terme n'a donc pas toujours une connotation négative :

Es vos poingnant un veneor

Deseur un ronci caceor.

Ses ciens sivoit, son cor tenoit, Inconnu, 1305-7.

Et lors encontrerent .I. chevalier armé sor .I. grant ronchi. Lancelot, III, LI, 17.

Lors voit sor un ronci ferrant

Un veneor qui vient crïant

Aprés chiens qui courent le bois. Durmart, 3773-5.

(...), il li avint k'il encontra un cacheour, tout seul chevauchant sour un grant ronchin, et portoit deriere soi un chevrel toursé ; TP, IV, 79, 2-4.

A tant oï un damoisel Venir le trot sor son roncin,

Devers le bos tot le cemin ; Atre, 766-8.

Cil est montés sor son roncin,

Qui est grant et fort et isnel, ib., 1470-1. Cf. ib., 2155.

Baillerai toi un ronchi fort et cras, Bueve, III, 2423.

Mais Jehans hiaume n'escu n'ot

Ne tel cheval comm' ot li quens ;

Nepourquant ses roncins ert boens. Jehan, 4150-2.

Soit monté sur un gros roncin

Bas, bien trotant et bon et fin, Deduis, 10297-8.

Cil descent jus de son roncin $M R$, Lxx, 74 .

.II. et .II. montent es roncins

mis sire Guillaume et Boidins ; Dole, 1802-3.

« Signor, » che dist li maistres, « je voi .I. chevalier

«Armé et fervesti sor .I. ronchi trotier. Aiol, 1825-6. 
Or voz proi je et manaide et merceis

Por cel Seignor qui en la crois fu mis

Que me prestéz palefroi ou roncin,

Itele beste qui porter me poïst,

Et bonnes armes por mon cors garantir,

Si m'en irai combatre au Sarrazin. Jourdain, 1702-7.

Et Tybers et la vielle n'ont cure d'arrester,

Ains font Bertain lor dame sor un ronchi lever ; Berte, 540-1.

Mais il y a quand même beaucoup plus d'exemples où les roncins servent de monture aux valets ; en voici un petit nombre de ceux que nous avons relevés :

Car je voi ça venir corant

Seur .I. molt grant roncin ferrant

.I. vallet par mi une porte, $M R, \mathrm{LV}, 115^{-7}$.

Et avoec li chevauchoit uns esquiers sour un grant roncin, (...) TP, VI, 114, 7-8.

Quant li esquiers, qui illuec estoit, oï ceste aventure, il descent de son roncin et se laisse caoir a ses piés (...) ib., VI, 121, 1-2.

Et quant il est armez, si monte en son cheval et prant son escu et son hiaume et son glaive et se part de leanz et lui et son escuier sus son roncin, et s'en vait aprés lui. Lancelot, vI, cVI, 27. Cf. $i b .$, I, X, 4 ; I, XIII, 6 et passim.

- Sire, fait li vallés, montés sour mon ronchin qui est fors et remuans; se vous le poés conquerre, vostres soit li roncins et je menrai le ceval a mon signeur pour sauver ma vie. - Et ton roncin, fait Percevaus, conment ravroies tu ? car se je pooie le ceval gaaignier, de ton roncin n'aroie je puis cure. - Sire, fait li vallés, je vous suivrai a pié et se vous conquerés le cheval, je prendrai mon ronchin, et li cevaus soit vostres, ib., vIII, 45, 19-26.

« Ja est chou li vallès al bai ronci Aiol, 3256 .

Et avoeques lui ensement

Ot bien jusqu'a dis damoisiaus

Sour bons roncis fors et isniaus,

Et li vallet sont grant et fort, Amadas, 4276-9.

Il envoie tot le bessié,

sor un roncin rade et movant, 
un de ses escuiers avant

dire a la dame qu'il venoit Dole, 3254-7.

Dont rueve un escuier descendre

Et a Gliglois le ronchin prendre, Gliglois, 1315-6.

Et lors vint laiens sour un grant ronchin uns escuiiers

(...) Merlin, II, 200.

Voir aussi : Protheselaus, 11510-1; $12454 ;$ MR, CxLvII, 104-5 ; TP, VII, 9, 32-3 ; VII, 135, 1-2 ; VII, App., II, 1, 11-4 ; VIII, 44, 1-3 ; VIII, 46, 1-3 ; vIII, 46, 9-12 ; Gliglois, 2069-71 ; Aiol, 613-9 ; 4807-8 ; Joufroi, 2841-3 ; Queste, 35, 15-6 ;88, 22-4 ; 90, 5 et passim ; Charrete, 22837 ; Perceval, 6982-3 ; 7045-9 et passim ; Mort Artu, 41, 77-8 ; 91, 2931 ; 143, 7-9 ; Méliador, 18549-50 ; Erec, 145-6 ; Raguidel, 3912-3 ; Wistasse, 1028-9 ; 1095-6 ; Laurin, 12147-8.

Le chevalier Sarraz, après avoir été renversé de son destrier, est bafoué par la foule quand il est obligé de monter sur le roncin de son écuyer, donc sur une monture indigne d'un chevalier ; cette scène est à comparer à celle où Gauvain, prisonnier de Caradoc, est mis tout nu sur un roncin :

Si s'en revient a son escuier tot a pié, qui l'atandoit an l'oreille de la forest, si en fet .I. descendre et monte en son roncin. Et lors conmance la criee assez greingnor que devant, car il le voient sus le roncin monté, si li escrient : «Ha, dans mauvés chevalier recreant, vos estes ore, Deu merci, tant amandez que vos estes venus de destrier a roncin ! » Lancelot, v, xcIII, 39.

(...) : si dist li contes que quant il ot enloignié une lieue de la ou il l'ot pris, si le despoilla tot nu et le fist lever sor un roncin dur trotant, (...) ib., I, xIV, 1.

Les messagers, qui devaient certainement se déplacer vite, montent parfois des roncins. Dans ces cas-là, ceux-ci doivent naturellement être en bon état :

Atant se part de laiens, qu'il n'i fait autre delaiance, et vient a son roncin et monte, et devale de la roce, et fait tant qu'il vient jusc'a Palamidés. TP, vi, 27, 78-80.

Si se part de leanz et la roine li donna roncin beau et fort et le vesti bien an guise d'escuier, et s'en vait par mer et par terre tant qu'il vint a la cort Claudas l'andemain de Pasques. Lancelot, v, xcIV, 20. 
et s'il est a tornoi ne guerre

por ton roncin mengier, au querre

ne fines, si l'aies trové

et le brief de par moi mostré.

(...)

el roncin monte, si s'en torne

et se seigne a l'issir de l'uis. Dole, 891-4 et 9o6-7.

Sor .I. rocin ferrant font le serjant monter,

Se li ont fais sor sainz et plevir et jurer

Qu'il ne finera mais, si ert au dus clamez.

Li serjant s'an torna de la bone cité ;

Il a tant le rocin point et esperoné

Que il ataint le duc a un tertre monter. Parise, 2080-5.

Et li ualles ne targe mie,

Ains se met tantost au chemin,

Ou grant troton met le roncin

Por son mesaige tost parfaire. Espees, 8890-3.

Les roncins servent de chevaux de charge, et nous les trouvons parallèlement à d'autres bêtes de somme :

«Boin ronchi i aroit a caretier ;

"S'amenés de la laine en cel(e) markiet,

« Nous en acaterons molt volentiers,

«U vous portés carbon a vo somier : Aiol, 2855-6.

Lo jor li sunt vengut trei cent roncin

Carjat d'eital aver com esterlin, Roussillon, 6054-5.

« Le matin, par som l'aube, quant il ert ajorné,

«Vos manré .IIIc. mars de cumin et de sel,

« Et de poivre molu .III ${ }^{\mathrm{c}}$. ro[n] cins trossez

« Et .m. buefz et .m. vaiches et .m. bacons salez, Roche, 3709-

12. Cf. ib., 3958-9.

par la garanne et par la sable

la est la terre gaaingnable :

de la vint li blez et li vins,

o mulz, o soumiers, o roncins. Thèbes, 8195-8.

Je te donrai cent mars de con or fin

Et d'esterlins tout carchié un roncin ; Bueve, II, 10477-8. 
Li quens vint errant a la porte

Et vit un escuier ki porte

Un mort sangler sor un ronci. Poitiers, 899-901.

Ugon, qui veut voir mourir Aceline, la duchesse de Beauvais, sur le bûcher, la fait transporter au lieu du supplice sur un roncin, certainement pour l'humilier; nous avons une situation semblable dans Mort Aymeri, où le protagoniste prisonnier est envoyé en Espagne :

Et quant Hugues l'antant, le sanc cuide marrir.

Lors l'a traite de terre, si l'ait fait desfuïr :

A cinc serjans la fit tot maintenant sasir,

Une chape li fait afubler et vestir,

Sor un roncin la fit a reboison ceïr. Orson, 2014-8.

Et en Espaigne envoia Aymeri

Que cuide fere a grant honte morir ;

Si l'ont monté sor un povre roncin. Mort Aymeri, 1725-7.

On se sert aussi de roncins pour porter des litières :

(...), et la litiere aloit moult soef, car doi des millors ronchis qu'il covenist querre le portoient. Ensi s'en va li chevaliers en la litiere. Lancelot, vII, xxxIIIa, 15.

Tout ainsi que Bruians chevauchoit ou chemin,

Par dedens sa litiére, et que doy fort romcin

Le portoient en air, sans noisse et sans hutin, Brun, 2671-3.

Dans deux exemples tirés de $T P$, on a l'impression qu'en dépréciant les chevaux, l'auteur désire montrer comment est méprisable la situation des chevaliers prisonniers. Mais dans une troisième situation semblable, du même texte, le roncin est grand et fort :

Et a Palamidés loient les piés et les mains et le metent sour un roncin caitif et assés povre. TP, vI, 17, 41-3.

Engennés, qui fort l'encauce la u il aloit aprés lui poignant, regarde avant et voit venir .vi. homes et trois damoiseles qui estoient en lour compaingnie. Entr'aus estoit Galaad montés sour un povre roncin et li avoient les mains loïes deriere le dos. $i b .$, VII, 105, 14-8.

Et quant il ot erré jusc'a midi, si li avint une aventure mout merveilleuse, car il encontra au quarrefour de .II. cemins .II. 
cevaliers tous armés qui menoient Lyonnel, son frere, tous nu em braies, sus un roncin grant et fort, les mains loiies deriere le dos $i b$., viII, 110, 22-6.

Nous relevons, dans Rigomer, un cas analogue : le chevalier est en très mauvais état, mais l'auteur préfère décrire celui de son roncin, ce qui nous donne l'occasion de lire la description d'un très mauvais cheval. Un autre cheval, tout aussi mauvais, se trouve dans Espees. Tous les deux sont bien différents des excellents chevaux qui nous ont été présentés aux pages 19ss.

A tant fu des foriés issans

Uns chevaliers de tel maniere

Qu'i malseant avoit la ciere ;

Sor .I. ronci venoit le pas,

Qui avoit lent cors et lent pas ;

Si avoit gales et soros,

Si avoit redousié le dos.

Magre avoit le crupe et l'esquine

Et les costés et le poitrine,

Grelle le col et grose tieste ;

Mesaessie estoit la bieste ;

Car de soif moroit et de fain.

(...)

Les esperons qu'il ot cauciés,

Avoit rebois et redousiés ;

Neporquant en ot depanés

A son ronci tox les costés.

(...)

Ataces i ot d'un cordiel.

Son ronci en a acovert,

Dont li costé furent overt. Rigomer, 15536-47, 15561-4 et 15600-2.

Il sont de la forest issu

En une lande et ont ueu

.I. ronchi ki ert atacies

A un pin, s'ert tous dehacies

Ne n'ot cuir as esperonaus,

Et il ert grailles, lons et haus

Et ot perchies les enclumes

Et ot toutes les enquetumes,

$\mathrm{K}^{\prime} \mathrm{il}$ fu camores tout por uoir

S'ot le col lonc et grosse tieste,

S'ert si hideus que nule bieste

$\mathrm{Ne}$ fu onques de tel laidure, 
Et toute le hanaskeure

Ki sus estoit ne ualoit mie

Le montant d'une nois pourie. Espees, 6139-54. Cf. ib., 6172-4.

L'idée du peu de valeur des roncins, mentionnée par A.-J. Greimas, se retrouve dans plusieurs textes dont quelques-uns soulignent le fait que les roncins ne valent pas, évidemment, les destriers comme chevaux de bataille et sont estimés en conséquence. C'est entre autres le cas dans l'extrait d'Ille où, pensons-nous, il faut comprendre que le protagoniste, lorsqu'il s'arme, monte sur un destrier au lieu de continuer son chemin monté sur un roncin :

Ylles s'arma al miols qu'il pot

De ses viés armes que il ot,

Et monte en son ceval d'Espagne

Qu'il ot amené d'Alemagne,

Si l'ot cevalcié por ronci ; Ille, 1436-40.

Li chevaus desox lui nen est roncins ; Roussillon, 2442. Cf. ib., 9051-2.

«Ainz que j'aie perdu ne roncin ne somier,

« Par le mien escient ja le comperront chier Renaut, 303-4.

En tote la rote n'ot roncin ne somier,

Ni si mavés garçon qui n'eüst .I. destrier. $i b .$, 4894-5.

«Ses pieres escuiers ert de gris afublez,

« Ne chevauchoient mie les roncins atrotez,

«Mes buens chevaux de pris, coranz et abrivez, ib., 6065-7.

« Ne chevauchoie mie ronchin achaitivé,

« Mes boens chevaux d'Espaigne coranz et abrivé. ib., 8323-4.

N'avoit mie ronsin amblant,

Ains sist en grant destrier d'Espaigne. Chauvency, 756-7.

Gerard s'adube des armes al chemin ;

Le runcin laisset, al bon cheval s'asist.

$\mathrm{E}$ Tedbald se redresse cum home esturdi,

Devant li garde, si choisist le runcin,

Prent sei al estriu, entre les arçuns s'asist ;

Quant fu munté, menbre fut del fuir, Guillaume, 384-9.

Et il lor dist, senz point de l'atargier,

Qu'il n'aient cure de chevals espargnier :

Qui pert roncin, il li rendra destrier. Couronnement, 1505-7. 
«S'il ont cheval afolé ne maumis,

« Por .j. roncin rendrai destrier de pris, Aymeri, 2613-4. Cf. ib., 2632.

Et Lion li donnait telz cop de l'acerin

Toute cez armure ne li vallent ung ronsin. Lion, 22920-1.

De tous ses chevaliers prisons

Ne retient vaillant un ronchie, Gliglois, 2674-5.

« Ne quier avoir qe ij. povres roncis ; Raoul, 5190. Cf. ib.,

662-4.

« Mauvais roncins faillis! » a son cheval clamé, Lanson, 5329.

L'ostes prist son roncin qui mout ert maigroiez ; Gautier

d'Aupais, 24.

« Ne vous en chaut, se li viex est ocis. »

Monter le font sor .I. povre roncin, $\mathrm{Mez}, 7729-30$.

Et dist Gerins : « Tout ce laissiés ester

Por .I. roncin a maleïçon Dé,

Que de millors en avrés vos assés. »ib., 7078-9.

Le dernier extrait de $M e z$ veut exprimer que ce n'est pas la peine de s'échauffer à cause d'un mauvais cheval.

L'empereur, pour qui Eracle a acheté un jeune cheval très précieux (voir supra, poulain), n'apprécie pas du tout cet animal et l'appelle par conséquent roncin :

«Eracle, je vous aim petit,

- Sire, fait il, por Dieu merchi,

por coi est çou ? - Por le ronchi

dont tu as erré folement, Eracle, 1520-3.

Joufroi et son garçon, déguisés en ermites, sont montés sur de mauvais chevaux, appelés tantôt roncin, tantôt cheval, tantôt palefroi :

Chascuns chevaucha gros ronchin ;

(...)

L'ostel et lo[s] chevals atorne. Joufroi, 1535 et 1543.

Venez monter sus mon roncin,

Et si nos metons el chemin,

(...) 
Si est sus lo roncin montez,

Et li vaslet deriers lui monte, ib., 2609-10 et 2638-9. Cf. p. 43

$i b ., 2568-71$.

Le cheval du jeune Gautier d'Aupais est appelé tantôt roncin, tantôt destrier, tantôt chaceor :

"Ostes, combien doi je ? A mon roncin son blé ? Gautier

d'Aupais, 39.

Gautiers vint a l'estable por veoir son destrier, ib., 47. Cf. $i b$. , 57 .

Por ce qu'a un tornoi perdi mon chaceor

Me bati et fist lait : tornai m'en par iror ; $i b ., 527-8$.

D'autres textes indiquent une différence de sens entre cheval et roncin, entre destrier et roncin, ou entre palefroi et roncin. Le seul fait que plusieurs dénominations se trouvent côte à côte est signe que l'on fait la disctinction entre les espèces :

pernent palefreis e destriers,

trossent roncins, chargent somiers, Rou, III, 4959-6o.

« Je n'ai roncin, palefroi ne destrier, Aymeri, 347.

Destriers, roncin et palefroi

ierent si angoisseux de soi, Thèbes, 2129-30.

N'en menrez cheval ne ronchin,

N'arme nule n'emportez

Quant vous de moi departirez. Cont. P., II, 7744-6.

N'i ot celui qui eüst pain ne vin,

Fain ne avainne a cheval n'a roncin. Mez, 9203-4.

Et si ont tuit bons destriers arrabis.

N'a en lor route ne mulet ne roncin. » $i b ., 3602-3$.

Mais il n'i truevent palefroi ne roncin,

Ne mul ne mule ne aferrant de pris, Enfances G., 941-2. Cf. ib., 94-7.

Si ont veü venir Joffroi

Qui le palefroi maine en destre,

Et son roncin maine à senestre : $M R$, LXVII, 338-40. 
Ne contoit ung bouton d'aloweir ung florin

Ne d'ossire a la joste pallefroy ne ronsin. Lion, 921-2.

«Se gaing palefroi ne boin ronchin,

«Baille cha ta main destre : je te plevi

"Que je te donrai tant al revenir,

« L'acordanche en ert faite a ton plaisir : Aiol, 2803-6.

Lorsqu'on se trouve dans un danger imminent il n'est pas question d'être difficile quant au choix de la monture pour la fuite, le premier cheval trouvé est bon :

Qui roncin trueve, destrier n'i vet querant ;

Chascuns sessist ce qu'il trova avant,

Li uns roncin, li autres auferrant,

Li tiers somier, li carz mulet enblant. Aymeri, 3191-4.

La punition d'un criminel ou d'un ennemi qui consiste à le traîner derrière un cheval, attaché à la queue de celui-ci, ou d'être écartelé, se trouve mentionnée par plusieurs textes. Elle peut être réalisée avec des roncins aussi bien qu'avec des destriers ou des palefrois (voir supra). Quelques-uns de nos exemples montrent que le choix des termes dépend (aussi) de la rime :

Mal jugement avés fait cest matin

De dos haus omes qui sont de rice lin,

C'on les traïne a keuës de roncin. Aspremont, 6476-8. Cf. ib.,

6752-4.

« Donc il sera del tot vergondez et honis,

«Et si frere detrait a coes de roncins, Renaut, 5348-9.

Pendus serez ou escorchiez toz vis,

Et li viellarz traïnez a roncins. Mez, 5807-9.

Par tel convens con ja porés oïr,

Que, se vous estes ne vencus ne maumis,

Je les ferai traïner a roncis. » Huon, 1450-2.

Et ne trovit nul home, se il an Deu créist,

Qu'il ne vosist ocire et les mambres tolir,

Ou pandre au autes forches ou detraire à roncins. Floovant, 6-8. Cf. ib., 1732-3; 1918-9.

Ardoir en feu u detraire a roncis ; Bueve, II, 2568. 
A deus ronchis les a fait ateler,

Parmi la vile les a fait träiner, $i b$., III, 6778-9.

Malgré le mépris pour les roncins qu'expriment régulièrement les auteurs, nous voyons aussi des scènes où une personne qui distribue des richesses ne dédaigne pas d'en faire don - et, au moins pour des paysans, cela vaut toujours la peine de les voler :

Car il y met a abandon

Or et argent, robes et dras,

Roncins, destriers, pallefroiz cras,

Et armes, et autre richesse. Galeran, 4932-5.

N'i ot si povre jougleour

.IIII. mars d'argent n'ait le jour

et boin ronci et un mantel. Floire, 3155-7.

Menestrel orent bons loiers

Tel com le vorrent demander,

Car Floriant lor fist doner

Robes et roncins et deniers. Floriant, 6312-5.

"Puis me fist mes parains laver et baptisier,

«Et chevauz et rocins et palefrois doner. Parise, 1470-1.

Païsant sont de la montaingne

qui sont descenduz en la plaingne

pour encombrer nostre chemin

et nous tolir aucun roncin. Thèbes, 7137-40.

En cas de disette, p. ex. pendant un siège, l'on est parfois obligé de sacrifier les chevaux, à la fois parce qu'on ne trouve pas à les nourrir et parce qu'on manque de viande. Voir aussi page 24 .

Ançois que vaingne ne Gerbers ne Gerins

Ne l'enperere ne li preus Mauvoisins,

Par droite force mengerez vos roncins. » Mez, 5800-2.

Il n'ont mes que mengier ne cheval ne roncin. Renaut, 11996.

Cf. $i b ., 11855$.

Dans Renart, le roncin se trouve parmi les animaux qui vont au procès contre Renart. Là il est appelé " seigneur » et il a un nom propre - il s'agit donc d'un personnage important :

Qui donques veïst Isangrin

et le mouton sire Belin 
et Brun l'ors et Tibert le chat

et monseignor Pelé le rat

et Chantecler et dame Pinte,

si con il vint a cort soi quinte,

et seignor Ferrant le roncin

et dant Roonnel le matin Renart, I, 1611-8.

L'exemple suivant, tiré de Lion, nous cause un certain embarras, car il est le seul où notre terme est placé à côté de palefroi. Comme nous l'avons déjà signalé à la page 17 , nous pensons que la rime y est pour beaucoup dans le choix du terme et que celui-ci tient, avec palefroi, la place de noyau dans le syntagme substantif. Peuton lire roussin, avec le sens de "roux » - et peut-être même y voir un premier pas vers la substitution de roncin par roussin? Pour oser soutenir cette thèse il faudrait avoir le manuscrit sous les yeux et relever d'autres occurrences du phénomène :

Et il fiert du tinelz en menant grant huttin,

Ne laissoit approchier le pallefroy ronsin ; Lion, 2949-50.

Bérinus présente trois exemples de roussin, synonymes de roncin (une occurrence) :

Atant fu le charriot attellé a quatre fors roussins, (...) Bérinus, 406.

Atant Guymars les ot si aprouchiez qu'il ataingnit Gallopin qui s'en aloit derriere son maistre, car son roussin ne pouoit si tost courir comme les destriers, (...) ib., 504.

Et puis dist a Galopin : «Or prenez ce destrier pour vostre roussin que vous avez perdu. $-i b ., 505$.

(...), fors de Gallopin seulement qui fu monté sur un roncin ; ib., 491.

Roussillon présente un exemple de la forme féminine du terme, probablement à cause de l'assonance :

Ele en donet taus mil, cascuns l'encline,

$\mathrm{E}$ al rei la meitat, per quei s'afine.

E Folche nol deit gins fil de roncine. Roussillon, 9048-50.

Voir aussi deux occurrences du diminutif roncinet à la page 311 (Bueve, II, 16421-9). 


\section{r. sambuer.}

Une sambue est une selle, spécialement pour les chevaux que montent les femmes. D'où l'on peut conclure que le sambuer est un palefroi qui sert de monture aux femmes, ce qui est corroboré par notre exemple où Guiborc prête son palefroi au jeune Gui :

Ele li ameine Balzan, sun sambuer ;

Bone est la sele, mais curt sunt li estriver,

Unc Guiborc nel prestad a chevaler. Guillaume, 1548-50.

\section{s. sommier.}

Le terme désigne normalement la bête de somme, plus rarement la charge de celle-ci, le bagage, l'équipage. Voir p. ex. deux occurrences dans Amadas : au vers 4610, il est indubitablement question d'un cheval, tandis qu'au vers 3029 il s'agit probablement de bagages ; les vers de Berte et de Couci nous semblent équivoques.

Ja sont venu li esquier

Et tui li keu et li soumier

Et Garines o le harnois. Amadas, 4609-11.

Coffres et bouges et somiers,

Et dras et vassiaus et deniers

Maine avant li com faire doit, $i b .$, 3029-31.

Cel jour ot la roÿne travail et paine male ;

N'i ot sonmiers a cofres ne dras troussez en male, Berte,

734-5.

Et lors que venrons au sommier,

Je me penerai d'esploitier

A repairier plus que le pas,

Si aporterai autres dras

Que ma dame pora viestir,

Et irai son ceval couvrir. » Couci, 6330-5.

Généralement il n'est pas possible de savoir si le terme désigne une espèce particulière ou si n'importe quel cheval peut servir de bête de somme. Nous avons déjà noté, plus haut, que les palefrois et les roncins servent à porter des charges : dans le vers 8198 de Thèbes, nous avons vu roncin avec mul et sommier (p. 64). Dans Aiol, on charge des malles sur des sommiers, appelés, une trentaine de vers plus loin, destriers :

«Et torserons les males sor les plus fors somiers, Aiol, 9448. 
Puis torserent les malles sor les destrier[s] grenus, $i b ., 9479$.

Notons aussi, dans Narbonnais, que les mulets des vers 753 et 881 sont les mêmes animaux que les sommiers des vers 729,827 et 860. Aux vers 1355 et 1661 du même texte, d'autres mulets servent de bêtes de somme. Les sommiers des vers 5981, 5993, 6080, 6083, 6091 sont les mêmes que les roncins du vers 6606 et les muls du vers 6035 :

«Va, si me charches .iiii. mulez de pris

D'or et d'argent et de ver et de gris, Narbonnais, 753-4.

. IIII. somiers tot a vostre talant ! » $i b ., 729$.

Chargerai vos .vii. mulez de Sulie

D'or et d'argent et d'autre menentie. » ib., 1355-6.

I lor demendent : «A qui sont cil somier ? » ib., 5993.

Se antrer pueent li conte de franc lin

Dedanz Nerbone o grant pales mabrin

Et la vitaille que portent li roncin,

Asez avront et pain et char et vin ; $i b ., 6004^{-7}$.

I lor demende : « Qui sont cil mul trosé ib., 6035.

Par contre, dans un assez grand nombre d'autres exemples, on fait bien la distinction entre sommier et p. ex. palefroi et destrier :

Uns clers (...)

Escuiers et sommier avoit,

Et bel palefroi chevauchant, $M R, \mathrm{IV}, 24^{-5}$.

Quant li jours vint, si s'aprestérent,

Et sour les palefrois montérent ;

Ne firent mener c'un sommier,

Et si n'eurent c'un escuiier. Couci, 6262-5.

De la le fis et jeter et cacier.

Ens Castelfort covint fuïr Ogier,

Si n'emmena palefroi ne sommier, Huon, 108-10.

Li cheval estoient d'Espaigne

Molt par ierent bel li destrier ;

Puis lor donna .xII. somier

Chargiez et de vair et de gris. Claris, 8204-7. 
Si vous donrai .xx. destriers d'Arragon

Et .ii. somiers chargiez de bons mangons. » Mez, 13494-5.

Et il esgarde k'il menoient

Et sommiers et cheuaus en destre ; Espees, 9382-3.

Chevaliers manda dusk'a trente,

Qui od lui iront sans atente.

Chevaus de pris ne biaus sommiers

Cargiés de soie, de deniers

Ne vaut laissier, que il peüst,

Que bien quatre vint n'en eüst,

Moitié sommiers, l'autre cevaus. Jehan, 5423-9.

En une bone nef qu'il vit

Fist mettre sommiers et destriers,

Roncins, palefrois et deniers, ib., 5466-8.

Hastivement s'est aturnez

De riche[s] dras e de deniers,

De palefreiz e de sumers ; Lais, vI, 122-4.

Dans Barbastre, les chevaliers se déguisent en marchands - et leurs chevaux (destriers ?) sont transformés en sommiers. Lion présente une situation semblable :

Chascun tient son destrier par les resnes noez,

Toz coiement les moinent, qu'il ne sont derveez.

Des chevaus font somiers, estes les aroutez ;

A loi de marcheant se sont acheminé, Barbastre, 3020-3.

Elle ait fait cez jossialz trosser et chargier

Par dessus ung chevalx en guise de solmier. Lion, 11498-9.

Dans un certain nombre de cas, le terme est employé parallèlement à mul(e)/mulet, ce qui montre que ces derniers servaient de bêtes de somme, avec les sommiers. Peut-être certaines occurrences du terme sommier désignent-elles même des mul(e)s/mulets et non des chevaux ? Cela pourrait être le cas dans le dernier extrait de Renaut.

Et rechargierent les sommiers et les murles,

L'or et l'argent, les riches vesteüres, $A m i$, 1977-8.

Se je vif tant que veingne a l'esclairier,

Il n'en menra ne murlet ne sommier, $i b ., 2019-20$. 
« Gardés demain à l'aube soiés apareilliez,

«Et si faites trosser les murs et les somierz : Gui de B., 28-9.

"Salués moi le roi, mon seignor droiturier ;

« Je li envoierai .m. murs et .M. somiers, ib., 3108-9.

Dont fait trosser et mulés et somiers. Mez, 12759.

Et fait trestot trosser li mur et li somer. Renaut, 2288. Cf. ib., $2304 ; 2456$.

«.x. mulez vos dorrai qui sont tuit anbleor,

«Chargiez d'or et d'argent, que nus ne vit meillor.

(...)

Berges prist .x. somiers qui erent sejorné,

Au roi les envoia a Bordeax sa cité, ib., 4195-6 et 4206-7.

Les vers suivants, tirés de Roche, sont intéressants : il est évident qu'il s'agit de la même scène, mais est-ce que les sommiers du premier extrait sont les mêmes animaux que les dromadaires du second ? La différence du nombre (30 sommiers et 20 dromadaires) peut être causée par une inadvertance de l'auteur ou du scribe :

Li bons rois Alixandre li dona grant soudée,

Chargiez .xxx. somiers de l'or de sa contrée

(...)

Li bons rois Alixandre li dona avoir tant,

Chargiez .xx. dromadaire[s] d'or fin et de besanz. Roche, 2898-9 et 2906-7.

Les sommiers étant des animaux très forts, ils sont bien aptes à l'écartèlement :

«Si les faites noer as keues des somiers, Gui de B., 1110.

Richart sera detrait a coe de somier, Renaut, 2416.

« Et Aalart voz frere sera vis escorchiés,

« Richart et Guischart traiz a coes de somiers, ib., 5513-4.

Et quant vos i avrés tant jut

Que del tout vos verai deçut,

Dont vos detrairai a somiers ; Rigomer, 7413-5.

On amène les sommiers à des parties de chasse afin qu'ils portent la proie : 
Renaut le fiz Aymon repaire de chacier,

Et avoit .iiii. cers trossez sor .I. somier : Renaut, 6396-6.

Dans Aspremont, nous voyons Rollandin descendre de sa monture, qui est un sommier, pour monter sur un destrier, c'est-à-dire sur un cheval de bataille. Or, nous l'avons déjà vu à la page 51, si les destriers viennent à manquer, on peut s'en passer et monter sur des palefrois ; Enfances $O$. montre que l'on peut même se contenter de sommiers quoique ceux-ci fussent certainement bien lourds et lents pour la bataille. Dans Fouke, un valet monte sur le premier cheval qu'il aperçoit, et qui se trouve être un sommier :

Rollandins sist cel jor sor un somiers,

(...)

Rollandins garde, si trova un destrier ;

Sen somier lait, se li salt en l'estrier. Aspremont, 5532 et

5542-3.

As armes keurent chamberlenc et huissier,

Et eschançon et keu et bouteillier

De la maisnie Charlon au cuer entier

Et de la gent duc Namlon de Baivier ;

Qui n'ot cheval, si monta seur sommier. Enfances O., 1057-61.

(...), si vint a une estable qe ert delees la posterne par ount home vet vers la ryvere, e trova la un somer. Yl mounta meyntenant le somer e s'en issist par la posterne e passa bientost la ryvere e vynt al champ ou son seignour fust abatu de son destrer e en poynt de estre ocys, s'yl ne ust survenu. Fouke, 14, 25-30.

Roland montre que le sommier est, malgré son importance comme cheval de charge, ou peut-être justement parce qu'on en trouve tant, un animal dédaigné et mésestimé :

Menet serez dreit ad Ais le siet.

Vus n'i avrez palefreid ne destrer,

Ne mul ne mule que puissez chevalcher ;

Getet serez sur un malvais sumer. Roland, 478-81.

Ben le batirent a fuz e a bastuns

E si li metent le col un caeignun,

Si l'encaeinent altresi cum un urs ;

Sur un sumer l'unt mis a deshonor. ib., 1825-8.

Il y a lieu d'attirer l'attention sur le fait que de nombreux occurrences du terme qui indiquent la fonction de bête de somme don- 
nent aussi le nombre exacte des animaux en question. Quelques nombres reviennent plus souvent que d'autres, p. ex. 4 et 30. Voici des exemples :

Et vous ferai un bon somier torser Huon, 6448.

Mener fait aprés lui doulx solmier ou quarmin. Lion, 924.

N'urent mes fors deus somers,

Chargés de draps e de deners ; Ipomedon, 313-4. Cf. Hunbau, 2857 ; Gaufrey, 6126.

.III. somiers a robes et armes

orent, et granz chevax de pris. Dole, 1276-7.

Mais faites prendre .c. destriers sejornez,

.c. palefrois et .c. muls afautrez,

iiii. somiers d'or et d'argent trossez. Mez, 9630-2.

« Voir, je vous en donrai .IIII. somiers torsés, Aiol, 9755.

Li vallez prist l'escu, et avec li bailla Lancelos quatre somiers touz chargiez d'avoir, (...) Mort Artu, 121, 1-2.

A tant s'en vont. Et Gorges li membrés

A de vitaille .IIII. somiers toursés

Des miudres mes qu'il avoit conquestés Auberon, 1968-70. Cf.

Gaydon, 4023 ; Gaufrey, 4955.

comme marceans le querrai,

.vII. somiers avoec moi menrai,

les .II. cargiés d'or et d'argent Floire, 1141-3. Cf. ib., 1151.

Et fist chargier les diz somers

D'or et d'argent et de deniers ; Joufroi, 2545-6. Cf. ib., 2533.

.x. sonmiers lor tilire[n[t qui tuit erent trossé Lanson, 3918. Cf.

$i b ., 4001 ; 4973$ et passim.

.xv. somiers chargiez de fin or an avrez; Parise, 592. Cf. Aymeri, 3385-6.

Pur lui me unt offert asez,

Quinze chastels e set citez

E quinze somers d'or e d'argent. Gui de W., 4779-81. 
« Or e argent tant vus durrai,

«Vint somers charger vus ferai. » $i b ., 8123-4$.

Ce chastel vous donray ou il a tant d'or mier

Que point ne le menroient en ung an .xx. sommier ; Tristan de N., 663-4. Cf. Gaufrey, 4803.

Si grant avoir en orent aporté,

.xxv. somiers d'or et d'arjent trossez ; Mort Aymeri, 2965-6.

Trente sommiers blans comme noiz

Font chargier l'endemain de draps, Galeran, 3298-9.

Or t'amenons et lor pain et lor vin,

Trente somiers de lor mellor or fin ; Aspremont, 3417-8.

Menés o vous desc'a trente somiers

Que vous ferés de mon avoir cargier. Huon, 407-8. Cf. Aymeri, 2099-101; Gaufrey, 8009.

Et li charge seissante omes a armes,

D'or et d'argent trente somiers li baille. Couronnement, 251-2.

Cf. ib., $1427 ; 1444$.

D'or et d'arjant quarante somiers torse, Enfances G., 142.

Quarante et cinc somiers i ait meney

D'or et d'arjant, de richoise trocey. $i b .$, 319-20.

Les .xxx. choisirent venant

Les .L. somiers menant ; Claris, 1182-3. Cf. Gaufrey, 7973.

D'or et d'argant soissante somiers charge,

De sandal et de poupres, de tires et de paile. $i b ., 161-2$.

Et soissante solmier que lon cherroy suyoient la ; Lion, 16269.

Et coisi .c. sommiers c'on avoit fet trousser. Gaufrey, 354.

.cc. somiers font troser et chargier. Narbonnais, 5981. Cf. ib., $6080 ; 6083 ; 6091$.

Ensemble o els mainnent trois cenz somiers. Charroi, 764 .

- plus le nombre exorbitant de 1700 : 
Et en aprés mil et set cens somier,

D'or et d'argent les faites tost cargier, Aspremont, 7835-6.

Voir aussi : Renart, I, 2109 ; Aiol, 2857-8 ; 4867-8 ; 8520-2 ; Cont. P., II, 9182-3 ; Joufroi, 121 ; 916-7 ; Aspremont, 10643-4 ; Couci, 498993 ; 5002-3 ; 6272-3 ; Inconnu, 3844-5 ; Renaut, 2444 ; 3831 ; Anjou, 671-3 ; 5246-9 ; Dole, 2584-5 ; Gui de B., 168 ; Barbastre, 2306-8 ; 3179 ; 3350-1 ; Parise, 35-7 ; Galeran, 906-8 ; 2824-5 ; 6785 ; Tristan de N., 12058-60 ; Lion, 3613-4 ; 4203-4 ; Gliglois, 2017-9; Godin, 18670 ; Mez, 6482-3; 13222-3 ; Helcanus, 5; Auberon, 1982-3 ; 2001 ; Berte, 1843-4 ; 2340-1 ; Thèbes, 8217-8 ; 8363 ; Roche, 1722 ; Huon, 6448 ; Florimont, 475-7 ; 4833-4 ;Floriant, 3556-61 ; 7348-50 ; 7400 ; Aliscans, 2150-2 ; 4396-7 et passim ; Eneas, 3757-9 ; 3774-6 ; Florence, 132-4 ; 164-5 ; Saisnes, 1124 ; 1646 (62-3) ; Partonopeu, 1992 ; 2014-5 ; Durmart, 6633 ; 6646 ; Lancelot, vII, Ia, 16 ; Iva, 4 et passim ; Perceval, 4146 ; Yder, 5702-3 ; Orson, 2854 ; Wistasse, 640 ; Simon, 1344 ; Bueve, I, 2700 ; 3466 ; II, 3885 ; 9970 ; III, 3708 ; 3770 et passim ; Chauvency, 4515 ; Erec, $1849 ; 1855$ et passim ; Laurin, 3606-7 ; Bérinus, 282.

\subsubsection{Les termes qui sont employés comme substantifs ou comme adjectifs}

a. ambleor $=$ cheval qui va l'amble (R. Grandsaigne d'Hauterive, op. cit., p. 21).

\section{Celi qui pres de li manoit}

Querroit il sour cel ambleeur. Escoufle, 6678-9.

b. arabi.

Le terme désigne à la fois ce qui est arabe et ce qui est rapide. Dans les cas particuliers, il est pratiquement impossible de savoir si l'auteur distingue entre race/origine et qualité, ou si au contraire il veut dire que les chevaux arabes sont tous rapides, ce qui signifierait qu'un cheval arabi devient synonyme d'un cheval coursier. Dans quelques-uns de nos exemples, nous constatons que la rapidité est soulignée. Que le cheval Bayart soit appelé et arabi et aragon (voir infra) peut être causé par une inadvertance ou, plutôt, par la rime. Mais cela peut aussi signifier que le terme arabi désigne la rapidité et aragon l'origine.

Voici d'abord une série d'extraits où le terme se trouve avec article :

Por qoi sui je desus cest arrabi

Que ja par moi n'iert ceval porsali,

Lance levee, ne fort escu croissi ? Aspremont, 8389-91. 
Guillaumes au cort nes sailli del arrabiz, Barbastre, 4974. Cf. ib. 6640 .

Et Genfroi l'Engevin sor l'arrabi corant, ib., 178.

Pus munte en l'arrabit corant, Gui de W., 11762.

A tant brochent les arrabiz Athis, 12383 .

Et pour savoir de Charlon au corraige herdit

L'estat et la maniere dont brochait l'arabit. Lion, 21028-9. Cf. ib., 31029 .

De tant con porent corre li arrabi

Li dui vassal se vont entreferir. Mez, 6895-6. Cf. ib., 6931-2 ; 14248.

Andui joingnent li arrabi,

li uns l'autre pas ne failli. Thèbes, 5671-2.

uns danziaux amenoit de l'ost

un arrabi qui court mout tost, $i b ., 5757-8$.

As armes connut son ami

Qui siet sor le bon arrabi Blancandin, 4729-30.

Son fil dona li rois son arrabi, Chevalerie d'O., 7279.

Cligés desor l'arrabi blanc

S'an monte armez de totes armes ; Cligés, 3982-3. Cf. ib., 3574$5 ; 3668$ et passim.

Enfances G., Renaut, Bueve, Raoul et Chevalerie d'O. présentent des exemples où le terme, avec article, forme apposition à Bauçant, Bayart, Arondel, Fauvel et Broiefort, les noms des chevaux de Guillaume, de Renaut de Montauban, de Bueve, de Raoul de Cambrai et d'Ogier. 1.1.2.q., nous verrons une construction identique avec le terme coursier ; ceci montre qu'arabi peut être synonyme de « rapide » :

L'escu a col sor Bauçant l'arabi. Enfances G., 949.

" Mes se veoit Renaut sor Baiart l'arabi, Renaut, 8048. Cf. ib., $8056 ; 12344 ; 13447$.

Jou li donrai Arondel l'arabi Bueve, I, 116o. Cf. ib., 9399. 
« La cele est mise sor Fauvel l'arabi ; Raoul, 2300.

Om li amaine Broiefort l'arrabi ; Chevalerie d'O., 6968.

Et voici quelques extraits où arabi est adjectif :

Lion est dessandut du destrier arabit ; Lion, 22606.

Montent es selles des destriers arrabis, Ami, 1114 .

«Que vos ferai verser del cheval arrabi. » Renaut, 2581.

Les lances en lor mainz tenanz,

Sor les chevaus arrabioiz ; Claris, 15953-4.

Se li garda un destrier arabi, Bueve, I, 10059.

«Ore pri vus, sire, pur la tue merci,

Que vus me rendez mun destrer arabi, Guillaume, 1906-7.

Voir aussi : Chevalerie d'O., 7305 ; 7357 ; Aymeri, 4107 ; Raoul, 530 ; 1992 ; 3527 ; Narbonnais, 4843 ; 6156 et passim ; Mort Aymeri, 1548 ; $1775 ; 1858$ et passim.

Dans Roussillon, nous avons relevé un exemple d'arabion, à la rime :

Fait venir Chabaucam, l'arrabion,

Et li cons i montet fors au perron Roussillon, 8103-4.

Dans les exemples de l'Arabi (avec majuscule), tirés de Narbonnais, nous ne voyons pas un nom propre : il s'agit indubitablement du substantif arabi. Voir aussi p. 28, amoravi, ib., 4868-9 :

Par mi la porte, que le portier ovry,

S'an ist mout tost armé sor l'Arabi, Narbonnais, 4171-2.

Tant a chascun brochié son Arasbi ib., 7329. Cf. ib., 7282 ;

7293.

c. aragon.

Ce terme indique l'origine des chevaux en question. Rien ne nous renseigne sur les qualités de ces chevaux, mais il doit s'agir en général de chevaux de bataille, entre autres choses parce qu'il se trouve assez souvent épithète à destrier et que les bons chevaux Flori, Bayart, Briefort et Arondel sont des aragons. (Cf. p. 81) : 
Gerbers devant sor Flori, l'arragon, Mez, 14221. Cf. ib., 11717.

Donc regrete Renaut Bayart son arragon Renaut, 6993.

«Va, si me met la sele sor Bayart l'arragon, ib., 7633. Cf. ib., $7640 ; 7651-2$.

« Hé ! Ogier, ce dist Kalles, bien oi vostre raison :

« Mis voudrïez avoir Briefort l'arragon

«Que je fusse or en France, a Rains ou a Sesson, ib., 5695-7.

Et si vous doins Arondel l'aragon, Bueve, I, 1186. Cf. ib., I, $6412 ; 9170-1$ et passim.

Prendent les armes, montent en aragon Macaire, 2686. Cf. ib., $2672 ; 3001$.

.v. mille sont armé, cescun sur l'aragon ; Lanson, 1603. Cf. ib., $1254^{-5}$.

Lors remonta chascuns en l'arragon. Ami, 3488.

Carahués vint poignant seur l'arragon, Enfances O., 2720.

Et Sadoines estoit montés sur l'arragon

$\mathrm{Ou}$ il se combatoit de champïon, Tristan de N., 2257-8. Cf. ib., 4802-5; 16689-9o.

Du roy partirent li baron,

Armez chaucun sor l'arragon ; Claris, 13648-9.

(Il broche l'aragon des esperons d'ormier) Roche, 2475.

Cinc aultre chevalier saisirent l'aragon, Lion, 7132.

Tot trabuche en un mont, et lui et l'arragon. Saisnes, 3326. Cf. $i b ., 4137$.

Grant honte ot quant cheü le virent li baron, E de ce que il ot perdu son aragon ; Aye, 420-1.

Nous ne voyons pas pourquoi Orson écrit l'Aragon. Gaston Paris traduit par « le cheval aragonais »; nous supposons qu'il faut simplement lire l'aragon :

La porte fait ovrir sans nulle arestison,

Et Miles s'an issi armez sor l'Aragon ; Orson, 1399-400. 
Le même phénomène se trouve dans Gaydon où la moitié des exemples présentent le terme à majuscule. Ici aussi nous lisons aragon. Il ne peut pas s'agir d'un nom propre, avant toute chose à cause de l'article indéfini du vers 9437 et du fait qu'aux vers 7788-9 il est question de trente cavaliers : ceux-ci peuvent bien être montés sur trente chevaux aragonais - mais pas sur trente chevaux portant le même nom :

« J'en voi la .II. desor les arragons. Gaydon, 2051. Cf. ib., 3478 ; 6319 et passim.

« Bien furent .xxx., tuit par election,

« Moult bien armé, chascuns sor l'Aragon. ib., 7788-9.

Atant ez voz Ogier sor l'Arragon. ib., 8485. Cf. ib., $8705 ; 9508$ et passim.

Et Ferraus broche, si prent un Arragon ; ib., 9437.

L'extrait ci-après, de Lion, est intéressant par le fait que le même cheval est appelé d'abord destrier gascon, ensuite aragon ; il confirme donc que notre terme désigne un cheval de bataille - mais il montre aussi que gascon et aragon sont confondus (c'est probablement le nombre de syllabes qui en commande le choix) :

Li duc issit avec que Herpin ot a nom,

Richement fuit arméz sur le destrier gascon ;

O lez aultre Romain vait brochant l'aragon. Lion, 3047-9.

Présentons maintenant quelques extraits où aragon est épithète. Comme dans les deux derniers, il s'accole régulièrement à $m u$ lets : ici le terme ne désigne que l'origine des animaux :

Montent es selles des destriers arragons, Ami, 214. Cf. ib., 1648.

Le cors do Saisne aportent .II. destrier arragon. Saisnes, 5530.

«Sire, dit li portiers, une rien sachiez vos,

"Qu'il sont bien .iiii.c. a vers elmes reonz,

«Et chascuns desoz soi bon destrier arragon, Renaut, 453-5.

« Ou est ore Bayart, vo destrier arragon? ib., 7048.

Et Richart point et broche le destrier arragon. ib., 9899 .

Il saut en Brieguerre son cheval arragon ; $i b ., 9726$. 
« Et seront trestuit .iiii. es mulez arragon,

« Ja n'i porteront armes, espié ne gonfanon, ib., 6176-7.

Ne se tenist a lui .I. mulet arragon. $i b ., 8950$.

Voir aussi : Aymeri, 2738 ; 2827 ; Macaire, 2493 ; 2668 ; 2893 ; 3032 ; Lanson, $1562 ; 1567$; Gaydon, 2057.

d. arami.

R. Grandsaignes d'Hauterive, op. cit., p. 32, traduit le terme par impétueux, sauvage. Nous n'en avons relevé qu'une seule occurrence :

Es vous les païsans desaus li arramis, Doon, 2847.

e. aufage/aufaigne/aufajois.

Nous n'avons relevé que peu d'occurrences de ce terme, dont l'étymologie serait l'arabe al faras = cheval. Il est évident qu'il s'agit d'un cheval arabe; dans Aliscans, le terme est féminin et désigne une jument :

Li amustant de Cordres se fu forment hastez,

Par desus l'aufajois qu'est de corre abrivez ; Barbastre,

5241-2.

Danebur l'orgueilleus sor l'aufaje corsier, $i b ., 5838$.

A tant ez un message sor un roncin aufainne, Saisnes, 1646

(20).

Mout fu prodom Palamedés

Et d'armes pot sofrir grant fes.

Sor un destrier sist brun, alfainne ; Athis, 12541-3.

Cheval ot bon et en sa lance ensaigne ;

Et Desreez chevauchoit une aufeigne. Aliscans, 1762-3.

Le vers 5234 d'Aliscans donne aufage brehaigne. L'éditeur Claude Régnier pense que le terme aufage a ici le sens de "sarrasin » et qu'il détermine brehaigne. Nous renvoyons à la discussion sur ce syntagme p. 96.

\section{f. auferrant.}

De même que le terme arabi, celui-ci est à double sens : il indique en effet la couleur grise et l'impétuosité. Les exemples que nous avons relevés, et dont nous présentons ci-après un petit choix, 
montrent qu'il s'agit de chevaux de bataille, ce qui correspond très bien au caractère impétueux des animaux.

«Car se Jhesu me done par son commant

"Que je m'en puise issir sor l'auferant, Aiol, 2436-7. Cf. ib., 2442-3; 4807-8 et passim.

On li amaine l'ausferrant, lors monte, n'i a plus targié, Cont. P., III, 15852-3. Cf. ib., I, 3221-2.

Li cuens Amiles enmi le pré se jut,

Devant lui ot son aufferrant quernu, Ami, 960-1.

Paien ensielent richement l'auferrant

D'arçons a or et de siele a argent. Aspremont, 2186-7. Cf. ib., 5750-1; 9846 .

Cil s'esvelle isnellement,

Se li amainne l'auferrant. Inconnu, 683-4.

(Même cheval : v. $682=$ destrier et v. $686=$ cheval.) Cf. ib., 751 .

A l'autre cop a feru l'auferant,

Le chié du bu li ala dessevrant. Otinel, 445-6. Cf. ib., 1648 ;

$1657 ; 1798 ; 1836$.

«Vez li sor l'auferant, sor lo destrier armé, Parise, 2128.

«Ainz combatroie armez sor l'auferrant Raoul, 690.

Savaris monte par desor Ataingnant ;

Quant montez fu, si broche l'auferrant, Gaydon, 4513-4. Cf.ib., 6023.

«Mes hausferans est durs, moult en sui aïrés ; Fierabras, 519.

Chascuns tot maintenant monta an l'auferrant ; Saisnes, 1201. Cf. ib., 1591-2 et passim.

Voir aussi : Renaut, 212-3; 427; 2734; 12384; Gui de B., 592 ; 2624 ; 2654-5 ; Floovant, 1118 ; 2493 ; Huon, 334 ; 1088-90 ; Meliacin, 8464; 12438-9 ; 12532-3 ; Enfances O., 5491-2 ; 6181; Tristan de N., 21783 ; Mez, 253 ; 263 ; 456 ; 3171 ; Lion, 6480 ; 8680 ; 19708 ; 32157 ; Auberon, 987 ; Gui de N., 1013 ; 2445-6 ; 2457 ; Orson, 2229 ; 3540-1; Enfances G., 389；407-8；664-5；757-8；941-2；1018； 2561 ; Roche, 3663-4 ; Thèbes, 3488-9 ; 3656-7 ; 4741-3 ; Rigomer, 7286-7 ; Violette, 5950-1 ; Roussillon, 2558 ; 2568 ; 7381 ; Aye, 2023 ; 
Charroi, 83 ; 91 ; 552 ; Jourdain, 1766 ; 1972 ; Athis, 7461 ; Protheselaus, 960 ; 1103 ; 4258 et passim ; Simon, 99 ; 292 ; Godin, 10217 ; 11931 ; 13353 et passim ; Gaufrey, 4237-8 ; Lanson, 2956 ; Aymeri, $487-8 ; 1695 ; 1891-2$ et passim ; Narbonnais, $4898 ; 5628 ; 6225$ et passim ; Poitiers, 496 ; Brun, 1139 ; 1253 ; 2119 et passim ; Mort Aymeri, $1166 ; 1226 ; 1231$ et passim ; Bueve, I, 3691; $7377 ; 7599$ et passim ; Guillaume, $1895 ; 1898$ et passim ; Ipomedon, 4733 ; 8989 ; 10102 ; Blancandin, $5716 ; 6304 ; 6342$.

Dans Aiol, le terme est apposition au bon cheval Marchegai, ailleurs appelé destrier et cheval :

Il vint a Marchegai son auferant. Aiol, 2399.

Aliscans montre que les auferrants peuvent être utilisés à tirer des voitures : le terme limonier désigne l'animal attaché au limon (cf. p. 188, où il est question de bœufs) :

D'un auferrant fist Guiborc limonnier,

Et .II. roncins i ot et .I. somier

Por tost aler et por mielz esploitier. Aliscans, 4944-6.

Les extraits suivants présentent notre terme avec coursier et gascon. Nous notons que l'ordre des mots est toujours le même : auferrant vient toujours en premier lieu - à notre avis cela signifie qu'il est le noyau du système substantif, d'autant plus que lorsque coursier s'accole à d'autres termes il se place toujours après le noyau (voir p. 101-102) :

A pié descent de l'aufferrant corsier, Ami, 1935.

«Et desoz vos chaet li auferrant corsier, Renaut, 6697.

Carahués l'ot, vis cuida enragier,

De maltalent prist couleur a changier,

Arrier retorne son auferrant coursier. Enfances O., 2706-8.

Floovans a broichié son auferrant corsier, Floovant, 404.

Par ces chanz fuient cil auferrant corsier. Mez, 9012.

On li amainne un auferrant coursier, Bueve III, 5780.

Son escuier commande que tost soit ensellés

Son aufferrant coursier ; et il fut aprestés. Tristan de N., 9808-9. Cf. ib., 14211. 
Puis monterent es sele[s] des auferans corsier[s] Aiol, 4634. Cf. $i b .4807-8$.

Mais il l'a abatu de l'auferant gascon. $i b ., 9003$.

Ocis li ont son auferrant gascon. Aspremont, 1890.

Si a le jor hasté son auferrant gascon,

Suant ot le col, le piz et le crepon. Barbastre, 127-8.

Dedens ont mis les auferrans gascons, Huon, 6752.

Dans les extraits suivants par contre, auferrant se présente comme épithète dans un système substantif, dans certains cas accompagné d'autres termes. Notons que l'ordre des termes varie, parfois certainement dû à la rime :

Et si sont tuit sanglant lor auferant destrier ; Parise, 2004.

E mulz e palefrois e auferrans destriers Aye, 3189.

A pié sont dessendu des auferans destrier[s] : Aiol, 6125. Cf. ib., $6769 ; 6875$.

Il dessendi a terre del destrier auferrant, ib., 5797.

Lors broche Baudoïns le destrier auferrant Saisnes, 1596. Cf. ib., $2553 ; 3904$.

Tante sale voidie de destrié auferran,

Tant cheval estraier ses reines traïnant. Floovant, 541-2.

Et si ont mis lor destriers auferrant, Huon, 6041.

Li destrier auferant font lever le poudrier. Renaut, 5224.

«Et bons chevaux d'Espaigne auferranz et corsiers. ib., 6478.

Et montent es chevalx auferranz et gascons. ib., 9200. Cf. $i b$. 1050 .

Et soient es chevax auferrans et quernus, Gui de N., 2136.

Voir aussi : Charroi, 242; 1415 ; Couronnement, 603; Brun, 702 ; Bueve, I, 6260 ; Guillaume, 2349 ; 2549 ; Simon, 2171 ; Raoul, 2415 ; $2485 ; 2606$ et passim ; Poitiers, 614 ; Narbonnais, $579 ; 2313 ; 4372$ 
et passim ; Mort Aymeri, 2611；2680；2711; Aymeri, 1199; 1716 ; 3204 ; Gaydon, $5282 ; 10743 ; 10756$ et passim.

g. bai.

Le terme indique la couleur du cheval. A.-J. Greimas, op. cit., p. 58, écrit : " D'un poil roux tirant sur le blanc (en parlant du cheval). ", mais, comme rien dans nos textes ne corrobore cette définition, nous pensons qu'il peut aussi bien s'agir d'une robe brun-rouge - telle la définition du Petit Robert. Annette Brasseur écrit, Saisnes, p. 932 : « cheval d'un rouge brun ».

Nous n'en avons relevé qu'un nombre restreint d'exemples ; il y a à peu près égalité entre substantifs et adjectifs :

« Faites moi ci .I. cheval amener!»

Et dist Seguins : « Je l'ai tot apresté.

En cest païs ne sai mie son per,

Le bai d'Arrabe qui molt fait a loer. Mez, 8954-7.

Le bai d'Espagne point et broce ; Ille, 1458 .

Aymeris s'est armé, si sailli el baucent,

Et Guillaumes d'Orenge desor le bai corant, Barbastre, 172-3.

On li a amené le bai de Monsenie,

E Auboïns y monte de si grant legerie Aye, 363-4.

Le bai de Gascogne eslaisça, Atre, 2614 .

Et commande c'on li ameint

Le bai de Gascoigne enselé. Erec, 2658-9. Cf. ib., 733 ; 2153.

Il nos rendront tot le passage

Ne nos tendront ne brun ne bai ; Athis, 11808-9. Cf. ib., 1181920 ; 16226-7; 16781-2.

Par une posterne est issu

Un vallet sor un bai tondu ; Protheselaus, 2262-3. Cf. ib., 9180 ; 9635 .

Pentalis, qu[i] sis uncles fu,

Sist sor un cheval bai tondu ; ib., 7978-9. Cf. ib., 9699-701.

«Amis chevalier sire au cheval bai, Aiol, 3069. Cf. ib., 4267-8.

« Ja est chou li vallès al bai ronci $i b ., 3256$.

Perchevaus point le cheval bai Cont. P., I, 1200. 
Et des Illes li rois Bruisans

Seur destriers sors, bais et bauçans, Inconnu, 5549-50.

La veïsciés tant destrier bai et sor. Aspremont, 3793 .

Un lundi par matin, por voir le vous dirai,

Orent Bertain montee sor un palefroi bai. Berte, 197-8

Voir aussi : Ipomedon, 4044 ; 4630 ; Brun, 2580; Aymeri, 3151; Raoul, 2782 ; 3161 ; 3165 ; Bueve, I, 8472 ; III, 7571 ; 10183.

Dans Saisnes, nous avons relevé quatre exemples du terme, tous substantifs. On note que, dans tous les quatre, le terme est suivi d'un syntagme prépositionnel indiquant l'origine du cheval, mais que celui de Justamont vient tantôt de Cornouaille, tantôt de Russie (deux premiers extraits) - c'est l'assonance qui dirige le choix :

Ai tant Justamonz broche le bai de Cornoaille ;

Plus randone menu qu'espreviers ne grant caille. Saisnes,

3594-5.

Baudoïns point le vair, cil le bai de Rossie,

As targes s'antrefierent. chascune en est croissie. ib., 3669-70.

Les resnes abandone do bon bai de Rossie, $i b ., 4003$.

Murgafier remonta sor un bai d'Orcanie $i b ., 4667$.

Dans Ogier et Jouvencel, nous avons relevé des occurrences de bayart adjectif qui doit avoir le même sens que bai :

Si lui demanda quel cheval il avoit. Si lui dist qu'il estoit bayart, et qu'il avoit quatre piez blans. Ogier, 148.

Sy s'en alla à Luc et obtint le seellé de son cappitaine pour la delivrance d'eulx deux, ouquel estoit promis rendre et baillier au seigneur de Crathor ung cheval baiart, qui estoit meilleur et de plus grant pris de tous les chevaulx qui estoient au cappitaine de Luc. Jouvencel, I, 75.

h. barbari.

Nous pensons qu'il s'agit d'un cheval dont la provenance est la Barbarie, c'est-à-dire le pays des Berbères.

L'un saut el brun baucent e l'autre el barbari, Aye, 2784. 


\section{i. barzelon.}

Selon W. Mary Hackett, le terme désigne un cheval espagnol. Dans notre exemple, il est apposition à Baucan, le nom du cheval de Fouque.

E presentet Baucan lo barzelon. Roussillon, 9259.

j. bauçant.

R. Grandsaignes d'Hauterive définit, op. cit., p. 51 : " [Cheval] de robe foncée, ou rayée de blanc (...) » et A.-J. Greimas, op. cit., p. 60 : « $1^{\circ}$ Blanc et noir, tacheté, pie. $-2^{\circ} \mathrm{n}$. m. Cheval pie. »

Notons les deux premiers extraits : dans Enfances G., bauçant a donné le nom propre Bauçant au cheval ; Renart présente la forme féminine balçane. Aux vers 1933 et 1942 de Guillaume, on pourrait lire Balçan. Cf. 1.1.3. Bauçant.

Mais d'une chose suix je griez et ireiz,

D'un brun bauçant ke ci m'est eschapeiz,

Ke je cuida Orable remener,

Desoz Oranges et poindre et trestorner.

- Filz, » dist li peires, « laisiez Bauçant ester,

Car vostre peires le vos ait bien gardé. » Enfances G., 1403-8.

Ez vos .II. prestres a eslais

qui en aloient au saint sane :

li uns ot une viez balçane

et li autres ot desoz soi

un souef anblant palefroi. Renart, II, 5142-6.

« Ohi, balçan, que je vus poei ja tant amer ! Guillaume, 1933.

« Ha, balçan, bon destrer, tant mar fustes, ib., 1942. Cf. ib., $2054 ; 2179$.

Et mesire Gavains adrece

Viers lui le cief de l'auferant,

Et il guencist sor le bauçant, Rigomer, 7286-8.

Et Garin d'Anseüne sor le baucent crenu, Barbastre, 5078. Cf. $i b ., 172$.

Rollans, li enfes, et Hue, li canu,

Et Graelens, sor le bauçant grenu, Aspremont, 10075-6.

A tant s'en part sor le bauçant Atre, 187 [28].

A la cowe dairier de son riche bauchant 
Atellait le glouton, puez le vait traynant

Jusques a Lion son perre, et li dit en riant : Lion, 25788-90.

On li ameine le balcent en la place ; Couronnement, 409.

Mort le trebuche del cheval,

Si l'abati del noir bauçan Athis, 13176-7.

Esperuns d'argent mer at os bauzan. Roussillon, 3829.

Es vos Tieiri cobrat le baucan ner ; $i b ., 2866$.

E crabentat lo mort del baucan var. ib., 6601.

Li rois Lohot li done le brun baucent crenu Saisnes, 1669.

Dans les deux derniers exemples de Roussillon, l'on pourrait voir dans bauçant un adjectif, mais nous préférons considérer noir et vair comme tels, de même que brun dans l'extrait de Saisnes. Cf. barbari et les extraits suivants.

Dans les extraits suivants, d'Athis, le terme est apposition à destrier :

Nule saiete qui descoche

Ne vet plus tost que li destriers,

Uns bruns baucenz qui mout ert chiers ; Athis, 11662-4.

Sor un destrier sist, baucent brun ; ib., 15797.

Dans l'Escoufle, le jeune Guillaume appelle le mulet d'Aélis baucent (au vers 487 le terme est épithète à destrier) :

"Ahi ! fait il, baucent, baucent,

Com g'ere lores en bon point

Quant cele qui au cuer me point

Vous amena sans mon seü ! Escoufle, 6286-9.

Bauçant se trouve assez souvent avec sor. Selon A.-J. Greimas, op. cit., p. 606, sor désigne un "Nom de couleur, à dominance de lustré, de brillant, corresp. aujourd'hui à fauve, châtain foncé, alezan, roux-brun » - un cheval sor bauçant serait donc peut-être un rouan ou un cheval pie dont la robe tirait sur le brun ? Ceci semble corroboré par le vers 1404 de notre extrait de Enfances $G$. ci-dessus. L'ordre des deux termes semble facultatif, quoique nous n'ayons trouvé qu'une seule occurrence de bauçant sor : 
Perchevaus point le sor bauchant

Qui molt tres surement le porte. Cont. P., I, 4502-3.

Puis prant le sor baucent, met la resne an son bras. Saisnes, 2212.

Car li escus estoit tous blans

Et li chevaus un sors bauçans. Rigomer, 341-2.

Li sors bauçans qui mout fu fors

De cief, de poitrine et de cors,

Hurte l'autre si qu'i l'abat ib., 11271-3. Cf. ib., 12927 ; 13472 ;

$13487 ; 13503 ; 13511$.

Li chevals sor coi il seoit

Ert .I. sors bauçans de Castiele. Raguidel, 4210-1. Cf. ib., 3256$7 ; 5716-7$ et passim.

Adonques li fu amenez .I. sor bauçant qui moult li plot durement. Laurin, 10332-3.

Lors a dit à son sor beaucent.

Dit li quens : « Ne teste autre foiz!»

Ne l'entendi li palefroiz ;

A chief de pose retesta. $M R$, cxuIx, 284-7.

Ne se desroie ne desflece

Mais aussi drois com uns bougons

Es estriers afficiés et lons,

Et seoit sour un baucant sor. Couci, 1275-8.

Nous pensons que dans les exemples de sor bauçant et dans celui de bauçant sor, notre terme est adjectif (voir aussi à la page 116) - il l'est sans aucun doute dans les extraits suivants :

Et Graelent sor le bauçant destrier Aspremont, 9653.

Puis monte en un cheval balchant, Cont. P., I, 2230. Cf. ib., III, 15082-4.

Une pucele chevalchant

Desor un parlefroi bauchant ; $i b .$, II, 9837-8.

Sor un cheval baucent estoit montés, Enfances O., 5140.

Girarz monte en chaval baucan crenut. Roussillon, 6879. Cf. ib., 3946. 
.I. fort destrier bauçant li ont fait amener, Parise, 496.

Puis si s'areste, si descent

Del petit palefroi bauchent Perceval, 7261-2.

Et Resus let cheval aler,

(...)

Si fet Prophilias le suen,

(...)

Sor et baucent, bien fet, d'Espaingne ; Athis, 12725-9. Cf. ib., 12395 .

Vint Jofrois d'Aixe chevauchent

Un grant destrier sor et bauçant Chauvency, 3207-8.

Il l'est probablement aussi dans l'extrait suivant qu'il faudrait donc lire : «j'ai là un [(autre) cheval qui est] pie et pommelé » :

"Se je lais cest enfant ce bon cheval mener,

«Mes fils li ocira, je le sai de verté ;

« Mais j'en ai là jus .I. bauçant et poumelé,

«Celui li chargerai, se vous le me loés. Gui de B., 2257-6o.

Voir aussi : Raoul, 2782 ; 2876 ; Gaydon, 6826 ; Ipomedon, 5055-6 ; 5366-7 ; Claris, 1361 ; 12786 ; Bueve, I, 7728 ; 7862 ; III, 2991 ; 3795 et passim ; Mort Aymeri, 709 ; Chevalerie d'O., $5636 ; 10711 ; 10727$ et passim.

k. blanc/blanchart/blanchet.

Peu de textes nous présentent des exemples où cette dénomination qui, évidemment, désigne un cheval blanc, est substantif. Les exemples de blanc adjectif sont quasi innombrables. Dans tous les cas, les chevaux blancs sont hautement estimés :

Desus le blanc d'Arrabe isnelement monta. Barbastre, 6558.

Sor le blanc de Rossie qui les sauz li porprent ; $i b ., 6908$.

Ves en chi un sor cel blanc pumelé Bueve, I 4596.

« Nous avons ci chevax fors et courans :

«Prenés cel noir et je panrai cel blanc ; Raoul, 7516-7.

Li palefrois sont apresté,

Richemant furent atorné.

Sor un blanchet mout avenant

Et mout bien fet et de biau grant 
Ont montee la dameisele. Athis, 6937-41.

Lors l'en conduist au palefroi.

Sor le blanchet l'ont remontee ; ib., 7666-7. Cf. ib., 8773-4.

La pucelle monterent adonc sur ung blanchart ; Tristan de $N$., 4408.

Regarda devant lui, s'a veü acourant

Ung moult fier chevalier qui en vint chevauchant

Dessus ung grant blanchart qui valloit maint besant, $i b .$, 912931.

Mort le tresbuche a terre du bon cheval blanchart, $i b ., 2776$.

Cf. $i b ., 2776$.

.I. chevalier tout desarmé

Plus blanc que une flor de pré.

Blanc sont li drap, blanc li cevaus, Rigomer, 5027-9. Cf. ib., 12989-92; 13027-8.

Tous armés sor le blanc cheval

Se met les rues contraval. Diable, 4137-8. Cf. ib., 1796-7.

Il sist sour le corant destrier

Qu'acata li ostes courtois :

Plus blans est tous que nule nois

Fors que le pié destre avoit brun. Amadas, 4264-7.

Vo blanc destrier me faites delivrer

Ki l'autre soir vos vint d'oltre la mer. Aspremont, 2174-5. Cf.

ib., 362-3; $2211 ; 2689-90 ; 7580-1$.

Lors regarde tout contremont la rive et voit venir une damoisele, ausi conme a besoing, montee sour un palefroi blanc, (...) TP, vI, 105, 1-3. Cf. ib., vIII, 82, 3-4.

L'endemeyn le marchant prist un palefroy blanc, si bel ne fust en tote le roialme, e le presenta a le roy Johan, qe molt leement le reçust pur sa belté. Fouke, 79,28-80,1. Cf. ib., 70, 23-6.

Voir aussi : Enfances O., 8146-7 ; 5401-5 ; 5537-40 ; Helcanus, 130 ; Inconnu, 3942-5; Renaut, 4757 ; Gui de B., 2325-6; Mez, 1417 ; 3146 ; Amadas, 5635-6 ; Cont. P., I, 1984-5 ; Barbastre, 2814 ; Erec, 2167 ;Ipomedon, 3532 ; 3596 et passim ; Thèbes, 5656-7 ; 6299-300 ; Dole, 2492-5 ; Queste, 252, 3-9 ; Blancandin, 1218 ; Cligés, 3567-71 ; 3982 ; Méliador, 4822-5 ; 3060-1 ; Lancelot, viı, xxIa, 23. 


\section{1. brehaigne.}

Dans Aliscans, la brehaigne est une jument stérile que monte Desramé. Il est à remarquer qu'elle lui sert pour les batailles. Il en est de même dans Aymeri, où le terme est épithète :

Atant ez vos Desramé, lor seignor,

Sus la breaigne, qui li cort de vigor. Aliscans, 33-4.

Desramez vient poignant toz irascuz

Sor la brehaigne, qui fet les sauz menus ; ib., 6106-7.

Desramez vet parmi l'estor poignant

Sor la brehaigne, qui soz lui vet bruiant. ib., 6129-30.

Il esperonne la grant ive brehangne

.I. Alemanz, desor une ive grant, Aymeri, 1777-8.

L'extrait suivant présente le syntagme aufage brehaigne, où Claude Régnier, p. 332 de son édition de Aliscans, traduit aufage par «sarrasin "; il voit donc dans brehaigne le noyau du syntagme, ce qui donnerait la lecture suivante : " jument stérile sarrasine ». Une autre possibilité serait d'y voir aufage comme le noyau avec le sens de « jument » (cf. p. 85, Aliscans, 1762-3) et brehaigne comme un adjectif, avec le sens de "stérile », et donc lire simplement « jument stérile ». Il ne nous semble pas possible de décider définitivement quelle analyse préférer :

Puis est montez en l'aufage brehaigne,

N'ot tel cheval en France n'Alemaigne,

Ne recreroit a pui ne a montaigne,

Plus cort par tertres qu'autre ne fet par plaigne. Aliscans,

5234-7.

m. brun.

Une autre dénomination qui montre la couleur du cheval. Comme substantif, le terme est très rare. Dans l'extrait de Buevon, nous n'osons affirmer lequel des termes brun et bai est le noyau du syntagme (nous sommes enclin à penser que c'est brun ; voir aussi : bai et morel).

Et Looïs desserre sor lo brun de Pavie ; Barbastre, 6933.

Tuit fussent pris, n'en alast uns,

N'en eschapast ne bais ne bruns, Athis, 11819-20. 
Devant lor armes ne garisent

Ne brun ne bai. Fierent, trebuchent, ib., 16226-7. Cf. $i b$., 16781-2.

C'ert uns bruns bais d'Espaigne, mais a droit souhaidier Ne trouvast on meillor pour ses armes baillier. Buevon, 2879-80.

S'estoit chascuns montés sur un bon cheval mor,

Nul n'en ist a pié : chascun ot brun ou sor. Brun, 1164-5.

Bien fu armés sor le brun d'Abilant ; Bueve, I, 8511.

Les cas où le terme est adjectif sont évidemment plus nombreux, sans fourmiller pourtant. Nous pensons que dans l'extrait de Florence, et brun et bai sont épithètes à destrier et qu'il faut lire « des destriers dont les uns sont bruns, les autres bais ».

Cil montent es chevaux bruns et bauceins et noirs, Renaut,

6317. Cf. ib., $11364 ; 12253$.

Alexis ot un cheval brun, Thèbes, 5577. Cf. ib., 5659-62.

Trenche le col del brun cheval Athis, 12616.

Esmerez vint a Romme, mout par fu liez et gais

Et jure Damedeu et le cors saint Gervais

Qu'ancor fera Grifons coreseus et irais

Et conquera destriers et des bruns et des bais. Florence,

2207-10.

n. chaceor.

Le chaceor est un cheval rapide utilisé spécialement pour la chasse et pour la course. A cause de sa rapidité, il peut servir de monture aux messagers. Le terme est normalement substantif (Friedrich Bangert, op. cit., p. 9, le met dans la catégorie des termes substantifs). Voici la plupart des exemples que nous en avons relevés :

Lanselos monte el cacheor

A grant paine et a grant dolor. Rigomer, 2071-2.

Mais n'öi hüer ne huchier

Ne cor ne chien ne caceor

Ne forestier ne veneor, $i b ., 1382-4$. 
Un serjant sour un caceour,

Si com apert l'aube dou jor,

Envoie por les compaignons. Amadas, 6809-11.

Montez fu sus un chaceour,

assez tost fu a son seignour. Cleomadés, 6707-8. Cf. ib., 6844-5.

Crompart firent monter errant

sor un des chaceours le roi ;

son chevalet prist deles soi, $i b ., 6844^{-6}$.

Palefreiz, chasçurs e desters

Lur dona il mult volenters, Gui de W., 157-8.

Le chaceor monte et s'en torne ; Tristan, 3366.

Se leva, et ne li fu paine

Que il sa sele ne meïst

Sor son chaceor et preïst

Trois gavelos, et tout issi

Fors del manoir sa mere issi. Perceval, 76-8o.

Cil venoit le hiaume lacié

et a sa venison trossee,

tel con Dex li avoit donee,

sor un grant chaceor ferrant. Charrete, 2018-21.

Et vit venir molt tost errant

Sour un grant cacheour ferrant

Un escuier gent et adroit. Violette, 4664-6.

(...) ; et devant els tot le chemin venoit .I. nains sor .I.

chaceor. Lancelot, II, LV, 1.

Et il sist sor .I. moult boin cacheor, si s'en parti del castel entre noune et vespres por les noveles porter a cort, (...) ib., vII, xxva, 1.

Por cele honte qui t'atent

Te donrai je autant d'onor

Se tu me renz mon chaceor! » Meraugis, 1444-6.

La nuit s'ambla de cort desor un chaceor,

Et a tant chevauchié par plain et par destor

Qu'il est venuz a Trape ou sont li vangeor

Qui vangeront la mort Guiteclin lor seignor. Saisnes, 5615-8. 
Li mes vint la poignant desor un chaceor. ib., 6846. Cf. ib., 7362 .

Es vos poingnant un veneor

Deseur un ronci caceor. Inconnu, 1305-6.

Sor un vair destrier cacheor

Est li enpereres montés. Diable, 2138-9.

Dans Partonopeu et Durmart, nous remarquons que le chaceor est aussi appelé roncin; dans Lancelot, aussi roncin et cheval; voir aussi p. 69 des extraits de Gautier d'Aupais :

Son cacheor en a mené ;

Li roncis est maigres et las, Partonopeu, 776-7. Cf. ib., 610-1 ;

$643-4 ; 647-8 ; 666 ; 686-7 ; 1632$.

Mon chaceor prist en sa main,

Et si le bailla a son nain. » Durmart, 3405-6.

Me toli ore mon ronci $i b ., 3391$.

Et quant la caure se rebaissa, si monta sor son chaceor et s'en revint au lac, (...) puis vint a son ronchin, (...) Et il descent, et ele prent sont cheval, sel fait establer. Lancelot, viı, xxia, 3-6.

Voir aussi : Atre, 914-5. ; Yder, 24-5 ; 243-4 ; Ipomedon, 2697-700 ; 4279-8o et passim ; Erec, 73-4 ; 123-4 ; Guillaume d'A., 2621-4 ; Raguidel, $1610 ; 1671 ; 1882$.

o. courant.

Ce terme est employé très souvent comme adjectif pour indiquer qu'un cheval est rapide. Puisque courant s'accole dans tous ces cas à cheval ou à destrier, il y a lieu de supposer que le courant est toujours un cheval de bataille. L'ordre des mots est facultatif :

« Issons noz en, biax frere, ce voz pri,

Sor les chevax coranz et arrabiz. » Mez, 825-6. Cf. ib., 3894 ;

14198 .

Leur chevaus furent courans et roides, (...) Helcanus, 114.

Sus .I. destrier courant né d'Egremorte,

Monte Judas qui pas ne se deporte ; Auberon, 499-500.

Qu'atout .L. chevaliers

Armez sor les coranz destriers Claris, 1090-1. Cf. ib., 16614-5. 
« Or et argent et pailes i a à grant planté,

« Et maint destrier corant et maint faucon mué. Gui de B.,

19-20.

Li Sarasins monta sor .I. corant destrier. ib., 426. Cf. $i b ., 495$.

Voir aussi : Lion, 12923-4 ; Enfances O., 874-6 ; Huon, 292-3 ; 3225 ; Otinel, 334 ; Floovant, 96-7 ; 586-7 ; Barbastre, 94-5 ; Aspremont, 2260-1 ; 7224 ; Renaut, 115-6 ; 457-8 ; Inconnu, 614 ; Enfances G., 368 ; Roche, 2286 ; 2431 ; 2455 ; Tristan de N., 5330 ; Galeran, 56101 ; Couci, 1208-9 ; TP, v, 130, 20 ; Aiol, 3848 ; 4713 ; Rigomer, 160679 ; Couronnement, 2483 ; 2628 ; Saisnes, 4319 ; Partonopeu, 3488 ; Charroi, 333 ; 358 ; 702 ; Athis, 7706 ; Brut, 750 ; 3230 ; 4102 ; Brun, 1178 ; 3341 ; 3406 ; Bueve, I, 1042 ; 1713 ; 1812 ; III, 3811 ; 3100 ; 4320 et passim ; Erec, 2019 ; 2391 ; Gautier d'Aupais, 57 ; 835 ; Lanson, 1523 ; 4047; 5713 et passim; Aymeri, 1905; 2028; 3196; 3819; Mort Aymeri, 1214 ; 1548 ; 1806 ; 2603 ; Raoul, 1533 ; 2057 ; 2549 et passim ; Narbonnais, $6290 ; 6293$.

Comme substantif, il est beaucoup plus rare. Nous ne sommes même pas sûr qu'on puisse y voir un substantif dans les exemples de $\mathrm{Mez}$, de Couronnement, de Bueve, de Chevalerie d'O. et dans le premier exemple de Saisnes, où il est apposition à Flori, à Alion, à Arondel, à Broiefort et à Vairon : dans les cinq cas, on pourrait sans difficulté le remplacer par l'adjectif « rapide » :

Devant la fille au roy qui fuit de noble orine

Demoustre son corrant, poissant lez une espine ; Lion, 5404-5.

Fiert soi dedens sor Flori, le corant. Mez, 5137.

On li ameine Alion le corant, Couronnement, 2503.

Et Tiers prist Arondel le corant, Bueve, I, 8079. Cf. ib., I, 7738.

Et Ogier broce Broiefort li corant ; Chevalerie d'O. 7908. Cf. ib., $10070 ; 1+240 ; 11013$.

A tant ez Baudoïn sor Vairon le corrant, Saisnes, 4977.

Cil corrant arrabi demainent grant podror. $i b ., 6100$.

p. coureor.

Un autre terme qui indique la rapidité et qui, lui aussi, s'emploie pour désigner des chevaux de bataille. Nous n'en avons relevé que peu d'exemples : 
Li vesques de Forois dessus le courëour, Bâtard, 174 .

Quant l'ot li dous, li frans, s'en ot au cuer tendrour,

Plore de ses biaus iex, si change sa color,

Et fiert des esperons Fauvel le coureour. Partonopeu- $C$, 2526-8.

Et aresna Bauçant le coreor. Mort Aymeri, 135. Cf. ib., 1582.

Et Gaudin point et broche le coraor Isnel, Florence, 1388.

Et chevauchent destriers coraors de Sulie, $i b ., 3436$.

Et de Flori, le destrier coureor. Mez, 4277.

Et Lancelot chevaucha tout le jor par mi la forest jusqu'a ore de prime et tant qu'il encontra .I. chevalier armé de toutes armes et du montez sor .I. grant destrier coureor. Lancelot, IV, LXXXIII, 20.

Veuren cau la ferent cist vantador,

La maisnade Girart, prefolador,

C'ont les chevaus noovens e corador. Roussillon, 3631-3.

Kalles vint à l'estout à mout riche compengne

Et sist seur .I. cheval coureor d'Alemaigne Gui de N., 2462-3.

Voir aussi : Narbonnais, 766 ; Aymeri, 2935 ; 3151.

q. coursier.

R. Grandsaignes d'Hauterive, op. cit., p. 138, explique: " I. Qui court bien, rapide (épith. souvent accolée à cheval et à nef) : (...) II. Cheval ; coursier (...) » Le terme est donc sensiblement synonyme des deux précédents et, comme eux, il sert à souligner la rapidité de chevaux de bataille ou de chasse.

Il se présente seul, avec ou sans article :

Il broche l'auferrant, et rois Guis le corsier ; Gui de B., 592. Cf. ib., 576 .

Si fu legier a aprester

Pour cheminer et pour errer,

Quer pallefrois n'ot a fferrer

Ne coursier c'on li maine en destre, Anjou, 5592-5.

Dedens l'estable menerent le coursier, Bueve, II, 10960. 
A oié descent del fauvelet corcier. Raoul, 1543.

Vous m'avez bien servir sans avoir nulz loier.

Li ung donne ung ronsin, li altre ung courcier, Lion, 1078-9.

Vous avez san raison affolléz mon corcier, ib., 12655. Cf. $i b$., 24565 .

Quant on dut laissier le jouster,

Li sires de Couchi monter

En va errant sour un coursier. Couci, 1808-10.

Mais moult ert biaus et bel sot chevauchier,

Montés estoit sus .I. moult bel coursier,

.I. palefrois le sievoit par derier. Auberon, 845-7.

Ceulx qui veullent boire bevront

Legierement, puis l'en amaine

Coursiers, qui ont mengié avoine,

Si verrés monter ven[e]eurs, Deduis, 8004-7. Cf. ib., 8016-7 ;

8021.

(...) ; si tost qu'il eut fait adouber son coursier Broyfort, monta au palais (...) Ogier, 83 .

Dans les extraits suivants, nous voyons en coursier une apposition aux noms propres :

Et Renaut est monté sor Bayart le corsier, Renaut, 5250. Cf. ib., 6646.

Et vous fussiez montez sor Flori le corsier, Saisnes, 3135.

Baudoïns est cheüz de Vairon le corsier, $i b ., 6761$.

Bueve descent d'Arondel le coursier, Bueve, III, 1805. Cf. ib., $3818 ; 3929$.

Voir aussi : Brun, 206 ; $2074 ; 2504$ et passim ; Laurin, 5842-5 ; Lanson, 625 ; Gaydon, 7413 ; Doon, 2940 ; Godin, 9112 ; 9177 ; 10397 et passim ; Chevalerie d'O., 6310 ; 10214 ; Jouvencel, II, 238 ; Jehan de S., 99, 6 ; 100, 13 et passim ; Jehan de P., 54, $10 ; 54,28$ et passim.

Dans les syntagmes substantifs accolés à auferrant, il est probablement épithète (voir d'autres exemples pp. 87-88), comme c'est le cas avec cheval, destrier et mulet : 
Aprés brocha chascun son auferrant corsier ; Barbastre, 2272. Cf. $i b ., 5838$.

Fain et avaine a l'auferrant corsier. Charroi, 552.

Mort le trebuche de l'auferrant coursier. Bueve, II, 6173. Cf. ib., 3387.

Si roidement s'aficha es estriers

Que soz li ploie ses auferrans corsiers. Narbonnais, 7046-7.

« Amis, » fait il, « pour Dieu vous vueill priier

$\mathrm{K}$ 'a ce besoing nous vousissiez aidier,

Que vousissiez vostre cheval coursier

Un petitet des esperons brochier, Enfances O., 1683-6.

Jus le trebuchent du bon cheval corsier ; Mez, 9038 .

Et al fais des cevaus arabi[s] et corsier[s]

Se sont tant fierement anbedui acointié Aiol, 6803-4.

Et montent es chevaus arabis et corsiers, Roche, 2469.

E chevauche un cheval corser braideuc, Roussillon, 5097. Cf. $i b ., 5080$.

Et maint cheval corsier de Gascoigne ou d'Espaigne. Saisnes, 847 .

Chevaus delivres et corsiers Athis, 9903.

Amené li ont un destrier

Fort et hardi et bien coursier. Rigomer, 835-6.

Lors font metre les seles sor li mulet corsier, Renaut, 6785 .

Voir aussi : Brun, 830 ; 3473 ; Bueve, I, 5041 ; 6195 ; 8061 ; II, 2167-8 ; Raoul, 1363 ; 2275 ; 3063 ; Aymeri, 1763 ; 2859 ; 3742 ; Doon, 3265 ; 6692 ; 8429 ; Gaufrey, 9497 ; Gaydon, 5516 ; 6023 .

\section{r. courtaut.}

Le terme peut s'accoler à chien et cheval ou être substantif. Dans tous ces cas, il désigne un animal à qui l'on a coupé la queue et les oreilles. On le connaît depuis le $\mathrm{xv}^{\mathrm{e}}$ siècle.

Ses gens hodez et traveillez, et leurs chevaulx aussi ne contredirent pas a monseigneur, qui picque son courtaut et fait tant 
en peu d'heure qu'il est en la basse court de son hostel descendu, ou il trouva ung varlet qui le deffist de son cheval. Cent, $16,70-5$.

s. crenu.

Ce terme est normalement un adjectif signifiant « à la belle crinière et aux longs crins (en parlant d'un cheval) " (Annette Brasseur, Saisnes, p. 1038). Nous en avons relevé un seul exemple où il est substantif :

L'escu a anbracié et broché le crenu

Et tint le branc d'acier dedanz son poig tot nu, Saisnes,

6533-4.

Et li vassax descent de l'auferrant crenu. ib., 6547. Cf. $i b$. , $7579 ; 1669$ et passim.

A destre uns chevaliers leur sourt

Sour un destrier crenu qui cort

Plus que ne destent uns quariaus. Amadas, 4629-31.

La veïssiez tant bons destriers crenuz, Aliscans, 6096.

Li soudans est montés le blanc destrier crenu ; Partonopeu- $C$, 2615 .

Rechainglent fermement les auferrans grenus, Bâtard, 5535 .

Voir aussi : Bueve, I, 2908 ; II, 5053 ; Erec, 1411 ; Simon, 14 ; 292 ; Brun, 247-8 ; Aymeri, 4170 ; Mort Aymeri, 1166.

t. esclavon.

Le terme désigne un cheval slave ; il s'accole aussi, comme adjectif, à d'autres animaux.

Quant le vit Esmerez, si broche l'esclavon ; Florence, 838.

Cele place font traire mainte mule esclavonne, $i b ., 3281$.

u. espagnol.

Nous n'avons relevé que peu d'occurrences de ce terme. Il doit indiquer la race des chevaux en question, mais nos exemples ne nous paraissent pas tous très clairs. L'espainol dans Yder est appelé vair espainol au vers 1289 :

Yder li feit le fust sentir,

Oltre l'empeint de tel ahir 
De l'espainol que il l'en serre

La lance entire contre terre. Yder, 1313-6.

Hutebon point et broche l'epagnol d'Oriant, Barbastre, 6006.

Lances e gonfanons, escuz de Bles,

Grans chavaus e corsers e espanes Roussillon, 4775-6.

Et chevauchent chevaus gascons et espanois, Renaut, 1771.

De cendaul sont covert li destrier espenois Florence, 1217. Cf. ib., 2009 .

armes et pallefroiz et chevals espanois, Rou, II, 4128 .

v. fauve/fauvel/fauvelet $=$ cheval fauve.

De même que W. Mary Hackett, nous nous demandons si, vu le défaut d'article, il ne faudrait pas lire Fauve dans l'exemple de Roussillon :

Envers Teuri tornet fauve el buort, Roussillon, 2856.

En mi la place es vos un chevalier.

Descendus est d'un grant fauve destrier. Aspremont, 194-5.

Et Cligés est venuz atant,

Plus verz que n'est erbe de pré,

Sor un fauve destrier comé.

La ou Cligés vint sor le fauve,

N'i ot ne chevelu ne chauve

Qui a mervoilles ne l'esgart, Cligés, 4714-9.

$\mathrm{Du}$ tresor en porterent tant conme il lor plot,

.III. chevaus en menerent, .ii. fauves et .I. sor. Aye, 2472-3.

Il broche le destrier des tranchans esperons,

E Garniers le fauvel qui li cort de rendon, ib., 408-9. Cf. $i b$., 1317.

Et Morans sist sor un destrier fauvel. Gaydon, 5191.

A pié descent del fauvelet corcier. Raoul, 1543.

w. ferrant.

Chez A.-J. Greimas, op. cit., p. 283, s.v. ferant, nous lisons : « adj. (...) avec infl. possible de alferant, coursier). Couleur gris de fer, 
grisonnant, épithète fréquente du cheval et de la barbe (...) n. m.

(...) Coursier, cheval de bataille. »Cf. 1.1.3. Ferrant.

Voici des exemples où le terme est substantif :

Rois Amalgons et li rois Esperrant,

L'uns sist el noir, l'altres sist el ferrant, Aspremont, 6175-6. Cf. $i b ., 8771$.

Et cil s'en est torné sor ferrant de Montir, Renaut, 1942.

Meïsme Karlemaigne les sivi de randon

Sus ferrant d'Alisandre qui va comme faucon. $i b ., 2214^{-5}$. Cf. bi., 13784 .

Apoingnant vint Garsy de Marre et sist sor ferrant de Navarre. Thèbes, 4661-2. Cf. ib., 5529-32.

« Tote l'une moitié de nostre bone gant

" S'en voit tot cest lariz avaul esperonant,

"Si que n'en saichent jai Sarazins et Persanz

« Deci que desor auz soïmen nos feranz,

« Si les acoilons bien à nos espiez troinchanz. Floovant, 195963.

Li rois Bilas siet el ferrant ;

Ainz ne vëistes plus corrant. Athis, 7705-6. Cf. ib., 12236-7 ; 12466.

Devant tous ses pers vint dans Butor de Montir

Sor un petit ferrant qui porte par aïr Partonopeu-C, 1748-9.

Et li palefrois sor qu'il sist

Estoit uns ferrans pumelés. Escoufle,7162-3.

Li bons chevaus ke il set desus

Esteit uns ferrans pomelez,

Plus beaus destrer ne fust une nez, Ipomedon, 5044-6.

Melander veit venir avant,

Mult coveite le bon ferrant. Protheselaus, 7980-1. Cf. ib., 9052

En voici d'autres où il sert d'épithète. Les deux extraits d'Aspremont donnent une telle quantité de nuances qu'il est finalement assez difficile de savoir exactement de quelle couleur est la robe du cheval d'Ulïen - elle est probablement rouge-gris pommelée : 
Uliens sist el rous lïart ferrant,

Li viels Galindres sor un mulet amblant ; Aspremont, 8022-3.

Ulïens sist el ros lïart ferrant ; $i b ., 8734$.

Et l'amiraus fait Bauçant enseler :

C'est uns cevax ferrans et pumelé,

N'ot si courant en trestout le rené. Huon, 6515-7.

un cheval ot ferrant obscur, Thèbes, 5503. Cf. ib., 6292.

«Vez le vos la as armes, sor cel ferrant destrier, Parise, 1901.

Sor .I. rocin ferrant font le serjant monter, $i b ., 2080$.

sor un grant chaceor ferrant. Charrete, 2021.

Et sist sor un cheval ferant. Protheselaus, 1095. Cf. ib., 7975 ;

8699 et passim.

Lors li vient un garçons poinant

Desor un grant cheval ferrant Yder, 1223-4.

Voir aussi : Blancandin, 3372 ; Erec, 1122 ; Rou, II, 3247 ; Simon, 426.

Dans les extraits de Violette, où il est question du cheval de Gerart de Nevers (et non d'un cheval indéterminé, comme p. ex. dans celui de Thèbes), nous sommes tenté de lire Ferrant au lieu de ferrant, à cause de l'article manquant. D.L. Buffum n'a pas de commentaire à ce sujet et nous gardons donc le texte de son édition. Cf. nos remarques au sujet de l'extrait de Florence, p. 120.

Molt par ont rechut liement

Gerart, car l'ostes s'entremet

De lui servir et se li met

L'esprevier a la perche errant,

Et si fait establer ferrant. Violette, 2519-23.

Des espourons broche ferrant, $i b ., 2766$.

D'espouronner ferrant ne fine, $i b ., 4866$.

x. gascon.

De même qu'aragon, ce terme indique l'origine des chevaux en question et rien d'autre. Sauf que le contexte nous renseigne qu'il 
s'agit de destriers, car le terme apparaît dans des scènes de bataille et s'accole à destrier :

Ly Sarrasin l'ont pris et versés du gascon

Et puis l'en ont mené tout droit au pavillon Tristan de $N$., 2263-4. Cf. ib., 19675-6.

Mais Lion fuit ligier et de gente faichon,

Tout dairiere le prince li salt sur le gascon. Lion, 7134-5. Cf.

ib., $12564 ; 15230 ; 27161-2$.

La veyssiez baitaille et ung estour felon,

Tant gentilz chevalier abaitre du gascon ib., 22901-2.

Les menuz salz i prent a returner,

Plus tost n'i fust pas un gascoin sojurnez. Guillaume, 2767-8.

Tant de gent averay a bien courte saison

Qu'i[l] seront .iii.c. mille montez sur le gascon! » Lanson, 1897-8. Cf. ib., 2973.

Bien fu armés sur le gascont,

Un bel ceval de molt grant pris. Inconnu, 1714-5. Cf. ib., 596o.

Et sist sor un ceval gascon ; ib., 1092.

Et montent es chevaux auferrant et gascon. Renaut, 1050. Cf. $i b ., 1771 ; 9200$.

« Et maines avec toi .v.c. de tes barons

"Adobez de lor armes sor les destriers gascons, ib., 585-6. Cf.

ib., 77-8.

'Feitez moi tost armer .c. de vos compengnons

Et vestir les haubers desous les auquetons

Et monter es chevax auferrans et gascons ; Gui de N., 969-71.

et sist sor un cheval gascon Thèbes, 9732 .

Son chevals li ont amené,

Un mult buen vair destrier gascon, Joufroi, 402-3. Cf. ib., 3080-1.

Monte en la selle dou bon destrier gascon, Ami, 1657.

Ayglentine est montee u palefroi gascon, Gui de N., 1359. 
Un extrait d'Athis a attiré notre attention et son analyse nous a causé quelques difficultés. Nous pensons que destrier souligne la qualité du cheval en question et que gascon indique que cette race fournit les meilleurs chevaux de bataille :

Point le cheval des esperons,

Qui fu destriers come gascons,

Brandist la lance et l'escu prent ;

Et li chevaus bruit et destent. Athis, 13015-8.

Voir aussi : Gui de B., 3527 ; Enfances O., 2732-3 ; Tristan de N., 16674-5; Mez, 3207 ; Gormont, 283-5; 551.

y. gris.

L'uns monte el vair et li autres el gris.

Y. el noir q'en l'estor fu conquis, Raoul, 3861-2.

Es destriers montent, sors et baucenz et grises. Mort Aymeri, 709 .

La pucelle monterent sus ung destrier gris. Lion, 28184 .

Avecques li veneoit .j. courtois jouvencel

Qui bien estoit montés sus un cheval grisel. Brun, 1877-8.

Si eussiez veu venir six cens hommes tous montez sus grisons d'ung poil et d'une sorte, (...)

Jehan de P., 65, 2-3.

Une pecheurs me rivela

Tout outre le rivier dela,

Et mon palefroi griolé ; Jehan, 3131-3.

z. liart.

Voici encore un terme indiquant la couleur du cheval : gris pommelé. Les exemples ne sont pas très nombreux, et il est parfois malaisé de préciser si liart est noyau ou épithète dans les syntagmes substantifs. Voir à ce propos ros. Cf. 1.1.3. Liart.

El liart sist d'Esclavonie

qui fu bons pour chevalerie. Thèbes, 6327-8.

La contesse leverent sor un liart gascon ; Barbastre, 246.

Athis i vient sor le liart, Athis, 12659. Cf. ib., $12731 ; 18143$. 
Le vavasour ount sucurru,

Mes le bon lyard ad perdu ; Protheselaus, 11852-3.

Destrier ot bel et bons qui ert liars. Enfances O., 5047.

Le terme est indubitablement épithète dans les exemples suivants :

C'estoit de tous li mieus montés :

sour un cheval liart seoit, Cleomadés, 676-7.

Il sist sor un destrier liart, Florimont, 6719.

A tant ez Baudoïn sor un destrier lïard, Saisnes, 1737.

Il sist sur un chival liard. Protheselaus, 11732. Cf. ib., 11795.

Athis repoint a destre part,

Qui sist sor un cheval liart: Athis, 16771-2. Cf. ib., 16893.

De son cheval liart per mort le loigne. Roussillon, 2772. Cf. ib., 2859 ; 6010 .

En meine le liart cheval. » Ipomedon, 5408. Cf. ib., 5098.

Voir aussi : Blancandin, 4552 ;Aymeri, 4141 ; Chevalerie d'O., 5133 ; 5146 ; Gaufrey, 4058.

aa. missaudor.

Un missaudor est un cheval de bataille de grande valeur. Louis Demaison, Aymeri de Narbonne, II, p. 236, définit : « (...) [destrier] de prix (valant mille sous) ». Il est donc tout à fait naturel que le terme se trouve régulièrement accolé à destrier :

Rollans, li enfes, qui sist el misordor,

Durendal tint entainte sans color. Aspremont, 8907-8.

Pres de la pucele se traist,

Se li consele par amor

$\mathrm{Au}$ nain qui tint le misaudor.

Li nains le palefroi caçoit

Que la pucele cevaucoit. Inconnu, 2762-6.

Il n'ancontrait vaissalz, filz de roy n'amaissour

Qu'il ne getaist a terre prandant le misadour Lion, 7680-1. 
Et li escuiers toute voie

Va tant, qu'il trouva son seignor,

Bien armez sor .I. missodor ; Claris, 23374-6.

Atant i point le missodor, Thèbes, 8693. Cf. ib., 705-7.

Gaydes li dus broche le missoudor, Gaydon, 7324. Cf. ib., 5049 ; 5056.

Aprés sont remonté et chevauchent le jour,

Tour droit vers Ermenie s'en vont li missoudour. Tristan de N., 6535-6.

Cf. ib., $11734:(\ldots)$ destrier missoudour.

"Et si n'aiez onques de ce poor,

"Que tant com puisse monter el misodor,

« N'avront paien ancontre moi sejor. Aymeri, 1267-9. Cf. ib., $2950-1 ; 1258 ; 2926 ; 3149$.

Devant les autres vint seur le missoudour

K'en champ conquist a Roume l'autre jour, Enfances O., 5286-

7. Cf. ib., 6307.

Laissiés li a le missaudour

Mult volontiers sans contredit. Amadas, 4172-3.

Les trenceüres, ce m'est vis,

Furent larges pour la blancor

Veoir dou ceval missaudor, $i b ., 4272-4$.

Et Perchevax du misaldor

descent, et puis l'a athachié Cont. P., III, 16870-1.

tant que ce vint a l'avespree,

qu'il encontra un vavasor

de sor un destrier misaldor, ib., III, 16430-2.

Renaut sist sor Bayart, le destrier misoudor, Renaut, 11468. Cf. ib., 11685 .

Or furent li baron es destriers misodors. Gui de B., 2606. Cf. $i b ., 4139$.

Vez ci Ogier qui nous fist l'autre jour

Maint Sarrazin morir a grant dolour ;

Bien reconnois ce destrier missoudour. » Enfances O., 1744-6.

Cf. $i b ., 4527-8$. 
Voir aussi: Athis, 11823 ; Partonopeu, 8839-40 ; Saisnes, 2722 ; 2515；7761; Godin, 12666；14792；16311； 17558; Mort Aymeri, 341 ; 436 ; Lanson, 407 ; 410 ; Blancandin, 4864 ; Bueve, II, 60 ; 307 ; III, 26 ; 233 ; 1713 ; 7048 ; Simon, 1998 ; Raoul, 4127-8 ; Narbonnais, 3563 .

ab. mor/morel.

R. Grandsaignes d'Hauterive, op. cit., p. 422, écrit que ce terme désigne les couleurs noire et brune et aussi « Cheval maure ; cheval noir (...) ».Il cite notre exemple tiré de Gormont et Isembart :

Car veça Gaudion, le chevalier loyel,

Le plus hardi paien qu'ains montast sur morel. Tristan de N., 2660-1.

ja l'eust mort icist, pur veir, quant a lui lança un Ireis ;

suz li, ocist sun bon moreis. Gormont, 99-101. Cf. ib., 104.

Il trait l'espée dou fouriel

Et esperonne le moriel Méliador, 10582-3.

Estouls de Lengres sist ou vair de Castaingne,

Et Bernars sist sor le vair d'Alemaingne,

Et Viviens en morel d'Aquitaingne. Gaydon, 5446-8.

Cil prendent la fille al Norois

Et maint cheval avoec morois, Silence, 231-2.

Li damoisiaus Laurin de Costantinnoble, qui fu montez sus morel, s'enpaint aprés son pere (...)

Laurin, 367-9. Cf. ib., 1083-4; 1991-3 ; 3420-1; 11404.

Et a ceste parole vint Dannemont sur ung grant cheval moreau (...) Ogier, 21.

Done mon pere la couverte

Qui est sus mon cheval morel. MR, v, 320-1.

Sinagon point et broche le bon destrier morois Florence, 2023.

S'estoit chascuns montés sur un bon cheval mor, Brun, 1164. Cf. $i b ., 2467$.

Si issi hors as chans sur .j. morel coursier ; $i b ., 2074$. Cf. $i b$. , 3210. 
Dans Roussillon, W. Mary Hackett donne les définitions « cheval arabe " pour maurel et « cheval more " pour moresc. A notre avis, il y a confusion entre race/origine et couleur. Nous hésitons devant l'analyse des syntagmes bai moresc et brun moresc, dans lesquels les trois termes peuvent être noyau ou épithète :

E desrauchet l'envers del maurel ner. Roussillon, 2871.

E donrai vos sempreres icest mores. ib., 9328. Cf. ib., 8422-3.

E desrauchet l'envers del bai moresc ib., 2732. Cf. ib., 1323.

A tant Folche lai ven per lo calmesc

E descendet a pie del brun moresc. $i b .$, 9299-300.

ac. mouvant.

Vers aus adrece Broiefort le movant, Chevalerie d'O., 6376. Cf. ib., $7865 ; 10995$.

Turnus point le destrier movant, Eneas, 9701.

ad. noir.

Il est évident que noir se trouve souvent avec cheval, destrier, palefroi pour en désigner la couleur. Dans les exemples d'Athis, d'Aspremont et d'Aye, on a l'impression que les auteurs auraient pu mettre cheval devant noir, ferrant et fauvel:

Rois Abïerne sist el noir

Qu'il ne donast por nul avoir, Athis, 7307-8.

Rois Amalgons et li rois Esperrant,

L'uns sist el noir, l'altres sist el ferrant, Aspremont, 6175-6.

Garniers sist el fauvel e Haguenon el noir, Aye, 1317.

Gaugain broche le neir kernu

E fiert Yder sor son escu ; Yder, 2267-8. Cf. ib., 2294-6.

Yder fiert lui de tel ahir

Qu'il feit al neir destrier ferir

Ensemple toz les quatre pez, ib., 2277-9. Cf. ib., 2478.

Je irai joster, nel leroie

Por riens. Ça mes armes! » Tantost

L'arment et cil s'en vet de l'ost

Sor .I. cheval plus noir que more. Meraugis, 5400-3. 
Puis li ameinent un neir destrier muvant Otinel, 882.

Luez droit i mistrent tante sele

en destriers noirs, sors et baucens Dole, 2226-7.

Ou palefroi noir come meure

monta, quant il ot pris congié $: i b ., 3380-1$.

Voir aussi : Ipomedon, $2680 ; 5543 ; 6626-7$ et passim ; Blancandin, 3464 ; Raoul, 7516-7.

ae. ros.

A.-J. Greimas écrit, op. cit., p. 572 : « I, ros n. m. (1190 J. Bod. ; allem. Ross, coursier). Cheval » et il cite l'exemple de la Geste des Loherins : «Beraus s'est mis sor le ros d'Oriant »; quelques lignes plus bas, on lit « III. ros adj. (...) Roux. » R. Grandsaignes d'Hauterive, op. cit., p. 521, ne mentionne que la seconde de ces significations, tandis que Jacqueline Picoche, op. cit., p. 438, s. v. Rosse écrit : « XII s. subst. masc. "mauvais cheval", $\mathrm{xv}^{\mathrm{e}}$ s. fém., (...) » et, plus bas dans le même article : "Roussin $\mathrm{xvI}^{\mathrm{e}} \mathrm{s}$. : altération, sous l'influence de roux, de l'anc. fr. roucin, du bas lat. rǔncīnus, var. ruccinus, p.-ê. d'origine germ. et apparenté à rosse. »

Guy Renaud de Lage, dans les notes de son édition de Thèbes, commente le vers

Le lïart rouz prist par la serse, Thèbes, 4763.

et écrit que rouz est un substantif dérivé du germanique et que lïart est un adjectif signifiant "gris pommelé ».

A cause de la graphie, Povl Skårup, qui a attiré notre attention sur les mentions du terme dans Tobler-Lommatsch, pense que dans le vers que nous venons de citer il s'agit plutôt de l'adjectif "roux » (< russus, et avec la prononciation fermée de l'o) que d'un substantif d'origine germanique ; il va jusqu'à douter de l'existence d'un terme ròs (< Roß, à prononciation ouverte de l'o) en ancien français. Comme lui, nous pensons que les exemples cités dans Godefroy vir ne prouvent pas l'existence d'un terme ròs < Roß. Nous suivons l'interprétation d'Annette Brasseur, donnée dans son édition de Saisnes, p. 794 : « L'emploi, si fréquent dans la Chanson des Saisnes, de l'adjectif rous comme substantif, ne paraît pas courant dans la littérature médiévale (...) » :

Aufarz de Denemarche veant tote sa gent,

Plus d'une arbelestee sor le rox se destant. Saisnes, 2679-80.

Ainz point le rox d'Orcane q'amprunta au roi Daire. ib., 2703. 
Puis prant le rox d'Orcane, vers Rune s'an repaire. $i b ., 2712$.

Qar le rox tint an destre par la renne d'or mier

Et sor son poig senestre tenoit son esprevier. $i b ., 2744^{-5}$.

Mes dites ou preïstes cel rox et l'esprevier. $i b .2752$.

De cest rox organois le fis jus trabuchier. $i b ., 2758$.

A mervoille esgarderent le bon rox orquenois $i b ., 2795$.

Dans des exemples tirés de Protheselaus, de Mort Aymeri et d'Aspremont, le terme se trouve accolé respectivement à cheval et à destrier ; nous ne doutons pas qu'il s'agit de l'adjectif «roux »:

Ly [rois] Thesëus est rescus

E mounta sur un chival rus. Protheselaus, 12132-3.

Bien fu armez desor un destrier ros ; Mort Aymeri, 1479.

Ulïens monte desor un ceval ros ; Aspremont, 7735.

Uliens sist el ros lïart destrier. $i b ., 7786$.

Un peu plus loin, dans Aspremont, le terme revient trois fois, accolé, comme dans Thèbes, à liart, mais placé devant celui-ci, d'abord écrit rous, ensuite ros. Les deux termes liart (qui se trouve dans tous les trois exemples) et ferrant (qui se trouve dans deux d'entre eux) pouvant avoir la fonction de substantifs, ne nous aident pas à préciser lequel des mots ros, liart et ferrant est le noyau dans ces syntagmes, mais la graphie rous dans le premier extrait nous fait penser qu'il faut y voir - et, par analogie, aussi dans ros - une épithète au sens de « roux»:

Ulïens sist el rous lïart ferrant,

Li viels Galindres sor un mulet amblant ; Aspremont, 8022-3.

Ulïens sist el ros lïart ferrant ; $i b ., 8734$.

Qant Ulïens se sist el ros lïart, ib., 9227.

Le roman d'Yder présente des exemples semblables. Son éditeur, Heinrich Gelzer, est de l'avis de Guy Renaud de Lage : il traduit ros, au vers 1348, par " Pferd », et liart, vv. 1348 et 1366, par " graufarben » (il considère donc ce terme comme un adjectif). Il ne commente pas la forme rus, du vers 1366 ; à notre avis, cette dernière forme, qui doit indiquer la prononciation fermée de l'o, 
corrobore le point de vue de Povl Skårup, et nous préférons voir dans l'expression « ros/rus liart » un syntagme substantif dans lequel liart est le noyau et ros/rus l'épithète, et traduire par « cheval rouan »:

Cheval ot $b[u] e n$, un ros liart,

D'Espaine fu et buens e beaus, Yder, 1348-9.

E Luguains saut al rus liart ; ib., 1366.

af. sor.

Le terme désigne la robe jaune brun.

Ni Grandsaignes d'Hauterive ni Greimas ne le donnent que comme adjectif. Dans les extraits de l'Escoufle et de Méliador, il est sans aucun doute substantif, et nous pensons que p. ex. dans les syntagmes sor gascon et sor bauçant, dans les extraits d'Ipomedon et de Roussillon, on peut le mettre dans la place du noyau. Voir d'autres exemples p. 93.

$\mathrm{Il}$ a tos les cochons mandés

Ki en la vile sont et mainent,

Ki tant vairs et sors les amainent Escoufle, 436-8.

La veissiés tant sor et tant bauchant

Fuir par le campaigne frains trainant, Aiol, 3199-200.

Or s'en chevauce Agamanor

Sus .I. cheval, .I. petit sor, Méliador, 4509-10. Cf. ib., 13743-4.

Defors pendent les lenges d'un gofanon ;

Autre en ac per la teste del sor gascon. Roussillon, 6357-8.

Il est sors bausans, li destrers, Ipomedon, 4555. Cf. ib., 5055-6.

L'extrait de l'Atre périlleux permet, à notre avis, deux manières d'analyse : le plus facile est de considérer sor comme un substantif précédé de l'article indéfini ; mais on pourrait répéter destrier du premier vers devant sor, qui serait alors épithète du syntagme destrier sor, et la construction des deux vers serait parallèle :

Li uns sist sor un destrier blanc,

Et li autres sist sour un sor. Atre, 5894-5.

Il est vrai que les exemples où le terme est adjectif prédominent : 
Qu'a force Claris remonterent

Sor .I. destrier d'Espaigne sor, Claris, 1535-6.

Un damoisel vit qu'an sa main

Menoit un cheval sor, basain. Florimont, 4439-40. Cf. ib., 6659 ;

10747 .

Bien le seroit an destre li riches destriers sors. Saisnes, 2226.

Cf. $i b ., 2212$.

Uns chevaliers auques d'ahé

estoit de l'autre part del pré

sor un cheval d'Espaigne sor ; Charrete, 1649-51.

Jus l'abati del cheval sor. Athis, 15508.

Voir aussi : Protheselaus, 4404 ; Méliador, 6715-6 ; 14044 ; 28278-9 ; Ipomedon, $2667 ; 4527 ; 4551$ et passim ; Blancandin, 5767 ; Jehan, 3114 ; Cligés, 4243 ; 4780 ; Mort Aymeri, 709 ; 1184 ; Aiol, 4268.

ag. vair/vairon.

A.-J. Greimas, op. cit., p. 654, écrit que vair adjectif peut signifier " gris pommelé, en parlant d'un cheval », et que le nom peut désigner un cheval tacheté. Quant à vairon, il note respectivement «De deux couleurs, tacheté » et « Cheval pommelé ». Nos textes montrent que vair est plus fréquent que vairon. Cf. 1.1.3. Vairon.

Montez est sor le vair qui bien est abrivez. Renaut, 3907. Cf.

ib., 4111-4.

Thiebaus montait ou vair movant d'Arabe ; Enfances G., 1509.

Cf. $i b ., 3059-60$.

Li amiraus du Coine sist seur .I. ver d'Espengne, Gui de N., 2465 .

Quois monte sur le vair d'Espaine ; Yder, 1283. Cf. ib., 1289.

Baudoïns li niés Charle venoit toz sox errier,

Qui s'ert faiz an sa tante richemant herbergier,

Et sist ou vair d'Espaigne qi mout fist a prisier, Saisnes, 1454-

6. Cf. ib., $1482 ; 1612$ et passim.

Puis monte ou vair destrier ou mervoille se fie, $i b ., 2162$.

Et vos, sire vasaus, sor ce vair montenier, Barbastre, 5843. Cf.

ib., 6581-2; 209 . 
Renier de Monhermer sor le vair Baligant, ib., 176.

Et sist deseur un vair destrier Inconnu, 5773；147.

Miles point toz premiers, cui l'anceigne est balie,

Et fu mout bien armez ou vair de Paenie ; Orson, 1664-5.

Si vos donrai un cheval ver, Partonopeu, 6818. Cf. ib., 6909-10 ; 6923 ; Partonopeu-C, 2044.

Li sires del Lit as merveilles,

Nathanaors d'Esquanaron,

I siet sur le meilleur vairon

Qui sur doz eüst onques selle ; Galeran, 5624-7.

Il estoit seur .I. destrier vairon que Josias li ot donné qui valoit l'ouneur d'un chastel.

Helcanus, 171 (p. 221).

Li sires de Gorre si sist sus .I. cheval vairon (...) Laurin, 1988-9.

Voir aussi : Rigomer, 16067 ; Aiol, 4489 ; Cont. P., II, 8058-9 ; Joufroi, 402-3 ; Diable, 2138 ; Dole, 2726-8 ; Gui de B., 2318 ; Escoufle, 438 ; Erec, 2944 ; Raoul, 3861-2 ; Mort Aymeri, 1795 ; Gaydon, 5446-7 ; Ipomedon, 5098 ; Bueve, III, 10144.

Dans les deux exemples suivants, tirés de Laurin, il est possible, vu le défaut d'article, de lire Vairon, c'est-à-dire de considérer le terme comme un nom propre (cf. Ferrant p. 124-125 et Vairon p. 128) :

Il broche vairon de grant ravine (...) Laurin, 844-5.

Atant laisse courre vairon (...) $i b ., 2349$.

\subsubsection{Noms propres}

Dans plusieurs textes se trouvent des énumérations de guerriers avec indication du cheval de chacun. Ces chevaux sont le plus souvent désignés par leur nom propre :

Aymeris s'est armé, si sailli le baucent,

Et Guillaumes d'Orenge desor le bai corant,

Bueves del Conmarchis sor le doine poisant,

Et Sanses sor Liart, et Hue en Atengnant, 
Renier de Monhermer sor le vair Baligant,

Navari de Toulouse desor le sor Bruiant, Barbastre, 172-7.

Dukez y est de Quornehout,

Qui est du linaige au Morhout,

Et siet sur Ferrant de Venisce ;

Gornemans de la Male Lice

Y siet sur Malreé le bay ;

Baudous, li filz au duc d'Angay,

Y est sur le courant Liart :

(...)

Sor le Mor y est de Tudele

Blandins de la Forest Oscure ;

Li Blonz des Illez d'aventure

Siet sur le Sor de Portigal ;

Encoste li le preu Rigal,

Qui filz est au forestier Blou :

Oncques en pié n'ot fer ne clou

Ses destriers Fauveaus de Tolete ;

Li fillastres Gorfrain la Brete,

Porfillionz du Gué Trenchant,

Arondel y va chevauchant,

Qui oncques ne gousta d'aveine,

N'oncques ne fu seignez de vaine,

Si court plus que ne vole aronde ;

Tallas de la Lande Reonde

Siet sur Volant de Bonivent

Qui ne lait a courre pour vent,

Ne pour montaigne, ne pour roche,

Quant on le point a droit en broche ;

Sur l'Orgueilleux siet Hardibrans, Galeran, 5615-21 et 5628-47.

Les ouz s'entrencontrerent trés en mi un vaucel.

Agravain point et broche son destrier Isnel,

Et Clamador Ferrent, et Berart le Favel,

Et Sanson le Baiart, et Manduz le Soreil.

A tant es vos Aliaume, armé desus Morel ;

Et Gaudin point et broche le coraor Isnel,

Et li dus d'Agenois son bon destrier Isnel,

Et Bruhan de Venice desus Trenchequarel,

Galerans sus Brunfort, et Jonas sus Tracel,

Et Sances de Biterne broche le gris Potrel,

Et li dus d'Auquitaigne son destrier Plançonel,

Et Mille de Hongrie rebroche Mirabel.

Esmerez fut devant, armez sus Leoncel ; Florence, 1383-95. 
Nous notons que dans l'extrait de Florence, quelques-uns des noms ont l'article défini, p. ex. le Favel. Il serait donc possible de l'écrire avec $\underline{\mathrm{f}}$ minuscule et de lire « le fauve », c'est-à-dire « le destrier fauve ». De même on pourrait écrire isnel au lieu de Isnel, p. ex. au vers 1389, et donc lire "son bon destrier rapide ». L'éditeur A. Wallensköld ne commente pas le passage, mais il indique l'emploi de minuscules dans d'autres manuscrits. Nous avons préféré suivre son édition du texte.

Dans l'Espinette amoureuse, Jean Froissart donne même un nom propre au cheval de bois qu'il avait dans son enfance; nous pensons que le nom Grisiel, indiquant la couleur, devait être copié sur un nom de cheval assez courant car, sinon, pourquoi le garçon le choisirait-il pour son jouet?

\section{Et s'ai souvent d'un bastonciel}

Fait un cheval nommé Grisiel, Espinette, 213-4.

Dans la majorité des exemples que nous avons relevés dans nos textes est montrée la couleur des chevaux en question. D'autres noms soulignent la rapidité et l'ardeur ou bien la force. Reste un petit groupe de noms dans lesquels se cachent d'autres qualités. A. Tobler, op. cit., p. 223, présente une liste de noms propres qu'il a relevés dans différents textes. Une liste encore plus longue se trouve dans la dissertation de Fr. Schmidt, op. cit., pp. 76-84. Il fait remarquer que tous les noms qu'il a relevés appartiennent à des destriers. C'est le cas aussi des 92 noms relevés par Friedrich Bangert, op. cit., pp. 31-45.

\section{a. couleur.}

Nous remarquons qu'un assez grand nombre de noms s'appliquent aux chevaux blancs. Cela confirme ce qu'écrit Michel Pastoureau, op. cit., p. 123, au moins en partie, car p. ex. les chevaux Fauvel et Bayart sont très appréciés : « Poètes et romanciers décrivent également avec force détails la robe de ces chevaux. Les plus prisés sont les tout blancs et les tout noirs ; ensuite viennent les baucents, c'est-à-dire d'une couleur quelconque mais largement tachés de blanc ; puis les liards, vairs ou ferrands, c'est-à-dire gris avec différentes manières de pommelures. En revanche les bais à pelage bure (brun) et les alezans à robe sore (fauve) sont quelque peu dédaignés. » Cf. Friedrich Bangert, op. cit., p. 57.

Abîme $=$ cheval noir. (?)

Uns escuiers li tint son arragon,

N'ot tel cheval jusques es pres Noiron,

Non ot Abismes, noirs fu come charbon,

En Femenie l'engendra uns luiton, Bueve, II, 17146-9. 
L'Aubagu = cheval blanc. (?)

Note p. 323: «Nom variable selon les manuscrits du cheval d'Arthur. Erec est le seul roman arthurien à le mentionner. L'origine reste obscure (allusion à la robe blanche - alba - du cheval ?). »

Sor l'abacu monte li rois, Erec, 4125.

Bauçant = cheval bauçant.

Et l'amiraus fait Bauçant enseler: Huon, 6515. Cf. ib. 8072.

Cel jor sist Guinemers seur Baucent l'arabi Aye, 1097.

Or avoit Ogier si grant envie du cheval Bruhier, nommé Bauchant, qui estoit si grant et si puissant ; et le sien estoit desia ung peu cassé. Ogier, 146. Cf. ib., 150 et passim.

L'en li amainne son auferrant destrier.

Ce fu Bauçans, qui tant fist a prisier. Mez, 4944-5. Cf. ib., 5091 ; 5731.

Dont sist Rigaus el Bauçant arabi. ib., 384.

Baucent et Roienaus li viautres, Renart, vI, 16241.

An fue torne si grepese Bausant Enfances G., 487. Cf. ib., 949.

Baucent l'entent, si commence a henir. Aliscans, 1146.

Le sor Baucent a forment regreté. ib., 1870.

Mort le trebuche, puiz a saizi Bucant,

Dou destrier fit present a Rodoant, Bueve, III, 14301-2.

Guiot point Balçan, si li laissad la reisne ; Guillaume, 1557. Cf. ib., $1661 ; 1667$ et passim.

Ne puis mes armes manier ne sustenir,

Brandir ma hanste, ne le Balçan tenir, ib., 1741-2.

Broche Bauçant par andox les costez, Mort Aymeri, 496. Cf. ib., $93 ; 111 ; 135$

Bausant amainnent, à la sele dorée ; Gaydon, 1063. Cf. ib., $1075-6 ; 2763$. 
Che fu Bauçant qe il per amoit chi, Chevalerie d'O., 7280. Cf. $i b ., 7330 ; 7711$ et passim.

Nous notons que dans quelques-uns des extraits ci-dessus, le nom est précédé par l'article défini ; à la page 17, nous en avons cité un autre exemple, tiré d'Enfances $G$. Dans ces cas, on pourrait lire bauçant ; voir aussi Ferrant, Liart et Sor/Sorel.

Bayart $=$ cheval bai. Voir aussi p. 119.

Lor chevaux estancherent, ne valent .I. boton,

Mais Bayart ne dolut ne janbe ne jambon. Renaut, 2220-1. Cf. $i b ., 2234 ; 5115 ; 5420 ; 6750 ; 6993 ; 7633 ; 7640 ; 7651$ et passim.

Il broche le cheval, dez esperons l'argue,

$\mathrm{Au}$ bon cheval Baiart a la teste tolue. Gui de N., 1085-6. Cf. ib., 1010-2.

Bien lor cuide Baiart fierement chalengier. Buevon, 2886. Cf. $i b ., 3561$.

Baiart porprent grans sauz per cans araz ; Roussillon, 4972. Cf. ib., 5120 .

A force Baiart broche des esperons des piez, Saisnes, 3743.

Lores vient a Souplice, si li rendi Baiart Partonopeu-C, 1769 .

Ainc en si bon ne monte nus frans hons,

Fors seul Baiart, Renalt le fil Haimon ; Chevalerie d'O., 9628-9.

Blancardin/Blanchart/Blanchenue $=$ cheval blanc.

Blancardin fist ens la sale amener,

Plus blans estoit que ne soit flor de pré. Huon, 7691-2. Cf. ib., $7783 ; 8024 ; 8098 ; 8369$.

La sele est mise sus Bla[n]chart le corant,

Qui plus tot court que esperver n'est volant. Otinel, 308-9.

Et mon riche destrier Blanchart il avera, Bâtard, 3664. Cf. ib., $3733 ; 4112$.

Et lors fit aller ung escuyer pour avoir le meilleur cheval qui fust en l'escuyrie, nommé Blanchart, (...) Ogier, 307. Cf. ib., 308. 
Et Dos remonte sor Blanchart l'arragon, Bueve, III, 5858.

Tint .I. espié, si a brochié Blanchart,

Le bon destrier que Ferrans li donna. Gaydon, 5134-5.

Le sielt li rois sor Blancart d'Alïer ; Chevalerie d'O., 3268. Cf. ib., 6313 .

Puis li ameinent Blanchenue

que li tramist l'autre an sa drue ; Thèbes, 6241-2.

Blanchenue fu chevax genz

et fu plus blanz que fins argenz ; ib., 6245-6. Cf. ib., 6264 ;

$8596 ; 9772$.

\section{Brun de Surie.}

(...) croyez que se la bataille estoit entre les gens, aussi estoit elle entre les chevaulx. Car le cheval Ogier s'en alla vers Brun de Surie qui estoit a Justamon. Si s'entretuerent tellement et a si grant force que Bauchant de la grant puissance que il avoit luy donna coups au Brun de Surie des deux piedz de derriere que il lui creva le cueur du ventre, si mourut la. Ogier, 176.

Cabauchan = cheval bauçant $?$

Il s'agit du cheval de Fouque, appelé Baucan au vers 9259 (voir barzelon).

Pren Cabauchan per fren, Folcon lo rent : Roussillon, 8415.

Cabauchanz fu chevaus ferranz e bais ;

Fu demis arabiz, l'autre morois. ib., 8422-3. Cf. ib., $8103 ; 8538$.

Carbonel $=$ cheval noir

Ere s'en vait li reis sor Carbonel, Roussillon, 1377.

Fauvel $=$ cheval fauve. Voir aussi p. 119 et Martin : Wistasse, 200-2.

Arthur Långfors, Le Roman de Fauvel, après avoir mentionné les expressions " fauve anesse » et "fauve jument », écrit, p. LXxxv : "L'ânesse ou la jument symbolique est désignée par le nom masculin de Fauvain, où l'équivoque est encore renforcée par la terminaison vain (vide). (...) La forme masculine, Fauvel, est très fréquente dans les textes médiévaux d'une certaine ancienneté, mais seulement comme nom de cheval. Il ne semble pas 
avoir, avant le Roman de Fauvel, personnifié la fausseté et l'hypocrisie. »

Baudüins seoit sor Fauviel Rigomer, 12051. Cf. ib., 12064-5 ; 12068.

$\mathrm{Li}$ amiraus s'eslesse à Olivier,

Et li quens broche Fauvel, son bon destrier. Otinel, 1681-2.

Fauvel l'emporte tost ; paien l'ont tant cremu

Que Fauvel ont reté : par lui sont confondu. Partonopeu-C, 2609-10. Cf. ib., 2526-8.

Diex, con il fu armez seur Fauvel son destrier! Aye, 1300. Cf. ib., $1326 ; 3059 ; 3977 ; 3086$.

Fauveax va en travers et la resne traïne. Gui de N., 1453.

Tiebaut sist seur Fauvel et Hernaut sus Ferrant

et Gui sist seur Vairon, he Dex, il l'ama tant ! ib., 1468-9.

Ens en la place li amainne on Fauvel ; Bueve, II, 4926. Cf. ib., II, 6018.

«La cele a mise sor Fauvel l'arabi ; Raoul, 2300.

Ferrant $=$ cheval gris. Voir aussi p. 119.

Mes Ferrant de Castele a la crope tieulee

Brise le col a l'autre, qui chiet mort en la pree. Renaut,

13777-8.

Et aprés point Girart Ferrant qu'ert pomelez ; Barbastre, 267.

Cf. ib., 1059 .

Gerars broche Ferrant, qui vint de Terrascone, Buevon, 210, Cf. ib., 1882 et passim.

Salemonz point Ferrant qi porprant les granz sax, Saisnes, 2404 .

En va touz seus sor Ferrant en la place Jourdain, 1081.

Qui fist l'arriegarde, il et si compengnon,

Descendi de Ferrant, si monta sor Veiron - Gui de N., 1363-

4. Cf. ib., 1468-9; 1917-8. 
Claires, biax niés, or montés sor Ferrant ; Aspremont, 10647.

Un cheval li tienent Ferrant

Que li dona sa damoisele. Gliglois, 2392-3.

Il point Ferrant des esperons, ib., 2431. Cf. ib., 2457 ; 2559.

et seignor Ferrant le roncin

et dant Roonnel le matin Renart, I, 1617-8.

Voir aussi : Bueve, III, 5467 ; 14889 ; Mort Aymeri, 757-9 ; 800 ; 821 et passim ; Laurin, 2354 ; 9684 ; Gaydon, 4085-6 ; 9491 ; 9508 ; Chevalerie d'O., 2458 ; 10279 ; Raoul, 2079.

Dans le premier extrait de Gliglois, on pourrait peut-être lire ferrant et y voir une épithète à cheval. Cf. Liart: Renaut, 11166 et Sor/Sorel.

Dans Raoul, un cheval est appelé Ferrant de Paris :

Bien fu armés sor Ferrant de Paris. Raoul, 2079. Cf. ib., 2517.

Florent/Florentin/Florescele/Flori $=$ cheval blanc.

« Je connois bien ce destrier que avez:

«Florent a non, si fu Gaudin l'escler, Mort Aymeri, 2977-8. Cf. $i b ., 1949 ; 2137$.

Quant Aÿmers a choisi le mastin,

Des esperons a brochié Florentin ; Aliscans, 5398-9. Cf. ib., $5403 ; 5432-3$.

«Amenés moi tost cza mon destrier Florentin, Simon, 1991.

Puis li ont Florentin sin destrier amené,

Plus blanc que nule nois, sachiez de verité ; Lanson, 5247-

8. Cf. ib., $5259 ; 5372$.

«Ohi, Florescele, bon cheval de nature,

Unc destrer ne vi tele criature ! Guillaume, 2201-2. Cf. ib., $2151 ; 2180-1$.

.xIIII. roi li ont Flori rendu, Mort Aymeri, 929.

Otinel broche Flori, son bon destrier, Otinel, 1093.

En celle guerre .I. roi lor i ocist, 
Ou il conquist le bon cheval Flori, Mez, 2481-2.

Tendi sa main, si prist Flori, le blanc, ib., 4054. Cf. ib., 4031 ; $4213 ; 4550$ et passim.

Mort l'abat du cheval qui ot à nom Floris ; Gui de N., 2760.

Galope le cheval Flori les sauz menuz, Saisnes, 3173. Cf. ib., $3135 ; 3310 ; 3358 ; 4154$.

Liart $=$ cheval gris pommelé. Voir aussi p. 118.

Bien fu montés deseur Liart

Et Jehans sist deseur Morel. Jehan, 4580-1.

Entre les quisses li gettent mort Liard ; Guillaume, 1806.

«Alez, ce dist Renaut, amenez moi Liart. » Renaut, 11163.

« Des or poez monter sor le cheval Liart. » ib., 11166.

Dans le dernier extrait il serait possible de lire liart et y voir un adjectif. Cf. Ferrant : Gliglois, 2392-3 et Sor/Sorel.

Malreé = cheval rayé, de couleur mate. Voir p. 119.

Marmorie $=$ cheval tacheté

Siet el cheval que il cleimet Marmorie, Roland, 1615.

Mor $/$ Morel $=$ cheval noir. Voir aussi p. 119.

La fréquence du nom indique sans aucun doute la faveur dont jouissent les chevaux noirs.

Moriel li ont molt bien apparellié, Aspremont, 1906. Cf. ib., $1908 ; 1928-9$ et passim.

De Maugis vos dirai coment a esploitié :

Il sist desor Morel qui mult fist a proisier, Renaut, 5252-3. Cf.

ib., 10127 .

Vint en l'angarde poingnant desor Morel. Jourdain, 1662.

Garniers s'en vait pasmant sor Morel son destrier ; Aye, 2814.

Et Guis sist sor Morel, n'ot tel jusqu'a Baionne ; Buevon, 211.

Cf. $i b ., 2011 ; 2640$. 
Makabrés vint avant sor Morel d'Alcasés Partonopeu-C, 1721. Cf. $i b ., 1896-7 ; 1941-2$ et passim.

Le destrier souvent sous li lanche,

Bien valoit .c. mars de monnee,

Non avoit Moriau[s] le Desree ; Lycorne, 6727-9. Cf. ib., 6213$9 ; 6288-90$.

Descendez del cheval, que trop l'avez laidi ;

Si montez sor Morel, que onques tel ne vi. Barbastre, 2177-8.

Lez esperons à or a fet sentir Morel ; Gui de N., 1425. Cf. ib., $2002 ; 2023 ; 2060 ; 2068-9$.

Voir aussi :Chauvency, 2878 ; $3980 ; 3994$; 3995 ;Jehan, 4130 ; 4182 ; 4230 et passim ; Cligés, 4614-5; 4619; Wistasse, 550-2; $556 ; 562$ et passim ; Gaydon, 5190-1 ; Doon, 8524 ; Bérinus, 257 ; 261 ; 273 et passim ; Chevalerie d'O., $2457 ; 5723 ; 6534 ; 10088$.

Pomelé $=$ cheval pommelé

Et si envoie, par .I. moinne rieuglé,

.II. des chevax, Ferrant et Pomelé, Gaydon, 4085-6.

Rosenet $=$ cheval roux. (?)

On li a amené Rosenet le bausant. Aye, 386.

Saigremor $=$ cheval noir. (?)

Quant est montés sor Saigremor

Ne l'atendriés por cent mars d'or

Qu'il ne vous ferist de sa lance. Poitiers, 209-11.

Sor/Sorel $=$ cheval alezan. Voir aussi p. 119.

Sorel, dans Roland, pourrait s'écrire sorel et être épithète à cheval. Cf. Bauçant, Ferrant, Liart et p. 119, Galeran, 5630-1 (Sor).

Li quens Gerins set le ceval Sorel Roland, 1379.

Lors a hurté Sorel, qui sous li va saillant. Doon, 8628. Cf. ib., 8798.

Tachebrun = cheval à taches brunes.

En Tachebrun, sun destrer, est munted ; Roland, 347. 
Vairet/Vairon/Varrain = cheval vair.

Joifroi d'Angiou li Vairet afilé, Chevalerie d'O., 2459. Cf. ib., 4689 .

De guenchir erraument s'efforce tant qu'il tint par le frain Vairon,

sel dona lors a un prison Dole, 2748-50.

Li vallet de Nantueil avala les degrés

Et monta seur Veiron qui li fu amenés ; Gui de N., 414-5.

Cf. ib., 496-7 ; 963-4; 1468-9; 1363-4; 1917-8; 2011-3.

Puis a fait un eslais por Vairon assaier.

Fort le trueve et seür, remüant et legier. Saisnes, 1462-3. Cf. $i b ., 1578 ; 2817$ et passim.

Et Amanfroi armé desor Vairon, Gaydon, 2290. Cf. ib., 2441 ; $2670 ; 2882 ; 2977$.

Varrain trova le destrier ademis ;

Li quens Guillelmes l'a par la resne pris. Aliscans, 5470-1.

\section{b. rapidité/ardeur.}

Adreçant $=$ cheval rapide .

Le destrier ou il sist va les sanz porprenant,

En l'ost n'a si isnel, seu cleinment Adreçant. Barbastre, 657980 .

Afilart/Afilé = cheval rapide

Atant si regarde Basins vers un vivier

S'a veü chevax paistre contreval .I. santier.

Li dus Jehanz i ot Afilart son destrier ;

Enz an pré le gardoient .xvii. escuier. Lanson, 2837-40. Cf. ib., $2893 ; 2930$.

Sus Afile monta, à la croupe tieulée. Gaufrey, 315.

Atant es vous Gaufrey monté sus Afilé, ib., 8158.

Alion $=$ cheval rapide (ailé $?$ ).

Dans les variantes, Ernest Langlois indique que le même cheval est aussi appelé Arondel. 
On li ameine Alion son destrier ;

A grant merveille par fu li chavals fiers, Couronnement, 644-5.

Cf. ib., 2503 et passim.

Arondel $=$ cheval rapide (comme une aronde). Voir aussi p. 119.

Sor Arondel a la crupe trieulee,

Que plus coroit que aronde enpenee, Bueve, I, 1060-1. Cf. ib., $906 ; 927$ et passim.

A propos des deux vers suivants, Albert Stimming observe que les chevaux changent de poils avant l'été et avant l'hiver et qu'il y en a qui prennent, à cette occasion, une couleur différente :

Si l'a ochis Arondiaus li müés, Bueve, I, 6502.

Forment me poisse d'Arondel le müé $i b .$, I, 7154.

Baligant $=$ cheval courant

Es vos a icés pongnant le roi Fabu

Sor le fier Baligant, qui forment cort menu ; Barbastre, 5884-5.

Nerbonois pasent Sore, Aymeris va avant

Un arpant mesuré sor le vair Baligant ; ib., 6574-5. Cf. p. 101, $i b ., 176$.

Et Malcuidans point le brun Baligant ; Bueve, I, 8543.

Bien fu armés sor le bai Baligant, ib., III, 10183. Cf. ib., III, 10212

Baudifer/Bondifer $=$ cheval courant.

Lion estoit arméz d'un riche jozerant

Et bonnez armeures et destrier corrant :

Baudiffer ot a nom, se dient li acquant. Lion, 19717-9.

Son cheval Bondifer li fu aparelliez, Florence, 1108. Cf. ib., 1597.

Prent le chival l'alfage, s'est el[s] arçons volez,

Si laise Bondifer, qui meilz valoit assez. Simon, 1766-7.

Brandi $=$ cheval actif $(<$ brandir $?)$. 
Et voit celui qui si tost s'en füi

Sor son cheval, que on noma Brandi, Bueve, I, 6080-1.

Clinevent $=$ cheval rapide (qui fonce contre ou comme le vent $?$ ).

Sor Alion est montes, son destrier,

Prent Clinevent, qu'il ne le volt laissier ; Couronnement,

2517-8.

Li dus brocha Clinevent son destrier, Gaydon, 2682. Cf. ib., $2799 ; 2869 ; 2894 ; 7413$.

Isnel $=$ cheval rapide. Voir p. 119.

Marchegai $=$ cheval rapide

Dont broche Marchegai son boin destrier,

Et Marchegai li saut .xIIII. piés : Aiol, 581-2.

Il vint a Marchegai son auferant. ib., $2399 ; 89 ; 778-80 ; 2135$

et passim.

Marchepalu $=$ cheval impétueux (qui traverse les marais).

Et vint devant sa gent dessus Marchepalu :

Ch'est .I. destrier d'Arable qui vaut .m. mars fondu, Gaufrey, 2872-3. Cf. ib., 2887 ; 3009.

\section{Marchepierre.}

C'ert Lucanus et siet sor Marcepiere, Bueve, I 9398. Cf. ib., I, 9407 ; III, $10953 ; 10962$.

Devant les autres, parmi une jonchiere,

Vint Tolomas, poignant sus Machepere ; Aliscans, 662-3. Cf. $i b ., 674$.

\section{Marchevallée.}

Quant le souldan Noradin l'aperceut, si lui dist :Vieil chevalier morien, mon amy, ne vous courroucez point car ie vous bailleray mon cheval Marchevalee, duquel n'a le pareil en tout ce monde vivant. Ogier, 188 . Cf. ib., $189 ; 190 ; 200$ et passim.

Migrados $=$ cheval rapide $($ cf. Arondel $)$. 
La sel' est mise sus Migrados l'inel,

Qui plus tost court que ne vole arondel, Otinel, 371-2.

Il lesse corre le bon destrier corrant,

Et Otinel Migrados le brivant. ib., 422-3.

Passeavant/Passevant $=$ cheval rapide et impétueux.

Lance ou poign, qui n'est mie torte,

Tient le damoisel de Bretaigne,

Qui siet sur Passeavant d'Espaigne

Dont li bons ducs li a fait don. Galeran, 4844-7. Cf. ib., 5606-7 ;

5924-5.

Un boin ceval li ont en la place amené,

Cil ert le roi meisme, Passeavant fu clamé[s]. Aiol, 9910-1.

Aiols point le destrier c'on claime Pasavent : ib., 9927. Cf. ib., $10021 ; 10051$.

Lors a Brias pris Passevant

Par les resnes, qui sont d'argent, Cristal, 3663-4. Cf. ib., 3734$5 ; 3927$ et passim.

Passecerf $=$ cheval rapide (plus rapide qu'un cerf).

Li quens Gerins set le ceval Sorel

E sis cumpainz Gerers en Passecerf. Roland, 1379-80.

Passelande $=$ cheval rapide (cheval d'Arthur).

Li rois monta sor Passelande, Tristan, 3522.

Pennepie $=$ cheval rapide (« qui marche sur la pointe de pieds »).

Atant est venu Carmel de Tabarie,

Un Sarrazin qui tus les altres guie ;

Bien est armé, si set sur Pennepie. Otinel, 943-5.

\section{Pennevaire.}

C'est Pennevaire, n'ot tel ceval sous ciel. Chevalerie d'O., 4605. Cf. ib., $10101 ; 11454$ et passim.

Regibet. $(<$ regiber $=$ ruer ?). 
On li a amené Regibet son destrier,

Par chen ot non Regibet que ja m'orrés nunchier,

Que homme ne cheval n'en oseit aprechier,

Fors cheli qui le garde et li donne à mengier, Gaufrey, 219-22.

Salt Perdut $=$ cheval qui saute (?).

Siet le ceval qu'il cleimet Salt Perdut : Roland, 1597.

Tost Courant $=$ cheval rapide $($ comme oiseau $)$.

Oncques sy bonne beste vo corps ne chevaucha, Car plus tost va courant qu'oyseaulx qui vollera.

Tost Courant l'appellon, pour ce que sy tost va. Tristan de N., 10438-40. Cf. ib., 10463 et passim.

Tracel $=$ cheval à grande allure (?). Voir p. 119.

Volant $=$ cheval rapide. Voir p. 119.

c. force.

Broiefort/Broieguerre $=$ cheval rapide qui brise toute force, toute résistance.

Lors se retourne Ogier, si apperçoit Brunamon monté sur son cheval Broyfort qui de plain sault sailloit trente piedz de long Ogier, 54. Cf. ib., $55 ; 56 ; 66$ et passim.

On li amaine Broiefort enselé ; Chevalerie d'O., 2419. Cf. ib., $2736 ; 2748$ et passim.

[Et Oger Broyefort, qui de corre est hastez] ; Fierabras, 3553.

Montés estoit sor un destrier ferrant, Fort et isnel et aspre et tost corant,

Que Broiefort nommoient li auquant. Enfances O., 874-6.

Carahués broche le pendant d'un costal

Seur un cheval meilleur de Bucifal ;

Fors Broiefort ainc hom ne vit ital,

C'ert li chevaus Brunamon l'amiral,

Puis le conquist Ogiers en champ mortal, ib., 1765-9. Cf. $i b$., 3758-9 et passim.

Et Ogier y antrait sur Broiefort le grant ; Lion, 22095. 
Puis li amainnent Broiefort l'arrabi. Gaydon, 7719.

Quant Ogier l'a veü si dolent ne fu on,

Il saut en Bruiefort, en sa main .I. baston, Renaut, 6882-3. Cf.

$i b ., 5695-7 ; 7462$ et passim.

Maugis point Broieguerre et vait au tref joster, $i b ., 10144$.

Il broche Brieguerre par andui les costez ib., 10166. Cf. $i b .$, $9213 ; 9726$.

Brü̈nent $=$ cheval bruyant (?).

La bone espeie ceinte a son senestre lez,

Et vint a Bruïnent qui totz fu ensellez ; Simon, 1616-7.

Brunfort $=$ cheval fort (de robe brune ?). Voir p. 119.

Bruyant $=$ grand cheval qui fait du bruit en galopant. Voir p. 119.

Estoudin $=$ cheval de bataille $(<$ estor $?)$.

Le jor sist Berengiers seur Estoudin norois,

Qui le chief e le col ot si blanc conme noif,

E Girars de Riviers en Argon le danois. Aye, 1087-9.

Gramimund = cheval grimpe-montagne (?).

Siet le cheval qu'il cleimet Gramimund, Roland, 1571.

(Le) Gringalet $=$ cheval beau et fort.

Dans son édition d'Erec, Jean-Marie Fritz note, p. $311:$ "Nom du cheval de Gauvain. Le mot vient du gallois Kein-Kaled : “beau et vigoureux [cheval]" et n'a donc sans doute aucun rapport avec le français moderne gringalet (à partir de 1611), (...) ».

La Vengeance Raguidel est le seul texte à présenter le terme sans article, mais Mathias Friedwagner, son éditeur, remarque dans une note, p. 210, que l'exemple (v. 983) est unique et probablement dû à une faute du manuscrit. Il donne la même explication du nom que J.-M. Fritz et trouve une explication de l'emploi de l'article dans le fait que le terme n'est pas encore tout à fait considéré comme un nom propre (7).

Dehe[t] ait qui portera foi

A Gringalet qu'il ne l'ochie! Raguidel, 982-3. 
Lors fait ses esperons sentir

Al Gringalet qui tost le porte $i b ., 2652-3$.

Gauvains monte en son gringalet. Erec, 4081. Cf. b., 3951 ; 3961.

Dont s'arma me sire Gavains

Molt tost, et quant il fu armez

Sor le Gringalet est montez, Cont. P., I, 3550-2.

Et lors point me sire Gavains

Le Gringalet qui tost li saut. ib., II, 12944-5. Cf. ib., II, 12804-5 ;

12381-3;12959 et passim.

Et a trestoz ses vallés dist

Que en sa terre s'en ralaissent

Et ses chevax en remenassent

Trestoz, fors sol le Gringalet. Perceval, 6206-9. Cf. ib., 7136.

Et Gavains des esperons fiert

Le Gringalet cele part droit Atre, 2570-1. Cf. ib., 2354-5 et passim.

Leoncel $=$ cheval fort comme un lion. Voir p. 119.

Matefelon = cheval qui vainc les perfides, les méchants

Li viels Girars broce Matefelon, Aspremont, 5026.

Orgueilleux $=$ cheval sans peur. Voir p. 119.

Passebrueil $=$ cheval fort (qui passe à travers les bois).

Il s'en vait droit en mi la court et monte sour Passebrueil, son ceval que il n'amoit mie petit.

$T P$, II, 2, 33-4.

Pliemont $=$ cheval fort (qui subjugue tout le monde ?).

Li rois Guiberz chevauche Pliemont, Mort Aymeri, 1794.

Lessen Florent, s'a Ploiemont mené. ib., 2137. Cf. ib., 2300 ; 3340 .

Ploiegant $=$ cheval fort

Monte sor Ploiegant, onques millor ne vi ; Aiol, 10009. 
Et saisi Ploigant que molt ot covoitié : ib., 10067. Cf. ib., 10070 ; 10112 et passim.

Ramunt = cheval fort (qui ramone, c'est-à-dire balaie tout en faisant son chemin?).

Ere s'en vait Girarz sus en Ramunt,

Sobre un cheval tout bon, tant le semunt, Roussillon, 1031-2.

Cf. $i b ., 1323-4$.

Taillefer.

Encor pert les les desrubans,

Par ou Taillefers s'en ala,

Li bons cevaus qui li dona

Grains d'or, li fix de sa seror. Poitiers, 18-21.

Tranchemor $=$ cheval qui traverse le marécage avec fougue (ou cheval noir sans peur?).

Rois fu de Niques et cosins l'amirant ;

Cheval ot bon, merveillos et corant :

Trestoz fu noirs mès que les piez ot blans,

Et les orilles et les nasiers devant :

Paien l'apelent Tranchemor lo bruiant ;

N'a tel destrier deci en Orient. Mort Aymeri, 1213-8.

Tranchequarel $=$ cheval sans peur. Voir p. 119.

Tencendor $=$ cheval batailleur.

En Tencendur, sun bon cheval, puis muntet Roland, 2993. Cf.

$i b ., 3342 ; 3622$.

Vielantif: cheval vieux, par conséquent expérimenté (cheval de Roland).

Es vos par la bataille, sor Vielantif, Rollant : Renaut, 12380.

La bone espee Durendal le vallant

Et Vielantiu qui tant estoit corant. Aspremont, 6077-8.

As porz d'Espaigne en est passet Rollant

Sur Veillantif, sun bon cheval curant. Roland, 1152-3. Cf. ib., 2032 et passim. 
d. autres qualités.

Alosé = cheval estimé.

Enmi la place li tienent Alosé,

Un bon destrier courant et abrievé ; Bueve, II, 2931, 2.

On li ensele maintenant Alosé, ib., III, 2811. Cf. ib., $2821 ; 2963$ et passim.

Argon $=$ cheval aragonnais (?). Voir p. 133, Estoudin .

L'adjectif danois ne correspond pas du tout à notre explication du nom, mais nous n'en voyons pas d'autre.

Ataignant $=$ cheval qui atteint le but. Voir aussi p. 118 .

Broce Ataignant, qui grans saus li pourprent, Bueve, III, 13277.

Et Ferraus broche Ataignant l'arragon ; Gaydon, 2312. Cf. ib., $2749 ; 2825 ; 3111$ et passim.

Audigon = le cheval d'Audigier.

En la place li traient son bon destrier

et ce fu Audigon qu'il ot tant chier ; Audigier, 196-7. Cf. ib., $242-3 ; 245 ; 374$.

Barbamusche $=$ cheval à poils au museau (?).

Siet le ceval qu'il cleimet Barbamusche, Roland, 1534.

Bel Joeor $=$ beau cheval vif.

Arire s'en torne le pas,

Mot par out bel cheval et cras.

Tristran rot le Bel Joeor : Tristan, 3995-7.

Bucifal = Bucéphale, le cheval d'Alexandre le Grand. Les chevaux des textes sont comparés à ce cheval pourtant incomparable.

Li amiraus s'eslesse et sist seur .I. cheval -

Mout par dut estre biaus qu'il fu fix Bucifal,

Le destrier Alixandre qui tant souffri de mal. Gui de N.,

2395-7. 
Il ot un mout riche cheval qui fu pere au bon Bucifal

que Alixandre tint meint jor, Thèbes, 6307-9.

Carahués broche le pendant d'un costal

Seur un cheval meilleur de Bucifal ; Enfances O., 1765-6.

Qant ge ai recovré Ferrant, mon bon cheval.

Certes je l'ai plus chier que nule rien charnal

Fors que le cors Buevon, le chevalier loial,

Que ge cuit qu'il vaut plus que ne fist Bucifal. Barbastre, 1059-62.

Quant l'ont armé, li uns d'ax vait amener un destrier d'Espaigne tel qui plus tost cort par chanpaigne, par bois, par tertres et par vax, que ne fist li boens Bucifax. Charrete, 6776-80.

Facebele $=$ beau cheval.

Folchers venc apoignant en Facebele,

Sobre un cheval movent de Conpostele. Roussillon, 2739-40.

Folatile $=$ cheval capricieux (?).

Ha, Folatille, tant jorz vos ai gardez ! Aliscans, 1694. Cf. ib., $1827 ; 2472-3$.

Bien s'en alast li quens sor Folatin, $i b ., 1802$.

Veit s'en Guillelmes, li marchis au cort nes,

Sor Folatise son destrier sejornez, ib., 1855-6.

Gaignon = cheval bien ordinaire (?).

Il s'agit du cheval de Marsile, l'ennemi de Charlemagne, qu'il y a lieu de déprécier. Le terme gaignon désigne normalement le chien de garde, souvent méprisé pour son métier. Voir p. 157.

As vus Marsilie en guise de barunt.

Siet le cheval qu'il apelet Gaignun, Roland, 1889-9o.

Galaté $=$ le cheval du héros Hector.

Hector monta sor Galaste, qui fu le plus biauz destriers dou monde ne qui jamais soit veüs ; et bien estoit dignes de tel maistre. Troie, 99, 44-6. 
Adont issi Hector de la ville et le heaume lacié monte sur

Galaté (...) ib., 100, 2-3.

Cf. ib., 117, 41-3; 158, 10-1.

Gascon $=$ cheval gascon.

Ricement fu armés du hauberc fremillon,

Et sist sor .I. ceval c'on apele Gascon. Fierabras, 5452-3.

\section{Justamont.}

Je voz donrai .I. bon branc aceré

Et le cheval Justamont, le faé, Mez, 3789-9o.

Laufage/Laufagoinne = cheval arabe. Nous pensons qu'il faudrait plutôt lire l'Aufage/l'Aufagoinne, d'autant plus qu'il s'agit du cheval de Sinados, le neveu du roi de Perse. Dans tous les exemples on pourrait même lire l'aufage/l'aufagoinne ; cf. p. 85 .

Desor Laufage en est li glous montés,

Cent Sarrasin sont avoec lui armé, Bueve, III, 2807-8. Cf. ib., 2816.

Sor Laufagoinne, qui li bruit et destent ; ib., III, 2839.

Lor seignour voient gesir sor le rochier

Et Laufagoine füir tout eslaissié, ib., III, 2961-2.

Martin.

Diu commencha a renoier

Et ses chevaus a manechier :

« Hari ! Martin ! hari ! Fauviel ! Wistasse, 200-2.

Mirabel $=$ cheval merveilleux. Voir p. 119.

\section{Murgalie.}

Monte el cheval, qui ot non Murgalie, Bueve, II, 15954.

Norrois $=$ cheval nordique

Il point a broce durement li Norrois, Chevalerie d'O., 1812.

Papillon = cheval léger (?). 
La coucha Ogier la nuit mais ce ne fut pas sans penser a son cheval Papillon lequel estoit luiton et avoit esté ung grant prince, (...) Ogier, 269. Cf. ib., 270 ; 273 et passim.

Pegasus $=$ Pégase

De laquelle tres sainte victoire la nouvelle ala par tout, ainsin que fist de Perseus par Pegasus, le cheval voulant, (...) Jehan de $S ., 221,27-9$.

Potrel $=$ cheval jeune. Voir p. 119.

Valantin $=$ cheval de valeur

Il broce Valantin par an .II. les costés. Fierabras, 2407.

\subsubsection{Adjectifs et syntagmes prépositionnels désignant l'origine, la robe ou d'autres qualités des chevaux}

Nous ne présentons en principe qu'un seul exemple de chacun des termes en question. La plupart d'entre eux se retrouvent dans un très grand nombre de textes, accolés aux différentes dénominations des chevaux.

Notons que parfois plusieurs adjectifs (surtout pour indiquer la robe des chevaux) se trouvent côte à côte, comme dans les exemples suivants, où il est question tantôt de plusieurs chevaux, tantôt d'un seul cheval à plusieurs qualités/plusieurs couleurs. Voir aussi p. 27:

La fu mainz bons chevax sors et bruns et bauçans, Saisnes, 936.

Puis montent as chevax sors et baucens et bais, $i b . .979$.

Et maint cheval couvert, sor et bai et bauçant, $i b ., 1188$.

Et cil monte ou destrier fort et isnel et gras, $i b ., 2198$.

E chevauge un chaval sor baucan laur. Roussillon, 6530.

E chevauge un chaval sor baucan ner. ib., 7019 .

Quant s'en cuide venir, es li sergant Morart

Et siet sor un destrier rous, bauchant et lïart. Partonopeu-C, 1759-60. 
Es chevax montent bruns et baucens et gris, Gaydon, 4936 .

a. origine.

Nous avons jugé utile de noter aussi les expressions d'origine dont on trouve des équivalents ci-dessus, p. ex. de Gascogne correspondant à gascon.

Sor .I. ceval le torse corant norois ; Aiol, 3848.

en avoit jusqu'a .xxiij.

armez, sor boens chevax irois. Charrete, 1661-2.

Li varlés est montez ou destrier de Surie, Brun, 240.

Et que li dux en moine lo destrier de Suleie, Simon, 186.

Et fu mout bien armetz el bai de Pincenie ; ib., 190.

A ce que l'emperere demenoit ploroison,

Estes vos .I. mesage sor .I. cheval breton ; Renaut, 1105-6.

Mort l'a jus abatu del cheval de Hongrie, ib., 1894.

« Fetes apareillier .c. destriers orcanois, ib., 2550.

Et l'enfes Guis let courre le destrier d'Orcanie ; Gui de B., 553. Cf. Simon, 63.

Je li donrait chevalz d'Allemaigne ou de Brie Lion, 1383.

Chevalz et pallefroy et destrier d'Arcanie $i b ., 5711$.

A Ganor ot bailliér doulx chevalz de Dammas ib., 6701 .

Il est montés, l'elme en son cief,

Sor .I. bauçant de Cornouaille. Raguidel, 5716-7.

A tant es vos pongnant par merveilleus outrage

Tenpeste de Leride sor un destrier d'Arcage. Barbastre, 5517-

8. Cf. Loquifer, 3755.

Li cheval desoz els sunt de Vaumers, Roussillon, 1108.

Un cheval sor bauzan de Balager. ib., 3946.

E sist en l'auferant amoravit, $i b ., 2558$. 
Sist le cheval goton, a l'amoraive ; ib., 2949.

E sist le cheval bai godemucon, $i b ., 2531$.

[Bon che]val arrabi ne castelan, ib., 5368.

En un cheval de prez de Maroc, ib., 5946.

Sobre un cheval movent de Conpostele. ib., 2740.

Il broiche le cheval, qui li vint de Cartaige Floovant, 380. Cf.

Raoul, 141.

Bien fu brochiés li destriers de Niors. ib, 2340.

Et Loeys el destrier castelois, $i b ., 3387$.

(...), et un tresbel coursier puilloiz. Jehan de S. 171,18-9.

Gautiers giete la main, s'a la crine combree

De Morel d'Alcasés qui vait teste levee ; Partonopeu, 1941-2.

Li amirans guenchist le destrier de Nubie, Gui de N., 2609.

Si que del bon ceval de Frisse

Le trebucha ens el sablon. Inconnu, 5882-3.

Oncques en pié n'ot fer ne clou

Ses destriers Fauveaus de Tolete ; Galeran, 5634-5.

Li Blonz des Illez d'aventure

Siet sur le Sor de Portigal ; ib., 5630-1.

Et siet sur Ferrant de Venisce ; $i b ., 5617$.

Esperonant sor le destrer d'Arage ; Chevalerie d'O., 1212.

Sor un cheval d'Arrabe sist

que li rois Daires li tramist: Thèbes, 5649-50.

Sor un cheval sist de Castele

qui plus tost cort que arondele ib., 5789-90. Cf. Escoufle,

1108. ; Raoul, 1178 ; 1775 .

Garsi est montez el destrier

qui fu amenez de Baivier ; ib., 4705-6. 
Sor un cheval sist de Gascoingne ib., 6349. Cf. Erec, 2659.

Sor un cheval de Capadoce Erec, 1965.

Et sist sor un cheval d'Illande $i b ., 2172$.

et cis i vint si fierement

sor un ceval d'Espaigne sor Eracle, 6124-5.

Es chevaus montent de Puille et de Rossie. Orange, 1301.

Et voit venir ung Turc sur le destrier d'Orbrie. Tristan de N., 8846 .

Jules Cezars son voloir li otrie,

Sor .I. destrier monte de Lombardie, Auberon, 2043-4.

b. robe.

Aux pages 21-22 nous avons présenté la longue description du palefroi de la jeune reine Camille. Ce cheval est un vrai tableau des couleurs possibles et l'on s'imagine difficilement un animal si bariolé.

Aux pages 139-140 se trouvent présentés d'autres exemples de chevaux de plusieurs couleurs, mais normalement la robe est unie :

et chevaucha un cheval grile, Thèbes, 6287. Cf. Enfances G., $275-6$.

El destrier est montez qui estoit pomelez, Renaut, 165.

Et fu armés sor un cheval flori, Bueve, I, 6061. Cf. Narbonnais, 6186 ; Lanson, 4142.

Puis i prist .j. destrier a le crupe tieullee, Bâtard, 683.

S'en menrois un bon cheval blanc

Dont l'oroille a color de sanc, Partonopeu, 7739-40.

Desus son palefroi roussel

La trousse et lie derriere soi, $M R$, LXVII, 110-1.

c. autres qualités.

Delez le tre ai je fait atachier

Un palefroi ambleour bel et chier, Enfances O., 2682-3. 
La veïssiez maint destrier abrievé, $i b ., 750$.

Et Basins esperone le destrier afilant,

Et li chevax li saut .xv. piez d'un tenant. Lanson, 2943-4.

Aprés le heaume venoit Saintré sur un tresbel et fringant destrier (...) Jehan de S., 111, 26-7.

Puis l'ont monté isnelement

Sus un souef amblant destrier, Amadas, 1906-7.

Les sieles metent es palefrois amblant, Huon, 316.

Et si prendés ces deus frans mesagiers,

Por palefrois lor donnés grans destriers $i b ., 415-6$.

Entre ses quisses out un ignel cheval, Guillaume, 774.

Rois Otes esperonne Bondifer l'alousé, Florence, 1597.

Or dist li contes que quant Lanselos se fu partis, et li rois Pellés vit qu'il s'en fu alés, il prist Galaad, si le fait metre sour un rice palefroi, (...) TP, vi, 84, 1-3.

Et enmena avoeuc li Galaad qui a mervelles estoit biaus enfes de son aage ; si le porta uns esquiers devant lui sour un palefroi fort et isnel et bien portant. $i b ., \mathrm{vI}, 49,24^{-} 7$.

Quant ele fu a tere venue, ele monta sour un palefroi ki mout estoit biaus et cointes et ricement apareilliés de toutes coses, (...) ib., II, 80, 4-6.

Et sachiés k'il fu assés grevés de celui caoir, car li cevaus, ki grans estoit et pesans a desmesure, li fu du tout caüs sour le cors, (...) ib., III, 196, 12-4.

Celes montent eneslopas

[De]sor les beaus palefroiz gras, Joufroi, 2143-4.

Maint bon cheval destré conré

Veïst on et mainte biele arme. - Violette, 5961-2.

Gerars li a requis une eure,

C'aler s'en velt, cheval li prest ;

Dist li sires : «Ja l'arés prest,

Grant et isniel, fort et adroit ; $i b ., 4985-8$. 
E chevauche un cheval corser braidenc, Roussillon, 5097.

Pois cobret au cheval de bon ale[u]. ib., 5112.

(...), si voit li tiers qui vien devant les autres molt grant piece sor un merveillus cheval ; Lancelot, I, xxx, 11.

Chascuns ot bon destrier, fort et isnel et sain. Buevon, 114 .

Cheval li amenerent et fort et legier $i b ., 2370$.

Guiteclins de Soissoigne sist ou destrier gaillart, Saisnes, 1746.

« Li cevaus est molt maigres et deshaitié[s] Aiol, 225.

Et li borgois fu sages et apensés :

Sor .I. boin palefroi en est montés, ib., 1259-60.

Lors virent tres parmi la plaingne

Trestoute seule sans compaigne

Une pucele chevauchier

Sor un palefroi bien et chier. Rigomer, 37-40.

Amené li ont un destrier

Fort et hardi et bien coursier. $i b ., 835-6$.

Il a pris le cheval, qui fu grans et corssus, Rome, 996. Cf. Loquifer, 3749.

Ernouls [re]fiert Sorné entre lui et Alos

Que mort l'a craventé du cheval rabinous. Partonopeu-C, 2646-7. Cf. Mort Aymeri, 1806.

Li cheval ravineus i traïnent lor bus. Aye, 1000.

«Car j'ai destrié et bon et remuant, Otinel, 331.

Sus .I. cheval trotant l'ont maintenant montée, Doon, 1250.

Li chevalz fu grans et apers, Durmart, 2409. Cf. ib., 5767.

Et grant destrier rade et corant $i b ., 9289$.

Del sergant et de l'esquier

Vult avoir le ronci trotier. $i b .$, 3267-8. Cf. ib., 556. 
Il monte sor un grant destrier

Vigeroz et fort et ligier ; $i b ., 4639-40$.

Au conte Amile devommez retorner.

Sist en la selle dou destrier sejorné, Ami, 79-80.

Montés estoit sor un destrier ferrant,

Fort et isnel et aspre et tost corant, $i b ., 874-5$.

Sor un destrier sist plus blanc que toaille,

Fort et seür et de tres fine taille, $i b ., 5403-4$.

Et li rois eschapa sor un cheval arvoine. Barbastre, 90.

Leur chevaus furent courans et roides, (...) Helcanus, 114.

Pués li amoinent auferrant bradi ; Enfances G., 2561.

Puis venoit ung homme d'armes, monté sus un grant coursier bardé, (...) Jehan de P., 59, 25-6.

mais trop ot petit palefroi,

si n'i pot ataindre en seant ; Renart, II, 5220-1.

Blancheflour la roÿne ont en litiere mis

Entre deus palefrois qui furent de grant pris, Berte, 2367-8.

Cant sunt ensanble si furent bien .xx.m.

A belles armes et as chevax de pris. Mez, 59-60.

Li chevaus rustes et saillant

Par son orgueil va sautelant Chauvency, 1104-5.

«Forment tient au cheval fers! ib., 3580.

Sor un fauve destrier comé ! Cligés, 4716.

Callot laist corre le bon destrer muable ; Chevalerie d'O., 1202.

1 Cf. Friedrich Bangert, op. cit., p. 8 : «Unter allen Tieren spielt im altfranzösischen Epos das Pferd die erste Rolle, da ein Held einer Chanson de geste kaum ohne Pferd denkbar ist. »

2 Cf. Francis Dubost, De quelques chevaux extraordinaires dans le médiéval : esquisse d'une configuration imaginaire, (Senefiance 32, p. 190) et Adolf Tobler, Vermischte Beiträge, v, pp. 223-229. 
3 Cf. Friedrich Bangert, op. cit., p. 9: «Die Appellativnamen, von denen ich 61 [corrigé, à la page 233, en 62] gefunden habe, teilen sich wieder in zwei Gruppen ein, in echte Substantive und in solche, welche zugleich Adjektive sind oder von Adjektiven gebildet wurden. Erstere kennzeichnen entweder die Verwendung des Pferdes im Dienste des Menschen oder sein Geschlecht, letztere entweder die Farbe, die Herkunft, die Laufgeschwindigkeit oder den Wert. (...) Nach ihrer Verwendung sind zunächst drei Hauptarten von Pferden zu unterscheiden : das Streitross, das Reisepferd und der Arbeitsgaul. » 4 Cette affirmation (émise aussi par Michel Pastoureau, voir p. 14) doit être modifiée, car quelques textes présentent des juments chevaux de bataille. Cf. brehaigne et jument. Friedrich Bangert est plus prudent ; il écrit, op. cit., p. 21 : « Da aber die Streitrosse in der Regel Hengste waren, (...) ».

5 Peter Dinzelbacher, op. cit., p. 199 : « Die rechts geführte Lanze konnte nur dann so gehandhabt werden, daß sie effektvoll und ohne Selbstverletzung durch den Rückstoß beim Anprall einzusetzen war, wenn das Roß im Rechtsgalopp lief, weswegen der Hengst (nur ein solcher wurde in der Schlacht geritten) im Mittellateinischen "dextrarius" und im Altfranzösischen "destrier" hieß. »

6 Concernant les chansons de geste, Friedrich Bangert écrit même, op. cit., p. 23, qu'il n'en a pas relevé une seule occurrence où il désigne le petit; et d'ajouter : "In der Regel ist darunter ein schon als Streitross verwandtes Pferd zu verstehen : (...)».

7 « (...) hier ohne Artikel, was unbelegt ist und daher wohl auf einem Fehler der Hs. beruhen wird, vgl. 2653 (...) ; denn der Name dieses berühmten Pferdes, aus substantivierten Adjektiven (kelt. kein - caled "schön" + "hart" d. h. ausdauernd) gebildet, wurde noch nicht völlig als Eigenname gefühlt, obgleich man ihn nicht mehr verstand (...)». 


\section{Les animaux II}

\subsection{Autres mammifères}

Dans le domaine des mammifères, on peut faire la distinction, en gros, entre les chevaux et « les autres », ceux-là appartenant, nous l'avons vu, presque exclusivement aux classes supérieures de la société, auxquelles ils sont indispensables, ceux-ci faisant partie, dans une large mesure, de l'entourage des roturiers, notamment des paysans.

Un extrait de Renart nous donne une idée sur la question de savoir quels animaux vivaient dans la ferme :

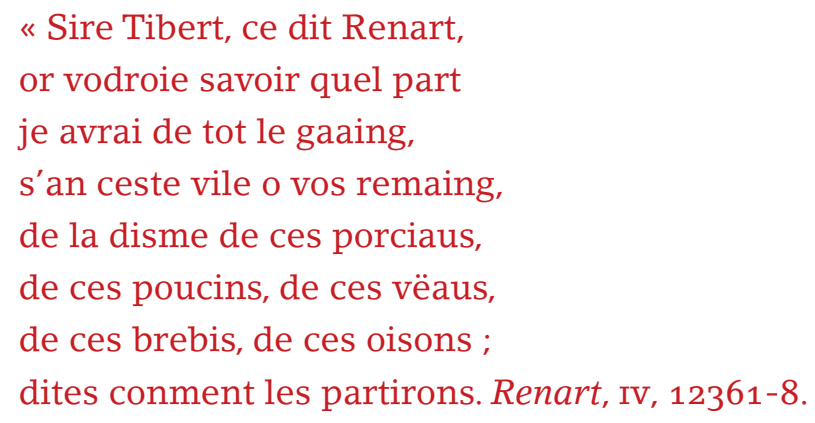

Mais il faut se prononcer avec prudence sur ce sujet, car p. ex. les chiens se retrouvent dans tous les milieux, quoiqu'il ne s'agisse certainement pas d'animaux de la même race. Les mulets sont utilisés par les nobles aussi bien que par les roturiers, tandis que les bovins et les ovins se présentent presque exclusivement en compagnie de paysans. Les textes s'occupant surtout de la vie des nobles, bœufs et brebis apparaissent beaucoup moins souvent que les chevaux, mais cela ne signifie certainement pas que ces animaux étaient de peu d'importance dans la vie des gens médiévaux, bien au contraire ils jouaient un grand rôle dans la vie de tout le monde.

Contrairement à ce que nous avons constaté pour les chevaux, ni la robe ni l'origine des autres mammifères ne jouent aucun rôle.

\subsubsection{Chiens}

Si nous présentons d'abord les chiens, c'est que ce sont là, à côté des chevaux, les seuls animaux dont les textes distinguent différentes races. Par contre, ils n'indiquent que rarement une dis- 
tinction des individus par des noms propres ; ceux-ci se trouvent notamment dans Renart et dans les fabliaux.

Pour les nobles, les chiens servent surtout pour la chasse, les gens simples ont des chiens de garde et les bergers les utilisent pour garder les troupeaux. Gace de la Buigne nous fait savoir qu'au « plat pays » chaque particulier a un chien :

Ou plat païs chascun a chien

Communalment, s'il vit du sien,

Les uns grans, les autres petis,

L'un est mastin, l'autre est mestis. Deduis, 8997-900.

Friedrich Bangert, op. cit, 172, cite 14 dénominations différentes de chiens. Il affirme que ce grand nombre de dénominations dans les chansons de geste est signe de l'intimité entre chien et maître et que, dans la hiérarchie des animaux des chevaliers, le chien tient la place numéro deux, juste après le cheval. Nous pensons que les chevaliers apprécient leurs oiseaux de chasse (au moins) autant que leurs chiens de chasse (voir pp. 200 et 236) mais, comme le montre Deduis, il y a des partisans de chacune des deux catégories ; la majeure partie du roman traite de la question de savoir « Qui est plus noble, oyseaux ou chiens ? "(vv. 5384 et 5631).

Gace de la Buigne énumère toutes les bonnes qualités des chiens. Il est vrai qu'aux vers 6147-72 du même texte, il énumère des qualités négatives : les chiens sont orgueilleux et félons, avares et jaloux, ils mordent et volent, sont traîtres et paresseux.

Chien est loyal a son seignour,

Chien est de bonne et vraye amour,

Chien est de bon entendement,

Chien saige a bien vray jugement,

Chien a force, chien a bonté,

Chien a hardiesse et beauté.

Chien est beste moult amiable,

Chien sage est beste veritable.

Chien a memoire souverain

(...)

Bon chien est viste et tost alant,

Et si est tost appercevant.

Chien a langue medicinable.

Amour de chien n'est pas muable.

A chien trouverez bonne gueulle.

(...)

Chien aime humaine compaignie,

Chien sauve a son maistre la vie.

Chien fait tout ce que on li apprent.

Chien est bien a comandement. 
L'en a de chien plusieurs soullas,

Les uns saillent par sur le bras,

Et les autres servent a table.

Tieux y a qui gardent l'estable,

Qui sceivent moult bien chevauchier,

Et des autres l'en fait sommier.

(...)

Il recongnoissent bien leur nom.

Autres bestes et oyseaulx nom.

Ilz gardent les bestes le jour,

La nuit l'ostel de leur seignour. Deduis, 5659-67, 5671-77,

5681-90 et 5701-4. (Voir la suite).

C'est également dans Deduis que se trouve un long passage qui prouve l'intelligence et la fidélité des chiens. Il s'agit du lévrier d'Aubery de Montdidier dont les exploits sont décrits aux vers 5811 à 5902 ; nous n'en citerons que ceux qui nous semblent les plus importants. Aux vers 836-862 de Macaire, nous lisons la même histoire.

Macaire tue le maître du lévrier :

Maiz, quant le chien vit qu'estoit mors,

Tout de foilles couvri le corps.

La se tint jusqu'a l'endemain,

Et adoncques le prist la fain

(...)

Le chien a Machaire trouvé

Seant a la table du roy,

(...)

Pour le mordre li couru seure

Et l'eüst du tout affollé,

(...)

Si regarderent la maniere,

Que le levrier un pain hapa

Sur la table, qu'il emporta

Tout droit a son maistre Aubery,

Qui gisoit mort ou bois foilli.

Et l'endemain et le tiers jour

Le levrier fist yceluy tour

En venant querrir a mangier,

Aussi pour son maistre vengier

Car, la ou il trouvoit Machaire,

Tout diz li vouloit il mal faire.

A la bouche Aubery mettoit

De la viande qu'enportoit. $i b ., 5837-40,5846-7,5852-3$ et 5858-70. 
Les chiens sont souvent méchants - peut-être parce qu'ils sont maltraités ou mal nourris mais, affirment les textes, surtout parce que c'est dans leur nature de s'attaquer aux faibles :

En la vile entra sanz demeure.

Lors li sont maint chien couru seure ;

Mordre le vont et envaïr,

Quer povre gent seulent haïr ; Anjou, 5423-6.

Com Amadas endroit li passe,

Voiant ses oels, d'une maison

Vit hors salir un grant gaignon,

Une grant hart entor son col.

Quant il voit Amadas, le fol,

Qui devant les autres couroit,

Si fait tout çou que faire doit

Sa nature tresfelenesse,

Devant Ydoine la contesse

Et joint ses piés et fait un saut

Et saisi Amadas bien haut,

Qu'il le vit nu et descouvert ;

Par une espaule as dens l'aërt,

Qu'il avoit maigre et descarnue. Amadas, 3166-79.

Dans un certain nombre d'extraits on exprime du mépris pour des personnes en les comparant à des chiens. Certains termes qui désignent des chiens (surtout mâtin) servent d'injures (voir chapitre 5).

D'un autre côté, les chiens peuvent être donnés en cadeau, de même que chevaux et oiseaux, p. ex. Huon apporte des cadeaux pour Charlemagne et celui-ci envoie Huon chez l'émir Gaudise à qui il demandera entre autres « mille veautres » :

Et ciens et viautres emmena il asés ;

Ostoirs, faucons et espreviers mués

En fait li enfes porter a grant plenté. Huon, 543-5.

De moie part l'amiral rouverés

Que il m'envoit mil espreviers mués,

Mil ours, mil viautres tres bien encaenés, ib., 2364-6.

Si li dona .r. blanc levrier,

.I. confanon et .I. destrier. Rigomer, 6545-6.

Pran mon levrier et mon faucon,

a Pollinicés an fai don ; Thèbes, 7439-40. Cf. ib., 7451-4. 
Plusieurs textes notent le caractère sauvage des chiens, cf. 5.1.2.

Dans Guillaume d'A., le roi est chassé par des marchands qui sont encore plus féroces que des chiens :

Li rois, de doel et de fain pales,

Vint la u les vit amassés ;

Mais moult li venist mix assés

Que sor kiens se fust enbatus :

Tres bien i dut estre batus. Guillaume d'A., 950-5.

Voici la liste des différentes appellations de chiens que nous avons relevées dans nos textes :

a. alan $=$ chien de chasse.

Lequel Deduit vint au secours

Du commun pueple tout le cours

Avecques levriers et alans

Et grans matins et chiens courans Deduis, 7769-72.

b. berseret $=$ chien de chasse.

.I. cerf ramu plenier et grant.

Cil fu bersés et sel sivoit

.I. berserés qui le caçoit ; Rigomer, 7608-10. Cf. ib., 7637-8.

Lor mestiers ert cortois et biax,

Chiens afaitoient et oisiaus,

Levriés afaitent et brakés

Et loiemiers et berserés, ib., 14229-32.

puis est montez el chaceor

et li autre montent es lor.

Son arc porta uns suens vallet

et uns autres son berseret ; Eneas, 3573-6.

E li cons i montet fors au perron

E prist un berseret tres son archon. Roussillon, 8104-5.

A propos du vers 86 du lai Guigemar, Alfred Ewert note, p. 166 de son édition, que berseret désigne normalement un chien de chasse, mais que dans le cas présent il propose le sens de carquois, étant donné que le terme peut désigner un objet du trousseau d'un chasseur (1) :

Sun arc li portë un vallez,

Sun ansac e sun berserez. Lais, I, 85-6. 


\section{c. brahon/braon.}

Le terme, dont les occurrences sont bien rares, désigne un chien de chasse, peut-être un chien braque. Heinrich Gelzer, dans ses notes à Yder, p. 216, propose de lire brachon et traduit le terme par «Brache, Spürhund ».

A propos du terme orsier des extraits d'Yder, il y a lieu de faire les remarques suivantes: Heinrich Gelzer, l'éditeur, n'est pas conséquent avec lui-même : dans son glossaire, il traduit le terme par " auf Bären dressierter Hund » et propose une analogie avec lévrier. Mais, dans ses notes au vers 3338, il appelle les orsiers «Besitzer ». Nous pensons qu'il s'agit, en effet, des propriétaires de l'ours et non pas d'une espèce de chiens, car au vers 3341 il est question du collier de l'animal qui a probablement eu sa place dans une ménagerie et que ses propriétaires ont fait danser devant un public :

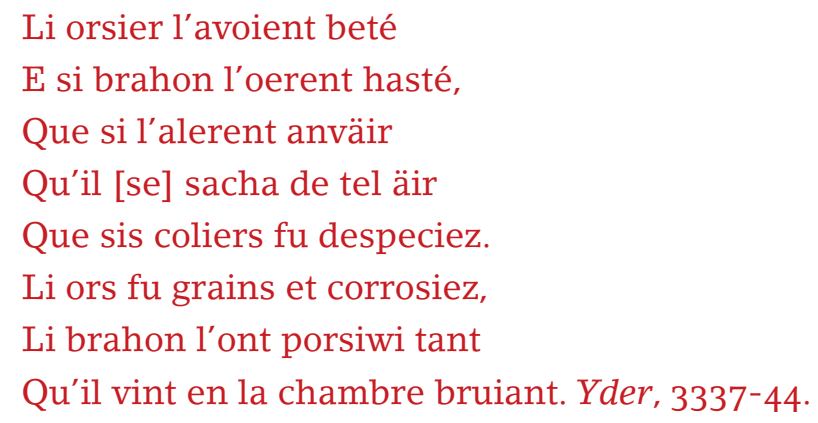

La le pöent li orsier prendre,

Se li ors les [i] volt atendre, $i b ., 3389-90$.

Trente braons l'asaillent, que viennent a estri, Florence, 4635.

d. braquet $=$ " Chien de chasse à poils ras et à oreilles pendantes ; très bon chien d'arrêt » (Petit Robert).

A tant ont Renart escrié

li braconnier qui l'ont veü,

et li brachet sont esmeü, Renart, Iv, 11696-8. Cf. ib., v, 13401-

2 ; VI, 16113-8.

Mais or me dites, mes deus braqués que je tant amoie, les emporta ele avoec li ? »

TP, III, 125, 8-10. Cf. ib., 125, 13-5; 125, 17-8 et passim.

(...), car il va tous les jours en cache, ore a bracés, ore as levriers. ib., v, 8, 6-7.

Cf. ib., 31, 17-20 ; 41, 9-11 ; I, 1, 25-8 ; VI, 170, 17-9 ; VIII, 51, 4-6. 
Quant ilz commencerent a approuchier du chastel, ilz encontrerent seur un roncin un vallet qui portoit un esprevier et menoit .II. brachés, (...) ib., vII, App. II, 1, 11-4. Cf. ib., 119-21.

Li ceval[s] vit les armes mal atirees :

Il fronche des narines, la geule bee ;

Aiols li tient les resne estroit seree,

Ausi porte [la] teste en haut levee

Que li cers que on cache a la menee,

Quant li bracet le cacent a la ramee. Aiol, 896-901. Cf. ib., 4696.

Al novisme jor encontrai

un cherf ramu qui fu bersez,

que uns brachés bien acorsez

chachoit et sivoit de molt pres. Cont. P., III, 14888-91.

Lors voit venir grant aleure

.I. porc senglier vers soi corant

et .III. brachez apres criant ; Claris, 12124-6.

La se defant tant acemeemant

Con li singleirs qui les brochas atant. Enfances G., 419-20. Cf. ib., 1889-92.

Et lors deschendi por tourser le chervel deriere lui ; et devant lui anportoit son brachet qui tote jor avoit seü son chevreil devant les autres. Lancelot, vII, IXa, 8. Cf. ib., vII, IXa, 18.

(...) et li autres portoit .I. arc et .I. carcois et .I. brachet deriere lui et .I. cor a son col.

$i b$., II, xxxvIII, 3 .

Plus loin nous verrons que les dames avaient des petits chiens pour leur amusement. Dans Lancelot, plusieurs dames ont un chien braque, entre autres la reine Guenièvre qui aimait la chasse :

(...) .I. escuier qui portoit un brachet la roine qu'ele faisoit tos jors mener o soi por amor a la dame del Lac qui doné li avoit ; Lancelot, II, LII, 2.

Einsinc ont chevauchié dusqu'aprés prime par mi l'ombre del bois tant qu'il encontrent .I. damoisele qui portoit .I. brachet entre ses braz. $i b .$, IV, LXXIX, 32 .

Lors encontra .II. damoiseles qui portoient .I. brachet ; $i b .$, IV, LXXX, 28 et passim. 
Les extraits suivants de Merlin montrent que ce texte ne distingue pas lévriers et braques :

Et pour chou que on sache l'ochoison de nostre bataille couvient il que tu en portes od toi les deus levriers que tu occhesis, l'un seur le col de ton cheval et l'autre derriere, (...) Et quant il a son duel mené grand pieche, il monte seur un grant cheval que uns vallès li amena, et puis prent les deus brakès et en met un devant et l'autre derriere, (...) Merlin, II, 90.

Voir aussi : Ami, 2343 ; Inconnu, 1283-6 et passim ; Dole, 435-6 ; Roche, 3274-7 ; 3301-2 ; Floriant, 288-90 ; Durmart, 10456 ; 10501 ; Yvain, 1264-7 ; Laurin, 9327 ; 9332 ; Godin, 10819-20 ; 10827 ; Cristal, $3865 ; 3903$ et passim.

e. caigne.

R. Grandsaignes d'Hauterive, op. cit., p. 87, définit : " $1^{\circ}$ Chienne (...). $-2^{\circ}$ Mauvais chien. » Il donne comme étymologie l'italien cagna ; sa date d'entrée en français serait le $\mathrm{xv}^{\mathrm{e}}$ siècle.

« Passez, passez, orde caigne que vous estes; par Dieu, vous n'y entrerez meshuy, meschante beste que vous estes ! » Et en ce disant, ferma l'huys. Et la royne, qui l'oyt, demanda : «A qui parlez vous, m'amye ? - C'est a ce paillard chien, madame, qui m'a fait tant de peine de le querir ; Cent, 28, 155-61.

f. chien.

Nous ne présentons qu'une très petite partie des nombreuses occurrences du terme relevées dans nos textes :

Or est Renart en male frape, car li chien le tienent en trape et li vilains venoit aprés ; Renart, II, 4765-7.

Li lievres, qui les chiens douta, $M R$, LxxxIII, 19.

Li chers, ki mout estoit isniaus durement, s'en vait devant et li chien aprés (...) TP, I, 183, 12-3. Cf. ib., 187, 10-4 ; 187, 41-2.

Un jour avint qu'il cachoit en .I. bois qui duroit jusc'a la mer, et tant qu'il perdi ses ciens et ses veneours et ses homes, tous fors un seul qui estoit ses cousins germains. $i b$., vIII, 189, 5-8. Cf. ib., 152, 16-20. 
Tant escouta li preus, li gens,

Qu'il öi cors et chiens et gens

Qui grant noise mainent ensanble. Rigomer, 1195-7. Cf. ib., $1216-8 ; 1382-4$ et passim.

Quant il eut quatorze anz passés,

Sy fu molt biaux ly damoisiax ;

Molt sot de chienz et molt d'oysiax, Gliglois, 10-2.

Li chien glatissent environ Floriant, 8187.

par le veneur font les kiens destoier.

Qui dont oïst Henri les kiens huchier,

quant les ooit glatir et abaiier, Godin, 10789-91. Cf. ib., 10797 ;

10806 et passim.

De même que le terme cheval servait de dénominateur commun dans l'univers équestre, celui de chien a cette fonction dans le domaine canin, p. ex. dans Bueve, où les chiens du vers 4413 sont les mêmes que ceux du vers 4379 :

Et vint levriers, vint brakés acoplés Bueve, I, 4379.

Les chiens a on as veneors livrés. ib., I, 4413.

Mais nous le voyons employé parallèlement aux autres dénominations, sans qu'il ne nous soit possible de déterminer de quelle espèce de chiens il s'agit :

Ele a mandé ses veneors,

enseler fait ses chaceors,

prennent lor ars, corz et levriers,

chiens et viautres et liemiers. Eneas, 1457-6o.

Adonc s'esmuevent quatre cens loiemier

Et chien et vetre et brochait et livrier. Enfances G., 1889-90.

Li chevalier alloit per le boix chevalchant ;

Cez chien et cez livrier, cez gens le vont suyant. Lion, 514-5.

La Chastelaine de Vergi nous montre que les "belles dames" avaient des petits chiens pour leur amusement ; le chien en question est mentionné neuf fois dans le texte et il est appelé petit chien une fois (v. 358), chienet six fois (vv. 380, 383, 654, 736, 880 et 908), petit chienet deux fois (vv. 34 et 718). Chienet se trouve aussi dans le fabliau Du chevalier de le robe vermeille, dans Laurin et dans Perceval où il désigne un chien de chasse : 
Et a cele amor otroier

deviserent qu'en un vergier

li chevaliers toz jors vendroit

au terme qu'ele li metroit,

ne ne se mouvroit d'un anglet

de si que un petit chienet

verroit par le vergier aler ; Vergi, 29-35.

Lors li a toutes acontees

ses venues et ses alees,

et la couvenance premiere,

et du petit chien la maniere. $i b ., 355^{-8}$.

Et prist son esprevier mué,

Que il meïsmes ot mué,

Et maine .II. chienès petiz, $M R$, LVII, 37-9.

Ainsi comme il se durent asseoir a digner, avint que uns chiennés vint en la sale. Laurin, 8657-8. Cf. ib., 8662; 12888 et passim.

Le champ devant lui le vergier

Avoit deus chienés a aoisiax. Perceval, 8538-9.

Les vers indiquant l'origine d'un chien sont extrêmement rares. Le second exemple tiré de Deduis indique probablement un (petit) épagneul :

Et, quant il a fame esposée,

Si a la teste plus mellée

Assez que ne soit chiens de Flandres, $M R$, crv, 25-7.

De ses saiges chiens d'Alemaigne

Et de ses bons chiens de Breteigne Deduis, 7981-2.

Quatre chenés ait bien querans

D'Espaigne et tres bien retrouvans $i b .$, 10301-2.

Le terme chael, désignant le petit du chien, se rencontre sporadiquement :

Que que il iert en cel martire,

si oit tel chose qui l'esmaie,

car uns chaiaus de loing l'abaie, Renart, II, 5076-8.

qu'il me douroit a grant planté

de se braiches et de son miel, 
que je aim plus que riens soz ciel,

se ses .II. chaiaus li rendoie,

qu'au soir a mengier atandoie. $i b .$, IV, 9534-8. Cf. Proverbes,

$2298 ; 2312$.

coc, chapon ne guelinne ne viel chien ne chael, Rou, 4187.

Le terme chienaille désigne l'ensemble des chiens. Dans Diable il semble avoir une connotation négative, dans Ogier il est injurieux :

Desos la vaute, o le chienaile,

La fache on ja le lit au fol, Diable, 1208-9.

Et Robers s'en va sor la paile

Couchier ensamble la chienaile. $i b ., 2407-8$.

Haa, mauldicte chiennaille, se vous passés plus oultre il est fait de vostre vie. Ogier, 194.

g. épagneul.

Chiens courans y a enfondus,

Espaignaux pour roigne tondus, Deduis, 9079-80.

h. frebau.

D'après Joseph Gildea, ce terme désigne un chien notamment utilisé pour la chasse aux cerfs. Nous n'en avons relevé qu'un exemple, dans Partonopeu, accolé à chien :

Dont verrés venir loiemiers

Et chiens frebaus et bons levriers ; Partonopeu, 1797-8.

i. gaignon = mâtin, dogue, chien de basse-cour.

Les gaignons servent à protéger les poules etc. contre le renard ; ils surveillent les activités à la ferme et assistent les paysans à la chasse. Comme les gaignons ne se rencontrent qu'avec les vilains, le terme peut avoir une connotation négative : les gaignons sont souvent méprisés à cause de leur statut inférieur.

Chez Friedrich Bangert nous lisons, op. cit., p. 173, que contrairement aux chiens nobles (lévrier, braquet etc.), les termes gaignon et mâtin désignent le chien ordinaire qui sert comme chien de garde, chien de boucher ou chien de berger, mais qui n'est pas utilisé pour la chasse.

Tost porroie a mon cors faillir,

s'il me huioit .III. gaignons : 
j'avroie en aus maus conpaignons ; Renart, IV, 11180-2. Cf. ib., 10394.

.vIr. gaingnon vienent descouplé,

en aprés vienent venaor,

anbaletier et chaceor,

et li venieres huit et crie. ib., v, 15404-7. Cf. ib., 15204-8 et

passim.

Et puis le fis en un vivier

tote une nuit haranc peschier

jusqu'au matin que un vilain

i vint, sa conquiee en sa main,

qui avec lui ot un gaingnon

qui li refist mau peliçon,

et si li peliça le pel : ib., III, 8923-9.

Si les gaignons sont méprisés c'est en particulier dû à leur couardise et leur méchanceté (voir supra et cf. 5.1.2) :

Dist Perchevaus : « Or puis retraire

Que il resamblent de gaignon,

Qui cort sus a son copaignon

Quant il l'a rescous du ferain : Cont. P. , I, 1046-9.

«Mais j'ai fait comme le gaignon,

Dist il, qui aboie en son close,

Et puis par defors issir n'ose,

Et ne fait que le manechier. Meliacin, 7466-9. Cf. ib., 7491.

La merite li a rendue

Que fait li mastins que on nage

A chelui quil porte al rivage :

Si tost com a tere l'a mis,

Ne veut plus estre ses amis,

Ains l'abaie et mordre le vait.

« Tout ensement, » che dist, « ai fait.

Assés sui pire que gaignons. » Diable, 3558-65.

Li rois, pour ce que tant l'a chier,

adoubé l'a a chevalier ;

envie en ont si compaingnon,

a fol le tiennent et gaingnon. Thèbes, 145-8.

Paien glatissent et ullent com gaignon ; Aliscans, 5808. Cf. ib., 5842. 
Les gaignons sont apparemment toujours prêts à mordre :ils montrent leurs dents qu'ils ont très aiguës. Leur voracité est décrite de façon très réaliste dans Bueve : Fromont et Haton, après avoir été torturés, sont livrés à la merci de quatre gaignons - appelés d'ailleurs dans le même passage limiers, à cause de l'assonance (?), qui les déchirent complètement avec leurs dents. Rou présente une scène semblable : les Danois ravageant le pays se servent d'ours et de chiens (A.J. Holden pense que brohuns désignent des chiens ; cf. p. 152, brahon/braon) pour tuer les femmes captivées :

Quant cil voient lor compaignon

Dens esteigniés comme gaignon,

Chascuns d'iaus vers Gerart acourt. Violette, 3735-7.

Puis a fait Bueve quatre gaignons mander,

Si lor a fait cordes en pié nouer

Puis i a fait les gloutons ateler.

Li frans dus Bueve forment äiriés,

$\mathrm{Il}$ a fait prendre quatre grans loiemiers,

Les deus gloutons les atacha as piés,

La saus as fourkes les fait Bueve hauchier ;

Quant li gaignon se sentirent quaissiet,

Cuident que fachent li cors as chevaliers ;

Ki lor vëist defouler et mengier,

Bras et espaules et grans braons sachier

Et cuers et foies et atraire et maschier,

Tant qu'entr'iaus deus ne se porent aidier! Bueve, III, 6786-

98.

Les dames e les damaiseles

enfuicient jusque as mameles ;

puis amenoent les guainuns,

ors enchenez e brohuns,

ki lur traeient les cerveles

e derumpeient les mameles. Rou, III, 1223-8.

Le rêve de la reine Esclarmonde, dans Godin, semble indiquer que l'on craint aussi la voracité des lévriers :

Si faitement la roïne sonjoit.

Avis li fu que lés sen fil veoit

.r. grant levrier qui le geule beoit ;

bien li sanbloit que mengier le voloit,

mais li levriers doucement le lechoit

et a l'enfant Godin s'umelioit. Godin, 8537-42. 
Quand l'auteur d'Eneas veut décrire la laideur de Cerbère, il le compare à un gaignon. Dans Loquifer, les dents du monstre Chapalu, qui a la tête d'un chat et le corps d'un cheval, sont comparées à celles d'un gaignon :

janbes et piez a toz veluz

et les artolz a toz crochuz ;

tels ongles a com de grifon

et coëz est come gaignon ; Eneas, 2565-8. Cf. ib., 2577-9.

les dans agus aseis plus d'un gaignon. Loquifer, 3795.

Le mépris pour les gaignons apparaît dans la description de la situation humiliante de Fromont emprisonné :

En un fossé, la vi gesir Fromont,

Entor le col ot loiié deus gaignons,

Qui li compissent le vis et le menton, Bueve, II, 5405-7.

Le même texte présente des occurrences de gaignart qu'Albert Stimming traduit par " hündisch » et, au sens figurée, "räuberisch ; grausam, gewalttätig », c'est-à-dire "infâme, rude, brutal »:

Si m'äit dieus, moult est fel et gaignars. » Bueve, II, 4101.

En nule terre plus wagnart ne savon, ib., II, 10910.

j. lévrier.

Les lévriers sont des chiens de chasse, particulièrement de la chasse aux lièvres, mais dans Renart et Deduis nous les voyons aussi chasser et renard et loup. Il n'est donc pas surprenant de les rencontrer dans de nombreuses descriptions de scènes de chasse ; on souligne parfois leur vitesse :

fuiant s'an torne, si baaille,

qu'il n'i ose plus demorer

por les levriers qu'il vit mener. Renart, Iv, 11506-8. Cf. ib., 9961-2; 9999-10001.

Que qu'Isangrins se va frotent,

este vos .I. garçon trotent,

.II. levriés tint en une laisse,

voit Isangrin, vers lui s'eslaisse $i b ., v, 13385-8$. Cf. $i b ., 15416$ et passim. 
Maiz les leux qui aux levriers vont

Ancor plus grant plaisance font,

Car on regarde vollentiers

Bien tost aler vistes levriers. Deduis, 8537-40.

Maiz le lievre besse l'oreille

Quant voit les deux levriers venir

Pour le prendre et pour le tenir, ib., 8740-2.

Avec li vavasor estoit uns escuiers qui avoit un daim trossé et deus lievres et menoit deus levrers en laisse. Lancelot, I, xxxIII, 6 .

« Done moi de la ceue de ton destrier :

« S'en ferai une laise a .r. levrier ; Aiol, 2893-4. Cf. ib., 6681.

Tel se fye en aultruy, s'il en avoit mestier,

Qu'aussy tost l'averoit quë ou bois ung levrier. Tristan de N., 5025. Cf. $i b ., 1145^{-6} ; 9620$.

La firent lez paien teillement esmaier

Qu'i s'anfuyent fuant assitost que livrier ; Lion, 17904-5. Cf. ib., 514-5.

A la dame va environ

Comme levriers qui lievre cache, $M R$, cxxIII, 112-3. Cf. passim.

(...) ; si n'ourent pas gramment chevauchié qu'ilz encontrerent un chevalier qui chevauchoit tout desarmé fors que d'espee et menoit levriers et brachés jusques a .vi. TP, vII, App. II, $1,119-21$.

Deduis décrit un lévrier idéal - et extraordinaire :

Ne resambloient pas mastin,

Car onc nature ne fourma

Nul plus beau levrier qu'il y a,

Par especial un levrier,

Qui estoit d'un bon escuier,

Duquel je vous diray la taille.

Museau de lus avoit sanz faille,

Harpe de lion, col de cingne.

Ancore y avoit autre signe,

Car il avoit oil d'esprevier,

Et tout blanc estoit le levrier.

Oreille de serpent avoit,

Qui sur la teste li gisoit, 
Espaule de chevrel sauvaigne,

Costé de bisse de boscaige,

Loigne de cerf, queue de rat,

Cuisse de lievre et pié de chat. Deduis,8826-42.

Voir aussi : Dole, 954-6 ; Espees, 8870-1 ; Enfances G., 280-1 ; Lancelot, II, LI, 8 ; Aliscans, 6365 ; TP , v, 8, 6-7 ; 41, 9-11;Cent, 2, 30 ; 9 , 103 ; Floriant, 288-90 ; Eneas, 1457-60 ; Florimont, 1768-74 ; Thèbes, 7439-40 ; 7445-8 ; Poitiers, 1357 ; Mort Aymeri, 359-60 ; Laurin, 8881-4 et passim.

La forme féminine levriere (= levrette) se trouve régulièrement, surtout pour montrer la rapidité d'un cheval (2) (voir aussi 5.1.5.) :

Et la vielle i keurt conme levriere, Berte, 359.

Et li chevaus s'en fuit comme levriere Aliscans, 6365 .

Point Folatile, qui plus cort de levriere, $i b ., 1827$.

Un arabi isnel comme levriere ; Bueve, I, 9399.

Le grant destrier qui pas ne cloche

Ainz va ainsi comme levriere. Chauvency, 1910-1.

Car plus les redoutent ne fait lievres levriere Buevon, 180o. Cf. ib., 2382-3.

Quar Esmeraude, sa levriere,

Le sesi aus naces derriere, $M R, \mathrm{cvI}, 891-2$.

" En une large borse seront li denier mis,

«Au col d'une levriere et lié et assis : Renaut, 7992-3.

(...) ; la royne a une levriere, comme vous savez, dont elle est beaucoup assotée, et la fait coucher en sa chambre ; (...) Cent, 28, 58-6o. Cf. ib., 28, 67 et passim.

Levrerette se trouve une fois, dans Deduis :

Maiz une levrerete tainqre

Et un[e] autre tant estriverent

Que au lievre le chemin osterent. Deduis, 8956-8.

Dans Durmart, le chien " savant " est appelé levriere cinq fois, levrier une fois : 
Vos enmenrés ceste levriere,

Si gardés qu'en nule maniere

Ne tornés voie cha ne la

Se celi non ou ele ira.

S'ele vient a chemin forchié,

Tost avera le chief baissié.

S'ele arreste, si arrestés ;

La ou ele va, si alés. Durmart, 1739-46. Cf. ib., 1595 ; 1654-5 ;

$1813 ; 1988$.

Mesire Durmars prent la lasse ;

Atot le levrier s'en torna, $i b ., 1764-5$.

Un extrait de Tristan de $N$. semble indiquer que les lévriers sont aussi peureux que les gaignons - à moins que ce ne soit la rapidité que l'on veuille souligner :

Mais au commancement de son regne premier

$\mathrm{N}^{\prime}$ avoit en tout le monde nul sy couart berger

Que d'une seulle espee ou baston ou levier

Eüst on fait Tristan fouÿr come levrier. Tristan de N., 6604-7.

Un autre extrait, de Roche, nous apprend que les lévriers (et les mâtins) sont utilisés pour garder le bétail et qu'on les loge dans des niches :

Davant la maistre porte sus .j. perron s'asiet,

Desoz l'ombre d'un chasne grant et gros et plainnier,

Et voit de totes parz son avoir repairier,

Entrer enz en ces parz et berbis et bergiers,

Et gesir en ces loiges et ma[s] tins et levriers,

Et ces bues et ces vaiches dont il i a miliers. Roche, 3200-5.

k. limier.

Ce terme désigne proprement un chien tenu en laisse. Nous le voyons régulièrement employé dans des scènes de chasse, où il désigne le chien dressé à trouver la trace du gibier et à diriger ensuite la meute de chiens dans la bonne direction, comme on le voit dans l'extrait de Deduis. (Voir Friedrich Borchert, op. cit., p. 24 et Friedrich Bangert, op. cit., p. 173).

Le deuxième extrait d'Ogier mentionne un trait caractéristique du limier : les oreilles pendantes, ce qui nous fait penser que le limier est ordinairement un chien braque :

Si traient les chiens au limier, Deduis, 8067. Cf. ib., 7936-7 ;

7951 et passim. 
Et si vous dy bien, sire, se dist l'archevesque, que vous n'avez quatre grans lymiers les plus affamez que vous ayez en vostre court qui si fort mengassent a deux repas que il feroit bien a ung, (...) Ogier, 116.

Et a celle heure devint Ogier palle mort et deffait et lui pendoient les pieces du visage longues comme les orailles d'ung limier de chasse, (...) ib., 311.

Renaut le fiz Aymon repaire de chacier,

Et avoit .iiii. cers trossez sor .I. somier :

A chacier les ot pris a .IIII. liemiers. Renaut, 6395-7.

as uns a proié qu'il alassent

boissoner ovoec les archiers ;

et li autrë as liëmiers

porsievre, qui sont bon as cers ; Dole, 178-81.

Levriés afaitent et brakés

Et loiemiers et berserés,

Si afaitoient les faucons

Por prendre grues et hairons, Rigomer, 1431-4.

Les iex roïlle ausi com liemier ; Otinel, 542.

Uns veneres siut le sengler ;

Li liemiers l'en fait aler. Partonopeu, 585-6. Cf. ib., 1797-8.

Adonc s'esmuevent quatre cens loiemier

Et chien et vetre et brochait et livrier.

Li veneour sor les corans destriez Enfances G., 1889-91.

Puis a ses veneors mandez,

Ses brachés et ses loienmiers

Et ses vaitres et ses levriers. Floriant, 288-90.

Voir aussi : Cent, 2, 31 ; Diable, 1095 ; 1087-91.

1. lisse.

La lisse (lice) est une chienne.

Chascun puet v[e]oir clerement

Qu'il [n'est] nulle beste si ville

Comme lisse, car par la ville

S'en va fuyant pour amasser

Tous les chiens qu'elle puet trouver. Deduis, 6572-6. 
Il y a de chiens et de lices,

Ce m'est avis, de pluseurs guises. ib., 9073-4.

Pour quoy la laissa courre comme une lisse entre deux douzaines de chiens, et accomplir tous ses vouloirs et desordonnez desirs. Cent, 91, 55-8.

m. mâtin.

Le mâtin est un gros chien que nous voyons garder la maison et les bestiaux mais aussi participer à la chasse aux loups, aux renards et aux ours.

Chez Jean Verdon, Les loisirs au Moyen Age, nous lisons, à la page 63 : «Les mâtins ont pour rôle de garder les bestiaux et la maison de leur maître, mais certains chassent toutes les bêtes. Ils ne sont pas très appréciés. »

un matin vit delez le feu,

delez la couche a fait son leu, par un pou que au feu ne touche, mais li essonbres de la couche ne laissa veoir Isangrin. Renart, I, 2553-7.

li mastin crïent et abaient. ib., III, 6548. Cf. ib., 6511-8 ; 6735-6 et passim.

Qant Isangrin le vit lever, vit qu'il vost le feu alumer, un petitest se trait arriere, par la nage le prant derriere.

Li vilains a geté un cri ; li matins l'ot tantost oï : Isangrin a pris par la coille, enpaint et sache et tire et roile, tout erraige ce que il pant. Mais Isangrin mout pres se prant derrier as naches au vilain, mes de ce avoit le cuer vain et sa dolor li angraignoit que li chiens as danz le tenoit. $i b .$, I, 2613-26.

Trois mastins les meillors de France

(li pires des trois ne les dote),

qui laienz sont en cele croute, amenez conme vezïez a vostre granche toz liez, et gardez que bons liens aient. Donez lor dou pain qu'il n'abaient, 
que bien porroient esmaier

dant Renart par lor abaier,

qu'il s'enfuiroit a son reçoit :

(...)

les matins faites bien tenir

a vostre garçonet toz trois

a l'uis de la granche detrois.

Qant Renart iert bien aprochiez,

les chiens maintenant li huiez,

si les laissiez aler aprés ;

(...)

Robinet, va tost deslïer

les .III. matins et si les hue. »

Li gars sa chape a terre rue,

as matins corut en la granche,

chascun lien pres del col tranche,

les mastins huie et aprés cort.

Li matin saillent en la cort, $i b .$, IV, 10326-35, 10340-5 et 10556-

62. Cf. passim.

Fres et novel venront ja li mastin Bueve, III, 1791.

Vos chevaus a fait sa jornee ;

Li mastin ont sa mort juree,

Faire en volront lor quaresmel ; Cont. P., I, 4417-9.

Et si y a de mastineaux,

Qui tous ont mengié les museaux, Deduis, 9081-2.

Nous avons l'impression que l'extrait suivant ne présente pas nécessairement une image réaliste du comportement des matins, mais que l'expression « à guise de mastin » a été choisie à cause de la rime :

L'une chambe tient droite et l'autrë en declin,

Les oilz ovre et reclot a guise de mastin, Simon, 1980-1.

De même que gaignon, le terme mâtin peut avoir une connotation négative, également surtout pour la couardise et la méchanceté de l'animal. Ceci apparaît très clairement dans un passage de Deduis dans lequel Gace de la Buigne compare les mâtins aux lévriers. Voir aussi 5.1.2. :

Pour ce couvient il distinguer

Entre chien mastin et levrier

Et les autres chiens de deduit,

Car les mastins vilain son tuit, 
Ne maiz aucuns qui vont suivant

Les nobles chiens quant vont chassant,

Qui pour la noble compaignie

S'ennoblissent, je n'en doubt mie.

Se li mastin sont traïteur

Et mauvaiz, ce leur est honneur, Deduis, 6687-96.

Se il welt tensier en charriere,

Ussaige fait de chamberiere

Que d'anemin di(s)t son voloir

Quant del vengier nen ait pooir :

Vengier ne s'ose et di(s)t en lait,

Si com mavais mastins le fait :

C'il sent le louf fors en sa plasce,

Davant s'escont, puels le manasse :

Ne vient a leu, qu'il n'est si os,

Puels l'abaie quant est enclos. Florimont, 2869-78.

Li quenz Fromons, qui a cuer de mastin, Mez, 237.

L'envoioit le scien pere par Grignart son cousin,

Car n'ot sy lait paien dela l'eaue du Rin :

Le corps avoit plus gros que destrier ne roncin,

Les yeulx avoit enfflés et rouges con mastin, Tristan de $N$.,

4413-6.

n. pradant.

Sen vel(e)tres tot primers e sen pradenc, Roussillon, 1752.

o. seu.

ne li est mie aviz qu'il soit alé cachier

ne que il tieinge a traistre ne seüs ne levrier. Rou, II, 3910-1.

As veneürs e a vatlez

fist mener seüz e brachez

e lïemiers, par autre veie

les fist aler, que l'en ne veie ; $i b .$, III, 523-6.

p. veautre.

A.-J. Greimas, op. cit., p. 657, et R. Grandsaignes d'Hauterive, op. cit., p. 581, expliquent: « (...), chien qui chasse l'ours et le sanglier ». Ceci est confirmé par Friedrich Borchert, qui écrit , op. cit., p. 67, que le veautre est un grand chien fort qui sert notamment à la chasse au sanglier. Dans les scènes de chasse, les veautres 
se trouvent souvent avec d'autres chiens. Voir aussi braquet : Inconnu, 1283-6 ; Floriant, 288-9o et limier : Enfances G., 1889-9o.

Braie livriers et si vaitres glatise,

Ci espriviers desor ses peirches crie : Enfances G., 280-1.

prennent lor ars, corz et levriers,

chiens et viautres et liemiers. Eneas, 1459-60.

On fist as noces beter ors

Et vers et a chiens et a viautres. Escoufle, 1710-1.

Dans quelques textes, où il est question de comparaisons et de rêves, ils sont mentionnés à côté de lions ce qui indique qu'on leur attribue une certaine sauvagerie et férocité :

Plus iriez que leons et veautres

Va criant davant toz les autres : Joufroi, 4499-500.

De sun paleis uns veltres i acurt : Roland, 2563.

Bueves gist en Barbastre, en un lit tregité,

Si a songié un songe dont il est esfreé

Que il desoz Barbastre avoit pris un sengler,

Sa venoison desfet, sor lui s'est aresté,

Qant trente viautre saillent, corant et abrivé

Sa venoison li tolent, n'ot en lui c'aïrer. Barbastre, 2742-7.

Un lion et deus viautres, blanc conme noif negie,

En l'eve se feroient tuit en une saillie, $i b ., 5670-1$.

Noms propres.

Ce n'est que dans Renart, dans quelques fabliaux, dans différents textes qui relatent la vie de Tristan, dans Partonopeu-C, où Anselot sauve un beau lévrier blanc sur le point de se noyer lors d'un naufrage, dans Ipomedon, où le protagoniste se vante des performances (inventées) de ses chiens, et dans Guillaume d'A., que nous trouvons des noms propres de chiens

Dans Renart, le nom de Roenel désigne les chiens en général (comme Renart désigne les renards en général) : le nom est donné à un représentant de l'espèce plutôt qu'à un chien particulier. Il s'accole d'ailleurs à plusieurs termes : gaignon, mastin, veautre.

et seignor Ferrant le roncin

et dant Roonel le matin Renart, I, 1617-8. 
c'iert diemanche par matin

devant Roënel le mastin. ib., III, 6735-6. Cf. ib., 6785 et 7625 ;

VI, 17221-2.

devant Roënel le gaingnon,

le chien Froibert de la Fontaine, $i b .$, III, 6748-9. Cf. ib., vI,

17437-42 et passim.

Baucent et Roienaus li viautres, ib., vI, 16241.

Apele le chien de meson !

Estula avoit non li chiens ; $M R$, xCvI, 42-3. Cf. $i b .$, 61-2.

Or ot chascuns d'aus grant paor.

Si s'esploitent de tost fuir,

Et chien commencent à venir ;

Balouart, le chien au provost,

Le sesi aus jambes tantost ;

Si en porta plain sa goule.

Le prestre rest en male foule,

Quar Esmeraude, sa levriere,

Le sesi aus naces derriere, $i b .$, cvI, 884-92.

(...), que nus ne le reconnoissoit ne ja n'i fust reconeüs a mon escient, se ne fust Hudenc son braquet, ki le reconnut tout maintenant que il le vit. La u li home ne le pooient reconnoistre, le reconnut li braqués. $T P, \mathrm{I}, 1,25-8$.

Ahi ! Husdent, ja tex brechetz

N'ert mais trové, qui tant set prez

Ne tel duel face por seignor ; Tristan, 1457-9. Cf. ib., 1444 et passim.

Tristans et maistre Govrenaus

Et Yseus et ses chiens Hudains, Escoufle, 590-1.

Noon l'apelai por la mer

Ou gel vi a dolour noër. Partonopeu-C, 483-4.

Noons, en qui j'oi mai fiance,

En avoit au cuer esmaiance ; $i b ., 779-80$. Cf. $i b ., 655,664$ et passim.

Mut ad ui ben curu Nublet,

E Ridel e tuit mi brachet,

Si ke treis granz cerfs ai hui pris ;

Mes de trestuz mes chens le pris, 
De ceus ke geui unt curu,

Enporte Baucan le velu, Ipomedon, 4425-30. Cf. ib., 4465-6 ;

5473-4.

E je vus redirrai tut veir,

Un men brachet neir Baailemunt

Mes chens venqui quanque il sunt. ib., 6512-4.

Li oïrent escrier tuit :

« $\mathrm{Hu}$ ! Hu ! Bliaut, cis cers s'en fuit. » Guillaume d'A., 2573-4.

\subsubsection{Chats}

Le chat Tibert joue un rôle important dans Renart ; nous y apprenons qu'il mange les souris et les rats et qu'après avoir mangé il aime se mettre à l'aise. Voici quelques-unes des nombreuses occurrences du terme :

Et se sire Tibert li chaz

menja les soriz et les raz, Renart, I, 1275-6. Cf. ib., I, 1743-6 ;

III, 7446-50.

il esgarda, si a choisi

Tibert le chat, qui se gisoit

sor une roche et rostisoiz

sa pance encontre le soloil. ib., IV, 11532-5. Cf. ib., IV, 11549-53.

Lors s'est Tibert en piez dreciez,

si gite sor son col sa qeue

et sa lange aguise et desneue

por bien parler, et si herice

trestoz les peus de sa pelice. $i b .$, vI, 17108-12.

Ailleurs dans Renart, et dans les autres textes, c'est encore la chasse aux souris et aux rats qui revient régulièrement; mais, dans la plupart des cas, le chat apparaît dans des dictons, des proverbes et des comparaisons (voir chapitre 5) :

Et vos, sire Pelez li raz,

je vos fis ja tenir as chaz,

qant vos eüstes mangié l'orge :

il vos estraintrent cele gorge. Renart, I, 1743-6.

Li vilains reproche du chat

Qu'il set bien qui barbes il lèche ; $M R, \mathrm{xv}, 196-7$. Cf. Prover-

bes, $264 ; 1063$. 
«Diva, sont cuites les pertris?

- Sire, dist-ele, ainçois va pis,

Quar mengies les a li chas. » $i b .$, xvII, 57-9.

Et se li covient huches,

Et corbeillons et cruches,

Le chat aus souris prendre

Por les huches desfendre ib., XLIII, 127-30.

De la cuisine s'en ist plus que le pas,

Por la savor se deleche com chaz, Aliscans, 4836-7.

Dist Renoart : « Mout ai or le cuer mat

Por seulement la morsure d'un chat. » ib., 6299-300.

\subsubsection{Muls/mulets/mules/mulettes}

Les mulets et les mules sont souvent mentionnés avec les chevaux. Ceci n'est que naturel car, comme ceux-ci, ils servent au transport des hommes et des marchandises. Nous pouvons constater que l'on se sert du verbe chevaucher même quand il s'agit de mules et de mulets, p. ex. :

En son dos ot vestu un chier bliaut de Frisse

Et chevache la mule, que n'iert blanche ne bisse, Florence, 5059-60.

Il est à remarquer que contrairement à la langue moderne qui ne connaît que deux termes : mulet (le mâle) et mule (la femelle), l'ancien français en connaît trois : mule (forme féminine), mul et mulet (formes masculines). Notons au passage que dans le tome II de son édition de Bueve de Hantone, Albert Stimming fait la distinction entre mul qu'il traduit par " Maulesel ", c'est-à-dire " petit mulet produit de l'accouplement du cheval et de l'ânesse ", et mulet qu'il traduit par "Maultier ", c'est-à-dire " produit de l'accouplement de l'âne mâle et de la jument ». Ni le Dictionnaire Général (définition de mulet: " Produit mâle de l'accouplement d'un baudet avec une jument, ou d'un cheval avec une ânesse »), ni Littré (définition de mulet : "Quadrupède engendré d'un âne et d'une jument, ou d'un cheval et d'une ânesse, et qui n'engendre point, (...) »), ni nos dictionnaires étymologiques ne connaissent une telle distinction; ils donnent mulet comme diminutif de la forme $m u l<$ latin mulum. - Dans le tome iı de Bueve, Stimming traduit d'ailleurs les deux termes par « Maulesel».

Nous trouvons dans nos textes un grand nombre d'exemples de tous ces termes, mais nous n'en présentons qu'un petit choix. 
Voici d'abord des extraits où il est question d'animaux de selle ; nous remarquons que bien souvent il s'agit de la monture d'une dame et que plusieurs textes soulignent la vitesse des animaux en question :

Et la roïnne sor un murl sejorné Ami, 1450. Cf. ib., 1993 ; 24456 et passim.

Quant atorné ara son oire

La roïne d'a cort aler

Et fera ses muls enseler.

Ses palefrois et ses cevals, Inconnu, 3780-3.

Paien issent des nes, la pute gent haïe.

Sor un mul est montez la bele Malatrie, Barbastre, 1677-8. Cf. ib., $1727-8 ; 2280$ et passim.

Lors esgarda auant son uis,

Et uit .I. moine cheuauchant

Sour .I. mul, (...) Espees, 330-2. Cf. ib., 390-5; 585 et passim.

Maudaranz et Maudoires et li rois Mauqueruz

Le fiz le roi de France leverent sur .I. mul ; Floovant, 807-8. Cf.

ib. 884-5.

Li rois estoit montez sor .I. mulet anblant,

Si auloit oïr messe au moustier sain Vinçant. ib., 873-4. Cf. $i b$., 1403-4.

Et Foques si s'estoit vestu et atorné,

Il monte en .I. mulet qui li fu apresté,

Et porte .I. rain d'olive par grant humilité ; Renaut, 2083-5.

Bordon ot et escarpe, paume et espi,

Et boin mulet anblant a son plaisir, Aiol, 1536-7. Cf. ib., 4091-

$2 ; 4656$ et passim.

Et au quart, endroit eure de tierche, si truevent en lor voie .I. homme qui chevauchoit .I. mulet, une cape bleue afublee. Lancelot, vII, xxva, 5.

Si tost com il s'est partis du roi, si trueve une damoisele moult tost chevauchant sor une mule corsiere. ib., vII, xxxva, 3. Cf. $i b .$, I, XXXII, 11 ; II, XXXVI, 39 et passim. 
Lors vit par les pres chevalchant

Une dame sor une mule, Cont. P., I, 1622-3. Cf. ib., 1887-9 ;

1909-11.

Que que cil merci li demande,

a tant ez vos, par mi la lande,

une pucele l'anbleüre

venir sor une fauve mure,

desafublee et desliee ;

et si tenoit une corgiee

don la mule feroit grant cos,

et nus chevax les granz galos,

por verité, si tost n'alast

que la mule plus tost n'anblast. Charrete, 2779-88.

Tant a la mule esperonee

par mi le fonz d'une valee

que venuz est au mes Renart. Renart, I, 759-61. Cf. ib., 1211-2 ;

VI, 18406-8.

Et maintenant la dame envoie

Son ami à grant aleüre,

Puis saut et deslie la mure : $M R, \mathrm{xcIV}, 130-2$.

Adont vont la pucelle sur la mulle montant. Tristan de N., 535 .

Cf. $i b ., 541 ; 764$.

(...), atant es vous de cele part venir une pucele mout bien apareillie, et encore avoit ele plus en li, car ele estoit de si tres merveilleuse biauté que nule plus. Et cevauchoit une mule trop bien amblant, et avoeuc çou avoit la mule une trop riche sambue, si que çou estoit merveille de li regarder. TP, vI, 114, $2-7$.

Le palefroi li vellarz monte,

Et la dame cortoise et franche

Sor une mule tote blanche; Athis, 9960-2.

Veez cy sa mulette qui n'attent aultre choses que je soie en voye, pour porter son maistre ou l'on ne veult pas que je soye. Cent, 31, 53-5. Cf. ib., 17, 142-3 et passim.

Que les mule(t)s soient la monture des dames pas excellence se voit de façon pertinente dans Mort Aymeri ; pour entrer dans Narbonne, il se sert d'une ruse : lui et ses guerriers s'habillent en femmes, troquant leurs destriers contre des mulets pour berner les Sarrasins maîtres de la ville : 
«Et lesserons les destriers sejornez

«Et monterons es mulez afeutrez ;

«Comme puceles chanjerons nostre aler ; Mort Aymeri,

2391-3.

Comme puceles muerent lor senblant :

Si ont lessié les bons destriers coranz

Et sont monté sor les mulez anblanz. ib., 2602-4.

Voir aussi : Partonopeu, 5900 ; Atre, 6420-4 ; Protheselaus, 1486-7 ; 6097-9; Yder, 70-1; 3740 ; 3787-8 et passim ; Méliador, 13140-1; Ipomedon, 7942-3 ; Papegau, 1, 9-11 ; 3, 16-7 et passim ; Blancandin, 647-9 ; Lais, v, 510-2 ; Brun, 1178 ; Erec, 5168-9 ; 5172 ; Godin, 18547 ; Guillaume, 3535 ; Simon, 1029-30 ; Narbonnais, 675-6 ; 1003 ; 2548 ; Mort Aymeri, 997 ; 1047 ; 2638 ; Poitiers, 449-51 ; 6646 ; Aymeri, 130 ; 1104 ; 2295 ; 4422 ; Laurin, 8706-8 ; Lanson, 4490 ; Gaufrey, $6319 ; 6912 ; 7459$ et passim.

Les mulets sont rapides, par conséquent ils servent de monture aux messagers :

Lors en monterent li mesagier tuit dis

Suz les mulez, n'i ot plus terme quis ; Aymeri, 2689-90.

Il est montez sor un mul aragon,

Par mi les rues s'en vait a esperon, Couronnement, 1799-80o.

A propos de l'extrait suivant de Perceval, nous remarquons une connotation négative de l'adjectif fauve : la demoiselle qui monte la mule est extrêmement laide. Cf. sa description pp. 345-346 et aussi les remarques concernant fauve p. 182-183 :

Et l'endemain autel i firent,

Dusques al tier jor que il virent

Une damoisele qui vint

Sor une faulve mule, et tint

En sa main destre une corgie. Perceval, 4609-13.

En voici d'autres où les mulets et les mules servent de bêtes de somme. Dans les derniers extraits, les mulets portent une litière :

Et rechargirent les sommiers et les murles, Ami, 1977.

Laiés le vivre, se grant treü vos rent,

Murs et cevals desc'a mil et set cent

Qui trestot soient cargié d'or et d'argent Aspremont, 7713-5. 
«Que la vitaille aportent as murs et a somiers. Renaut, 2456. Cf. $i b ., 2288 ; 2304$.

Del tresor fist chargier cent mulez enbleor ; Barbastre, 1634.

" Gardés demain à l'aube soiés apareilliez,

« Et si faites trosser les murs et les somierz : Gui de B., 28-9.

Clarembauz n'estoit mie d'avoir trop agrevez ;

.III. mulez lor a fait d'or et d'argent troser,

Et ont fait la duchesce gentement conreer ;

Sor .I. mulet anblant font la dame monter. Parise, 747-50.

Sonmiers et mules font no baron torser,

Dessi a trente en ont fait aprester. Huon, 8717-8.

Li rois Enpires me donna avoir grant,

Chargiez .iii. muls entre or fin et arjant. Mez, 3475-6. Cf. ib., $4142 ; 12759$.

Quant tot orent aparoillié, quatre buens murs ont atachiez por la biere soëf porter. Eneas, 7487-9.

Hermanfroiz qui fu mort n'i volent oblier :

Desor une lettiere le font bien conreer,

Sor .ii. mulez l'ont fet tot maintenant monter. Renaut, 3366-8.

Voir aussi : Orson, 340-1 ; Chevalerie d'O., 976 ; 9055 ; Turpin, 11956 ; Mort Aymeri, 1553 ; Narbonnais, 753-4 ; 1355-6 ; 1661-3 ; 6035.

Les mulets sont régulièrement donnés à une personne que l'on désire récompenser ou honorer. Nous avons l'impression qu'on n'en fait pas cadeau à des chevaliers (qui ont plutôt besoin de destriers), mais surtout p. ex. à des ménestrels ; il y a pourtant des exceptions :

Chascun donnéz cent livres de deniers

Et un murlet chascun por lor cors aaisier. Ami, 246-7.

Et, se il violt ne cendal ne orfroi

Ne mul ne mule, destrier ne palefroi,

Done l'ent, ber, si con Carles fist toi. » Aspremont, 2567-9.

«.x. mulez vos dorrai qui sont tuit anbleor,

"Chargiez d'or et d'argent, que nus ne vit meillor. Renaut, 4195-6. Cf. ib., 11029. 
« De par le roi de France .I. present vos faison

« De . ${ }^{\mathrm{m}}$. mulès trossés de garison, Gui de B., 956-7. Cf. ib.,

$654-5 ; 3108-9$.

Il a en France envoié grant treü,

Or et arjent et palefrois et muls Mez, 3023-4. Cf. ib., 6425-7 ;

6601-3 et passim.

Si li donne sa mule lues

Ou li hernaps est frois et nefs,

Que son frere li achata. Galeran, 4087-9.

Tu tenoies la grant mesinee,

tu la fesoies baude et liee,

car tu donnoies les courroiz,

donnoies mulz et palefroiz,

armes, robes et couvertors,

bliauz et deduiz et otors ; Thèbes, 6013-8.

car vostre volenté ferai,

çou que mestiers ert vos donrai,

ciers pailes et or et argent,

biax dras et mules en present. Floire, 1135-8.

Li suens ostages fu bien guerredonez,

Quar .cc. mars li a la nuit donez,

Et .II. destriers et .II. muls sejornez,

Et bons blïauz et mantiax angolez ; Aliscans, 3831-4.

Dans plusieurs exemples, nous voyons indiquée l'origine - nous trouvons quelques-unes des indications que nous avons déjà rencontrées pour les chevaux :

«Et Olive seoit sus .j. mul sulien Roche, 3086. Cf. ib., 2972 ;

3002 .

L'amirant fu monté sor un mul de Surie, Barbastre, 6146. Cf.

Tristan de N., 13947-8 ; Lion, 30973.

A tant es un mesage sor un mul aufarin ; ib., 5466. Cf. Mort

Aymeri, 1553.

Un mul d'Afrique li firent amener. Aspremont, 7730.

Quatre mulet des plus fors d'Arragon

Pas ne portassent icele oblation. ib., 7605-6. 
Cele place font traire mainte mule esclavonne, Florence, 3281.

Li rois i va et li autres baron

Et la roïnne sor un murl arragon Ami, 1652-3.

Cf. Tristan de N., 6221 ; Renaut, 6176-7 ; 8950 ; Bueve, I, 8736 ;

Doon, 8051.

Je voz donrai mon murlet arrabi

Et trente livres de deniers parisis. ib., 2123-4. Cf. Orson, 3404.

Paien i fissent une offrande si large,

Thiebaus offrit un boin mulet d'Arabe. Enfances G., 1515-6.

Pluis i ot or et arjant et vaisele

Ke trante mules des millors de Biterne

Ne porteroient demei lue de terre. $i b ., 1786-8$.

A ces paroles ez un mesaige ou vint

Grant aleüre sour un mur sarasin. $i b ., 53-4$.

Cause sei e vesti com far solie,

E montet en un mur de Bogerie ; Roussillon, 1202-3.

Il est moult tost montez, e de ses barons .xxx.,

Es palefrois anblans e es mulz d'Aquilande, Aye, 1448-9. ib., 2648.

Belisent munte sur un mul de Hungrie,

Que plus tost veit l'ambléure serrie

Ke par la mer ne veit nef ne galie. Otinel, 721-3.

Tote la terre raenplissent d'avoir,

D'or et d'arjent et de muls espanois. Mez, 3853-4. Cf. Thèbes, 4125 .

Assez i orent harpeor et jugler Et dras de soie et hermins engoulez Et muls d'Espaigne et destrier sejornez. Orange, 1883-5. Cf. Bueve, I, 9447.

On trouve aussi d'autres termes que nous avons déjà vu employés pour qualifier les chevaux :

Lors font metre les seles sor li mulet corsier, Renaut, 6785. Cf. Bueve, III, 3251 ; 14734 .

«Montauban vos dourai et les murs qui sunt bloi, $i b ., 11029$. 
E montez en un mul amblant ferrant, Roussillon, 148.

Li cent baron monterent es muls gaillars ; $i b ., 193$.

Et le monterent sor un mul aufarin ; Mort Aymeri, 1553.

.M. ors privez et .M. murs anbleors, $i b ., 1583$.

Li quens ne l'osa ramproner,

Ains fist la contesse monter

Desor un sor mulet amblant. Poitiers, 449-51.

Et lors s'encontrent une damoisele sor un mul amblant

(...) Lancelot, II, XxxvI, 39.

Et quant vint endroit none, si encontrerent une damoisele qui chevalchoit une blanche mule. ib., II, LVIII, 1.

Bien et tost et a droit apareilla son oirre

Et fu tres bien montez sor une mule noire, Berte, 1601-2.

Quant le vit la pucele, moult li fist lie chiere,

Contre lui est venue sor la mule coursiere ; Buevon, 2390-1.

Lors monta la pucele, qu'ele plus n'atendi,

Sor sa mule qui ot le poil blanc et flori ; ib., 2703-4.

Et chevache la mulle, que n'iert blanche ne bisse,

Ainz iert fauve et ferrande ; la destre espale ot grise. Florence, 5060-1.

Unne mule chevauchoit brune,

onc ne veïstes meillor une. Thèbes, 4105-6.

Il monte sus .j. mul corant et arabi, Roche, 322.

Dans l'Escoufle, le terme bauçant est employé par Guillaume qui s'adresse au mulet d'Aélis :

«Ahi ! fait il, baucent, baucent,

Com g'ere lores en bon point Escoufle, 6286-7.

Dans Cent se trouve le terme mulette, employé parallèlement à mule; dans Roche, le terme montenière désigne peut-être une mule de montagne : 
Quand ce bon escuier fut en bas descendu, il trouva une petite mulette au pié des degrez du chasteau, et ne vit ame qui la gardast. Cent, 31, 46-9. Cf. ib., 31, 53-4 et passim.

Et la bonne mule le mena par rues et ruelle, (...) $i b ., 31,61-2$.

La chargent la vitaille, le pain et la farine,

As montenieres montent, cil char braient et crient ; Roche, 2969-70.

L'extrait suivant, de Roche, est intéressant pour deux raisons : assis sur une mule (aux vers 3765 et 3771 appelée mulet), qui ne semble pas douée pour le combat, Landri lutte contre Hardré ; pour montrer combien peu elle vaut à ses yeux, il l'appelle âne. Dans des situations extrêmes, on arrive donc à confondre ânes et mulets/mules. Cette confusion n'est d'ailleurs pas étonnante étant donné que ceux-ci sont des « hybride[s] (...) de l'âne et de la jument ou du cheval et de l'ânesse. » (Petit Robert) :

Devant lui voit la mule, si [li] commence a dire :

« Ha[i] mule d'Espaigne, Damedieu[s] te maudie !

« Diex confunde la tere ou vous fust[es] norie ;

« Molt fait li hons que fox qui en asne se fie ! "Roche, 3774-7.

Un extrait de Gui de W. corrobore le fait que les mulets ne valent rien pour le combat: le protagoniste s'en sert pour se déplacer mais dès qu'il est question de se battre il en descend et monte sur son cheval (qu'il a donc ménagé jusque là) :

Ore vait Gui, suef errant,

Sur un petit mulet amblant ;

(...)

Del mulet mult tost descent,

Sun cheval munte, ses armes prent, Gui de W., 1229-30 et 1239-40.

\subsubsection{Anes}

Tandis que le mulet peut remplir les fonctions les plus nobles, l'âne est un animal plus humble qui travaille dur pour tous, mais peut-être surtout pour les classes sociales inférieures. Nous le rencontrons plus souvent dans Renart et dans les fabliaux que dans les chansons de geste et dans les romans, et il est régulièrement l'objet du mépris, des injures et des coups. Quand une personne est comparée à un âne c'est pour la déprécier. 
l'asne acoillent a la polie, qui de trere pas ne s'oublie :

li randu le vont menacent

et li anes va fort traient. Renart, II, 3635-8.

mais de ce n'as tu or corage

ne de aler en pelerinage,

ainz aimes mieuz tot oen mes

de la buche porter grant fais

et granz sachiees de charbon,

et si avras de l'aguillon

tot le crepon desus pelé,

et qant se vandra en esté

que de mouches sera grant nombre

lors ne durras neïs en l'onbre. ib., III, 9043-52. (tu = l'âne)

mes Tiemers, qui est dure beste

et qui trop mal endurer puet,

ne se remue ne remuet. $i b .$, IV, 10942-4. (T. = l'âne)

Wistasse aguillonne Romer Wistasse, 1040. (R. = un âne)

- Galestrot, vien ça, pute asnesse, $M R$, cvi, 729. (G. = la ser-

vante du vilain)

Il avint ja à Monpellier

C'un vilain estoit costumier

De fiens chargier et amasser

A .II. asnes terre fumer.

.I. jor ot ses asnes chargiez ;

Maintenant ne s'est atargiez :

El borc entra, ses asnes maine,

Devant lui chaçoit à grant paine,

Souvent li estuet dire : « Hez! » ib., cxIv, 1-9. Cf. ib., 22-5 ;

37-8.

En la maison ot une anesse

Qui se gisoit en mi la cort

Qui ne vost pas estre encorpée ; ib., cxxIv, 218-20.

Fame soferoit plus de cous

Que une asnesse de .II. anz

De mal et de poine .II. tanz. $i b .$, cxxiv, 244-6.

Il esgarda devant lui et vit venir .I. païsant qui menoit .I. asne devant lui. Laurin, 5929-30. 
(...) il cevaucha trusc'a eure de sonne. Lors ataint un home de grant aage, vestu de robe de religion qui cevauchoit un asne,

TP, vIII, 2-4.

Et li vallés tant chevalcha

Qu'il vit un carbonier venant,

Un asne devant lui menant. Perceval, 834-6.

Et il esgarde, si voit venir un ermite desus un grant aisne et entra le bois moult pres de lui, (...) Lancelot, vII, XxIxa, 1.

Lors lieve sus, un grant peron jeta,

Qui bien le some d'un grant asne pessa, Bueve, I, 3117-8.

Venus est au glouton, dist lui en haste :

« Trai toi en sus, lechieres, Dex mal te face!

« Ne faire nul desroi par ceste sale :

« Ja t'aroi[e] batu si com un asne. » Aiol, 4015-8. Cf. ib., 8860-3.

et si cevalca Nostre Sire

le plus vil bieste c'on puist dire,

por demoustrer humelité

qui doit estre en humanité :

ce fu de l'ane le femiele. Eracle, 6109-13.

L'ânon a les mêmes fonctions :

Et Perchevaus a entendu

A son cheval faire esforcier,

Mais qui le devoit escorchier

N'iroit se le petit pas non.

Mius li venist sor un anon

Estre montez, par saint Sevestre ; Cont. P., I, 4350-5.

Il portast bien le fais a un asnon. Aspremont, 1829 .

Un extrait de Tristan en prose montre clairement la différence entre le cheval et l'âne : le premier est noble, le second est vil (comme d'autres animaux). Cette idée, qui fait d'ailleurs dire à un proverbe qu'on ne doit pas lier les ânes avec les chevaux (Proverbes, 1494), correspond bien à celle qu'on avait des hommes : les chevaliers et leurs enfants sont nobles par nature, les roturiers resteront inférieurs :

La court le roi Artu est tout autresi com la fontainne douce et boine $\mathrm{u}$ cascuns vient pour estaindre son soif. Et autresi font 
les bestes et cheval et asne ; quant li chevaus vient a la fontainne et il s'i est reposés et aaisiés, il s'en part assés plus biaus qu'il n'i vint ; li asnes i vient vix et ors, et autresi viex et mauvais s'em depart: ce ne vient pas de la teche de la fontainne, anchois vient de la nature de la beste et du vil estat de li. TP, II, 203, 15-23.

Les ânes ont peur de passer un pont ; il faut les battre pour les faire avancer :

Demande, ou ge te batrai tant,

Que mielz ne fu asnes à pont. $M R, \mathrm{cxxxv}, 66-7$.

la le troverent li bergier,

sel batirent com asne a pont. Renart, I, 1066-7.

Dans Renart nous avons trouvé deux exemples où l'origine est indiquée. Est-ce à cause de la rime qu'il est espagnol dans les deux cas? :

et puis s'en vint la matinee,

si menoit un asne espanois

et compaignons jusques a .III. Renart, II, 3630-2.

Tant con li vilains se demente,

Tiemer, ses asnes espanois,

qui ne crient gelee ne nois,

oï dementer son saignor : $i b ., \mathrm{IV}, 10820-3$.

Le membre de l'âne a la réputation d'être très grand :

Quant li vallés ot la promesse,

Si trait le vit, dont une anesse

Péust bien estre vertoillie. $M R$, xxI, 133-5.

(...) «Veez cy le petit asnon de ceans, qui n'a gueres d'aage avec demy an, et si a l'instrument grand et gros de la longueur d'un braz. » Cent, 80, 61-4.

A propos de fauve ânesse, il faut attirer l'attention sur des extraits de Renart :

trop savez de la fauve anesse, Renart, I, 1323.

Renart, qui fait mainte promesse

et mout set de la fauve anesse, $i b$., III, 7433-4. 
et sur la note à la page $170 \mathrm{du}$ tome $\mathrm{I}:$ " symbole de la tromperie ». Dans le Roman de Fauvel par Gervais du Bus, l'éditeur Arthur Långfors écrit, p. Lxxxiv : «Au moyen âge, l'adjectif fauve, par suite d'un rapprochement facile avec faus (falsus), avait pris un sens défavorable. Dès le $\mathrm{xII}^{\mathrm{e}}$ siècle, la méchanceté de ce monde est personnifiée sous l'apparence d'une ânesse ou d'une jument fauve. »Cf. 1.1.3. Fauvel.

\subsubsection{Chameaux et dromadaires}

Les occurrences des chameaux et des dromadaires dans nos textes ne sont pas si rares que l'on aurait pu le penser étant donné qu'il s'agit d'animaux non originaires d'Europe. Il faut pourtant noter que, dans la majorité des occurrences, il est question d'animaux appartenant à des Sarrasins ; dans tous les cas, ils se rencontrent parmi les classes des riches, ce qui indique leur grande valeur.

Il ne nous a pas été possible de distinguer exactement les uns des autres et nous pensons que les auteurs eux-mêmes n'ont pas fait de distinction. Nous constatons que les deux espèces servent comme bêtes de somme et comme montures. Les textes soulignent parfois leur rapidité ; à propos de l'occurrence unique de Durmart, où le roi Nogant veut s'enfuir à toute vitesse, Joseph Gildea note, II, p. 142 : « (...) ce mot, qui suggère l'idée de la vitesse, n'est pas forcément réservé aux chameaux. " L'auteur de Narbonnais semble conscient que tout le monde ne peut pas connaître le dromadaire : il mentionne la difficulté de le diriger et sa grande rapidité.

Sor un dromadaire est montés

Qui mout tost li fu aprestés ;

Maintenant s'eslonge de l'ost,

Si entre en la forest mout tost ; Durmart, 14697-700.

Les dromadaires font mout tost aprester ;

C'est une beste, qui la savroit guiër,

Ainz que veïst les .iiii. mois paser,

Trestote terre porroit avironer. Narbonnais, 3616-9.

Vos li durrez urs e leons e chens,

Set cenz camelz e mil hosturs muers, Roland, 30-1. Cf. ib., 184.

Eins que sil en mi vie les dous mullers,

En a lo reis en France des messagiers ;

Et furunt vint, que ant chevaus doublers,

Palefreis e chames e muls corsers. Roussillon, 311-4. 
[E] dous mil chames carjanz amblanz ; ib., 299.

«Amaugis les porchace, qui mult par est preudon,

«Vitaille lor aporte assez et a foison,

« Chevaux et palefroiz et chamelx qui sunt bon. Renaut, $5685-7$.

A tant ez vos Salatré, un Persant,

Par la bataille sor un chamelin blanc : Mort Aymeri, 2678-9.

Polydamas, cil de Thesaire,

sist sor un corant dromadaire ;

covert l'ot d'une porpre bise ; Thèbes, 6311-3.

Illoec conquist le bon ceval

Qui plus va tost et pui et val

Que dromadaires por besongne. Amadas, 1535-7.

Quant Salehadins vit Taillefort de Valcois,

Sorbrun et Acherét, l'amustant des Nydois,

Et les .xv. courreurs felons et maleois,

Le riche dromadaire galopa li Turquois, Bâtard, 5722-5.

Trois cens chamois, ainz graignors ne veïstes,

Tuit sont chargié de pain et de farine,

De char salee et de vin sor la lie. Enfances G., 2060-2.

« Prenez. $\mathrm{x}^{\mathrm{m}}$. murs amblans et sejornez,

« Et autretant chameus et de bugles assez, Gui de B., 654-5.

Cf. $i b ., 956-8$.

Son riche dromadaire fist chargier en la tor. Barbastre, 1635.

Corsont de Tabarie de Barbatre est enblez,

Desus un dromadaire qui ja n'en iert passez. ib., 1098-9.

Li bons rois Alixandre li done avoir tant,

Chargiez .xx. dromadaire[s] d'or fin et de besanz. Roche, 2906-7.

- Par foi, » dist Salmadrine, « molt avez bien parlé.

"Quex chevaus menrez vos ? gardez ne[1] me celez,

- Par foi, noz deus mulez, » si li a dit Outrez.

- Je ai .ij. dromadaires coranz et abrivez

«Que faiz en .j. celier, bien a .vij. anz, garder.

«En .viij. jorz et demi serez vos retorné,

« Car il iront plus tost c'oisiaus ne puet voler. » ib., 1560-6. 
Les dromadaires ont du celier amenez,

Et lacent les cu[i]ries et les chanfreins dorez,

Que mal ne lor puist faire li venz ne li orez,

Car il iront plus tost c' oisiaus ne puet voler. $i b ., 1581-4$. Cf. $i b$. , $1624^{-7} ; 1696$ et passim.

Il s'en issi san nule domoraile

Et est monteiz desus un dromadaire

Ke plus tost cort ke alondre volaige. Enfances G., 1863-5.

Loquifiers ist premerains de sa nef,

avoc lui sont .IIII. roi coronés ;

son dromadaire ont devent lui moné,

selle ot le dos de fin acié tenpré,

li estrier sont de fin or noielé,

li anés ot .xxir. pouz de les ;

il n'est cheval de la soe bonté,

il n'ait si grant en la crestienté.

Ans cort autant con .r. falcons müé, Loquifer, 998-1006.

Troie présente un exemple d'une voiture tirée par deux dromadaires :

Et li chars estoit covert tous d'on cuir d'olifant boilli, si le traioient dous dromadaires, (...) Troie, 99, 30-2.

Voir aussi : Protheselaus, 2847-9; Blancandin, 1101-3 ; 2025-6 ; 2073-5 et passim ; Bueve, III, 3622 ; Simon, 606 ; 952 ; Aymeri, 3648 ; $3669 ; 3688 ; 3698$; Gaufrey, $4047 ; 4097 ; 4113$ et passim.

\subsubsection{Olifants}

Peu d'exemples du terme. Dans Ogier, l'animal en question sert de monture à l'enseigne :

Or avoit en sa charge ledit Caraheu cent mille combatans. Et estoit Rubien sur ung ollifant qui portoit l'estendart en son nom. Ogier, 123.

(...) le cheval estoit bien aussi grant comme ung olifant,

(...) Papegau, 15, 3 .

La oïssiez cors d'olifenz soner,

Cors et buissines mout hautement corner,

Ces olifanz et glatir et uler. Narbonnais, 3634-6. 
(...), et y multiplient les olifans que en autre partie du monde. Jehan de $S ., 213,12-4$.

«Par Dieu, mon amy, il fauldroit avoir des holifans grans planté a porter tant de maisons.

Jehan de P., 39, 23-4.

\subsubsection{Bovins}

Les bovins sont utiles de plusieurs manières, surtout aux paysans. Il n'est donc pas étonnant que nous les rencontrions dans les descriptions des milieux ruraux. Dans les milieux nobles, ce n'est guère que la viande de bœuf qui intéresse.

Les textes ne distinguent pas les races (quoiqu'il ait dû y avoir des différences d'une région à l'autre du pays), parlant seulement de bœufs, de taureaux, de vaches etc. Nous trouvons un grand nombre d'occurrences des termes bœuf et vache, beaucoup moins des autres. Les noms propres sont assez rares.

Les propriétaires des troupeaux (de bovins et d'ovins) sont fiers de leurs animaux. Jean Bichon, op. cit., p. 299 en cite un exemple.

Voici quelques exemples de chaque terme :

a. aumaille $=$ gros bétail .

ceenz puez veoir mainte aumaille

et buez et vaches et moutons,

espreviers, ostors et faucons. » Renart, II, 3528-30.

mais de tant me recort, sanz faille,

que ça jus a une valee,

entre .II. monz en une pree,

ou l'en amaine sovent pestre

l'aumaille de ceste chanpestre

vile qui est ici delez. ib., vı, 16346-51. Cf. ib., 16412-7 ; $16592-7$.

Il n'i ot buef ne vache ne aumaille praee

Se ne fu a argent ou a or achatee! Renaut, 5617-8.

les berbiz pernent e l'almaille,

les maisuns ardent e destruisent, Rou, III, 1084-5.

b. bœuf.

Dans Renart, le bœuf s'appelle Rognel; dans Aucassin, le bœuf qu'a perdu le pauvre valet s'appelle Roget ; là, comme ailleurs, on parle des différentes tâches qu'ont les bœufs : 
Cil vilain dont je vos conmanz

a conter merveilles romanz

.viIr. bues a sa charue avoit :

an la contree on ne savoit

meillor bues qu'estoient li suen ; Renart, Iv, 9287-91.

.I. buef arai seul a ma part,

Roignel qui fu seignor Lietart. ib., IV, 9351-2. Cf. ib., 9361-2 ;

9409-14 et passim.

Buez et vaches, brebis et bleiz

Avoi tant c'on n'en savoit conte, $M R$, LXIX, 10-1. Cf. LXVIII, 34-5 et passim.

(...) perdi le mellor de mes bués, Roget, le mellor de ma carue ; (...) Aucassin, xxiv, 51-2.

Mainte riche vile ont robee,

Prennent bues, vaches et berbiz ; Claris, 14198-9. Cf. ib., 6870.

Li veneor n'orent pas honte

s'il orent boef au premier mes

as bons aus, destrempé d'aigrés, Dole, 479-81.

Or chevauce li rois et il et si guerrier.

Les buez font charoier et les chars atirier, Gui de B., 392-3.

Un extrait d'Aliscans nous apprend qu'on écornait les bœufs pour les rendre moins offensifs :

Par mer s'en fuit le fort roi Desramé,

Et nos fuions comme buef escorné. Aliscans, 7015-6.

Le toponyme Gué des Bœufs, dans Lancelot, nous montre que les bœufs se déplaçaient régulièrement, soit qu'ils changeaient de pâturage, soit qu'ils servaient à transporter des marchandises, et que pour cela ils étaient obligés de traverser les courants d'eau (3) :

(...) chastel qui est a set lieues englesches pres del lieu que Merlins apele le chastel del Gué des Bues, (...) Lancelot, I, IV, 24 .

Quant à Gondebuef, nom d'un chevalier allemand, nous pensons qu'il est question d'une déformation : l'auteur (ou le scribe) a peut-être pensé à Godefroy, nom allemand courant à l'époque. Voir Aymeri, tome III, p. 266 : 
Il en apele Gondebeuf l'Alemant : Aymeri, 473.

Charroi présente deux bœufs par leurs noms propres; ils sont particuliers parce qu'ils sont attelés à la première charrette du cortège. Remarquons aussi qu'on les appelle limoniers, c'est-àdire « animal attaché au limon»:

Et Harpins hauce, si a Baillet tüé,

Et puis Lonel, qui estoit par delez

(Cil dui estoient li mestre limonier), Charroi, 1276-8.

c. bugle = jeune bœuf.

«Et autretant chameus et de bugles assez, Gui de B., 655 .

«Et trestous ces chameus et bugles qui i sont. $i b ., 958$

d. génisse.

Le terme a le même sens que dans la langue moderne, pourtant on semble pouvoir l'employer aussi comme synonyme de veau, voir infra : $M R$, cxxiv, 83-90.

«Sire, fait il, se Diex me gart,

li miaux si est que je i voie

que vos de ceste bele proie

retaingniez a vostre eus cest tor,

et cele genice encor

a ma dame Once l'orgeilleuse,

bone li ert et savoreuse,

qar ele est mout et tandre et grasse,

et je, qui ne voil pas a masse,

si avrai sanz plus ce veel, Renart, vI, 16698-706.

e. tor/torel.

et vos, sire Bruianz li tors,

reconmandez l'ame dou cors Renart, I, 415-6. Cf. ib., , 8218 ;

16696-701 et passim.

«Va querre les coilles d'un tor,

Les coillons atout le forcel $M R$, cxLIx, 472-3.

Il demanda au pastorel,

Qui mainte vache et maint torel

Avoit gardé en sa jonece : ib., LxxxIv, 105-7.

Dure ot la teste plus que toriaus ne ors ; Aspremont, 6031. 
An cel païs est arivee ;

au prince vint de la contree,

por grant angin li ala querre

qu'il li vaudist tant de sa terre

com porpendroit un cuir de tor, Eneas, 391-5.

L'ostel gueires esloignié n'oi,

Quant je trovai an uns essarz

Tors sauvages et espaarz,

Qui s'antreconbatoient tuit

Et demenoient si grant bruit

Et tel fierté et tel orguel,

Se le voir conter vos an vuel,

Que de peor me tres arriere ;

Que nule beste n'est tant fiere

Ne plus orguelleuse de tor. Yvain, 278-87.

Voir aussi : Laurin, 538-9 ; 4281-2.

\section{f. vache.}

Nous avons relevé des occurrences du terme surtout dans les fabliaux. Dans De Brunain la Vache au Prestre, se trouvent des exemples de vaches ayant un nom propre.

La maison sist joste un plaisié,

qui estoit richement garnie

de tout lou bien que terre crie,

si con de vaches et de bués,

de berbiz et de lait et de ués ; Renart, vi, 15560-4.

je cuit bien nos est avenu,

car je voi venir, ce me sanble,

.I. tor et une vache ensanble

qui a avec lui son vael, ib., vI, 16378-81. Cf. ib., 16683-7 et

passim.

Si sai bien faire frains à vaches

Et ganz à chiens, coifes à chièvres. $M R, \mathrm{I}, 123-4$.

Li prestres comande en oirre

$C^{\prime}$ 'on fasse pour aprivoisier

Blerain avoec Brunain lier,

La seue grant vache demaine.

Li clers en lor jardin la maine,

Lor vache trueve, ce me samble. $i b ., x, 38-43$. Cf. $i b ., 14-7 ; 22-4$ et passim. 
- Nous li donrons une vakielle

Et .I. petitet de no terre ; ib., xLIV, 123-4.

Bouchier qui welent tüer vaches

Ne firent si grans cops qu'il font Cont. P., II, 11802-3. Cf. ib., 10058-61.

Pansez de la nourrir, si ne li faillez ja,

Car je croy quant il serait grant que bon serait

A bien garder mez vaiche, grant mestier en arait. " Lion,

15367-9. Cf. ib., 24139-41; 24218-9 et passim.

les la forest en mi le pré,

la ou gerroit la vache vere, Thèbes, 9198-9.

De .xv. liues el rivache

ne remest ainc ne bués ne vace,

ne castel ne vile en estant ;

vilains n'i va son boef querant. Floire, 71-4.

g. veau.

« J'ai à mes povres parentiaus

Doné brebis, vaces et viaus, $M R$, LXIX, 91-2.

.I. veel ot en la maison

Qui fut loiez à un baston

Et estachiez à la cordele :

Une jenice fu mout bele.

La dame vint, si la desnoe,

Puis la saisie par la coe,

Et ses amis devers la teste :

En la cuve revient la beste $i b .$, cxxiv, 83-90. Cf. $i b ., 94-5$ et passim.

et vostre fil, qui mes n'aleste,

qui a oen esté sevrez,

avra, se ainsi le voles,

a son mangier ce vaelet

qui est tandre et de lait,

n'avra encor .viII. jorz demain, Renart, vI, 16754-9. 


\subsubsection{Ovins et caprins}

Tandis que les bovins servent de plusieurs façons leurs propriétaires (les bœufs comme animaux de traite, les vaches comme fournisseuses de lait etc.), les moutons et les chèvres ne leur sont utiles que par leurs produits : le lait pour la fabrication de fromage, la laine et les peaux pour celle des vêtements, la viande pour la table.

Nous présentons quelques exemples de chacune des dénominations relevées dans nos textes :

a. agneau.

Jel fis cheoir en la loviere,

la ou il vost mangier l'aignel : Renart, I, 1060-1.

En son toit Percheval mena ;

descendu l'a et desarmé,

et d'un sorcot l'a affublé

d'un blanc drap, et la penne fu

d'aigniax noirs ; puis a fait le fu, Cont. P., III, 14136-40.

Li enfes rit, la mere pleure :

« Tu ris, et tu plorer deüssez,

Se point de senz en toi eüssez,

Quer on te maine perdre vie

Con l'aignel a l'escorcherie. Anjou, 4078-82.

L'agneau est connu pour sa douceur et sa faiblesse :

Et mout cuident, qu'il soit prodon,

Por la conpaignie au lion,

Qui aussi doucemant se gist

Lez lui, come uns aigniaus feïst. Yvain, 4009-12.

S'il empoigne ung homme d'armes, il emportera aussi franchement comme il feroye ung agneau ; vous ne pourriez croire les faitz d'armes qu'il fait. Ogier, 121.

Conut vos ai, com li leus fet l'aignel. Aliscans, 6220.

Les os li froisse ausiz com .I. aignel, Gaydon, 7972. 


\section{b. bouc}

Si dent sambloient miol d'oef,

De color tant estoient rous,

Et si ot barbe come bous. Perceval, 4628-30.

\section{c. brebis.}

si a assez de gras fromages,

grosses brebiz et grasses vaches ; Renart, I, 1789-90. Cf. ib., II, 3779-866 et passim.

Brebis et vaches, et deniers

Ot à mines et à setiers, $M R$, LXxxvI, 19-20. Cf. ib., XCVI, 21-3.

.I. fil avoit qui menoit pestre

Toute jor en champ ses brebis ; $i b .$, xcvIII, 4-5.

Enmi sa voie encontra un garson

Qui gardoit bestes el chemin la amont,

Pors et berbis et aval et amont. Ami, 147-9.

A tant virent un juvencel gardant berbis, (...) Fouke, 60, 3-4.

Les brebis sont des êtres faibles et peureux (voir aussi 5.1.1., 5.1.2. et 5.1.3.) :

Et si sai je tout chertainnement que je vi ja caoir en une fosse assés parfonde le leu et le berbis, la meïsmes u li leu l'emportoit. Et quant li leus vit et reconnut k'il estoit caüs en la fosse dont il ne pooit issir et il se vit emprisonné, il devint si mauvais et si couars durement k'il ne fourfist rien a la berbis, la u il estoit encore en la fosse avoeuc lui seul a seul. Et se la berbis eüst point de hardement en soi, je croi bien tout chertainnement k'il n'i eüst ja desfensse qu'ele ne le peüst ochirre, si avoit li leus du tout perdu le cuer et le hardement. TP. III, 151, 22-31.

Malement sunt Bordelois desconfiz.

Parmi ces chanz fuient conme brebiz. Mez, 2439-40.

Une brebis stérile n'a que peu de valeur :

Vaillant une berbis brehaigne

Ne redoute Percheval point. Cont. P., II, 11188-9. 
d. chèvre.

Le terme se trouve sporadiquement dans nos textes, avant tout dans Renart et les fabliaux :

- Oil, fait il, a un viez lievre

et a dame Hersent la chievre

mout bien et mout tres saintement. Renart, II, 3567-9.

Or me dites ci orandroit

se savez par ou chievre poit.

- Par le cul, qant il est overt.

- Mes par la corne, dit Tibert. ib., IV, 11901-4.

(...), le traïstre me mist a un mestier dont neant ne savoie, car il m'envoioit au bois garder ses chievres et ses moutons ;

(...) Bérinus, 427.

Il s'agit parfois d'images :

Quar les veaus si sont liépars,

Et les chièvres si sont lions. $M R$, xII, 18-9. Cf. ib., I, 123-4 ;

cXXXIII, 82-4.

Ileqe vist Fouke beste venimouse, q'avoit teste de mastyn,

barbe e pees come chevre, (...)

Fouke, 63, 15-6.

e. mouton.

Le terme désigne l'animal mâle, tandis que la brebis est la femelle et l'agneau le petit.

Si con il fu dou bois issuz,

deus moutons a es chans veüz : Renart, II, 5303-4.

Or oiez que li bouchiers fist :

Si coiement .I. mouton prist

Que li paistres ne s'en perçut ; $M R$, LxxxIv, 111-3. Cf. $i b ., 126-$ $8 ; 132-3$ et passim

Dont n'i remest pain ne gastel,

Ne vins, ne bareus, ne bouchiaus,

Vache, ne moutons, ne porciaus,

Qui ne soit et chastel menez, Cont. P., II, 10058-61.

«Un monstre vous dona cornu et bien lané,

« Si dist : Biaus dous amis, che moton retenés : Aiol, 6262-4. 
Dans les extraits suivants on note que le mouton s'appelle Belin, nom qui est le terme ancien pour désigner le bélier :

Li rois apele un chapelain,

monsaingnor Belin le mouston, Renart, III, 8334-5. Cf. ib., I, 1611-8 ; III, 9075-6 et passim.

garde aval en une chanpaingne

et voit une mout grant conpangne

de brebiz paissent un gaïn :

entr'eles fu mestre Belin

le moston, qui se reposoit :

tant ot mangé que las estoit. ib., III, 8967-72.

Et dist Belin : « Je n'en puis mes,

je sers a un vilain felon

qui ainz ne me fist se mal non.

Ces berbiz ai ge engendrees

que tu voiz ici asamblees ; $i b .$, III, 8976-8o.

Les moutons sont faibles et peureux, comme nous l'avons déjà noté au sujet des brebis (voir supra et cf. 5.1.1). Cette qualité fait qu'on les méprise ; dans Guillaume, le mépris qu'on éprouve pour Tiébaut, à cause de sa fuite, est souligné par la «proie » indigne d'un chevalier :

De sus al tertre vit un fuc de brebiz ;

Par mi la herde l'en avint a fuir,

En sun estriu se fert un motun gris.

En son estriu se fiert un gris motun.

Tant le turnad e les vals e les munz,

Quant Tedbald vint a Burges al punt,

N'out al estriu quel chef del motun ;

Une tele preie ne portad mes gentilz hom. Guillaume, 395-

402.

Bien sçay que vous avés hardement de mouton. Tristan de N., 7965.

Ainsiz l'affronte com féist un mouton. Gaydon, 8060.

Unaut, le magicien sarrasin, est capable de transformer un mouton en un destrier, c'est-à-dire qu'il change sa couardise et sa faiblesse en courage et force :

Cil sot de nigromance, ben estoit doctrinez,

Qu'il feiseit .I. muton .I. destrier enselle, Simon, 1115-6. 
Dans plusieurs fabliaux, on fait le rapprochement entre les testicules de l'homme et les moutons, ou bien on parle de celles des moutons :

Hersens i vint par aventure ;

Ses mains geta sor ses coillons ;

Si cuide que ce soit moutons $M R$, xxIv, 348-50.

Tant que tu fusses ausi mox

Com une coille de mouton. $i b .$, I, 154-5.

La pucele sanz contredit

Li dist : «Frere, se Deus m'aïst,

Ce sont .II. coilles de mouton, ib., cVII, 145-7.

Turpin présente un exemple du terme pour désigner la poutre servant à battre les murailles en brèche, le bélier :

Il asist sis moys e au setieme fist adresser ses engyns, perreres, e mangoneus, e moutons e autres engynes bones a chasteuz prendre. Turpin, 302-4.

f. ouaille.

Ce terme s'emploie le plus souvent au pluriel pour désigner l'ensemble des moutons, le troupeau. Il se trouve néanmoins aussi au singulier :

Lors li escrie : « Esta! esta !», uns paistres qui gardoit oeilles. Cont. P., III, 15494-5.

Tout la ou il vint ne pot nuls cops endurer, ainçoiz fuioient touz devant lui, aussi comme font les oiselez devant oisel gentil et les oueilles devant le leu ravissant. Laurin, 4417-20.

ad oailles vait envirun, Rou, III, 1658 .

« Signor, » fait il, « alez a moi.

Ancui vairez mout grant mervelle :

Meler le lycon a l'oëlle. » Florimont, 3354-6.

qu'en la terre ne prenge beuf ne porc ne oeille ; $i b .$, II, 2722.

\subsubsection{Porcs}

Comme l'on s'y attend, les porcs apparaissent régulièrement dans nos textes quand il est question de viande et de repas. 
Voici quelques exemples des différents termes qui s'y trouvent :

a. cochon.

Nous n'avons relevé qu'un seul exemple de ce terme, dans un texte assez tardif. A ne pas confondre avec cochon, < cocionem, désignant un marchand, un maquignon, p. ex. $M R$, LXxxIV, 19.

Allez a l'offrande tous les dimenches, et a chacune messe, celles qui en ont la puissance, paiez loyaument les dismes a Dieu, comme de fruiz de poulles, d'aigneaux, de cochons, et autres telz usages accoustumez. Cent, 32, 275-9.

b. porc.

Ce terme, aussi bien que ceux de pourceau et porcelet, revient fréquemment, surtout dans les fabliaux. Les porcs vivent librement dans la nature, comme les moutons (voir supra, s.v. brebis : Ami, 147-9). Il faut d'ailleurs faire attention, car le terme peut désigner l'animal sauvage aussi bien que l'animal domestique.

Li chars de porc et li connins

Aporta on, por .II. mès faire $M R$, xxxIv, 314-5.

Tant aquist et tant conquesta,

Qu'il ot assez et .I. et el,

.I. bacon fist contre Noel

$\mathrm{D}^{\prime}$ un porc qu'il ot en sa meson

Norri trestoute la seson :

Bien ot plain paume de lart. ib., xcvII, 124-9. Cf. ib., cxxxII, 46$7 ; 146-56$ et passim.

Et tels i a vont en Bretaingne

Buès et pors, vaches achater $i b .$, xxxvII, 52-3.

Aiol[s] li fist porter .v. pains entier[s],

De trestout le menor, sans nul dangier,

Se peust .I. vilains bien aaisier,

Et .II. hastes de porc lonc de .II. piés, Aiol, 4037-40.

Cil pert molt bien ses margeries

Qui devant les pors les espant, Cont. P., II, 8600-1.

Tu nen as loi ne $\mathrm{k}^{\prime} \mathrm{a}$ uns pors

et tu morras ci par mon fer ; Eracle, 5634-5. 
(...), je viz le larron que je avoie delivré de la prison mon pere, qui s'embati sur moy et avoit grant foison de pors, (...) Bérinus, 428. Cf. ib., 429 .

c. porcelet.

"Se guerpir volés, sans nul plait,

Le porcelet ke nouri as,

Gesir te ferai en ses bras. $M R$, xxxIII, 202-4. Cf. $i b ., 268-9$; 286-9.

- Sire, » fait ele, « or ne vous griet

Que porcelez voldra mangier : ib., CI, 30-1. Cf. ib., 38-9.

\section{d. pourceau.}

Certes j'ai un porchiel nouri,

Il a passé .v. mois entiers ; $M R$, xxxIII, 196-7. Cf. ib., 243-4 ;

266 et passim.

Tant a chevalchié le chemin

qu'il encontra un pelerin

qui vin avoit en un bouchel,

et d'une espalle de porcel

avoit une piece en s'esquerpe, Cont. P., III, 15273-7.

Et de la fleur fait ses gastials,

Et del tercuel torte a porcials, Silence, 1821-2.

De la car fist si grant pieces taillier

Que le porcel fait mettre en dus moitiés, Chevalerie d'O., 9510-1.

Et li mengiers fu delitables

Et nes ; car tartes auant orent

De gayn, ki mout bien lor plorent,

Et puis apres porciaus farsis Espees, 8614-7.

Le destrier voit venir le damoisel,

Bien reconoist son seignour Otinel,

Henist et grate [ausi] comme porcel. Otinel, 373-5.

Si qu'a sollail levant atant est ung vaichier

Que per les boix [alloit] pour paistre et pour mengier

Vaiche, berbis, porcialz, car c'est son mestier Lion, 15314-6. 
Un extrait de Lion nous montre que les pourceaux vivent dans les tas de fumier :

Sus ung fumier s'areste ou il avoit estrain,

Delés pourcialz et true qui estoient malsain

Se couchait la duchesse qui le cuer ot certain. Lion, 2747-9.

Dans Berte, les valets à qui il avait été ordonné de tuer Berte mais qui n'exécutent par l'ordre présentent le cœur d'un pourceau au lieu de celui de la jeune reine, pour prouver leur obéissance. C'est là un ancien témoignage de la ressemblance du cœur d'un cochon et du cœur humain :

Je lo que nous le cuer d'un pourcel enportons,

A ma dame Margiste si le presenterons ;

Par iceste maniere bien nous escuserons,

Et si savez bien tuit $k^{\prime}$ en couvent li avons

Que le cuer de celi raporter li devons. Berte, 658-62.

e. truie.

Le terme semble toujours avoir une connotation négative et inviter au mépris. Nous l'avons constaté ci-dessus, dans Lion, 2747-9, et nous le voyons aussi dans les exemples suivants : qu'il s'agisse du prêtre illettré dans Renart, du membre viril dont parle la femme dans le fabliau LXIII, des truies des proverbes et d'Audigier ou du nain Crompart que Cleomadés trouve trop laid (voir aussi 5.4.) :

viellarz estoit auques li prestres, ne fu onques de lestre mestre plus savoit de truie enfondue que de lestre deporveüe. Renart, v, 14849-52.

Ce est la riens qui plus m'anuie, Mengié l'eüst ore une truie, Mès que vous n'en eüssiez mort. MR, LXIII, 59-61.

True ne songe se bren non. Proverbes, 2432. Cf. ib., 320.

La dame est acouchiee lez un seü ou truies et porceaus orent geü, por la chaleur du fiens qu'ele ot sentu. Audigier, 124-6.

Dist Cleomadés : « Mout m'anuie que cis hom a ce nes de truie doit avoir Marine ma suer. Cleomadés, 2059-61. 


\subsubsection{Lapins}

Dans notre introduction nous avons exprimé notre hésitation à considérer tous les lapins des textes comme des animaux domestiques. Les lapins restant à la limite de notre sujet, nous ne présentons que quelques extraits où il nous semble au moins possible de penser qu'ils ont été élevés dans des garennes surveillées. Il s'agit dans tous les exemples de scènes de repas :

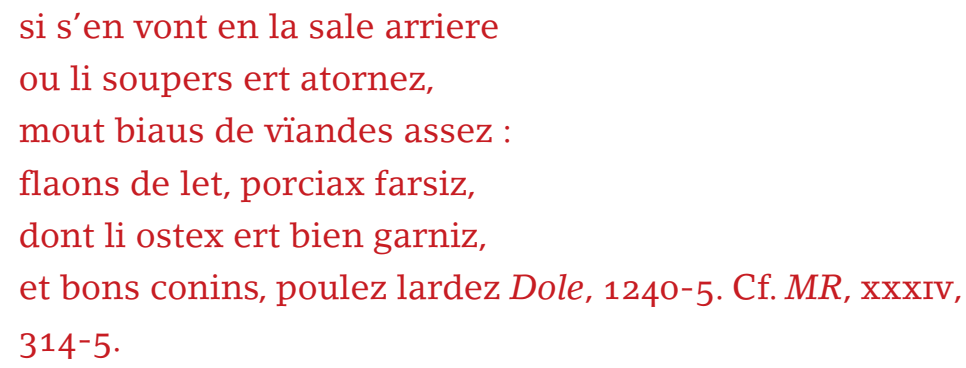

Devant li mectent un pasté.

Galopin l'a tantost tasté ;

C'est connin, et si y a poivre ; Anjou, 3637-9.

Dans Deduis est décrite une scène de chasse au renard qui vole poules et lapins :

De le prendre on a plus grant feste,

Car est malicieuse beste,

Et pour ce qu'il est poulaillier

Et qu'il seult les connins mengier. Deduis, 8597-60o.

\subsubsection{Animaux de distraction}

Quelques textes présentent des dames possédant des chiens pour leur plaisir (voir pp. 155-156 et 307), de même que plusieurs personnes - hommes et femmes - se plaisent à s'occuper d'oiseaux, dont beaucoup leur servent pour la chasse.

Plus rares sont les animaux mammifères apprivoisés, tels singes ou ours, qui se trouvent à l'intérieur des châteaux et même des monastères. Ils ne peuvent servir qu'à la distraction des habitants. Cf. 2.2.3. 
Es sales trovent mil destriers coreors,

.M. ors privez et .M. murs anbleors,

Quatre vint sinjes et aitretant fuirons,

Ostoirs de mue, esperviers et faucons, Mort Aymeri, 1582-5.

.xIIII. mil s'en couroient de beles

Des plus cortoises qui furent en la terre.

Portent oisiax et moinent fieres bestes,

Sinjes privez et calandres et melles ; ib., 1677-8o. Cf. Deduis, 1797-1809.

Il m'est avis que vous seriez bon menestereux pour ours mener, (...) Bérinus, 192.

\subsection{Les oiseaux}

Nous divisons les oiseaux domestiques en trois groupes: les oiseaux prédateurs qui servent à la chasse au vol et qui appartiennent exclusivement aux membres des classes nobles et qui sont très souvent donnés en prix aux gagnants des joutes et des tournois ; les oiseaux de basse-cour qui fournissent à tout le monde particulièrement de la viande, mais aussi des plumes et des œufs, et que l'on trouve mentionnés par conséquent avant tout dans des descriptions de repas ; les oiseaux de distraction.

Quant aux problèmes que pose la classification de certains oiseaux, nous renvoyons à l'introduction.

\subsubsection{Oiseaux de chasse}

Les oiseaux prédateurs, qu'élèvent les nobles dans le but de s'en servir pour la chasse, ont une très grande valeur, car leur « éducation » est longue et difficile (voir Friedrich Borchert., op. cit., pp. 16 et 77-80). On comprend donc que ces animaux sont très appréciés et que leurs propriétaires en sont très fiers. Pour plus de détails, nous renvoyons aux pages 63-66 de Jean Verdon, op. cit.

Quelques textes indiquent la couleur vair des yeux des oiseaux. Quant au terme sor, que nous avons rencontré souvent à propos de la robe (roux-brun ou châtain) des chevaux, il signifie " non mué ». Friedrich Borchert, op. cit., p. 8o, écrit que le faucon non mué, qui n'a pas encore un an, est toujours appelé "sor ", à cause de sa couleur. Cf. ce qu'écrit A.J. Holden, p. 601, à propos de notre exemple tiré d'Ipomedon, : " terme de fauconnerie, qui n'a pas encore mué. » 
Od lui en meine treis destrers

$\mathrm{E}$ bons brachés e bons levrers

E osturs e beaus esparvers,

Ben volanz e sors e muers. Ipomedon, 2641-4.

Des .iii. faucons sors premiers s'avisa Godin, 10614.

« Nous y portons .I. sor faucon. » Méliador, 13963.

Tu auras faucons et laniers

Niez, ramages, sors, muiers, Deduis, 797-8. Cf. ib., 207 ; 1732 ;

2692-3 et passim.

.I. faucon sor et .j. muier. Escoufle, 6693. Cf. ib., 6684 .

Li un paissoient par ces rues

Espreviers et faucons de mues,

Et li autre portoient fors

Terceus, oistors muez et sors. Erec, 351-4. Cf. ib., 5354.

Ainz que Gerbers otast ses esperons

Li envoia la roïne .I. faucon

Et la pucelle .I. sor esmerillon,

Et a Gerin un bon destrier gascon, $\mathrm{Mez}, 3720-3$.

Je voz donrai mon sor esmerillon. $i b ., 5585$.

Ses gans es mains cousuz a or,

Ung esprevier de plume sor

Tient sur son poing bien affaité. Galeran, 2059-61.

Si afaitoient les faucons

Por prendre grues et hairons,

Les ostoirs et les espriviers

Et les tiercels sors et muiiers

Et tous les bons oisiaus de proie. Rigomer, 14233-7.

Nous présentons un choix restreint des extraits de textes où se trouvent mentionnées les différentes espèces de ces oiseaux. Il est à remarquer qu'ils n'apparaissent pas dans les textes qui concernent les milieux inférieurs.

a. alerion = espèce d'aigle.

Aussi, si treuvez milion

Ou turquet ou alerion,

En ta court les faces porter, Deduis, 811-3. 


\section{b. autour.}

Li chevaliers ot un ostoir Rigomer, 9559. Cf. ib., 14472 et passim.

Set cenz cameilz e mil hosturs muabless, Roland, 184 .

Toloit la cace, le deduit de l'ostor Aspremont, 6616. Cf. ib., $3852-3 ; 6267-8 ; 7657-8$.

Sur son vivier un grant arbre esgarda.

Vit un ostoir fourmé qui manoit la ;

Vit qu'il miautit, vers l'iaue s'envolla.

.I. grant malart saisi et empieta,

A la terre l'ocist et devora. Auberon, 111-5. Cf. ib., 122-3 ;

1034-9.

Dans Do tint en son poing .j. ostor de .v. mues,

Aler doit en riviere por le sien cors desduire ; Roche, 2185-6.

E quant va en riviere, o lui maine Garnier :

Ou il porte l'ostor ou le faucon gruier. Aye, 8-9.

« Et si sai bien mestier d'ostoir et d'esprevier ;

«Si resai bien conduire une meute de ciens, Aiol, 9331-2.

Les damoissials monter a fait,

Puis fist monter ses conpaignons,

Et portent ostoirs et faucons

Et gerfaus et bons espreviers. Inconnu, 3840-3. Cf. ib., 3939-41.

Li .xx. plus viel que ge vos ai nommé,

Portoit chascuns .j. bon ostoir mué ;

Et li puisné qui sont de grant barné,

Chascuns de ceus porte .j. faucon ramé ;

Et li .xx. juenne bacheler redouté,

Chascuns d'aus a .j. esprevier porté. Aymeri, 1594-9.

Le terme se trouve souvent dans des comparaisons ; celles-ci nous renseignent sur les qualités et les habitudes des oiseaux :

Mais li ostoirs qui joint a l'anne

Ne se paine plus ne ahane

De restraindre, quant il a fain,

Que cil enfans, prisons a l'ain,

Ne painne plus estre escapés,

Que li vallés quist atrapés. Silence, 3863-8. Cf. ib., 91-4. 
« Daphus, dist il, n'est pas merveille se vous le me demandez, quar trop longuement ai esté en mue. Voirement resamble je le mauvais ostoir qui longuement se tient en sa mauvaise plume. Helcanus, 274.

Les euz vairs et rianz si conme ostoir müer, Barbastre, 1814 .

Cf. $i b ., 1944$.

D'a lui conbatre, de cho n'i a nul jor

Plus que mallart o le müé ostor. Aspremont, 358-9.

« Nient plus que li escoufle[s] peut l'ostoir resambler,

« Ne se peut li mieus fiex a son sens atorner. Aiol, 7122-3.

— « Ne t'esmaier, » ce dist Gerin, « cosins !

Ferons entr'aus con ostoirs en pertris ! Mez, 7262-3.

gieté serïens hors de mue

autrement c'on ne fait ostoir,

se savoient cest ovreoir! Eracle, 4610-2.

Voici des exemples où les autours sont offerts en cadeau. Nous notons, dans l'extrait de Durmart, l'indication de l'origine de l'oiseau donné à Arthur (c'est, à notre connaissance, le seul cas où norrois ne s'accole pas à un cheval); nous y notons aussi que les oiseaux prédateurs sont donnés aux " hauts barons " - aux vers précédents, Durmart a offert de l'or, de l'argent, des chevaux et des coupes :

Une dame l'ostoir li done ; Claris, 21478.

L'or et l'arjent et les chevax de pris

Et les ostors, les faucons montardiz. Mez, 2927-8. Cf. ib., 2693.

Les bials ostoirs, maint falcon mostardin, Aspremont, 130.

Gerfauz et ostoirs et faucons

Ce donoit il as hauz barons.

Des jöeaz prent li rois Artus

Un bel ostoir norois sens plus. Durmart, 15191-4.

c. émerillon = petit faucon .

Bernars l'anneis tenoit un emeril, Enfances G., 36.

Desor son poing tenoit un esmeri, Bueve, II, 5348. 
Desus sa main ot un esmerillon, $i b ., 5400$.

Chascunz enmi le renc se lance

Les saus menus et les galos

Es bons escus ferréz ados ;

Et puis batent des esperons

Ainsi comme uns esmerillons

Que chasce tant aprés sa proie. Chauvency, 1786-91.

adont veïssiés vos faucons

et ostoirs et esmerillons

et molt grant plenté de mouskés

voler aprés les oiselés. Floire, 3191-4.

Ne n'i ot nul, quelx que il fust,

Qui faucon ou terçuel n'eüst,

Esmerillon ou esprevier,

Ou ostoir sor ou bien manier. Erec, 1977-8o.

Voir aussi : Inconnu, 3939-41 ; Deduis, $823 ; 1711$; 9708 et passim ; Mez, 3720-2.

d. épervier.

Dans la plupart de nos exemples, il est question de chasse ; dans d'autres, nous voyons un homme ou une femme tenant l'oiseau au poing :

Son esprevier tint sor son poing. Rigomer, 15229. Cf. ib.,

15341-2 et passim.

A cest besoing n'a cure de garçon

Ne d'espervier ne de vol d'oisellon,

De nul deduit se de l'espee non. " Aspremont, 1062-4. Cf. ib., 3852-3.

Tout quidastes avoir trové

Quant vous alastes l'esprevier

Por une vielle chalengier

Qui estoit fronchie et ridee.

Vous l'aviez molt esgardee

Quant vous vostre amour li donastes

Et a l'esprevier le menastes

Por desraisnier et por prover

C'on ne porroit mie trover

Plus bele. Mais coment avint

Quant Erech et Enyde i vint ?

Vous lor laissastes l'esprevier. » Cont. P., I, 1532-43. 
Endementres qu'il parloient ensi, si voient vers aus venir une damoisele qui s'aloit esbanoiant par mi la riviere et portoit sour son poing un esprevier qui ert mout biaus. TP, vI, 77, 23-

6. Cf. ib., vII, 120, 11-3.

Baillié avoit son escuier,

Si comme il dit, son espervier, $M R$, cxv, 195-6. Cf. ib., LVII, 37$9 ; 48-9$ et passim.

La pucelle desvaule contreval le plainchié,

Nu piez, eschavolée, portoit .I. espervier. Floovant, 501-2. Cf. ib., 978-9.

Comme les autours, les éperviers sont l'objet de dons et de prix :

« Et li rois li donoit .I. esprevier mué. Renaut, 4385. Cf. ib., 4397.

Vos arés ciens bos, praieres, Bonnes roubes, bieles rivieres, Hostoirs, espreviers et gerfaus,

Faucons, gentius et bons cevals ; Inconnu, 3573-6. Cf. ib., 1586$91 ; 3840-3 ; 3939-41$.

A tous ceulx qui voudroient jouster sur le destrier, Et donrroit on ung pris qui moult fait a priser :

Ung cheval de .c. mars, et ung noble esprivier, Et ung noble chastel seant sur ung rocher, Tristan de N., 5041-4.

Por tant que ce fust la plus bele, N'i avra il ja damoisele

Qui ait l'espervier se li non ; Meraugis, 183-5. Cf. ib., 237-9 ; $342-5 ; 374-5$.

Je li anvoi per vos un esprivier

De tierce mue, n'ait millor desous ciel. » Enfances G., 572-3.

Cf. $i b ., 581-3$.

Contre monsaignor Cardroain

Covient son chevalier combatre, Et s'il l'en puet l'orguel abatre, Yde a perdu son espervier Et li atre l'a sens plaidier. Durmart, 2028-32. Cf. ib., 2015-6 ; $2020 ; 2023$ et passim. 
(...) vous envoient l'esprevier parmi l'esguart de ceuz de qui il est jugiez, que vous le miex l'avez fait (...) Laurin, 11507-9.

[Une] perce y fu mise et .I. esprevier de iiij. mues moult bel et moult gent : et fu devisé que cele qui seroit la plus bele et avroit seignor ou ami qui miex le feroit et plus bel, cil emporteroit l'esprevier. $i b ., 14777-80$

Plusieurs exemples soulignent la rapidité des éperviers et leur aptitude à la chasse ; voir aussi 5.1.5. :

Que, tout ainsi comme on afaite

Un courtois esprivier gentil,

A ele le cuer si soutil

A tout bien faire et si haitié,

Si courtois et si afaitié

Que nus n'i saroit que reprendre. Meliacin, 3478-83.

Espreviers, quant il vole a quaille,

Ne destent de gringor ravine

Que il vers la gent sarrasine

Ne voist plus durement assés. Diable, 1854-7.

Il le fait si mater et justicier

Come on afaite le ramage espervier. Aspremont, 30-1. Cf. ib., 9006-7.

Et plus tres volentiers ne l'oe

Que espreviers ne prenne aloe. Rigomer, 15341-2.

Li vens lor vient qui par vigor i fiert,

Ainsiz les mainne com l'aloe esprevier. Ami, 2659-6o.

Gace de la Buigne souligne qu'ils sont faciles à dresser :

Ceste rieule de moy tenez

Que l'esprevier en juing est nés

Communalment, maiz bien est vray

Que li aucun sont né en may,

Et ou mois d'aoust ensuivant

En main d'ommë est bien volant. Deduis, 6493-8.

e. faucon.

Gace de la Buigne prétend que de tous les oiseaux de chasse ce sont les faucons qui font le plus grand plaisir : 
Je commenceray aux seigneurs,

Car deües leur sont honneurs,

En traittant tout premierement

Des faucons, car certainement

De tous autres oyseaulx ce sont

Ceulx qui plus grant plaisance font. Deduis, 9401-6.

Il est donc naturel que le terme se trouve dans de nombreuses descriptions de scènes de chasse :

Ainz qu'aüst sa raison finee,

Entre li cuens par lo palais,

Qui portoit un faucon montais. Joufroi, 3646-8.

Ez voz Girart qui vient de giboier,

En Blaivies entre par la porte derrier,

Sor son poing porte un faucon montenier. Ami, 3391-3. Cf.

Gaufrey, 4957 ; 5050.

Cele part tourne le palefroi amblant,

Sor son poing tient un grant faucon volant.

Si com li rois va la dame aprochant, Bueve, III, 12106-8.

Aprés nonne, solail baisant,

Vi chevaliers venir passant

Faucons, trois si come je cuit. Chauvency, 1159-61.

Mout fu deduisans leur sejors ;

Es rivieres vont as faucons Jehan, 6052-3.

As perches sisent li faucon,

Hostoir, girfaut, esmerillon. Poitiers, 1358-9.

«Une autre chose me vint en avison ;

« En riviere ère alez o $u$ faucon,

« S'avoie pris une ane et un mallon, Mort Aymeri, 335-7. Cf.

ib., 427-8.

As tables vont aucun juer

U as eschiés, pour deporter.

$\mathrm{Li}$ aucuns faucon vont loirier ;

Cescuns s'en va esbanoiier. Couci, 477-80.

« En trestoute la terre n'a riviere petite

"Que n'aie à mon faucon ane ou sorceille prise. Gui de B., 120-1. Cf. ib., 19-20. 
s'avint adont sifaitement

c'uns de lor faucons abati

une corneille pres d'iki

ou Clarmondine ert et Crompars ; Cleomadés, 6654-7. Cf. ib.,

6665-6 et passim.

A icelle parolle le bastart repaira,

Ung faucon sur son poing que moult bel affaitta,

Du cheval dessendi, dedens l'ostel entra,

Le faucon mist a perche et gorge fait lui a.

Le filz au forestier, a qui moult anoya,

Ahardi le faucon, le col lui debrisa,

Et puis li dist : « Trouvés, mal ait qui vous porta

Ne qui en cest hostel ycy vous amena !

Fault il des gentilz homes en ce païs deça ?»

Quant le bastart ouÿ que 'trouvé' l'appella,

Et vit son faucon mort, forment lui ennoya. Tristan de N.,

4882-92.

Si tenoit sur son poing un faucon joli,

Si vit en un vivier .j. malart acroupi :

Si a geté l'oissel et li malart sailli.

Li oyssiaus vola tant que le malart choisi :

Il le toucha de l'elle, a terre l'abati ; Brun, 2758-62.

Comme les autres oiseaux de proie, les faucons se trouvent dans des comparaisons. Ici encore, la rapidité est soulignée (voir aussi 5.1.3. et 5.1.5.) :

Autresin con li fauz qui del pong est voles,

De ferir es oisiaus qant est antalentez,

Se fiert li cuens entr'aus, ne s'est aseürez. Barbastre, 264-6.

Cf. $i b ., 302-3$.

Lor armes bruient comme faucon volant. Otinel, 1857.

Des c'on parla de mort, n'y fist arrestison,

Devers le bois s'en fuit plus leger que faucon. Tristan de $N$.,

6196-7. Cf. ib., 3026-7.

Les eulx avoit riant et vairs comme ung falcon, Lion, 596. Cf. $i b ., 4340-1$ et passim.

Ils se trouvent également l'objet de prix et de dons :

Li roi des hiraus qui la furent

Errent pour veü si qu'il durent, 
Et li principal de la fieste,

Pour donner le pris plus honnieste

D'un faucon faitich et plaisant. Couci, 2002-6. Cf. ib., 2013-4 ;

2061-4.

Envoiez li .xx. destriers sejornez,

.xxx. faucons de .iii. mues ostez

Et .iiii. muls d'or et d'arjent trossez. Mez, 2911-3. Cf. ib., 2693 ;

3720-3 et passim.

Pollinicés voit le prison

qui li aporte le faucon ;

enaprés le levrier esgarde :

«Ci a, fet il, mout bele garde! Thèbes, 7451-4.

Dans Lycorne, un chevalier est appelé le « Chevalier au Blanc Faucon ». Nous ne savons pas exactement pourquoi, mais nous supposons que c'est parce qu'il possédait un faucon blanc. Dans le même texte il y a d'ailleurs d'autres chevaliers qui tiennent leur nom d'animaux domestiques: le "Chevalier au Coulon Blanc/Blanc Coulon » (vv. 5334 et 5563), le " Chevalier au Chien d'Argent » (v. 5826, peut-être fautif pour « Chief d'Argent », cf. v. 5340) :

Li chevaliers secons ot non

Le Chevalier au Blanc Faucon Lycorne, 5327-8. Cf. ib., 5562.

Dans $\mathrm{Mez}$, nous avons relevé des exemples de fauconnet et de fauconcel; quoique les deux termes désignent un petit faucon ils s'accompagnent de l'épithète petit :

.I. chevaliers ala le brant saisir

Et par raison molt fierement li dist :

«Par Dieu, Fromons, trop es maltalentiz,

Fel orguillox, et si en vax trop pis !

Je ne te pris vaillant .I. parisis !

A molt grant tort as ce baron ocis.

La costume as al fauconnet petit

Qui ert laniers, malvais et alentiz,

Que ja par lui ne sera oisiax pris ;

Tant que il voit que autre l'ont saisi,

Dont se esgete, qu'il lor cuide tollir. Mez, 8783-93.

« La costume est al fauconcel petit

Qui l'oisel porte as piez, cant il l'a pris ; $i b .$, 10351-2. 


\section{f. gerfaut.}

Les exemples de ce terme ne sont pas bien nombreux :

Galerans aprint d'autre part,

Par le conseil Lohier son maistre,

Comment l'en doit ung oyseau pestre,

Gerfaut, oustour ou esprevier,

Faucon ou gentil ou lannier,

Et l'aprint a laisser aller

Et poursuïr et rappeller

Et comment l'en le garde en mue,

Et quant l'en l'oste et remue ;

Des chiens sot, s'en ama la feste ;

S'aprint a deffaire la beste, Galeran, 1174-84.

(...), aparçust le roy Johan aprocher la marche, si manda au roy un destrer gras e beal e un girfaut tut blanc muer. Le roy le mercia mout de le present. Fouke, 31, 23-6.

Hostoirs, espreviers et gerfaus,

Faucons, gentius et bons cevals ; Inconnu, 3575-6. Cf. ib., 3840-3.

U plus haut arbre parché a

Un gierfaut qui tout droit se tient Lycorne, 5748-9.

Ber ert cil quil conquerra,

Un girefalc tut blanc muer,

De meillur n'oistes parler,

E un destrer de grant pris, Gui de W., 766-9.

Ne qu'il prengne ane ne heron

Ne de gerfaut ne de faucon. Deduis, 8477-8. Cf. ib., $9449 ; 9488$ et passim.

g. hobereau.

R. Grandsaignes d'Hauterive, op. cit., p. 353, explique: "Petit oiseau de proie. $»$

Et aussi que Deduit d'Oyseaulx

Li faisoit porter hobereaux

Et le menoit parmi les champs

Qu'il n'avoit encor que neuf ans. Deduis, 5563-6. Cf. ib., 823 ;

1713. 


\section{f. lanier/laneret.}

Le lanier est une espèce de faucon. Parce qu'une deuxième signification du terme est « paresseux, lent » (voir faucon : Mez, 878393 et gerfaut: Galeran, 1174-82, où il sert d'épithète respectivement à fauconnet et à faucon), il doit s'agir d'un mauvais oiseau car, comme nous l'avons vu, les faucons sont normalement très rapides. Dans notre extrait d'Anjou, pourtant, l'adjectif gentil procure au terme une nuance positive.

Albert Stimming traduit le terme par «Würgfalke » et écrit que cet oiseau est difficile à dresser pour la fauconnerie. A la page 233 du tome II de son édition d'Aymeri de Narbonne, Louis Demaison note : « terme désignant primitivement une espèce de faucon peu estimé pour la chasse. »

Avec le sens d'oiseau de proie, le terme est assez rare. Ce n'est que dans Deduis que nous en avons relevé beaucoup d'occurrences, ainsi que de laneret, désignant le mâle.

Tantost le varlet a geté

Le lanier, qui estoit mué Deduis, 257-8. Cf. ib., 253-4 ; 269 et passim

Et avec lui ot un varllet

Qui portoit un bon laneret, ib., 219-20. Cf. ib., $273 ; 280$ et passim.

N'oublierent pas lez oisiaux :

Gierfaus, faucons, gentilz laniers

Et tercees, qui sont maniers

Des heirons et des ennes prendre. Anjou, 512-5.

Les ieus ot vairs comme faucons laniers ; Bueve, III, 2154.

i. milion $=$ « oiseau de proie oriental $»($ Deduis, p. 606).

Et si y a de milions,

De turqués et d'alerions,

Tuniciens de Barbarie

Qui refont bonne vollerie,

Maiz ne sont pas communalment

Vers les parties d'Occident.

Les milions prennent les grues

Et oes grosses et menues,

De plumaige a l'aigle resamblent,

Maiz plus gens et plus petis samblent. Deduis, 10477-86. Cf.

$i b ., 811 ; 817$. 
j. mouchet/mousket = émouchet.

Nous n'avons relevé que peu d'exemples du terme. Voir aussi émerillon : Floire, 3191-4.

Et qui s'en saura bien jouer,

Esmerillons et hobereaux

Mouchés pour ses enfans mouviaulx, Deduis, 822-4.

Les oiseaux qui sont afaitables,

Qui a prendre autres sont plus ables,

Comme espreviers, esmerillons,

Toute maniere de faucons,

Ostours et hobers et mouchés, ib., 1709-13.

De la noblesce des faucons

Et d'ostoers et d'esmerillons

Et de mouchez et de hobez ib., 6527-9.

k. pasqueret $=$ espèce d'épervier. Synonyme de ramaget .

1. ramaget $=$ " épervier pris au printemps de sa seconde année » (Deduis, p. 627).

Au commencement de juillet

Aiez esprevier ramaget,

Que aucuns appellent pasquerés, Deduis, 11507-9.

Et puis aurez un ramaget,

Tres bel, de l'annee soret, $i b ., 11523-4$.

m. sacre/sacret = espèce de faucon.

Un sacre et un nïez faucon

Fist geter amont le felon Deduis, 389-90.

De lanerez plus de deux paire,

Aussi de sacres, de sacrez

Et de ces bien grans tercelez, ib., 802-4.

n. taharote $=$ espèce de faucon originaire d'Afrique.

Taharotes se font nonmer,

De Barbarie d'oultre mer.

Il sont petis et vermeillez

Aussi comme deux tercelez, Deduis, 9945-8. 
o. tercelet $=$ faucon mâle.

Voir aussi lanier : Anjou, 512-5 et émerillon : Erec, 1977-80.

Espreviers portent et faucons,

Ostoirs, tercels, esmerillons,

Car il venoient de jebiers. Inconnu, 3939-41.

Que j'ai bien en riviere eü

.x. faucons, estre les terciaus, Escoufle, 7118-9.

Maiz il veult ancor regarder

Sur les beaux grans marchés voller

Ces tres bons tercelés gentis, Deduis, 9617-9. Cf. ib., 9638 ;

$9647 ; 9656$ et passim.

p. tunisien $=$ faucon africain .

Voir milion : Deduis, 10479.

q. turquet $=$ faucon turc.

Les turqués, les alerions

Sont vistes comme esmerillons Deduis, 10487-8. Cf. ib., 812 ; 10478.

\subsubsection{Oiseaux de basse-cour}

Si les oiseaux prédateurs sont la propriété de la seule noblesse, ceux de la basse-cour se trouvent dans les fermes aussi bien que dans les châteaux. Ils fournissent avant toute chose de la viande pour la table des riches, mais aussi d'autres produits.

Nous présentons d'abord deux extraits de Renart : le premier montre que si un roturier n'a pas de grosses bêtes il a au moins des représentants de la basse-cour ; dans le second, le vilain Lietart promet un choix de volaille à Renart. Suit la liste des différentes dénominations que nous avons trouvées dans nos textes; elle contient une petite partie des occurrences relevées :

si l'avoit gité de granz biens

que il n'avoit ne buef ne vache

ne autre beste que je sache,

fors .x. gelines de parage.

Renart l'en avoit fait domage

de .II. gelines et d'un coc, Renart, I, 854-9.

De vos prandrai ge tele cure

que tout iert en vostre saisine, 
ane, chapon, coc et geline.

Chascun jor avrez a planté

et selonc vostre volanté

la char que vos plus desirrez :

des .x. poucins saisiz serez

et de Blanchart ja sanz demeure ; ib., IV, 11266-73. Cf. ib., II, 4069-76. (Jars)

a. ane $=$ canard.

Dans le fabliau De deux Angloys et de l'anel, un Anglais parle mal français et confond asnel (= ânon) et anet (= caneton).

Cet exemple suffit pour prouver que ce qu'affirme Friedrich Bangert concernant les chansons de geste, à savoir que les termes malart et ane semblent s'employer seulement pour désigner les canards sauvages (4), n'est pas vrai de toute la littérature narrative. Voir aussi malart.

- Triant, » fait il, « par seint Tomas,

Se tu avez .I. anel cras

Mi porra bien mengier, ce croi. $M R$, xLvI, 25-7. Cf. passim.

Anes, grues, cappons, pawons vait achetant Lion, 5267 .

b. biset.

Salverda de Grave pense que le terme désigne peut-être un pigeon. Nous ne l'avons trouvé que dans l'extrait suivant où la mère de Lavine accuse Eneas d'homosexualité :

il ne velt pas biset mangier,

molt par aimme char de maslon ;

il priseroit mialz un garçon

que toit ne altre acoler ;

o feme ne set il joër,

ne parlerast pas a guichet ;

molt aime fraire de vallet ; Eneas, 8570-6.

c. chapon.

Renart, qui des chapons sovient,

ne les pot mestre en oblïence : Renart, III, 8734-5. Cf. passim.

Pois au lart orent et chapons. $M R$, II, 78. Cf. ib., IV, 101 ; xxxIV, 270-1 et passim.

Lors reservent de mes noviax,

lardez et chiques, et oisiax 
de riviere, et chapons rostis ; Cont. P., III, 16653-5. Cf. ib., I, $687-9 ; 2669-71$.

Il orent assez a mengier, quar Goutez avoit assez tuez de chapons. Laurin, 7194-5.

Cf. ib., $9452 ; 13311-2 ; 14545$.

Et Galet a ung chapon pris ;

Pour li le tue et cuist en roust, Galeran, 754-5.

Elle faisoit le feu et tornoit lez chappon ; Lion, 1432. Cf. ib., $2318 ; 5603-4$ et passim.

Aus chapons vont, si en tuerent, Poules ont pris a grant plenté

Et poucins a lor volonté ; Claris, 18426-8. Cf. ib., 24302.

Ainc n'i laissierent qu'acater

Ne car ne capon ne geline ;

Tout envoient a la cuisine Gliglois, 2176-8.

d. colombe.

Nos textes ne mentionnent guère les colombes que dans des cas où elles ont la valeur de symboles de la pureté, comme nous le voyons également dans un proverbe : Le coulomb n'a point de fiel (Proverbes, 1067). Dans MR, Bueve, Mort Aymeri et Aiol, il s'agit d'un rêve ; dans TP et Lancelot, nous assistons à la scène où le saint graal apparaît à Lancelot, à Gauvain et à Bohort (voir aussi 5.6.) :

Et puis me vint en mon avis,

Mais je le conte mult envis,

Chaiens venoit .I. colenbiaus,

Ki mult estoit et gens et biaus, $M R$, XLIX, 77-80.

Si dius m'äit, moult en fui effraés,

Quant doi coulon, qui moult furent privé,

De lour aletes m'eurent acouveté, Bueve, III, 747-9.

« Un grant estoire trovoit de colons blans,

« En vers lo ciel l'enmenoient volant. Mort Aymeri, 328-9.

« Puis vi de li issir .II. colons blans.

« Dont m'esvellai del songe, n'en sai avant. Aiol, 388-9.

Endementres qu'il parloient ensi, si se regarda Lanselos et vit laiens entrer par mi une fenestre le coulon que mesire Gau- 
vains avoit veü autre fois, si portoit en son bec un encensier d'or mout rice. $T P, \mathrm{vI}, 32,1-4$.

Endementiers qu'il parloient issi, se regarde mesire Gauvains et voit par mi une verriere un blanc colon qui aportoit en sa boche I. encenser d'or trop riche. Lancelot, II, LXVI, 11.

Endementres qu'il parloient einsi, si entra laienz li coulons qui portoit en son bec .I. ancensier d'or et se feri en la chambre ou il entroit acoustumeement. $i b ., \mathrm{v}, \mathrm{xcvIII}, 24$. Cf. $i b ., 41$.

Mais dans la $88^{\mathrm{e}}$ nouvelle de Cent, nous lisons d'un colombier dans lequel le mari cocu se cache avec de vraies colombes :

- Seroye je point bien, dit l'autre, en nostre colombier ? qui me chasseroit la ? » (...) Ce vaillant homme monta en ce colombier, qui se fermoit pardehors a clef, et se fist illec enfermer, (...) Nostre bonne bourgoise habandonna son mary, et le laissa toute la nuyt rencouller avec les colons, a qui ne plaisoit gueres, (...) Cent, 88, 82-3, 91-3, 95-7.

Dans Ombre, nous pensons qu'il s'agit de colombes sauvages :

Miex vous venist avoir tendu

la fors une roi aus colons ; Ombre, 438-9.

e. coq.

Dans Renart, le coq Chantecler est un personnage fier qui est prêt à défendre ses poules :

Chascune d'ele s'en deshaite, qant sire Chanteclés li quois, en une sente lez le bois, entre deus pieus rala arriere : s'estoit mis en une poudriere.

Mout fierement lor vint devant, la plume ou pié, le col tendant, si demande par quel raison eles fuioient en maison.

Pinte respont, qui plus savoit, cele qui les grous eus ponoit, qui pres dou coc juchoit a destre ;

si li a raconté son estre Renart, II, 4120-33. Cf. ib., 4424-8.

et Chanteclers revint volant

a ses gelines maintenant, 
qui mout l'avoient atandu, ib., v, 13557-9. Cf. ib., 13494-501 ; 13661-2 et passim.

Dans une autre partie du roman se trouve le coq Blanchart/Blanchet (à cause de sa couleur), qui appartient au vilain Lietart :

Renart vers le vilain s'eslaisse,

si li dit : « Dex te saut, Lietart !

va moi querre le coc Blanchart ; ib., Iv, 10412-4. Cf. ib., 10430 ;

10440-3.

noz gelines a soes tient

et noz poucin et quide et croit

que nostre coq Blanchet suen soit, ib., IV, 10288-90.

Par leur chant, les coqs annoncent le lever du soleil - à moins qu'ils ne chantent encore plus tôt :

Et l'autres clers si s'aparoille,

Qant il oït le coc chanter,

Car il cuidoit trop demorer. $M R$, cxIx, 256-8.

Quant il oï le premier cot canter,

Huelin a maintenant apielé :

«Frere, fait il, or sus! se vos hastés !

Il a grant piece que li kos a canté. » Huon, 9127-30.

Dormoient les deux dames ou moult de beauté a ;

Mais droit a mÿenuyt, sy con le coq chanta,

Vint Galaffrë au lit, ung grant coustel sacha, Tristan de N.,

3933-5. Cf. ib., 10605-7.

- «Quoi ! dame, quoi ! ja s'en est ille

Alee anuit des premiers cos. Escoufle, 4108-9.

Tout ensement parlant pour eux enamourer

Furent dessus le lit pour yaulz a deviser,

Tant que de l'ajornee oyt lez cos chanter. Lion, 6236-8. Cf. ib., 13924-5 et passim.

Au matin, par som l'aube, l'est alé esveillier,

A .iiij. chaudelabres, et il et sa moillier.

"Sire duc de bon aire, vestez vos et chauciez,

«Tant a li cos chanté que jours[s] est esclairciez. » Roche,

3484-7. 
A la ferté vienent al coc cantant Bueve, I, 4516. Cf. ib., 857 ;

877 .

Voir aussi : Lanson, 6164-5 ; Bérinus, 54 ; 84.

Un coquet doit être un jeune coq, mais le terme désigne aussi la girouette :

Ele n'avoit mie autel cuer

Com maintes femmes par le mont

Qui corages remuans ont

Et tout aussi les vont tornant

Comme li cokés torne au vent. Jehan, 2222-6.

f. $g a l=$ coq

La nuit, quant chanterent li gal,

Rest dedenz Monreal entrez, Floriant, 2946-7.

A mie nuit, endroit le gal canter, Aspremont, 7021.

Endroit la miennuit, si com chante le gal,

Se parti deux Aymon de cel grant ost roial, Renaut, 12079-80.

g. geline $=$ poule.

Dans Renart, nous trouvons les poules Pinte et Rousette en face du coq Chantecler dans de nombreuses scènes; elles sont toujours convoitées par le renard:

ez vos les gelines a tant

et Chantecler paumes batant.

Pinte s'escrie premeraine

et les autres a longe alaine : Renart, I, 313-6.

Mes li gloz ne se pot tenir ;

vit les gelines ou paillier, ib., III, 6470-1.

dame Rousete la geline

et dame Pinte sa cousine. ib., III, 7751-2 et passim.

On mange les gelines :

Se voloit disner par matin

Mais n'ot geline ne pocin

Ne à mangier qui gaires vaille, $M R$, LXxv, 7-9. 
Son mestre keus mist a raison,

Et li commande estroitement

Qu'il se painne esforciement

D'un couleïch si atourner

Que on n'i sace qu'amender,

De ghelinnes et de capons, Couci, 8020-5.

Et puis menga, ke mout li sist,

D'un paste de gheline froit Espees, 3600-1. Cf. ib., 3762-5.

Tout le monde sent que les gelines s'enfuient devant le moindre danger :

Quant il vit le Sauvage qui de fere aatine

N'avoit de ceur neant plus comme une geline Tristan de N., 7665-6.

h. jars.

Le jars se trouve, à côté d'autres espèces de volaille, dans $R e$ nart :

La vile si ert en un bois ;

mout i ot gelines et cois,

eunes, malarz et gars et oues

et messires Coutenz des Noes,

uns vilains qui mout iert garniz,

manoit mout pres dou plaissaïz.

Plantaïve estoit sa maisons

de gelines et de chapons ; Renart, II, 4069-76.

i. malart = canard mâle.

Il s'agit normalement de canards sauvages (voir ane) mais, dans l'extrait ci-dessus, nous pensons qu'il est question d'un canard domestique, étant donné qu'il se trouve en compagnie de tant d'autres oiseaux de la basse-cour.

j. oie/oison.

Comme les poules, les oies servent de nourriture au renard aussi bien qu'aux hommes. De nombreux extraits de Renart et des fabliaux en témoignent. Un oison est une jeune oie.

Et Renart escotoit defors,

et si se panse en son corage

$c^{\prime}$ or puet il bien faire domage

sire Gonbert d'une grasse oe ; Renart, III, 7092-5. 
danz Grinberz a une grase oie,

a Noel la quide mangier, $i b ., \mathrm{v}, 14426-7$ et passim.

Vos demorroiz ci avuec nos

A disner, et ferons grant joie.

Quar véz ci au feu la grasse oie, » $M R$, xxxi, 58-6o. Cf. ib.,

254-6.

- Dame, li chapon sont tout cuit,

Et les .II. oies en .I. haste, »

Ce dist Ysane qui les haste. ib., cxvi, 234-6 et passim.

Chapons, oues, lart et poree

Et grant feu a la cheminee

Avions et vin de Vertus, Deduis, 8985-7.

Je sui legiers a departir

de vous et de vostre maison.

Miex ameroie un cras oison. Barisel, 232-4. Cf. MR, xxxiv,

751-5.

k. paon.

Tandis que les poules et les oies font partie de la cuisine des roturiers et des nobles, les paons semblent réservés à la table des derniers. On les sert rôtis, lardés et poivrés. Cf. Friedrich Bangert, op. cit., p. 215.

Voici un petit choix des nombreux exemples :

Sus au palais montarent a droiture,

Assez i ot des poons et des grues, Ami, 1998-9. Cf. ib., 2259-61.

Adonc vit il venir les metz que on aportoit pour mettre sur la table. Et voyant qu'il ne faisait la riens, si print ung entremez d'un pan qu'on portoit devant le roy (...) Ogier, 161-2. Cf. ib., 214.

Si li aporte deus blans pains buletés

Et un paon bien rosti et pevré Bueve, II, 3331-2. Cf. ib., II, 3620 ; III, $3710 ; 4885$.

Si li aportad un grant poun rosti, Guillaume, 1409. Cf. ib., 1428.

On lor aporte pain et vin,

Si se desjunent al matin.

Avoec ço lor aporta on

Cascun la cuisse d'un paon. Rigomer, 5511-4. 
Simeneaus buletez li aportent adonc

Et plain pot de boen vin et .I. rosti poon ; Renaut, 9025-6. Cf.

$i b ., 9051-2$ et passim.

Un pasté de .II. paons manz

fet li a doner en grant haste. Dole, 2216-7. Cf. ib., 3261-3 ;

5449-52.

Atan ez les serjanz qui portent lo mengier.

Li un porte .I. paon roti an un astier ; Parise, 2289-90.

Li uns portoit plainne boz de vin viez,

L'autre .I. poon rosti et afaitié, $M e z, 10966-7$. Cf. ib., 10456-9 ; 10970-1.

A planté orent venissons,

Sers et grues, jantes, poons, Florimont, 9571-2. Cf. ib., 6252-6.

Si vos servi come pulcele ;

le poun mis en l'asquiele :

unkes n'en mustes la maissele. Gormont, 244-6. Cf. ib., 259-61.

Voir aussi : Simon, 1481-2 ; Narbonnais, 5451 ; Raguidel, 756.

Une plume de queue d'un paon sert à embellir le heaume du héros Partonopeu :

L'autre revit assez le jour

Parthonopiex joindre en l'estor ;

a val le cognut el sablon,

car une queue de poon

ot en l'iaume lacié derriere

et quant il point par la riviere,

la plume au vent s'espant et euvre

et de devant un peu le ceuvre. Thèbes, 5871-8.

\section{1. pigeon.}

(...), puis les grans plas tous combles de lappereaulx, perdriaux et gros pijons d'ostel, (...) Jehan de S, 236, 7-9.

m. poulaille.

(...), de perdriz, de gras chappons, de poulailles et de pijons

(...) Jehan de $S ., 245,2-4$. 
Les gens du roy d'Angleterre firent tuer beufz et montons, et de vieille poulaille qu'ilz trouvoient ; vous povez pencer si elle estoit fort tendre. Jehan de P., 33,31 - 34,2.

n. poule.

Le terme est moins fréquent que son synonyme geline. Voir aussi chapon : Claris, 18426-8.

Dist Lanselos : « Jou vos donroie

A mangier d'une crasse molle.

Encor ai jou une telle poille

Qui orains fu rostie a poivre ; Rigomer, 14094-7.

Mais qu'il me cuit dame Avinée,

Et demain, à la matinée,

Li ferai un conroi nouviel

Boin, et plaisant et sain et biel,

De tendres poulles et d'oisons. $M R$, xxxIv, 751-5.

o. poulet/pouletel.

.I. escoufles sour .j. de ciaus

Tenoit .j. poulet qu'il manja. Escoufle, 6812-3.

Au vert jus de nouvele grape

Li donna Blonde un froit poulet, Jehan, 1352-3. Cf. ib., 1359.

tout hors menga le branc d'un pouletel. Godin, 8663. Cf. ib., 15404 .

anchois menga tous hors .ii. pouletiaus ib., 8720 .

p. poussin/poussinet.

Nous avons l'impression que les termes désignent le plus souvent un poulet (p. ex. Renart, IV, 11383), mais quelquefois un très jeune poussin (p. ex. Meliacin, 275 et 283 [voir 5.6.]).

Toujorz ai esté pautoniers

et aloie mout volantiers

la ou je savoie hastis

les gelines et les poucins : Renart, III, 8821-4.

A tant prant Renart, si manoie,

Blancharz et les .x. poucinez

qu'aportez li a Martinez ib., IV, 11382-4 et passim. 
Une table trouva garnie

De viandes et de bons vins,

De pain, de char et de pocins. Claris, 9621-3.

Molt en pense Constance de vrai cuer enterin

Et ses filles andeus, Dieus leur doinst bon destin !

Li une li aporte a mengier d'un poucin

Et l'autre li retrempe de fresche aigue son vin ; Berte, 1359-

62.

« Nos l'avons en costume, en France et a Poitiers,

"Quant hons hauberge autrui, qu'il le conroie bien,

« De pocinez pevrés et [de] gastiaus broiez

« Et vin et ysopé tant com li est mestiers. Roche, 3282-5. Cf.

ib., 3332-4; 3899-902.

Les poussins sont faibles, et leurs plumes ne valent rien :

Mais la beste estoit forte, sy savoit moult d'engin,

Les Sarrasins occist con se fussent poussin. Tristan de N.,

6253-4.

Pués recouvrait ung cop a loy de pallasin,

Ausi le deffroissait comme se fuit ung puissin. Lion, 2955-6.

Devant Corsuble feri un Barbarin

Si tres grant coup sor l'iaume outremarin

Qu'il n'i valut vaillant un roumoisin,

Ne li haubers la plume d'un poucin ; Enfances O., 5951-4.

Dans Deduis, poussin désigne le petit des oiseaux de chasse ; dans Queste, 184, 20 et 25, des oisillons sauvages (cf. ib., 168, 2) :

Et tantost aprés nidefient

Et font eufs et puis pullefient.

Assés tost vient l'amour nouvelle,

Et au malle et a la femelle,

Envés leurs poucins, que nature

Y met tout pour leur norreture, Deduis, 6561-6.

\subsubsection{Oiseaux de distraction}

En plus des oiseaux prédateurs apprivoisés, dont les nobles hommes et femmes - tirent un grand plaisir à la chasse, nous trouvons quelques exemples d'autres oiseaux tenus en cages ou en volières et qui égaient leurs propriétaires par leur chant. Cf. 
Friedrich Bangert, op. cit., p. 210 : « Singvögel wurden zur Unterhaltung in den Wohnungen gehalten. »

Philippe Contamine déclare, dans le tome 2 de l'Histoire de la vie privée, pp. 468-469, que les paons sont tenus comme « oyseaux a plaisance », certainement à cause de leur beau plumage : " Dans la description de Guillebert de Mez, l'accent est mis, à dessein, sur les dimensions militaires de l'hôtel (pourvu d'une vraie salle d'armes), sur sa commodité et son confort, sur son rejet de ce qui pourrait paraître trop strictement utilitaire (dans la cour, des oyseaux a plaisance, des paons, et non des poules ou des canards), (...) ».

Mainte viële i oïssiez tentir,

De maint oisiax i oïssiez lo cri,

Chante la melle avueques lo mauviz,

Jais et calandres, chascuns en son latin. Mort Aymeri, 2568-71.

Le chevalier a fame avoit

Une dame que monlt amoit,

Car elle estoit et bonne et belle,

C'est grant tresor de l'avoir telle,

Laquelle avoit un estournel,

Qui parloit si bien et si bel

Qu'a tres grans merveilles avoient

Ceulx qui si bien parler l' [ou] oyent.

Or avint en une journee

Qu'il faisoit froide matinee

Que la dame la cage prist

Et l'estournel pres du feu mist,

Maiz il s'en yssi de la caige, Deduis, 6429-41. Cf. ib., 6458 ;

$6467 ; 6472$.

«En la chambre roial, où le roussignol trie,

« Là coucheron Doon entre les bras d'amie, Doon, 10504-5.

Un vers de Perceval nous fait penser que le perroquet, cet oiseau exotique, se trouvait sporadiquement dans les maisons riches où l'on l'appréciait à cause de sa beauté. Nous remarquons qu'il est mentionné avec un épervier, autre oiseau très estimé. L'oiseau qui a donné son nom au roman du Chevalier du Papegau sait parler et réfléchir - il est tenu dans une belle cage :

Et la pucele vint plus cointe

Et plus acesmee et plus jointe

Que espreviers ne papegaus. Perceval, 1795-7. 
(...) celluy qui avra la plus belle amye et le pourra monstrer pour armes, si avra ung papegault que ung nain la apporte chascun an, (...) Papegau, 5, 21-3.

(...) aprés eulx venoit ung nain qui estoit vestus d'escarlate fouree de vair, qui chassoit devant luy ung pallefroy qui pourtoit une cage la ou estoit dedens le papegau, (...) ib., 7, 23-5.

Mais nul ne vous pouvoit dire la noise que faisoit le papegaulx ; car il dist au nain qu'il le meine au plus hault lieu qu'il pourra, et si cria : "Nains, nains, porte moy veoir le meudre chevalier du monde ! (...)ib., 10, 36 - 11, 4.

(...) ; car les quatre escharbocles de la caige au papegaulx gitoient si grant resplendeur parmy le palais, que c'estoit merveille a veoir. Et le papegau mesmes disoit en chantant tous les fais que son chevalier avoit fait (...) ib., 21, 14-8.

1 «berserez as a subst. normally means 'hunting-dog'; and it is a fact that certain hunting-dogs were carried to the chase; but it appears from the examples cited by Tobler-Lommatsch, Altfr. $W b$., that it also denoted some part of the huntsman's equipment, most probably the quiver, as in the present instance. »

2 Cf. Albert Stimming, Bueve, III, 2, p. 438: "Das Femininum levriere wird mehrfach des Reimes wegen verwandt, besonders in Vergleichen, (...) ».

3 Jean-Pierre Leguay, La rue au Moyen Age, p. 95, cite des rues qui tirent leur nom d'animaux domestiques.

4 Op. cit., p. 220 : " Die Enten kommen unter den Bezeichnungen mallart (mastart, malart, mellart), ane und anete in den Ch. de g. vor, und zwar ist unter den männlichen Wörtern offenbar der Enterich, unter den weiblichen die weibliche Ente zu verstehen. Alle aber werden, wie es scheint, nur auf wilde Enten angewandt. » 


\section{La vie des animaux}

Les chapitres précédents montrent que les animaux domestiques sont bien présents dans les textes littéraires narratifs - certains très fréquemment, d'autres rarement ou même exceptionnellement.

Nous allons nous pencher sur la question de savoir comment vivaient ces animaux. Comment les soignait-on ? Quelle était leur nourriture ? Quels animaux avaient une relation intime avec leur maître/maîtresse et quels animaux menaient une existence plus libre ? etc.

Les réponses à toutes ces questions ne seront certainement pas faciles à donner car, si les textes ne sont pas parcimonieux quant à mentionner les animaux, ils sont assez réticents quand il s'agit de nous renseigner sur leur vie. De sorte que nous sommes obligé de glaner des petites informations par-ci par-là et d'essayer de nous faire ainsi une image plus complète de cette vie.

\subsection{Appréciation des animaux}

Un extrait de Durmart prouve qu'un vrai chevalier ne peut pas exister sans son cheval ; il serait fou de l'abandonner et d'y renoncer :

- «Par Jhesu, le haut roi poissant,

Fait messires Durmars li fiers,

Ensemble o moi iert mes destriers.

Sens mon cheval n'irai je mie,

Car ce me senbleroit folie

Se je en la cité estoie,

Et ge mon cheval n'i avoie.

Je seroie tos esbahis,

Car se bataille u poigneïs

Commençoit devant la cité,

Se tot li autre erent monté

Et je a pié i demoroie,

Dolans et coreciés seroie ;

Car chevaliers qui veut valoir

Doit bien a grant besoing paroir Durmart, 11026-40. 
Puisque les chevaliers n'étaient rien sans leurs chevaux (1) - leur réputation dépendait de leur courage mais aussi de la force et de l'agilité de leurs montures - on constate une grande intimité entre homme et animal et même d'un grand amour de l'homme pour son cheval ; il est plein de pitié lorsque celui-ci est malade ou mal soigné, il lui donne un nom et il regrette le cheval absent ou mort (2).

Friedrich Bangert, op. cit., p. 8, écrit que les relations étroites entre l'homme et son cheval procurent à ce dernier quelque chose d'humain. Il n'est pas seulement le compagnon inséparable et le serviteur de son maître, mais aussi son confident.

Dans son Dictionnaire des symboles, à l'article « cheval », Chevalier-Gheerbrant écrit : « (...) le cheval n'est pas un animal comme les autres. Il est la monture, le véhicule, le vaisseau, et son destin est donc inséparable de celui de l'homme. Entre les deux intervient une dialectique particulière, source de paix ou de conflit, (...) » (nous citons d'après Begoña Aguiriano, Le cheval et le départ en aventure dans les romans de Chrétien de Troyes, Senefiance, 32, p. 27).

Son chevaul li ameinent, qui pas mengié n'avoit

S'avoine ne son foin qu'aporté li avoit.

Quant Richiers l'ai véu, mout joianz an estoit. Floovant, 1096-8.

Et dist li rois : « Laissiez ester Flori.

Nel me devez rover ne requerir.

Saisis en sui ; si ne le voel guerpir. »

A maleür li dus Gerbers a dit :

«Viltance sanble de mon cheval tolir.

Gel vos baillai et devisai isi,

.I. soir l'aiez, et rendez al matin.

Or me volez de covenant folir.

Mais par Celui, qui le mont establi,

.I. parole voz sai dire de fi :

Se je ne rai le bon cheval Flori,

Encor t'en puet molt granz max avenir. » Mez, 4345-56.

Et ont li doi compaignon requis l'ermite se il la nuit de riens les porroit aaisier, eulz et leur chevaus. Il dist que les chevaus porroit il bien aaisier, mais il n'estoit mie bien pourveu pour eulz. Il distrent que plus leur estoit de leur chevaus que de eulz. Helcanus, 135.

Einz n'aresta Girart, si vint a son destrier.

Li chevaus se gisoit, ne se pot aïdier,

Et qant Girart le voit, pres fu del enragier : 
« Ferrant, ce dist Girart, or me doi aïrier,

Que ge vos voi einsi del tot afleboier.

Hahi! Quieus estiez por vo seignor aidier,

Por foïr a besong et por bien enchaucier !

Por un jor porter armes ne l'esteüst changier. Barbastre,

4468-75.

Donc regrete Renaut Bayart son arragon Renaut, 6993. Cf. ib., 13447.

Mais de vostre destrier Broifort ie ne sçay qu'il devint quant vous fustes prins empres la cité d'ivoyre. Adonc Ogier se conmença a conplaindre de son cheval et disoit : $\mathrm{Ha}$, mon bon cheval, iamais ie n'en pourrai trouver ung tel qui me puisse si bien porter. Ogier, 139.

Le sor Bauchent a forment regreté. Aliscans, 1870.

Aupatris est devant que li soudans a chier ;

Gautiers siet sor Morel, sel trueve molt legier :

Bien riches amiraus nel porroit esligier ;

Aupatris l'aime plus qu'il ne fait sa moillier

Et Gautier vait sans frain por Morel aesier. Partonopeu, 195660 .

Aupatris aime donc le cheval plus qu'il n'aime sa femme, ce qui correspond d'ailleurs à ce que nous lisons chez Christina Álvares, Gauvain, les femmes et le cheval, Senefiance 32, p. 37 : « - car, évidemment, le Gringalet vaut davantage qu'une femme. » Quant à Lancelot, il n'est pas douteux qu'il apprécie les chevaux plus que ses domestiques :

Et Lancelos li conte comment il estoit herbergiés et comment .I. chevaliers vint laiens ou il menjoit, qui prist a force .I. vaslet et le mist sor le col de son cheval. « Et encore m'a il plus meffet, fet Lancelos, kar cil qui avec lui vindrent enporterent mes armes et emmenerent mon cheval. Lancelot, II, L, 3.

L'extrait suivant, d'Aiol, montre que le protagoniste est fier de son destrier Marchegai et parie qu'il est supérieur au cheval de Makaire ; la suite lui donnera raison. La monture de Rohars lui vaut certainement :

« Vos estes riches hom, je sui un[s] bris ;

« Mais del ceval me poise c'avés laidit :

« Il est et biaus et cras et bien garnis,

«Si n'en a nul millor en ces pais 
«Fors seulement le vair roi Loeys.

"Sire, chelui ne voil mie aatir :

« Chou est mon droit signor, nel voil laidir ;

«Mais encontre le tien bien l'aatis

« Por une liewe corre tout .I. chemin ;

«Et se li miens peut vaintre, si me plevis

«.M. mars de blanc argent et .c. d'or fin,

«Et del destrier a faire tout mon plaisir ;

«Se li tien[s] vaint le mien, jel fac ausi. Aiol, 4240-51.

Mais Rohars ot un cheval si tres bon

qu'en tout le mont millor ne trovast on. Godin, 14324-5.

A cause de l'estime et de l'amour qu'on ressent pour les chevaux, on méprise le chevalier qui, au cours d'un combat frappe (exprès ou non) le cheval plutôt que le cavalier :

Lors a son cheval hurté des esperons et vint a lui l'espee ou poing et l'a feru amont la ou il le cuidoit miex assener. Cilz vit le cop venir qui a merveilles estoit pesdanz, si se guenchi ; et li cops chaï sus son cheval et le consuÿ de tele force que, vousist l'armeure ou non, li a le col a tout le chief rasé jusques aus ars. Dont couvint le cheval et Calcas tout cheoir en .I. mont. Quant li empereres vit ce, si fu si courouciez du cheval Calcas, quant il le vit ainsi atourné. Adont est Calcas des arçons issus et dist a l'empereour : "Par Dieu, lierres traitres, mar y atouchastes le cheval et moi pour lui ocirre si ne vous avoit riens mesfait. » Ainz qu'il ot ce mot pardit fu li empereres descenduz. Il vint en sa presence et dist : "Calcas sachiez que du cheval me poise. Mais le coup n'osastes vous atendre si l'a li chevaus comperé et vous aussi le comperrez quant vous murdrier m'avez apelé tant ne le fusse je onques. » Helcanus, 115-6.

Ha, ce dist il, faulx traytre que tu es ne sçavoyes tu assener sur moy sans blecier mon cheval ? Ogier, 130.

Si consivi le cheval si qu'il le pourfent jusques ou pis. Li chevaus chaÿ mors. Li chevaliers s'est desmontez moult isnelement et dit : "Certes, mauvais chevaliers, de grant faintise vous est venu quant onques ferites le cheval qui onques riens ne vous forfist. Ce ne fu il mie que vous abati, si le comperrez chien. » Laurin, 2017-22.

Or oés que il fist, li cuvers desloiaus :

Par mi les janbes fiert le bon destrier isniau ; $\mathrm{Au}$ premier cop li a tranchié les piés. 
"Vasaus, » ce dist Berars, " mont par estes leniers

Quant moi avés laissié mon cheval tuez! Lanson, 5725-9.

Gauvain, accusé d'avoir tué un cheval, répond qu'il l'a fait pour ne pas être tué lui-même. Lancelot affirme n'avoir jamais tué de cheval exprès, sinon pour se sauver d'un grand danger. Persidés qui a tué le cheval de Hector agit contrairement aux « coutumes $\mathrm{du}$ bon chevalier » et les hommes qui veulent tuer le cheval de Bohort sont appelés « mauvaises gens » et «larrons » :

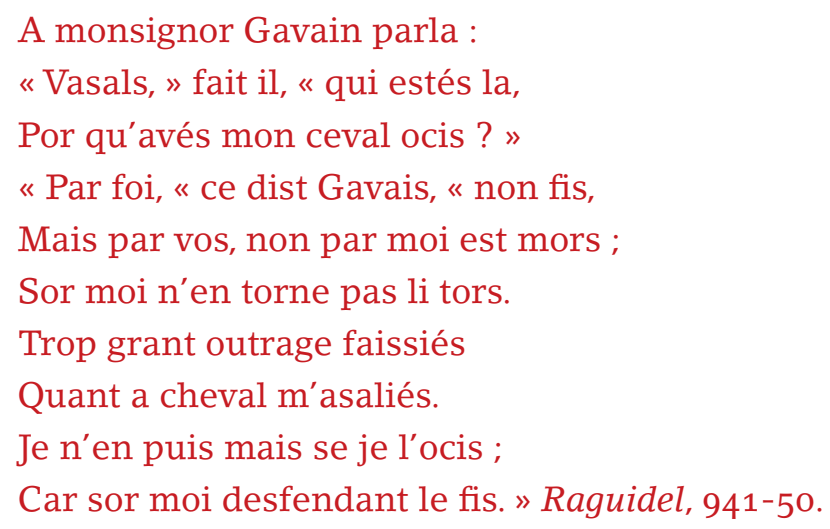

"Sire chevaliers, se vos descendiés, je overroie la barre et nos combatrions a pié. » Et cil dist que rien n'en fera. "Donc vos ocirai je, fet Lancelos, vostre cheval, si avrois plus perdu. - Se vos le poez ocire, fet cil, si l'ociés. - M'aït Diex, fet cil, je n'ocis onques cheval a escient, se ma mort n'i vi ou mon grant damage, ançois vos laisserai je avant venir. »Lancelot, II, XxxvIII, 14.

Et lors trait Hector l'espee, si li cort sus tout a cheval, et chil se cuevre de son escu et fiert le cheval par mi la teste, si le jete mort. « Dehais ait, fait Hectors, qui au millor chevalier del monde vous tient, car chi avés vous fait .I. poi de malvaistié, quant vous mon cheval avés ochis, et che n'est pas coustume de tres boin chevalier (...) ib., vIII, LXVa, 16.

Quant Boorz les voit en tel manniere, si cuide bien que ce soient mauvaises gent, (...) Et quant il voit la damoisele delivre, si point as autres, et quant il le voient apoindre, si dist li uns a l'autre : «Occions li son cheval et le metons a pié, qu'il n'avra ja plus duree. » $i b ., \mathrm{v}$, xcvIII, 4 .

Il ne faut pourtant pas oublier qu'aux combats il est régulièrement nécessaire de changer de cheval. Cf. à ce propos p. 14 (Jean Flori) et notons deux petits extraits :

(...) il n'est pas merveilles se uns chevaliers pert son cheval en la bataille : (...) Troie, 152, 46-8. 
Mes Bilas ot reprise aleinne ;

Novel cheval et frois en meinne. Athis, 16697-8.

Il faut également prendre en considération que parfois les chevaliers semblent apprécier leurs armes et tout leur équipement plus que leurs chevaux et que dans certains cas nous connaissons le nom de l'arme d'un héros mais pas celui de son cheval.

Dans Ogier, le jeune chevalier Gautier veut absolument avoir Marchevallée, le destrier de Noradin, pour en faire cadeau à Ogier - et pour avoir lui-même le destrier de celui-ci. L'auteur nous apprend que les chevaliers « aventureux " n'apprécient rien plus haut que les beaux chevaux mais, deux pages plus loin, il fait dire à Ogier qu'il ne donnerait pas son épée pour un royaume :

Car c'estoit le plus deliberé cheval qu'on sceust iamais trouver, car il s'entendoit franchement de l'avoir pour en faire ung present a son oncle Ogier affin que il eust Bauchant, le cheval de son oncle ; car il n'est riens en quoy telz chevaliers adventureux prennent plaisirs plus grans ne felicité qu'en beaulx chevaulx. Ogier, 254 .

Dea, sire, prisez vous si peu mon espee ; ie vous prometz et iure en bonne foy que ie ne la donneroye pour la valeur d'un royaume, (...) ib., 256.

Le grand guerrier Rainouart constitue certainement un cas particulier : non seulement il préfère se battre avec sa massue, il prétend même qu'il hait les chevaux :

Chevals e armes par matin vus durrai.

- Ne place Deu, dame, » dist Reneward,

"Sus ciel n'ad rien qui tant hace cun cheval. Guillaume, 283840.

Nous notons aussi les deux opinions différentes du « damoisel » et de Gauvain concernant les chevaux : le premier propose de les laisser paître la nuit et de les retrouver le matin, mais Gauvain a peur que le sien, qui ne connaît pas le pays, ne s'égare ; et il refuse de le quitter, ce que le « damoisel » ne comprend pas :

- Li miens, fait Gavains, que fera,

Qui ne connoist pas le paiis ?

S'or le m'avoient leu ocis,

$\mathrm{U}$ aucune beste sauvage,

Il me servit tout mon eage,

(Qu'il ne poroit estre celé)

En ma contree reprové 
Que je l'aroie seul laiscié

Comme couart par mauvaistié,

Si l'aroient estranglé lex.

Certes ja n'i remanra sex,

Ains prendrai o lui bien et mal.

- Se vous, fait il, por un ceval,

Vous i laisciés de gré morir,

Por fol vous en porrés tenir.

Cevax recouverrés assés,

Mais se vous croire me volés

Vous en venrés ensanle o moi

A ce castel u aler doi. »

Gavains li dist : " Ce est l'estrox ;

Je n'irai pas ensanble vox

Quant mes cevax n'i enterroit. Atre, 852-73

On apprécie aussi les chevaux d'une façon objective : on estime leur valeur et loue leurs qualités. Citons un bref passage de l'Histoire de la France rurale, I, p. 456 : «Un cheval coûtait très cher à l'achat et pour sa nourriture, sans doute trois ou quatre fois plus que le bœuf. Si les grands exploitants ont pu rapidement utiliser ses services, la plupart des manants ne le purent, (...) ».

Les chevaux mentionnés ci-après sont tous d'une très grande valeur. On le constate encore mieux si l'on compare leur prix à celui du cheval maigre qui tire la charrette où a été mis Gauvain (voir p. 293, Lancelot, II, LXVI, 31) :

Este me vos, $\mathrm{u}$ vient del bos

Uns chevaliers achevaucant

Desour un sor cheval bauçant,

Qui bien valoit .c. mars d'argent ;

Si faisoit il, mien ensiant,

Mil livres avec .I. preudome.

N'avoit millor desi a Rome, Rigomer, 7214-20. Cf. ib. 10761-5.

Ung moult fier chevalier qui en vint chevauchant

Dessus ung grant blanchart qui valloit maint besant, Tristan

de N., 9130-1.

Et met la lance le feutre et point

Le cheval qui cent mars valoit, Perceval, 1442-3.

Si garde et voit venir aval

La rue un mult rice ceval

Qui bien cent livres u plus vaut ; Amadas, 4127-9. 
Puis est montez $u$ cheval sor

qui bien valoit un besant d'or. Thèbes, 1821-2.

Be demora feris, li chivaler morust e soun cosyn vendÿ le chival .c. soutz (...) Turpin, 199-200.

Ce dient li roial : « Garnier est li eschas.

Tiex .m. chevaus enmeinne e sejornez e cras

Le pire fust vendus a Pavie .c. mars. » Aye, 2867-9.

Il avoit son cheval molt chier,

Quar .xL. livres valoit, $M R$, xcIV, 75-6.

De lower averez, seint Thomas,

Un cheval qe vandra dis mars! $i b$., XLVIII, 93-4.

Le cheval sur qui sist valoit .xx. mars d'argent, Doon, 4903.

Un ceval i ot l'emperere

qui bien valt por vendre a son frere

deus cens mars d'argent plainnement, Eracle, 1283-5.

et cis i vint si fierement

sor un ceval d'Espaigne sor

qui valt plus de cent onces d'or, ib., 6124-6.

Et li autres vers lui randonne

Le bon destrier d'Espaigne sor ;

Qui valoit .I. trop grant tresor ; Claris, 17595-7.

Il estoit seur .I. destrier vairon que Josias li ot donné qui valoit l'ouneur d'un chastel. Helcanus, 171.

Les mulets peuvent valoir une somme importante :

Et Hermanjart qui le cuer ot irié

Monte en un mul qui .xx. mars fu proisié. Narbonnais, 675-6.

Le jeune Aiol était pauvre et son cheval maigre ne valait pas grand-chose ; plus tard il peut faire don de chevaux précieux :

«Marchegai ne valut que .xiii. sous ou quinse. Aiol, 8044 .

« Li chevals vaut .c. livres c'Aiols li a doné. ib., 7431. 
Laurin présente de nombreux chevaux précieux; les prix indiqués sont soit réalistes soit fantaisistes :

(...) je ne voudroie mie pour .I. cheval de .c. livres que je ne le seüsse, (...) Laurin, 1348-9.

Il sist sus .I. cheval de quoi il ne vausist mie tenir .iiij. cens livres. ib., 2988-9.

Atant hurte li chevaliers qui sires est d'eulz tous le cheval qui bien valoit .iij.[.c.] mars (...) $i b ., 1736-8$.

(...), lors laissa courre le cheval qui bien valoit .j. paÿs, (...) ib., 1768-9.

Marques laisse courre le cheval qui bien valoit .I. chastel (...) ib., 2327-8. Cf. ib., 5230-2; 7342.

Les chevaux représentant dans tous les cas une certaine valeur, ils peuvent servir de mise aux jeux - où on peut les perdre, comme le jeune Gautier d'Aupais :

Si s'est assis au geu a son grant encombrier,

Si a perdu sa robe et son corant destrier : Gautier d'Aupais,

56-7.

Dans Bueve, le jeune prince est prêt à payer presque n'importe combien pour avoir les destrier Arondel, mais Bueve refuse toujours. Le sultan Noradin paie une rançon exorbitante pour son cheval Marchevallée :

Li fieus le roi est avoec acostés,

De lui fu Bueves molt sovent acolés ;

« Bueves, » dist il, « molt boin cheval avés,

Vendés le moi u cangiés u donés,

De Colecestre vous renderai les clés,

Par signorie en arés mil fievés

S'arés cent livres de deniers monaés,

D'or en avant serés mes plus privés,

Devens mes cambres et servis et amés,

Bien ert servis que vaurés amener

Et mal venus qui äir i volés,

Car je sui jovenes et novel adoubés ; Bueve, I, 6294-305.

" Sire, » dist Bueves, « autre cosse rovés,

C'a mon destrier avenir ne pöés ;

Jou n'en prendroie l'onor de cinc chités 
Ne les castiaus ne trestous les fievés ;

Donés me fu, quant je fui adoubés,

Sel me dona Yosiane al vis cler, ib., I, 6311-6.

«Sire, d dist Bueves, « laissiés le plaidier,

Jou nel donroie por plain un val d'or mier ; ib., I, 6397-8.

(...), et pour son coursier Marchevallee que il veult ravoir randra dix pucelles d'honneur, dix faucons, dix espreviers, dix ieunes Sarrazins pour faire baptizer, dix courciers de pris, dix cendaulx bien ouvrez d'ouvrage turquin avecques dix aubers doubles et dix espees. Ogier, 254.

Ceci est valable pour d'autres animaux aussi, par exemple les oiseaux. Mais on voit aussi que les prix varient beaucoup selon les conjonctures : lorsqu'il y a un trop-plein de denrées, p. ex. quand une armée a fait un butin riche, les prix baissent, tandis qu'ils montent en temps de disette :

Illuec ont asis l'esprevier,

Sor une perce tote d'or.

Li espreviers vaut un tresor. Inconnu, 1586-8.

Mès le fossier si avoit mis

En son braeul

.c. et .II. soulz, que il avoit

Receu d'un buef qui cras estoit. $M R$, xLV, 116-9.

- Certes, tu es de bon confort, biax frere; que benois soies tu ! Et que valoit tes bués ? - Sire, vint sous m'en demande on ; (...) Aucassin, xxiv, 63-5.

La veïssiez tante vile brisier,

Tant buef tuer, tante vache escorchier.

Et Loheren en firent fol marchié,

Que .I. vache donent por .vii. deniers,

iiii. moutons por .I. angevin viez. Mez, 2270-4.

Tels quatre pains donent pour un denier.

Assés en ont eu l'un doi chevalier ;

Et por dos sols a on un buef entier. Aspremont, 3462-4.

Un sol pain vendent quinse besans d'or mier

Et d'un molton valt dis sols un qartier. ib., 3469-70. 
Ce vous ai je dit por les Grizois, que il avoient par poi tous leur desirs et meillor esperance de lor henemis conquerre qu'il n'avoient devant eü ne onques mais, mais adonc lor avient grant chierté en l'ost, que un pains i valoit un besans et la char d'un bues dis mars d'argent, si pristrent conseil de porchacier viande. Troie, 177, 13-9.

Del mangier a parler n'estuet,

Car si furent li mes plenier

Con s'an eüst buef a denier. Cligés, 4982-4.

Les marchands profitent des bonnes occasions : il arrive des gens riches dans la ville et immédiatement les prix augmentent :

Et la perdriz vendoit on .j. mengon,

Et la geline .x. solz, o le chapon.

Ainz ne troverent si chiere venoison,

Si chier oisel, char fresche ne poisson,

Tant chier lor fust vendue a desreson,

Qu'il an prisassent la chierté .j. bouton. Aymeri, 2159-64.

Comme les chevaux représentent une grande valeur, on ne s'étonne pas de voir qu'ils étaient convoités par les voleurs. Voir à ce propos tome 2 de l'Histoire de la vie privée, p. 498 : «Les lits se trouvaient dans les chambres (...) et dans leurs annexes (garderobes, voire retraits), et aussi dans certaines pièces de service, surtout les écuries, peut-être pour éviter le vol des chevaux. »

Quatre larrons avoit le bos,

Felons de mal afaitement.

Onques ne sai con faitement

Or ont Lanselot espiié,

Mais cele part sont adrecié ;

Car son cheval vaurent embler. Rigomer, 3350-5.

Puis dist : « Sire proudons, ou yray establant

Mon cheval pour le froit, c'on ne le voit amblant?

Car larrons vont en bois voulentiers conversant.

- Je lo, dist ly ermites, que ceans voit entrant. » Tristan de N., 21223-6.

Les chevaliers aiment non seulement leurs chevaux, mais aussi leurs chiens et leurs oiseaux, c'est-à-dire des animaux qui leur sont indispensables pour la chasse. Il se crée une intimité entre l'homme et l'animal qui se manifeste de plusieurs manières (très rarement par un nom propre du chien) : 
Mais or me dites, mes deus braqués que je tant amoie, les emporta ele avoec li ? Helcanus, 125, 8-10.

- Sire, fait mesire Tristrans, je atent un mien braquet ki est alés ne sai quel part, que je ne laisseroie mie volentiers ariere pour que je ravoir le peüsse, car je l'aim mout. ib., v, 31, 17-20. Cf. ib., vIII, 51, 4-6.

Chiens et oiseauz amait ; Troie, 73, 45 .

A tant courut por l'esprevier ;

Ne cuic k'eüst dusqu'a Pevier

Miels volant, ne dusqu'a Angiers. Violette, 2460-2.

De Brunehaut vous os bien tesmoignier

Que sor son pong tenoit .I. esprevier ;

Onques nus hom ne vit plus bel muier. Auberon, 1534-6. Cf.

Ib., 1554-5;1721-3;1714-8.

Jean Froissart écrit qu'il aime ceux qui aiment leurs chiens et leurs oiseaux ce qui nous fait penser qu'il y en avait qui les aimaient moins :

Et se m'a Nature introduit

Que d'amer par amours tous chiaus

Qui aimment et chiens et oisiaus. Espinette, 32-4. Cf. Méliador, 20441.

On s'aperçoit que les oiseaux devaient être plus appréciés que les chiens (probablement parce que plus difficiles à éduquer (3)) en regardant la carrière de Gerlin : de braconnier, c'est-à-dire valet des chiens, il devient ensuite fauconnier, pour finir sénéchal (vers 347). Ogier est très fier de son fils qui s'y connaît en fauconnerie, comme Richard de Normandie :

Braconnier maistre en fist li rois Pepins.

Les chiens li baille; cil volentiers les prist.

Li dus Gerbers volentiers en servi.

Celui mestier li rois li retoli ;

Fauconnier maistre de ses oisiax en fist. Mez, 340-4.

Quant Ogier fut party d'avecques le roy et qu'il vit que le roy le prenoit en si grant amour, si en fut terriblement ioyeux. Si vit ung espervier en perche, si le voulut manier. Et son pere lui demanda s'il estoit bon faulconnier et il dist que ouy et en tou- 
tes chasses et en tous gibbiers, dont son pere fut plus ioyeulx que devant. Ogier, 65.

Richart sout escremier o verge et o baston,

bien sout esprevier duire et ostor et faucon, Rou, II, 3084-5.

Si les hommes aiment leurs chiens, ceux-ci montrent aussi leur affection pour leur maître. Le lion si affectueux de Gui de Warewic se comporte comme on a l'habitude de le voir chez les lévriers ; il en est de même du cheval fée d'Ogier :

Li leuns errament sus sailli ;

Dunc le comence a esjoir,

Cum un levrer sur li saillir. Gui de W., 4174-6.

Adonc le cheval Papillon fut tres ioyeux, ainsi qu'il le monstroit par ses signes, car il le venoit cotoyant ne plus ne moins que si se fut esté ung petit chien. Si se coucha a quatre piedz (...)

Ogier, 281

Le dévouement de Husdent, le chien braque de Tristan, est remarquable : lorsque son maître a dû l'abandonner, il refuse toute nourriture ; mis en liberté, il cherche Tristan et le trouve, après quoi il l'aide à chasser le gibier. Toute cette histoire s'étend sur les vers 1440-1636 de Tristan; nous en citons quelques extraits :

Parler m'orez d'un buen brachet :

Qens ne rois n'out tel berseret,

Il ert isneaus et toz tens prez,

Quar il ert bauz, isneaus, non lenz,

Et si avoit a non Husdanz.

Liez estoit en un landon.

Li chiens gardoit par le donjon ;

Qar mis estoit an grant freor,

Qant il ne voiet son seignor.

Ne vout mengier ne pain ne past

Ne nule rien q'en li donast ;

Grignout et si feroit du pié,

Des uiz lermant. Dex ! qel pitié

Faisoit a mainte gent li chiens! Tristan, 1440-53.

Tantost com il fu desliez,

Par mié les renz cort, esvelliez,

Que onques n'i demora plus.

De la sale s'en ist par l'us,

Vint a l'ostel ou il soloit

Trover Tristran. Li rois le voit, 
Et li autre qui aprés vont.

Li chiens escrie, sovent gront,

Mot par demeine grant dolor.

Encontré a de son seignor :

Onques Tristan ne fist un pas

Qant il fu pris, qu'il dut estre ars,

Que li brachez nen aut aprés ;

Et dit chascun de venir mes.

Husdant an la chanbrë est mis

O Tristran fu trait et pris ; $i b ., 1489-504$.

Les autres chiens, on ne les estime pas très haut, on les méprise même. Il semble donc tout naturel de les maltraiter, p. ex. en les nourrissant mal ou en les laissant trouver eux-mêmes leur nourriture, et aussi d'employer le terme chien comme une injure. Le pauvre valet qui a perdu un bœuf, ce qui représente pour lui une catastrophe, ne comprend pas qu'Aucassin pleure la perte d'un lévrier : pour lui ce n'est qu'un chien puant :

Je la requis d'amors, car je l'amoie tant.

El n'ot cure de moi ne que d'un chien puant, Florence, 6318-9.

(...) : je vig hui matin cacier en cest forest, s'avoie un blanc levrer, le plus bel del siecle, si l'ai perdu : por ce pleur jou. - Os ! fait cil, por le cuer que cil Sires eut en son ventre! que vos plorastes por un cien puant! (...) Et vos plorastes por un cien de longaigne ? Aucassin, xxiv, 39-43 et 61 .

Un extrait de Gui de B. indique que les chiens ne se laissent pas faire, qu'ils sont toujours prêts à se défendre, ne serait-ce que contre les mouches :

«Véistes onques chien que tant soit deschirés

«Qu'il ne se muet des mousches garantir ne tenser? Gui de

B., 1935-6.

Quant aux classes sociales non nobles, nous trouvons une certaine intimité entre le vilain et ses bœufs, qui lui sont aussi indispensables à lui pour le labourage que les destriers aux chevaliers pour le combat; on a pourtant l'impression que les sentiments du vilain pour ses animaux sont d'un caractère plus utilitaire, que le vilain ne pense guère qu'au profit qu'il peut tirer de ses animaux. 


\subsection{Traitement des animaux}

Les chevaux étant indispensables aux chevaliers, ceux-ci sont obligés de les soigner tant que possible; ce travail est effectué par leurs écuyers et valets, qui les étrillent et épongent, les mettent dans les écuries et leur fournissent la litière, leur donnent à manger et à boire, les saignent, soignent leurs blessures s'il y a lieu, les font ferrer etc. Quand un chevalier ou des seigneurs sont accueillis dans un château étranger, à la cour, chez des amis, dans un couvent, chez un ermite etc., il y a normalement des valets pour s'occuper des montures :

Lors descent a l'osteil, et ung varlet vint la

Que son destrier corrant en l'estauble menait ;

Dou fain et de l'awainne a planteit li donnait. Lion, 21106-8.

Atant les damoiselles prinsdrent leurs chevaulx et les menerent establer, et Gallopin mesmes s'en entremist avecques elles, et firent tant que les chevaux furent bien aisiez et conreés. Bérinus, 495 .

Je voldrai iestre li vostres despansiers Et, s'il voz plaist, g'iere vostre escuiers, Por selles maitre, por roncins aplaingnier, Ne por ferrer de touz les quatre piés.

Quant monteréz, si tenrai vostre estrier,

Vos esperons voz voldrai deschaucier. Jourdain, 2102-7.

Li vallet out les destrers pris,

En une estable les out mis, Yder, 151-2. Cf. ib., 61-3; 605 ; 1570.

Yder avra bon esquier,

La pucele prent son destrier.

Lié l'ad, puis li tolt le frein,

$\mathrm{E}$ an(a)prés le done del fein. ib., 4510-3. Cf. ib., 6004-8.

La ont fait lor chevaus sainnier et ventouser, Lanson, 4746.

Marchegai font ferer et bien sainier, Aiol, 1483.

Dittes à tous noz gens qu'ilz facent ferrer leurs chevaulx, quant il sera heure ; (...) Jouvencel, I, 102.

Aiols est montés ens el solier.

La bele Lusiane al cors legier

Un escuier commande le sien destrier : 
Si l'a trop bien froté et estrillié,

Torchié et abevré et aaisié ;

En l'estable le maine por herbergier ;

Le frain li abati qu'il ot el chief,

Al kavestre de cerf l'a atachié ;

Del fain et de l'avaine a al mengier. Aiol, 2054-62.

La pucele vaillant al cors sené

Del solier avala tous les degrés

Et vint a Marchegai por esgarder,

S'aplanoie ses crins et ses costés ;

Et a un escuier o lui mené :

Garder li fait les piés s'ils sont feré,

Et on le trova bien encor(e) clavé.

Lors s'en torna la bele al cors molé

Et laise le ceval bien ostelé,

Del feure et de l'avaine li done assés. ib., 2133-42. Cf. ib., 227-

$8 ; 997-8 ; 1759-60 ; 6127$.

Un petis garçonés acourt,

Qui mult ert preus et bien apris.

Le cheval a par le frain pris.

D'une part le vergié l'en maine,

Se li donna herbe et avaine,

Sel torce et conroie et fait bien.

$\mathrm{Au}$ cheval ne faut nule rien. Rigomer, 2110-6. Cf. ib., 2557-61.

S'ay de l'avaine trente aissins,

Et du mien pré bon faing novel. » Galeran, 6734-5. Cf. ib.,

6791.

Il a fait les chevauz tot de novel ferrer,

De coi il porront bien an lor païs aler. Parise, 2919-20.

Done moi une couverture

De qoi tu cuevres ton cheval, $M R$. v, 308-9.

Et.v. au fain, tout sans l'avaine,

Et .v. à la litière sainne,

C'on mist desous vos .II. chevaus ; $i b .$, xxxIv, 379-81.

Et puis penserent des cevaus au miex qu'il le porent faire, puis regarderent lour plaies et les bendent et les estraignent au miex qu'il pueent. TP, vI, 7, 52-4.

Si descent, et il s'a pene

$\mathrm{Au}$ miex k'il pot a lui aidier 
Desarmer et fait estoier

Son cheual a un grant uilain,

Noir cenu et de noir pelain,

Ki en l'estable le mena

Et le frain tantost li osta

Et la siele, et le do li frote

De l'erbe et apres de sa cote,

Cil se met a paistre, (...) Espees, 3742-51.

Atant .IIII. escuier descendent

Du pales, dont li dui entendent

Au corpois Claris desarmer,

Li autre dui a l'establer

Son cheval ; assez li donerent

Foin et avaine, qu'il troverent,

Et li font bonne litiere

Et par devant et par derriere. Claris, 10509-16. Cf. ib. 17925-7.

Molt veïssiez as escuiers

Mener palefroiz et destriers,

Fere estables, peissons fichier,

Chevaus mener, chevaus lier,

Chevaus forbir et abevrer,

Avoinne, fuerre, herbe porter. Brut, 1801-6.

Uns varlés est vers lui venus,

Qui volt son ceval establer. Couci, 430-1.

Dedens une marescauchie

Li ont son cheval establé,

Si li donnent avainne et blé ; Violette, 5087-9. Cf. ib., 1557-61 ;

2489-93.

Li vallet devant ces estables

Torchent les chevax et estrillent, Erec, 358-9.

« Bele douce fille, prenez

Cest cheval et si le menez

En cel estable avec les miens.

Gardez que ne li faille riens,

Ostez li la sele et le frain ;

Se li donnez avoinne et fain,

Conreez le et estrilliez,

Si qu'il soit bien aparoilliez. » ib. , 451-8.

Tant le garde qu'il [l'ot] ml't bel

Et ml't gros de fainc et d'avaine. 
Il [1']avoit [fait] sainnier de vaine,

Et des costés et des pastures.

En mains d'un mois de ses pointures

Fu tous garis et respassés. Escoufle, 6668-73. Cf. Galeran, 639.

Voir aussi : Cont. P., II, 7116-7 ; Rigomer, 2505-8 ; 10467-70 ; Amadis, 2693-5 ; Aymeri, 3911 ; Otinel, 737-8 ; Cleomadés, 9083-9 ; Aliscans, 594-5 ; 2723-5 ; 3818-9 ; 4263 ; Floriant, 6905-8 ; Huon, 8024 ; Inconnu, 6059; Durmart, 6612-4; Queste, 81, 26-7; 133, 7-10 ; Charrete, 2530-1 ; Gautier d'Aupais, 24-6.

L'Escoufle présente une scène analogue de la vie roturière :

Et la meschine a le mul pris

Ki jadis ot de .c. le pris.

Ml't par estoit cortoise et franche ;

Ele le maine ens en la granche,

Le frain li oste, si le lasce,

Puis l'a liié a une estace

D'un chevestre qu'il ot le chief :

Ne l'alonga pas jusc'au chief

Por son harnas qu'il ne malmete.

Ne cuidiés pas que ne li mete

Du fain devant lui a fuison. Escoufle, 5011-21.

On soigne aussi les chevaux avant de partir pour la guerre et pour le voyage :

Cez fers de ces espiez an frainsnes anhanter

Et ces chevax de garde torchier et conraer! Saisnes, 797-8. Cf. ib., 802 .

Li chevals fu bien enfernés

Et bien estrains et bien çainglés ; Durmart, 1383-4.

vint Guillame, le filz Osber,

son cheval tot covert de fer. Rou, III, 7489-90.

Lors eslit li rois de tous ses varlés chelui ou plus se fioit et li dist que il gart que rien ne faille en son ronchin, car chevauchier li couvenra encor anuit. Lancelot, vII, Ia, 14.

Ses chevaus fu tost estrilliés,

Car li forestier li aida. Méliador, 19933-4.

Aiol présente le cheval Marchegai exceptionnel qui n'aime pas les autres chevaux : 
"Che[s] chevaus vos convient a departir :

"Atachi[é]s Marchegai desous cel pin

"Qui mort et brait [toujours], fiert et henist,

« Ne peust autres chevaus lés lui sofrir. Aiol, 4909-12.

Les chevaux de bataille mènent une vie dure et dangereuse : les cavaliers les éperonnent pour les faire courir vite, les adversaires les renversent, les blessent, les tuent parfois :

Tant ot corut tot le jor l'alferrant

Ne puet aler, sos lui va recreant ; Aspremont, 5750-1. Cf. ib., 1890.

Soz Aymon de Dordone ont mort son auferrant ; Renaut, 2734.

Bayart s'en vet clochant, bien resenble espiné. $i b ., 5115$.

En contreval vers terre va li cous descendant,

Par de devant les jambes consuï l'auferrant,

Le col li a tranchié et l'espaulle ensemant.

Li chevaus chiet le pré, Danemont vait chéant. Gui de B.,

2654-7.

Girard s'en turne par mi le coin d'un tertre ;

Cinc liwes trove tant encunbree presse

Que unc n'alad un sul arpent de terre

Qu'il n'abatist Sarazin de la sele,

$\mathrm{E}$ qu'il ne trenchad pé u poig u teste.

E quant il issi de la dolente presse

Sun bon cheval li creve suz sa sele.

Del dolent chanp quant Girard fu turné

Desuz ses alves est sun cheval crevé. Guillaume, 696-704.

Des esperons a hurté l'auferrant, Otinel, 1648. Cf. ib., 1657.

Des espuruns point l'auferant,

qu'il en fist raer le sanc ; Gormont, 16-7.

An trante leu navrere l'aferrant,

Li chevals chiét, Ainmeris an desant ; Enfances G., 407-8.

Sanglanz est li chevaus, la sele de Sardaine ;

Au bon courant destrier fu faillie l'alaine : Barbastre, 94-5.

Et quant le duc Naymes vit que il n'y avoit remede que de fuyr si hurte son destrier des esperons par telle maniere qu'il vint courir sur Dannemont par tel effort que ainsi qu'il vint pour 
aprocher de lui le cheval de Dannemaont eut si grant paour que il se leva sur les deux piedz de derriere tout droit tellement que le duc Nayme perça le cheval oultre et getta homme et cheval par terre. Ogier, 21.

Et li ceval keurent de grant radeur, plus que mestiers ne fust, car il se ferirent des testes et des pis ensamble si durement qu'il caïrent mort a tere. TP, vII, 148, 27-9. Cf. ib., 44, 9-11.

Et li rois, ki boins cevaliers ert et hardis durement, li adrece la teste du ceval et li vient au ferir des esperons, bruiant conme foudres, et le fiert en son venir si durement k'il abat lui et le ceval a la tere. Et mout fu Dynadans debrisiés de cel caoir, car li cevaus caï sour lui, et nonpourquant il s'en releva mout tost. Ses cevaus s'en voloit fuir, mais il ne pot, car li esquier ki illuec estoient le reprisent tantost. $i b ., \mathrm{v}, 95,28-35$.

Et bien paroit k'il avoient venu grant oirre et a besoing, car lour ceval estoient caut et tressué. ib., v, 285, 6-7. Cf. ib., II, 116, 5-7 ; III, 8, 4-6.

(...) ; si apoingnoit sus .I. cheval grant et fort et il ert ja navrez en la teste et en l'espaule, si en est touz sanglanz; et ses chevaux n'est pas si sains qu'il n'eust a tout le mains .xx. plaies et qu'il n'eust ja esté feruz de .II. glaives ou de .III., que il en clochoit en la jambe senestre, se ne fust cil qui desus lui estoit que le destraingnoit des esperons aguz et le faisoit aler grant oirre a fine force. Lancelot, v, xcv, 35. Cf. ib., IV, Lxx, 17.

Li espee cheï par dessus l'auferrant,

La teste li trencha sans riens de remanant. Bâtard, 733-4.

Ez vous un chevalier, de l'esploitier se paine

Et fu navrés ou cors si que moult forment saine ;

Ses chevaus ert navrés d'un espiel parmi l'aine. Buevon, 147-

9. Cf. $i b ., 414-5 ; 2606-8$.

Si tres radement est jus dou hiaume avalee $\mathrm{K}^{\prime} \mathrm{au}$ cheval a la teste en deus moitiés coupee,

Tout abat en un mont seur l'erbe defoulee. $i b .1729-31$.

Bien sambloit qu'il eüst son cheval trop hasté,

Car forment li batoient li flanc et li costé ; ib., 2068-9.

Il estoit bien montez ; ausi estoit Lancelos ; si conmence en tel maniere la chace entr'eus deus qui dura jusqu'en la forest bien 
en parfont demie liue ; et lors recrut li chevax au conte et chiet morz desouz lui. Mort Artu, 198, 39-43.

Parthonopeu voloit ferir,

Mais ne l'en puet si avenir ;

Trop a son espié bas porté,

$\mathrm{Si}$ a en la teste asené

Le cheval noir desos le[s] iex,

Ens le cervel met les escuex ; Partonopeu, 3061-6.

Desos les iex fiert le cheval

Que le musel enporte aval,

Et ne remaint por le coliere

$\mathrm{Qu}$ 'en la goule le brant ne fiere.

Li chevaus chiet et Sornegurs ; ib., 3145-9

Puis chace Macabré par puis et par montaigne ;

Et cil fuit o Morel qui en suour se baigne, Partonopeu-C,

1896-7.

Ne sai que vos celasse, tant est la chose alee

Que Naymes de Baviere est a pié an la pree,

Sor l'escu ambrunchiez, si a traite l'espee ;

N’a mie de cheval, que la teste a copee. Saisnes, 7554-7.

Voir aussi : Durmart, 4696-8 ; Gui de W., 11154-7 ; Godin, 1789o1 ; Laurin, 4132-4 ; Aymeri, 1789-91 ; 2826-8 ; Turpin, 254 ; 323-4 ; Mort Aymeri, 922 ; 2312-5 ; Rou, III, 8782 ; Narbonnais, 4751-3.

Dans Cristal, le cheval du protagoniste mord et tue la monture de l'adversaire :

Et Passevent sailli avant

Et prist par le test l'auferrant.

As dens, qu'il ot et gros et fort,

Le destraint par si grant esfort,

Que la teste tot li esmie

Si com une pome porie. Cristal, 3927-32.

Le destrier de Lancelot est en si mauvais état après la bataille qu'il n'est même pas capable de manger mais reste exténué devant la mangeoire :

(...) ; si alerent tout avant en l'estable et voient le cheval qui avoit plaié la teste et le col et le pis et les jambes, et li os li pe- 
rent en plusor liex et se gisoit devant se maingnoire a moult malvaise chiere, que il ne mengoit ne ne bevoit. Lancelot, viII, La, 3 .

On ne se soucie pas toujours d'un cheval blessé ou tué ; il y en a normalement assez pour le remplacer (cf. p. 230) :

Sire, ja ne voz esmaiez

de vostre cheval, s'il est morz ;

car ceanz a chevax bien forz ;

tant voel que vos aiez del nostre :

tot le meillor, an leu del vostre, Charrete, 2974-8.

Pour punir sa femme qu'il croit adultère, un gentilhomme ne donne pas à manger à son palefroi à elle; le texte indique que l'on traite parfois ainsi un cheval emprunté :

Del palefrois li fu avis,

Tant estoit maigres et chetis,

Qu'il fust en males mains keüs.

Bien traveilliez et mal peüs

Sambloit que il eüst esté,

Si come on fait cheval presté,

Qui le jor est bien traveilliez

Et la nuit est mal aesiez.

Autel del palefroi sambloit :

Tant estoit maigres qu'il trambloit

Einsi com s'il fust enfondus. Perceval, 3695-405.

Un peu en contradiction à ce que nous avons affirmé à la page 227, et malgré tous les exemples de chevaux appréciés et aimés par leur maître, nous constatons donc que d'autres exemples semblent indiquer que cet amour est basé en grande partie sur le profit que l'on tire des animaux, que l'on y pense peut-être plus comme à un moyen de transport etc. que comme à un être vivant.

Dans Couronnement, Guillaume cherche des guerriers - même les plus pauvres qui ont de mauvais chevaux mal ferrés :

Et tuit i vieignent li povre bacheler,

A clos chevals, a destriers desferrez, Couronnement, 2256-7.

Nous venons de le voir: aux combats, les chevaux travaillent beaucoup et suent extrêmement ; on recommande aux chevaliers de les laisser se reposer parce qu'ils en ont besoin : 
De la fumee des chevauz

Torble li airs et li solauz ; Durmart, 8075-6.

Esventez voz chevaus que si avez laidie ; Barbastre, 6739 .

Dist l'emperere : « Tens est del herberger ;

En Rencesvals est tart del repairer.

Noz chevals sunt e las e ennuiez.

Tolez lur les seles, lé freins qu'il unt es chefs,

E par cez prez les laisez refreider. » Roland, 2482-6.

On les ménage aussi avant les batailles, car on sait qu'un cheval fatigué ne pourra pas tenir aussi longtemps qu'un cheval reposé :

Quant il orent ordenez lor homes en tel manniere com il virent qu'il le convenoit, si s'en tornerent cele part ou la bataille est et chevauchent par loisir por lor chevaux, qu'il ne fussent lassez au besoing. Lancelot, vI, cIV, 29.

Et si tost conme la nuit fu meslee au jor, tuit li .IIII. roi se firent armer et firent .IIII. mile homes fervestir et ne monterent mie en lor chevaux, por ce que il ne les i trovassent mie trop las au besoing, ainz les tindrent par les frains, chascun le suen delez lui, (...) ib., vI, CIV, 56.

Quant il orent mengié, si se misent au chemin et chevauchierent tout le jour belement et par loisir, pour ce que lor cheval ne fuissent trop las, de quele ore qu'il venissent a la bataille.

Mort Artu, 176, 50-3.

Un petit extrait de Perceval attire l'attention sur une différence entre la manière des chevaliers de faire avancer leurs chevaux et celle d'autres cavaliers : le jeune protagoniste récemment adoubé ne sait rien de l'utilisation des étriers et des éperons - jusque là il s'est servi de la cravache - comme la pucelle dans Laurin:

Puis li met le pié en l'estrier,

Si le fait monter le destrier.

Ains mais estriers veü n'avoit

Ne d'esperons rien ne savoit

Fors de cinglant ou de roorte. Perceval, 1185-9.

Lors fiert la pucelle son pallefroi des escorgies et s'en retourne aussi comme elle estoit venue. Laurin, 11584-5. 
Jean Froissart nous apprend qu'aux tournois il y a une odeur très forte provenant de la respiration des chevaux et des crottins l'air en est empesté :

La sont si grandes les alainnes

Des dis chevaus et les fumieres

Que ce samblent droites pourrieres,

Qui en l'air volent contremont. Méliador, 6818-21.

Les chevaux qui ont perdu leur maître, tué, blessé ou en fuite, quittent le champ de bataille, ou ils sont saisis par les guerriers triomphateurs ou par leurs valets. Ils constituent une partie importante du butin, et souvent les chevaliers en font cadeau à leurs amis et à leurs écuyers :

Li cevax saute sus, si s'aroute

Tout le cemin par u il vint. Atre, 288-9.

Le destrier saut et va sa voie,

Au travers parmi le forest.

A G. merveille desplest,

Car ne se sot pas conseillier ;

Qu'il ne veut pas seule laissier

Pour nul besoing la damoisele.

Le destrier traïne la sele,

Fuiant s'en va mout effraés ; ib., App., 644-51.

(...), et meint bon cheval corre tout estraié parmi le champ, qu'il n'estoit qui les retenist ; Mort Artu, 181, 47-9. Cf. ib., 151, 8-11.

Et li chevax sanz demoree

S'en vait fuiant, teste levee,

Vers les loges, et cil le voient

Qui en lor tentes se levoient, Perceval, 4269-72. Cf. ib., 4317-

21.

La poïssiés veoir froissier heaumes et esquarteller escus et chevaliers cheoir mors de lor chevaux et destriers aler sans leur meistres par mi le sablon. Troie, 29, 9-11.

Lors s'en va le cheval fuiant

Tout droit as dames hanissant ; Lycorne, 5532-3.

Lors se part sus ceste matere

Et chevauce apriès le cheval

De Corbilier qui en .I. val 
Paissoit et estoit arrestés. Méliador, 13676-9.

Meint cheval curt par cel estur,

Estraer void de sun seignur. Ipomedon, 4829-30.

Biaus palefroiz et bons destriers

Par les chans aler estraiers. Brut, 4413-4.

Chevals et muls, vaiseus ovrés,

Que li Romain orent covrés. Diable, 2097-8.

Antor que Baudoïns ot conqis l'auferrant,

Ez la rote des Saisnes a esperon brochant. Saisnes, 1591-2.

Je vois eschargaitant un povre soudoier

Qui ça outre est passez por roncins gaaignier ; $i b ., 3604-5$.

Monter le fist sor un cheval conquis,

Dont la ot maint, noir, sor et blanc et gris,

Parmi les chans fuians, ça cinc, ça dis. Enfances O., 6494-6.

« U est li grans esciés que mener soloies,

« Li mul, li palefroi et li corant destrier, Aiol, 6687-8.

Voir aussi : Orson, 1480-1 ; Narbonnais, 6952-3 ; 7570 ; Mort Aymeri, $1205 ; 2190$.

Aliscans nous présente une scène où le guerrier Renaut, furieux d'être tombé du cheval, frappe celui-ci très fort ; un nain veut punir le jeune chevalier Hector en frappant son cheval :

Onc n'en sot mot, si chaï par derriere ;

Si li chaï sa grant perche pleniere.

$\mathrm{Li}$ ber se tint a la queue derriere,

Et li chevaus le trait par la poudriere.

Onc ne fina si qu'an une riviere ;

Iluec lessa Renoart en l'ortiere.

Quant Renoart fu a terre chaüz,

Plus tost qu'il pout est en piez resailluz.

Le cheval prist, quar mout fu irascuz.

D'un de ses poinz l'a si .II. cox feruz

Que li chevaus est a terre cheüz. Aliscans, 6374-84.

Lors jete les mains, si aihert Hector au fraim et li vaut crachier en mi le vis et fiert son cheval d'un baston que il tient par mi la teste si que il l'abat a jenols. Et lors fu Hectors moult dolans, car il amoit moult le cheval (...) Lancelot, viII, LXIa, 98. 
Un extrait d'Aiol indique qu'un cheval dont on a coupé la queue est dédaigné. Plusieurs extraits de Lancelot présentent des chevaux dont on a coupé la queue, le toupet et les oreilles (cf. p. 293). Les chevaux en question sont mauvais (maigres, mal équipés, vieux) et, en combinaison avec d'autres manières de se comporter, une personne peut se servir d'une telle monture pour exprimer un état d'âme : chagrin et humilité chez la damoiselle de Honguefort :

Mais li destrier[s] Makaire est si menés

Qu'il ne se peut movoir ne remuer :

Par gas i sont venus cis baceler,

Sel vont batant de fust et de tinés,

La coe li manachen[t] a recoper

Et recreant ronchi l'ont apelé. Aiol, 4363-8.

(...), ne ne vestirai de robe qui enverse ne soit, ne ne chevalcherai cheval qui n' ait la coe coupee et le top, ne n'avra frain en teste fors .I. malvés chevestre de cordele. Lancelot, II, XLVI, 5 .

Les autres chevaux - et les mulets - aussi peuvent être fatigués, soit qu'ils ont été surmenés par leurs cavaliers, soit que ceuxci n'ont pas eu le temps de les soigner convenablement. Nous voyons pourtant le jeune Durmart et Lancelot interrompre leurs voyages pour permettre à leurs chevaux de se reposer :

Atant a li Galois veü

Un vallet qui tot a pié fu,

Si ot devant lui atachié

Son chaceor tot estanchié. Durmart, 4153-6.

Icele nuis giut li Galois

A la maison d'un vavassor

Qui li porta mout grant honor,

Et l'endemain i sejorna

Por son cheval qu'il reposa. ib., 8940-4.

Si a tant alé, d'ire et de courouz forsenez, qu'il vint a .I. haut tertre. Lors resgarde son cheval, si le voit si baingnié de sanc et de suor dou travail qu'il avoit fet que, s'il ne fust de si grant force, il fust pieça morz. Lors s'areste, car bien pense que cil ne l'ataindront meshui ; si resgarde souz lui et voit .II. pavillons tanduz desouz .I. orme, et delez avoit .I. loge galesche qui est fete de nouvel. Et il torne cele part son cheval, car grant mestier avoit de repos, si descent et l'atache a l'arbre, si tost com il est descenduz ; Lancelot, v, xcIX, 28. 
Aprés cest mot n'i atant plus Lanceloz, ainz broche le cheval des esperons aguz et tranchanz. ib., v, XcI, 13. Cf. ib., IV, LXXVI, 32 ; VII, IXa, 16 et passim.

(...) che fu le semaine de la mi aoust, si furent grans et parfondes les creveures et li chevax ne fu mie fres, car il ot alé grant jornee : si s'encombra des piés devant et caï en unes crevaches moult grans. $i b .$, vII, xxxIIa, 9.

Chiés .I. ostel lor cheval erent,

Qui mout lassé sont et estrait. $M R$, LXxxIx, 772-3.

Estes vous, poignant à droiture,

.I. vallet par mi une rue,

Son cheval d'angoisse tressue

Qui molt venoit à grant esploit. ib.,LV, 108-11.

La mesnie le roi s'est trop abandonnee -

Il sunt trop tost venu toute une randonnee -

N'i a si bon cheval n'ait la croupe escumee. Gui de N., 1401-3.

Si chevauchoit plus fort qu'oissiaus ne vole a vent,

Et plus tost c'uns bougons d'arc manier ne destent,

Et tant que le cheval fist es costez sanglent,

Dont sanc de toutes pars a la terre en descent.

Plus tost va li chevaus que foudre[s] avec vent. Brun, 63-7. Cf. $i b ., 124-7$.

Et dous messages poignant toz abrivez ;

De France vienent, s'ont lor chevals lassez

Et recreüz, confonduz et matez. Couronnement, 1384-6.

Partonopeus a tant chaciet

Que son ronci a estanciet ; Partonopeu, 643-4.

Tant erra k'il vint a Nevers.

Tout estoit de suour couvers

Li chevaus, tant ot eüt caut ;

Mais au messagier pas ne caut, Violette, 754-7.

Nos n'i prenons chastel ne n'assaillons defois,

Mout i a de tes homes malades et destrois,

Cheval sont amaigri et mul et palefrois. Saisnes, 1804-6.

Voir aussi : Queste, 1, 5-7 ; Charrete, 279-81 ; 296-8 ; Yvain, 5050-1 ; Protheselaus, 7635-9 ; Raguidel, 387-93. 
Guillaume et Aélis, dans l'Escoufle, interrompent leur voyage pour se reposer. Guillaume enlève les mors à leurs mulets mais leur met des entraves pour les empêcher d'aller trop loin :

Il a osté as muls les frains

Et enpasturés des chevestres. Escoufle, 4432-3.

Ele vient au mul, si deslace

Le chevestre dont ses amis

L'ot empasturé, puis ra mis

Son frain et tot apareillié. ib., 4746-9.

Il peut d'ailleurs arriver un accident comme à la monture du pèlerin, dans l'Escoufle, qui a marché sur un clou, ou à celle de Gauvain qui a perdu un fer; ou bien les cavaliers entrent dans un mauvais terrain, comme nous le voyons dans Tristan:

.I. jor passoi[en]t par devant

La maison pelerin françois :

A .j. de ceus, qui poins[t] anchois

En sa maison por ostel prendre,

.I. clous le fiert parmi le tendre

Del pié de la chevauceüre.

Ice fu grant malaventure

Et maleürtés et pechiés.

Li chevaus est si entechiés

Qu'il ne puet en l'ostel entrer.

$\mathrm{Al}$ marescal le fait mostrer,

Qu'i[1] veut savoir ce que peut estre.

Fait il : « Esgardés, sire mestre,

Com mes chevaus cloce orendroit. »

Il esgarde, si voit tout droit

Le clau en pié qui ml't est lons. Escoufle, 6626-41.

Et chaça tant que a bien pres

Le retenist et aretast,

Se ses chevax ne desferrast

D'un des piez devant tot a net.

Et mesire Gavains se met

Aprés son harnas a la voie,

Qu'il sent son cheval qui tendroie

Soz lui, si l'en anuie trop ;

Mais il ne set qui le fait clop,

S'estos le pié feru ne l'a.

Tantost Yvonet apela,

Si li comanda a descendre

Et de son cheval garde prendre, 
Qui molt cloche tres durement.

Et cil fait son comandement,

Tantost li lieve un pié en haut

Et trove que uns fers li faut,

Et dist : « Sire, il l'estuet ferrer ; Perceval, 5682-99.

A plain erre, chemin et sente,

Li chevalier vienent aprés.

Mot a grant presse en cel marchés ;

Esfondré l'ont, mos est li fans.

Li cheval entrent jusq'as flans,

Maint en chiet, qui que s'en traie. Tristan, 3668-73.

Mais la vie est dure pour d'autres catégories aussi. Citons un passage de Jean-Pierre Leguay, op. cit., p. 50, qui montre comment l'étroitesse et la sinuosité de la majorité des rues médiévales rendent la circulation en ville difficile et cause de fréquents accidents : "Le croisement des véhicules ou même des animaux de bât est difficile. (...) Des chevaux fougueux ou vicieux, blessés par les pointes qui traînent partout, deviennent brusquement agressifs, se battent en pleine rue, blessent leurs maîtres ou des passants. On cite le cas d'une rosse nantaise qui "cheyt en la dove", à la sortie d'un pont fréquenté, avec son conducteur et son chargement, "en ce combattant o les autres chevaulx et en mourit" "

Dans un monde bien différent de celui des destriers, les bêtes de somme ont des coups de fouet, les ânes sont habitués au travail fatigant et au mauvais traitement, les bœufs reçoivent des coups pour les pousser au travail :

Es les somiers par la voie bastue.

Li mesagier si ont chascun tenue

Une corgiee en sa main tote nue. Narbonnais, 860-2.

Mes Tiemers, qui est dure beste

et qui trop mal endurer puet,

ne se remue ne remuet. Renart, IV, 10942-4. Cf. ib., I, 1066-7 ;

IV, 10820-3.

« Ne faire nul desroi par ceste sale :

« Ja t'aroi[e] batu si com un asne. » Aiol, 4017-8.

Et li asne esturent tuit quoi

En mi la rue volentiers,

Quar l'asne n'est pas costumiers

$\mathrm{D}^{\prime}$ aler se l'en nel semonoit. $M R$, cxIV, 22-5. 
Dont prent la forche qu'il portoit,

A quoi il les asnes chaçoit : ib., cxIv, 37-8. Cf. ib., cxxxv, 66-7.

L'aguillon au buef poindre

N'i doit estre oubliez, $i b .$, XLIII, 144-5.

Un extrait de Wistasse décrit une scène où un chevalier essaie de faire avancer ses deux chevaux : il les frappe et leur crie et les insulte. L'extrait nous révèle par ailleurs les mots que l'on employait pour faire avancer les chevaux :

Ses chevals commenche a ferir,

Et il reculoient d'aïr.

Diu commencha a renoier

Et ses chevaus a menechier :

« Hari ! Martin! hari ! Fauviel !

Por les boiaus, pour le cerviel!

Huët ! avant vois, por les dens !

Pour poi que tous ne vous cravens.

Hari ! viels jumens estaïe,

Jamais de vous n'avra aïe. » Wistasse, 198-207.

Il est certain que les chiens étaient souvent mal traités (et mal nourris). L'emploi fréquent des termes chien, mâtin etc. pour insulter des adversaires corrobore le fait :

Mais de Lovel mie ne set,

Son bon ami, son compaignon

Que batu ot com un waignon

Dans Gousselins et traïné Guillaume d'A., 1494-7.

Elle ne croit Mahon niant plus c'un chien poris. Lion, 2841.

$\mathrm{Au}$ retour le ferai encruer con mastin. » Bâtard, 224. ib., 138-9.

Dans l'Histoire de la France rurale, I, p. 438, nous lisons que « paysans et vassaux pouvaient être tenus de loger et nourrir les chiens du maître chassant dans les parages. "; ces chiens étaient sûrement vus d'un mauvais œil par les vilains. Deux petits extraits de Gui de $W$. montrent que la vie de ces chiens de chasse n'était pas sans danger :

En un grant forest entrerent,

Un fier sengler i troverent,

Lur chiens tuz i descoplerent,

A grant esforz le chascerent.

Li sengler s'en va tost fuiant, 
Des chiens i fait damage grant,

Plus de cent en ad ocis,

Tost ad trespassé le pais ; Gui de W., 6817-24.

De tuz les chiens quil siweient

Recruz e occis esteient

Fors solement treis que bons sunt, $i b ., 6833^{-5}$.

A la guerre, les guerriers n'épargnent rien, comme le montre l'extrait suivant de Saisnes, qu'Annette Brasseur traduit ainsi (p. 750) : «"Ils ne trouvent bœuf ni vache dont il puisse être question par la suite", c'est-à-dire "ils tuent tous les bœufs et toutes les vaches qu'ils rencontrent." » :

Si com la rote dure est li païs desers :

Ne truevent buef ne vache que n'an soit bone pes, Saisnes, 836-7.

Buevon montre que les bergers rassemblent autour d'eux leur troupeau lorsque les loups sont dans les parages :

Moult le fait bien Guillaumes et li quens Aymeris,

Entour aus se ralient li chevalier de pris

Com entour le pastour font pour le leu brebis. Buevon, 492-4.

Si samble que il aient lor gent en garde pris

Conme li pastour font pour les leus lor brebis ; ib., 1599-600.

A la ferme, les animaux vivent assez librement ce qui cause parfois des dégâts que les hommes essaient d'empêcher ; dans l'extrait suivant nous voyons les comportements caractéristiques des porcelets et des poules : les uns fouillent, les autres grattent :

On leur aporte pute estrine,

.I. porcelet et .II. gelines.

Par chou perdront il leur cambrel

Que leur parens leur a presté ;

Li pourcelès i va fouant,

Les gelines i vont gratant,

Li boine fame les en cache,

Si les hue et si les manache ; $M R$, xLIV, 311-8.

Il n'y a certainement pas lieu de souligner une fois de plus le danger que courent les habitants de la basse-cour : le Roman de Renart nous présente une foule d'exemples où le renard s'offre un bon repas de poules, de poulets etc., comme dans ceux-ci : 
il avoit ja garni sa fouse

d'une geline grasse et grosse

et avoit mangié au matin

une cuise d'un gras poucin ; Renart, I, 503-6.

Renart, qui tant aime gelines,

de .II. chapons se rest dingnez

puis est ou cloitre retornez.

Les .III. menja que nus nel sot ; $i b .$, III, 8748-51.

Dans le même roman, nous voyons comment les paysans essaient de protéger leur propriété :

Les le bois avoit un manoir ;

la un vilains soloit manoir

qui mout avoit cois et jelines.

Renart en fist tes deceplines

que bien en menja plus de .xxx. ;

toute i a tornee s'entente.

Li vilains fait Renart gaitier ;

ses chiens avoit fait afaitier ;

ou bois n'avoit santier ne triege

ou il n'eüst cepel ou piege,

ou trebuchet ou laz tandu,

ou roiz ou raiseul estandu ; Renart, III, 6427-38.

Un seul exemple, de Roussillon, montre que les moutons ont des poux : nous pensons que c'est vrai aussi pour d'autres animaux :

E no le preizerie un berbegal, Roussillon, 4498.

Deduis nous montre comment on essaie de garder les oiseaux de chasse en bonne santé : on leur fait avaler des plumes pour les faire vomir et curer ainsi leurs intestins. Le même texte parle des vers qui, malgré toutes les précautions prises, attaquent les oiseaux :

Estoupes, drapeaux ne coton

Ne donnez pour cure au faucon,

Maiz plumez, jointes li donnez

D'oiseaulx et son droit li ferez,

Aussi le pié de lievre est bon

Aucune foiz pour le faucon, Deduis, 7399-404.

Il dit que mourir les convient

Quant le mal d'aguilles leur vient.

Je li confesse qu'il dit voir, 
Car on ne puet l'aguille avoir,

Pour ce qu'elle est haut en l'eschine, $i b ., 6927-31$. Cf- $i b$., $6788-9 ; 6943 ; 6977 ; 9673$.

\subsection{Nourriture des animaux}

Que mangent les animaux ? Plus haut, nous avons vu que les chevaux et les mulets se nourrissent d'avoine et de foin ; d'autres textes mentionnent aussi l'herbe des champs, l'orge et le froment:

Et montent es chevaux corans et abrievés :

Ne menjuent de fain, d'avoine ne de blés,

For que herbe de chans, d'araines et de prés. Gui de B., 809-

11.

(...), car li esquier de Dynadant avoient apareillié le cheval de Palamidés k'il avoient trouvé paissant en une mareschiere.

TP , IV, 92, 18-20. Cf. ib., vI, 133, 47-50 ; VIII, 166, 4-8.

Et ses chevax ot de l'estrain

Et de l'orge un bachin tot plain. Perceval, 6505-6. Cf. ib., 3071$2 ; 3477$.

Defors le mur erbergent, lonc la taillade,

E donent as chevaus erbe e ssivade. Roussillon, 1219-20.

Quel dus a comandat qu'al rein non cost,

Mais por fuerre a chevaus herbe l'an brost ; $i b .$, 9268-9.

Je croi que d'avaine et de fain

Il puet bien avoir eü fain,

Li bles li a petit cousté ! Meliacin, 2057-9.

Du palais si le desarmerent

Et son cheval li establerent,

Si li donnent foing et avaine Floriant, 2071-3. .

Fainc et avainne as chevaus li otroie. Gaydon, 3967. Cf. $i b$., $3997-8$.

Destrier n'i ot forrage, s'il n'i fu aporté ;

Tote nuit pesent l'erbe qui fu aval le pré. Barbastre, 4076-7. 
Marchegai son destrier li establerent,

Trés en mi le maison li assenerent.

De l'avaine et del feure se li don[e]rent : Aiol, 778-8o.

Et ses cheuaus, k'il n'ot pas prest

Ilueque auaine ne fain,

Peut l'erbe et il li ot le frain

Oste, por bien deliurement

Paistre ; (...) Espees, 3552-6.

Vaches et beus et chevals aherbés ; Bueve, I, 5113.

Avaine aportoit as chevax

(...)

Et leur cheval l'erbe paissoient Jehan, 3013 et 3023. Cf. ib., 3578-9.

Un petit passage de Laurin nous apprend que l'avoine est préférable à l'orge comme nourriture des chevaux. Un autre passage du même texte montre un cheval mangeant du pain :

(...) por mon cheval vous requier je que se vous avez avainne ne chose qui bonne li soit par amours que vous l'en donnez, et je vous en savrai moult gré. - Sire, d'avainne n'ai je pas, mais d'orge ai je assez çaiens, si l'en donrai moult volentiers. » Laurin, 11923-7.

Mirador si avoit pris .I. pain grant et merveilleuz de la ou il venoit. Il l'a trenchié par menus morsiaus par dessus son escu et a fait mengier a son cheval, qui grant mestier en avoit. $i b$., 9562-4.

Gauvain et Hector qui, avant de se coucher, avaient laissé leurs chevaux paître l'herbe autour d'une chapelle doivent les chercher le lendemain matin :

Puis ostent a lor chevax les frains et les seles et lessent pestre par la montaigne. (...) Quant li jorz fu venuz, il alerent veoir ou lor chevaus estoient, si les quistrent tant qu'il les trouverent ; (...) Queste, 148, $32-149,1$ et 151, 23-4.

Voir aussi : Floovant, 992-3 ; 998-1000 ; Roussillon, 663-4 ; Cont. P.,I, 3140-5 ; 4098-9 ; II, 7110-2 ; 8456-8 ; 9150-1 ; III, 14141-5 ; Rigomer, 282-3 ;Couci, 4369-72 ; Renaut, 3138 ; Parise, 1332-3 ; 14404 ; Galeran, 742-4 ; Huon, 3223-5 ; 7633-6 ; Tristan de N., 10974-5 ; Claris, 4309-11 ; 8703-8 et passim ; Gui de N., 1803-6 ; Roche, 16668 ; Floire, 1241-2 ; Eneas, 354-6 ; Saisnes, 2007-8 ; Lion, 1030-3 ; 
12760 ; 13313-6 ; Cleomadés, 13080 ; Mez, 397 ; Partonopeu, 6478 ; Escoufle, 3593-5 ; 4254-8 ; 4348-51 ; Atre, 762-4 ; 846-9 ; Yvain, 5358-9 ; Ogier, 112 ; Lais, v, 78-9 ; Merlin, I, 236 ; Cristal, 4409-10.

Assez rarement nous lisons qu'il faut donner à boire aux chevaux. Un extrait de Rigomer nous apprend que c'est une "folie » (vers 1968) de faire boire un cheval qui vient de manger de l'herbe fraîche. Le poète sait comment il faut traiter les chevaux - et il s'adresse à un public de connaisseurs :

Lors retorne a la fontaine por faire boire son cheval qui grant mestier en avoit ; Lancelot, IV, LXxvII, 4.

Les huissiers ont bien atornez

D'iague douce, de foin, d'avaigne. Florimont, 11798-9. Cf. ib., 11874-5.

Desqu'a la rivere est alé

Erranment [et] se met al gué,

Si ad abevré son cheval. Protheselaus, 5318-20.

Et trova hors en mi la place

Un garçon qui voloit mener

Son destrier a l'eve abevrer,

Atorné de frain et de sele. Erec, 4890-3.

« Biaus sire, » ço dist Lanselos,

«Que ferés vos de mon destrier?»

"Amis, tu n'as nul escuier,

Si le te voel mener au gués. »

« Biaus sire, » fait il, «non ferés !

Il ne bevera devant none,

Car ne manga annuit d'avone,

Mais la fresche herbe raverdie.

Jou n'en voel ore abevrer mie. » Rigomer, 1970-8.

(...) ; on y abreuve les chevaulx et tout le bestial de l'ost ;

(...) Jouvencel, I, 154. Cf- ib., 201.

En temps de disette, souvent causée par les guerres, les chevaux aussi bien que les hommes vivent misérablement de ce qu'ils trouvent ; après, quand la situation s'est améliorée, ils trouvent de quoi manger à leur faim :

Sor .iiii. chevalx erent, telx est lor povretez :

Entre elx toz n'en plus, ce est la veritez. 
Et cil ne vivent pas d'aveine ne de blez,

Ne de fein que nen ait de soleiz essorez ;

De fuille et de racine vivent, c'est lor plentez,

Et qui trove un herboi mult est boneürez.

Li cheval amaigroient et sunt tuit descharnez, Renaut, 3434-

40 .

Li cheval pessent l'herbe, qui les a amendez, $i b ., 3494$.

Le bétail et les moutons trouvent la nourriture dans des enclos ou dans les prés et les bois où ils sont gardés par des vachers et des bergers ; les gardiens mènent aussi abreuver leurs animaux à une fontaine. Dans l'Histoire de la France rurale, I, p. 567, nous lisons : «En raison de l'exiguïté presque générale des prairies, d'ailleurs souvent accaparées par le seigneur, l'on peut mener dans les bois pâturer le bétail, mais non dans les jeunes taillis et à l'exclusion, bien compréhensible, des chèvres. A cause de l'importance pour la nourriture des porcs des faînes et surtout des glands, des seigneurs, par exemple en Bourgogne, se réservent parfois la vente de ces fruits : lors de leur maturité, l'accès des bois est alors interdit aux troupeaux de porcs. »

Et Isangrin, qu'ot poil chenu, s'en vint traient a un mainil ; bestes vit paitre en un cortil, Renart, v, 13568-70.

Garde aval en une chanpaingne et voit une mout grant conpangne de brebiz paissent un gaïn : ib., III, 8967-9.

Lors a choisi par aventure

Bestes, qui aloient pesant ;

Cele part torne maintenant,

Le bergier prent a apeler Claris, 9419-22.

Des que li ponz fu avalez

$\mathrm{Au}$ main, quant les bestes menerent

Cil, qu'en pasture s'en alerent. ib., 26843-5.

En cel pré avoit un rastelier ou il menjoient cent et cinquante toriaus. Queste, 149, 15-6.

Mais aprés vespres regarda devant lui et vit a senestre partie .II. pastors qui gardoient bues et vaches. Lancelot, II, L, 24 . 
Lors esgarde li vaslet sor senestre un poi en sus le chemin, si a veu el chief de la forest vaches qui paissent et berbiz ; $i b .$, I, xxI, 4 .

Ele l'apele Ysabeline :

«Cueil ces vaches par cel porpris,

Maine les en par cel pastis,

Je m'en irai par l'uis derriere. » $M R$, LXIII, 148-51.

les bestes fist el bois mener

e as vilains les fist garder. Rou, III, 4839-40.

N'a entour la forest remés home vivant,

Chevalier ne bourjois, vilain ne paÿsant,

Sarteur ne charbonnier ne vilain ahanant,

Nes ceus qui sont des bestes en la forest gardant, Berte, 2537-

40 .

Et je li dis que je gardoie

Vaques et buès en la forest Raguidel, 612-3.

(...) ; et il entrerent

En une lande et esgarderent

Biestes uenir a grant plente,

Qu'en toute la crestienté

N'en auoit pas tant, ce quidoient,

De tel guise, et toutes menoient

D'abreuer a une fontaine Espees, 10479-85.

Les agneaux (et l'auteur pense probablement aux moutons en général) sont des animaux qui se contentent de peu et qui trouvent leur nourriture même dans les landes arides :

(...) ; mes ele estoit si durement seche que de totes les herbes del monde n'i avoit il tant dont l'en puist un aignel pestre. Lancelot, I, XXvI, 31.

Il n'est pas toujours aisé de garder les animaux dans les champs : ils cherchent la nourriture qui leur est défendue :

On ne se peut garder nullement d'un larron

Ne de beste qui paist aux champs en verdison

Ne voisent au blé pestre, veulle la garde ou non ; Tristan de

N., 337-9.

Voir aussi : Perceval, 242-4 ; Cent, 12,35-7 ; Lion, 15339；24225 ; 24338 ; Wistasse, 588-9 ; Raoul, 1395. 
Les chiens de chasse reçoivent la curée tout de suite après que le gibier a été tué. La curée, qui s'appelle aussi droiture, est, selon É. Littré, I, p. 935 : «Portion de la bête que l'on donne aux chiens après qu'elle est prise. » A la même page, il en donne l'étymologie suivante : "Cuir, parce que, comme on voit dans Modus, la curée se donnait dans un cuir. »

Les chasseurs semblent dans tous les cas bien nourrir leurs chiens :

Quant il orent le cerf escorcié, il prennent la droiture as ciens et lour donnent, (...) TP, vI, 26, 8-10.

Si sai cacier le cerf et le sangler ;

Quant jou l'ai pris, le prise sai corner

Et la droiture en sai as ciens donner. Huon, 7447-9. Cf. ib., 2915-8.

Li rois i est courant venus

L'espee traite, plus n'areste,

Si li a coupee la teste,

Et puis li a l'entraille ostee

Et la cuirie as chiens donee,

Puis a mis le cor a la bouche. Floriant, 322-7.

Li bois ne puet le porc garir,

Ains l'estuet as levriers salir.

Et cil en vient, li chien l'ont pris

Et l'ont molt tost a terre mis ;

Et il a tot as chiens doné, Partonopeu, 1845-9.

Mes son cerf ad mult tost deffet,

Son brachet pest, iloec le lest, Ipomedon, 655-6.

Vienent avant li veneor,

Qui al chien donent a mangier

Pain blanc, dont ne ffiert nul dangier ; Diable, 1128-30. Cf. ib., 1145-6.

On les voit aussi manger les boyaux et laper le sang des animaux tués à la chasse :

Et, quant on getté la bouelle

Que on crie aux chiens : «Appelle, appelle ! » Deduis, 8319-

20. 
Li chien furent appareillié qui le sanc commencierent a laper. Helcanus, 59.

De Husdent, le chien de Tristan, nous lisons que, privé de son maître, il refuse le pain et le past. Ernest Muret explique past ainsi : " mélange de farine et de son que l'on détrempe dans des lavures pour nourrir les chiens de chasse. »:

Ne vout mengier ne pain ne past

Ne nule rien q'en li donast ; Tristan, 1449-50.

Voir aussi : Yvain, 4251-3; Ogier, 68.

Dans les maisons, les chiens semblent avoir eu leur place près de la table ou même sous la table à laquelle mangeaient leurs maîtres - toujours prêts à s'emparer de ce qui tombait par terre et des reliefs des repas:

Il a boté la table laidement de son pié,

Trestot a repandu le boivre et le mangier,

Dont en tindrent lor feste et braichot et levrier. Roche, 3275-7.

« En la moie foi, sire, ne somes pas bergier

"Que nos de tel viande dëussiens aseier.

« En la terre de France n'en vi onques mangier ;

«Vos en verrez demain ces ma[s]tins enragier. $i b .$, 3278-81.

« No vitaille et nos tables ont boté en .j. mont,

«Or en tienent lor feste et braichot et waignon ; $i b .$, 3301-2.

Ja le menjuent brachet et leverier, Ami, 2343.

Ils mangent aussi les charognes et les cadavres ; ils ont dû être habitués à la mauvaise nourriture, «le pain des chiens (4)»:

« Portez, fet il, leanz cel cors,

que nel menjucent chien ça hors. » Thèbes, 5859-60.

car as Griex volt le cors tolir

qui nel puissent ensevelir ;

mengier le veut fere as mastins

et as voutoirs et a corbins ;

aprés sa mort s'en veut vengier

et as mastins faire mengier,

car mout grant deul cil en avront,

quant as chiens mengier le verront. ib., 6503-10. Cf. $i b$., 10096-8. 
« Li chien de ceste vile s'en sont gagié

«Qu'il mengeront le car de cel destrier. Aiol, 965-6.

« Et Marchegai est mors et a sa fin alés :

«Piecha que l'ont mengié li cien en .I. foussé, $i b ., 8262-3$.

Rou fist querre les suens, touz lez ensepeli,

lez autres mors as chiens et as oyseaux guerpi. Rou, II, 130-40.

Vo cheval arai malgré vostre,

Mais ce n'ert pas por chevalchier,

Ains le volrai faire escorchier,

Si en avront la char li chien Cont. P., I, 4448-51.

Pour quoi la lesse il tant vivre?

Face la a cevaus detraire

Et tous lez membres du cors traire,

Et puis la lesse au chienz mengier : Anjou, 7792-5.

En prison sont, en chartre obscure,

En font penitance molt dure ;

Du pain aus chienz ont molt petit,

S'en prennent il par apectit. ib., 4997-5000.

Ne te sovient il de celui

Cui tu feïs si grant anui

Que tu le feïs sor son pois

Avec les chienz mengier un mois, Perceval, 7111-4.

Manger avec les chiens peut constituer une punition :

Mais ce sacies de verité,

La termine qu'il i sera,

Que avoec un chien mangera,

Ja n'avra autre conpaignon,

Faire li 'stuet, ou voeille u non. Cristal, 3228-32.

Le pauvre palefroi maigre, dans Perceval, mourra bientôt de faim ou devra être abattu - la charogne sera mangée par les chiens :

Autel del palefroi sambloit :

Tant estoit maigres qu'il trambloit

Einsi com s'il fust enfondus.

Toz li caons li fu tondus

Et les oreilles li pendoient ;

Cuiriee et past $\mathrm{i}$ atendoient

Tout li mastin et li gaignon, 
Qu'il n'i avoit se le cuir non

Tant solement desor les os. Perceval, 3703-11.

Dans Roland, nous voyons que les porcs aussi mangent régulièrement des cadavres : Olivier rassure Roland que leurs cadavres ne connaîtront pas ce sort indigne. Un extrait de Turpin montre pourtant que le sien est si malmené qu'on le dirait rongé par des chiens :

Nostre Franceis i descendrunt a pied,

Truverunt nos e morz e detrenchez,

Leverunt nos en bieres sur sumers,

Si nus plurrunt de doel e de pitet,

Enfuerunt nos en aitres de musters ;

N'en mangerunt ne lu ne porc ne chen. » Roland, 1746-51.

E estoyt tot quassés de ordes coups de bastouns e dars et de setes, e fut aussy atornez come chyn attorneyt carogne. Turpin, 1150-2.

Les oiseaux de chasse ont des petits bouts de viande, souvent de petits oiseaux (cf. Friedrich Borchert, op. cit., p. 83), tandis que les poules, qui mangent du blé, grattent la terre pour trouver de quoi se nourrir :

Gerars li menres repaist un esprevier

Et li fait gorge de l'ele d'un plouvier. Huon, 338-9.

Un poi ensus pour esplumer

Li laist, un poi pour aamer,

Puis prent l'aloe et l'esprevier,

$C^{\prime}$ on ne tint mie a ravenir ;

De la cervele le repeut, Violette, 4213-7.

Et jou conme caitive et lasse

De grant folie m'entremis :

L'esprevier a disner enpris,

Ki d'oisiax moult petit savoie.

Endementiers que jel paisçoie

D'un oiselet qu'il avoit pris,

Li espreviers, qui ert assis

Sor mon puig, si m'est escapés. Atre, 2626-33.

Lez Erec s'est li cuens assis

Et la pucele o le cler vis,

Qui de l'alete d'un plovier 
Paissoit sor son poing l'esprevier

Por cui la bataille ot esté. Erec, 1301-5.

Biautés va Aharer gabant

Qui s'en aloit tout repaissant

Le faucon qu'avoec lui portoit,

D'un oisiel gorge li faisoit.

Quant l'ot repeut, sy l'aplanoie. Gliglois, 1347-51.

Sor le hauberc est le cop devalé,

.v. .c. des mailles en a au branc osté

Et de l'espaule a un braon sevré,

.iiii. faucon en fusent bien disné. Narbonnais, 4761-4.

Et je vois en la boucherie

Acheter un cuer de mouton

De quoy je paitray mon faucon. »-Deduis, 1870-2.

Et cuer de porc lui est char bonne,

Si tien pour saige que li donne.

Buef tendre, qui soit bien lavez,

Li poués donner, se l'avez. ib., 7413-6. Cf. ib., 9825-6.

Vers Soibaut va con hons de grant proesce,

dou branc le fiert et tellement l'empece

que de sa char li oste une tel lesce

que nus faucon qui de fain a destraice

ne prendroit tant de char de porc fresce

con fait Soibaut estre a mout grant destrece. Godin, 17754-9.

Lors descendi li cois a pié,

qui dou froment a tant mangié

q'ainz ne se pot d'ileuc partir : Renart, v, 13683-5.

s'en est venuz jusqu'a la haie,

de leanz antrer mout s'esmaie,

qar les chapons voit ou soleil

et Chantecler qui clingne l'oil

et les poucins et les gelines

qui erent lez un tas d'espines

en un paillier ou el gratoient, $i b .$, vI, 15603-9. Cf. $i b$. 15647-50.

\subsubsection{Gardiens}

Nous ouvrons ici une parenthèse pour jeter un coup d'œil sur les personnes qui gardaient les animaux dans les champs. Parlons 
d'abord des bergers et des bergères. L'on sait qu'il s'agissait fréquemment de jeunes et même de grands enfants qui menaient paître leurs bêtes, souvent très loin de toute habitation et pour un salaire minime ou même pour rien d'autre que leur nourriture. Tout cela explique le peu d'estime que l'on avait en général de ces gens : ils étaient faibles et très pauvres et, comme ils vivaient très isolés, ils ne savaient rien du monde et des autres hommes - on les trouvait stupides et farouches. Quant aux bergères, on les considérait apparemment parfois de mœurs légères.

Au chief dou chanp s'esbeneoient

et de lor cornes se hurtoient.

Que qu'il fesoient lor mellee,

lor bergiere s'en iert alee ;

li bergers les ot oubliez ; Renart, II, 5307-11.

De par son petit fil Herbert

(Jou Hierbers qui li gare ses biestes), Rigomer, 3314-5.

« Ja n'en ateindron .I. ne soit a mort livrez:

« Il ne nos avront mie come bergiers trovez ! Renaut, 4735-6.

Cf. $i b ., 8424-5$.

Chou est li fieus un vilain päissant,

A brebis l'ont trové si fierement

Et pour mieus vendre l'ont atorné si gent. » Bueve, I, 767-9.

A Aiglentine dist : « Tout ce devés lesser.

Je vous tieng moult a folle, ceur avés de berger,

Qui reffusés ung roy pour ung simple guerrier ; Tristan de $N$., 2134-6. Cf. ib., 2128-30;6604-7; 8141-2 et passim.

Cel jour vesti ses filz les dras a ses bergiers,

Si n'orent nulles chausse[s], escapins ont chaucié. Roche, 3504-5. Cf. ib., 3278-9.

Je croy que se n'est pas pawillon de bergier,

Ains est d'un riche princier ; je l'irait esvillier ! Lion, 17856-7.

Cf. ib. $10015-6$ et passim.

Et li prince respont : « Cuer avez de bregier, Et pués que ceste guerre vollez ansi laissier,

A Jhesu vous commant qui se laissait player, ib., 13154-6. 
Li lorain erent fait d'uevre riche et legiere,

N'estoit pas acesmee conme povre bregiere. Buevon, 2394-5.

Cf. ib., 3433-5.

Mes freres disoit voir, sans pensee bregiere ;

Vous ne li devés pas donner de vous lasniere. » Bâtard,

1787-8.

Ne doi amer par legerie

dont l'em puisse dire folie ;

ainsi doit on prier berchieres

ou ces autres fames legieres. Thèbes, 4167-70.

Voir aussi: Aliscans, 1973; Violette, 1548-50 ; Saisnes, 7402-4 ;

Yvain, 333-4 ; Cent, 82, 4-7.

Quelques extraits semblent indiquer que l'on estimait les vachers, les charretiers et les laboureurs aussi peu :

Il n'a vacher en ces bruyeres,

Tant soit fol ne tant soir cornant,

Qui ne congnoisse ane ne mallart Deduis, 8388-90.

Et cil qui le conduient ne furent pas garson

Ne del tot atorné a loi de charreton ;

N'i a cel qui n'ait vestu boin hermin pelison

Ou bon bliaut de poelle ou riche ciglaton ; Florence, 1259-62.

Et tu diz, tu es chevaliers ;

Mielz sembles estre charruiers,

Qui reviegne de la charrue ; Claris, 22767-9. Cf. ib., 22774-6.

Du destrier a terre le verse ;

Et puis li dist en reprouvier :

«Mal semble cist coux de bouvier,

Qui a ale a la charrue. »ib., 22866-9.

Gueres n'eurent esté en leur hostellerie que veez cy venir quatre gros charruyers ou bouviers plus villains encores, et entrerent baudement en cest hostel, demandans rigoreusement ou estoit la ribauldelle que ung ruffien nagueres avoit amenée derriere luy sur ung cheval, et qu'il failloit qu'ilz bussent avec elle et a leur tour la gouverner. Cent, 98, 91-8.

Mais de nombreux exemples montrent de l'estime pour les vachers et les bouviers. Bien souvent ceux-ci possèdent une partie des animaux qu'ils gardent et dirigent, c'est-à-dire qu'ils peuvent 
être très aisés. Comme ils doivent maîtriser de grosses bêtes, ils sont en général forts. N'oublions pas que les bouviers ne font pas que garder les bœufs, ils les utilisent aussi pour le labourage etc. et jouent par leur travail un rôle important dans la vie champêtre.

Estes vous poingnant à droiture

Contre lui son bouvier Robet :

«Qu'as tu ? » fet il, « qu'as tu, vallet?

Qu'as tu? » fet il, « comment vas tu?

- Sire, mal vous est avenue :

Li forestiers vos bues en maine. $M R$, CvI, 312-7.

Quar je leverai orendroit

Por ces bouviers fere lever,

Jà sera tans d'en champ aler

Por nos terres à gaaignier. ib., xxIV, 188-91.

Atan tez .I. vilain Raoul,

Un bouvier qui vient de charrue ; $i b .$, LXxx, 84-5.

Puis prist un brant, dont le pont est d'or mier,

Une grant toise i puet en embracier,

S'ot pleine paume de lé a un bovier ; Aliscans, 4674-6.

Encor avoit Bernarz .x. filz de sa moillier :

Proz [furent] et cortois, li .v. sont chevalier,

Et a .xxx. charrues dont il fait gaainnier,

Que sui .v. fil maintiennent a .lx. boviers. Roche, 3191-4.

Nous avons relevé quelques exemples du terme vachère. Il n'est pas tout à fait clair s'il s'agit de la femme du vacher qui aide celuici à garder les vaches :

Atant est la vaichiere dont oyr vous avés ; Lion, 24688. Cf. ib., 33628 .

Cil ot une vachiere qi mout ot cler le vis, Saisnes, 2034.

Nous ne savons pas de quels animaux il est question dans Lion, qui raconte que ce sont les femmes qui s'occupent des bêtes :

Il n'ait en cest tour personne se moy nom,

Se se ne sont trois femme qui gardent la maison,

Qui norissent lez beste dont ceans ait foison ; Lion, 4298-300. 


\subsection{Etables, bergeries etc.}

Nous l'avons vu plus haut : les chevaux sont régulièrement mis à l'écurie après avoir accompli leur tâche. Mais les moutons, les porcs et les vaches, que l'on mène paître dans les champs et les prés, parfois dans les bois, eux aussi on les met à l'abri pendant la nuit et pendant la saison hivernale où l'herbe se fait rare, pour les protéger contre le froid et la pluie - et contre les loups.

Les textes parlent d'étables pour les chevaux et les vaches (ce n'est qu'au $\mathrm{xvI}^{\mathrm{e}}$ siècle que le mot écurie a pris le sens moderne (5)), de bergeries pour les moutons et les porcheries pour les porcs. Mais, comme ils ne donnent pas de descriptions, nous ne savons pas de quelle nature étaient ces constructions, s'il s'agissait de simples abris, de cabanes, de toitures (en chaume ou en tuiles), ou si l'on construisait plus solidement. Il devait y avoir des différences considérables entre les étables des riches et celles des pauvres.

Jean-Pierre Leguay, op. cit., pp. 59-61, mentionne différents animaux domestiques élevés en ville; il écrit que "des chevaux, des mulets, des ânes (...) ont leurs "estables" (sic) dans les dépendances des hôtels particuliers, en plein cœur des cités. »

L'Histoire de la vie privée, tome 2, p. 450, mentionne un colombier, un gelinier et " une estable a mettre pourceau » ; à la page 451, une étable à chevaux, deux autres pour le bétail, une bergerie, un colombier (à l'étage) et une porcherie (au rez-de-chaussée du même bâtiment) et, p. 458, une étable à bœufs (boal), une bergerie (cortal), un colombier et une porcherie.

Eranment d'une estable issi

Moult vistement un sien vallet,

Si li tramist le Gringalet, Atre, 4358-60.

Et fis mes vallés entremetre

De son ceval mettre a estable. ib., 5036-7.

(...), elle manda secretement le filz d'un cordonnier son voisin, et le fist venir en l'estable des chevaulx de son pere (...) Cent, $55,113-5$.

(...) elles se vindrent rendre en la maisonnette du bergier de leens, qui estoit auprès d'un large et grand parcq a mettre les brebiz, et trouverent la le maistre bergier qui besoignoit entour de ce parcq. $i b ., 57,21-5$.

Ilueques a granz bercheries

et granz tourbes de porcheries ; Thèbes, 6985-6. Cf. ib., 8193-4. 
Or n'a li prestres de réduit,

Fors tant qu'il entre en .I. toitel

Où brebis gisent et aignel ; $M R, \mathrm{xxIV}, 294-6$.

Cil sont povre, li riches fols

En son cortil avoit des chols,

Et en l'estable des brebis : $i b .$, xcvI, 21-3.

L'autres se trest vers le bercil

Por l'uis ouvrir : tant fet qu'il l'uevre, ib., xcvI, 31-2.

Tot ansement Turnus feisoit

come li lous qui est a plain

et vient a la faude al vilain :

environ vait molt famoillos,

les berbiz voit dont est gelos,

les aigniaus ot dedens beller,

garde par ou porroit antrer. Eneas, 5370-6.

Il est ensy comme ly leux

Quy de mengier est desireux

Quant est devant le faudeïs

Et voit devant luy lez brebiz, Gliglois, 683-6.

Mais Ogier de prime arrivee se alla fourrer du premier sault au meillieu de la bataille comme ung loup affamé en ung parc de brebis. Ogier, 33 .

Pour Ollivier le dit, a la chiere herdie,

Que loingtempz fuit noris delez la vacquerie ; Lion, 25242-

3. Cf. ib., $26133 ; 26136 ; 26151$.

Pour empêcher les chevaux de s'en aller, p. ex. quand on les laisse paître dans un pré, on leur met un paturon, c'est-à-dire une chaîne (cf. p. 253 : Escoufle, 4746-9) :

Mais il est drois que uous ales

A Caradigan en prison

A la roine, et soupecon

N'aies, ke uous mal i aies

Autre, fors c'aues en uos pies

Vnes pastures que i'ai ci. »Espees, 11498-503. Cf. ib., 11526-

$31 ; 11577 ; 11582$ et passim.

Les chiens ont des niches ou ils doivent se contenter d'une (mauvaise) couche de paille : 
Et voit de totes parz son avoir repairier,

Entrer enz en ces parz et berbis et bergiers,

Et gesir en ces loiges et ma[s]tins et levriers,

Et ces bues et ces vaiches dont il i a miliers. Roche, 3202-5.

Et se coucha dedens l'autre couche qui n'estoit pas paree. Et eust on dit que c'estoit pour les chiens. Ogier, 109.

Et Robers s'en va sor la paile

Couchier ensanble la chienaile. Diable, 2407-8. Cf. ib., 1208-9.

Quant aux poules et aux autres membres de la basse-cour, il fallait nécessairement les protéger la nuit contre les renards et d'autres prédateurs. Renart mentionne un poulailler, sans en indiquer la nature ; du colombier dans Cent nous savons qu'il devait être assez grand, puisque le mari pouvait s'y installer, et qu'il se fermait à clé (à cause des voleurs ?) :

Cest cortil fut mout tres bien clous

de pieus de chesne aguz et grous,

bien fu fermez d'aubes espines :

laienz avoit mis ses gelines

danz Coutenz por la forteresce. Renart, II, 4085-9.

Qant il oï Renart rungier,

mout durement se mervoilla,

et en aprés se porpansa

que gorpil estoit ou taissons

qui venuz estoit as chapons ;

au gelinier en vint errant, ib., v, 14280-5. Cf. ib., v, 14240-51.

et, se il puet trouver le gelinier,

il s'en vorra otout les hués aler. Audigier, 223-4. Cf. ib., 326-7.

Ce vaillant homme monta en ce colombier, qui se fermoit pardehors a clef, et se fist illec enfermer, (...) Cent, 88, 91-3.

(...), et luy dist qu'elle prinst les deux meilleurs chapons de la chaponnerie de l'ostel, (...) ib., 59, 102-4.

Le perroquet dans Papegau a une cage richement décorée :

(...) ; car les quatre escharbocles de la caige au papegaulx gitoient si grant resplendeur parmy le palais, que c'estoit merveille a veoir. Papegau, 21, 14-6. Cf. ib., 7, 23-5. 


\section{5. « Langues»}

Les textes présentent des spécimens des « langues » des animaux. Au chapitre précédent, nous avons vu que les coqs chantaient très tôt le matin et réveillaient ainsi les gens, mais nous lisons aussi que les chevaux hennissaient, que les chiens aboyaient, etc.

Bayart conut Maugis, a henir commença, Renaut, 10550.

Ainz des destriers uns ne se remua ;

Cil au paien henist et braidona, Aliscans, 1543-4. Cf. ib., 4900-1; 5120-1 et passim.

Entre Galeran en la ville

Ou il oit de destriers dix mille

Parmy ces rues cler hanir, Galeran, 3339-41.

Cil cheval, cil roncin henissent Escoufle, 290.

La peussiez veoir maint corrant palleffroy

Et maint riche destrier que pas ne furent quoy,

Hignissent et si braient et moyne[n]t grant effroit ; Lion, 6505-7.

(...) ; il escoute et ot un ceval henir assés pres de lui ; et heni si fort que toute la forest en retentit pres d'illuec. TP, I, 97, 33-5. Cf. $i b ., 98,1-3 ; 114,9-12 ; 169,13-6$; III, 28, 1-2.

Et quant ele aloit, il issoit de son ventre un si tres grant glatissement conme se ele eüst dedens li jusques a .xx. brakés. De tel fachon estoit la Beste Glatissant. ib., vi, 170, 17-9.

Car buefs muient, chevaulx henissent,

Chiens abaient, lions rugissent, Deduis, 10629-30.

Qant il s'ala esbenoier,

les berbiz oit ou chanp beller ; Renart, II, 5300-1. Cf. Eneas, 5370-6.

Li chien ullent et si font noise,

cuident que il voille descendre ; $i b .$, IV, 11806-7.

Cil cor sounoient mos a mos

Ces gens hüent, cil chien glatissent,

Que toutes les forés tentissent, Rigomer, 1216-8.

Ciens n'i abaie ne kos n'i puet canter ; Huon, 2912. 
A tant en sunt el bois entrez,

Li chien commencent a tracier,

A galtir et abaïer. Floriant, 296-8. Cf. ib., 300-1.

Lors voit venir grant aleure

.I. porc senglier vers soi corant

Et .III. brachez apres criant ; Claris, 12124-6.

Lors oïssiez tant cor, tante buisine,

Braient cil ors et cil viautre glatissent,

Cil mul rechanent et cil destrier rechinent,

Cil espervier desus cez perches crïent,

Bien les puet l'en oïr d'une grant liue. Orange, 1313-7. Cf.

Mort Aymeri, 699-700.

S'i estiez le premier jor d'esté,

Lors orrïez les oseillons chanter,

Crïer faucons et cez ostoirs müez,

Chevaus hennir et cez muls rechaner, ib., 245-8. Cf. ib., 409-

11.

Quant l'a veu, si prist a rire,

Tieus ris, conme uns asnes feroit,

Quant l'ainesse venir verroit. Claris, 10182-4.

Et, ainsi que le bon homme eut ouvert la huche, et que cest asne veist la lumiere, il commença a recaner si hideusement qu'il n'y eut la si hardy qui ne perdist sens et memoire. Cent, 61, 177-80. Cf. ib., 79, 56-9.

roxignous ne oiseaus pas n'i chantot ;

laienz ot une asnesse qui rechanot

et une vielle lisse qui lors ulloit ;

et une chate borgne de faim braioit. Audigier, 137-40.

Voir aussi :Cont. P., II, 10838-9 ;Diable, 353-5 ;2537-8 ; Anjou, 7042 ; Enfances O., 1615 ; Espees, 6184-5 ; Florimont, 4366-8 ; Partonopeu, 603-6 ; 5841-2 ; 5851 ; Athis, 12019 ; Ipomedon, 4489 ; 4498 ; Blancandin, 5313 ; Cligés, 4874-7 ; Narbonnais, 3654; Aymeri, 3601 ; Doon, 2790-1 ; Bérinus, 97.

Le fabliau xuvi présente des onomatopées pour illustrer les voix de la brebis et de l'âne :

Ainelet a petite l'os,

Corte l'eschine et cort le dos ;

Cestui n'est mie fils bèhè 
Quoi dites vos, Alein, que est ?

Ce ne fu mie fielz berbis

- Tu dites voir, par saint Felix.

Foi que ge doi à seint Jobon,

Cestui fu filz ihan, ihan ;

Encor fu d'anesse en maison

Et ge vos porte ci d'asnon. MR, xLVI, 101-10.

Cf. Adolf Tobler, op. cit., V, pp. 218 et 222-223.

Cf. Fr. Schmidt, op. cit., pp. 66-68.

3 Cf. Robert Delort, La Vie au Moyen Age, p. 179 : « La plus belle et captivante, très accessible aux dames, est la chasse par oiseau interposé ; (...) L'art de fauconnerie était l'un des plus délicats qui soient (...) »Voir aussi p. 200.

4 Cf. Friedrich Bangert, op. cit., p. 181 : «Hundefrass dient als Bild schlechter Nahrung : (...) ».

5 Dans l'extrait d'Ogier, p. 307, du xve siècle, écurie a le sens de « ensemble de chevaux »: Et lors fit aller ung escuyer pour avoir le meilleur cheval qui fust en l'escuyrie, (...). 


\section{Utilisation des animaux}

Nous avons pu voir que les hommes aiment leurs animaux, s'en occupent et les soignent et les regrettent quand ils les perdent. Et pourtant : si le chevalier aime son cheval presque autant qu'un être humain, s'il apprécie tant son épervier, si le vilain promet à son âne du foin et de l'avoine - n'est-ce pas avant toute chose parce qu'ils savent tous que les animaux leur sont utiles et même nécessaires ? Nous avons déjà affirmé que le chevalier n'est rien sans son destrier; de même le chasseur au vol ne peut rien obtenir sans un bon oiseau et le vilain ne peut pas labourer ses champs sans bœufs. Dans une très large mesure, c'est l'utilité des animaux domestiques qui crée l'intimité entre hommes et bêtes.

Jean Bichon, op. cit., p. 5, l'exprime très bien : « (...), l'animal est encore un auxiliaire et par là un associé plus ou moins permanent. Il garde la maison, le troupeau ; il aide le chasseur et lui est même indispensable pour certaines chasses (animal suivi à la trace grâce au flair du chien, tenue en laisse - c'est le limier - ou suivi grâce à ces aboiements significatifs ; fauconnerie) ; il porte les fardeaux, tire les charrettes et les charrues; il sert de monture, soit pour le voyage, soit pour le tournoi et la guerre (...) » et pp. 17-18: « La richesse du paysan se mesure au nombre de ses bêtes. Le pauvre a une vache, ou une chèvre, ou quelques brebis ; des lapins, quelques volailles; un chat, un chien. Le riche a de grands troupeaux. L'animal domestique joue un grand rôle dans l'alimentation (viande de porc ; produits laitiers) ; un rôle capital dans le vêtement (le coton, la soie, sont des produits exotiques très rares ; le lin, le chanvre, ne tiennent pas chaud ; les matériaux principaux sont, dans un pays froid aux maisons mal chauffées, la laine et les fourrures). Si l'on excepte les moulins à eau et à vent, les animaux sont l'unique source extra-humaine d'énergie ; sans eux pas de labours (sinon à la pioche), pas de transports lourds, pas de voyages (sinon le voyage à pied, qui reste, d'ailleurs, le voyage ordinaire du pauvre ; et, sur les rivières, le voyage en bateau). Sans le bœuf, le cheval, le chien, la vie quotidienne s'écroulerait ; disparaîtraient ces activités nobles que sont la chasse, le tournoi, la guerre. »

Ce sont des différentes manières d'utiliser ces animaux dont nous allons parler dans le présent chapitre. Nous le divisons en trois parties: dans la première, nous montrerons comment les hommes utilisent les animaux vivants ; dans une deuxième, nous parlerons des différentes manières d'utiliser les produits des animaux vivants, comme p. ex. le lait ; dans la troisième partie, enfin, 
sera montré comment les hommes savent utiliser les produits des animaux morts, comme p. ex. la viande et les plumes.

\subsection{Utilisation des animaux vivants}

Un nombre considérable des extraits de textes présentés aux chapitres 1 et 2 donnent une image variée des domaines où les animaux servent les hommes. Nous avons vu, en effet, les destriers présents dans des scènes de bataille, les dames voyageant montées sur des palefrois, les chiens chassant le gibier, les bœufs tirant les charrettes etc.

Deux brefs passages de Deduis énumèrent des animaux « qui servent a homme » - les mêmes dans les deux cas :

Telx bestes, comme je vous nonme

Aux bestes qui servent a homme,

Comme buefs, vaches et pourceaux,

Chevaulx et brebis et aigneaulx,

Sont anemies pour certain Deduis, 7801-5.

Les bestes qui servent a homme

Comme sont brebis et aigneaux,

Chevaulx, buefs, vaches et pourceaux. ib., 9228-30.

Nous allons présenter un grand nombre d'autres exemples dont certains sont plus particuliers et plus précis ou qui montrent comment les hommes sont conscients des activités - quotidiennes ou exceptionnelles - que les animaux leur permettent de réaliser.

Étant donné que les textes montrent beaucoup de scènes de guerre et de tournoi, il est évident que nous y voyons de nombreux chevaliers montés sur des chevaux de bataille et beaucoup de chevaux préparés pour la bataille. En voici quelques exemples :

Ja n'avez vous escange le meillor chevalier Qui onques portast armes ne montast sor destrier. Gui de N., 50-1.

Se il veoit un chevelier

Armei(s) sor son courant destrier,

Ja por ce ne lairoit sa proie Florimont, 515-7.

Li varlet et li escuier

Courent enseler lor destrier. Floriant, 3117-8. 
Et maint riche destrier torchier et conraer, Saisnes, 802.

De la ville s'en issent arméz sur l'aufferant, Lion, 8680.

Et Sadoines estoit montés sur l'arragon

Ou il se combatoit a loy de champïon, Tristan de N., 2257-8.

Les deus chevaliers regardoient

Qui sor les grans chevas seoient,

Tot armé, les elmes laciés. Durmart, 10201-3.

Les cavaliers sont fiers de leur habileté et aiment montrer qu'ils maîtrisent souverainement leur monture :

Or sont passez les champions françois lo riviere, et sont dedens l'isle ou ilz font bondir et faire pennades aux chevaulx. Car naturellement les François sont plus dextres a chevaucher et mieulx leur advient que a toute autre nacion. Ogier, 39 .

Le guerrier qui remporte la victoire d'une bataille y gagne aussi un butin qui comprend normalement un grand nombre d'animaux. Ce butin est important pour le parti victorieux, car il s'y trouve des chevaux remplaçant ceux qui ont été tués ou blessés, mais aussi d'autres animaux comme bœufs et moutons qui serviront à nourrir l'armée :

Mil auferrans François i conquesterent,

Quatre cens mules ke sont bien affautrees. Enfances G., 1018-9.

Mes les armes et les espees,

Broisnes, targes a or listees,

Les palefroiz et les destriers

Et muls et mules et somiers,

Les riches trez, les pavellons,

Le riche escec que fet avons,

Tot devant nos ferons mener, Athis, 8415-21.

Dis chevaliers trestos montés

Et quinze destriers enselés

Ont cil des molins gaagnié Durmart, 11841-3.

Pour ce abati cent vassaus

Et gaaigna cinc cens chevaus ; Amadas, 1469-70.

Qui vost avoir ou armes ou destrier,

Assez en pot celui jor gaengnier ; Aymeri, 1951-2. 
Il et si oncle maint destrier milsoldor

En amenerent c'ont conquis en l'estor. Raoul, 4127-8. Cf. ib., 3861-2.

La proie accoille[n]t, mains hom en fu chaitis,

Et bues et vaiches et chevaus et roncis. $i b ., 3854^{-5}$.

De pain, de vin, de char salee,

Et de farine buletee,

De bues, de pors riches et biax,

De brebiz, de moutons, d'ainiax,

D'or et d'argent, de dras de soir ! Claris, 6868,72.

Daneiz furent felons et a mal fere isnel,

ne lessent en Chartrain ne en Duneiz bordel,

ne maison en estant qui soit fors du chastel,

ne lessent beuff ne vache, genice ne torel,

porc ne brebis ne oe ne chievre ne aignel,

coc, chapon ne guelinne ne viel chien ne chael,

ne forment en guernier ne bon vin en tonnel. Rou, II,

4182-8. Cf. ib., 2720-2; III, 1084.

Il est normal qu'une armée dévaste le pays conquis et y vole le bétail, de même que l'on se procure bœufs et vaches etc. pour nourrir les assiégeants :

I[1] lor arst viles et maisons,

Si prist lor bués et lor motons

Et lor homes et lor vilains ; Durmart, 10937-9.

Ne remaint buef ne vache de ci a Monpelier

Ne jusques a Biaucaire qu'il ne facent praier,

Ne jusqu'a Saint Cointin qui an Vermondois siet,

$\mathrm{Qu}$ 'au siege de Clermont ne facent achaier. Orson, 2659-62.

Trestout entor la chité me corés

Et bués et vaches et chevals amenés,

Les pastureurs, gardés, vous n'adessés,

Mi home sont, si en ai grant pité,

Ciaus a cheval mar vous esparngerés, Bueve, I, 5055-9.

Bueves i va armés, sor Arondel,

La proie acuellent par devers le castel,

Que bués, que vaches, que roncis, que pourcel, $i b .$, II, 4917-9.

Cf. $i b .$, II, 4625-6 ; 4727-8; III, 5715-6. 
La veïssiez tante vile brisier,

Tant buef tuer, tante vache escorchier. Mez, 2270-1.

Grant proie amainent qu'il ont prise et robee ;

De buès, de vaches i a grant asamblee, Narbonnais, 5939-

40. Cf. ib., 6401-2.

mout grant avoir ont par dedens conchis :

bués et pourchiaus et vaques et brebis

et autre avoir qu'il ont sor les cars mis. Godin, 13165-7.

«Vous me lairés ces vakes et ces tors

" et ces moutons, ces brebis et ces pors. ib., 13479-80. Cf. ib.,

$12782-5 ; 12942-3$.

Dans les combats particuliers et les tournois, le gagnant obtient le cheval/les chevaux de son adversaire. En plus, on lui accorde un prix qui est très souvent un oiseau, mais qui peut compter aussi chevaux et chiens :

Tant fist Gliglois et tant jousta

Que deux chevaus i gaaigna. Gliglois, 2479-80.

N'el tournoy n'ot deux chevaliers

Qui tant guaignaissent de destriers

Comme il tos sol par son corps fist. ib., 2539-41.

A tant es vos illuec Biauté

Sor un mulet a esperon ;

Desor son puing tint le faucon

Quy par voie li fut donnés.

Ainc plus biaus oisiaus ne fu nes.

Ne tant ne quant ne s'aresta,

Vint a Gliglois, se lui puira,

Desor le puing li a assis.

«Tenés, fait elle, biaus amis.

Certes, fait elle, Dieu merchy,

Vous l'avés tres bien deservi ! » ib., 2646-56. Cf. ib., 1362-5.

Il g[a]aina les dous destriers

E ssi occist les dous guerriers

E de la mort me guaranti ; Yder, 173-5.

Il prist hier soir dis chevaliers

Et gaaigna quinze destriers. » Blancandin, 1489-90. 
Mais seur tous le pris enporta

Meniadus, c'on li donna

l'esprevier pour le mieus lançant

et pour le plus bel chevauchant. Cleomadés, 17513-6.

(...) ; et ont establi entr'els que cil qui al meillor de tos i sera esleus avra .I. esprevier et .I. faucon en reconnoissance de victoire. Lancelot, II, LXV, 6.

Et la est la dame qui garde

.I. esprivier biel et joli,

Et le donra, ce dist, celi,

Sans doubte se li aÿt Diex,

Qui ce jour tournira mieulz. Méliador, 5803-7. Cf. ib., 7248-52 ; 7684-5 et passim.

Ki le turneiement veintra,

E d'ambes parz le pris avra,

Le girefalc e le blanc destrer,

Qui tant fait a preiser,

E les levrers qui tant bons sunt,

Abandoné lui serrunt, Gui de W., 775-80. Cf. ib., 766-73 ; 913-4.

A tous ceulx qui voudroient jouster sur le destrier,

Et dourroit on ung pris qui moult fait a priser :

Ung cheval de .c. mars, et ung noble esprivier, Tristan de N., 5041-3.

«Sire, en mon paÿs si a une coustume, je ne sai se elle est en cestui, que le premier cheval que cil avoit du gieu gaaignié que cilz le devoit avoir qui le present avoit aporté. Laurin, 1151921.

$\mathrm{Au}$ tournoi à Blanches Mores, dans Durmart, les chevaux ainsi gagnés sont offerts immédiatement aux dames et pucelles qui y assistent; dans Perceval il y a des scènes analogues. Ipomedon offre les chevaux gagnés à la Fiere, Butor donne le cheval gagné à sa femme, Diomedès à son amie, Marques à sa future femme :

Les dames ont maint prisonier,

Et si ont maint riche destrier,

Car qui cheval i gaaignoit

U qui chevalier i prendoit

As dames en faisoit present

Et as puceles ensement. Durmart, 7651 . 
Et mesire Gavains fiert lui

Si qu'il li fait molt grant anui,

Que tot envers l'en porte al plain.

Et tent a son cheval la main,

Sel prent au frain et si le baille

A un vallet et dist qu'il aille

A celi por cui il tornoie,

Si li die qu'il li envoie

Le premier gaaing qu'il a fait

Le jor, qu'il velt que ele l'ait.

Et li vallés atout la sele

Le cheval mainne a la pucele, Perceval, 5517-28.

Onques de gaaignier destriers

Ne fu mais si entalentez,

Quatre en le jor presentez

Que il gaaigna de sa main :

S'en envoia le premerain

A la damoisele petite ;

De l'autre a la feme s'aquite

Au vavasor, qui il molt plot ;

Une de ses deus filles ot

Le tierç, et l'autre rot le quart. $i b ., 5574-83$.

Le destrer prent Ipomedon

E si l'ad baillé a Jason,

Di li ke il face sun present

A la Fere delivrement. Ipomedon, 4135-8. Cf. ib., 3730-1.

Mais Butor s'en ala au hourdis maintenant,

Sa feme presenta le cheval en rïant. Brun, 2122-3.

Tantost s'areste et le cheval combra,

Un vallet voit, sa dame l'envoia, Bueve, I, 1005-6. Cf. ib., I, 1055 .

Diomedès ala joster a Troïlus por l'amor s'amie et le tresbucha a terre. Puis prent le destrier et le baille a un damoisel et dist : «Va t'en isnellement a paveillon Calcas de Troye et presente le destrier de par moi a sa fille. Troie, 148, 49-53.

Marques a commandé que on feïst un present a Dyogenne du cheval le conte de Prouvence. Laurin, 5460-1.

Dans Erec, un épervier constitue le prix offert au chevalier qui présente la plus belle « amie » et qui ose la défendre : 
Car devant trestote la gent

Iert sor une perche d'argent

Uns espreviers mout bien assis

Ou de cinq meues ou de sis,

Li mieudres c'on porra savoir.

Qui l'esprevier voudra avoir,

Avoir li covendra amie

Bele et sage sanz vilenie.

S'il i a chevalier tant os

Qui vuille le pris et le los

De la plus bele desranier,

S'amie fera l'esprevier

Devant touz a la perche prendre,

S'autres ne li ose desfendre. Erec, 565-78.

Nous avons vu, aux chapitres 1 et 2, que les gens riches offrent souvent des chevaux, des chiens, des oiseaux à leurs amis ou à leurs alliés. Les exemples d'une telle générosité sont très nombreux; un peu moins souvent on pense aux gens démunis, p. ex. à ceux qui ont tout perdu à cause d'une guerre, aux jongleurs ou aux serviteurs. On donne aussi d'autres animaux, comme le montre l'extrait de Roland :

Vos li durrez urs e leons e chens,

Set cenz camelz e mil hosturs muers,

D'or e d'argent .IIII.c. muls cargez, Roland, 30-2.

Li rois Ladon par grant honnor

Donna .xxirII. chevaus

A[us] .xII. nobiles vassaus, Claris, 8200-2. Cf. ib., 29677-8.

Aus gentis homes de la terre

Qui povre furent por la guerre

Donoient armes et destriers,

Robes, palefroiz et deniers Joufroi, 3365-8. Cf. ib., 179-8o.

Et ge lor dorrai volentiers

Palefroiz et dras et deniers ; Blancandin, 4269-70.

Au matin, quant il fu grant jour,

Furent paié les jougleor.

Li un orent biax palefrois,

Beles robes et biaus agrois, Atre, 6653-6.

Li bon menestreu de haut pris

Orent palefrois et roncis

Et beaz jöeaz et bons doniers. Durmart, 15131-3. 
Quant il vait en tornoi, li gentiz Alemanz,

S'i gaaigne chevaus, si les donne as serjanz

Et as chevaliers povres qui d'avoir n'ont nïent. Roche, 17-9.

Cf. $i b ., 104-6 ; 4420-1$.

Armes et destriers et chevals

En dona a cent por s'amor Diable, 268-9.

Joiax, dras de soie et destriers

lor donoit il a grant plenté. Dole, 94-5.

Dix destriers fait mener en destre,

Que li donna le roy d'Espaigne. Galeran, 3308-9. Cf. ib., 2726-

$31 ; 4932-5$.

Yder od seisi le destrier

Ci.l done a Mordrec le portier, Yder, 1477-8. Cf. ib., 1517-24.

« Berarz de Mondidier, fait ele, alez vos ant

$C^{\prime}$ on ne vos truise ci mout ascherïemant !

Tant voi an vos proësce, valor et hardemant,

Mon esprevier vos doing qi ne vole pas lant.

La roïne Lucaire m'an fist arsoir presant ;

Aufarz de Danemarche, qi l'aime duremant,

Par amors li tramist et par acointemant. Saisnes, 2651-7.

Amour envoya nostre marchant devers sa patiente, et luy presenta comme aultrefoiz chiens et oyseaux, (...) Cent, 22, 42-4.

A l'occasion du couronnement d'Erec, on lui offre plusieurs animaux :

Le jor ot Erec mainz presenz

De chevaliers et de borjois :

De l'un un palefroi norrois

Et de l'autre une cope d'or ;

Cil li presente un oistor sor,

Cil un brachet, cil un levrier,

Et cil autres un esprevier,

Cil un corrant destrier d'Espaigne, Erec, 2384-91.

La jeune veuve du prince Guillaume d'Angleterre est renvoyée chez son père, pourvue de riches dons pour celui-ci :

Quant li reis ou son filz perdu,

fille al conte d'Angou, sa bru,

od mult noble apareillement 
e od vaissels d'or e d'argent,

od palefreiz e od destriers

e od grant numbre de deniers,

enveia a Facon son pere Rou, III, 10263-9.

Le roi Bilas arrive à Rome où il compte trouver la femme qui lui a été promise ; il apporte beaucoup de richesses, avant tout à l'intention d'Evas, le père de la promise. Mais celle-ci lui sera refusée et pour l'adoucir et pour le faire renoncer à la belle, on lui propose de très riches dons :

Et maint cheval bon et isnel

Fait amener ansenble o soi,

Maint mul, maint riche palefroi,

Que il donra an cest päis,

Ou doit feire noviaus amis. Athis, 4994-8.

Se li ofrez premieremant

Chiens et ostors, or et argent,

Chevaus et murs et palefroiz

Et riches dras et biaus couroiz,

Cendaus, tirez et ver et gris

Et les avoirs de cest päis,

Por clamer quite la fience

Dont antre nos a covenence. $i b ., 6259-66$.

Vint mile mars d'or au grant pois

Et cent pailles et cent orfrois,

Cent palefroiz et cent destriers

Et cent ostors et cent levriers

Vos en donra, ce vos a dit. ib., 6343-7.

Butor fait de riches dons à la nourrice de son fils Brun, Doon paie Bueve pour son service, Auloris promet à un garçon une récompense pour le sien - dans ces cas, les animaux en question constituent une sorte de salaire :

"Chevaus et palefrois et dras fourrés de gris

«Vous d[o]nrai a plenté, du tout a vo devis. » Brun, 1990-1.

Vous trametrai vint destriers sejornés,

Vint palefrois richement enselés

Et vint ostoirs et vint faucons müés

Et vint levriers, vint brakés acoplés Bueve, I, 4376-9. 
" $\mathrm{Au}$ revenir, auraz gent guerredon :

« Je te donrai mon hermin pelison,

« Mon palefroi et mon esmerillon. Gaydon, 152-4.

Jourdain de Blaye donne des destriers au pauvre pêcheur qui l'a sauvé ; lorsqu'il retrouve sa femme Oriabel, il organise une grande fête et fait de beaux cadeaux aux jongleurs :

Jordain ramembre dou pescheor a certez

Que il trouva desor mer a un vespre

Et l'an mena la nuit a sa harberge.

Moult fist Jordains que chevaliers honestes :

Dis bons destriers li donna par les resnes

Et dis mantiaus et dis pelices vaires, Jourdain, 2065-70.

Jordains li anfes si grans noces i fist

Conme le jor que il premiers la prinst.

Cil jougleor n'i ont mie failli,

Qui palefrois, qui murlet arrabi. ib., 2507-10.

Le prud'homme qui donne des leçons à Arthur lui recommande de donner des chevaux aux hommes pauvres qui les ont mérités par leur " prouesse ». A ses vavasseurs il donnera robes et palefrois. Dans les deux cas, il aura soin de donner des animaux qu'il a déjà montés lui-même, car cela les rend plus précieux. Les " hauts hommes " (rois, ducs) auront des joyaux et des vases, mais aussi des oiseaux et des chevaux (Lancelot, VIII, XLIxa, 27-28).

Voir aussi : Mez, 2911-3；6601-3；13494-5; Florimont, 6444-6 ; 6495-6 ; Floriant, 6288-92 ; Floovant, 96-7 ; MR, LxxIV, 371-2 ; Violette, 542-3; Gui de N., 1948-9 ; Berte, 132-4; Aspremont, 142 ; Amadas, 1516-20 ; Escoufle, 138-9 ; Méliador, 2539-41 ; Ipomedon, 3858-61 ; Erec, 5354-7 ; Lais, III, 20-4 ; Brut, 2054 ; 2063-5 ; Brun, 2572-4 ; 2580 ; 2636 ; Bueve, III, 4936 ; Guillaume, 1570-2 ; Cligés, 408-11 ; 1125-6 ; Raoul, 2275-6 ; 3063-4 ; Guillaume d'A., 154-5 ; Macaire, 1684-5 ; Deduis, 12195-6 ; Lanson, 2188-9.

On trouve des exemples où différents animaux servent, avec des produits de nutrition, de rançon :

(...) : vos ne me sarés ja demander or ni argent, cevaus ne palefrois, ne vair ne gris, ciens ne oisiax, que je ne vos doinse. Aucassin, x, 67-9.

Quarante barges de fromant bureté,

Et autretant de vin et de claré,

Et autretant de bacons et de sel. 
Et mil destriers et mil mul sejorneiz,

Mil chiens, mil vetres, mil ors anchaeneiz.

Puis voz randrai le fil a l'amiré. » Enfances G., 2223-8.

Dans Yder, un épervier est donné chaque année en « droit de reconnaissance » de l'hommage promis :

Li rois feit a Talac son buen

Pur ço qu'il li out feit le suen ;

Par les cles le seisist ariere

Del Rogemont en tel maniere

Ke Talac l'en face al(e)ïance

$\mathrm{E}$ rente de reconnissance

De fieu un esprevier müés,

Il et si eir chescun an mes.

Talac feit al rei son homage

E la rente del fié li gage, Yder, 2500-9.

Pour se déplacer sur terre, on se sert de différents moyens. Le plus courant est de monter sur un cheval, un mulet etc. :

Del roi ont pris congié andui ;

Puis sont es palefroiz monté ; Joufroi, 2926-7.

Sor deus mout riches palefrois

La roïne monte et li rois ; Durmart, 1061-2.

Et de la cambre s'est partie,

Et sour son palefroi monta ; Couci, 6377-8.

Es uous sor .I. grant palefroi

Vn chevalier grant aleure, Espees, 166-7.

Puels la montait le palefroi,

Amblant s'en vet par le gravi. Florimont, 2665-6.

Lors se vestent sanz plus atendre,

.xvi. palefrois a fet prendre,

Moradas les fist sus monter, Floriant, 1179-81.

E Aye chevaucha le jor .I. fauve mul, Aye, 55 .

Estes vos .xIr. pers de la terre de France,

Les escharpes es cous, sor les muloz qui anblent. Floovant, 1403-4. 
Sur la mule qui souef amble

S'en va Fresne et depart a tant ; Galeran, 4120-1. Cf. ib.,

4136-7.

Ydain fait venir en la place

Une mule bien afeutree.

Tote sa cose a comandee

A sa mainnie, lors monta ;

Un esprevier sans plus porta

Et .ii. levriers o li en mainne. Raguidel, 3782-7.

Une dame sor .I. mure. $i b .$, 3993. Cf. ib., 3620-1.

(...) Il cevaucha trusc'a eure de nonne. Lors ataint un home de grant aage, vestu de robe de religion qui cevauchoit un asne (...) TP, VIII, 100, 2-4.

Atant ez vos Alimodés,

Un roi felon et molt engrés,

Et chevalchoit un dromadaire. Blancandin, 1101-3.

Plusieurs scènes de Lancelot montrent que les chevaliers chevauchant sous le soleil souffraient de la chaleur dans leurs armures et cherchaient souvent la fraîcheur sous un arbre ou dans les forêts. La reine de Sorestan se fait protéger sous un dais :

(...) par illuec passoit une bele dame qui estoit reine de la terre de Sorestan qui marchisoit a Norgales par devers Sorelois, et menoit avec lui plus de .LX. chevaliers armez, si portoient par desus lui .IIII. valez a cheval .I. tapiz sor .III. lances por le chaut, qui mal li feist. Lancelot, Iv, LxxvIII, 1.

Pour telle raison ou pour telle autre, deux (ou trois) personnes montent sur le même cheval :

Et mesire Gauvain prie cheli qui est a cheval que il port chelui qui est a pié et il si fait. (...) Et quant il voit qu'il ne se puet mais tenir le cheval, si monte mesire Gauvain deriere lui, si le soustient, (...) Lancelot, viII, LxIva, 17 et 31.

Quoy dea ! dist ung de ceulx d'Escallon, ilz sont deux à deux sur ung cheval ! Jouvencel, I, 139.

Le cheval Gui sunt puis munté,

Alez s'en sunt a la cité. Gui de W., 1603-4. 
Le cheval Rainbrun andui munterent, $i b ., 12450$.

Montez sunt ja li chevaler

Andui sor cel sul destrer,

Prothes[e]läus en la sele. Protheselaus, 4726-8.

Li chevax andeus les en porte, Erec, 4903.

«Et porroiz vos, fet il, chevauchier ? » Et il dist qu'il s'i essaiera. Si se drece si navrez come il estoit, et li vaslez li aide tant qu'il sont venu au cheval dont li rois estoit chaüz. Si monte li rois devant et li vaslez derriere, por tenir le par mi les flans : car il cuide bien qu'il chaïst autrement, et si feist i sanz faille. Queste, 30, 11-6. Cf. ib., 43, 21-3; 153, 7-9.

Dans l'Escoufle, le terme chevauchure apparaît plusieurs fois avec le sens de «monture », sans préciser la nature de celle-ci :

Il n'avoit en toute la terre

.II. si riches cevauceür[e]s Escoufle, 5966-7.

Or se rebaudist et enhaite

Li pelerins et aseüre

Pour la bonne chevaucheüre

Ou li vassals ne claime rien. $i b ., 6552-5$. Cf. ib., 6631.

Les personnes malades et les prisonniers - et aussi les cadavres - sont transportés sur des chevaux ou sur des litières portées par des chevaux ou des mulets :

Et lui dist le roy qu'il n'espargnast or ne argent pour le faire amener sur ung destrier conme ung larron (...) Ogier, 73.

Si vous mande de par moy ceste letre et vous dire de bouche que luy envoyez sur ung destrier lié et estroictement senglé en maniere d'ung larron. $i b ., 74$.

Et Melander monter l'ad fait

Un palefrai qu[i] süef vait.

Et cil l'en menent mult süef, Protheselaus, 11188-9o.

Car quant le vit sor le cheval liier,

Toute pasmee remest deseur l'erbier. Enfances O., 412-3.

Les mors cerchierent par chans et par larris,

Et les navrés ont a cheval remis ; ib., 1253-4. 
Quens Aymeris en avoit grant dolor

Qui oit mener sa jent a desenor.

Son un somier lo leverent garçon,

Si li lierent et les piez et les poinz. Mort Aymeri, 1596-9.

Pour Gerart sont en grant esfroi ;

Par dedesus un palefroi

Le font monter, et puis s'en vinrent

Vers le chastiel, et quant les virent

Cil dou chastiel, s'en issent fors ; Violette, 4966-70.

Vistement avoit fait .ii. chevaus encouplier,

Si troserent Loher qui tant fist a loer,

Dedenz une leitiere ont fait le cors poser ; Renaut, 8oo-2. Cf.

ib., 3366-8.

Ses plaies li font reloier

Et la letiere apareillier ;

Si l'emportent sor deus chevaus. MR, CXLIX, 601-3.

Lors voit par mout fiere mervelle

Mout cler ardoir une candelle

Trestout droit au chief d'une biere

Qui venoit par mi la bruiere

Mout tos et par mons et par vaus,

S'estoit mise sor .ij. chevaus. Rigomer, 1233-8. Cf. ib., 1243-5.

Cil .xx. cheualier amenoient

Vne litiere a .ij. cheuaus, Espees, 2004-5. Cf. ib., 1902-5.

En une grant litiere font Makaire chargier,

Mente et fenoil i mistrent por le soef flarir ;

Dui somier le porterent, cel conduit Mensier : Florence,

5961-3. Cf. ib., 4224-6.

(...) ; et andemantres qu'il se dormoit, si passa par illuec devant

.I. chevalier en .I. litiere que .II. palefroi portoient ; si estoit li

chevaliers navrez (...) Lancelot, v, LxxxviII, 7. Cf. ib., I, xxIv, 47 ;

V, xcIII, 31.

Voir aussi : TP, vI, 69, 66-7 ; Meliacin, 6258-61 ; Claris, 16980-2 ; 17021 et passim ; Meraugis, 4746-50 ; Eneas, 6125-7 ; 7487-9 ; Floriant, 1947-51 ; Roland, 1746-8 ; Wistasse, 1095-6 ; Queste, 87, 135; Yder, 2466-83 ; Méliador, 27843-4 ; Raguidel, 3616-7 ; Turpin, 1182-3; 1195-6 ; Merlin, II, 212. 
Le futur héros Lancelot et ses deux cousins, bébés, sont transportés dans des berceaux à dos de cheval :

Par chele cauchie s'en va li rois Bans, s'enmaine sa feme sour .I. palefroi grant et bel et amblant soef et un escuier moult preu et de grant service plain qui l'enfant portoit devant lui sour .I. moult grant ronchin en un bercheul. Lancelot, viI. Ia, 16 .

Et il fait prendre les .II. enfants qui estoient en .II. bercheus sour .I. soumier, $i b$., vII, IVa, 4.

Ce n'est que sporadiquement que nous voyons des personnes montées sur des voitures, celles-ci étant réservées plutôt au transport des marchandises. Nous remarquons que dans quasi tous les cas il s'agit de dames. Sans que cela soit dit expressément, nous le tenons pour donné que ces voitures sont tirées par des chevaux ou par des bœufs, comme l'indiquent les deux extraits de Laurin :

« En chars et en charetes les fesons charier ; Gui de B., 1257

Et les dames des chars commencent à crier, ib., 1554. Cf. ib., 1562 et passim.

Le char a la dame fu astellez. Laurin, 6185.

Dyogenne est montee en son char entre lui et Galienne sanz plus ; et les autres dames et les pucelles sont montees es autres chars. ib., 3609-11. Cf. ib., 3456-7; 13832-3.

Dedens ung char encourtinee

D'un tapiz qui fu faiz a Rains,

Li sisiesme de ses nonnains, Galeran, 902-4. Cf. ib., 948-9.

La roïne le voit venir,

Son careton fait coi tenir,

Blonde fist avoec li entrer. Jehan, 5711-3.

Ainssi comme il l'ot aprochie, si esgarda et vit que on menoit en .I. char une dame de grant aage. Et estoit liee par les flans au char d'une grant merveilleuse chaienne. Helcanus, 71.

En une curre est Amphïaras, Thèbes, 4951. Cf. ib., 5001 et passim.

Les personnes qui sont condamnées à mort sont transportées au lieu du supplice sur une charrette : 
Le lendemain, des le point du jour, le bourreau avec sa charrette fut devant la prison, ou il n'eust gueres esté que veez cy venir le bailly a cheval et ses sergens et grand nombre de gens pour l'accompaigner ; et fut nostre homme mis, troussé et lyé sur la charrette, (...) Cent, 75, 57-62.

C'est probablement de cette manière de transporter les condamnés que vient l'idée qu'il est honteux pour un homme estimé et sans reproches - un chevalier - de monter sur une charrette. L'exemple le plus fameux est celui de Lancelot, mais il y en a d'autres. C'est là une des formes de montrer son dédain pour quelqu'un - une autre façon est de placer la personne sur un mauvais cheval dont on a parfois coupé la queue et les oreilles:

Et li nains passe tot le chastel, et quant il est outre, si dist mesire Gauvain a Lancelot : "Sire chevaliers, dont ne seriés vos plus honorablement sor un cheval que sor cele charete?

Lancelot, II, XxxvI, 26.

Et lors esgarde, si voit venir une charete et avoit .I. cheval es limons qui avoit la coe coupee et les .II. oreilles de la teste, (...) et en la charete avoit .I. chevaliers, les mains liiees triés le dos, et une chemise sale et despanee et fu par les .Ir. jambes liés as .II. limons de la charete (...) ib., II, XL, 9.

(...) : ne des lors en avant, tant com li rois vesqui, ne fu nus hom dampnés mis en charete, ains avoit en chescune vile un viel roncin sans coe et sans oreilles, si i montoit l'en cels que l'en voloit honir et si les menoit l'en par totes les rues. ib., II, $\mathrm{XL}, 23$.

Al matin, quant li solaus fu levez, s'esveilla mesire Gauvain, si se trova en la charete la plus laide del mont et vit son escu lié as limons de la charete et son cheval atachié a la coe de la charete; mais es limons devant avoit .I. cheval si maigre et si chaitif qu'il valoit a paines par samblant .III. deniers. ib., II, LXVI, 31.

Et vit le chevalier en cors,

Deschau[z] et nu sor un roncin,

Con s'il fust pris a larrecin,

Les mains liees et les piez. Erec, 4380-3.

Les cadavres des héros morts à Roncevaux sont transportés sur des charrettes : 
Li reis cumandet Tedbalt e Gebuin,

Milun le cunte e Otes le marchis :

« En .III. carettes les guiez ... » Roland, 2970-2.

Pour le transport de marchandises on se sert le plus souvent de bêtes de somme - des chevaux grands et forts, des mulets, des ânes, des chameaux et des dromadaires. Cf. Perrine Manne, op. cit., p. 345 : « Dans le transport des hommes et des marchandises, le cheval joue un rôle primordial. Cependant, qu'il soit bâté ou non, le cheval est en compétition avec d'autres animaux. Pendant tout le Moyen Age, les lourds charrois de bœufs sillonnent les routes et les ânes sont largement enrôlés. »

Ainz aimes mieuz tot oen mes

de la buche porter grant fais

et granz sachiees de charbon, Renart, III, 9045-7. (un âne)

Il avint ja à Monpellier

C'un vilain estoit costumier

De fiens chargier et amasser

A .II. asnes terre fumer.

.I. jor ot ses asnes chargiez; $M R$, cxIV, 1-5.

Li carbonniers .j. asne avoit,

Dont son carbon vendre portoit. Wistasse, 1006-7. Cf. ib., $1014 ; 1028-9$.

Vint chameus toz chargiés entr'or fin et argent, Florence, 207.

Cf. $i b ., 3176$.

.vir.c. cameilz, d'or e d'argent cargiez, Roland, 645. Cf. ib., 32 ; 130.

Por lui dorroie grant tresor :

Chargiez set dramadaires d'or. Blancandin, 2025-6. Cf. $i b$., 2073-5.

Il ly enveyerent trente chivaus chargés de oor e de argent e de autres richesses de Espayne, e carante chivauz chargés de vyn dous e mile beles Sarazynes. Il parleunt a counseyl a Genyloun e ly promistren(s) vint chivaus chargez de oor e de argent e de pailes pur (...) Turpin, 918-22.

Un cheval chargié de besans

Moenrons o nos, » dist Solymans, Florimont, 12439-40. Cf. ib., 12468-9. 
E chargent les sonmiers qui la vitaille portent. Aye, 1403.

Ha ! nobles empereres, prenez de nostre avoir,

Rouge or e blanc argent e bons pailes grejois,

Plus que ne porteront .iii. mul espeingnois. » $i b ., 2646-8$.

Ne deïst un sol mot por .x. muls d'or chargiez. Saisnes, 37772.

Comme le montre Fr. Schmidt, op. cit., p. 104, on utilise aussi, mais moins souvent, des charrettes, tirées par des chevaux ou par des bœufs. Ces animaux peuvent être appelés limoniers (cf. p. 188) :

La veïssiez tant tré, tante tante chargie

An chars et an charretes et sor murs de Hongrie! Saisnes, 5805-6.

Si me fai faire un char molt noble,

D'or et d'azur et de synoble,

Garni de cinc si fors chevaux

Que ne lez tieigne mons ne vaux ; Anjou, 2735-8.

En ceste terre a il charroi assez, Chars et charretes i a a grant planté.

Fetes voz genz arriere retorner

Par Ricordane, ou nos somes passé,

Si faites prendre les bués par poesté. » Charroi, 949-53. Cf. ib., 958.

(...) ; mais il ne fut pas si tost a Paris, a sa derreniere charetée, que la porte a ses talons ne fust fermée. Il fut tresbien venu et receu de l'orfevre. Et, après que son charbon fut deschargé et ses chevaulx mis en l'estable, il voult soupper tout a loysir,

(...) Cent, 7, 11-6. Cf. ib., 73, 69-72.

J. careton aconsivi

Qui une carete menoit

A .iiij. chevals qu'il avoit. Wistasse, 160-2.

S'i en despant d'avoir plus que n'en puist pourter

Ung char a sis chevalz quant que porait mener, Lion, 6274-5.

Cf. ib., 13060-1.

Et fit l'empereur faire conmandement de charir vivres a l'ost qui tantost fust assemblee. Ogier, 17 
Et des .xxxIr. chevaliers eurent de finance tant conme deux chariotz pouoient porter. $i b ., 72$.

Chargent charretes, chargent chars Claris, 13506. Cf. ib., $14859 ; 28706$.

Et lors le roy de Navarre cria a ceulx que les chevaulx dedicts charietz conduisoient, (...) Jehan de P., 55, 5-6.

Et on y aporte et amaine,

Et sur charretes et sur chars,

Cerfs et cengliers et autres chars, Galeran, 6782-4.

Merlin recommande d'utiliser des chevaux pour transporter de la terre. L'extrait de Bâtard, ne montre pas très clairement si la charge de charbon est placée sur le cheval ou si celui-ci tire une voiture avec les sacs de charbon - ou peut-être les deux ? :

Or m'enseigne dont comment je ferai la terre oster. » Et Merlins dist : «Tu le feras oster a chevaus et a charetes et as hommes (et) au col et (faire) porter loing. » Merlin, I, 54 .

Li Bastars de Buillon se prist a regarder

Et voit .j. carbonnier les .j. cheval aler,

La ou il avoit fait sas de carbon poser,

Que droit a Mont Oscur il les devoit mener.

Li Bastars esporonne, lors le va saluer,

Puis li a dit briement : "Ne me voeilliés cheler :

Et ou devés vous ore le voiture mener ? " Bâtard, 5842-8. Cf.

$i b ., 5869 ; 5873 ; 5894$.

Broifort, le bon destrier d'Ogier, doit transporter des pierres pour la construction d'une abbaye pendant l'emprisonnement de son maître. Ogier est indigné que l'on ait osé utiliser un destrier à une telle tâche :

(...), et son cheval pour ce qu'il estoit grant et fort fut mis a charier la pierre de l'eglise, ou il demoura par l'espace de sept ans. Ogier. 115.

Et ainsi que Ogier se deconfortoit de son cheval ung moine qui estoit en sa presence, lequel estoit de l'abbaye de saint Pharon de Meaulx, conmença a dire : Sire Ogier, vostre bon cheval Broifort est a Meaulx, lequel depuis que vous fustes prins n'a fait autre chose que charier la pierre en l'abbaye de saint Pharon, laquelle l'abbé fait édifier tout de neuf. A ce dist Ogier : ce- 
lui la qui mist mon cheval a tirer la pierre me prisé a bien peu, car vraiement oncques si bon cheval ne tira pierre. Maudis soit il qui luy a aprins le mestier. $i b ., 140$.

Les bêtes de somme constituent la seule richesse de quelques paysans ; ceux-ci sont donc livrés à pauvreté quand ils sont privés de leurs animaux :

« Dame, merci pour Dieu, de vo fille me plain!

N'avoie c'un cheval dont gaignoie mon pain,

Dont je me garissoie et ma fenme Margain

Et mes petis enfans, qui or morront de fain ;

A Paris en portoie chaume et buche et estrain,

Soisante sous cousta, un an a, en certain,

Or le m'a fait tolir, Dieus li doinst mal demain !

A meschief l'ai norri cest yver de mon grain. Berte, 1759-66.

Lors encontra .I. vilain qui menoit .I. asne charchié de buche.

(...) Et quant je reving la ou je avoie leissié mon asne, si trouvai dusques a .vi. leus qui ja l'avoient estranglé et le voloient mengier. Et quant je ving la, si ne sai quel part aler, ains m'an vois a mon ostel tel duel faisant come vos poez veoir. Et je n'ai mie tort, se je em plor, car je n'avoie aide a mon pain gaingnier se l'asne non. Lancelot, IV, LXxI, 1 et 9.

Pour tirer les charrues et les herses, nos textes ne présentent presque que de gros bœufs. L'extrait de Perceval nous apprend qu'il faut deux bœufs pour tirer une herse.

Citons, à propos de la charrue, ce qu'écrit Robert Delort, op. cit., pp. 126 et 128 : « La lourde charrue de fer - ou, du moins, dont les pièces majeures (soc, versoir, coutre) sont en fer - a amené une révolution sur de nombreux points : chère, elle est la propriété de quelques-uns ou d'une communauté dont la solidarité est ainsi renforcée ; lourde, elle doit être tirée par plusieurs bêtes (bœufs ou surtout chevaux) et pose donc des problèmes de l'attelage, du gros bétail et de la main-d'œuvre paysanne : un bouvier pour exciter les bêtes, un laboureur aux mancherons. (...) L'usage de la force motrice traditionnelle, fournie par les animaux de trait, a été à son tour grandement facilité par la diffusion de nouvelles techniques. La lourde charrue posait par exemple le problème de l'attelage : bêtes plus nombreuses, plus fortes, plus rapides, plus vigoureuses. On ferre les bœufs, on emploie, si on le peut, des chevaux, jusque-là réservés à la guerre encore plus qu'aux charrois. L'extension de cet élevage spécialisé est freinée par la routine, par la fragilité de ces bêtes, donc leur prix, mais la diffusion de la culture de l'avoine à cette époque (milieu et fin du xir ${ }^{\mathrm{e}}$ siècle en Picardie, par exemple) est sûrement en relation avec leur utilisation 
plus fréquente. De son côté, le bœuf, plus lent mais plus fruste et très robuste, disparaît de quelques régions à l'agriculture très avancée. Mais au xiıI siècle le cheval ne l'a nullement éliminé du Bassin parisien, a fortiori des pays méridionaux. »

Tout ceci est confirmé par Georges Duby, Féodalité, p. 192 : « (...), dans certaines provinces, les cultivateurs choisirent de substituer le cheval au bœuf pour les travaux agricoles. Cette mutation s'est produite sans doute dans les contrées les plus fertiles de l'Occident pendant la seconde moitié du xiI ${ }^{\mathrm{e}}$ siècle. (...) L'avantage du cheval est sa rapidité. L'atteler à la charrue, c'était accélérer sensiblement les façons de la terre, c'était par conséquent se donner le moyen à la fois de multiplier les labours et de pratiquer le hersage : déjà , la "tapisserie" de Bayeux, à la fin du xi siècle, montre une herse tirée par un cheval. »Cf. Perrine Manne, op. cit., pp. 341-342. L'Histoire de la France rurale, nous apprend, I, p. 453, que " la charrue (...) requiert un fort attelage, deux paires de bœufs ou davantage si le sol est lourd; au minimum deux chevaux, ou trois, voire quatre, en Ile-de-France ou ailleurs, lorsque la terre est très grasse. » et, p. 455 : «En liaison avec l'expansion de la charrue, et plus importants qu'elle encore, puisque les effets s'en firent sentir dans tous les transports routiers, furent les progrès de l'attelage et l'usage du cheval à des fins de traction et non plus surtout militaires. » (voir aussi la suite).

An plorant li dist, sel deslie

Rognel si main, que sa jornee

iert tote a noient atornee,

que nul esploit ne porra faire,

que li .vir. buef ne püent traire,

que trop est fort la terre et dure. Renart, IV, 9484-9. Cf. ib.,

9440-3.

Ier main m'iert avis que j'avoie

trop en .virI. bues a ma charrue. ib., IV, 9666-7. Cf. ib., 9409-14 ;

9922-8.

J'estoie luiés a un rice vilain, si caçoie se carue, quatre bués i avoit. Or a trois jors qu'il m'avint une grande malaventure, que je perdi le mellor de nos bués, Roget, le mellor de ma carue ; si le vois querant, si ne mengai ne ne buc trois jors a passés ; si n'os aler a le vile, c'on me metroit en prison, que je ne l'ai de quoi saure : Aucassin, xxiv, 49-53.

Il me vendra mes bues requerre,

Quant il voudra arer sa terre, $M R$, cxvI, 43-4. 
A Longueville avoit un villain païssant qui avoit sez beaux beuff et sa charue arant ; Rou, II, 12367. Cf. $b ., 693-5$; III, 11135-8.

Dous bués avommes en l'aree,

Ses maingerons a la pevree. Florimont, 4403-4. Cf. ib., 505-8.

«Mal semble cist coux de bouvier,

Qui a ale a la charrue. »Claris, 22868-9.

Del mestier de charrue vous savriiez aidier,

De la keue tenir ne les chevaus chacier ? Gautier d'Aupais, 201-2.

«Vostre chevaus n'est mie des miex corans :

« L'autre jor nen ert mie si rabiant,

"Ains resambloit ronchin a paisant,

« Destelé de kerue l'as, recreant Aiol, 4228-31.

Car vos porpansez, damoisele,

de ce vilain qui si vos tue

et vos fait traire a la charrue ; Renart, v, 15018-20.

Une charrue adès avoit,

Tos tens par lui la maintenoit

D'une jument et d'un roncin ; $M R$, LXxIV, 3-5. Cf. ib., XIII,

121-5.

Et li R., ce sevent li auquant,

$\mathrm{Fu}$ asez graindre, par le mien esciant,

Qe d'un torel a charue traiant. Raoul, 3244-6.

Il ne sot tant son cheval esforcier

Ne le passast .j. roncins charuier. $i b .$, 3400-1.

Il pensa que veoir iroit

Herceors que sa mere avoit,

Qui ses avaines li semoient :

Bués doze et sis herces avoient. Perceval, 81-4. Cf. ib., 300-10 ; $323 ; 327$

Quelques textes mentionnent que les bœufs - estimés très forts - tirent les voitures lourdes ou servent à tirer de lourds objets, tels des troncs d'arbre : 
Encor ai ge un tresor eissi grant

Ne le trairoient .x. bues en charoiant ; Aliscans, 3101-2.

(...) . xx. arbres et plus, si grans que quatre buefz avroyent assez a faire de traire le meindre, (...) Papegau, 17, 9-11.

Nous voyons l'âne chargé de tirer l'eau du puits :

L'asne acoillent a la polie,

qui de trere pas ne s'oublie :

li randu le vont menacent

et li anes va fort traient. Renart, II, 3635-8.

Une manière courante de punir les criminels, les ennemis vaincus ou toute autre personne qui s'est opposée à la loi ou à la morale, est de les traîner à la queue d'un cheval (pour les pendre ou les brûler après) ou de les écarteler en leur attachant jambes et bras à la queue de quatre chevaux qu'ensuite on fait courir chacun dans sa direction :

Sor tuit li altre l'unt otriet li Franc

Que Guenes moerget par merveillus ahan.

Quatre destrers funt amener avant,

Puis si lient e les piez e les mains.

Li cheval sunt orgoillus e curant :

Quatre serjanz les acoeillent devant

Devers un' ewe ki est en mi un camp.

Guenes est turnet a perdiciun grant ;

Trestuit si nerf mult li sunt estendant

E tuit li membre de sun cors derumpant :

Sur l'erbe verte en espant li cler sanc. Roland, 3962-72. Tur-

pin, 1176-8.

Adonques fu Hervix en la place emené,

A .iiii. buens chevaux qui furent abrivé ;

Le traïtor ont pris si l'ont si atorné

Com vos porrez oïr s'entendre le volez :

Et es piez et es poinz a granz seïns noez,

A chascune des menbres fu .I. cheval coplez,

Toz les .iiii. chevaux ont brochiez et hastez ;

Iluec fu li traïtres et morz et afolez. Renaut, 2984-91.

Demain lor iert randu por Charle nostre irors,

Qar issir les ferai de lor piax a rebors,

La char oindre de miel, sa donrai a mes ors,

Et derompre a chevax antre .II. carrefors. » Saisnes, 637-40. 
« Je le pandrai as fourches, ja n'an ert trestornés,

« Et si sera vilment à chevaus traïnés

« De Rainc jusc'à Orliens, voiant tot le barné. » Gui de B.,

1001-3.

«Vos revanrez à Basme les jugemans oïr

«A l'amiraul de Perse, qui vos ferai morir

« Et detraire à chevaus et les manbres tolir,

« Puis vos ferai toz pandre ardoir et anfoïr ; Floovant, 1837-40.

De la prison l'osta avecques ses privés

Et a .iiii. chevaulx fut tantost atellés.

Tout contreval la ville fut il bien traïnés. Tristan de N., 16367-

9. Cf. ib., 2918-9; 12454-5.

A la cowe dairier de son riche bauchant

Atellait le glouton, puez le vait trayant

Jusques a Lion son perre, et li dit en riant : Lion, 25788-90. Cf.

ib., 3506-7; 13936-7.

Et quant le rois oï ce, si les fist despoillier tos nus et trainier aval la vile a coes de chevals et lors les fist mener a une crois (...) Lancelot, II, LX, 22.

Et pour itant di par raison

Qu'il soit a chevaus traïnnez

Tant qu'il soit trestous demenbrez, Floriant, 5766-8. Cf. ib., 5789-91.

Atant les fist prendre tous douze, si les a fait liier a douze chevaus, et tant les fist detraire que pau en remest ensamble. Merlin, I, 37.

La est Guibors la contesse honoree,

demain doit estre a chevaus traïnee. Loquifer, 2692-3.

Madame de Vendôme propose la punition suivante de la "dame » de Jehan de Saintré, pour son infidélité :

«J'en dy, beaus amis, c'on la devroit lyer sur un asne, le vis devers la queue, et mener par la ville a grant desrision. » Jehan de $S ., 305,27-9$.

Dans Lancelot, nous voyons Mordret, « le chevalier vaincu », victime d'une punition plus méprisante que cruelle : lié sur un mauvais cheval, il est mené par les rues où on lui jette du fumier et des ordures : 
(...) si voient un home aler en braies par mi la rue et estoit montez sor .I. cheval chaitif et megre et avoit liez les piez par desouz d'une corte. Après lui venoient plus de .c. ribaut qui tout le huoient et crioient après lui et lui getoient fiens et boe et ordure ; (...) Lancelot, v, Lxxxv, 12.

Dans le même texte, un mari trompé punit sa femme adultère en la traînant par ses tresses pendant qu'elle court à côté de son cheval, et la dame de Roestoc veut punir le nain qui a si souvent insulté Gauvain :

Devant le pavillon avoit .I. chevalier tout armé sor son cheval et tenoit encoste de lui .r. damoisele toute nue en sa chemise qu'il aloit batant et trainant par mi les tresces tout a cheval et li faisoit toute la honte et toute la vilonnie qu'il pooit sanz li occirre. (...) et pris la damoisele et la tres hors del pavillon, si l'aloie traiant par les treces et batant encoste de mon cheval, (...) $i b .$, IV, LXXXIII, 42 et 70 .

Et si sache que por la honte qu'il dist au chevalier en prendrai je venjance, que a l'entree de toutes les viles ou je venrai li ferai liier .I. cavestre au col et a la coe de mon palefroi, si le trainerai aprés moi, ne ja pour lui n'apeticherai m'aleure. $i b .$, vIII, LVIa, 52. Cf. ib., viII, LVIIIa, 3.

Voir aussi : Aliscans, 4159-60 ; Eracle, 1149-51 ; Thèbes, 2407-8 ; Mez, 10197-9 ; Claris, 2455-8 ; Cont. P., II, 11900-1 ; 13246-9 ; Rigomer , 7399-400 ; Amadas, 745-9 ; 6833-4 ; Silence, 4230-1 ; 66546 ; Anjou, 7792-6 ; Parise, 841-3 ; Huon, 9985-8 ; Charrete, 3454-5 ; Bueve, I, 1972 ; 5608-9 ; Graal, 1891-7 ;Jourdain, 4123-6 ; Athis, 732 ; Ogier, 162 ; 167 ; 264 ; Blancandin, 1021-3 ; 5492-3 ; Godin, 862-9 ; Doon, 9658-9 ; Cligés, 1481-5 ; Simon, 2071 ; Orson, 2688 ; Laurin, 4486-8 ; Lanson, 776 ; Cristal, 8294 ; 9005 ; Bérinus, 461.

L'un des grands plaisirs des nobles est la chasse. On la pratique de plusieurs manières, en fonction de la nature du gibier. Le grand gibier (sanglier, cerf) est poursuivi à cheval et avec une meute de chiens jusqu'à ce que la fatigue l'oblige à se présenter au combat final. Lièvres, lapins etc. sont attrapés par les chiens (d'où le terme lévrier), mais aussi par les oiseaux, qui chassent également perdrix, canards sauvages etc.

Citons Robert Delort, op. cit., p. 179: " La chasse est à la fois un excellent entraînement, un jeu passionnant et un sport utile, détruisant des fauves ou des nuisibles et fournissant à la table seigneuriale une nourriture riche et carnée qui maintient ou augmente la vigueur des commensaux. Elle exige un équipement coûteux et un personnel nombreux. La plus belle et captivante, 
très accessible aux dames, est la chasse par oiseau interposé ; des rapaces de haut vol (gerfaut, faucon) ou de bas vol (autour), minutieusement dressés, interceptent des rongeurs type lapin ou lièvre et surtout de grands oiseaux : hérons, grues, canards ; et des chiens particulièrement entraînés, après avoir levé ce gibier, se précipitent au secours du faucon dès qu'il est à terre, pour achever sa victime en évitant de le blesser. » Voir aussi Jean Verdon, op. cit., pp. 61-63.

Le jeune Blancandin apprend, entre beaucoup d'autres choses, comment tenir les chiens et les oiseaux de chasse :

Li latiniers par fu tant saiges

Qu'il li aprist de toz langaiges ;

D'eschés, de tables, de deduiz,

De chiens et d'oiseax bien fu duiz. Blancandin, 39-42.

Aux vers 559 à 662 d'Ipomedon, Hue de Roetlande nous donne une belle description d'une chasse. Nous n'en présentons qu'un petit extrait, suivi d'autres exemples montrant des chiens (et parfois, des chevaux) en action :

Par tot fet venir e mander

Ses veneors et ses vallez,

Ove levrers, ove brachés,

Ove veltres, ove limers,

E cil i venent volenters ; Ipomedon, 566-70. Cf. ib., 581-2 et passim.

Tholomeu reest aprés venu

Sur un grant chaceür tundu,

Pruz ert e forz cil chaceür

E cil semble ben veneür ; ib., 2697-70o.

Tantost la dame a commandé

Que li chien soient acouplé ;

Enseler fait ses caceours

Et atorner ses venours. Guillaume d'A., 2621-4.

Aprés le sivent liement

Bracet, viautre qui vont saillant,

Qui vont aprés le cerf braiant. Inconnu, 1283-5.

Puis a ses veneors mandez,

Ses brachés et ses loienmiers Floriant, 288-9. 
Li quens ala un jor chacier,

Avesques lui troi chevalier :

Les chiens meinent li veneor. $M R$, cxLIX, 55-7. Cf. ib., 459-6o.

Li cers estoit mout legiers a merveilles et si isniaus qu'il avoit tant fui et alé que tout li cien l'avoient perdu. TP, vI, 23, 11-3.

Cf. $i b .$, vI, 23, 17-9; v, 31, 6-11 et passim.

A un matinet se leva

Et o sa gent cachier ala ;

Mutes de chiens i fait mener

Et viautres por prendre sengler. Partonopeu, 531-4. Cf. Parto-

nopeu-C, 53-7.

Et il n'avoit de riens envie

Fors que de mener bone vie,

D'aler as chiens et as oisiaux. Floriant, 6595-7.

Si prent ses chiens et ses levriers,

Ses brakes et ses loiemiers,

Et se met en droite ordenance

Pour cacier, il y a fiance,

A .I. cerf qui est en ses bois ; Méliador, 1072-6. Cf. ib., 117-22 ;

161-4;3321-2.

Voir aussi : Lais, I, 81-2 ; IV, 139-42 ; Erec, 120 ; Lancelot, I, IX, 7 ; Roussillon, 650 ; 6640 ; Protheselaus, 1776-8 ; 1872-3 ; Rou, III, 51922 ; Cligés, 2749-51;6342-4.

Et voici des exemples avec des oiseaux. Dans le long extrait de Durmart, le jeune protagoniste observe des chevaliers qui s'amusent à la chasse avec des faucons; la chasse dure jusqu'au coucher du soleil :

Atant e vous Lion que sur son poing portait Ung moult bel esprivier ou il se delitait.

Entrér est en la saulle ; de voller repairait. Lion, 1286-8.

Tant que un jour li prist envie

$\mathrm{D}$ 'aler en gibier pour deduire

Et pour son esprevier miex duire

A prendre l'aloe et la quaille ; Violette, 4146-9.

Chascuns mete an son dos son hauberc fremillon

Et monte an son cheval auferrant ou gascon,

Et port chascuns oisel, esprevier ou faucon,

Aval lez la riviere deduire nos alon! " Saisnes, 1442-5. 
N'oublierent pas lez oisiaux :

Gierfaus, faucons, gentilz laniers

Et tercellés, qui sont maniers

Des heirons et des ennes prendre. Anjou, 512-5.

Por salf conduit avrés si riche don,

car nostre maistres a ancor tel folcon

qui prent lou lievre, la grue et lou hairon ; Loquifer , 71-3.

Et si getames nos ostoirs el laris ;

Un en perdimes ersoir a l'avesprir. Huon, 1384-5.

Il se leva un juesdi devant prime,

Esbanoier s'en va lez en marinne,

A douz faucons ont abatu un cisne, Jourdain, 2271-3.

Devant lui regarde, si voit

Chevaliers qui vont riverant ;

A pié coroient li alquant,

Grant noise mainnent et grant bruit

Cil qui la sunt en lor desduit.

Quatre faucons en haut jeterent,

Et cil qui de ce se meslerent

Fisent bien sordre les oiseaz ;

Mout lor senbloit li desduis beaz,

Car quant li un faucon montoient,

Li autre faucon descendoient.

Si tost se vont aval coler

Que la ne lor puet eschaper

Hairons ne ane ne mallars.

(...)

Adont vont lor faucons loier

Cil qui les orent a garder ;

Vers l'ostel welent retorner,

Car le solel voient couchier. Durmart, 9108-25 et 9162-5.

Claudas préfère les faucons, qui volent haut, aux autours :

Il amoit riviere seur tous deduis et plus les faucons que les ostoirs (...) Lancelot, vII, viIIa, 3.

Voir aussi : Blancandin, 3464-7 ; Rome, 336-8.

Dans Protheselaus, Jonas, le valet de la reine Medea, se sert de son épervier pour envoyer au protagoniste une lettre de la reine ;Protheselaus se sert du même messager pour expédier la réponse : 
Jonas fu ben apris et sages

Et saveit [de] plusurs languages.

Od lui p[or]rta un esperver,

De quatre feiz esteit müer.

(...)

Sor son poing porte l'esperver.

(...)

$\mathrm{Li}$ bons vallez tost aparceit

Que cil ben le conui[s]t et veit.

Il en a trait les brés s'amie,

$\mathrm{A}[\mathrm{s}]$ gez de l'esperver les lie.

Le poing [et] le braz li tendi,

L'esperver de loinz li offri.

Mult ert de lai lé et parfunt,

Grant vol ot desque la [a]munt ;

Mais Jonas fu mult enseinné,

L'esperver ad ben afaité,

D'asez loin a[1] reclaim veneit,

Kar alques a[i]gre le teneit.

(...)

Prothes[e]läus ben saveit

Que vers lui l'esperver tendeit.

(...)

Il ad escrit brés a dreiture

$\mathrm{Al}$ melz qu'il deviser les sot

Et al plus bel qu'il faire pot.

A l'esperver les ad chargié[z]

Et as gez les ad ferm lié[z].

A l'endemain as kernels vent

Et sor sun poin[g] l'esperver tent. Protheselaus, 7410-3, 7425,

7440-51, 7456-7 et 7557-63.

Si les chiens des nobles sont utiles et même indispensables pour la chasse, les roturiers, eux en ont pour garder leur propriété contre les intrus de toute sorte : voleurs aussi bien que bêtes sauvages. Il n'est donc pas étonnant que les renards et les loups craignent les chiens de garde du vilain :

Adam la verge reprisse a,

en la mer fier par maltalant ;

un chien en saut hastivement.

Qant vit le leu, si laisse corre

por la berbiz qu'il vost rescorre ;

il li resqueut : mout a enviz

la laissa li leus la berbiz ; Renart, II, 3798-804. 
Li garz qui suit Renart premiers,

qant il choisi les liemiers,

voit le convers, si li escrie :

«Deslie, va, les chiens deslie ! » ib., II, 4599-602. Cf. ib., 9682-

96 et passim.

Aux pages 155-156, nous avons vu que les dames nobles avaient des (petits) chiens pour leur divertissement. En voici un autre exemple, tiré de Lion :

Se sciet une nonnain sur l'erbe qui verdie ;

Abbausse fuit du lieu et de la manandie.

Toute soulle fuit la, a ung chien s'abanie. Lion, 10680-2.

La reine Medea possède deux chiens : un petit chien braque et un grand lévrier ; celui-ci est si sauvage que tout le monde a peur de l'approcher. Quand il est sur le point de tuer le petit braque, il est lui-même tué par Protheselaus. La belle dame du lai Lanval possède un lévrier et un épervier :

La rëine aveit un brachet

Ne mie grant, mes petitet.

Et l'ot lunges süef gardé :

Ipomedon 1[i] ot doné,

Tant cum il fu son chevaler ;

Et n'ot un[e m]es aver si cher.

De le cambre est venu juant,

Un lien d'argent träinant.

La rëine rot un levrer

Ki mult ert grant, hardi et fer ;

Le secle n'aveit si grant chen,

Si resteit fel sor tute ren.

N'ot beste el mond, [s'a lui] mellast,

Qu'il a force ne devorast.

Il n'ot en la curt chevaler,

Vallet [ne[ serjant n'esquïer

Qui pur ren l'osast manïer,

Pur qu'il le vëist curucer.

La u li levrers un os tent,

Li brachet par devant li vent.

Li levrers esteit famillus

Et fu mult felet envïus :

Salt al brachet [et si] le prent,

Et la rëine en halt s'escrie :

« Mis brachet mort, pur deu äie !

Aidez, cheles, a mon brachet!"

N'i ot chevaler ne vallet 
Qui tucher osast le levrer,

Kar mult ert fel et paltener.

$\mathrm{N}$ 'i ad nul qui socurs li face,

Estranglé l' [ë]ust en la place.

Prothes[e]läus est irez,

Ultre la table salt junz pez,

Le levrer äert par le col :

[Li] plusors le tenent pur fol.

Li levrers le brachet guerpist

Et celui par le braz seisist,

A poi la dent parmi ne vient.

Prothes[e]läus ferm le tent

Et l'ad destreint par tel äir,

Tot li estot le braz guerpir.

A qui qu'il peist u qu'il mal face,

Estranglé l'ad en[mi] la place. Protheselaus, 3276-319.

Un espervier sur son poin tient,

E un levrer aprés li vient. Lais, v, 573-4. Voir aussi p. 231, Ra-

guidel, 3782-7 et cf. $i b ., 4474-5$.

D'autres personnes aussi gardent des chiens pour leur plaisir, tel le bon curé de l'extrait suivant :

Ce bon curé avoit ung chien qu'il avoit nourry de jeunesse et gardé, qui tous les aultres chiens du païs passoit d'aller en l'eaue querir le vireton, ung chappeau si son maistre l'oblyoit ou de fait apensé le laissoit quelque part. Bref, tout ce que bon et sage chien doit et scet faire il estoit le passe route ; (...) Cent, 96, 5-11.

Dans Helcanus, un chien meurt après avoir bu une boisson empoisonnée : on se sert de lui comme cobaye ; dans Parise, un porc a le même rôle :

Dont s'apenssa que il ensaieroit le beuvrage. Et il maintenant en a versé en .I. henap et en mist a .I. chien devant, qui grant soif avoit ; et si tost comme il en ot avalé, il chaÿ mort devant lui. Helcanus, 310.

Ancor tenoit la pome qu'en son poign ot saisie,

Que li dona la dame an sa chambre voutie.

Li dux li a osté, de ses mains l'a saisie,

A .r. porc la geta lez une chambre antie :

Li cuers li est partiz et li oil li saillirent. Parise, 157-61. 
Dans Bueve, un lévrier meurt après avoir mangé le poison destiné au jeune protagoniste :

Trestous les jors que le palais monta.

Or oiés ja, que la mere fera :

Grandes culevres et crapaus asambla,

Porir les mist et puist les pestela,

Trestous les jors a son fil les dona ;

Ainc n'i parut ne ainc ne s'en garda ;

Quant vient un jor, li enfes s'apensa,

A un levrier que Dos i amena

En a doné, et li chiens trebucha

Et cäi jus, car li mort le toucha ; Bueve, I, 679-88.

Comme les chiens aiment mordre, on peut les utiliser pour punir les personnes importuns :

Si voit en mi liu l'escacier

Que on devroit as cins kacier

U mout plus haut qu'.I. autre pendre. Hunbaut, 1379-81.

Nous venons de le voir : les chiens protègent les hommes et leurs propriétés, y inclus ses autres animaux. Les chats s'occupent des souris et des rats qui pourraient faire de gros dégâts en mangeant le blé, le fromage etc. et en rongeant les boiseries des maisons :

Le chat aus souris prendre

Por les huches desfendre $M R$, XLIII, 129-30.

Et li vilains le dist en ses escris :

« Li fix al cat doit prendre le soris. » Aspremont, 756-7.

Au tinel vont por essaier lor bras,

Mes nel meüssent, qui lor donast Damas,

Ne nul des autres, qui lor donast Baudas ;

Et cil le lieve comme soriz fet chaz. Aliscans, 4848-51. Cf. ib., 3916-7.

«Tibert, savez que nos ferons ?

Ce dist Renart ; ci maint un prestre,

si connois bien trestot son estre ;

assez a froment et avoine,

mais les soriz li font grant paine ;

mengié en ont ja pres d'un mui. Renart, I, 834-9.

Les coqs sont utiles à l'homme en ceci qu'ils lui annoncent le lever du soleil, c'est-à-dire le moment où lui-même doit se lever 
pour reprendre son travail. On remarque que, dans plusieurs cas, les auteurs les font chanter à minuit déjà ; probablement ne fautil pas prendre ces affirmations au pied de la lettre mais comprendre qu'il faisait encore noir.

Nous avons vu bien des exemples du chant des coqs aux pages 217-218, en voici d'autres :

Tout droit a mieneut que li cocque vont chantant Lion, 15589.

Car il dit et affie que droit au coq chantant

Le voleiste mordrir per neut en son dorment. ib., 13989-90. Cf. ib., 13924-5 et passim.

Dient Franceis : « Lais nus, lecchere, ester !

Mal seit l'ore qui li tuen cors fu né.

Uncor n'ad li cos, ço quid, que dous feiz chanté. » Guillaume, 2902-4.

Ainz que li cos chantast, Gerars i vint. Mez, 2110.

Quant ot chou fait, tantost li cos chanta, Auberon, 449.

Aprés la mie nuit que coc orent chanté,

Se leva Clarïons, n'i a plus sejorné. Buevon, 839-40.

Segnor, sien poiat del premer gal, Roussillon, 1610. Cf. ib., 933 ; 9588.

La meschine qui, la nuit, doit porter la future Fresne, encore bébé, dans une région étrangère, se trouve rassurée quand elle entend le chant des coqs et l'aboiement des chiens, signes qu'elle se trouve à proximité de la civilisation ; une scène semblable, avec le roi Arthur, se trouve dans Lancelot:

En un grant chemin est entré,

Ki en la forest l'ad mené.

Par mi le bois sa veie tint,

Od tut l'enfant utrë en vient ;

Unques del grant chemin ne eissi.

Bien loinz sur destre aveit oï

Chiens abaier e coks chanter :

Iloc purrat vile trover.

Cele part vet a grant espleit

U la noise des chiens oieit. Lais, III, 139-48.

La ou il depeçoient le porc, si oï li rois un coc chanter a destre et ne sambloit estre gaires loing. Li rois ot de mangier talant, 
si saut en son cheval et vet cele part ou il oï le coc chanter, (...) Et quant i fu un poi alés, si trova un porpris clos a la roonde de palis. Lancelot, I, IX, 8.

Au marché, les paysans viennent vendre leurs animaux, ou ils en achètent (pour l'importance des marchés, cf. infra, 4.2., Histoire de la France rurale, I, pp. 587-588). Les marchés sont également fréquentés par chevaliers et marchands :

Il ne trovoient bel avoir

a nule foire ou il alassent, ne biau cheval, qu'il n'achatassent

por presenter l'empereor. Dole, 605-8.

Et sachiez que Chevalerie

Doivent Marchéanz tenir chiers

Qu'il amainent les bons destriers

A Laingui, à Bar, à Provins $M R$, xxxvII, 24-7.

Et tels i a vont en Bretaingne

Buès et pors, vaches achater $i b .$, xxxvII, 52-3.

.xII. deniers en ot Giraus

Qui mes .II. bues m'aida à vendre. $i b .$, cxvI, 36-7.

Quanques ses barons aportoit

Si tresbien et si cier vendoit

Qu'ains que passaissent deus estés

Eurent deus kevaus acatés ; ib., cxLvI, 389-92. Cf. ib., cxLvII,

89-93.

Il n'orent mie quatre liues alé

Qu'en mi la voie ont un vilain trové ;

Vient de Saint Gile ou il ot conversé,

A quatre bués que il ot conquesté Charroi, 874-7.

Au marchié vient, qui devoit assambler,

Voit un vilain un roncinet mener

De bele taille sel prist a regarder,

Dedens son cuer le prist a goulouser,

Vint au vilain sel prent a apeler :

«Dites moi, sire, se dieus vos puist sauver,

Vaurriés me vos cel roncinet livrer

Pour nul denier que pëusse donner ?

Moult volentiers le vourroie acater, » Bueve, II, 16421-9. 
Et Thierris est ens le marchié alés

Et a tant cignes et paons achatés

Et cras capons, venisons et lardés, ib., III, 9845-7.

en amaser metoit sa cure,

einz laissast plumer ses guernons

qu'il menjast .I. de ses chapons,

ne qu'il eüst au feu cuisine

ne de chapon ne de geline,

einz les faisoit au marchié vandre ; Renart, vI, 15584-9.

$\mathrm{Il}$ a tos les cochons mandés

$\mathrm{Ki}$ en la vile sont et mainent,

Ki tant vairs et sors les amainent

Que tos en est plains li marchiés

Et defoulés et demarchiés. Escoufle, 436-40.

Quelques seigneurs cupides profitent de ces ventes : ils perçoivent des taxes sur les marchandises, que ce soient des animaux vivants ou des produits agricoles :

D'un chappon, ii. deniers ; de my lot de boullie

Paioit on une maille, c'estoit chose taillie. Tristan de N.,

1171-2.

Ung denier d'un chappon quant on l'ait achetér, Lion, 21091.

S'en avoit mis ses fielx le païs en servage :

Toute Jherusalem ot mis en tel quavage

Qu'il avoit .vi. deniers domnir chascun manage,

Ne on ne vendist point pour .v. sols de frommage

Dont il ne recheüst .j. denier d'avantage. Bâtard, 3747-51.

\subsection{Utilisation des produits d'animaux vivants}

Les vaches, les brebis et les chèvres fournissent du lait, les poules des œufs ; avec le pain, ces produits constituent la base de la nourriture des paysans médiévaux.

Les produits laitiers - et dans nos textes il est question surtout de lait et de fromage, tandis que le beurre n'est guère mentionné - semblent être mésestimés par les nobles et considérés donc comme une nourriture tout juste bonne pour les paysans, qui vivent près des vaches et des brebis - et parfois pour les bébés. Une scène d'Erec corrobore le fait et, dans Roche, nous voyons 
Doon et Jofroi renverser la table sur laquelle on veut leur servir du lait et du fromage ; ils appellent cela « nourriture de bergers ". Ce mépris peut expliquer le nombre relativement grand d'exemples tirés de textes où il est question de roturiers, comme p. ex. dans le fabliau xuIII qui présente la liste de ce dont un paysan a besoin :

Avec lui erent dui vallet

Qui portoient gasteax et vin

Et gras fromages de gaÿn

As prez le conte Galoain

A ceus qui fenoient le fain. Erec, 3122-6.

Et la dame les moinne sus ou terrin celier

Ou on fait la cu[i]sine et la mesnie siet ;

De lait et de frommaige i sont li mès plenier

Et de flaons noviax et de pain tot musié ; Roche, 3266-9.

« En la moie foi, sire, ne somes pas bergier

«Que nos de tel viande dëussiens aseier.

« En la terre de France n'en vi onques mangier ;

«Vos en verrez demain ces ma[s]tins enragier. $i b ., 3278-81$.

« En la terre de France acostumé avons,

«Quant on hauberge autrui, que bel ostel li font

« De pocinèz pevrez, de haste de chapon

«Et vin et isopé assez et a foison ;

"Onques ne vi mangier fromaige ne maton;

«Vos en verrez demain enraigier ce[s] gaignons.

- Amis » ce dist li maire, « assez en donrons ; ib., 3332-8.

Ce pelichon emporteréz

Et vint sous dont acheteréz

Du lait pour vostre enfant repestre. Anjou, 4547-9.

Et, se il bien li plaist,

Si porchast, que il ait

Viaus, une vache à lait,

Qu'il nel mete en delait

A l'enfant alaitier,

Quant il en a mestier ; $M R$, XLIII, 229-34.

Pain et vin orent, et oes fris,

Et du fromage à grant plenté

Que li vilains ot amassé. ib., LXxiv, 76-8. 
Se m'aportiez .I. frommage

En vostre giron et .v. oes,

Bien cuideriez ravoir vos bues ; $i b .$, cvi, 332-4.

Ce dist Adam : " Dame, prenez

ceste berbiz, si la gardez :

tant vos donra lait et fromache

assez i avrons compenage. » Renart, II, 3783-6.

Prestres Martins estoit mout sages

de bien norrir, par cez erbages,

brebiz dont il ot maint fromage, $i b ., \mathrm{v}, 14853-5$.

«Ja Breton n'en ert liés s'il nen a pain de brent

«Et plain un pot de lait u fait son sopement. » Aiol, 8979-80.

La le nourry ung an de beurre et de fromaige

Qu'elle robe a la gent d'environ l'eritaige. Tristan de N., 808-9.

Pain orent assez a mangier

Et aigue a boivre et bon fromage,

Car li vilains par le boscage

Portoit adés sa garnison. Meliacin, 14494-7.

Après que le curé fut mussé ou l'on musse les œufs, le beurre, le fourmage et aultres telles vitailles, (...) Cent, 73, 115-7. Cf. ib., $73,209-11$.

(...), la dame fist mectre a la table ung tres beau fromage gras, et ung plat bien fourny de tartes, de pommes, et de fromage, avecques la belle piece de beurre frez, (...) $i b ., 83,80-3$.

Le jeune fils du vavasseur Gautier, parlant à son père, mentionne $\mathrm{du}$ lait bouilli et du fromage :

« Dou lait aurez qui ert sans [hues] boillis,

«Ja d'autre mes n'iert vos ventres farsiz.

«Se bien le faitez, par Deu de paradis,

"Assez aurez de moutons, de brebis,

" Pois et fromaige, bien iert chascuns servis ; Gaydon, 6989-

93.

L'empereur, dans Aiol, se moque de la nourriture des Lombards qui, dit-il, mangent, avec d'autres immondices, de la présure (cf. p. 322, Audigier, 17-20) : 
« Tant as mangiet compeus de soris et de rates,

« Et tant de le composte, de presure et de rapes, Aiol, 8861-2.

Dans Audigier, où sont consommées tant d'immondices, le fromage se trouve mentionné avec des rats :

et enprés si mengierent fromaiges frés,

puis ont eü emprés un autre més :

quatre raz eschaudéz fu entremés. Audigier, 86-8.

Dans le fabliau cıx, le vilain demande à sa femme du lait caillé, un met qui est méprisé par les nobles - cf. supra le troisième extrait de Roche :

- Erme, j'ai tel fain que je muir, »

Fet il, « sont boilli li maton ? MR, cIX, 32-3.

Néanmoins, il y a aussi des textes où les produits laitiers se trouvent au menu des nobles :

Pastez de chevrolz et lardez

(de ce i ert la plentez)

de chevriex, de cers et de dains,

et fromages et cras et sains

de la riviere de Clermont. Dole, 370-4.

si s'en vont en la sale arriere

ou li soupers ert atornez,

mout biaus de vïandes assez :

flaons de let, porciax farsiz,

dont li ostex ert bien garniz,

et bons conins, poulez lardez

(de ce estoit granz la plentez)

et poires et fromages viez. $i b ., 1240-7$.

Si revanrait icy mengier mon lait burér

Et boire le brasin, mengier bacon sallés Lion, 3649-50.

Maiz on n'y mist point d'oigonnet

En la dodine faite au let, Deduis, 3467-8.

Poissons orent et pain et vin

Et bon formages de gaïn. Joufroi, 1525-6.

La veïssez jeter fromajes et cartiers,

Et granz pieces de char, et granz costiauz d'acier ; Parise,

2305-6. 
Une nef ont chargie, comme cil sont sage,

De besquit et de vin, de pain et de fromage, Florence, 148-9.

il avoient aportés

des fromages fres assés

et puns de bos waumonés Aucassin, xxxi, 5-7.

Le mépris pour les fromages se manifeste dans des métaphores où le terme s'emploie pour expliquer la non-valeur ou la nonexistence de quelque chose (voir 5.3.).

Ceci est vrai, dans une mesure encore plus large, pour les œufs : dans la quasi-totalité de nos exemples du terme, il signifie " rien » et nous n'en avons pas relevé beaucoup où les œufs sont mentionnés avec d'autres denrées. Nous pensons qu'il s'agit toujours d'œufs de poules :

Mez, s'aucun me fesoit demande

Quiex mes ne quans elles mengierent,

Je di que pois reschauféz ierent

Du jour devant, et puis des oez. Anjou, 1330-3.

- Se vous pooiez mengier un buef,

On vous saoleroit d'un oef,

Quant de mes mains eschaperez. Cont. P., II, 8051-3. Cf. ib., III, 14428-30.

Or te doin je congié de boivre,

Et de mangier poucins au poivre,

Et oes quant tu en voudrax, $M R$, LxxviI, 180-2.

- Carbonées, fromage, oes fris,

Singnor, de chou vous puis aidier. $i b .$, LXxxIx, 804-5.

Et luy dist : «Et que pourrons nous soupper ? - Monseigneur, respondit il, je vous feray faire des œufs en plus de cent mille manieres; vous aurez aussi des pommes et des poires. Nostre hoste a aussi de bon fourmaige, et bien gras : (...) Cent, C, 33-8.

Dans Lion, nous avons relevé des exemples d'un emploi particulier d'œufs pourris :

Tout adés a sa cowe en avoit nuef ou dis.

L'un li gete bodare, l'autre des yeux poris, Lion, 2868-9. 
Sa femme per la rue qui des anffan petit

Estoit adés ruee et huee a hault cris ;

En maniere de sotte li get on d'uelx poris, $i b ., 3573-5$.

Voilà pour les denrées. Quant aux autres produits, les textes sont assez discrets. On sait que les crins des chevaux étaient utilisés de même que les cornes des bœufs; il est seulement question sporadiquement de la laine des moutons :

« Done moi de la ceue de ton destrier :

« S'en ferai une laise a .I. levrier ; Aiol, 2893-4.

Nos en feriiemes moult bien faire

De cascune quatorze paire

De gros aigniax et de cordé. Guillaume d'A., 3189-91.

Madame, qui les eust toutes oÿes, respondit : «Il souffit de prendre de sa berbis la laynne, (...)

Jehan de S., 254, 23-4.

Il en est de même quant au fumier, utilisé pour fertiliser les terres. Voici un extrait de Lancelot où il est question d'un tas de fumier près d'une abbaye. Cf. p. 363, Florimont, 4271-4 et p. 294, $M R$, cxiv, 1-5:

Et il estoit las et travilliez de l'errer qu'il avoit fait et estordiz de la fain qu'il avoit eue, si s'andormi sor .r. fumier delez la porte de l'abaie (...) Lancelot, v, LxxxIx, 4.

Dans un autre extrait du même texte, on lance du fumier sur Gauvain : la scène montre le dédain des serviteurs pour le célèbre chevalier (voir p. 332 un autre extrait de Lancelot où l'on lance des boyaux sur Bohort, dans une situation semblable) :

Et quant li menestrel voient le chevalier en la charete, si vont aprés, huant et criant, et li getent fiens et boes et chavates et totes les ordures qu'il truevent, (...) ib., II, LXVI, 32.

La chaleur du crottin de cheval est utilisée pour guérir les blessures (la fièvre ?) :

Mais si fort s'i sentoit blecé des coupz et des playes que autrefois avoit eues. Si se fist mettre et envelopper a ung fiens de chevaulx bien chauldement pour consolider tout son corps et passa la nuyt et fist tout recueillir pour eulx prendre leur repos. Ogier, 91. 
Le lecteur ne s'étonne pas que dans Audgier, cette " œuvre ordurière ", différentes espèces de fumier servent pour les repas et pour les dons :

Enprés icelui més ge vos di, orent quatre corbeaus de viéz rosti.

En bouse de vache furent falti : Audigier, 94-6.

Et enprés si mengierent lor raz lardéz,

puis ont eü aprés un bon civé

de merde de geline entremellé. $i b ., 503-5$.

et Audigier lor donne molt lieement :

trente crotes de chievre a chascun tent. $i b ., 515-6$.

On connaît l'importance des marchés urbains où les paysans venaient vendre leurs produits aux habitants des villes; « (...), presque toute l'année, ils [les marchés] pouvaient disposer d'excédents de poulets, d'œufs, de beurre, de fromage, de légumes, de laine. » (Histoire de la France rurale, I, pp. 587-588) :

Et nepourquant me dist Sirois

Que j'oi des bues .L. sous,

Qui les conta, si les reçut ;

Mès je ne sai s'il m'en deçut,

Ne s'il m'en a neant emblé,

Qu'entre .II. sestiere de blé,

Et ma jument et mes porciaus,

Et la laine de mes aigniaus

Me rendirent tout autretant. $M R$, cxvi, 69-77.

\subsection{Utilisation des produits d'animaux morts}

Le produit le plus utilisé est, on s'en doute, la viande. Car, si les bergers, et les paysans en général, sont obligés de se contenter des produits laitiers pour se nourrir, tout le monde - les pauvres aussi bien que les riches - préfère des plats plus substantiels comprenant de la viande de toute sorte. Et sur les tables des riches on voit toujours plusieurs espèces de viande : du bœuf, du porc, de la volaille et, évidemment, beaucoup de gibier.

Voici des exemples qui présentent la viande de bœuf, régulièrement servie avec celle d'autres animaux : 
Avoit ce soir prise poison,

Tant ot mengié bon buef as aus, $M R$, LXVIII, 34-5.

Qui mengier volt, ne li fu deveé,

Pain ot et vin et piument et claré

Et char de buef, venoison et saingler : Ami, 3255-7.

Li veneor n'orent pas honte

s'il orent boef au premier mes Dole, 479-8o.

Seignor, ce dist Girart, que portent li somier ?

- Char salee et vin viez, biau sire chevalier ;

Pein, buef cuit et farine, que feïmes chargier. Barbastre, 4286-8.

Ains que Pentecouste venist,

Li païs maint present li fist :

Li uns cras bués, li autres pors, Jehan, 5197-9. Cf. ib., 5984-9.

Si se sont au souper assis.

Assez orent, ce m'est avis,

Char de buef grace et venoison,

Gelinnes crasses et poisson, Floriant, 1139-42.

Et d'autre part font li qeu atorner

Riches vyandes et le feu alumer,

Buès escorchier et porciax eschauder, Narbonnais, 2430-2.

(...), et de prinsault apporterent la belle porée verte avecques beau lard, et belles trippes de porc, et une langue de beuf rostie, (...) Cent, , 83, 31-3.

Un extrait de l'Escoufle semble indiquer que la viande de vache était servie régulièrement à tout le monde - même aux domestiques - et que pour cela elle était moins appréciée que d'autres espèces de viande :

Venisons, lardés et daintiés,

Et lardés qui ne sont pas viés.

Poissons de douce eve et de mer

Ki ne sont porri ne amer

I ot en esseu et en rost,

Et tant qu'il n'i eut nul n'ait rost

Devant lui ; neïs li garçon

En orent tuit si grant fuison

Com se ce fust de char de vache. Escoufle, 713-21. 
La viande de porc se rencontre notamment dans les fabliaux, d'où l'on est autorisé à penser qu'elle se trouvait davantage sur la table du peuple que sur celle des nobles. Ceux-ci ne la méprisaient pourtant pas.

Elle apparaît souvent sous deux dénominations : le bacon, qui désigne le lard (salé) et le jambon (salé ou fumé), et la charbonée, qui désigne la viande grillée (sur du charbon); notre extrait cidessous du fabliau Lxxxix montre d'ailleurs que la charbonée se fait avec la viande de différents animaux :

\section{(...). Quant Thiery s'avisait}

Lez vin et le pain et lez bacon qui furent la,

Lez bleif et lez awainne, grant joie en demenait. Lion, 16272-4

Tant aquist et tant conquesta,

Qu'il ot assez et .I. et el,

.I. bacon fist contre Noel

D'un porc qu'il ot en sa meson

Norri trestoute la seson :

Bien ot plain paume de lart. $M R$, xcvII, 124-9. Cf. ib., cxxxvI, 526-8 et passim.

Ge croi bien se nos eüsson

Charbonée d'un cras bacon,

Que nos en beüssion molt mielz. » ib., cxxxvi, 579-81. Cf. $i b$.

cxxIII, 332-3 et passim.

Dient qu'il feront charbonées

Du bacon, et Travers l'entent. ib., xcvII, 420-1.

Ce fu un pou devant Noël

que l'en metoit bacons en sel, Renart, v, 13311-2.

Voir aussi : Raguidel, 1878 ; Bérinus, 269-70 ; 271-3 ; Protheselaus, 559-60 ; Bueve, I, 7959 ; II, 1127.

Garnier de Nanteuil blesse son adversaire Auboin et lui coupe un morceau de la cuisse, comparée par l'auteur à un rôti de viande de truie ; une scène analogue se trouve dans Yvain où le protagoniste coupe une « charbonée » de la joue d'un géant :

Par dessus le haubert descent la bone espee,

.c. mailles li trancha de sa broigne safree,

$\mathrm{E}$ l'en y a tailliee toute la gironnee.

Par devers le trumel l'espee est rendiflee.

Garniers l'en abat jus une tel charbonnee

Du braion d'une truie n'eüssiez tel denree ; Aye, 524-9. 
Et cil, qui tint l'espee treite,

$\mathrm{Li}$ a une anvaïe feite.

Del tranchant, non mie del plat,

Le fiert si, que il li abat

De la joe une charbonee. Yvain, 4211-5.

Évidemment on mange aussi la viande des moutons, comme le montrent les exemples suivants ; Ogier prisonnier en mange en grande quantité, tandis que Charlemagne mange aussi bien la viande d'autres animaux :

Mais l'archevesque lui dit qu'il estoit deliberé de lui faire cuire d'un sextier de blé chascun pain dont il auroit assez d'un quartier et une tasse de vin d'ung sextier. Et la piece d'ung mouton entier ; ne sera ce pas assez ? Ogier, 116. Cf. ib., $135 ; 168$.

Il manjout poy de payn, mes il manjast ben a un manger la quarte partye de un moutoun, ou deus gelynes, ou une owe, ou une espaule de porc, ou un pooun, ou un(g)e grue, ou un(e) [1] evere tot enteyr ; (...) Turpin, 879-82.

Toutes voies me suis garnis

Des ore dusqu'à l'an renuef,

De car de monton et de buef :

C'est bon por faire carbonées, $M R$, LXxxIX, 852-5.

De char de porc et de moton,

De bone vache de seison

I avoit il si largemant

Con se fust prise por nëant. Athis, 8861-4.

Mais Deu merci et Turpin le baron

Tant a mengié car de porc et molton : Chevalerie d'O., 9554-5.

On li ot fait apparillier

Assés a boire et a mangier

Car de porc et car de mouton Wistasse, 234-6.

le jüesdi de rovoison,

que l'en menjüe les mostons. Renart, III, 8993-4.

Il les fist tantost conduire en une tresbelle chambre, et envoya couvrir la table et faire beau feu et apporter la souppe, et la piece de mouton, et le vin blanc, (...) Cent, 26, 311-3. 
Et elle luy promist que, s'il tenoit bonne bouche, elle luy donneroit de la char et de mouton pour fournir son mesnage pour toute ceste année ; (...) $i b ., 40,128-31$.

Elle fist son commandement, car l.e jour de son partement se leva bien matin pour aller a la boucherie, et appoincta ung bon poussin et une piece de mouton, (...) ib., 93, 27-30.

Les viandes, la graisse et même la fressure des chèvres se trouvent au menu des protagonistes d'Audigier, "parodie de chanson de geste »; nous supposons que cet animal était généralement mésestimé comme fournisseur aux tables des nobles :

Du conte Turgibus orroiz la vie.

Onques n'ama riens tant come boulie

et, as festes envieus, chievre a l'alie

por ce qu'il fu noriz en Lonbardie Audigier, 17-20.

Il li font un chaudel d'ués couveïz ;

enprés si li donnerent oignons porriz

por ce que il eüst plus sain le piz ;

mais il n'en volt mengier s'il ne sont friz

en bon seïn de chievre ou de berbiz. ib., 165-9.

« Enquenuit la ferai mengier o toi :

« deus froissures de chievre aurons nous troi. ib., 471-2.

Le lardé est un morceau de viande ; il n'est pas précisé de quel animal :

Si prist un pasté et un pain,

Et un lardé a l'autre main, Atre, 4147-8.

Il est évident que l'on utilise aussi la graisse des porcs, et certainement en général plus intelligemment que dans les extraits suivants :

(...), furent les portes de le chastel, qe treblees erent, ars e espris par feu, qe fust illumee de bacons e de grece, (...) Foukes, 24, 21-2.

Les autres dient que l'en l'arde,

Mes tout avant que l'en la larde

De goutes de saïn ardant. Anjou, 7787-9.

Dans Bâtard, les Sarrasins, assiégés par Baudoin dans la ville de Mecque, souffrent de la faim ; l'un d'eux, Esclomart, dit qu'il leur 
manque de tout, aussi de "bacon salé ». Cela ne signifie probablement pas qu'ils mangent de la viande de porc mais, plutôt, que le terme bacon peut désigner aussi la viande d'autres animaux . Cf. infra, vv. 5561-8 du même texte :

"Nous somme si endroit enclos et enserré Par le gent crestienne, et s'ont chi tant esté Que chaient sont fali et li vin et le blé,

Li fain et les avaines et li bacon salé ; Bâtard, 2136-9.

Lors du siège de Montauban, Aymon aide ses fils menacés de famine en lançant des jambons dans la ville :

Ja n'eüst garantie, demor ne tensement, Ne fust Aymes lor pere qui lor fist .I. present, Qui .xxvi. bacons fist ruer a sa gent ; Renaut, 12025-7. Cf. ib., $12013-4 ; 12046$.

Ces derniers exemples, parmi d'autres, montrent qu'une armée a besoin non seulement de chevaux pour les batailles mais aussi de ravitaillement : on amène donc des bœufs et des porcs, des génisses et des brebis etc., qui seront abattus au fur et à mesure de la campagne ; parfois on attaque l'ennemi pour s'emparer de ses provisions, parfois on paie les animaux très cher au marché :

De pain bescuit, de char salee

Et de farinne buletee,

De bues, de pors et de chevax

Sor coi monteront les vassaus. Floriant, 2877-80.

Et bien .III bués qui bien furent nourris Gaufrey, 1343.

(...) ainsi que le roy Cormorand faisoit assembler le bestial du pays de l'antour, c'est assavoir chevaulx et beufz et vaches, moutons et brebis, dont il lui en avoit sans nombre ensemble les quinzes moienes prisonniers et tresor (...) Ogier, 223.

Puis si faites crier et vostre ban noncier :

Qui a buef nen genice, si l'amaint au marchié.

Se ele vaut .xx. sous, .xxvii. l'en paiés :

Enci verrois vitalle a l'ost acharoie[r]. Lanson, 3849-52. Cf. ib., 3859-62.

Tout droit a la journee, si que le jour vit on,

S'embusquerent no gent, par derrier maint buisson,

Pour atendre le proie de la chité de non.

Devant soleil levant virent le compaignon 
Issir de la chité boés, vaches a foison,

Et plus de mil brebis ; la furent maint mouton,

Mais n'i avoit nul porc, car li paien felon

N'en mengeroient d'un pour tout l'or Psalemon, Bâtard, 5561-8.

«Alés a le couverte, par haies et buissons,

Pour le nombre savoir des traïtours felons

Qu'ensi nous ont robés vaches, brebis, moutons.

Se je puis esploitier, nostre proie rarons ; $i b .$, 5666-9.

Et cil dient : « Marcheant somes,

Qui vitaille a vendre menomes :

Pain et vin et bacons salez,

Et bués et pors avons assez

Por tüer, se besoinz estoit. » Perceval, 2537-41. Cf. ib., 2568-70.

En temps de guerre, p. ex. lorsqu'une ville est assiégée et que les habitants commencent à souffrir de la faim, on peut être obligé de manger la viande de chats et on tue même les chevaux de bataille, pourtant si nécessaires et si précieux, pour en manger la viande (cf. Fr. Schmidt :, op. cit., p. 75) :

Et quant sa femme congneut ung iour qu'il n'y avoit remede et que a Maience la famine estoit si grande que tout le monde estoit contraint de manger chas et ratz, (...) Ogier, 60.

Or quant Ogier fut retourné au chasteau et que il vit que il n'avoit plus vivres ne gens il se print trestost a doulourer de la perte de ses gens et de ce qu'il n'avoit plus de quoy tirer avant. Si lui fut force d'escorcher ung cheval pour vivre si peu de tenps qu'il y pensoit demeurer et fist tant qu'il ordonna son fait, lava ses escuelles, mist son pot au feu, (...) $i b .$, 107. Cf. $i b$., 108.

Muerent de fain cil bon mulet anblant

Et li destrier en vont afoibloiant.

La les manjuënt cele gent mescreant. Aspremont, 2102-4.

Or ont tel tans qu'il n'ont pain ne forment,

Si est lor os enchierie forment,

Car de ceval manjuënt tolt sanglent. ib., 2842-4.

Tuit orent lor chevaus par la famine ocis ;

De toz ceus que avoient n'ont il que vint et sis. Barbastre, 4969-70. 
Mais ne leur valut riens; quar si leur fu leur viande faillie que leur chevaus leur couvenoit mengier de destresce de fain. Helcanus, 39.

Cil furent .c. qui se vont haubregier, Mais entr'aus toz n'orent que .v. destriers,

Que par famine les avoient mengiez. Mez, 11133-5.

Mais tant fu grans li peuples a Clermont otelez

Que toz lor faut li vivres et li pains et li blés ;

Mainjuent lor chevaus quant il les ont tuëz. Orson, 2673-5.

Mais, pendant le siège de Montauban, où nous voyons les assiégés obligés de tuer leurs chevaux pour survivre, Renaut ne peut pas tuer le sien; il se contente de le saigner et de préparer avec le sang un repas pour lui-même et ses enfants :

Puis mangerent chevalx et destrier misoudor,

(...)

La vitaille lor faut, chascun son cheval tue,

(...)

Il n'ont mes que mengier ne cheval ne roncin.

(...)

Quant Renaut l'a veü, si dolent ne fut onc,

Il ne le ferist puis por tot l'avoir del mont ;

Mes .I. bacin a pris Renaut le fiz Aymon,

Del sanc de lui osta, por verté le savon,

Puis fet boillir le sanc le palés contremont,

S'en mengierent li conte et li dui enfançon. Renaut, 11685,

11855,11996 et $12127-32$.

Tristan de $N$. nous apprend qu'il y a même des pays où l'on préfère la viande chevaline à celle des bœufs :

Car së est la coustume ou païs par dela

Que de cheval mengüent, de ce ne doubtés ja,

Mieulx l'aiment que d'un beuf, ja sy bon ne sera. Tristan de

N., 21418-20.

Quant à la viande des oiseaux, on remarque que les chapons ont dû être très appréciés : ils apparaissent à tout propos sur les tables des nobles aussi bien que sur celles des roturiers, même pour le petit déjeuner :

Lors fu li corps Flourence honneréz et siervis

De pain, de char, de vin, de bons cappons rostis ; Rome,

2824-5. 
Aprés la messe sanz targier

Li fait aporter a mengier

Un chapon a le salse en rost. Cont. P., I, 687-9.

Aprés est assis au disner.

Assez li avoit a doner

Sa suer venisons et chapons ; $i b ., 2669-71$.

La dameisele cort isnel

A sa chanbre et revint mout tost,

S'aporta un chapon an rost Yvain, 1046-8.

Talac demande pein e vin

E buens chapons en pain lardez,

Od ses compainons s'est disnez : Yder, 1548-50. Cf. ib., 5690-

$2 ; 6012-3$.

Il menga avuec le prevost

Pain et vin et chapons en rost. Blancandin, 987-8.

Et a tant chines et paons acatés

Et cras capons et venison assés, Bueve, I, 8162-3.

Pain, et char, pastéz et chapons, MR, IV, 101.

Au matin, quant l'aube est crevée,

S'est mult tost levée Auberée ;

Si atorne au mielz qu'ele pot

Char de porc et chapons en rat. ib., cx, 431-4.

(...), et luy dist qu'elle prinst les deux meilleurs chapons de la chaponnerie de l'ostel, et les appoinctast tresbien, et puis qu'elle allast a la boucherie querir le meilleur morseau de beuf qu'elle pourroit trouver, et si cuisist tout a une bonne eaue pour humer, ainsi qu'elle le saroit bien faire ; Cent, 59, 102-8.

Robin, le valet de Jehan, sert même des pâtés de chapon à son maître et à Blonde qui se cachent dans la forêt :

Avaine aportoit as chevax

Et as deus vrais amans gastiaus,

Pain blanc et de capons pastés, Jehan, 3013-5. Cf. ib., 635-7.

Or, dans un grand nombre de textes, on trouve aussi des exemples de la viande d'autres oiseaux, surtout celle des poulets, que les textes appellent poussins. Voir aussi paon, p. 220 : 
Et jà cuisoient, ce me sanle,

.IIII. capon et .II. gelines. $M R, \mathrm{xxxIV}, 270-1$.

- Triant, » fait-il « par seint Tomas,

Se tu avez .I. anel cras

Mi porra bien mengier, ce croi. ib., xLVI, 25-7.

Quant l'oe fu mout trés bien cuite,

A grant loisir et sanz grant luite,

Li prestre a s'oe coverte ;

Et le clerc a la sause fete

Blanche et espesse et bien molue,

Si que tot le cors l'en tressue : $i b .$, cxLIII, 19-24. Cf. $i b ., 8-11$;

46-8.

Li pasté sont envolepé

De l'une part en la besace ;

En l'autre tele eure est fouace

U char froide u geline en rost, Escoufle, 4300-3. Cf. ib., 4444-7.

(...) le jour de son partement se leva bien matin pour aller a la boucherie, et appoincta ung bon poussin et une piece de mouton, (...) Cent, 93, 28-30.

Premierement menjust ou pocin ou geline, Florence, 4248.

Li cos est trop durs a mangier,

se le volez a eschangier,

car il a bien .II. anz toz plains,

mais je vos randrai de mes mains

.III. poucins tendres, se volez, Renart, vI, 15937-41.

Pouchinés fruis au lart et à la gansaillie Doon, 10515. Cf. ib., $1857-9 ; 5959$.

Si se disnerent en seant

Sor frois joinz noveaz aportéz ;

Poucins ont tenres, bien lardés Durmart, 8276-8.

Lus et saumons, poucins farsis

Orent assés a cel disner, Violette, 2525-6.

De toute sa mesnie ne remaint il que vint,

Et cil demandent l'eve, au mangier sont assis,

A ce mangier $\mathrm{n}[\mathrm{i}]$ orent $\mathrm{ma}[\mathrm{i}] \mathrm{s} \mathrm{qu}^{\prime}$.j. poucin, Roche, 3899-

901. Cf. ib., 3282-5; 3332-5. 
Un pau le fant disner, laz un saucin,

De pastat de poisson e de polcin. Roussillon, 9719-20.

Une table trouva garnie

De viandes et de bons vins,

De pain, de char et de pocins. Claris, 9621-3.

Communement s'asieent au souper ;

Assez i orent venoison de sengler,

Grues et gentes et poons emprevez. Charroi, 811-3.

Et puis apres porciaus farsis

Et pigons en paste et rostis ; Espees, 8617-8.

Miex ameroie un cras oison. Barisel, 234.

Lorsque Athis et Prophilias préparent leur voyage à Athènes, ils font entre autres choses provisions de poulets qui seront tués au fur et à mesure du trajet :

Dates et figues et fromages

Et de polez pleinnes les cages Athis, 9415-6.

La viande très tendre des poussins est donnée à manger aux oiseaux de proie :

Desor son poing tenoit un esmeri,

Faisoit lui gorge de l'ele d'un poucin ; Bueve, II, 5348-9.

Les peaux de tous les animaux sont très appréciées, celles des animaux sauvages aussi bien que celles de presque tous les animaux domestiques : ânes, chèvres, moutons, bœufs etc. On les utilise surtout pour des vêtements :

S'achate .I. bon bliçon d'aigneaux $M R$, xxxI, 347 .

S'ot .II. peliçons bons et biaus,

L'un d'escuireus, l'autre d'aigniaus ; ib., cxxv, 15-6.

Vestu estoient comme gent mal senée :

Chascuns avoit une gonele lée

Et une jupe de gros agniax forrée, Aymeri, 1622-4.

Tot li trenca l'escut le cuir d'azer ; Roussillon, 2868.

Troberent lo saint ome qui per Deu pene.

Il non a drap vestit, mais pel cabrene, $i b .$, 7407-8. 
Chemise ot [et] neire malveise,

Une pels ot mult enfumee

De gros mutuns et mult usee. Protheselaus, 4833-5.

Piaus de moutons por peliçons ouvrer, Bueve, II, 16416. Cf. ib., II, 881-2.

c'est un merveilleus chaputois.

- Ha! Diex, con je seroie rois,

se le pooie as mains tenir

a mon chief por le froit couvrir ;

por ce que il bone pel a,

bon chapel et grant i avra. Renart, II, 5151-6.

S'avec moi voloies venir,

l'en ne feroit oen hosel

ne chaucemente de ta pel. ib., III, 9022-4.

Et vos, prestres, estièz ivre

qui lassiez vostre mestier

por aler un chat dechacier ;

en vos avet mavés apel :

de moi voliez avoir la pel,

s'estoit por metre ou peliçon

a vostre putain au maison. ib., IV, 11856-62.

Et dist Belin : «Je n'en puis mes,

je sers a un vilain felon

qui ainz ne me fist se mal non.

Ces berbiz ai ge engendrees

que tu voiz ici asamblees :

mal ai mon servise enploié,

quar li vilains m'a ostroié

a ses soieors a lor prise,

et si a il ma piau promise

a hosiaus faire a un preudome

qui les en doit porter a Rome. ib., III, 8976-86.

et li freres Chievres de Rains,

cil qui fait pingnes et lanternes,

ataint l'ors entre .II. citernes :

d'une vete de buef qu'il porte

li a toute l'eschine torte ; $i b .$, I, 692-6.

Escourgieez tient en sa main

Noueez et dures de ners,

Ne sçay ou de buef ou de cerfs. Galeran, 4110-2. 
Vo cheval arai malgré vostre,

Mais ce n'ert pas por chevalchier,

Ains le volrai faire escorchier,

Si en avront la char li chien

Et je le cuir, si vous di bien

Que j'en ferai faire un bahut, Cont. P., I, 4448-53.

Dans Charroi, Guillaume met de gros souliers en cuir de bœuf (de vache) pour faire croire qu'il est marchand, c'est-à-dire roturier, et non pas noble. Le pauvre valet dans Aucassin, lui aussi, porte des souliers en cuir de bœuf. Pour le rendre méconnaissable et faire croire qu'il soit le fils de son précepteur Soibaut, on chausse Bueve, le jeune noble, de la même façon (« à la paysanne »). Dans Yvain, un vilain s'habille de peaux de bœufs.

Il est évident que la noblesse méprise ce matériau, mais l'extrait du fabliau cxvi l'apprécie pour sa solidité :

Li cuens Guillelmes vesti une gonnele

De tel burel com il ot en la terre

Et en ses jambes unes granz chauces perses

Sollers de buef qui la chauce li serrent ; Charroi, 1036-9.

Li rois Otranz li dit par contraire :

« Di va, vilains, Mahomez mal te face!

Por quoi as or si granz sollers de vache,

Et ta gonele et tes conroiz si gates? ib., 1327-30. Cf. ib., 991.2 ; 1336.

(...) ; et estoit cauciés d'uns housiax et d'uns sollers de buef fretés de tille dusque deseure le genol, Aucassin, xxiv, 19-20.

Un pelichon de moutons le et grant

$\mathrm{Li}$ a vestu la dame maintenant,

Sollers de buef li cauche a päisant

Grans et mal fais et hideus et pesans ; Bueve, III, 844-7. Cf. ib., III, 815-7.

Vestuz de robe si estrange,

Qu'il n'i avoit ne lin ne lange,

Ainz ot a son col atachiez

Deus cuirs de novel escorchiez

De deus toriaus ou de deus bués. Yvain, 309-13.

Ses sollers ne sont mie à las,

Ainz sont de vache dur et fort ; $M R$, CxvI, 10-1. 
- Vos alés de noient parlant,

Qu'il ne me faut rien, que jou sace.

- Si fait : unes hueses de vace

Et esperons et cape a pluiue

Te donrai je (mes mout m'enuie),

Un ronchi et un palefroi :

Donc arai plus perdu en toi ! Guillaume d'A., 1610-6.

Un extrait de Gui de W. indique que l'on utilisait les peaux des chevaux pour en fabriquer les liens des boucliers - mais il est indubitable que la rime est importantes pour le choix du terme roncin :

Le col li pent un escu doblentin,

Les bendes a or erent faiz d'un runcin ; Gui de W., 10093-4.

Dans Cont. P., un paralytique est armé d'une vessie de bœuf dont il peut se servir pour se protéger contre des agresseurs et pour faire marcher la personne qui tire sa voiturette :

Moi et mon char vous covient traire

Par les rues, se Dieus me saut,

Et si arois ja un assaut

De chavates et de polmons,

Car al corner les ai semons,

Et s'ai chi ma grande vessie

De buef bien sofflee et tesie

Dont je vous donrai les la joe,

Se bien ne traiez par la boe

Et par les flos et par les tais. » Cont. P., II, 7718-27.

Nos textes ne mentionnent que très sporadiquement les plumes d'oiseaux. Elles ont dû être utilisées à autre chose qu'à la parure des casques, comme dans nos premiers extraits, mais nous n'en avons pas de témoignages :

car unne queue de poon

ot en l'iaume lacié derriere

et quant il point par la riviere,

la plume au vent s'espant et euvre Thèbes, 5874-7.

L'escu au col où ot point .I. lyon,

Et en sa lance fermé un confanon,

Et sor son elme la coe d'un paon. Gaydon, 9509-11.

(...), et sur leurs chiefs chascun un tresbel chappel de plumes

(...) Jehan de $S ., 99,33-4$. 
Qui donc veïst con li houlier

Plument chapons et plument oies, $M R$, cxvI, 184-5.

Espees (où il s'agit d'une dame), Durmart (où il s'agit du roi Arthur) et Ipomedon mentionnent des chapeaux de paon(s), sans nous renseigner le moins du monde de la nature de ces couvrechefs. Nous pensons qu'il est question de chapeaux ornés de plumes de paon :

Puis met .I. capel de paon

Sour son chief ke caus ne li griet,

Plaisaument sor le siele siet. Espees, 1142-4.

Sor son chief portoit un chapel

De paons mout riche et mout bel. Durmart, 7217-8.

E pur la hadle out un chapel,

De fresaus aturné mut bel,

A or purfreit, a beaus butuns,

Unc si blancs ne de poüns. Ipomedon, 2733-6.

Le mou et les boyaux ne servent apparemment à rien : dans Aiol, où le mou est mentionné parallèlement à des charognes et des ordures, et dans Lancelot, on en lance sur des personnes pour leur témoigner son dédain :

Borgois et macheclier l'ont molt gabé,

Des pomons de lor vakes l'ont il rué : Aiol, 2581-2.

Et li getent chavates et caillaus bis,

Et pomon et caronge et merde ausi. ib., 2766-7.

Atant s'en va contreval la vile par totes les rues, si est hués et arochiés et de boials et de chavates.

Lancelot, II, XL, 11. Cf. ib., II, LXVI, 32.

L'ivoire est utilisé entre autres choses pour fabriquer des cors. Les textes n'indiquent naturellement pas si l'ivoire provient d'animaux sauvages ou d'animaux domestiqués, mais n'est-il pas probable que ces derniers en ont fourni au moins une grande partie?

Bernart ses freres sonne un cor d'olifant, Narbonnais, 7234 .

Cf. ib., $7268 ; 3634$. 


\section{Animaux dans les idées, la langue et l'imagination}

Citons d'abord un petit extrait de la thèse de Jean Bichon (op. cit., pp. 20-21) : « (...) le monde animal a, pour l'homme du xiI ${ }^{\mathrm{e}}$ siècle, une présence intense. Il baigne de toutes parts le monde humain, il est étroitement mêlé aux besoins des hommes, à leur vie, à leur sensibilité. Pour certains animaux domestiques: cheval, chien, l'intégration au monde des hommes est profonde et engendre un compagnonnage quasi-humain : il n'est pas exorbitant de dire que ces animaux-là font partie de la société. »

$\mathrm{Vu}$ la place importante qu'occupent les animaux domestiques dans la vie des hommes, riches ou pauvres, qui s'étonnerait qu'ils aient influé sur les façons de penser et de parler?

Nos textes témoignent abondamment du fait. Il arrive que l'on prête à des animaux des traits humains, comme nous l'avons vu chez les chevaux extraordinaires (voir pages 19-21). Nous trouvons de nombreuses situations où une action ou une réaction humaine est comparée à celle qu'on a l'habitude de voir chez les animaux. Nous en trouvons d'autres où le caractère d'une espèce est soulignée afin de caractériser une personne ou un groupe de personnes; c'est en général pour injurier quelqu'un qu'on lui donne un nom d'animal (on dit p. ex. d'une personne qu'elle est sotte comme un mouton). Nous en trouvons aussi où la qualité spécifique d'une espèce animale sert à souligner la qualité d'un animal d'une autre espèce.

De tout cela naissent des métaphores à base d'animaux, de même que la langue s'est enrichie de dictons et de proverbes dans lesquels on voit comment l'homme a bien observé le monde de ces animaux et en a tiré des leçons.

Nous le considérons en dehors du domaine de notre étude d'examiner l'évolution du vocabulaire, p. ex. de poussin et de mouton, et aussi de parler de tout le vocabulaire autour des animaux, surtout cheval, qui engendre chevalier, chevaucher, déchevaucher, entrechevaucher etc. Nous avons déjà indiqué, p. 18, comment les verbes monter, descendre, poindre etc. s'emploient dans des scènes avec chevaux, sans mentionner ces derniers.

A travers toutes les comparaisons et les métaphores, le lecteur obtient une idée de l'opinion des hommes concernant les animaux domestiques. Nous apprenons ainsi que les brebis sont connues pour leur faiblesse et leur sottise, que l'on admire la rapidité des lévriers etc. De même, il nous est permis de jeter un coup d'œil 
sur la vie quotidienne où les paysans craignent toujours le vol de leurs brebis par le loup et les dégâts causés par les nombreuses souris. Nous avons une idée de l'importance de la chasse au vol en lisant d'une fois à l'autre qu'un chevalier (ou sa monture) se déplace aussi vite qu'un faucon vole, ou qu'il est craint par ses adversaires autant que l'épervier par les petits oiseaux.

\subsection{Comparaisons}

Par les comparaisons, nous apprenons bien des détails sur les qualités et les activités des animaux, $p$. ex. on nous renseigne que les faucons sont rapides et que les chiens se secouent le matin pour se réveiller :

Comme ung chien, qui ne fault que escourre la teste au matin quand il se leve qu'il ne soit prest, estoit monseigneur ; Cent, $27,243-5$.

\subsubsection{L'homme (ré)agit comme un animal}

Dans un nombre considérable de cas, nous voyons des guerriers comparés à un animal qui en poursuit un autre. Le guerrier s'attaque à son adversaire comme un épervier s'attaque à sa proie (1), ou comme le loup aux brebis. Or, si les comparaisons sont multiples, elles ne sont pas très variées.

Le point de vue peut changer : tantôt la scène est vue du côté de l'attaquant :

Il les saisit toz .IIII. par les bras,

Si les demaine comme soriz fet chas. Aliscans, 3916-7. Cf. ib., $4848-51 ; 5454-5$.

Ausi com esprevier, quant il vole a la quaille,

Se fiert entr'aus rois Otes, ne li chaut qui l'asaille ; Florence, 1378-9.

Ai tant Justamonz broche le bai de Cornoaille ;

Plus randone menu qu'espreviers ne prant caille. Saisnes, 3594-5.

Si com li vols d'un esprevier

Fait les petis oisiaus mucier,

Si fait ses anemins trambler. Florimont, 9401-3. 
Entresi con li faus fiert es oisiaus menuz,

Se fiert li cuens entr'aus, que il n'est esperduz. Barbastre,

302-3.

Quant Moranz vit dant Guilliaume venir,

Plus le desire que faucons la perdris. Mez, 1036-7. Cf. $i b$.,

5744-6; 5951-2;10019-20.

Crestien desrompirent le grant estour frarin,

Si se fierent en iaus qu'escoufles au pouchin. Bâtard, 248-9.

Onques n'en ot loisier, car le Bastart cuida

Prendre comme mouton, quand li leus a lui va. ib., 5406-7. Cf.

ib., 5703-5; 433-4; 3130-3.

(...) : il est ainsi com li leux qui vient del bois et se fiert soudainnement entre les berbiz qu'il occist et estrangle, ainz qu'eles se soient prises garde de lui : (...) Ils sont aussi esbahi conme li aingnelet, (...) Lancelot, IV, LxxxIII, 36. Cf. ib., v, Lxxxv, 13.

La bataille desire $\mathrm{k}^{\prime}$ ains fameilleus lions

Ne desira autant ne aigniaus ne moutons Buevon, 2582-3. Cf. ib., 924-7 ; Troie, 108, 4-7.

De totes pars de mer les acuellent tant fort

Con li chiens le cengler quant est navrez a mort ; Aye, 1502-3.

Mais riens ne leur valut, quar ceulz si les suirent tout aussi comme le chien fait le leu et ne les osoient aprochier. Laurin, 1689-90.

Comme Albert Stimming, nous nous étonnons de l'image de l'extrait suivant: on s'attendrait à voir les chiens poursuivre le gibier ; peut-être veut-on dire que les lévriers sont plus rapides que les braquets?:

Et Açopart les vait si festinant

Com li brakés vait le lievrier sivant ; Bueve, I, 5364-5.

Voir aussi : Lion, 8889-90 ; $17081 ; 21825 ; 31787-8$ et passim ; Floriant, 2282-3 ; 7174-5 ; Rome, 3063 ; Claris, 18305-6 ; Helcanus, 41 ; Silence, 5508-10 ; Enfances O., 2785-9 ; Athis, 11996-7 ; Yvain, 8804 ; 1264-7 ; 3192-5 ; Cent, 83, 38-41; Ogier, 33 ; Papegau, 46, 27-8 ; Tristan de N., 1267-8 ; 6947 ; 11479 ; Rous, II, 1060-1 ; App., 70510 ; Narbonnais, 1616-22 ; 7393-4 ; 7462 ; Laurin, 5496-8 ; 5984-6 et passim ; Gaufrey, 463 ; Cristal, 5473-4. 
Dans autant d'exemples et, dans une large mesure, dans les mêmes textes, la situation est vue du côté de l'attaqué :

Je ne sui pas si aise com li poissons qui noe, Pres sui k'en autel point que pinchons ou aloe K'espreviers fameilleus tient saisi en sa groe, Berte, 859-61.

paien li fuient con aloe esprevier ; Loquifer, 294. Cf. ib., 1422$3 ; 2470-1$.

Mes c'est des dons le roi, de mort vos deffion,

Car il vos doute plus que l'aloë faucon. Aye, 158-9.

As paiens fist mainte grief enviaille,

Plus le doutoient ne fait l'esprevier caille ; Enfances $0 ., 5413^{-}$

4. Cf. ib., 5367-8; 5982-4.

Si le fuient com gierfaut fait la grue. Buevon, 1578.

Car plus les redoutent ne fait lievres levriere $i b ., 1800$.

(...), car mesire Tristrans, ki pas ne recroit, a ja tant fait par fine force que cil d'Orcanie s'enfuient devant lui, tout autresi espoenté com li lievres s'en vait fuiant devant les ciens. TP, v, $238,2-5$.

Si l'ont li Grizois en poi d'oure conu come ceauz qui le redoutent trestous et s'epee ausi comme la mort, et fuient ausi devant lui comme le loup devant les chiens. Troie, 97, 3-6.

Si cum li cerfs s'en vait devant les chiens,

Devant Rollant si s'en fuient paiens. Roland, 1874-5.

Voir aussi : Lion, 11040-1 ;11343-4 ; Bâtard, 1387-8 ; Mez, 2439-40 ; Gui de N., 1136-7 ; Aliscans, 2727-8 ; 5808 ; 5823 ; 5842 ; Florence, 847 ; Violette, 3735-7 ; Roussillon, 2646 ; 4299 ; 6371-2 ; 8748-50 ; Lancelot, IV, LXxvIII, 32 ; Couronnement, 1072-4 ; Brut, 4341-2 ; Lycorne, 2574-5 ; Narbonnais, 5158-9 ; Aymeri, 2776-8 ; Laurin, 441720.

Synador, le héros, se défend tout seul contre la foule d'ennemis ; l'auteur le compare à un grand chien attaqué par des mâtins :

[A cestui mot] s'esvertue comme le grant chien que li petit mastin tien[nen]t, (...) Laurin, 1767-8. 
Certains exemples montrent la hardiesse du héros, comparé au sanglier se tournant contre les chiens (ou le chasseur) qui l'ont poursuivi :

Comme sanglers qui a estal livré

Enmi les chiens quant il l'ont arresté,

Se deffendoit Charles au cuer sené. Enfances O., 6020-2.

Ainc mais ne furent ses parelles,

Car aussi com quant est li pors

Escauffés des ciens par effors

Et il a tous estal leur livre

Tant que les pluiseurs a mort livre,

Ainsi Jehans seur le sablon

Leur a mis le cors a bandon. Jehan, 4372-8.

Li ber al branc d'achier lor avoit fait tel parc

Comme fait li sangler[s] qui as ciens se combat. Aiol,

10772-3.

Donc veïssiez jaiant desver !

Come sengler parmi espié,

Qant li chien l'ont auques chacié,

S'anbat contre le veneor,

Tot ansemant, par grant iror,

Corrut au roi se l'anbraça,

Einz por l'espee nel leissa. Brut, 2968-74.

Voir aussi : Troie, 128, 55-7 ; Laurin, 1781-2 ; 5980-2; TP, v, 239, 33-5; Charroi, 357.

La rage des hommes est comparée à celle des chiens ou des bœufs :

Adont conmencent durement a huchier,

Ullent et crient conme chien enrachié. Mez, 11115-6.

Comme chien anragié poignent por ralier. Saisnes, 4852. Cf.

$i b ., 4953$.

Il meïsme chevache, ambrons, l'eamë anclin,

Sa grant targe a son col, ou poig l'espee tint,

Que formant se dotoit de la gent Apolin

Qui par defors glatissent autresi com mastin. ib., 7073-6.

Toute nuit hulent ausi comme mastin. Enfances G., 1978. 
Bien veit ferir Guillaume comme beuf esragiez, Rou, II, 1478.

Quand il est en colère, le jeune Lancelot fronce le nez « comme un cheval»:

(...) ; et fronchoit del neis en sa grant ire autresi com mes chevaus et estregnoit les dens ensemble si que il croissoient moult durement, (...) Lancelot, viI, Ixa, 4.

La malheureuse Berte boite comme un cheval encloué :

La s'apoia la bele qui de plorer fu roe,

Car de paine clochoit com chevaus con encloe. Berte, 843-4.

Ceux qui, du haut des murs, regardent comment Yvain tue le géant descendent en toute vitesse après le combat ; Chrétien de Troyes les compare à des chiens de chasse se ruant sur la curée :

Cest cop vostrent mout tuit veoir

Cil, qui estoient as creniaus.

Lors i parut li plus isniaus ;

Car tuit corent a la cuiriee

Si con li chien, qui ont chaciee

La beste tant que il l'ont prise. Yvain, 4248-53.

\subsubsection{L'homme a les traits de caractère d'un animal}

Les chiens peuvent être méchants ; quelques extraits montrent que parfois ils mordent même la main qui les a sauvés, d'autres soulignent leur couardise. Mais les hommes ne sont pas meilleurs :

Et li muniers prant à passer,

Or seroit il pire que chiens

S'il ne lor faisoit aucun bien

Del lor, car il lo puet bien faire. $M R$, cxIx, 150-3.

"Il sont plus haigrez que waignon,

« De grant orguel et de fierté. » Chauvency, 3668-9.

et leur sire est remés cha fors,

qui mout estoit crueus et fors

et fel et fiers et plus irous

ke ciens dervés ne leu warous. Barisel, 159-62.

Bien tanceroit a un estrange,

Qui ranposne son conpaignon. 
Ne vuel pas sanbler le gaignon,

Qui se hericë et regringne,

Quant autre mastins le rechingne. » Yvain, 644-8.

Li quenz Fromons, qui a cuer de mastin, Mez, 237.

Bien apert que vos estes mauvais mastins provez. Saisnes, 7479. Cf. ib., 7635 .

- Qui ore ne s'en fuit, tost i puet mort gisir ;

Alum nus ent pur noz vies garir. »

Dist Vivien : « Ore oi parler mastin. » Guillaume, 256-8.

Dist Perchevaus : « Or puis retraire

Que il resamblent de gaignon,

Qui cort sus a son copaignon

Quant il l'a rescous du ferain : Cont. P., I, 1046-9.

J'ai la costume au felon chien :

Quant en li a le gué passé

Et il se sant a fermeté,

Celui abaie et sel vialt mordre. Athis, 590-3. Cf. ib., 11303-4.

Mais quant il ad en un païs

Hummë u femme de grant pris,

Cil ki de sun bien unt envie

Sovent en dient vileinie ;

Sun pris li volent abeisser :

Pur ceo comencent le mestier

Del malveis chien coart felun,

Ki mort la gent par traïsun. Lais, I, 7-14.

On peut constater que les individus qui font le plus de bruit et semblent les plus zélés sont souvent les plus paresseux et les plus lents quand il en vient aux efforts - ceci est vrai pour les chiens et pour les hommes :

Il fu de lui com du mastin

Qui vaint tous les cris au matin ;

$\mathrm{Au}$ descoupler est li premiers

Et au besoing li plus laniers. Partonopeu-C, 345-8.

« Mais j'ai fait comme le gaignon,

Dist il, qui aboie en son close,

Et puis par defors issir n'ose,

Et ne fait que le manechier. Meliacin, 7466-9. Cf. ib., 7491. 
vos avez la teche al vilain,

qui la androit hue son chien

ou il n'ose aler por rien, Eneas, 6888-90.

Chez d'autres animaux, c'est la simplicité, l'humilité ou la faiblesse que soulignent les auteurs pour nous faire voir celle des personnes dont ils parlent :

Eracles forment s'umelie, ne fait sanlant qu'il le desvoelle ;

le toup devant a une fuelle

con cevaus que on maine vendre

por faire le gent a entendre ;

onques aigniaus plus simplement

n'ala $u$ liu $u$ on le vent

con fait li tendre cars, li biele,

le col estraint de la cordiele. Eracle, 424-32.

Li baron de Herupe ne sont mie muart,

Ainz li seront bien estre et felon et gaingnart

Et simple com aignel et fier comme liepart. Saisnes, 670-2.

O la biauté orent amor,

De corteisie grant valor,

Franches paroles, douz respons,

Sinples les cuers come colons. Athis, 2653-6.

Ses enfans en sus de li chace

Et bece ausi con la geline

Ki desouz le cok s'ageline ; $M R$, xLIX, 194-6.

Li roys si les mainne ferant devant lui tout aussi comme se ce fussent brebiz. Laurin, 5990-1. Cf. ib., 13737-9 ; 13746-7.

Puis vint aux dames et damoiselles, aux moynnes et a leurs autres gens qui comme brebis aux coins de la sale estoient plorans et leur dist : (...) Jehan de S. 298, 22-4. Cf. ib., 220, 21-2.

L'auteur de Queste exprime que l'âne symbolise l'humilité et par conséquent il en fait la monture d'un religieux :

Puis le monta sus un asne, ce est la beste d'umilité, et bien fu aparant chose, que Nostre Sires le chevaucha quant il vint en sa cité de Jherusalem, (...) Queste. 158, 21-3. ib., 70, 1-2. 
Lors ateint un home de grant aage qui ert vestuz de robe de religion et chevauchoit un asne, (...) ib., 162, 5-6.

Ailleurs, c'est le caractère difficile de l'âne qui se retrouve chez quelques hommes :

Qant li baron l'antandent, chascun s'est errier trais,

Tot ansi com li asnes qi regarde le fais ;

De lor seignor aidier firent samblant mauvais. Saisnes, 344-6.

D'autres hommes doivent être comparés à des porcs qui ne pensent qu'à se remplir l'estomac ou à dormir :

Et li vilains, comme porciaus,

S'encressoit, et plains ses bouciaus

Bevoit de vin en larrecin,

Maint cras chapon et maint poucin

Menja toz seus en sa despensse ; $M R$, Lxxx, 49-53.

(...), ainsin que le Philosophe dit, que l'on doit seullement mangier et boire pour vivre et non pas vivre pour boire ou pour mangier, comme les gens pourceaux font. Jehan de S., 25, 22-5.

Il ne respondit mot, mais s'endormit comme ung pourceau. Cent, 26, 371-2.

La jeune Alemandine, dans Athis, a été déçue par Artumides à qui elle reproche son instabilité : à un moment il est fort (comme un faucon), le moment d'après peureux et faible (comme un canard et un pigeon) - et enfin tout à fait indigne (comme une corneille) :

S'il fust oiseaus, il fust faucons,

Si chanjast d'anes a colons

Et des colons a la corneille ! Athis, 19097-9.

Lancelot, dans Rigomer, est devenu abêti par le travail de cuisinier, indigne d'un chevalier. Remarquons que le cheval, quoique noble, reste quand même un animal - comme le chameau :

Mais si par estoit biestïaus

Comme uns camex ou .I. chevaus. Rigomer, 14013-4. 


\subsubsection{L’homme a des qualités généralement attribuées à un animal}

Les qualités peuvent être positives, comme la vitesse et la grandeur :

L'en fist lués aprés souper

et les letres et les escriz,

et sealer en or massiz,

q'en envoie per les contrees

par garçons qui les ont portees

qui sont plus isnel que cheval. Dole, 3121-6.

As vos poignant Malprimis de Brigant :

Plus curt a piet que ne fait un cheval. Roland, 889-90.

Li gloz en va, onques mot ne parla,

Qui plus tost cort que brachez ne chevals. Mort Aymeri, 653-4.

La firent lez paien teillement esmaier

Qu'i s'anfuyent assitost que livrier ; Lion, 17904-5.

L'autre, plus esveillé qu'un rat et viste comme ung levrier, part et s'en va, (...) Cent, 9, 102-3.

Et la vielle meïsmes i keurt conme levriere, Berte, 359. Cf.

Gaufrey, 3179 .

Et Franceis se desrengnent a guise de faucon, Renaut, 8785 .

Cf. $i b ., 9920$.

Berengiers vint poingnant a guise de faucon ; Aye, 2125.

Et cort et saut par un pre enverdi!

Li espreviers qui vole a la pertri

Ne vait si tost, par verté le vous die. Bueve, I, 6086-8.

Dont est en lui bon chevalier,

Dont est hardis plus que lions

Et guenchissans plus que falcons. Partonopeu, 10244-6.

Ainssi s'en est oultrepassez joint comme .r. faucons ; Helca-

nus, 173.

Plus est movant qu'esmerillon

Et plus hardis est que lion. Cristal, 2951-2. 
Devant tous plus joins c'uns faucons

s'en vint Cleomadés brochant, Cleomadés, 8676-7. Cf. ib.,

11380-1 ; Laurin, 2014 ; 8311.

et Renoars saut con falcons de mue : Loquifer, 2340.

lou cors a grant conme destriers d'Arrage, $i b ., 3755$.

Dure ot la teste plus que toriaus ne ors ; Aspremont, 6031.

O lui sont Borgoignon qi sont dur com toriax ; Saisnes, 5182.

Neel, vicomte du Cotentin, tient son surnom de sa vitesse :

Por bonté e por isnelece,

pr hardement e por proëce

fu apelez Chief de Faucon,

Neel Chief de Faucon out non. Rou, III, 4127-30.

Voir aussi :Joufroi, 455-6 ; Aspremont, 640-1 ; Enfances O., 5982-4 ; 2755-9 ; Brun, 123 ; Chauvency, 1786-91 ; 1876-9 ; Raoul, 5595-6 ; Narbonnais, 6122.

Dame Eremborc, en prison avec son mari, rêve d'être un faucon : ainsi elle pourrait s'envoler et obtenir la liberté. Yvain et Jehan expriment une idée semblable lorsqu'ils quittent leur dame : ils voudraient être une colombe pour pouvoir aller vite chez elle :

Car pleüst Deu qui forma tout le mont, Que je volaisse ausiz com uns faucons

De ceste chartre ou je sui en prison, Jourdain, 413-5.

Mes sire Yvains plore et sospire

Si fort, qu'a painnes li puet dire :

« Dame! cist termes est trop lons.

Se je pooie estre colons

Totes les foiz, que je voldroie,

Mout sovant avuec vos seroie. Yvain, 2579-84.

Se je pooie estre uns coulons

Toutes les fois que je vaurroie,

Mout sovent avoec vous seroie,

Mais c'est çou que estre ne puet,

Autrement a faire l'estuet. Jehan, 1934-8.

Une qualité positive très souvent mentionnée est celle de la couleur vair des yeux, comme ceux des oiseaux de chasse : 
Et ont les iauz plus vars c'ostors n'esmerilons :

N'ont plus bel bacheler jusqu'a Cafarnaon. Orson, 1568-9.

La char ot blanche come flor en esté

Et les eulz vers come faucon mué ; Narbonnais, 4430-1.

Les euz ot verz et clers comme faucon, Mort Aymeri, 1298. Cf. $i b ., 161 ; 3468$.

Quant Gloriaus le vit, si l'a moult regardé,

C'onques si bel veillart ne vit en son aé :

De jambes fu bien fet et de cors bien moullé,

Et si ot les iex vers comme faucon mué ; Gaufrey, 1486-9.

Les ieux ot vairs comme faucons, Floriant, 739.

Et esgardent Florence o la clere façon,

Que ot la char plus blanche que n'est ost de poison,

Et les iaus ot toz vars en guise de faucon, Florence, 225-7. Cf.

$i b ., 968 ; 2286$.

Et s'a les yeux plus vairs que chil de nul faucon. Rome, 240.

$N^{\prime}$ en painie qu'en i sache trover :

Bel a le cors, eschevi et mollé,

Et vairs les eulz comme faucon müé. Orange, 255-7.

Anthoine se leva pour la voir ou menton,

Les yeulx lui regarda, qui sont vers con faucon, Tristan de N., 3026-7.

Les yeulx avoit plus vair que esprivier ne faulcon. Lion, 33505. Cf. ib., $596 ; 4566$ et passim.

Une jeune fille est comparée aux éperviers et aux perroquets. A la suite de l'extrait que nous présentons se trouve une longue description de ses beaux vêtements - c'est donc le beau plumage des oiseaux qui compte ici :

Et la pucele vint plus cointe

Et plus acesmee et plus jointe

Que espreviers ne papegaus. Perceval, 1795-7.

Nous trouvons aussi quelques exemples de qualités négatives ; ces comparaisons ont tendance à devenir de véritables injures : 
N'avrïez vos force vers nos

Ne c'uns aigneax contre deus lous. Erec, 4427-8.

Des que Milon fu mors, sa gent fu esbahie,

Con tropiaus de brebis est d'une part fouÿe ; Aye, 4031-2.

Fait Alpatris : « Bien ai veü

Que de vilain non a on bien

Fors cil quel tient vil come chien. Florimont, 12062-4.

Li faus juïf par lor envie,

C'on devroit tüer come chiens, Perceval, 6292-3.

Li rois monta, et il li tint l'estrier,

Si s'en foui comme coart levrier, Charroi, 360-1.

- Gloz, » dist Guillelmes, « Deus te doinst encombrier ! Je ne te pris plus qu'un chien enragié. »Couronnement, 2142-3.

- Bau sire Dex, aïe ! ce dit li rois Floram, «Ja estoient il pire que [ne] sont chiens puanz ; Floovant, 1985-6. Cf. ib., 846 .

Mais, avant que feusse venu, Avoit ja mengié et beü Tant char, tant pain, tant aulx, tant vin Qu'il puoit comme un viel mastin. Deduis, 1763-6.

Toit et tes gens n'estes que chievrez. ib., 2889.

Quant il vit le Sauvage qui de fere aatine N'avoit de ceur neant plus comme une geline Tristan de $N$., 7665-6.

Des guerriers sarrasins, dans Roland, ressemblent physiquement à des porcs ; nous pensons que ce trait indique aussi leur caractère - on les méprise :

E l'altre aprés de Micenes as chefs gros ;

Sur les eschines qu'il unt en mi le dos

Cil sunt seiet ensement cume porc ; Roland, 3221-3.

Dans d'autres textes aussi, on juge les gens d'après leur extérieur : ils sont laids, par conséquent ils sont méchants, féroces même : 
Et s'estut ce encore del mains

A l'autre laidece qu'ele ot,

Que si oeil estoient dui clot,

Petit ausi com oeil de rat.

Ses nez fu de singe ou de chat,

Et ses levres d'asne ou de buef :

Si dent sambloient miol d'oef,

De color tant estoient rous,

Et si ot barbe come bous. Perceval, 4621-30.

«Si voirent vos aït Deus, »

Fait un hiraus fel come wains, Chauvency, 1802-3.

Je m'aprochai vers le vilain,

Si vi qu'il ot grosse la teste

Plus que roncins ne autre beste,

Chevos meschiez et front pelé,

S'ot plus de deus espanz de le,

Oroilles mossues et granz,

Autés come a uns olifanz,

Les sorciz granz et le vis plat,

Iauz de çüete et nes de chat, Yvain, 294-302.

Ja fuissent desconfi li moine,

Qant une gent lor acouru,

Qui le maintin et secouru ;

Mais n'estoient pas crestien :

Musiaus avoient comme cien, Rigomer, 10406-10. Cf. ib.

13698-9.

Et si sont plus velu que .I. gaignon betez. Renaut, 3464. Cf. ib., 3700 .

C'est une gent qui ne goustent de blé,

Mais le char crue, comme gainon dervé ;

Tot adés gisent au vent et a l'oré,

Plus sont velu que viautre ne sengler, Huon, 2915-8.

Il en est un peu différent du géant Açopart qui, par la suite, sera un serviteur fidèle de Bueve ; tout le monde a d'abord peur de lui à cause de sa laideur, mais ensuite on l'apprécie :

La bouche ot grant que baee porta,

Des dens devant ronchin feré sambla,

De ceus deriere sanglers qui escuma, Bueve, I, 3910-2. 
Le géant Morachier est laid: entre autres choses il a une très grosse tête et dans ses narines peuvent entrer des œufs d'oie :

La teste avoit plus grosse assez d'un buef plenier,

(...)

En une des narines du nés, lés le joier,

Pourroit on largement un oef d'oue muchier ; Gaufrey, 2971 et 2976-7.

La rapidité de l'épervier, si souvent soulignée comme une qualité positive, se trouve au négatif quand on y compare le caractère féminin : comme il est difficile de saisir l'oiseau, il est difficile de saisir la nature de la femme, si volage et instable :

« Li corages de fame si est vains e legiers,

Car ensement se torne conme li espreviers :

Qui mieus le cuide avoir si est tost senestriers. Aye, 1149-51.

Les hommes qui ne savent satisfaire les femmes sexuellement sont comparés à des chapons, mal vus par les poules. A moins qu'on ne les compare à un mauvais cheval épuisé, comme le fait la jeune épouse de Gui de Hantone. Or, les femmes coureuses sont comparées à des chiennes. Voir un exemple de Cent, à la page 165 et cf. Friedrich Bangert, op. cit., p. 181 : «Die Hündin ist das Bild gechlechtlicher Ausschweifung » :

Bien savez le coc chaponnez

Est as gelines mal venus :

Aussi homme qui est tenus

A mal ouvrier est deshaciez

Entre fames, bien le saciez,

Ce seront nonnains ou begines,

Si com chapons entre gelines. $M R$, Lxxxv, 104-10.

Il est tous vieus et chenus et ferrans,

N'a plus d'amour qu'un ronchis recreans, Bueve, III, 135-6. Cf. $i b .$, III, 100-1.

Dans Partonopeu, Perceval et Jehan, nous voyons une population entière qualifiée de stupide :

Loial chevalier sunt Breton,

Mais il sunt sot come mouton

Et fol parlier sens felonie. Partonopeu, 7293-5. 
- « Sire, sachiez tot entresait

Que Galois sont tot par nature

Plus fol que bestes en pasture ; Perceval, 242-4.

Saiciés vous tout voir, Francis sont

Plus sote c'un nice brebis. » Jehan, 2704-5.

A la bataille de Hastings, les Normands qualifient indirectement les Anglais de chiens parce qu'ils ne comprennent pas leur langue (cf. Friedrich Bangert, op. cit., p. 180) :

Normand dient qu'Engleis abaient

por la parole qu'il n'entendent. Rou, III, 8068-9.

ço lor ert vis qu'il glatisseient

ker lor langage n'entendeient. ib., III, 8231-2.

Voulant prouver la force du géant Ferragut, Turpin lui fait emporter Ogier le Danois comme un agneau :

E cum li jeaunt l'oust esgardé, il ala souef juste ly e l'embrasa en ces bras tot armez e l'enporta veyaunt touz en soun chacthel autresi cum un aignelet. Turpin, 623-5.

Pour montrer que la peur serait naturelle chez une jeune fille (Fenice), mais qu'elle ne convient pas à un héros courageux (Cligés), Chrétien de Troyes donne des exemples du monde des animaux « à l'envers »: les animaux forts (chiens, castors, loups, aigles) fuient devant les animaux faibles (lièvres, tourterelles, agneaux, colombes). Wace fait dire aux messagers de l'empereur de Rome qu'il serait contraire à la nature que l'empereur fuie devant le roi Arthur - comme si l'on voyait le lévrier fuir devant le lièvre, le lion devant la brebis et le loup devant la chèvre :

Se cele comancier ne l'ose,

N'est mervoille, car sinple chose

Doit estre pucele et coarde.

Mais il qu'atant, de coi se tarde,

Qui por li est par tot hardiz,

S'est vers li seule acoardiz ?

Dex, ceste criemme don li vient,

C'une pucele seule tient,

Simple et coarde, foible et quoie ?

A ce me sanble que je voie

Les chiens foïr devant le lievre,

Et la turtre chacier le bievre,

L'aignel le lou, li colons l'aigle, 
Et si fust li vilains sa maigle,

Dom il vit et dom il s'ahane,

Et si fuit li faucons por l'ane,

Et li gripons por le heiron,

Et li luz fuit por le veiron,

Et le lyon chace li cers,

Si vont les choses a envers. Cligés, 3793-812.

Se tu longues les puez tenir

Que nos nel te faciens guerpir,

Dire porras, si ert mervoille,

Que li lyons fuit por l'ooille

Et que li lous fuit por la chievre

Et li levriers fuit por le lievre.

Ne puet mie ensi avenir

Ne nature nel puet sofrir. Brut, 2119-26

Dans Deduis, Gace de la Buigne mentionne deux espèces de vin : le vin de truie qui donne sommeil, et le vin de singe qui, bien au contraire, rend gai :

Or a trouvé un autre vin

Que boit de soir et de matin

Que l'en appelle vin de truie, Deduis, 1789-91.

Encore y a un autre vin,

(...)

Vin de singe se fait nonmer

Vin qui ainsi fait demener

Ceulz qui en boivent a oultrage. $i b ., 1797$ et 1807-9.

\subsubsection{L'homme est traité comme un animal}

De rares extraits expriment que parfois des hommes et des femmes étaient très mal traités, comme si c'étaient des animaux, parce qu'on les méprisait et qu'on voulait les humilier et punir. Cf. Friedrich Bangert, op. cit., p. 180. Il est à remarquer que dans la majorité des cas il est question de chiens, si souvent l'objet du mépris des hommes. Nous verrons plus loin, 5.3., que quelques termes désignant des animaux servent à injurier directement ou indirectement ces personnes :

Bertain ont abatue, n'i ont plus arresté,

A fource li ouvrirent sa bouche estre son gre,

A guise de cheval que on a afrené

Li ont mis cele corde, ce fu grant cruauté ; Berte, 447-50. 
Hors le traient com .I. mors chien, $M R$, vIII, 198.

Au pié li chiet, et si se claime

De son fil qui gueres ne l'aime,

Ne ne li veut faire nul bien

Ne plus qu'il feroit à .I. chien. ib., cxxv, 51-4.

Je te ferai brisier et la teste et le piez,

Puis seras con un chien en un fossé lanciez. Barbastre, 6519-

20. Cf. ib., 4201.

Maintenant se part d'ilueques et il estoit ja nuiz oscure ; si chevauche tant la ou il cuide trover gent et abat avant soi trez et pavillons et loges et destruit quanqu'il ataint et occist chevaliers et dames et damoiseles ne n'en a plus pitié que de chiens. Lancelot, IV, LXxx, 36.

L'estandart n'eslongiés, seignour, pour nulle fin,

Car qui eslongna, foy que doi Apolin,

$\mathrm{Au}$ retour le ferai encruer con mastin. » Bâtard, 222-4.

L'en porte a terre tretot plat

Asi estendu comme .I. chat. Claris, 13213-4.

N'i avoient garant ne coife ne chapel,

Cez borjois ocioient com se fusient porcel. Saisnes, 238-9.

Fromont traïnnent jusqu'a un viés fossé,

Escorchié l'ont conme buef escorné.

A grant dolor a son tans afiné, Jourdain, 4129-31.

Maintenant l'a seisi parmi le caperon,

A son col le geta aussi comme .I. mouton ; Gaufrey, 4544-5.

maint felon ai danté comme cheval au frain ; Rou, II, 494.

Il le fait si mater et justicier

Come on afaite le ramage espervier. Aspremont, 30-1.

Yseut compare Tristan déguisé en lépreux à un âne parce qu'il la portera au Mal Pas ; plus loin, dans son serment, elle le compare à une bête de somme :

- Ne vuel mes dras enpalüer :

Asne seras de moi porter

Tot souavet par sus la planche. Tristan, 3917-9. 
Si m'aït Dex et saint Ylaire,

Ces reliques, cest saintuaire,

Totes celes qui ci ne sont

Et tuit icil de par le mont,

Qu'entre mes cuises n'entra home,

Fors le ladre qui fist soi some,

Qui me porta outre le guez,

Et li rois Marc mes esposez. $i b ., 4201-8$.

Après la bataille devant Rouen, les Normands dépouillent les morts qui couvrent le champ de bataille comme des porcs :

lez mors vont despoillant, si jurent comme pors,

nen ont graignor pitié qu'il eüssent des cors, Rou, II, 3295-6.

\subsubsection{Animal comparé à un autre animal}

Ces exemples, assez rares, dans lesquels il est question notamment de destriers comparés soit à des éperviers ou des faucons, soit à des lévriers ou des levrettes, servent avant tout à donner une impression de la vitesse des chevaux. On peut se demander si, dans un certain nombre des exemples présentés plus haut, 5.1.3., où la rapidité des chevaliers est comparée à celle d'un animal, il n'est pas question, en réalité, plutôt de celle de la monture que de celle du cavalier :

Et [sist] sor .I. destrier qui corroit de randon :

A lui ne valoit riens vols a esmerillon. Simon, 319-20.

«Mes montez sor Bayart le boen corant destrier,

"Qui plus tost vos corra que ne vole espervier, Renaut,

7594-5.

Et sist desor Bayart qui bruit come faucon, ib., 7666. Cf. ib., $2237 ; 9832$.

Le cheval fiert de l'esperun,

Qui plus randone que falcun ; Gui de W., 3381-2. Cf. ib., 6212-4.

Feraus lait corre le bon destrier gascon,

Qui plus tost cort que ne volent faucon. Gaydon, 9526-7.

Li oisel qui volent par l'air

Ne vont plus tost dou palefroi ; Erec, 1388-9. 
- Lor cheval sont corant, isnel conme faucon. Buevon, 181.

Limbanors fu armés de tres riche maniere

Et sist sor un destrier corant conme levriere, $i b ., 2382-3$. Cf.

Aymeri, 1755.

Il broce le cheval qui cuert comme levriere, Lanson, 1643.

(...) font sentir les esperons aus chevaus qui plus tost vont

que esmerillons a la quaille (...) Laurin, 2732-3. Cf. ib., 9024-5.

Puis broche le cheval corant

Qui plus tost cort, quant il s'eslesse,

Que uns levriers quant ist de lesse

Et il a le lievre acueilli. Cont. P., II, 12024-7.

Li lyons avoec li s'en vint,

Com .I. levrier o li se tin[t]. Lycorne, 6091-2. Cf. ib., 6077-9.

Car la avoit .I. grant sengler.

Le cors avoit grant con .I. tor, $i b ., 4172-3$.

Ce fu l'eschale d'une ançoine barbée,

Qui plus tost noe par mi la mer salée

Que fax gruiers ne vole a recelée Mort Aymeri, 3323-5.

Aymeri voit dans un rêve un oiseau grand comme un bœuf :

« Devant aloit .I. noirs oisels volanz,

« Grans come bues estoit en son estant, Mort Aymeri, 314-5.

Voir aussi : Roland, 1534-5 ; 1571-2 ; Couronnement, 656-8 ; Tristan de N., 15974-5 ; Renaut, 2237 ; 7594-5 ; 7666 et passim ; Aspremont, 30-1 ; 9006-7 ; Bueve, III, 1932 ; 10954 ; Brun, 2467-9 ; Barbastre, 5839-40 ; Otinel, 1637-8 ; Aliscans, 6365 ; Deduis, 3977-8.

Nous attirons l'attention sur les exemples suivants où c'est encore la vitesse qui est soulignée : dans Partonopeu, la vitesse d'un navire est comparée à celle du cerf fuyant devant le lévrier ; dans Blancandin à celle du faucon; dans Tristan, la flèche tirée par Tristan est deux fois plus rapide qu'un émerillon ; dans Meraugis et Chauvency, les chevaux ne sont pas comparés à des animaux rapides, mais à un éclair :

Sigle la nes jusc'a la nuit,

Plus tost que cers levrier ne fuit ; Partonopeu, 763-4. 
Plus tost que ne vole faucons

Vait li bateax as avirons. Blancandin, 4147-8.

La seete si tost s'en vait

Rien ne peüst de lui gandir.

Par mié l'uel la li fait brandir,

Trencha le test et la cervele.

Esmerillons ne arondele

De la moitié si tost ne vole ; Tristan, 4474-9.

Quant il cheïrent, li cheval

S'en fuïrent plus tost que foudre. Meraugis, 4484-5.

Et li cheval saillent com foudre, Chauvency, 1539.

Dans Saisnes, la robe du destrier est comparée aux plumes d'un paon, ses yeux à ceux d'un faucon ; le roncin de Gauvain est maigre comme un poussin :

L'ampereres de Rome, li prince et li baron

A mervoilles esgardent le bon destrier gascon :

Plus li luisoit li poils que pene de poon,

Si ot la teste maigre, l'oil vair comme faucon, Saisnes, 2233-6.

« Vassal, fait ele, or descendez

Et avec moi çaiens entrez

Atout vostre cheval ronchin,

Qui plus est megres d'un pochin, Perceval, 7273-6.

La biche surnaturelle de Papegau est comparée à la fois à un cheval et à une vache. Une comparaison particulière se trouve dans Gaydon où une massue a la forme d'une tête de brebis :

Si estoit une beste a merveilles grant, aussi grande come ung grant cheval, et avoit une corne enmy le front aussi tranchant come nul rasoir du monde. Et si avoit grans mameles .xiiij., dont la maindre estoit aussi grant come la mamelle d'une vasche. Papegau, 83, 19-24.

Prent sa massue au materon faitiz ;

Devant fu grosse com teste de brebis, Gaydon, 6350-1. 


\subsection{Métaphores}

A part les cas dans lesquels les termes expriment directement ou indirectement du dédain et insultent la personne visée, les métaphores à base d'animaux ne sont pas très nombreux. Dans Galeran, on comprend que la dame avait la langue bien pendue ; dans Partonopeu- $C$, il est question de la faiblesse des personnes et, dans Florimont, le lait désigne la douceur de la générosité :

De son non ne doy plus parler,

Car le faisoit trop tost aller

Le cheval de sa langue e courre,

Que nulz ne lui povoit rescourre

Quant elle estoit en haulte alaine : Galeran, 35-9.

Or voit de quel pié Fresne cloche

La dame qui son cheval broche ;

Ire la transporte et ardure,

Sa langue broche oultre mesure

Qui li desvoie le courage ; $i b ., 3913-7$.

Devant l'empereor leans

Ert humbles com uns innocens,

Si simples et si souplians

Et si aigniaus, si enclinans, Partonopeu-C, 555-8. Cf. ib., 3146.

Et Damas li respont : " Molt avés divers dis,

Mais se je sui creüs, ja ne rerons berbis. » $i b ., 2239-40$

Li soudans atant li repont :

«Ce me samble l'aignel qui gront. ib., 3497-8.

Biaus fils, mes ne poroit descrire

Le bien que largeté ait fet :

Se tu wels boivre de son lait,

Il est plus dous que atre chose

Et si plus fres que n'est rose. Florimont, 1928-32.

Il faut toutefois réserver une place particulière aux fabliaux et aux Cent nouvelles nouvelles, où se trouvent pas mal de métaphores. A la page 10, nous avons mentionné le terme poulain comme métaphore du sexe masculin, en voici des exemples :

- Bele, » fet il, « c'est mes poulains.

(...)

Mès ja por ce mar aurez garde

Que n'i puissiez bien amener 
Vo poulain pestre et abevrer. $M R, \mathrm{LxV}, 61$ et 94-6.

- Dame, » fait il, « c'est mes polains, ib., cxI, 173.

Por la fille au vilain le di,

Qui tantost si se converti,

Que le poulain au bacheler

Fist à sa fontaine abevrer. $i b .$, cVII, 231-4.

La doulce maison fut treslargement troublée, quand en la garenne que plus chere tenoient lesdictz parens, avoient osé lascher les levriers et limiers ce desplaisant mal, et que plus est, touché sa proye en dangereux et dommageable lieu. Cent, 2, 28-32.

Car, comme le poulain s'eschauffe sentant la jument, et se dresse et demaine aussi faisoit le sien, levant la teste contremont si tres prochain de l'aurfavresse. $i b ., 7,44^{-7}$. Cf. $i b ., 53$; $59 ; 66$.

Mais je veil ravoir mon enfant ; vostre maistre ara la vache, et j'aray le veau, moy! ib., 22, 102-4. Cf. ib., 29, 107-10.

Quant à l'extrait suivant, nous pensons qu'il s'agit d'une métaphore signifiant que la femme querelleuse, fâchée parce que son mari ne tenait pas compte de ses paroles, se mit encore davantage en colère :

(...), elle se mist en la queste de luy et partout le suyvoit, Dieu scet disant quelx motz; et l'aultre se taisoit et picquoit son chemin. Et elle tant plus montoit sur son chevalet, et disoit de maulx et maledictions a son pouvre mary, (...) $i b ., 84,18-22$.

Les verbes chevaucher (< cheval) et ronciner (<roncin) expriment bien comment l'homme fait l'amour à la femme :

Elle maudit qui oncques luy fist requerre d'estre chevauchée : " Je le vous disoye bien, » dit lors son mary. Après la prend entre ses braz, et la roncina tresbien, (...) ib., 44, 237-40. Cf. ib., 20, 164-6; 50, 53-6.

Dans Eneas se trouve un emploi métaphorique de conin symbolisant le sexe féminin (Eneas est accusé d'homosexualité) :

il n'aime pas poil de conin. Eneas, 8595 . 


\subsubsection{Métaphores de mépris}

Voici d'abord quelques exemples, où les terme mâtin et chien ont la fonction d' injures (2) :

«A ! reis, ja Dex non vaille, cors de mastin ! » Roussillon, 2677.

«Fil a putain, malvais mastin puant, Loquifer, 2038.

Ahi, Guillelmes fel traïtre mastin, Aliscans, 1809.

Ha, faulx chien mastin, or congnois ta loy estre faulse et dampnable (...) Ogier, 202. Cf. ib., $156 ; 176 ; 203$.

« Hui, hui, fait ele, vilenaille,

Chien esragié, pute servaille ! Perceval, 5955-6.

Mais les exemples des « injures indirectes » sont bien plus nombreux. Ici encore, ce sont les chiens qui dominent; nous n'avons, en effet, relevé qu'un exemple d'un autre animal et deux avec du fromage, visiblement toujours l'objet de mépris (cf. pp. 362-363) :

N'ot point d'escu, fors l'auberc doblantin,

Mes tot li orent depecié li mastin, Aliscans, 333-4.

Quant Aÿmers a choisi le mastin,

Des esperons a brochié Florentin ; ib., 5398-9.

Bien apert que vos estes mauvais mastins provez. Saisnes,

7479. Cf. ib., 7073-6.

Se je ne te foutoie bien,

Tu me harroies plus c'un chien. $M R$, LXIII, 45-6.

Quant ce virent li chien puant

Si se sunt de cele part treit, Graal, 526-7.

Puis acieut Robers les gaignons

La u les treve cha et la : Diable, 2636-7. (Turcs)

Cel jor en ot ml't le noaus

Mahons, lor Diex, et Tervagans,

Que ainc mais ne fu fais lagans

Veüs de tex chiens .j. seul jor. Escoufle, 1266-9. (Turcs) 
« Franceis doivent le chien et batre et ferir

« Tant qu'il viege au pié Kalles, ileques doit garir! Renaut,

7994-5. (Ogier)

« Baron, or tost as chiens qui devant nos se mirent! » $i b$.,

13373. (Sarrasins)

Alons dessus ches chiens : il ne valent noient,

Car il despitent Dieu et le sien sacrement. Bâtard, 138-9. (Sarrasins)

« Leres, leres, fait li hermites,

tu es pires ke sodomites,

ne chiens ne leus ne autre beste. Barisel, 771-3.

car ta creance ne pris jou .I. bouton ;

tes dex ne fait ne mercis ne pardon;

an crois fut mors, de verté lou seit on.

Je n'i creroie neque'an .I. guaïgnon ! » Loquifer, 3327-30.

Conment vous parjuréz Jhesucris et les sains !

Nient plus de foy n'avéz que chevaus ne poullains, Rome,

2204-5.

Vez la .I. chevalier, ou vient.

L'escu par les enarmes tient ;

Mes molt est blanche s'armeüre,

Ce samble .I. fromage em presure

Qui soit de la foissele issus, Floriant, 2169-73.

Et Flohart a la ventaille saisie,

As deuz li a del hauberc esrachie ;

Ausi trangloit com ce fust formagie. Aliscans, 6767-9.

Voir aussi : Bueve, I, 1399-400 ; Ogier, 32 ; 43 ; 63 et passim ; Godin, 9623-4 ; 13936-7 ; 15297 ; Floriant, 6318-9 ; Bâtard, 2401-3.

\subsection{Proverbes, dictons, locutions}

Dans son livre Proverbes français antérieurs au Xve siècle, Joseph Morawski présente une série considérable de proverbes à base d'animaux domestiques. Un certain nombre d'entre eux se retrouvent dans nos textes, qui en fournissent aussi quelques autres : 
Mais malvaise haste n'est preus :

S'en puis dire un mot veritable,

Que trop a tart ferme on l'estable

Quant li chevaus en est perdus. » Cont. P., I, 1660-3.

Quant en a le cheval perdu

A tart va l'en fermer l'estable. Galeran, 1452-3.

cil qui n'a talant de mantir,

li vilains, dit bien chose estable :

que trop a tart ferme an l'estable

quant li chevax an est menez. Charrete, 6954-7.

« Li asnes ciet par la sorsomme,

Fait Gavains, ç'ai oï retraire ; Atre, 4004-5.

(...) ; et a ce propos peut on dire : " De chiens, d'oiseaulx,

d'armes, d'amours : Pour ung plaisir mille douleurs. » Cent, 59,

209-11.

Li rois n'a pas fet ke desvé :

Ki n'a ch[e]val, si aut a pié. » Yder, 1343-4.

Ensement dit Marie, maix bien s'an doit cesser ;

Aprés le chevalz perdut, vuelt l'estable fermer. Lion, 11662-3.

Che que la truie fait, les porchiaus demand'on. » Bâtard, 5352.

Et li vilains le dist en ses escris :

« Li fix al cat doit prendre le soris. » Aspremont, 256-7.

Ce dist l'archevesque Turpin : il fait mal reveiller le chat qui dort. Ogier, 73.

Un proverbe assez répandu mentionne la chèvre ; il signifie à peu près : on peut faire une chose si longtemps que le résultat en sera le contraire de ce qu'on voulait - on peut tout exagérer (cf. Proverbes, 2297) :

tant grate chievre que mal gist. Renart, IV, 9632. Cf. ib., vI, 15768.

Tant grate chievre que mal gist Erec, 2584.

Dans Tristan de N., où Clarinde veut épouser Blanchandin(e), qui est encore une femme, nous lisons un proverbe disant que deux personnes de la même force (du même sexe) ne peuvent 
pas s'entr'aider - il faut un coq ! Le second proverbe reste dans le même ordre d'idées: c'est un mauvais mari qui ne fait pas l'amour à sa femme. Nous notons que l'auteur emploie les deux termes poule et geline et qu'ils désignent la même chose :

Maisement est aidee poule de la geline. Tristan de N., 13391.

Mal ait telz coquellés qui a poulles ne va ! »ib., 15549.

Il est parfois malaisé de distinguer les dictons des proverbes. Où ceux-ci se présentent à nous sous une forme assez fixe, toutes les fois qu'ils apparaissent, les premiers semblent mieux adopter leur forme aux situations dans lesquelles on les emploie. Toutefois, pour les uns comme pour les autres, il s'agit d'idées reçues et de connaissances acquises par une longue expérience de plusieurs générations :

Mot sont li chien de grant servise ! Tristan, 1636.

« Hachet li chevalier en plache !

«Chiens qui ne trueve qui le base,

« Tuit vont a lui et il a tous.

« Bas! Wartéz com il est estous!

« Ch'est uns droit kienz de baquerie. Chauvency, 2095-9.

Li chevaus, qui ne va pas lant,

S'esforce, quant an l'esperone. Yvain, 2146-7.

On dit que ses paroles pert

Souventes fois qui cort a ciens. Atre, 4126-7.

Malves gäeing fet an gibier,

Qui pert l'aloe et l'esprevier.

Tel le ferons, ce m'est avis,

S'i perdons vos et noz amis. » Athis, 8275-8.

Mangier me devroient mastin

Quant de vilain fis palasin. Partonopeu, 2563-4.

Partonopeu présente un autre dicton dont Joseph Gildea écrit, tome $\mathrm{II}^{2}$, p. 62: "We must admit that the verse is as mystifying to us as it evidently was to the scribes. We forego any attempt to elucidate it even if $B$ is taken at its face value. A proverb, elusive as far as we are concerned, may account for the difficulty. (...) »:

Ensi va de ceste besoigne

Com on fiert chien qui truie engroingne. » $i b .$, 7053-4. 
Dans une note, à la page 104 de son édition de Dole, Félix Lecoy écrit que pour le dicton suivant il s'agit d'une «locution inconnue »:

c'iert en l'eure qui li chiens cort

qu'il i sera ja mes amez ! Dole, 5615-6.

Il faut comprendre l'extrait suivant ainsi : quand le maître aime son chien, tous les autres en font autant. Dans le cas du texte : l'empereur aime Eracle, et tous ses gens l'aiment aussi :

car puis que sire assaut son cien,

tout li autre le sivent bien ; Eracle, 1901-2.

Les animaux domestiques et des produits apparaissent dans des locutions devenues plus ou moins des clichés :

Que il viengne entre chien et leu, $M R$, xIx, 90.

Je di et si vuoil bien c'on saiche

Que Ferris cuidoit toutevois

Que li rois li gardet sez oies

Tant estoit de fier contenant. Chauvency, 490-3.

vos resamblez le chien qui crie

ainz que la piere soit cheüe. Renart, II, 4228-9.

Bien lor savoit chat en sac vendre. Joufroi, 1676 .

Car se mon mautalent acrois,

Jo te batrai conme anne a pont. » Hunbaut, 2086-7.

(...) ; et de bien venir, bouta sa teste dedans la cheminée, ou il vit nostre bouchiere plus simple $\mathrm{q}[\mathrm{u}]$ 'un chat baigné, dont il fut tres esbahy. Cent, 40, 113-6. Cf. ib., 61, 164-6.

Si l'aultre son compaignon, le derrenier venu, avoit bien fait du mauvais cheval et en maintien et en parolles, encores en fist il plus ; (...) ib., 33, 185-7.

Elle estoit fort amoureuse d'un gros chanoine qui avoit plus d'argent que ung vieil chien n'a de puces ; (...) $i b ., 92,11-3$.

Pour quoy la laissa courre comme une lisse entre deux douzaines de chiens, et accomplir tous ses vouloirs et desordonnes desirs. $i b ., 91,55^{-8}$. 
« Nostre Dame! ou m'a bien baillé de l'oye, et si ne m'en doubtoie gueres ; $i b ., 33,99-100$.

Et tant fist par son engin, qui point oyseux n'estoit, qu'il advisa une maniere par laquelle bien luy sembloit, s'il en pouvoit venir a chef, que monseigneur raroit beurre pour œufs. $i b ., 3$, 151-4.

Il n'eut gueres esté en son logis,luy qui cognoissoitmousche en laict, qu'il ne percesut tantost que la chambriere de leans estoit femme qui devoit faire pour les gens. ib., 18, 7-10.

Si va le char davant les buefs ; Galeran, 2149.

D’Ysabel n'ai jou fait nul conte

K'il n'est pas mestiers ne œus,

N'on ne doit pas devant les bués

Metre le char quant on caroie. Escoufle, 6162-5.

Nous avons relevé un grand nombre d'occurrences de locutions qui toutes signifient « peu de chose » ou «rien ». Il est à remarquer que les locutions contenant le terme ouf, au singulier ou au pluriel, seul ou avec une épithète, sont de loin les plus nombreux. Si certains animaux domestiques et certains produits se retrouvent si fréquemment dans de telles expressions, c'est certainement parce que l'on les mésestimait ou qu'en réalité ils étaient de très peu de valeur. Nous avons déjà vu, p. 313, combien les chevaliers méprisaient le fromage, et nous pensons que dans la société campagnarde les œufs se trouvaient à prix bas à cause de leur abondance : on les donnait pour presque rien.

Voici d'abord des exemples avec le terme œuf:

Aussi qu'il ne coustast .I. oef ; $M R$, LXxx, 81. Cf. $i b .$, LXxxII, 12 ; XIX, 457.

Et cil de son comandement

Ne donroient vaillant deus oez,

Car il les ont trespassez lués Cont. P., II, 87, 10-2.

Li .I. dit au duc, l'autres l'a desloé,

Que mar fera por Karle vaillant .I. ouef pelé, Renaut, 338-9.

Cf. $i b ., 396 ; 11772 ; 12740 ; 13821$.

François sont si preudome et vasal aduré,

Se il avoient ore ce grant palés listé,

Ne m'en donroient il vaillant un oef pelé. Barbastre, 601-3. 
« Tot son pooir ne vaut .II. aux pelez. Otinel, 1385.

Mais avant vous n'en ruis deus oés. Violette, 4992. Cf. ib., 5980-1.

Tout le ga[a]ing done et depart,

C'onques riens ne tient a sa part

Qui vausist un oef de geline. Diable, 3593-5.

Deux extraits, dans lesquels on ne parle pas du peu de valeur de l'œuf, mais qui insiste sur le peu de temps qu'il faut pour cuire un œuf :

\section{(...) Einsi s'esmaie \\ Et demente qu'onques la nuit \\ Ne dormi tant qu'en eüst cuit \\ .I. oef. (...) Meraugis, 5340-3.}

Mais ne dura mie cil tans,

par le mien escient, je cuit,

tant que on eüst un oef quit. Cont. P., III, 14428-30.

Voir aussi : Galeran, 3435-6 ; Huon, 6583-5 ; Meliacin, 10415-8 ; Enfances O., 4059-61 ; 6937-8 ; 7982-4 ; Alisacans, 1280-4 ; 70814 ; Eneas, 795-800 ; Buevon, 2917 ; Roussillon, 602-3 ; 4200 ; 5628 ; Loquifer, 1937-8 ; 2923-4 ; Charroi, 427 ; Athis, 6901-2 ; Bueve, III, 2082 ; 8373 ; 8521 ; Yvain, 3891-4 ; Ogier, 37 ; Hunbaut, 2954 ; Gaufrey, $971 ; 2554 ; 6357 ;$ Doon, $2091 ; 2671 ; 3348$ et passim.

Ensuite des exemples avec le terme fromage, toujours précédé de vaillissant, c'est-à-dire " ayant la valeur de » :

« Damage n'i avrez vaillessant .I. formage, Renaut, 1333.

«Frere, venés vous ent, sy lessés ce bocage,

Car je vous partiray a mon grant heritage ;

Je n'en tenray sans vous vaillissant ung fromage. Tristan de N., 21459-61.

Et s'arait bon chevalz, c'est ceu qui me sollaige,

Qui ne me cousterait vaillissant ung fromaige ; Lion, 2999300.

ne doute oré vaillissant .I. fromage, Loquifer, 467.

Ne troverez en trestot vostre eage

Qui vos en toille vaillissant un fromage ; Couronnement, 484-5. 
Foi que doi Dieu qui nos fist a s'ymaje,

Ja n'en avroiz vaillesant un fromaje ; Narbonnais, 164-5.

N'i val li cercles d'or vaillissant .I. fromage ; Simon, 230.

La générosité dite « de fumier » n'a aucune valeur car on ne donne que pour être payé de retour :

$\mathrm{S}[\mathrm{e}] \mathrm{u}$ est largesce de fumier ;

Seu fet li vilains volentiers :

Le fumier donet a la terre

Por plus de bleif qu'il welt conquerre. Florimont, 4271-4.

Et voici des exemples avec des animaux. Il y a lieu de souligner qu'il s'agit exclusivement d'animaux fréquemment méprisés et que les chiens dominent. Dans les deux derniers extraits de $\mathrm{Si}$ mon et dans ceux de Loquifer, il est question de charognes :

Mais ly crestien sont sy bien sur leur saisine

C'on ne guaagne sur eulx vaillant une geline, Tristan de N., 22050-1.

Donqe dirra vostre veysin :

"Cesti ne vaut plus qe un mastyn ;

Si Dieu me doint de son bien,

Cesti ne vaut plus que un chien. » $M R$, LII, 354-7.

Dist Renoart : « Sire, lessiez m'ester !

Ge ne dorroie un chien de vo parler. Aliscans, 7775-6.

"Se il ne conquiert à brief terme nommé,

« On ne le droit priser .I. pouchin escaudé. Doon, 11453-4. Cf.

Godin, $8613 ; 9197$ et passim.

Ja mon signor, ce sai jou bien,

Ne prisers vaillant un chien

Que on a mort la u il est. Guillaume d'A., 1283-5

Et s'il a Mahomet de noient irascu,

Si li dont por son droit mil marcs d'argent fondu.

- Por foi, dist Synadors, ja ne vos ert teü.

Ne li feroie droit plus que .I. mastin velu, Simon, 752-5.

J'ai guerpi Mahomet et si l'ai defié ;

Ne pris mais sa poissance vaillant .I. chien tué, $i b .$, 530-1. 
Que Mahomet ne vaut .I. deners moneez,

For solemant li ors qu'ensemps est assemblez.

Ausi l'en porroit on giter par ces fossés,

Comme [on] feroit .I. chien, se il estoit tuez. ib., 990-3.

ce est une brogne dont li pors est molt lé,

dont li cors est de fin or esmeré,

tuit li anel de fin acier tenpré ;

ne crient cop d'arme vaillant .I. chien tüé. Loquifer, 1546-9.

canque tu dis ne pris pas .I. festu,

toi ne tes deus vaillant .I. chien pandu. ib., 1625-6.

« Tais toi, » dist il, « ton deu, je lou defi,

car n'i creroie nec'an .I. chien pori. » ib., 3238-9.

Le fabliau Le roi d'Angleterre et le jongleor d'Ely montre du mépris pour le roncin du jongleur : il ne vaut pas plus que du crottin :

Ataunz usse je de or real

Com il se tient valer fient de cheval ! MR, LII, 269-70.

\subsection{Allusions à fables et légendes}

Dans quelques textes se trouvent des allusions à des histoires qui devaient être connues par le public à qui on s'adressait. Ces allusions étant parfois très vagues, il n'est pas toujours possible de dire à quoi pense l'auteur.

Les deux extraits suivants, de Tristan de N., renvoie à une légende dont K.V. Sinclair écrit, dans une note à son édition, p. 750 : "Ces vers renferment une allusion à la légende de Macaire, personnage d'une chanson de geste sur la Reine Sebile et qui fut battu en champ clos par un lévrier. (...) En mentionnant le lévrier et la défaite de Macaire, notre trouvère ne fait que souligner la mauvaise réputation du personnage traître de sa propre composition. » :

Seigneurs, ycil Maquaires qui tant ot seignorie

Fist le champ au levrier devant la baronnye, Tristan de N.,

1145-6.

Entre lui et Maquaire estoient compaignon,

Que le levrier mata a loy de champïon. ib., 9619-20. 
Gaston Paris, à la fin d'un petit article dans Romania, xxxI, (1902), pp. 100-103, intitulée «Une fable à retrouver », écrit : « Reste à savoir quelle est cette fable. Je ne la connais pas, (...) » Il s'agit de la fable mentionnée dans Aliscans et dans Violette. Douglas Labaree Buffum appelle une «fable obscure » (note pp. 275-276) :

Guillelmes l'ot, si taint comme charbon ;

De mautalant en froncist le guernon.

«Comment, deable! dist il, si plaideron?

Ce est la fable du tor et del mouton ; Aliscans, 3431-4.

Chou est la fable dou tor et dou mouton. Violette, 1423.

Par contre, l'extrait de Dole rappelle une histoire bien connue :

Quant il furent amdui ensamble,

si le fet lors parlement

e paroles ou il ment:

par passer les chievres, les chous,

sachiez qu'il n'estoit mie fous. Dole, 3468-72.

Galeran et Tristan de $N$. mentionnent la même histoire du roi Arthur et du chat; nous ne savons pas à quoi font allusion les auteurs :

Et cil qui jeu souffrir ne puet,

Par si grant ire s'en esmuet,

Qu'il le ledenge de contrueve,

Et le roy Artu li repreuve,

Que le chat occist par enchaus. Galeran, 5067-71.

Artus le nous approuve, qui tant ot baronnye,

Car au temps qu'il regna, pour voir le vous affie,

Se combati au chat qu'aleita en sa vie

Du let d'une seraine qui en mer fut peschie.

Mes le chat devvint tel, ne vous mentiray mye,

Que nulz homs ne duroit en la soye partye

Qu'i ne mesist a ffin, a deul et a hachie. Tristan de N., 1651-7.

Dans une note au vers 8800 de Cristal, Hermann Breuer suppose que ce vers indique que le sage Salomon a pris la fille après la mère. Il ne dit pas sur quoi s'appuie sa supposition dont nous n'avons pas pu trouver de confirmation :

N'entendés mie a Salomon,

Qui manga l'owe et puis l'oison! »Cristal, 8799-80o. 
L'extrait suivant fait allusion à une fable d'Esope qui se retrouve chez La Fontaine (Livre I, fable 10 : Le Loup et l'Agneau) :

Et se de moi volez enquerre

La començaille de la guerre

Ne l'acheison de la meslee :

L'eve, ce dit, avez troblee,

Come li lous dist au moton. Athis, 10911-5.

Dans Tristan de N., p. 751, se trouve la note suivante : «1531 ss. Les renseignements sur la mort de Mahomet fournis à cet endroit et aux vv. 6480-86, 17433-4 s'accordent en général avec ceux qu'on relève dans d'autres poèmes épiques, (...) ».

Voici deux des exemples indiqués et quelques exemples analogues :

- Par foy, se dist Anthoine, ung dieu avés vaillant,

Car ly pourcel l'allerent sur ung fiens estranglant

Pour ce qu'il estoit yvres. On le treuve lisant. Tristan de N.,

1531-3.

Sur ung fiens se coucha, ort, et waste et puant,

Et la endroit l'allerent ly pourcel devorant.

La mourut wastement dessus ung fiens puant. ib., 6484-6.

Il vint a Meques trestot premierement,

Mais il but trop par son enivrement,

Puis le mangierent porcel vilainement. Couronnement, 850-2.

Pour cui en paienie on het tous les pourchiaus, Bâtard, 3127.

De Mahomet ne doit nus hom pledier.

Verité fu, nostre sire l'ot chier,

O les profetes l'envoia preeschier,

Par lui nos dut nostre loi ensegnier ;

Mes i but bien de fort vin un setier,

Puis se coucha dormir en un fumier,

Tant que porciau l'i alerent mengier. » Narbonnais, 5762-8.

Mais n'i avoit nul porc, car li paien felon

N'en mengeroient d'un pour tout l'or Psalemon,

Pour che qu'il estranlerent sus .j. fumier Mahon. ib., 5567-9.

Sus ung fumier puant fist tant, se sceit on,

L'estranglerent porcialz a grant destrucion ;

Pour ce ne mengez point de chair de bacon. » Lion, 17616-8. 
Bien sai qu'estranglés fut per dessus ung fumier

De porciaulz, et por tant n'an pot il mengier ;

Ains se lairoit ung Turc de famine esraigier

Qu'il mengaist d'un porcelz ne piece ne quartier. $i b ., 20821-4$.

Car vous adorés ung dieu nommé Mahon, lequel fut de vie mauldicte et demnable et le monstra bien a la fin. Car il fut estranglé honteusement et miserablement d'ung pourceau en ung fumier, dont il m'esmerveille grandement comme vous estes si abusez d'y avoir creance. Ogier, 303.

Je ne pris Mahomet deus deniers mouneez, Que truies l'ont mengié deus mil anz a pasé ; Barbastre, 1360-1.

«Car toi ne Mahoumot ne pris pas .I. denier.

«Bien a pasé .c. anz que truies l'ont maingié. » Floovant,

373-4.

Concernant l'extrait suivant de Roland, où ce n'est pas Mahomet mais une statue qui est mordue par les chiens et les porcs - ce qui pourrait être l'origine de la légende des exemples ci-dessus - nous renvoyons à la longue argumentation de Friedrich Bangert, op. cit., pp. 161-162 :

Et Tervagan tolent sun escarbuncle

E Mahomet enz en un fosset butent

E porc e chen le mordent e defulent. Roland, 2589-91.

\subsection{Jeux d'enfants}

Jean Froissart énumère, parmi une longue série de jeux d'enfants, quelques-uns dans lesquels se trouvent mentionnés des animaux domestiques. A l'exeption du cheval de bois, ces jeux nous sont inconnus ; Anthime Fourrier se demande si le jeu " à l'agnelet » indique que les enfants jouaient au saute-mouton :

Et s'ai souvent d'un bastonciel

Fait un cheval nommé Grisiel,

Et ossi souvent fait avons

Hïaumes de nos caperons,

(...)

Jeuiens nous au roi qui ne ment,

Aux barres et a l'agnelet,

(..) 
Au mulet, au salir plus haut

Et a le carette Michaut,

(...)

A le corne de buef au sel, Espinette, 213-6, 220-1, 229-30 et

235 .

\subsection{Euvres d'art}

Dans quelques textes se révèle l'esprit imaginatif de l'homme : des oiseaux tiennent dans leur bec une chaîne à laquelle est attachée une source de lumière (3) :

Li autres chiés de la chaene qui la lanpe conduist et meine, a un piler de travers vint, uns colons d'or le bec la tint, soldez estoit sor la cimaise, de la tonbe ert assez a aise. Ja mais la lanpe ne charra tant com li colons la tanra ; il la tanroit toz tens mes biens, se ne estoit sole une rien : un archier ot de l'autre part, tresgitez fu par grant esgart ; androit lo colun ert asis sor un perron de marbre bis ; Eneas, 7685-98.

Et ou milieu de la chambre estoit une pierre entaillie en forme d'un faulcon, et avoit en son bec une chaïnete d'or qui pendoit bien jus ung espan, ou estoit estachié ung escharbocles qui gitoit de nuit si grant resplendeur qu'il sembloit que la chambre fust toute embrasee. Papegau, 28, 3-8.

Dans Cleomadés et Meliacin se trouve un automate en forme de poule et une image héraldique :

On deviserait les joiaus, mout fu chascuns riches et biaus. Une geline, ce m'est vis, de fin or fist Melocandis et trois poucinés qui sivoient cele geline et si chantoient si doucement que il sambloit Chascun qui chanter les ooit k'ains n'oÿ si grant melodie. Cleomadés, 1585-93. Cf. ib., 2120. 
Gados portoit l'escu d'ermine

a une vermeille geline,

a une ourle de witecos ; ib., 11321-3.

Un joel en atraist mout bel :

Une geline en un platel

Et avoeques de poucins sis ;

Mais sachiés que d'or fin massis

Furent compos et tresjeté.

Li clers, qui avoit volenté

C'on seüst laiens son convine,

A mise a terre la geline,

Qui tantost se prist a escourre

Et commença partout à courre,

Et si poucinet aprés li. Meliacin, 273-83.

Jehan de $S$. présente des exemples d'animaux employés comme termes de blason :

(...) et diray des autres nouvelles que Saintré fist a l'encontre du seigneur de Loisselench, baron de Poulayne, qui porte d'argent a un boeuf rampant de gueules, corné et onglé de sable ; (...) Jehan de S., 143, 19-23. Cf. ib., 153, 2 ; 153, 7.

(...), le seigneur de Nulz, ui porte d'or a une teste de beuf de sable, (...) $i b ., 144,3-5$.

Le conte de Bearn, qui portoit d'or a deux vaichez de guelles, (...) $i b ., 193,11-2$.

Le reliquaire dont se sert Guillaume le Conquérant est appelé « oeil de bœuf » :

desus out mis un filatiere,

tot le meillor qu'il pot eslire

e le plus chier qu'il pout trover,

oil de boef l'ai oï nomer. Rou, III, 5691-4.

1 En ce qui concerne les exemples où il est question d'oiseaux, il n'est pas sûr que tous présentent des oiseaux domestiqués. Nous avons préféré néanmoins en tenir compte.

2 Il n'est donc pas vrai, comme l'écrit Friedrich Borchert, op. cit., p. 23, que seul le terme mâtin, désignant le chien de garde, mais pas le terme chien, plus noble, soit employé comme injure. Cf. Friedrich Bangert, op. cit., p. 173 : « (...) so werden die diese Hunde [gaignon et mâtin] bezeichnenden Wör- 
ter fast nur im üblen Sinne, und zwar mastin hauptsächlich als Schimpfwort gebraucht. » et, ib., p. 179 : « Das Wort gaignon kommt merkwürdigerweise als Schimpfwort nicht vor, sehr häufig aber das wahrscheinlich mit dem verwandten Wort gaignart. »

3 Notons qu'aux vers 3293 à 3306 d'Ipomedon, se trouve une lampe semblable, mais où l'oiseau est un aigle. 


\section{Conclusion}

Nous l'avons annoncé dans notre introduction : les animaux domestiques sont bien présents dans les textes narratifs, surtout les chevaux. Dans sa thèse sur L'animal dans la littérature française au XII et au XIII siècles, Jean Bichon affirme, p. 14: "Il n'y a pas de chanson de geste sans cheval ». Notre étude a montré que ceci est vrai pour presque la totalité des autres textes narratifs médiévaux. Il est vrai aussi que le nombre des autres animaux domestiques est bien inférieur à celui des chevaux et que nous les trouvons surtout dans des textes assez tardifs et montrant la vie des paysans ou des bourgeois. Les nobles s'occupent quasi exclusivement de leurs chevaux et, dans une moindre mesure, des chiens et des oiseaux de chasse, tandis que les roturiers tirent plus de profit des vaches, des brebis etc.

C'est ainsi que nous avons pu constater la grande intimité qui existe entre les chevaux et leurs propriétaires, intimité qui se montre p. ex. dans le fait que beaucoup de ces chevaux ont un nom propre (phénomène extrêmement rare chez les autres animaux, à l'exception du Roman de Renart). Cette intimité, qu'on pourrait dans bien des cas qualifier d'amour, nous ne la voyons pas se traduire tellement par les paroles mais plutôt par les actes : on soigne bien ses chevaux (ou mieux : on les fait soigner par des écuyers), on leur accorde du repos après les courses et les batailles, on leur donne à manger et à boire, les étrille et les ferre, les met dans des écuries avec de la litière, etc.

C'est aussi chez les chevaux que les textes distinguent origines et races et que l'on mentionne régulièrement la couleur de la robe. Parmi les autres animaux domestiques, seuls les chiens se distinguent aussi par la race, mais on ne connaît ni leur origine ni leur couleur. Et si l'on prend en considération la race, c'est essentiellement pour distinguer entre chiens de chasse (des nobles) et chiens de garde (des paysans), ceux-ci étant plus ou moins méprisés et mal traités.

Quant aux oiseaux, nous constatons une distinction semblable : les oiseaux de proie (faucons, éperviers etc.) appartiennent aux gens nobles et sont réservés aux plaisirs des riches, tandis que les oiseaux de basse-cour se trouvent aussi chez les paysans et sont utiles à tout le monde en fournissant viande, œufs et plumes.

La plupart de nos textes ayant pour théâtre la société noble, et les actions se déroulant donc dans le monde des chevaliers, il est évident que nous lisons surtout des chevaux de bataille, des chiens de chasse et des oiseaux de chasse - et beaucoup moins 
des bœufs, des moutons, des poules etc. Et il est tout aussi évident que nous avons des connaissances parfois assez détaillées des qualités, de la nourriture et du traitement des animaux des nobles, tandis qu'on ne nous renseigne qu'incidemment sur le traitement des autres animaux domestiques.

Tous les animaux sont utiles aux hommes, chaque catégorie à sa manière, mais le point de vue se trouvant du côté des chevaliers, il est clair que nous y lisons régulièrement des qualités exquises des destriers, et que l'on se contente de nous faire savoir $\mathrm{p}$. ex. que les chapons sont gras - ce qui d'ailleurs ne nous apprend rien de nouveau, c'est une épithète de nature.

Tous les animaux sont utiles, disons-nous. Les chevaux servent de monture aux guerriers aussi bien dans les batailles que dans leurs déplacements (mais les dénominations ne sont pas les mêmes : le destrier est un cheval de bataille, le palefroi un cheval de marche) - ils sont montés par des hommes et par des dames, plus rarement par des roturiers, car ils sont coûteux à l'achat et à l'entretien. On les utilise aussi comme bêtes de somme, mais peu de nos textes les montrent comme animaux de traite dans l'agriculture : là, les paysans utilisent traditionnellement les bœufs. A côté des chevaux, nous voyons les mulets, qui servent de monture à toutes les classes. Les chameaux et les dromadaires apparaissent sporadiquement, dans le monde des riches, comme moyen de transport des hommes et des marchandises, les gens modestes se contentent des ânes.

Nous avons mentionné le rôle des oiseaux et des chiens dans les scènes de chasse. Les derniers servent aussi à garder les habitations et surtout à protéger poules, brebis etc. contre les attaques des renards et des loups. Les chats chassent les souris et les rats.

Nos textes abondent en descriptions de repas. C'est ainsi que nous voyons les variétés de viande consommée : on sert beaucoup de chapons, de paons et de poulets, mais aussi de viande de brebis, de bœuf et de porc (le dernier animal semble apprécié surtout chez les paysans, moins chez les nobles). Dans des situations extrêmes, comme pendant des famines et de longs sièges, les gens sont parfois obligés de manger la viande de leurs chevaux. Les œufs, dont un grand nombre de locutions mentionnent le peu de valeur, doivent entrer dans beaucoup de plats, sans être mentionnés, p. ex. dans les nombreux gâteaux. Cela doit être le cas du lait aussi, mais nous avons vu que le lait et les produits laitiers (le fromage) sont jugés indignes comme nourriture par des nobles - ils sont bons pour les bergers.

Les gens du Moyen Age vivant près de leurs animaux, ce qui cause une grande intimité et une grande expérience, ont très bien observé leurs qualités spécifiques. Ils ont constaté que l'âne est rétif, que le taureau est fort, la brebis faible et peureuse de même que la poule, que le chat aime bien se prélasser et que les chiens 
sont souvent méchants etc. Lorsqu'un auteur veut décrire une personne il n'est pas rare de le voir la comparer à un animal, il écrit p. ex. qu'un tel est méchant comme un chien ou rapide comme un épervier, que tel autre réagit comme le faucon chassant une perdrix. Les catégories d'animaux sont aussi comparées entre elles : un cheval court aussi vite qu'un lévrier ou comme vole un faucon.

De là viennent des proverbes et des dictons qui révèlent cette expérience et ces observations : les paysans savent qu'il ne faut pas acheter chat en poche et qu'il faut fermer l'écurie avant que le cheval ne s'enfuie ou ne soit volé. De là naissent aussi une série de locutions ayant trait aux animaux domestiques. Il est à remarquer que les proverbes ont leur origine dans le monde des paysans, tandis que les comparaisons se font presque uniquement entre nobles et animaux : ceux-là sont des vérités " éternelles » basées sur l'expérience de plusieurs générations, ceux-ci ont été créées exprès, par un auteur, pour une situation particulière. 


\section{Tables}

\subsection{Table I}

Cette table indique le nombre d'occurrences de termes indiquant les chevaux (toutes les dénominations, y compris les noms propres), les autres mammifères, (également y compris les (rares) noms propres), et les oiseaux (prédateurs, de basse-cour et de distraction), ainsi que la totalité d'occurrences de termes indiquant les animaux domestiques et le pourcentage des chevaux sur ce nombre.

\begin{tabular}{|c|c|c|c|c|c|}
\hline Техте & CHEVAUX & $\begin{array}{r}\text { AUTRES } \\
\text { MAMMIFÈRES }\end{array}$ & OISEAUX & TOTAL & $\begin{array}{r}\% \mathrm{DE} \\
\text { CHEVAUX }\end{array}$ \\
\hline Aiol & 446 & 29 & 5 & 480 & 92,91 \\
\hline Aliscans & 240 & 21 & 3 & 264 & 90,90 \\
\hline Amadas & 72 & 3 & 1 & 76 & 94,74 \\
\hline Ami & 40 & 23 & 4 & 67 & 59,70 \\
\hline Anjou & 22 & 8 & 7 & 37 & 59,46 \\
\hline Aspremont & 268 & 20 & 19 & 307 & 87,30 \\
\hline Athis & 359 & 18 & 15 & 392 & 91,58 \\
\hline Atre & 178 & 8 & 15 & 201 & 88,56 \\
\hline Auberon & 25 & 6 & 9 & 40 & 62,50 \\
\hline Aucassin & 28 & 16 & o & 44 & 63,64 \\
\hline Audigier & 12 & 15 & 2 & 29 & 41,38 \\
\hline Aye & 83 & 15 & 13 & 111 & 74,77 \\
\hline Aymeri & 128 & 21 & 10 & 159 & 80,50 \\
\hline Barbastre & 271 & 34 & 6 & 311 & 87,14 \\
\hline Barisel & 2 & 2 & 1 & 5 & 40,00 \\
\hline Bâtard & 130 & 22 & 3 & 155 & 83,87 \\
\hline Bérinus & 215 & 8 & 2 & 225 & 95,55 \\
\hline Berte & 16 & 4 & 2 & 22 & 72,73 \\
\hline Blancandin & 115 & 7 & 5 & 127 & 90,55 \\
\hline Brun & 138 & 3 & 2 & 143 & 96,50 \\
\hline Brut & 57 & 8 & 1 & 66 & 86,36 \\
\hline Bueve I & 363 & 29 & 13 & 405 & 89,63 \\
\hline Buevon & 101 & 8 & 5 & 114 & 88,60 \\
\hline Cent & 53 & 111 & 11 & 175 & 30,29 \\
\hline Charrete & 105 & 5 & 0 & 109 & 95,45 \\
\hline
\end{tabular}




\begin{tabular}{|c|c|c|c|c|c|}
\hline Texte & CHEVAUX & $\begin{array}{r}\text { AUTRES } \\
\text { MAMMIFÈRES }\end{array}$ & OISEAUX & TOTAL & $\begin{array}{r}\% \mathrm{DE} \\
\text { CHEVAUX }\end{array}$ \\
\hline Charroi & 24 & 20 & 1 & 45 & 53,33 \\
\hline Chauvency & 89 & 5 & 4 & 98 & 90,82 \\
\hline Chevalerie d'O. & 500 & 19 & 7 & 526 & 95,06 \\
\hline Claris & 539 & 12 & 9 & 560 & 96,25 \\
\hline Cleomadés & 254 & 5 & 24 & 283 & 89,75 \\
\hline Cligés & 50 & 7 & 7 & 64 & 78,12 \\
\hline Cont. P. & 337 & 31 & 6 & 374 & 90,11 \\
\hline Couci & 62 & 1 & 6 & 69 & 89,86 \\
\hline Couronnement & 72 & 11 & 1 & 84 & 85,71 \\
\hline Cristal & 101 & 8 & 15 & 124 & 81,45 \\
\hline Deduis & 71 & 520 & 393 & 984 & 7,22 \\
\hline Diable & 37 & 23 & 2 & 62 & 59,68 \\
\hline Dole & 65 & 10 & 6 & 81 & 80,25 \\
\hline Doon & 177 & 15 & 6 & 198 & 89,39 \\
\hline Durmart & 322 & 21 & 53 & 396 & 81,31 \\
\hline Eneas & 75 & 19 & 6 & 100 & 75,00 \\
\hline Enfances G. & 139 & 41 & 13 & 193 & 72,02 \\
\hline Enfances 0. & 156 & 2 & 5 & 163 & 95,71 \\
\hline Eracle & 85 & 6 & 1 & 92 & 92,39 \\
\hline Erec & 146 & 12 & 35 & 193 & 75,65 \\
\hline Escoufle & 57 & 53 & 36 & 146 & 32,12 \\
\hline Espees & 137 & 20 & 4 & 161 & 85,09 \\
\hline Espinette & 5 & 4 & o & 9 & 55,56 \\
\hline Fierabras & 140 & 7 & 8 & 155 & 90,32 \\
\hline Floire & 13 & 6 & 6 & 25 & 52,00 \\
\hline Floovant & 86 & 8 & 4 & 98 & 87,76 \\
\hline Florence & 171 & 47 & 14 & 232 & 73,71 \\
\hline Floriant & 121 & 14 & 3 & 138 & 87,68 \\
\hline Florimont & 158 & 20 & 5 & 183 & 86,34 \\
\hline Fouke & 57 & 5 & 2 & 64 & 89,06 \\
\hline Galeran & 76 & 23 & 11 & 110 & 69,09 \\
\hline Gaufrey & 183 & 37 & 5 & 225 & 81,33 \\
\hline Gautier d'Aupais & 15 & 1 & 1 & 17 & 88,24 \\
\hline Gaydon & 291 & 17 & 12 & 320 & 90,94 \\
\hline Gliglois & 43 & 5 & 7 & 55 & 78,18 \\
\hline Godin & 235 & 35 & 16 & 286 & 82,17 \\
\hline Gormont & 29 & o & 2 & 31 & 93,55 \\
\hline Graal & 2 & 1 & o & 3 & 66,67 \\
\hline Gui de B. & 110 & 14 & 9 & 133 & 82,71 \\
\hline Gui de N. & 112 & 5 & 1 & 118 & 94,92 \\
\hline
\end{tabular}




\begin{tabular}{|c|c|c|c|c|c|}
\hline Texte & CHEVAUX & $\begin{array}{r}\text { AUTRES } \\
\text { MAMMIFÈRES }\end{array}$ & OISEAUX & TOTAL & $\begin{array}{r}\% \mathrm{DE} \\
\text { CHEVAUX }\end{array}$ \\
\hline Guillaume & 117 & 9 & 7 & 133 & 87,97 \\
\hline Guillaume d'A. & 22 & 20 & 2 & 44 & 50,00 \\
\hline Helcanus & 130 & 9 & 3 & 142 & 91,55 \\
\hline Hunbaut & 45 & 4 & o & 49 & 89,80 \\
\hline Huon & 174 & 15 & 13 & 202 & 86,14 \\
\hline Ille & 44 & o & o & 44 & 100,00 \\
\hline Inconnu & 161 & 29 & 43 & 233 & 69,10 \\
\hline Ipomedon & 231 & 61 & 6 & 298 & $77,5^{2}$ \\
\hline Jehan & 74 & 6 & 6 & 86 & 86,05 \\
\hline Jehan de P. & 41 & 3 & 1 & 45 & 91,11 \\
\hline Jehan de S. & 248 & 20 & 12 & 280 & 87,57 \\
\hline Jourdain & 61 & 5 & 2 & 68 & 89,71 \\
\hline Jouvenvel & 162 & 6 & $\mathrm{o}$ & 168 & 96,43 \\
\hline Lais & 29 & 12 & 4 & 45 & 64,44 \\
\hline Lancelot & 1982 & 92 & 25 & 2099 & 94,43 \\
\hline Lanson & 129 & 11 & 6 & 146 & 88,36 \\
\hline Laurin & 584 & 44 & 29 & 657 & 88,89 \\
\hline Lion & 437 & $5^{2}$ & 52 & 541 & 80,78 \\
\hline Loquifer & 30 & 18 & 10 & 58 & 51,72 \\
\hline Lycorne & 78 & 4 & 23 & 105 & 74,29 \\
\hline Macaire & 67 & 34 & 1 & 102 & 65,69 \\
\hline Mahomet & 7 & 11 & o & 18 & 38,89 \\
\hline Meliacin & 180 & 2 & 9 & 191 & 94,24 \\
\hline Méliador & 239 & 18 & 24 & 281 & 85,05 \\
\hline Meraugis & 45 & 3 & 5 & 53 & 84,91 \\
\hline Merlin & 151 & 61 & 1 & 213 & 70,89 \\
\hline Mez & 471 & 32 & 26 & 529 & 89,04 \\
\hline Mort Artu & 107 & o & o & 107 & 100,00 \\
\hline Mort Aymeri & 122 & 26 & 20 & 168 & 72,62 \\
\hline Narbonnais & 172 & 36 & 6 & 214 & 80,37 \\
\hline Ogier & 548 & 32 & 14 & 594 & 92,26 \\
\hline Ombre & 2 & o & o & 2 & 100,00 \\
\hline Orange & 16 & 6 & 8 & 30 & 53,33 \\
\hline Orson & 53 & 7 & 3 & 63 & 84,13 \\
\hline Otinel & 72 & 4 & 2 & 78 & 92,31 \\
\hline Papegau & 74 & 13 & 319 & 406 & 18,23 \\
\hline Parise & 80 & 4 & 2 & 86 & 93,02 \\
\hline Partonopeu & 126 & 39 & 16 & 181 & 69,61 \\
\hline Partonopeu-C & 86 & 42 & o & 128 & 67,18 \\
\hline Perceval & 169 & 21 & 5 & 195 & 86,67 \\
\hline
\end{tabular}




\begin{tabular}{|c|c|c|c|c|c|}
\hline Texte & CHEVAUX & $\begin{array}{r}\text { AUTRES } \\
\text { MAMMIFÈRES }\end{array}$ & OISEAUX & TOTAL & $\begin{array}{r}\% \mathrm{DE} \\
\text { CHEVAUX }\end{array}$ \\
\hline Poitiers & 16 & 6 & 4 & 26 & 61,54 \\
\hline Pontieu & 8 & 0 & 0 & 8 & 100,00 \\
\hline Protheselaus & 245 & 28 & 17 & 290 & 84,48 \\
\hline Queste & 164 & 20 & o & 184 & 89,13 \\
\hline Raguidel & 130 & 20 & 5 & 155 & 83,37 \\
\hline Raoul & 210 & 16 & 3 & 229 & 91,70 \\
\hline Renaut & 606 & 32 & o & 638 & 94,80 \\
\hline Rigomer & 322 & 19 & 25 & 366 & 87,97 \\
\hline Roche & 87 & 50 & 10 & 147 & 59,18 \\
\hline Roland & 109 & 36 & 5 & 150 & 72,67 \\
\hline Rome & 53 & 1 & 6 & 60 & 88,33 \\
\hline Rou & 171 & 51 & 11 & 233 & 73,39 \\
\hline Roussillon & 190 & 46 & 15 & 251 & 75,70 \\
\hline Saisnes & 285 & 21 & 14 & 320 & 89,06 \\
\hline Silence & 15 & 5 & 5 & 25 & 60,00 \\
\hline Simon & 41 & 8 & 2 & 51 & 80,39 \\
\hline Thèbes & 194 & 17 & 7 & 218 & 88,99 \\
\hline Tristan & 48 & 71 & 1 & 120 & 40,00 \\
\hline Tristan de N. & 278 & 23 & 23 & 324 & 85,80 \\
\hline Troie & 84 & 18 & o & 102 & 82,35 \\
\hline Turpin & 35 & 6 & 3 & 44 & 79,55 \\
\hline Vair $\mathbf{P}$. & 42 & o & o & 42 & 100,00 \\
\hline Vergi & o & 9 & o & 9 & 0,00 \\
\hline Violette & 81 & 4 & 18 & 103 & 78,64 \\
\hline Wistasse & 76 & 13 & o & 89 & 85,39 \\
\hline Yder & $15^{1}$ & 7 & 4 & 162 & 93,21 \\
\hline Yvain & 59 & 12 & 6 & 77 & 76,62 \\
\hline
\end{tabular}

\subsection{Table II}

Cette table indique le nombre d'occurrences des différents termes désignant les chevaux, dans un choix de nos textes. Il reste bien entendu que nous ne tenons compte que des occurrences où les termes sont employés comme substantifs, c'est-à-dire que p. ex. dans destrier auferrant, nous n'avons compté que destrier, tandis que p. ex. dans ... col de l'auferrant crenu, nous comptons auferrant (mais pas crenu!).

Concernant les noms propres, nous renvoyons à 1.1.3. 


\begin{tabular}{|c|c|}
\hline ambleor & $1:$ Escoufle \\
\hline amoravi & $1:$ Narbonnais, Partonopeu-C. \\
\hline arabi & $\begin{array}{l}5 \text { : Cligés, Gui de W. } \\
4 \text { : Narbonnais, Renaut } \\
3 \text { : Barbastre, Chevalerie d'O., Macaire, Mez } \\
2 \text { : Roussillon, Thèbes } \\
1 \text { : Aliscans, Aspremont, Athis, Blancandin, Bueve I, Gaydon, Lion, } \\
\quad \text { Raoul }\end{array}$ \\
\hline aragon & $\begin{array}{l}20 \text { : Lion, Renaut } \\
12 \text { : Gaydon } \\
5: \text { Chevalerie d'O. } \\
3: \text { Aye, Saisnes } \\
2: \text { Bueve I, Lanson, Mez } \\
1: \text { Ami, Barbastre, Claris, Enfances O., Orson, Roche }\end{array}$ \\
\hline arami & $1:$ Doon \\
\hline aufage & $\begin{array}{l}2: \text { Barbastre } \\
1: \text { Aliscans }\end{array}$ \\
\hline auferrant & $\begin{array}{l}18 \text { : Aliscans } \\
14 \text { : Bueve I. } \\
11 \text { : Aiol, Chevalerie d'O., Enfances G., Raoul } \\
10 \text { : Mez } \\
9 \text { : Aymeri, Brun, Narbonnais, Saisnes } \\
8 \text { : Protheselaus } \\
7 \text { : Aspremont, Fierabras } \\
6 \text { : Bâtard, Gaydon, Godin, Guillaume, Macaire, Mort Aymeri, Re- } \\
\quad \text { naut } \\
5 \text { : Gaufrey, Huon } \\
4 \text { : Lion } \\
3 \text { : Barbastre, Blancandin, Charroi, Enfances O., Gui de B., Gui de } \\
\quad \text { N., Ipomedon, Roussillon, Thèbes, Tristan de N. } \\
2 \text { : Ami, Cont. P., Floovant, Gui de W., Inconnu, Jourdain, Orson, } \\
\quad \text { Simon } \\
1 \text { : Athis, Auberon, Aye, Cristal, Doon, Gormont, Lanson, Parise, } \\
\quad \text { Poitiers, Rigomer, Roche, Violette }\end{array}$ \\
\hline augalie & $1:$ Barbastre \\
\hline bai & $\begin{array}{l}7 \text { : Saisnes } \\
4 \text { : Athis } \\
3 \text { : Protheselaus } \\
1 \text { : Atre, Aye, Aymeri, Barbastre, Bueve I, Erec, Ille, Mez, Raoul, } \\
\quad \text { Roussillon, Simon }\end{array}$ \\
\hline barbari & $1:$ Aye \\
\hline barzelon & $1:$ Roussillon \\
\hline
\end{tabular}




\begin{tabular}{|c|c|}
\hline bauçant & $\begin{array}{l}8: \text { Fierabras, Rigomer } \\
6 \text { : Chevalerie d'O. } \\
5: \text { Bueve I. } \\
4 \text { : Guillaume } \\
3: \text { Aliscans, Athis, Raguidel, Roussillon, Saisnes } \\
2 \text { : Aye, Barbastre, Raoul } \\
\text { 1: Aiol, Aspremont, Atre, Couci, Couronnement, Enfances G., Gay- } \\
\quad \text { don, Gui de B., Lion }\end{array}$ \\
\hline $\begin{array}{l}\text { blanc/ } \\
\text { blanchart/ } \\
\text { blanchet }\end{array}$ & $\begin{array}{l}3: \text { Athis } \\
2: \text { Barbastre, Tristan de N. } \\
1: \text { Bueve I, Cligés, Raoul }\end{array}$ \\
\hline brehaigne & $4:$ Aliscans \\
\hline brun & $\begin{array}{l}2: \text { Athis. } \\
1: \text { Barbastre, Brun, Bueve I, Roussillon }\end{array}$ \\
\hline chaceor & $\begin{array}{l}12 \text { : Lancelot } \\
8 \text { : Perceval } \\
6 \text { : Partonopeu } \\
4: \text { Ipomedon } \\
3: \text { Charrete, Erec, Gui de W., Raguidel, Saisnes } \\
2: \text { Cleomadés, Durmart, Guillaume d'A., Rigomer, Tristan, Yder } \\
1: \text { Amadas, Atre, Brut, Gautier d'Aupais, Inconnu, Meraugis, Par- } \\
\quad \text { tonopeu-C, Violette }\end{array}$ \\
\hline cheval & $\begin{array}{l}1681: \text { Lancelot } \\
487: \text { Laurin } \\
330: \text { Ogier } \\
303: \text { Claris } \\
280: \text { Cont. } P . \\
260: \text { Rigomer } \\
246: \text { Renaut } \\
229: \text { Athis } \\
227: \text { Cleomadés } \\
212: \text { Mez } \\
208: \text { Lion } \\
203: \text { Durmart } \\
194: \text { Méliador } \\
169: \text { Bueve I. } \\
165: \text { Aiol } \\
164: \text { Chevalerie d'O. } \\
159: \text { Jouvencel } \\
151: \text { Doon } \\
149: \text { Bérinus, Godin } \\
141: \text { Queste } \\
140: \text { Gaydon } \\
139: \text { Jehan de S. }\end{array}$ \\
\hline
\end{tabular}




\begin{tabular}{|c|c|}
\hline- & 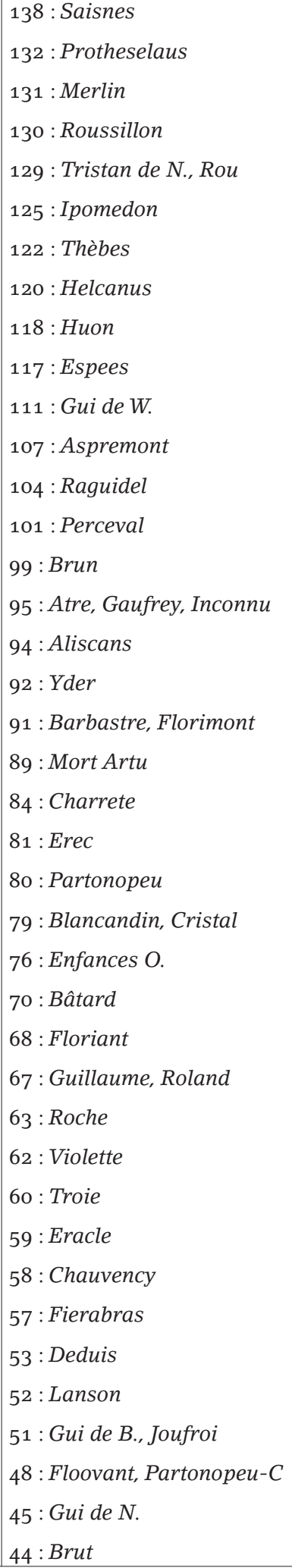 \\
\hline
\end{tabular}




\begin{tabular}{|c|c|}
\hline - & 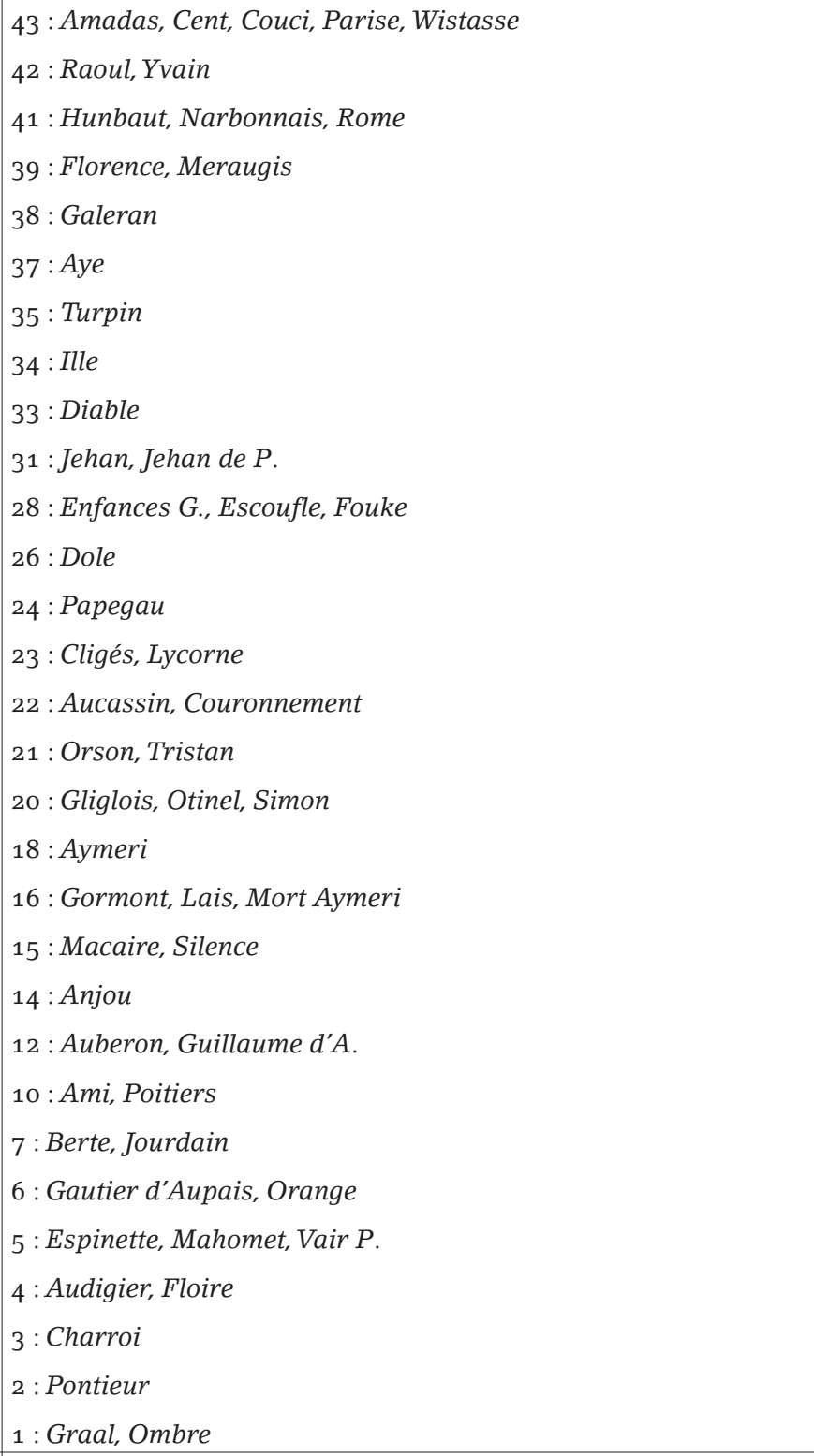 \\
\hline courant & $\begin{array}{l}4: \text { Chevalerie d'O. } \\
2 \text { : Bueve I, Saisnes } \\
1 \text { : Couronnement, Fierabras, Lion, Mez }\end{array}$ \\
\hline coureor & $1:$ Bâtard, Florence, Mort Aymeri, Partonopeu-C \\
\hline coursier & $\begin{array}{l}23 \text { : Ogier } \\
17 \text { : Jehan de S. } \\
14: \text { Méliador } \\
12 \text { : Deduis } \\
9: \text { Brun } \\
8: \text { Godin } \\
6 \text { : Jehan de P. } \\
5: \text { Barbastre } \\
4: \text { Renaut } \\
3: \text { Lion } \\
2: \text { Aliscans, Chevalerie d'O., Gui de B., Laurin, Roussillon, Saisnes } \\
1: \text { Anjou, Auberon, Couci, Doon, Fierabras, Floovant, Gaydon, } \\
\quad \text { Jouvencel, Lanson, Raoul }\end{array}$ \\
\hline
\end{tabular}




\begin{tabular}{|c|c|}
\hline courtaut & $1:$ Cent \\
\hline crenu & $1:$ Saisnes \\
\hline destrier & 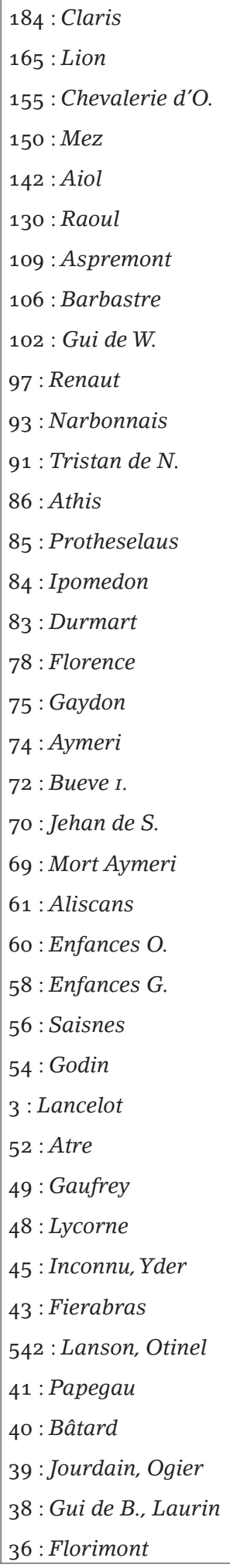 \\
\hline
\end{tabular}




\begin{tabular}{|c|c|}
\hline - & 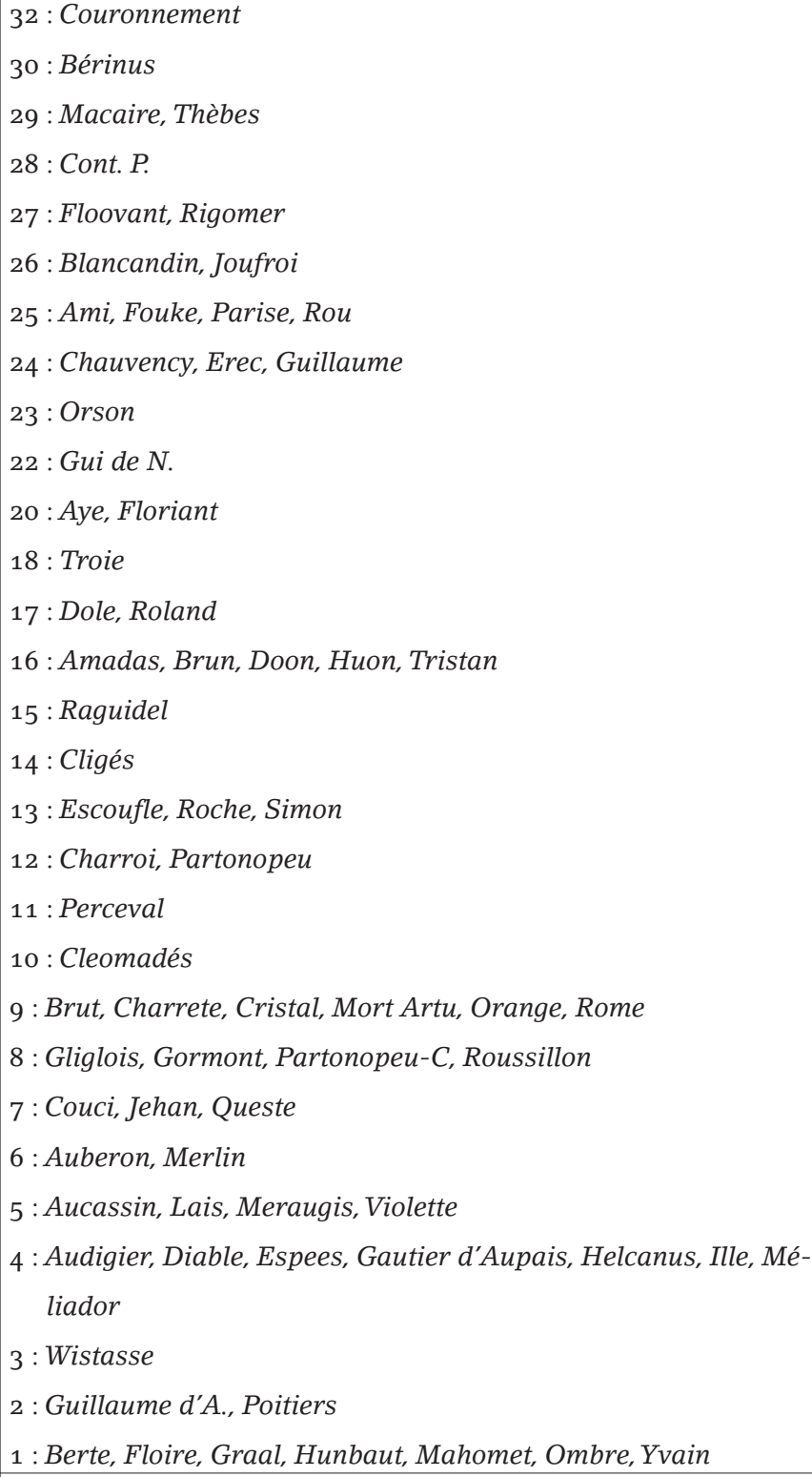 \\
\hline doine & $1:$ Barbastre \\
\hline esclavon & $1:$ Florence \\
\hline espagnol & $1:$ Roussillon, Yder \\
\hline estalon & $1:$ Méliador \\
\hline $\begin{array}{l}\text { fauve/ } \\
\text { fauvel }\end{array}$ & $\begin{array}{l}2: \text { Aye, Cligés } \\
1: \text { Roussillon }\end{array}$ \\
\hline ferrant & $\begin{array}{l}8: \text { Fierabras } \\
3: \text { Athis, Renaut, Violette } \\
2: \text { Aspremont, Protheselaus, Thèbes } \\
1: \text { Escoufle, Floovant, Ipomedon, Partonopeu-C, Roussillon }\end{array}$ \\
\hline gascon & $\begin{array}{l}5: \text { Lion } \\
2: \text { Gormont, Tristan de N. } \\
1: \text { Athis, Guillaume, Inconnu, Lanson }\end{array}$ \\
\hline gazele & $1:$ Barbastre \\
\hline genet & 1 : Jehan de S. \\
\hline gramadone & $1:$ Iпсоппи \\
\hline gris/grison & 1 : Jehan de P., Raoul \\
\hline
\end{tabular}




\begin{tabular}{|c|c|}
\hline haquenée & $\begin{array}{l}13: \text { Jehan de } S . \\
2: \text { Cent, Jehan de P., Méliador } \\
1: \text { Jouvencel }\end{array}$ \\
\hline iocor & $2:$ Espees \\
\hline ive & $\begin{array}{l}4: \text { Aymeri } \\
2: \text { Espees, Gui de B. } \\
1: \text { Aliscans, Perceval, Roche, Thèbes }\end{array}$ \\
\hline jument & $\begin{aligned} 3 & \text { : Gaydon, Laurin } \\
2 & \text { : Aliscans, Lancelot } \\
1 & \text { : Aiol, Charroi, Cent, Enfances G., Jouvencel, Merlin, Roussillon, } \\
& \text { Tristan de N., Violette, Wistasse }\end{aligned}$ \\
\hline liart & $\begin{array}{l}4: \text { Athis } \\
2: \text { Yder } \\
1: \text { Barbastre, Protheselaus, Roussillon, Thèbes }\end{array}$ \\
\hline limonier & $2:$ Aliscans \\
\hline missaudor & $\begin{aligned} 4 & \text { : Saisnes } \\
3 & \text { : Godin } \\
2 & \text { : Aymeri, Enfances O., Thèbes } \\
1 & : \text { Amadas, Aspremont, Athis, Blancandin, Claris, Cont. P., Gay- } \\
& \text { don, Inconnu, Lion, Partonopeu, Tristan de N. }\end{aligned}$ \\
\hline mor/morel & $\begin{array}{l}4: \text { Laurin, Roussillon } \\
2: \text { Gaydon, Gormont } \\
1: \text { Méliador, Ogier, Tristan de N. }\end{array}$ \\
\hline movant & 3: Chevalerie d'O. \\
\hline noir & $\begin{array}{l}2: \text { Raoul, Yder } \\
1: \text { Aspremont, Athis, Aye, Cligés }\end{array}$ \\
\hline palefroi & 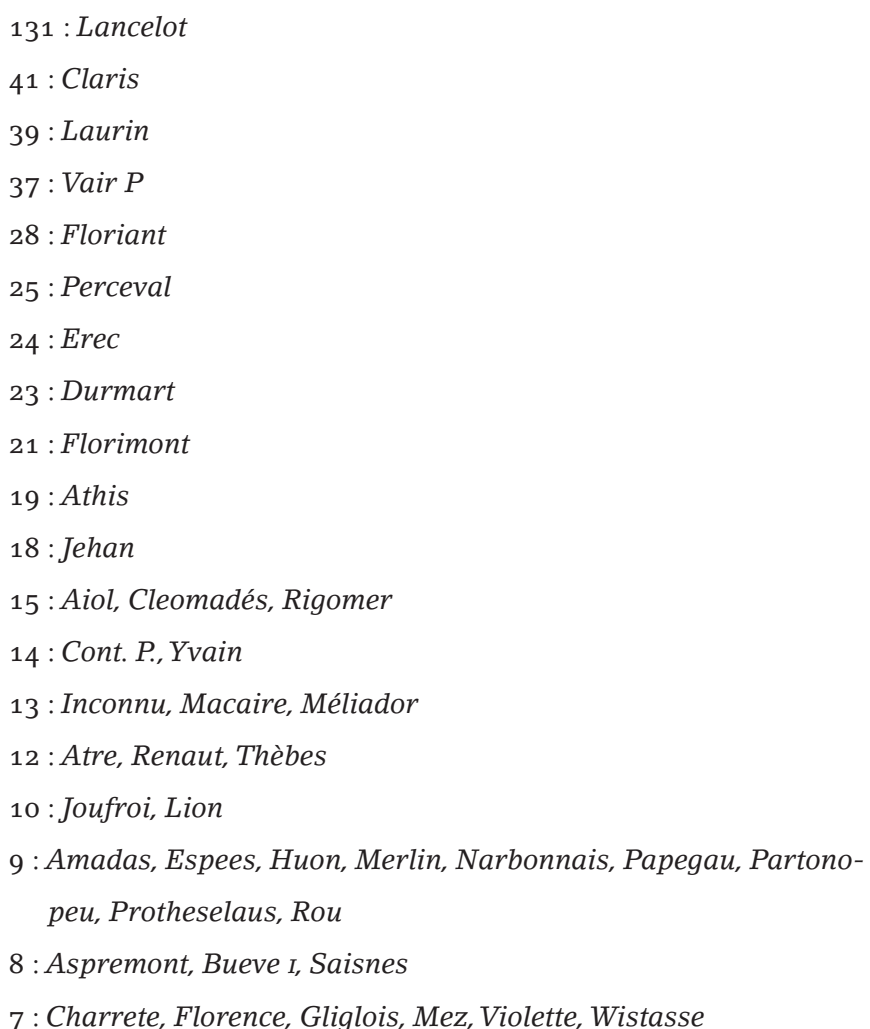 \\
\hline
\end{tabular}




\begin{tabular}{|c|c|}
\hline - & $\begin{array}{l}6 \text { : Chevalerie d'O., Dole, Escoufle, Jourdain, Parise, Queste, Rous- } \\
\quad \text { sillon, Tristan } \\
5 \text { : Anjou, Aymeri, Blancandin, Gaydon, Helcanus, Ipomedon } \\
4 \text { : Aye, Bâtard, Berte, Couci, Enfances G., Floovant, Gui de N., Gui } \\
\quad \text { de W., Mort Artu, Pontieu, Raoul } \\
3 \text { : Bérinus, Gaufrey, Godin, Partonopeu-C, Roland, Tristan de N., } \\
\quad \text { Yder } \\
2: \text { Aliscans, Auberon, Deduis, Enfances O., Floire, Fouke, Galeran, } \\
\quad \text { Guillaume d'A., Ille, Lanson, Lycorne, Orson, Raguidel, Roche, } \\
\quad \text { Rome Troie } \\
1 \text { : Barbastre, Brun, Charroi, Cligés, Couronnement, Eracle, Fie- } \\
\quad \text { rabras, Gautier d'Aupais, Gui de B., Hunbaut, Mort Aymeri, } \\
\text { Orange }\end{array}$ \\
\hline poulain & $\begin{array}{l}24: \text { Eracle } \\
3: \text { Thèbes } \\
2: \text { Lycorne } \\
1: \text { Bâtard, Chevalerie d'O., Ille, Rome, Roussillon }\end{array}$ \\
\hline poutrel & $\begin{array}{l}2 \text { : Florence, Gaydon, Godin, Rigomer, Roussillon } \\
1: \text { Brun, Gui de N., Jourdain }\end{array}$ \\
\hline roncin & $\begin{array}{l}20 \text { : Perceval } \\
16 \text { : Aiol } \\
10 \text { : Mez, Queste } \\
9 \text { : Atre, Durmart, Godin, Partonopeu } \\
7 \text { : Aliscans, Lion } \\
6 \text { : Raoul } \\
5: \text { Aymeri, Bâtard, Chevalerie d'O., Escoufle, Joufroi, Méliador, } \\
\quad \text { Wistasse } \\
4: \text { Aspremont, Bérinus, Espees, Gui de W., Guillaume d'A., Jour- } \\
\quad \text { dain, Merlin, Roussillon, Thèbes } \\
3: \text { Claris, Erec, Florence, Gautier d'Aupais, Gaydon, Gliglois, Ipo- } \\
\quad \text { medon, Lanson, Mort Artu, Mort Aymeri, Parise, Protheselaus, } \\
\quad \text { Rigomer, Roche, Rou, Saisnes } \\
2: \text { Cont. P., Couronnement, Deduis, Doon, Enfances G., Florimont, } \\
\quad \text { Gaufrey, Guillaume, Huon, Ille, Jehan, Orson, Partonopeu-C, } \\
\quad \text { Tristan de N., Yvain } \\
1 \text { : Amadas, Berte, Brun, Bueve I, Charrete, Chauvency, Cristal, } \\
\quad \text { Eracle, Floire, Floriant, Galeran, Hunbaut, Jehan de S., Laurin, } \\
\quad \text { Narbonnais, Poitiers, Pontieu, Raguidel,Roland, Violette, Yder }\end{array}$ \\
\hline ros & 7 :Saisnes \\
\hline sambuer & $1:$ Guillaume \\
\hline
\end{tabular}




\begin{tabular}{|c|c|}
\hline sommier & 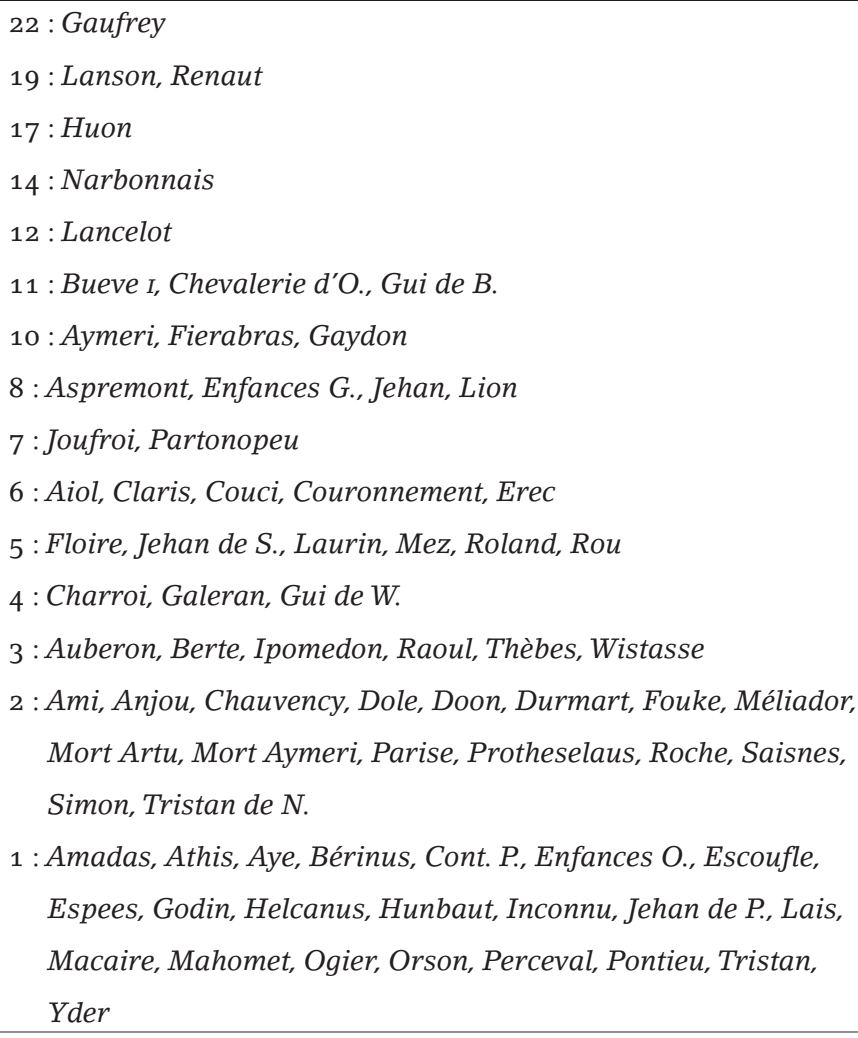 \\
\hline sor & $\begin{array}{l}2 \text { : Ipomedon, Méliador } \\
1 \text { : Aiol, Atre, Brun, Cligés, Escoufle, Laurin, Roussillon }\end{array}$ \\
\hline vair/vairon & $\begin{array}{l}8 \text { : Saisnes } \\
4 \text { : Renaut } \\
3 \text { : Barbastre } \\
2 \text { : Enfances G., Gaydon, Laurin, Partonopeu, Partonopeu-C, Yder } \\
1 \text { : Aiol, Escoufle, Galeran, Gui de N., Ipomedon, Orson, Raoul }\end{array}$ \\
\hline
\end{tabular}

\subsection{Table III}

Cette table indique le nombre d'occurrences des différents termes désignant les mammifères, dans un choix de nos textes (à l'exception des chevaux, voir table II).

Nous ne tenons pas compte des noms propres. 


\begin{tabular}{|c|c|}
\hline $\begin{array}{l}\text { agneau/ } \\
\text { agnelet }\end{array}$ & \begin{tabular}{|l}
3 : Cligés, Lancelot, Partonopeu-C, Saisnes \\
2 : Deduis, Gaydon, Rou \\
1 : Aliscans, Anjou, Aymeri, Bâtard, Buevon, Cent, Claris, Eracle, \\
$\quad$ Erec, Espees, Espinette, Guillaume d'A., Lanson, Ogier, Queste, \\
$\quad$ Troie, Turpin, Yvain
\end{tabular} \\
\hline $\begin{array}{l}\text { âne/ânesse/ } \\
\text { ânon }\end{array}$ & $\begin{array}{l}16 \text { : Cent } \\
7 \text { : Wistasse } \\
6 \text { : Lancelot. } \\
5 \text { : Queste } \\
3 \text { : Perceval } \\
2 \text { : Aiol, Atre, Tristan } \\
1 \text { : Amadas, Anjou, Aspremont, Audigier, Bueve I, Claris, Couron- } \\
\quad \text { nement, Doon, Eracle, Floire, Florence, Hunbaut, Jehan de S., } \\
\quad \text { Jouvencel, Laurin, Partonopeu-C, Roche, Roussillon, Saisnes, } \\
\quad \text { Thèbes, Violette }\end{array}$ \\
\hline aumaille & $2: \operatorname{Rou}$ \\
\hline $\begin{array}{l}\text { bauçant } \\
\text { (mulet) }\end{array}$ & 2: Escoufle \\
\hline berseret & $\begin{array}{l}4: \text { Tristan } \\
3: \text { Rigomer } \\
1: \text { Eneas, Roussillon }\end{array}$ \\
\hline bœuf & $\begin{array}{l}11 \text { : Charroi } \\
9 \text { : Cent } \\
8 \text { : Aucassin, Rou } \\
6 \text { : Deduis, Jehan de S. } \\
5 \text { : Troie } \\
4 \text { : Lion, Perceval } \\
3 \text { : Floire, Godin, Narbonnais, Tristan de N. } \\
2 \text { : Aliscans, Barbastre, Bâtard, Bueve I, Claris, Cligés, Dole, Flo- } \\
\quad \text { riant, Florimont, Galeran, Gaufrey, Gaydon, Jehan, Lanson, } \\
\quad \text { Mort Aymeri, Raoul, Rigomer, Roche, Roussillon } \\
1 \text { : Ami, Brut, Durmart, Eneas, Escoufle, Espinette, Fouke, Gui } \\
\quad \text { de B., Jehan de P., Jourdain, Lancelot, Laurin, Loquifer, Mez, } \\
\quad \text { Ogier, Orson, Papegau, Protheselaus, Queste, Raguidel, } \\
\quad \text { Saisnes, Yvain }\end{array}$ \\
\hline bouc & $1:$ Mahomet, Perceval \\
\hline brahon & $1:$ Florence \\
\hline
\end{tabular}




\begin{tabular}{|c|c|}
\hline braque & $\begin{array}{l}44 \text { : Merlin } \\
26 \text { : Ipomedon } \\
20 \text { : Lancelot } \\
11 \text { : Protheselaus } \\
5 \text { : Cristal } \\
4: \text { Tristan } \\
3 \text { : Rou } \\
2 \text { : Aiol, Bueve I, Doon, Durmart, Enfances G., Erec, Florence, Go- } \\
\quad \text { din, Laurin, Loquifer, Mort Aymeri, Roche, Roussillon, Yvain } \\
1 \text { : Ami, Aymeri, Cligés, Dole, Floriant, Hunbaut, Lais, Méliador, } \\
\quad \text { Rigomer }\end{array}$ \\
\hline brebis & $\begin{array}{l}6 \text { : Bâtard } \\
5 \text { : Laurin, Lion } \\
4 \text { : Cent, Godin, Lancelot } \\
3 \text { : Claris, Gaydon Jehan de S., Ogier, Papegau, Rou } \\
2 \text { : Buevon, Deduis, Tristan de N., Wistasse } \\
1 \text { : Aliscans, Ami, Aucassin, Audigier, Aye, Bérinus, Brut, Bue- } \\
\quad \text { ve I, Cristal, Eneas, Eracle, Fouke, Galeran, Gaufrey, Gliglois, } \\
\quad \text { Guillaume, Helcanus, Jehan, Merlin, Mez, Narbonnais, Queste, } \\
\quad \text { Roche, Rome, Roussillon }\end{array}$ \\
\hline bugle & 2 : Gui de B. \\
\hline caigne & $1:$ Cent \\
\hline chameau & $\begin{array}{l}5: \text { Roland } \\
4: \text { Aymeri } \\
3: \text { Florence } \\
2: \text { Enfances G., Gui de B., Narbonnais, Roussillon } \\
1: \text { Escoufle, Mort Aymeri, Protheselaus, Renaut }\end{array}$ \\
\hline chat & $\begin{array}{l}4: \text { Aliscans, Loquifer } \\
3: \text { Deduis } \\
2: \text { Cent, Ogier, Tristan de N. } \\
1: \text { Audigier, Claris, Galeran, Perceval, Yvain }\end{array}$ \\
\hline chèvre & $\begin{array}{l}4: \text { Audigier } \\
2: \text { Bérinus, Jouvencel } \\
1: \text { Aucassin, Brut, Deduis, Dole, Erec, Fouke, Rou }\end{array}$ \\
\hline
\end{tabular}




\begin{tabular}{|c|c|}
\hline $\begin{array}{l}\text { chien/ } \\
\text { chienet/ } \\
\text { chiot }\end{array}$ & 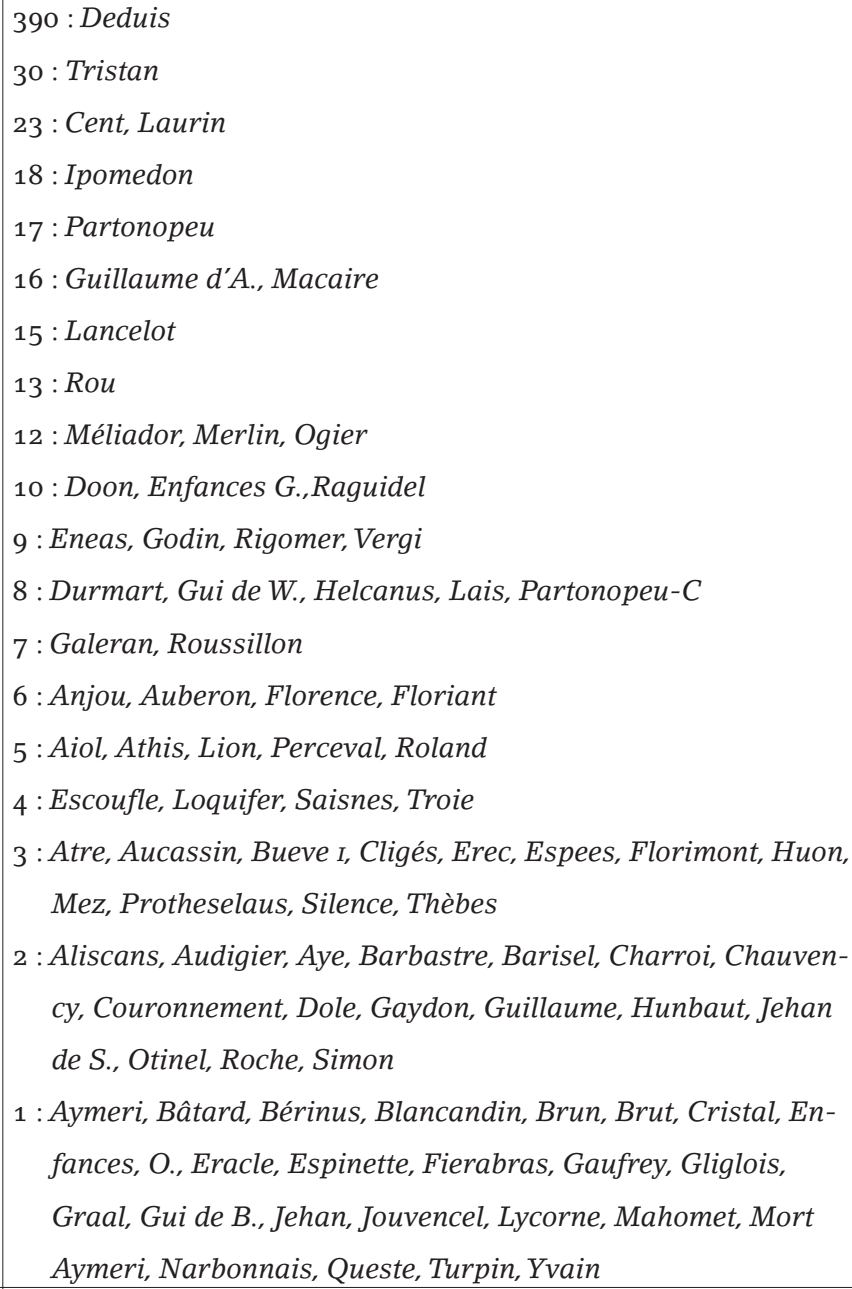 \\
\hline cochon & $1:$ Cent \\
\hline connin & $\begin{array}{l}2: \text { Deduis } \\
1: \text { Anjou, Cont. P., Dole, Eneas }\end{array}$ \\
\hline dromadaire & $\begin{array}{l}18: \text { Gaufrey } \\
8: \text { Roche } \\
5: \text { Blancandin } \\
3 \text { : Fierabras } \\
2 \text { : Simon } \\
1 \text { : Amadas, Barbastre, Bâtard, Durmart, Enfances G., Gautier } \\
\quad \text { d'Aupais, Gui de W., Loquifer, Narbonnais, Thèbes } \\
\end{array}$ \\
\hline épagneul & $1:$ Deduis \\
\hline fuiron & $1:$ Mort Aymeri \\
\hline gaignon & $\begin{array}{l}2 \text { : Aliscans, Chauvency, Loquifer, Roche } \\
1 \text { : Amadas, Eneas, Florence, Florimont, Huon, Guillaume d'A., } \\
\quad \text { Perceval, Rou, Thèbes, Violette, Yvain }\end{array}$ \\
\hline génisse & $\begin{array}{l}2: \text { Lanson } \\
1: \text { Mahomet, Rou }\end{array}$ \\
\hline
\end{tabular}




\begin{tabular}{|c|c|}
\hline $\begin{array}{l}\text { lévrier/ } \\
\text { lévrière/ } \\
\text { levrette }\end{array}$ & $\begin{array}{l}85 \text { : Deduis } \\
29 \text { : Lancelot } \\
17 \text { : Macaire } \\
14 \text { : Cent } \\
12 \text { : Partonopeu } \\
10 \text { : Bueve I } \\
8 \text { : Durmart, Protheselaus } \\
7 \text { : Ipomedon } \\
6 \text { : Chevalerie d'O., Gui de W., Partonopeu-C } \\
4 \text { : Godin, Laurin, Merlin, Tristan de N. } \\
3 \text { : Florimont, Mort Aymeri, Thèbes } \\
2 \text { : Aiol, Aymeri, Brut, Buevon, Couronnement, Enfances G., Erec, } \\
\quad \text { Lion, Loquifer, Lycorne, Méliador, Raguidel, Rigomer, Roche, } \\
\quad \text { Roussillon, Tristan } \\
1 \text { : Aliscans, Ami, Athis, Atre, Aucassin, Barbastre, Berte, Charroi, } \\
\quad \text { Chauvency, Dole, Escoufle. Espees, Fierabras, Floriant, Gau- } \\
\quad \text { frey, Jehan de S., Jourdain, Lais, Lanson, Narbonnais, Otinel, } \\
\quad \text { Poitiers, Rou }\end{array}$ \\
\hline limier & $\begin{array}{l}6 \text { : Deduis } \\
4 \text { : Partonopeu } \\
2 \text { : Florence, Méliador, Ogier } \\
1 \text { : Atre, Cent, Dole, Eneas, Floriant, Ipomedon, Otinel, Rigomer, } \\
\quad \text { Rou }\end{array}$ \\
\hline lisse & $\begin{array}{l}2: \text { Deduis } \\
1: \text { Audigier, Cent }\end{array}$ \\
\hline mastin & $\begin{array}{l}8 \text { : Deduis } \\
4 \text { : Saisnes } \\
3 \text { : Aliscans, Gaufrey, Godin } \\
2 \text { : Bueve I, Enfances G., Florimont, Ogier, Roche, Roussillon, Si- } \\
\quad \text { mon, Thèbes } \\
1 \text { : Bâtard, Doon, Enfances O., Fouke, Guillaume, Laurin, Loquifer, } \\
\quad \text { Mez, Partonopeu, Partonopeu-C, Perceval, Tristan de N., Yvain }\end{array}$ \\
\hline montenière & 1 : Roche \\
\hline mouton & $\begin{array}{l}7 \text { : Cent } \\
6 \text { : Lion, Troie } \\
4 \text { : Bâtard, Ogier, Tristan de N. } \\
3 \text { : Deduis, Guillaume } \\
2 \text { : Athis, Claris, Gaufrey, Gaydon } \\
1 \text { : Aiol, Aliscans, Bérinus, Buevon, Chevalerie d'O., Cleomadés, } \\
\quad \text { Dole, Durmart, Fierabras, Florimont, Godin, Jehan de P., Lan- } \\
\quad \text { son, Mez, Partonopeu, Protheselaus, Rou, Silence, Simon, Tur- } \\
\quad \text { pin, Violette, Wistasse }\end{array}$ \\
\hline
\end{tabular}




\begin{tabular}{|c|c|}
\hline $\begin{array}{l}\text { mul/mule } \\
\text { mulet/ } \\
\text { mulette }\end{array}$ & $\begin{array}{l}42 \text { : Escoufle } \\
31 \text { : Florence, Renaut } \\
25 \text { : Roche } \\
23 \text { : Mez } \\
22 \text { : Narbonnais } \\
21 \text { : Barbastre } \\
19 \text { : Roland } \\
18 \text { : Roussillon } \\
16 \text { : Cent, Enfances G. } \\
14 \text { : Aiol, Espees } \\
12 \text { : Aspremont, Galeran, Mort Aymeri, Raoul } \\
11 \text { : Aye, Aymeri } \\
9 \text { : Lancelot } \\
8 \text { : Athis, Chevalerie d'O., Gui de B. } \\
7 \text { : Huon, Papegau } \\
6 \text { : Bueve I, Gui de W., Thèbes } \\
5 \text { : Gaufrey, Gui de N., Orange, Orson, Poitiers, Raguidel, Saisnes, } \\
\quad \text { Tristan de N., Yder } \\
4 \text { : Charrete, Couronnement, Gaydon, Jehan de S. } \\
3 \text { : Aliscans, Erec, Gliglois, Jourdain, Lion, Meraugis, Parise, Par- } \\
\quad \text { tonopeu, Partonopeu-C, Perceval, Protheselaus } \\
2 \text { : Buevon, Charroi, Cleomadés, Eneas, Lais, Laurin } \\
1 \text { : Atre, Berte, Blancandin, Brun, Cristal, Diable, Doon, Espinette, } \\
\quad \text { Floire, Floovant, Godin, Guillaume, Inconnu, Ipomedon, Jou- } \\
\quad \text { vencel, Lanson, Macaire, Méliador, Ogier, Rou, Simon, Turpin }\end{array}$ \\
\hline oliphant & 1 :Fierabras, Jehan de P., Jehan de S., Narbonnais, Ogier, Papegau \\
\hline ouaille & $\begin{array}{l}3: \text { Rou } \\
1: \text { Brut, Cleomadés, Florimont, Laurin }\end{array}$ \\
\hline ours & $1:$ Bérinus, Mort Aymeri \\
\hline porc & $\begin{array}{l}4: \text { Rou } \\
3: \text { Roland } \\
2 \text { : Bérinus, Eracle, Godin, Perceval, Tristan de N., Wistasse } \\
1: \text { Aiol, Ami, Athis, Bâtard, Cent, Chevalerie d'O., Claris, Deduis, } \\
\quad \text { Dole, Jehan, Lanson, Lion, Parise, Raguidel, Silence, Turpin }\end{array}$ \\
\hline pourceau & $\begin{array}{l}5: \text { Lion } \\
3: \text { Deduis } \\
2 \text { : Audigier, Bâtard, Berte, Cent, Narbonnais, Ogier } \\
1 \text { : Chevalerie d'O., Couronnement, Espees, Godin, Jehan de S., } \\
\quad \text { Otinel, Saisnes }\end{array}$ \\
\hline pradant & $1:$ Roussillon \\
\hline seu & 2:Rou \\
\hline singe & 1 : Deduis, Mort Aymeri \\
\hline
\end{tabular}




\begin{tabular}{|c|c|}
\hline tor/torel & $\begin{array}{l}11 \text { : Queste } \\
6 \text { : Mahomet } \\
3 \text { : Gaufrey, Laurin } \\
1 \text { : Aliscans, Brun, Eneas, Godin, Gui de W., Lycorne, Raoul, Rou, } \\
\quad \text { Roussillon, Saisnes, Troie, Turpin, Violette, Yvain }\end{array}$ \\
\hline truie & $\begin{array}{l}2 \text { : Audigier } \\
1 \text { : Aye, Barbastre, Bâtard, Cleomadés, Deduis, Floovant, Lion }\end{array}$ \\
\hline vache & $\begin{aligned} 20 & \text { : Lion } \\
4 & \text { : Godin } \\
3 & \text { : Roussillon } \\
2 & \text { : Aiol, Aucassin, Bâtard, Bueve I, Cent, Charroi, Deduis, Gaydon, } \\
& \text { Lancelot, Mez, Narbonnais, Roche, Rou } \\
1 & \text { : Athis, Audigier, Brut, Charrete, Claris, Escoufle, Floire, Fouke, } \\
& \text { Gaufrey, Guillaume d'A., Jehan de S., Jouvencel, Laurin, Ogier, } \\
& \text { Orson, Papegau, Raguidel, Raoul, Rigomer, Saisnes, Thèbes }\end{aligned}$ \\
\hline veau & $\begin{array}{l}10: \text { Cent } \\
2: \text { Mahomet } \\
1: \text { Rou }\end{array}$ \\
\hline veautre & $\begin{aligned} 7 & \text { : Florimont } \\
5 & \text { : Barbastre, Enfances G. } \\
4 & \text { : Huon, Roland } \\
3 & \text { : Lancelot } \\
2 & : \text { Chevalerie d'O., Ipomedon, Roussillon } \\
1 & : \text { Aymeri, Couronnement, Eneas, Escoufle, Floriant, Gui de W., } \\
& \text { Guillaume, Loquifer, Orange, Partonopeu }\end{aligned}$ \\
\hline
\end{tabular}

\subsection{Table IV}

Cette table indique le nombre d'occurrences des différents termes désignant les oiseaux, dans un choix de nos textes.

\begin{tabular}{|c|c|}
\hline alerion & 3:Deduis \\
\hline autour & $\begin{array}{l}13 \text { : Deduis } \\
6 \text { : Partonopeu } \\
4 \text { : Athis } \\
3 \text { : Aiol, Auberon, Aye, Erec, Gaydon, Godin, Huon, Ipomedon, } \\
\quad \text { Mez, Roland, Rou, Silence } \\
2 \text { : Aymeri, Cligés, Durmart, Escoufle, Galeran, Gui de W., Lais, } \\
\quad \text { Lancelot, Mort Aymeri, Orange, Roussillon, Thèbes } \\
1 \text { : Bueve I, Claris, Eracle, Floire, Floovant, Florimont, Gui de B., } \\
\quad \text { Guillaume, Guillaume d'A., Loquifer, Ogier, Orson, Poitiers, } \\
\quad \text { Roche, Yvain }\end{array}$ \\
\hline
\end{tabular}




\begin{tabular}{|c|c|}
\hline biset & $1:$ Eneas \\
\hline calandre & $2:$ Mort Aymeri \\
\hline canard/cane & $1:$ Athis, Enfances G. \\
\hline chapon & $\begin{array}{l}11 \text { : Lion } \\
5 \text { : Deduis } \\
4 \text { : Laurin } \\
3 \text { : Yder } \\
2 \text { : Bueve I, Claris, Cent, Jehan, Tristan de N. } \\
1 \text { : Anjou, Audigier, Aymeri, Blancandin, Dole, Florence, Galeran, } \\
\quad \text { Gautier d'Aupais, Gaydon, Gliglois, Jehan de S., Roche, Rome, } \\
\quad \text { Rou,Yvain }\end{array}$ \\
\hline colombe & $\begin{array}{l}7: \text { Florence, Lancelot } \\
5: \text { Eneas } \\
3: \text { Athis } \\
2: \text { Lycorne } \\
1: \text { Aiol, Brut, Cent, Cligés, Jehan, Mort Aymeri, Ombre, Yvain }\end{array}$ \\
\hline $\operatorname{coq}$ & $\begin{array}{l}12 \text { : Lion } \\
3 \text { : Huon, Tristan de N. } \\
2 \text { : Bérinus } \\
1 \text { : Auberon, Bueve I, Buevon, Chevalerie d'O., Escoufle, Guillau- } \\
\quad \text { me, Lais, Lanson, Mez, Roche, Rou }\end{array}$ \\
\hline émerillon & $\begin{aligned} 6 & \text { : Deduis } \\
3 & \text { : Laurin } \\
1 & \text { : Aymeri, Chauvency, Cristal, Enfances G., Erec, Floire, Gaydon, } \\
& \text { Mez, Orson, Poitiers, Simon, Tristan }\end{aligned}$ \\
\hline épervier & $\begin{array}{l}61 \text { : Deduis } \\
43 \text { : Durmart } \\
26 \text { : Erec } \\
17 \text { : Protheselaus, Violette } \\
15 \text { : Atre } \\
12 \text { : Méliador } \\
10 \text { : Laurin, Saisnes } \\
9 \text { : Cristal, Enfances G., Lancelot } \\
8 \text { : Ogier } \\
7 \text { : Lion, Rigomer } \\
6 \text { : Huon } \\
5: \text { Meraugis } \\
4: \text { Auberon, Jehan de S., Partonopeu, Raguidel } \\
3 \text { : Aymeri, Bueve I, Claris, Cligés, Fierabras, Galeran, Gaydon, } \\
\quad \text { Loquifer, Mez, Mort Aymeri } \\
2: \text { Athis, Enfances O., Floovant, Florimont, Gui de B., Ipomedon, } \\
\quad \text { Perceval, Raoul, Thèbes, Tristan de N. } \\
1 \text { : Aiol, Ami, Aye, Berte, Brun, Buevon, Chevalerie d'O., Cleoma- } \\
\quad \text { dés, Couronnement, Diable, Florence, Guillaume, Helcanus, } \\
\quad \text { Lais, Lanson, Lycorne, Macaire, Narbonnais, Orange, Otinel, } \\
\quad \text { Roland, Rome, Rou, Yder }\end{array}$ \\
\hline
\end{tabular}




\begin{tabular}{|c|c|}
\hline étourneau & 5 : Deduis \\
\hline faucon & $\begin{aligned} 218 & \text { : Deduis } \\
29 & \text { : Escoufle } \\
12 & \text { : Laurin, Méliador, Mez } \\
10 & \text { : Lion } \\
9 & \text { : Aye } \\
8 & \text { : Mort Aymeri } \\
6 & \text { : Cleomadés, Loquifer, Papegau, Roussillon, Tristan de N. } \\
5 & \text { : Durmart, Gliglois, Godin } \\
4 & \text { : Athis, Blancandin, Chevalerie d'O., Gaufrey, Gui de W., Parto- } \\
& \text { nopeu, Rou } \\
3 & \text { : Bueve I, Fierabras, Florence, Gui de B., Narbonnais, Orange, } \\
& \text { Roche, Rome, Saisnes } \\
2 & : \text { Aymeri, Bâtard, Chauvency, Enfances O., Erec, Galeran, Gay- } \\
& \text { don, Jehan de S., Jourdain, Lancelot, Lycorne, Ogier, Silence, } \\
& \text { Thèbes, Yvain } \\
1 & \text { : Amadas, Ami, Anjou, Auberon, Brun, Buevon, Cligés, Cristal, } \\
& \text { nel, Parise, Perceval, Poitiers, Raoul, Rigomer, Roland }\end{aligned}$ \\
\hline gal & $1:$ Roussillon \\
\hline geai & $1:$ Mort Aymeri \\
\hline geline/poule & $\begin{array}{l}11 \text { : Deduis } \\
10 \text { : Cleomadés } \\
6 \text { : Tristan de N. } \\
2 \text { : Escoufle, Espees } \\
1 \text { : Anjou, Athis, Audigier, Aymeri, Cent, Claris, Cristal, Diable, } \\
\quad \text { Doon, Fierabras, Florence, Gliglois, Rigomer, Rou, Turpin }\end{array}$ \\
\hline gerfaut & $\begin{array}{l}18 \text { : Lycorne } \\
8 \text { : Gui de W. } \\
7 \text { : Deduis } \\
2 \text { : Buevon, Partonopeu } \\
1 \text { : Anjou, Durmart, Fouke, Galeran, Godin, Guillaume d'A., Nar- } \\
\quad \text { bonnais, Poitiers, Yvain }\end{array}$ \\
\hline hobereau & $6:$ Deduis \\
\hline lanier/ & $\begin{array}{l}25: \text { Deduis } \\
1: \text { Anjou }\end{array}$ \\
\hline mauviette & $1:$ Mort Aymeri \\
\hline $\begin{array}{l}\text { merle/ } \\
\text { merlette }\end{array}$ & $2:$ Mort Aymeri \\
\hline milion & 4 : Deduis \\
\hline $\begin{array}{l}\text { mouchet/ } \\
\text { mousket }\end{array}$ & $\begin{array}{l}3: \text { Deduis } \\
1: \text { Floire }\end{array}$ \\
\hline oie/oison & $\begin{array}{l}1 \text { : Barisel, Cent, Chauvency, Deduis, Dole, Galeran, Gaufrey, Tur- } \\
\quad \text { pin }\end{array}$ \\
\hline
\end{tabular}




\begin{tabular}{|c|c|}
\hline paon & $\begin{array}{l}11 \text { : Lion } \\
5 \text { : Mez } \\
3 \text { : Bueve I, Dole, Gui de B., Guillaume, Lancelot, Ogier, Tristan } \\
\quad \text { de N. } \\
2 \text { : Ami, Anjou, Enfances G., Florimont, Gaydon, Gormont, Jehan } \\
\quad \text { de S., Lanson, Orange, Roussillon } \\
1 \text { : Charroi, Chevalerie d'O., Cristal, Durmart, Espees, Fierabras, } \\
\quad \text { Floire, Florence, Fouke, Gui de W., Ipomedon, Narbonnais, Or- } \\
\quad \text { son, Parise, Raguidel, Rigomer, Roche, Rome, Saisnes, Simon, } \\
\quad \text { Thèbes, Turpin }\end{array}$ \\
\hline pasqueret & $1:$ Deduis \\
\hline perroquet & $\begin{array}{l}313 \text { : Papegau } \\
1: \text { Perceval }\end{array}$ \\
\hline pigeon & $\begin{array}{l}2: \text { Jehan de S. } \\
1: \text { Espees }\end{array}$ \\
\hline poulaille & $1:$ Jehan de P., Jehan de $S$. \\
\hline $\begin{array}{l}\text { poulet/ } \\
\text { pouletel }\end{array}$ & $\begin{array}{l}3: \text { Godin } \\
2: \text { Jehan } \\
1: \text { Dole, Escoufle }\end{array}$ \\
\hline poussin & $\begin{array}{l}7 \text { : Cleomadés } \\
5 \text { : Cent } \\
4 \text { : Doon, Godin } \\
3 \text { : Roche } \\
2 \text { : Claris, Deduis, Lanson } \\
1 \text { : Bâtard, Berte, Durmart, Enfances O., Floire, Florence, Guillau- } \\
\quad \text { me, Lion, Perceval, Roussillon, Tristan de N., Violette }\end{array}$ \\
\hline ramaget & $2:$ Deduis \\
\hline rossignol & $1:$ Doon \\
\hline sacre & 3:Deduis \\
\hline taharote & $6:$ Deduis \\
\hline $\begin{array}{l}\text { tercel/ } \\
\text { tercelet }\end{array}$ & $\begin{array}{l}7: \text { Deduis } \\
3: \text { Erec } \\
1: \text { Anjou, Escoufle }\end{array}$ \\
\hline tunisien & $1:$ Deduis \\
\hline turquet & $3:$ Deduis \\
\hline
\end{tabular}




\section{Index}

L'index contient les dénominations des différents animaux domestiques qui se trouvent dans nos extraits de textes cités. S'il y a plusieurs occurrences d'un terme à la même page nous n'en notons qu'une.

Pour faciliter l'utilisation de l'index, nous le divisons en trois parties. La première contient les termes désignant les chevaux, la deuxième ceux désignant les autres mammifères et la troisième ceux désignant les oiseaux.

Nous tenons à avertir le lecteur que certains termes, tels que arabi, noir, sor, vair, qui se trouvent placés dans la partie 8.1., servent aussi à caractériser des représentants des deux autres parties.

\subsection{Chevaux}

ambleor, 80

ambleeur, 80

anbleors, 178, 200

amoravi, 28, 82

l'Amoravy, 28

amoravit, 140 .

arabi, $80-82,85,103,121,124,162,178$

Arabi, 82

Arasbi, 82

arrabi, 39, 80-82, 100, 133, 141, 177, 287

arrabioiz, 82

arrabion, 82

arrabis, 69,82

arrabit, 81

arrabiz, 81, 99

arabis, 103

arabit, 81,82

arabiz, 123

aragon, 80, 82-84, 107, 174

Aragon, 83, 84

aragons, 23, 82

Arragon, 38, 75, 84, 176 
arragon, 24, 28, 35, 38, 83-85, 120, 123, 136, 177, 228, 279

arragons, 84

arami, 85

aufage, $85,96,138$

alfage, 129

alfainne, 85

aufainne, 85

aufaje, 85

aufajois, 85

aufeigne, 85

auferrant, 17, 70, 85-88, 101-104, 108, 121, 145, 244-246, 250, 304

aferrant, 69, 244

alferrant, 244

aufarin, 176, 178

auferans, 60,88

auferant, 17, 86-88, 91, 130, 140, 244

auferran, 88

auferrans, 32, 88, 103, 104, 108, 279

auferranz, 88

aufferant, 279

aufferrant, 86, 87

ausferrant, 86

hausferans, 86

augalie, 28

bai, 21, 39, 62, 89, 90, 96, 97, 113, 118, 122, 129, 139-141, 334

baiart, 90

bais, 27, 90, 96, 97, 120, 123, 139

bay, 119

bayart, 90

barbari, 90, 92

barzelon, 91, 123

bauçant, 17, 39, 91-94, 116, 121-123, 139, 140, 178, 232

balçan, 91

balçane, 91

balcent, 92

balchant, 93

basain, 117

bauçain, 27

baucan, 92, 93, 139

bauçan, 92

bauçans, 90, 93, 139

baucant, 93

bauceins, 97

baucens, 27, 114, 139, 140

baucent, 27, 39, 89-94, 118, 178

baucenz, 92, 109

bauchant, 59, 91, 93, 116, 139, 301 
bauchent, 94

bausans, 116

bausant, 127

bausent, 27

bauzan, 92,140

beaucent, 93

blanc, $7,20,22,27,31,35,41,47,48,52,58,81,89,91,94,95,104,116,120-$ $122,125,126,133,142,145,150,161,168,178,184,191,209,210,216$, $239,250,263,282,295,321,326$

blanchart, 94, 95, 232

blanche, 21, 22, 121, 171, 173, 178, 344, 357

blanchet, 94, 95

blans, 19, 22, 27, 45, 49, 50, 79, 90, 93, 95, 122, 135, 215, 220

blanz, 35, 123

breaigne, 96

brehaigne, 45, 85, 96, 146, 192

brehangne, 96

brun, 85, 89-92, 95-97, 113, 116, 120, 129, 200

brune, 112, 133, 178

bruns, 22, 92, 96, 97, 139, 140

chaceor, 69, 97-99, 107, 151, 158, 251

caceor, 61, 97, 99

caceour, 98

caceours, 303

cacheor, 97-99

cacheour, 61, 98

chaceors, 155

chaceour, 98

chaceours, 98

chaceür, 303

chasçurs, 98

cheval, 16, 19, 20, 22, 23, 25-34, 36-42, 44-46, 51-54, 56-59, 61, 62, 66-71, $73,74,77,80,82-97,99,101-110,112-118,120-145,148,154,155,160$, $171,179,181,182,185,199,203,205,208,226-234,236,238,241-254$, 258-261, 265, 269, 274, 276, 277, 280-283, 286, 288-290, 292-294, 296$302,304,311,317,324,325,330,333,338,341,342,347,349-355,358$, $360,364,367$ ceval, 16, 19, 20, 24, 26, 33, 51, 53, 56, 62, 67, 73, 80, 108, 111, 115, 127, $131,132,134,136,138,140-142,153,184,228,230,232,233,241,242$, $245,271,274,324$

cevals, $172,174,205,210$

cevaus, 19, 24, 26, 31, 53, 62, 75, 95, 103, 135, 143, 144, 241, 245, 265 , 287,340

cevax, 24, 29, 107, 232, 249

chavals, 129

chavaus, 105

cheual, 242 
cheuaus, 75, 259, 291

chevalet, 33, 98, 355

chevallet, 33

chevals, 24, 25, 29, 30, 54, 67, 68, 93, 105, 108, 233, 243, 244, 247, 248, $252,255,259,280,285,295,301,342$

chevalx, 75, 88, 260, 325

chevalz, 25, 140, 144, 295, 358, 362

chevas, 279

chevaul, 227

chevauls, 27

chevaulx, 11, 90, 103, 123, 231, 240, 254, 260, 271, 274, 279, 295, 296, $301,317,323$

chevaus, $8,10,20,21,24,26,30-32,50,59,67,75,82,93,99,101,103$, $105,106,109,123,132,138,142,145,162,166,182-184,227,229,233$, 240-246, 248-250, 252, 253, 255, 258, 259, 279-281, 284, 285, 291, 296, 299, 301, 324, 325, 338, 341, 352, 357-359

chevaux, 23, 25-27, 31-33, 39, 46-48, 50, 51, 53, 64-68, 71, 73, 75, 80-82, $84,86,88,94,97,100,101,104,107-109,118,120,122,126,129,136$, 139, 142, 145-147, 150, 171, 176, 177, 181, 200, 203, 228, 229, 231-234, 236, 240, 243-245, 247-249, 251, 254, 255, 258-260, 271, 272, 274, 276, $278,279,281,282,284,287,290,292,294-298,300,303,317,323-325$,

$331,333,351,352$

chevauz, 71, 241, 248

chevax, 20, 27, 29, 30, 32, 51, 78, 88, 94, 99, 108, 123, 127, 128, 134, 139, 140, 143, 145, 173, 203, 242, 243, 246, 247, 249, 252, 253, 258, 259, 290, $300,323,326,358$

chival, 110, 115, 129, 233

chivaus, 26, 294

chivauz, 294

çival, 31

kevaus, 311

courant, 16, 99, 107, 114, 119, 120, 129, 132, 136, 187, 244, 263, 278, 288

corans, 28, 59, 164, 258, 299

corant, 23, 29, 37, 39, 62, 81, 89, 95, 100, 118, 122, 132, 135, 140, 144,

$145,153,168,178,184,234,250,275,351,352$

coranz, 31, 67, 99, 174, 184

corrant, 26, 38, 100, 106, 129, 131, 240, 274, 285

courans, 5, 94, 99, 145, 151, 157

curant, 30, 135, 300

coureor, 16, 100, 101

corador, 101

coraor, 101, 119

coraors, 101

coreor, 101

coreors, 200

coureour, 101 
courëour, 101

courreurs, 184

coursier, 30, 40, 53, 59, 60, 80, 81, 87, 101-103, 105, 112, 114, 141, 144, 145 ,

235

corcier, 102, 105

corser, 103, 144

corsers, 105,183

corsier, $17,85,87,88,101-103,177$

corsiere, 42,172

corsiers, 6o, 88, 103

courcier, 102

courciers, 235

coursiere, 178

courtaut, 103

crenu, 91, 92, 104

crenus, 52

crenut, 93

crenuz, 104

grenu, 91

grenus, 74, 104

kernu, 113

quernu, 86

quernus, 88

destrier, 7, 14, 22, 26-39, 45, 46, 51, 53, 54, 59, 61, 63, 67-70, 72, 74, 75, 77, $82,84-88,90,92-95,97,99-105,107-132,135-137,139-146,150,161$, $162,167,175,177,194,197,201,205,227-229,231,233,234,240,244$, 246, 248-251, 259, 260, 265, 269, 274, 275, 277-280, 282, 283, 285, 290, $296,317,324,325,351-353$

desters, 98

destrer, 30, 31, 35, 77, 82, 91, 106, 125, 127, 141, 145, 210, 282, 283, 290

destrers, 19, 25, 34, 35, 116, 201, 240, 300

destrés, 34

destrié, 88,144

destriers, 13, 17, 19, 23-25, 27-30, 34, 35, 37-39, 46, 51, 52, 55, 67, 69-73, 75, 77, 78, 82, 84, 88, 90, 92, 97, 99, 101, 104, 108, 109, 111, 114, 117, 119, 120, 137, 140, 141, 143, 173-176, 200, 209, 226, 239, 242, 247, 249, 250, $254,274,278,279,281,283-288,311,325,343,351$

doine, 39, 40, 118

esclavon, 104

esclavonne, 104, 177

espagnol, 40, 91, 104, 182

epagnol, 105

espainol, 104, 105

espanes, 105

espanois, 105, 177, 182

espenois, 105 
estalon, 39

fauve, $27,38,92,105,120,123,145,173,174,178,182,183,288$

faulve, 174

fauvel, 105, 113, 383

fauvelet, 102, 105

fauves, 105, 302

ferrant, 27, 35, 47, 57, 61, 62, 64, 98, 105-107, 113, 115, 125, 132, 145, 178

ferant, 105, 107, 340

feranz, 106

ferrande, 178

ferrans, 27, 106, 107, 347

ferranz, 123

gascon, 30, 35, 48, 84, 87, 88, 107-109, 116, 138, 140, 201, 304, 351, 353

gascoin, 108

gascons, 32, 88, 105, 108, 109

gascont, 108

gazele, 39,40

genet, 40

genez, 40

gramadone, 40

gris, $27,67,74,105,106,109,114,117,119,120,124,126,140,194,250,286$,

287

grile, 142

griolé, 109

grise, 85,178

grisel, 109

grises, 109

grisons, 109

hacquenée, 40, 41

acquenees, 40

hacquenee, 40

hagenée, 40

haquenée, 40, 384

iocor, 41

ive, $41,42,45,96$

ieve, 42

yve, 41,42

jument, 39, 41-46, 56, 59, 85, 96, 123, 146, 171, 179, 183, 299, 318, 355

jumant, 44

jumens, 43, 45, 255

jumentes, 10

jumentiele, 43

jumenz, 43

liart, 109, 110, 115, 116, 126

liard, 110

lïard, 110

liars, 110 
lïart, 107, 114, 115, 139

lyard, 110

missaudor, 110, 111

milsoldor, 280

misadour, 110

misaldor, 111

misaudor, 110

misodor, 111

misodors, 111

misordor, 110

misoudor, 111, 325

missaudour, 111

missodor, 111

missoudor, 111

missoudour, 111

mor, 97, 112

maurel, 113

moreau, 112

moreis, 112

morel, 96, 112

mores, 113

moresc, 113

moriel, 112

morois, 112, 123

mouvant, 113

movant, 62, 113, 117, 342

muvant, 114

noir, 21, 27, 32, 33, 45, 47, 59, 91, 92, 94, 106, 109, 112-114, 120, 123, 126, 127 ,

$135,242,246,250,310$

neir, 113, 114, 170

ner, 92, 113, 139

noire, 21, 22, 112, 178

noirs, 22, 27, 48, 97, 114, 120, 126, 135, 191, 352

palefroi, 11, 16, 21, 28-34, 36, 37, 46-56, 62, 68-70, 72-74, 90, 91, 94, 95, $108-110,113,114,142-145,173,175,207,247,250,265,285-88,291$, $292,302,331,351$

palafroi, 31

palefrai, 290

palefreid, 77

palefreis, 69

palefreiz, 75, 286

palefrez, 51

palefrois, 29, 31, 35, 37, 38, 46-55, 70, 71, 73-75, 77, 78, 88, 94, 102, 106, $143,145,172,176,177,247,252,278,284,286-288$

palefroit, 31

palefroiz, 22, 27, 31, 35, 93, 143, 176, 184, 242, 250, 279, 284, 286, 288.

palefroy, 54, 95 
pallefrein, 47

pallefroi, 53, 248

pallefrois, 32, 101

pallefroiz, 53, 71, 105

pallefroy, 16, 70, 72, 140, 225

parlefroi, 47, 93

polain, 55, 56

polains, 29, 45, 56, 355

polein, 55

poulain, 41, 42, 45, 55-57, 68, 354, 355

poulains, 55, 56, 354

poulein, 56

poullains, 357

poutrel, 57

podrel, 57

poitrel, 57

potrel, 57

poutrels, 57

poutriel, 57

roncin, 43, 46, 51, 54, 56, 58-74, 85, 99, 125, 153, 167, 168, 274, 293, 299, 325,

$331,353,355,364$

rocin, 64,107

rocins, 71

romcin, 65

ronchi, 59, 61, 62, 64, 66, 68, 251, 331

ronchie, 68

ronchin, 16, 33, 59-63, 67-70, 99, 243, 292, 299, 346, 353

ronchis, $42,60,65,71,347$

ronci, 29, 59, 61, 62, 65-67, 71, 89, 99, 144, 252

roncié, 59

roncine, 72

roncinet, $58,72,311$

roncins, 54, 58, 60-65, 67-71, 73, 74, 87, 240, 250, 299, 346

roncis, $55,62,68,70,99,280,284$

ronsin, $16,67,68,70,72,102$

rouchin, 59

roussin, 72

roussins, 46, 72

runcin, 59, 67, 331

ros, $107,109,114-116$

rous, $107,114,115,139,192,346$

roussel, 142

rouz, 114

rox, 114, 115

rus, 115, 116

sambuer, 73 
sommier, 50, 51, 73-75, 77, 79, 149

solmier, $75,78,79$

some, $181,225,351$

somer, 76,77

somers, 78,79

somier, 37, 64, 67, 70, 74, 76-78, 80, 87, 164, 291, 319

somiers, 13, 38, 55, 69, 73-79, 175, 254, 279

somierz, 76, 175

sommiers, 50, 54, 58, 73-77, 79, 174

sonmiers, 73, 78, 295

soumier, 73, 292

soumiers, 64

sumer, 77

sumers, 75, 266

sor, $8,10,11,13,22,27-29,34-39,41-44,46,47,50,51,53-55,57,60-65$, $68,73,74,77,81-86,89-103,105-119,121,123-127,129-132,134,135$, $137-145,151,157,164,168,170,172-174,176-178,181,184,195,200$, $201,204,223,228,230,232,233,237,250,262,265,267,273,278-281$, $284,285,288-291,293,295,302,306,307,317,331,332,351,352,368$ sors, 27, 90, 93, 109, 114, 116, 117, 139, 201, 312 sort, 27,266

vair, 22, 23, 27, 30, 39, 74, 90, 92, 99, 104, 108, 109, 112, 117-119, 128, 129, $200,225,229,287,343,344,353$

vairon, $28,117,118,233$

vairs, 27, 116, 120, 203, 208, 211, 312, 344

var, 92, 114

veiron, 349

vers, 344

\subsection{Autres mammifères}

agneau, 191, 193, 348

agnelet, 367

agniax, 328

aigneaulx, 278

aigneaux, 196, 278, 328

aigneax, 345

aignel, 191, 262, 272, 280, 340, 348, 354

aignelet, 348

aigniaus, 191, 272, 318, 328, 335, 340, 354

aigniax, 191, 317

aingnelet, 335

ainiax, 280 
alan, 151

alans, 151

âne, 171, 179-182, 275, 277, 294, 300, 340, 341, 350.

ainesse, 275

aisne, 181

ane, 181, 207, 210, 214, 219, 225, 269, 305, 349

anes, 180, 300, 341

anesse, $123,180,182,276$

anne, 202, 360

anon, 181

asne, 45, 179-182, 254, 275, 289, 294, 297, 300, 301, 340, 341, 346

asnel, 45, 214

asnes, 44, 180, 182, 255, 275, 294, 341, 358

asnesse, 180, 275

asnon, 181, 182, 276

aumaille, 186

almaille, 186

berseret, 151, 238

berserés, 151, 164

berserez, 151, 225

bœuf, 46, 186, 188, 232, 239, 256, 277, 298, 318, 330, 331, 352, 369, 372

beuf, 195, 319, 325, 326, 338, 369

beuff, 280, 299

beufz, 11, 222, 323

beus, 259

boef, 187, 190, 319, 369

boés, 324

buef, 186, 187, 213, 235, 236, 255, 256, 280, 281, 298, 316, 319, 321, 323,

$329-331,346,347,350,368$

buefs, 274, 278, 361

buefz, 64, 300

bues, $163,187,236,261,270,273,280,298,300,311,314,318,323$,

352

buès, 262, 281

bués, 10, 187, 189, 190, 235, 280, 281, 295, 298, 299, 311, 319, 323, 324,

330, 361

buez, 186, 187

bouc, 192

bous, 192, 346

brahon, 152, 159

braon, 152, 159, 267

braons, 152, 159

brohuns, 159

braque, 152, 153, 163, 238, 307

bracés, 152

bracet, 153

brachés, 153, 161, 164, 201, 303 
brachet, 152, 153, 169, 170, 238, 263, 264, 285, 307, 308

brachez, 13, 153, 167, 239, 275, 342

braichot, 264

brakes, 304

brakès, 154

brakés, 151, 155, 164, 274, 286, 335

braqués, 152, 169, 237

braquet, 152, 157, 168, 169, 237

brechetz, 169

brochas, 153 .

brebis, 10, 11, 147, 187, 190, 192-194, 196, 256, 268, 272, 275, 277, 278, 280, $281,312,314,323,324,333,334,340,345,348,353$

berbis, 9, 163, 192, 197, 273, 276, 317, 354

berbiz, 186, 187, 189, 194, 262, 272, 274, 306, 314, 322, 329, 335

brebiz, 192, 194, 261, 271, 272, 280, 314, 340

bugle, 188

bugles, 184, 188

caigne, 154

chameau, 341

cameilz, 202, 294

camelz, 183, 284

camex, 341

chamelin, 184

chamelx, 184

chames, 183, 184

chameus, 184, 188, 294

chamois, 184

chat, $72,160,162,170,171,277,309,329,346,350,358,360,365$

cat, 309, 358

chas, 171, 324, 334

chaz, 170, 171, 309

chèvre, $193,277,348,358$

chevre, 193

chievre, 44, 193, 280, 318, 322, 349, 358

chievres, 193, 365

chièvres, 189, 193

chien, 97, 103, 137, 148-157, 162-169, 229, 236, 238, 239, 255, 263-265, 274, $275,277,280,303,306-308,330,333-340,345,350,356,357,359,360$, $363,364,369$

chael, 156, 157, 280

chaiaus, 156, 157

chen, 132, 266, 307, 367

chenés, 156

chens, 169, 170, 183, 284

chienès, 7,156

chienet, 155, 156, 389

chiennés, 156 
chiens, 5, 8, 13, 61, 147-157, 159, 160, 163-169, 189, 199, 210, 236-239, 255-257, 263-266, 272-274, 278, 281, 284, 285, 288, 302-304, 306-310,

$334-339,345,348-350,356-358,360,363,367$

chienz, 155, 265

chyn, 266

cien, 239, 265, 304, 346, 360

ciens, 61, 150, 154, 202, 263, 287, 336-338, 359

cins, 64, 309

kiens, 151, 155

cochon, 196, 198

cochons, 116, 196, 312

connin, 199

conin, 199, 355

conins, 5, 199, 315

connins, 196, 199

dromadaire, 31, 32, 76, 183-185, 289

dramadaires, 294

dromadaires, 31, 32, 76, 183-185, 294

dromedaire, 25

éléphant

holifans, 186

olifans, 186

olifant, 185, 332

olifanz, 185, 346

olifenz, 185

ollifant, 185

épagneul

espaignaux, 157

frebau, 157

frebaus, 157

fuiron

fuirons, 200

gaignon, 137, 150, 157-160, 166, 168, 265, 339, 346

gaignars, 160

gaignons, 157-160, 163, 313, 356

gaingnon, 158, 169

guaïgnon, 357

guainuns, 159

wagnart, 160

waignon, 255, 264, 338

génisse, 188

genice, 188, 280, 323

jenice, 190

lévrier, 149, 152, 157, 160, 161, 168, 239, 302, 307, 309, 348, 352, 364

leverier, 264

levrer, 238, 239, 307, 308

levrerete, 162 
levrers, 161, 201, 282, 303, 307, 308

levrier, 7, 149, 150, 159, 161-163, 166, 167, 209, 264, 285, 309, 317, 342,

$345,352,364$

levriere, 162, 163, 169, 225, 336, 342, 352

levriers, 13, 151, 152, 154, 155, 157, 159, 160, 161, 163, 164, 168, 263 ,

$273,286,289,304,349,352,355$

levriés, 160

lievrier, 335

livrier, 155, 161, 164, 342

livriers, 168

limier, 163, 164, 168, 277

liemier, 164

liemiers, 155, 164, 168

liëmiers, 164

lïemiers, 164, 167, 307

limers, 303

limiers, 159, 355

loiemier, 155, 164

loiemiers, 151, 157, 159, 164, 304

loienmiers, 164, 303

lymiers, 164

lisse, $164,165,275,360$

lices, 165

mâtin, 150, 157, 165, 166, 255, 356, 369

mastin, $125,148,161,165,166-169,255,265,336,337,339,345,350$,

$356,359,363$

mastineaux, 166

mastins, 158, 165-167, 264, 339, 356

mastyn, 193, 363

matin, 72, 125, 166, 168

matins, 151, 166

mouton, 71, 193-195, 267, 321, 322, 324, 327, 333, 335, 347, 350, 365, 367

molton, 235, 321

monton, 321

montons, 222

moston, 194

mostons, 321

moton, 193, 321, 366

motons, 280

motun, 194

mouston, 194

moutons, 11, 186, 191, 193-196, 235, 257, 261, 262, 271, 279-281, 314,

$317,321,323,324,328-330,335$

moutoun, 321

muton, 194

mutuns, 329 
mul, 46, 53, 69, 73-75, 77, 171, 172, 174-178, 233, 243, 250, 252, 253, 275, $286,288,295$

mule, 49, 51, 69, 77, 104, 171-179, 289

mules, 58, 171, 174-177, 179, 279

mulès, 176

mulés, 35, 76

mulet, 29, 35, 69, 70, 75, 85, 92, 102, 103, 107, 115, 171, 172, 175-179,

$281,288,324,368$

mulette, 173, 178, 179, 391

mulez, $74,76,85,174,175,184$

mulle, 173,178

mullers, 183

muloz, 288

muls, 74, 78, 172, 175-178, 183, 209, 250, 253, 275, 279, 284, 295

mulz, 64, 88, 176, 177

mur, $76,177,258$

mure, 173, 289

murl, 172, 177

murles, 75,174

murlet, 75, 175, 177, 287

murs, 23, 76, 175, 177, 178, 184, 200, 286, 295, 338

ouaille, 195

oailles, 195

oeille, 195

oeilles, 195

oëlle, 195

oueilles, 195

ours, $44,150,152,159,165,167,199,200$

ors, $6,72,152,159,168,178,182,188,200,275,288,300,329,343,364$

porc, $45,153,195,196,263,266,267,275,277,280,308,310,318-321,323$,

$324,326,345,366,367$

pors, $46,196,197,280,281,311,319,323,324,337,351,364$

pourceau, 196-198, 271, 341, 367

porcel, 197, 350, 366

porcelet, 196, 197, 256

porcelez, 197

porcelz, 367

porchiaus, 358

porchiel, 197

porcials, 197

porcialz, 197, 366

porciau, 366

porciaulz, 367

porciaus, 147, 193, 197, 318, 328, 341

porciax, 199, 315, 319

pourceaux, 198, 278, 341

pourcel, 198, 280, 366 
pourcelès, 256

pourchiaus, 281, 366

pourcialz, 198

pradant, 167

pradenc, 167

seu, 128, 167

seüs, 167

seüz, 167

singe, 346, 349

sinjes, 200

taureau

tor, $184,188,189,352,365$

torel, 188, 280, 299

toriaus, 188, 261, 330, 343

toriax, 343

tors, $188,230,281$

truie, 198, 320, 349, 358, 359

true, 198

truies, 198, 367

vache, $45,186,188-190,213,235,256,277,280,281,313,318,319,321,330$,

353, 355

vace, 190, 331

vaces, 10,190

vaches, 11, 186, 187, 189, 190-192, 196, 256, 261, 262, 270, 271, 278, 280,

$281,311,312,323,324$

vaiche, 190

vaiches, $64,163,273,280$

vaichez, 369

vakes, 281, 332

vakielle, 190

vaques, 281

vasche, 353

veau, 188, 190, 355

vael, 189

vaelet, 190

vëaus, 147

veel, 188, 190

viaus, 190

veautre, 167,168

vaitres, 164, 168

veautres, 150, 167, 168

veltres, 168, 303

vetre, 155, 164

viautre, $168,275,303,346$

viautres, 13, 121, 150, 155, 168, 169, 304 


\subsection{Oiseaux}

alerion, 201

alerions, 211, 213

autour, 21, 202, 256, 259, 303, 333

hosturs, 183, 202, 284

oistor, 285

oistors, 201

ostoers, 212

ostoir, 202-204

ostoirs, 8, 13, 201-204, 275, 286, 305

ostor, 202, 203, 238

ostors, 52, 186, 203, 286, 344

osturs, 201

otors, 176

oustour, 210

biset, 214

calandre

calandres, 200, 224

canard, 214, 219, 341

ane, 181, 207, 210, 214, 219, 225, 269, 305, 349

anel, 45, 214, 327, 364

anes, 180, 300, 341

chapon, 157, 214, 215, 220, 222, 236, 280, 312, 313, 326, 341

capon, 215, 327

capons, 219, 312, 326

cappons, 214, 325

chaponnez, 347

chapons, 214, 215, 219, 257, 267, 273, 312, 325, 326, 332, 347

chappon, 215, 312

chappons, 221

colombe, 215,343

colenbiaus, 215

colon, 216

colons, 215, 216, 340, 341, 343, 348, 368

colun, 368

coulon, 215

coulons, 216, 343

coq, 216-218, 310, 359

COC, 157, 213, 214, 216-218, 280, 310, 311, 347

cocque, 310

cois, 219, 257, 267

cok, 340

cokés, 218

coks, 310

coquellés, 359

cos, 173, 217, 310, 327 
kos, 217, 274

quois, 216

émerillon, 203, 212, 213, 352

emeril, 203

esmeri, 203, 328

esmerillon, 201, 204, 207, 287, 342, 351

esmerillons, 204, 212, 213, 352

épervier, 204, 212, 224, 277, 283, 288, 305, 307, 334, 347

esparvers, 201

esperver, 122, 306

espervier, 204-206, 237, 275, 308, 350, 351

esperviers, 200

esprevier, 7, 107, 115, 153, 156, 161, 201, 202, 204-206, 210, 212, 235,

$237,238,266,267,282,284,285,288,289,304,334,336,359$

espreviers, 13, 90, 150, 186, 202, 205, 206, 210, 212, 224, 235, 266, 284,

$334,336,342,344,347$

esprivier, 7, 205, 206, 282, 304, 344

espriviers, 168, 201

étourneau

estournel, 224

faucon, $6,7,100,106,150,200-204,206-213,238,257,267,281,282,303-$ $305,334,336,341-344,351-353$

falcon, 203, 208

falcons, 32, 185, 342, 343

falcun, 351

fauconcel, 209

fauconnet, 209, 211

faucons, 5-8, 13, 52, 150, 164, 186, 200-204, 206-209, 211-213, 235, 275,

$286,304,305,334,335,341-344,349,351,353$

faulcon, 344, 368

faus, $183,335,345$

fauz, 208

fax, 352

folcon, 305

gal, 218, 310

geai

jais, 224

geline, 214, 215, 218, 219, 222, 236, 257, 312, 318, 327, 340, 345, 359, 362, $363,368,369$

gelines, 5, 213, 216-219, 222, 256, 257, 267, 273, 327, 347.

gelynes, 321

gheline, 219

ghelinnes, 219

guelinne, 157, 280

jelines, 257

gerfaut, 210, 211, 303

gerfaus, 202, 205, 210 
gierfaut, 210, 336

girefalc, 210, 282

girfaut, 207, 210

hobereau, 210

hobereaux, 210, 212

hobers, 212

hobez, 212

jars, 219

gars, 166, 219

lanier, 211, 213

laneret, 211, 394

laniers, 201, 209, 211, 305, 339

mauviette

mauviz, 224

merle

melle, 224

melles, 200

milion, 201, 211, 213

milions, 211

mouchet, 212

mouchés, 212

mouchez, 212

mouskés, 204

oie, 9, 219, 220, 347

oe, 206, 219, 280, 327

oes, 27, 211, 313, 314, 316

oies, 219, 220, 332, 360

oison, 219, 220, 328, 365

oisons, 5, 147, 222

oue, 347

oues, 219, 220

owe, 321,365

oye, 361

paon, 220, 221, 326, 331, 332, 353

pan, 220

paons, 5, 220, 221, 224, 312, 326, 332

pawons, 214

poon, $6,221,331,353$

poons, $5,6,220,221,328$

pooun, 321

poun, 220, 221

poüns, 332

pasqueret, 212

pasquerés, 212

perroquet, 224, 273

papegau, 225

papegault, 225 
papegaulx, 225, 273

papegaus, 224, 344

pigeon, 214, 221, 341

pigons, 328

pijons, 221

poulaille, 221, 222

poulailles, 221

poule, $218,222,359,368$

poille, 222

poules, 157, 199, 216, 218-220, 224, 256, 266, 273, 312, 316, 347.

poulles, 196, 222, 359

poulet, 222

polez, 328

pouletel, 222

pouletiaus, 222

poulez, 199, 315

poussin, 222, 223, 322, 327, 333, 353

pochin, 16, 353

pocin, 218,327

pocinez, 223

pocinèz, 313

pocins, 223,328

polcin, 328

pouchin, 335, 363

poucin, 217, 223, 257, 327, 328, 341

poucinés, 368

poucinet, 369

poucinez, 222

poucins, $147,214,215,222,223,267,316,327,369$

puissin, 223

ramaget, 212

rossignol

roussignol, 224

roxignous, 275

sacre, 212

sacres, 212

taharote, 212

taharotes, 212

tercel

tercees, 211

tercelés, 213

tercelez, 212

tercellés, 305

tercels, 213

terciaus, 213

terçuel, 204

tiercels, 201 
tunisien, 213

tuniciens, 211

turquet, 201, 213

turqués, 211, 213 


\section{Bibliographie}

\subsection{Textes étudiés}

APF : Les Anciens Poëtes de la France.

BFR : Bibliothèque Française et Romane.

CFMA : Les Classiques Français du Moyen Age.

GRL : Gesellschaft für romanische Literatur.

$\mathrm{RB}$ : Romanische Bibliothek.

SATF : Société des Anciens Textes Français.

TLF : Textes Littéraires Français.

Abbréviation Titre

- $\quad$ Adenet le Roi. Voir : Berte. Buevon. Cleomadés. Enfances

O.

$-$

Aiol

Aimon de Varennes. Voir : Florimont.

Aiol. Chanson de geste. Publiée d'après le manuscrit unique de Paris par Jacques Normand \& Gaston Raynaud. Paris, Librairie de Firmin Didot et $C^{\mathrm{ie}}, 1877$. Réimpression New York, Johnson Reprint Corporation, 1966. (SATF).

- $\quad$ Alexandre du Pont. Voir : Mahomet.

Aliscans Aliscans. Publié par Claude Régnier. 2 vol. Paris, Librairie Honoré Champion, éditeur, 1990. (CFMA 110/111).

Amadas Amadas et Ydoine. Roman du xiII ${ }^{\mathrm{e}}$ siècle. Édité par John. R. Reinhard. Paris, Librairie ancienne Honoré Champion, éditeur, 1926. (CFMA 51).

Ami

Ami et Amile. Chanson de geste. Publiée par Peter F. Dembowski. Paris, Librairie Honoré Champion, éditeur, 1969. (CFMA 97).

Anjou Jehan Maillart : Le Roman du Comte d'Anjou. Édité par Mario Roques. Paris, Librairie Honoré Champion, éditeur, 1964. (CFMA 67).

- $\quad$ Antoine de la Sale. Voir : Jehan de S.

Aspremont La Chanson d'Aspremont. Chanson de geste du xir siècle. Texte du manuscrit de Wollaton Hall édité par Louis Brandin. Deuxième édition revue. 2 vol. Paris, Librairie Honoré Champion, éditeur, 1970. (CFMA 19/25).

Athis Li Romanz d'Athis et Prophilias (L'Estoire d'Athenes). Nach allen bekannten Handschriften zum ersten Male vollständig herausgegeben von Alfon Hilka. 2 vol. Dresden, 1912. (GRL 29/40). 

Brian Woledge. Paris, Librairie ancienne Honoré Champion, éditeur, 1936. (CFMA 76).

Auberon

Audigier

Aye

Aymeri

Barbastre

Barisel

Bâtard

Bérinus

Berte

Blancandin

Le Roman d'Auberon. Prologue de Huon de Bordeaux. Edition critique avec une introduction et des notes par Jean Subrenat. Genève, Librairie Droz, 1973. (TLF 202).

Aucassin et Nicolette. Chantefable du XIII ${ }^{\mathrm{e}}$ siècle. Éditée par Mario Roques. Deuxième édition, nouveau tirage revu et complété. Paris, Librairie Honoré Champion, éditeur, 1969. (CFMA 41).

Omer Jodogne : Audigier et la chansonde geste, avec une édition nouvelle du poème. Bruxelles, La Renaissance du Livre, 1960. (Le Moyen Age, Lxvi).

Aye d'Avignon. Chanson de geste anonyme. Edition critique par S.J. Borg. Genève, Librairie Droz, 1967. (TLF 134).

Aymeri de Narbonne. Chanson de geste. Publiée d'après les manuscrits de Londres et de Paris par Louis Demaison. 2 vol. Paris, Librairie de Firmin Didot et $C^{\text {ie }}$, 1887. Réimpression New York, Johnson Reprint Corporation, 1968. (SATF).

Le Siege de Barbastre. Chanson de geste du xiI ${ }^{\mathrm{e}}$ siècle. Éditée par J.L. Perrier. Paris, Librairie ancienne Honoré Champion, éditeur, 1926. (CFMA 54).

Le Chevalier au barisel. Conte pieux du xıII siècle. Édité d'après les manuscrits connus par Félix Lecoy. Paris, Librairie Honoré Champion, éditeur, 1967. (CFMA 82).

Le Bâtard de Bouillon. Chanson de geste. Une édition par Robert Francis Cook. Genève, Librairie Droz, 1972. (TLF)

Bérinus. Roman en prose du xIv ${ }^{\mathrm{e}}$ siècle. Publié par Robert Bossuat. 2 vol. Paris, 1931-1933. (SATF).

Béroul. Voir : Tristan.

Adenet le Roi : Berte as grans piés. Edition critique par Albert Henry. Genève, Librairie Droz, S.A., 1982. (TLF 305).

Blancandin et l'Orgueilleuse d'Amour. Roman d'aventure du XIII $^{\mathrm{e}}$ siècle. Nouvelle édition critique, d'après plusieurs manuscrits en vers par Franklin P. Sweetser. Genève/Paris, Librairie Droz/Librairie Minard, 1964. (TLF 112).

Bodel, Jehan. Voir : Saisnes.

Bretel, Jacques. Voir : Chauvency.

Brun de la Montaigne. Roman d'aventure. Publié pour la première fois, d'après le manuscrit unique de Paris, 
par Paul Meyer. Paris, Librairie de Firmin Didot et $C^{\text {ie, }}$ 1875. Réimpression New York, Johnson Reprint Corporation, 1966. (SATF).

Brut La partie Arthurienne du Roman de Brut. (Extrait du manuscrit B.N. fr. 794). Édition avec introduction, glossaire, notes et bibliographie par I.D.O. Arnold et M.M. Pelan. Paris, Librairie C. Klincksieck, 1962. (BFR, série B, Textes et documents, 1).

Bueve Der festländische Bueve de Hantone. Nach allen Handschriften mit Einleitung, An-merkungen und Glossar zum ersten Male herausgegeben von Albert Stimming. Fassung I. Dresden, 1911. (GRL 25). - - - Fassung II. Dresden, 1912/1918. (GRL 30/41). - - - Fassung III. Dresden, 1914/1920. (GRL 34/42).

Buevon Albert Henry: Les Euvres d'Adenet le Roi. Tome II : Buevon de Conmarchis. Bruges, « De Tempel », 1953. (Rijksuniversiteit te Gent, Werken uitgegeven door de Faculteit van de Wijsbegeerte en Letteren, $115^{\mathrm{e}}$ aflevering).

Cent Les Cent Nouvelles nouvelles. Edition critique par Franklin P. Sweetser. Genève, Librairie Droz, 1966. (TLF 127).

Charrete Les Romans de Chrétien de Troyes. Édités d'après la copie de Guiot. III : Le Chevalier de la Charrete. Publié par Mario Roques. Paris, Librairie Honoré Champion, éditeur, 1970. (CFMA 86).

Charroi Le Charroi de Nîmes. Chanson de geste du xire siècle. Éditée par J.-L. Perrier. Paris, Librairie Honoré Champion, éditeur, 1972. (CFMA 66).

Chauvency Jaques Bretel : Le Tournoi de Chauvency. Edition complète par Maurice Delbouille. Liège/Paris, H. VaillantCarmainel/Librairie E. Droz, 1932. (Bibliothèque de la Faculté de Philosophie et Lettres de l'Université de Liège, fascicule 49).

Chevalerie d'O. La Chevalerie d'Ogier de Danemarche. Canzone di gesta edita per cura di Mario Eusebi. Milano/Varese, Istituto editoriale cisalpino, 1963. (Testi e documenti di letteratura moderna vi).

- C Chrétien de Troyes. Voir : Charrete. Cligés. Erec. Guillaume d'A. Perceval. Yvain.

Claris Li Romans de Claris et Laris. Herausgegeben von Dr. Johann Alton. Tübingen 1884. (Bibliothek des Litterarischen Vereins CLXIX).

Cleomadés Albert Henry: Les Euvres d'Adenet le Roi. Tome V: Cleomadés. Bruxelles, Éditions de l'Université de Bruxelles, 1971. (Université Libre de Bruxelles, Tra- 
vaux de la Faculté de Philosophie et Lettres, tome XLVI).

Cligés Les Romans de Chrétien de Troyes. Édités d'après la copie de Guiot. II : Cligés. Publié par Alexandre Micha. Paris, Librairie ancienne Honoré Champion, éditeur, 1957. (CFMA 84).

Cont. P. Gerbert de Montreuil : La Continuation de Perceval. Éditée par Mary Williams (tomes I et II) et Marguerite Oswald (tome III) 3 vol. Paris, Librairie Honoré Champion, éditeur, 1922/1925/1975. (CFMA 28/50/101).

Couci Le Roman du Castelain de Couci et de la Dame de Fayel par Jakemes. Édition établie à l'aide des notes de John E. Matzke par Maurice Delbouille. Paris, 1936. (SATF).

Couronnement Le Couronnement de Louis. Chanson de geste du $\mathrm{xII}^{\mathrm{e}}$ siècle. Éditée par Ernest Langlois. Deuxième édition revue. Paris, Librairie Honoré Champion, éditeur, 1966. (CFMA 22).

Cristal Cristal et Clarie. Altfranzösischer Abenteuerroman des xiII. Jahrhunderts. Nach † Friedrich Apfelstedt's Abschrift der einzigen Arsenal-Handschrift (3516) und † Hugo von Feilitzen's Entlehnungsnachweisen mit Einleitung, Anmerkungen und Glossar zum ersten Male herausgegeben von Dr. Hermann Breuer. Dresden, 1915. (GRL 36).

Deduis Gace de la Buigne : Le Roman des Deduis. Edition critique d'après tous les manuscrits par Åke Blomquist. Karlshamn, 1951. (Studia Romanica Holmiensia III).

Diable Robert le diable. Roman d'aventures. Publié par E. Löseth. Paris, Librairie de Firmin Didot et Cie, 1903. (SATF).

Dole Jean Renart : Le Roman de la Rose ou de Guillaume de Dole. Édité par Félix Lecoy. Paris, Librarie Honoré Champion, éditeur, 1963. (CFMA 91).

Doon Doon de Maience. Chanson de geste. Publiée pour la première fois $d$ 'après les manuscrits de Montpellier et de Paris par A. Pey. Paris, F. Vieweg, Libraire-Editeur, 1859. (APF).

Durmart Durmart le Galois. Roman Arthurien du Treizième Siècle. Publié par Joseph Gildea, O.S.A. 2 vol. Villanova, The Villanova Press, 1965/1966.

Eneas Eneas. Roman du XII ${ }^{e}$ siècle. Édité par J.-J. Salverda de Grave. 2 vol. Paris, Librairie ancienne Édouard Champion, éditeur, 1925/1929. (CFMA 44/62).

Enfances G. Les Enfances Guillaume. Chanson de geste du xiII siècle. Publiée par Patrice Henry. Paris, 1935. (SATF).

Enfances O. $\quad$ Albert Henry : Les Euvres d'Adenet le Roi. Tome III : 
Les Enfances Ogier. Bruges, « De Tempel », 1956. (Rijksuniversiteit te Gent, Werken uitgegeven door de Faculteit van de Letteren en Wijsbegeerte, $121^{\mathrm{e}}$ aflevering).

Eracle Gautier d'Arras : Eracle. Publié par Guy Raynaud de Lage. Paris, Librairie Honoré Champion, éditeur, 1976. (CFMA 102).

Erec Chrétien de Troyes: Erec et Enide. Édition critique d'après le manuscrit B.N. fr. 1376, traduction, présentation et notes de Jean-Marie Fritz. Paris, Le Livre de Poche, 1992. (Lettres Gothiques).

Escoufle Jean Renart: L'Escoufle. Roman d'aventure. Nouvelle édition d'après le manujscrit 6565 de la Bibliothèque de l'Arsenal par Franklin Sweetser. Genève, Librairie Droz, 1974. (TLF).

Espees Li Chevaliers as deus espees. Altfranzösischer Abenteuerroman. Zum ersten Mal herausgegeben von Wendelin Foerster. Halle a/ Saale, Max Niemeyer, 1877. Réédition Amsterdam, Editions Rodopi, 1966.

Espinette Jean Froissart : L'Espinette Amoureuse. Seconde édition entièrement revue par Anthime Fourrier. Paris, Éditions Klincksieck, 1972. (BFR, série B, éditions critiques de textes, 2).

Fauvel Le Roman de Fauvel par Gervais du Bus. Publié d'après tous les manuscrits connus par Arthur Långfors. Paris, Librairie de Firmin Didot et C ${ }^{\text {ie }}$, 1914-1919. Réimpression New York, Johnson Reprint Corporation, 1968 (SATF).

Fierabras Fierabras. Chanson de geste. Publiée pour la première fois d'après les manuscrits de Paris, de Rome et de Londres par A. Krœber et G. Servois. Paris, F. Vieweg, Libraire-Editeur, 1860. (APF).

Floire Le Conte de Floire et Blancheflor. Édité par Jean-Luc Leclanche. Paris, Librairie Honoré Champion, éditeur, 1980. (CFMA 105).

Floovant Floovant. Chanson de geste. Publié pour la première fois d'après le manuscrit unique de Montpellier par F. Guessard et H. Michelant. Paris, F. Vieweg, libraireéditeur, 1859. Réimpression Nendeln, Liechtenstein, Kraus Reprint Ltd., 1966. (APF).

Florence Florence de Rome. Chanson d'aventure du premier quart du xIII ${ }^{\mathrm{e}}$ siècle. Publiée par A. Wallensköld. Tome second. Paris, Librairie de Firmin Didot et Cie, 1907. (SATF).

Floriant Floriant et Florete. Edited by Harry F. Williams. Ann Arbor, University of Michigan Press, 1947. (University 
of Michigan Publications, Language and Literature, XXIII).

Florimont Aimon de Varennes : Florimopnt. Ein altfranzösischer Abenteuerroman. Zum erstenmal mit Einleitung, Anmerkungen, Namenverzeichnis und Glossar unter Benutzung der von Alfred Risop gesammelten handschriftlichen Materialien herausgegeben von Alfons Hilka. Göttingen, 1932. (GRL 48).

Fouke $\quad$ Fouke Fitz Warin. Roman du xIv siècle. Édité par Louis Brandin. Paris, Librairie ancienne Honoré Champion, éditeur, 1930. (CFMA 63).

- $\quad$ Froissart, Jean. Voir : Espinette. Méliador.

- $\quad$ Gace de la Buigne. Voir : Deduis.

Galeran Jean Renart : Galeran de Bretagne. Roman du xıII ${ }^{\mathrm{e}}$ siècle. Édité par Lucien Foulet. Paris, Librairie ancienne Honoré Champion, éditeur, 1925. (CFMA 37).

Gaufrey Gaufrey. Chanson de geste. Publiée pour la première fois d'après le manuscrit unique de Montpellier par F. Guessard et P. Chabaille. Paris, F. Vieweg, LibraireEditeur, 1859. (APF).

Gautier d'Aupais Gautier d'Aupais. Poème courtois du XIII siècle. Édité par Edmond Farral. Paris. Librairie Honoré Champion, éditeur, 1970. (CFMA 20).

$-$ Gautier d'Arras. Voir : Ille. Eracle.

Gaydon Gaydon. Chanson de geste. Publiée pour la première fois d'après les trois manuscrits de Paris par F. Guessard et S. Luce. Paris, Librairie A. Franck, 1862. (APF).

- $\quad$ Gerbert de Montreuil. Voir : Cont. P. Violette.

- $\quad$ Gervais du Bus. Voir : Fauvel.

- $\quad$ Girart d'Amiens. Voir : Meliacin.

Gliglois Gliglois. A French Arthurian Romance of the Thirteenth Century. Edited with an Introduction by Charles H. Livingston. Cambridge, Harvard University Press, 1932. (Harvard Studies in Romance Languages, viII).

Godin La Chanson de Godin. Chanson de geste inédite. Publiée par Françoise Meunier. Louvain, 1958. (Université de Louvain, Recueil de travaux d'histoire et de philologie, $4^{\mathrm{e}}$ série, fascicule 14$)$.

Gormont Gormont et Isembart. Fragment de chanson de geste du XII ${ }^{\mathrm{e}}$ siècle. Édité par Alphonse Bayot. Paris, Librairie ancienne Honoré Champion, éditeur, 1914. (CFMA 14).

Graal Robert de Boron : Le Roman de l'Estoire dou Graal. Édité par William A. Nitze. Paris, Librairie Honoré Champion, éditeur, 1971. (CFMA 57).

Gui de B. Gui de Bourgogne. Chanson de geste. Publiée pour la première fois d'après les manuscrits de Tours et de 
Londres par F. Guessard et H. Michelant. Paris, F. Vieweg, libraire-éditeur, 1859. Réimpression Nendeln, Liechtenstein, Kraus Reprint Ltd., 1966. (APF).

Gui de N. Gui de Nanteuil. Chanson de geste. Edition critique par James R.M ${ }^{c}$ Cormack. (man. M). Genève, Librairie Droz, 1970. (TLF 161).

Gui de W. Gui de Warewic. Roman du xirI siècle. Édité par Alfred Ewert. 2 vol. Paris, Librairie ancienne Édouard Champion, éditeur, 1932/1933. (CFMA 74/75).

Guillaume La Chanson de Guillaume.Publiée par Duncan McMillan. 2 vol. Paris, Éditions A. \& J. Picard \& C $C^{\text {ie }}, 1949 / 1950$. (SATF).

Guillaume d'A. Chrétien de Troyes : Guillaume d'Angleterre. Roman du xII ${ }^{\mathrm{e}}$ siècle. Édité par Maurice Wilmotte. Paris, Librairie ancienne Honoré Champion, éditeur, 1927. (CFMA 55).

Helcanus Le Roman de Helcanus. Edition critique d'un texte du $\mathrm{xIII}^{\mathrm{e}}$ siècle par Henri Niedzielski. Genève/Paris, Librairie Droz/Librairie Minard, 1966. (TLF 121).

- $\quad$ Heldris de Cornuälle. Voir : Silence.

- $\quad$ Hue de Rotelande. Voir : Ipomedon. Protheselaus.

Hunbaut Hunbaut. Altfranzösischer Artusroman des xiII. Jahrhunderts. Nach Wendelin Foerster's Abschrift der einzigen Chantilly-Handschrift zum ersten Male kritisch bearbeitet von Jakob Stürzinger. Aus dessen Nachlass ergänzt herausgegeben von Dr. Hermann Breuer. Dresden, 1914 (GRL 35).

Huon

Huon de Bordeaux. Édité par Pierre Ruelle. Bruxelles/ Paris, Presses Universitaires, 1960. (Université Libre de Bruxelles, Travaux de la Faculté de Philosophie et Lettres, tome $\mathrm{xx}$ ).

- $\quad$ Huon le Roi. Voir : Vair P.

Ille Ille et Galeron. Par Gautier d'Arras. Publié par Frederick A.G. Cowper. Paris, Éditions A. \& J. Picard \& $C^{\text {ie, }}$ 1956. (SATF).

Inconnu Renaut de Beaujeu : Le Bel Inconnu. Roman d'aventures. Édité par G. Perrie Williams. Paris, Librairie ancienne Honoré Champion, éditeur, 1929. (CFMA 38).

Ipomedon Ipomedon. Poème de Hue de Rotelande (fin du XII ${ }^{\mathrm{e}}$ siècle). Édité avec introduction, notes et glossaire par A.J. Holden. Paris, Éditions Klincksieck, 1979. (BFR, série $\mathrm{B}$, éditions critiques de textes, 17).

- Jakemes. Voir : Couci.

- $\quad$ Jean de Bueil. Voir : Jouvencel.

Jehan Jehan et Blonde de Philippe de Rémi. Roman du xiII ${ }^{\mathrm{e}}$ siècle. Edité par Sylvie Lécuyer. Paris, Librairie Honoré Champion, éditeur, 1984. (CFMA 107). 


\begin{tabular}{|c|c|}
\hline- & Jehan. Voir : Rigomer. \\
\hline Jehan de $P$. & $\begin{array}{l}\text { Le Roman de Jehan de Paris. Publié d'après les manus- } \\
\text { crits par Edith Wickersheimer. Paris, Librairie ancien- } \\
\text { ne Édouard Champion, éditeur, 1923. (SATF). }\end{array}$ \\
\hline Jehan de S. & $\begin{array}{l}\text { Antoine de la Sale : Jehan de Saintré. Édité par Jean Mis- } \\
\text { rahi et Charles A. Knudson. Genève, Librairie Droz, } \\
\text { 1967. (TLF). }\end{array}$ \\
\hline Joufroi & $\begin{array}{l}\text { Joufroi de Poitiers. Roman d'aventures du xiII siècle. } \\
\text { Edition critique par Percival B. Fay † et John L. Grigsby. } \\
\text { Genève, Librairie Droz, 1972. (TLF 183). }\end{array}$ \\
\hline Jourdain & $\begin{array}{l}\text { Jourdain de Blaye (Jourdains de Blavies). Chanson de } \\
\text { geste. Nouvelle édition entièrement revue et corrigée. } \\
\text { Publiée par Peter F. Dembowski. Paris, Librairie Ho- } \\
\text { noré Champion, éditeur, 1991. (CFMA 112). }\end{array}$ \\
\hline Jouvencel & $\begin{array}{l}\text { Le Jouvencel par Jean de Bueil. Texte établi et annoté } \\
\text { par Léon Lecestre. } 2 \text { vol. Paris, Librairie Renouard, } \\
\text { 1887-1889. (Société de l’Histoire de France). }\end{array}$ \\
\hline Lais & $\begin{array}{l}\text { Marie de France : Lais. Edited by Alfred Ewert. Oxford, } \\
\text { Basil Blackwell, } 1952 .\end{array}$ \\
\hline Lancelot & $\begin{array}{l}\text { Lancelot. Roman en prose du xiII }{ }^{e} \text { siècle. Editon critique } \\
\text { par Alexandre Micha. } 9 \text { vol. Genève, Librairie Droz, } \\
\text { 1978-1983. (TLF 247/249/262/278/283/286/288/307/31 } \\
\text { 5). }\end{array}$ \\
\hline Lanson & $\begin{array}{l}\text { Jehan de Lanson. Chanson de geste of the 13th Century. } \\
\text { Edited after the manuscripts of Paris and Bern with } \\
\text { introduction, notes, table of proper names, and glos- } \\
\text { sary by John Vernon Myers. Chapel Hill, The Univer- } \\
\text { sity of North Carolina Press, 1965. (University of North } \\
\text { Carolina, Studies in the Romance Languages and Lite- } \\
\text { ratures, 53). }\end{array}$ \\
\hline Laurin & $\begin{array}{l}\text { Le Roman de Laurin, fils de Marques le Sénéchal. Edited } \\
\text { by Lewis Thorpe. Cambridge, W. Heffer \& Sons, Ltd. } \\
\text { 1960. (University of Nottingham Research Publica- } \\
\text { tion, 2). }\end{array}$ \\
\hline Lion & $\begin{array}{l}\text { Lion de Bourges. Poème épique du xIve siècle. Édition } \\
\text { critique par William W. Kibler, Jean-Louis G. Picheri et } \\
\text { Thelma S. Fenster. } 2 \text { vol. Genève, Librairie Droz S.A., } \\
\text { 1980. (TLF 285). }\end{array}$ \\
\hline Loquifer & $\begin{array}{l}\text { La Bataille Loquifer. By Monica Barnett. Oxford, Basil } \\
\text { Blackwell, } 1975 \text { (Medium Ævum Monographs, New } \\
\text { Series vi). }\end{array}$ \\
\hline Lycorne & $\begin{array}{l}\text { Le Romans de la Dame a la Lycorne et du Biau Chevalier } \\
\text { au Lyon. Ein Abenteuerroman aus dem ersten Drittel } \\
\text { des xIv. Jahrhunderts. Zum ersten Male herausgege- } \\
\text { ben von Friedrich Gennrich. Dresden, 1908. (GRL } \\
\text { 18). }\end{array}$ \\
\hline
\end{tabular}


Macaire

Mahomet

Meliacin

Méliador

Meraugis

Merlin

Mez

Mort Artu

Mort Aymeri

MR

Narbonnais
Macaire. Chanson de geste. Publiée d'après le manuscrit unique de Venise, avec un essai de restitution en regard par F. Guessard. Paris, Librairie A. Franck, 1866. (APF).

Le Roman de Mahomet de Alexandre du Pont. (1258). Éditon critique ... par Yvan G. Lepage. Paris, Éditions Klincksieck, 1977. (BFR, séire B, éditions critiques de textes, 16).

Maillart, Jehan. Voir : Anjou.

Marie de France. Voir : Lais.

Girart d'Amiens : Meliacin ou le Cheval de Fust. Edition critique par Antoinette Saly. Aix-en-Provence, Publications du CUERMA, 1990. (Senefiance 27).

Méliador par Jean Froissart. Roman comprenant les poésies lyriques de Wenceslas de Bohême, duc de Luxembourg et de Brabant, publié pour la première fois par Auguste Longnon. 3 vol. Paris, Librairie de Firmin Didot et $C^{\text {ie }}, 1895^{-1899 . ~ R e ́ i m p r e s s i o n ~ N e w ~ Y o r k, ~}$ Johnson Reprint Corporation, 1965. (SATF).

Meraugis von Portlesguez. Altfranzösischer Abenteuerroman von Raoul von Houdenc. Zum ersten Mal nach allen Handschriften herausgegeben von Dr. Mathias Friedwagner. Raoul von Houdenc Sämtliche Werke I. Halle, Max Niemeyer, 1897.

Merlin. Roman en prose du xIII siècle. Publié ... par Gaston Paris et Jacob Ulrich. 2 vol. Paris, Librairie de Firmin Didot et $\mathrm{C}^{\mathrm{ie}}$, 1886. (SATF).

Pauline Taylor : Gerbert de Mez. Chanson de geste du $\mathrm{XII}^{\mathrm{e}}$ siècle. Namur-Louvain-Lille, 1952. (Bibliothèque de la Faculté de Philosophie et Lettres de Namur, fascicule 11).

La Mort le Roi Artu. Roman du xIII siècle. Édité par Jean Frappier. Troisième édition. Genève/Paris, Librairie Droz/M.J. Minard, 1964. (TLF).

La Mort Aymeri de Narbonne. Chanson de geste. Publiée d'après les manuscrits de Londres et de Paris par J. Couraye du Parc. Paris, Librairie de Firmin Didot et Cie, 1884. Réimpression New York, Johnson Reprint Corporation, 1966. (SATF).

Recueil général et complet des Fabliaux des XIII et xIv ${ }^{\mathrm{e}}$ siècles, imprimés ou inédits. Publiés avec notes et variantes d'après les manuscrits par Anatole de Montaiglon et Gaston Raynaud. 6 vol. Paris, Librairie des Bibliophiles, 1872/1877/1878/1880/1883/ 1890.

Les Narbonnais. Chanson de geste publiée pour la première fois par Hermann Suchier. 2 vol. Paris, Librairie 
de Firmin Didot et C ${ }^{\text {ie }}$, 1898. Réimpression NewYork, Johnson Reprint Corporation, 1965. (SATF).

Ogier Ogier le Dannoys. Roman en prose du $\mathrm{xv}^{\mathrm{e}}$ siècle. Publié pour Det danske Sprog- og Litteraturselskab par Knud Togeby. Copenhague, Munksgaard, 1967.

Ombre Jean Renart : Le Lai de l'Ombre. Par Félix Lecoy. Paris, Librairie Honoré Champion, éditeur, 1979. (CFMA 104).

Orange La Prise d'Orange. Chanson de geste de la fin du XII siècle. Éditée d'après la rédaction $\mathrm{AB}$ avec introduction, notes et glossaire par Claude Régnier. Deuxième édition. Paris, Librairie C. Klincksieck, 1969. (BFR, série B, éditions critiques de textes, 5).

Orson Orson de Beauvais. Chanson de geste du xiI ${ }^{\mathrm{e}}$ siècle. $\mathrm{Pu}$ bliée d'après le manuscrit unique de Cheltenham par Gaston Paris. Paris, Librairie de Firmin Didot et Cie, 1899. Réimpression New York, Johnson Reprint Corporation, 1968. (SATF).

Otinel Otinel. Chanson de geste. Publiée pour la première fois d'après les manuscrits de Rome et de Middlehill par F. Guessard et H. Michelant. Paris, F. Vieweg, libraireéditeur, 1859. Réimpression Nendeln, Liechtenstein, Kraus Reprint Ltd., 1966. (APF).

Papegau Le Chevalier du Papegau. Nach der einzigen Pariser Handschrift zum ersten Mal herausgegeben von Ferdinand Heuckenkamp. Halle a. S., Verlag von Max Niemeyer, 1896.

Parise Parise la Duchesse (Chanson de geste du xims ${ }^{\mathrm{e}}$ siècle). Edition et commentaires par May Plouzeau. Aix-enProvence, Publications du CUERMA, 1986. (Senefiance 17).

Partonopeu Partonopeu de Blois. A French Romance of the Twelfth Century. Edited by Joseph Gildea, O.S.A. Volume I. Villanova, Villanova University Press, 1967.

Partonopeu-C Partonopeu de Blois. Edited by Joseph Gildea, O.S.A. Volume II, Part 1 : The Continuation. Villanova, Villanova University Press, 1968.

Perceval Chrétien de Troyes : Le Roman de Perceval ou Le Conte du Graal. Publié ... par William Roach. Seconde édition revue et augmentée. Genève/Paris, Librairie Droz/Librairie Minard, 1959. (TLF 58).

- $\quad$ Philippe de Rémi. Voir : Jehan.

Poitiers Le Roman du Comte de Poitiers. Poème français du xIII ${ }^{\mathrm{e}}$ siècle. Publié avec introduction, notes et glossaire par Bertil Malmberg. Lund/Copenhague, C.W.K. Gleerup/ Ejnar Munksgaard, 1940. (Études Romanes de Lund I). 
Pontieu

Protheselaus

Protheselaus

Proverbes

Queste

Raguidel

Raoul

$-$

Renart

Renaut

Rigomer

-

Roche

Roland

Rome
La Fille du Comte de Pontieu. Nouvelle du XIII ${ }^{\mathrm{e}}$ siècle. Éditée par Clovis Brunel. [Rédaction primitive]. Paris, Librairie ancienne Honoré Champion, éditeur, 1926. (CFMA 52).

Hue de Rotelande: Protheselaus. Ein altfranzösischer Abenteuerroman. Zum erstenmal mit Einleitung, Anmerkungen, Namenverzeichnis, Glossar und Index kritisch herausgegeben von Franz Kluckow. Göttingen, 1924. (GRL 45).

Proverbes français antérieurs $\mathrm{au} \mathrm{xv}^{\mathrm{e}}$ siècle. Édités par Joseph Morawski. Paris, Librairie ancienne Édouard Champion, éditeur, 1925. (CFMA 47).

La Queste del Saint Graal. Roman du XIII ${ }^{\mathrm{e}}$ siècle. Édité parr Albert Pauphilet. Paris, Librairie Honoré Champion, éditeur, 1967. (CFMA 33).

La Vengeance Raguidel. Altfranzösischer Abenteuerroman herausgegeben von Mathias Friedwagner. Raoul von Houdenc, Sämtliche Werke ir. Halle a.S., Max Niemayer, 1909.

Raoul de Cambrai. Chanson de geste. Publiée par P. Meyer \& A. Longnon. Paris, Librairie de Firmin Didot et $\mathrm{C}^{\mathrm{ie}}, 1882$. (SATF).

Raoul de Houdenc. Voir : Meraugis. Raguidel.

Le Roman de Renart. Branches I à xIx. Éditées d'après le manuscrit de Cangé par Mario Roques. 6 vol. Paris, Librairie Honoré Champion, éditeur, 1957-1972. (CFMA 78/79/81/85/88/90).

Renart, Jean. Voir : Dole. Escoufle. Galeran. Ombre.

Renaut de Montauban. Edition critique du manuscrit Douce par Jacques Thomas. Genève, Librairie Droz S.A., 1989. (TLF 371).

Renaut de Beaujeu. Voir : Inconnu.

Les Mervelles de Rigomer von Jehan. Altfranzösischer Artusroman des xiII. Jahrhunderts nach der einzigen Aumale-Handschrift in Chantilly zum ersten Mal herausgegeben von Wendelin Foerster. Band I : Text. Dresden, 1908. (GRL 19).

Robert de Boron. Voir : Graal.

Doon de la Roche. Chanson de geste. Publiée par Paul Meyer et Gédéon Huet. Paris, Librairie ancienne Honoré Champion, éditeur, 1921. Réimpression New York, Johnson Reprint Corporation, 1968. (SATF).

La Chanson de Roland. Publiée d'après le manuscrit d'Oxford et traduite par Joseph Bédier. Paris, L'Édition d'Art H. Piazza, 1947.

Florence de Rome. Chanson d'aventure du premier quart du xIII ${ }^{\mathrm{e}}$ siècle. Publiée par A. Wallensköld. Tome 
premier : Appendice : Roman de Florence de Rome. Paris, Librarie de Firmin Didot et $C^{\mathrm{ie}}$, 1909. (SATF).

Rou

Le Roman de Rou de Wace. Publié par A.J. Holden. 3 vol.

Paris, Éditions A. \& J. Picard et C $C^{\text {ie }}, 1970 / 1971 / 1973$. (SATF).

Roussillon Girart de Roussillon. Chanson de geste. Publiée par W. Mary Hackett. 3 vol. Paris, Éditions A. \& J. Picard \& Cie, 1953. (SATF).

Saisnes Jehan Bodel : La Chanson de Saisnes. Edition critique par Annette Brasseur. 2 vol. (rédaction L/T). Genève, Librairie Droz S.A., 1989. (TLF 369).

Silence Le Roman de Silence. A thirteenth-century Arthurian verse-romance by Heldris de Cornuälle. Edited by Lewis Thorpe. Cambridge, W. Heffer \& Sons Ltd., 1972.

Simon Simon de Pouille. Chanson de geste éditée d'après le manuscrit $n^{\circ} 4780$ de la Bibliothèque Nationale par Jeanne Baroin. Genève, Librairie Droz, 1968. (TLF).

Thèbes Le Roman de Thèbes. Publié par Guy Raynaud de Lage. 2 vol. Paris, Librairie Honoré Champion, éditeur, 1966/1967. (CFMA 94/96).

$\mathrm{TP}$

Le Roman de Tristan en prose. 8 vol. Tome I édité par Philippe Ménard. Tomes II-viII publiés sous la direction de Philippe Ménard. Tome ir édité par Marie-Luce Chênerie et Thierry Delcourt. Tome iı édité par Gilles Roussineau. Tome Iv édité par Jean-Claude Faucon. Tome v édité par Denis Lalande, avec la collaboration de Thierry Delcourt. Tome vi édité par Emmanuèle Baumgartner et Michèle Szkilnik. Tome vis édité par Danielle Quernel et Monique Santucci. Tome viIr édité par Bernard Guidot et Jean Subrenat. Genève, Librairie Droz S.A., 1987/1990/1991/1991/1992/1993/1994/19 95. (TLF 353/387/398/408/416/437/450/462).

Tristan Béroul : Le Roman de Tristan. Poème du XII ${ }^{\mathrm{e}}$ siècle. Édité par Ernest Muret. Quatrième édition revue par L.M. Defourques. Paris, Librairie Honoré Champion, éditeur, 1972. (CFMA 12).

Tristan de N. K.V. Sinclair : Tristan de Nanteuil, chanson de geste inédite. Assen, Van Gorcum \& Comp. n. V., 1971.

Troie Le Roman de Troie en prose. Édité par L. Constans et E. Faral. Tome I. Paris, Librairie ancienne Honoré Champion, éditeur, 1922. (CFMA 29).

Turpin André de Mandach : Naissance et développement de la chanson de geste en Europe, Ir. Chronique de Turpin. Texte anglo-normand inédit de Willem de Briane (Arundel 220). Genève, Librairie Droz, 1963. (Publications romanes et françaises LxxVII). 
Vair P.

Vergi

Violette

Yder

$$
-
$$

Wistasse

Yvain
Huon le Roi : Le Vair Palefroi. Avec deux versions de La Male Honte par Huon de Cambrai et par Guillaume. Fabliaux du XIII siècle. Édités par Arthur Långfors. Troisième édition revue. Paris, Librairie ancienne Honoré Champion, éditeur, 1927. (CFMA 8).

La Chastelaine de Vergi. Poème du XIII ${ }^{\mathrm{e}}$ siècle. Édité par Gaston Raynaud, $4^{\text {e }}$ édition revue par Lucien Foulet. Paris, Librairie Honoré Champion, éditeur, 1979. (CFMA 1).

Le Roman de Violette ou de Gerart de Nevers par Gerbert de Montreuil. Publié par Douglas Labaree Buffum. Paris, Librairie ancienne Honoré Champion, 1928. (SATF).

Wace. Voir : Brut. Rou.

Willem de Briane. Voir : Turpin.

Wistasse le Moine. Altfranzösischer Abenteuerroman des xIII. Jahrhunderts. Nach der einzigen Pariser Handschrift von neuem heraugegeben von Wendelin Foerster und Johann Trost. Halle a. S., Verlag von Max Niemeyer, 1891. (RB 4).

Der altfranzösische Yderroman. Nach der einzigen bekannten (...) Handschrift herausgegeben von Heinrich Gelzer. Dresden, 1913. (GRL 31).

Kristian von Troyes : Yvain (Der Löwenritter). Herausgegeben von Wendelin Foerster. Vierte verbesserte und vermehrte Auflage. Halle a. S., Verlag von Max Niemeyer, 1912. (RB 5).

\subsection{Ouvrages consultés}

Álvares Cristina, Gauvain, les femmes et le cheval. Dans : Senefiance 32.

Aguiriano Begoña, Le cheval et le départ en aventure dans Les Romans de Chrétien de Troyes. Dans : Senefiance 32.

Bangert Friedrich, Die Tiere im altfranzösischen Epos von Friedrich Bangert. Marburg, 1885. (Ausgaben und Abhandlungen aus dem Gebiete der romanischen Philologie xxxiv).

Bichon Jean, L'animal dans la littérature française au XII et au XIII siècles. 2 vol. Service de reproduction de thèses. Université de Lille III, 1976.

Borchert Friedrich, Die Jagd in der altfranzösischen Literatur (mit Ausschluss der Artus- und Abenteuer-Romane). Inaugural-Dissertation ... vorgeleget von Friedrich Borchert. Göttingen, 1909.

Closson Monique, La femme et le cheval du XII ${ }^{e}$ au Xvi siècles. Dans : Senefiance 32 . 
Colliot Régine, Les chevaux symboliques d'Amadas et Ydoine. Dans : Senefiance 32.

Combarieu du Grès Micheline, Le cheval dans la Chevalerie Ogier de Danemarche. Dans : Senefiance 32.

Couillet Reynald, Le motif du don du cheval dans le Lancelot en prose. Dans : Senefiance 32.

Delort Jean, La vie au Moyen Age. $3^{\mathrm{e}}$ édition. Paris, Éditions du Seuil, 1982. (Points-Histoire 62).

Dictionnaire des lettres françaises. Publié sous la direction du Cardinal Georges Grente. Le Moyen âge. Paris, Librairie Arthème Fayard, 1964.

Dinzelbacher Peter, Mittelalter. Dans : Mensch und Tier in der Geschichte Europas.

Dubost Francis, De quelques chevaux extraordinaires dans le médiéval: esquisse d'une configuration imaginaire. Dans : Senefiance 32.

Duby Georges, Féodalité. Éditions Gallimard, 1996. (Le Grand Livre du Mois, 1999).

Gouiran Gérard, Entre Sarrasins et Chrétiens, ou le cheval décapité. Dans : Senefiance 32.

Grandsaignes d'Hauterive R., Dictionnaire d'ancien français. Paris, Librairie Larousse, 1947.

Greimas A.- J., Dictionnaire de l'ancien français. Paris, Librairie Larousse, 1980.

Histoire de la France rurale. Sous la direction de Georges Duby et Armand Wallon. 1. La Formation des campagnes françaises des origines à 1340. Paris, Éditions du Seuil, 1975. (Points-Histoire 166).

Histoire de la vie privée. Sous la direction de Philippe Ariès et Georges Duby. 2. De l'Europe féodale à la Renaissance. Paris, Éditions du Seuil, 1999. (Points-Histoire 261).

Leguay Jean-Pierre, La rue au Moyen Age. Rennes, Éditions Ouest-France, 1984. (Le Grand Livre du Mois, 2002).

Manne Perrine, Images du cheval à la ferme. Dans : Senefiance 32.

Mensch und Tier in der Geschichte Europas. Heraugegeben von Peter Dinzelbacher. Stuttgart, Alfred Kröner Verlag, 2000. (Kröners Taschenausgabe, Bd. 342).

Paris Gaston, Une fable à retrouver. Dans : Romania, 1902.

Picoche Jacqueline, Dictionnaire étymologique du français. Paris, Dictionnaire Le Robert, 1992. (Le Grand Livre du Mois, 1999).

Romania. Publié par Paul Meyer et Gaston Paris. 31 année - 1902. Paris, Librairie Émile Bouillon, éditeur, 1902.

Schmidt Fr., Das Reiten und Fahren in der altfranzösischen Literatur. Ein Beitrag zur Kulturgeschichte des alten Frankreichs. Inaugural Dissertation ... vorgeleget von Fr. Schmidt. Göttingen, 1914.

Senefiance 32, Le cheval dans le monde médiéval. Aix-en-Provence, CUERMA, 1992. 
Tobler Adolf, Vermischte Beiträge. Der Vermischten Beiträge zur französischen Grammatik fünfte Reihe. Leipzig, Verlag von S. Hirzel, 1912. Réimpression Amsterdam, Rodopi N.V., 1971.

Verdon Jean, Les loisirs au Moyen Age (nouvelle édition mise à jour). Paris, Éditions Tallandier, 1996. (Le Grand Livre du Mois, 2000). 